\title{
Results of Time-Domain Electromagnetic Soundings in Miami-Dade and Southern Broward Counties, Florida
}

Open-File Report 2011-1299 


\section{Results of Time-Domain Electromagnetic Soundings in Miami-Dade and Southern Broward Counties, Florida}

By David V. Fitterman and Scott T. Prinos

Open-File Report 2011-1299 


\section{U.S. Department of the Interior \\ KEN SALAZAR, Secretary}

\section{U.S. Geological Survey \\ Marcia K. McNutt, Director}

U.S. Geological Survey, Reston, Virginia: 2011

For product and ordering information:

World Wide Web: http://www.usgs.gov/pubprod

Telephone: 1-888-ASK-USGS

For more information on the USGS-the Federal source for science about the Earth,

its natural and living resources, natural hazards, and the environment:

World Wide Web: http://www.usgs.gov

Telephone: 1-888-ASK-USGS

Suggested citation:

Fitterman, D.V., and Prinos, S.T., 2011, Results of time-domain electromagnetic soundings in Miami-Dade and southern Broward Counties, Florida: U.S. Geological Survey Open-File Report 2011-1299, 289 p.

Any use of trade, product, or firm names is for descriptive purposes only and does not imply endorsement by the U.S. Government.

Although this report is in the public domain, permission must be secured from the individual copyright owners to reproduce any copyrighted material contained within this report. 


\section{Contents}

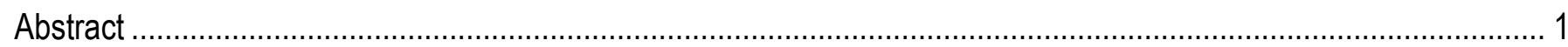

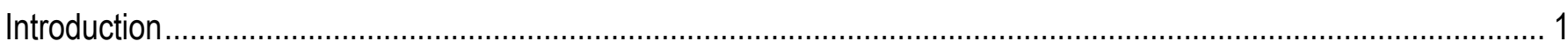

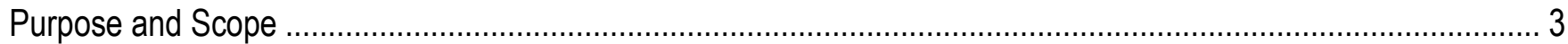

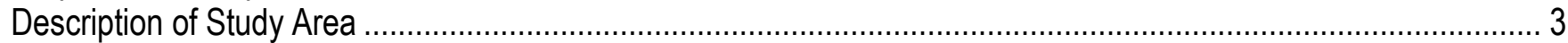

Time-Domain Electromagnetic Sounding Locations...................................................................................

Hydrogeologic Setting and Borehole Geophysical Information ....................................................................

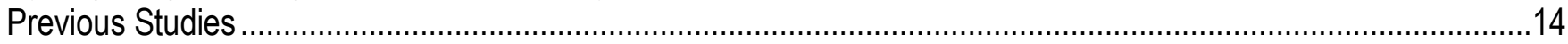

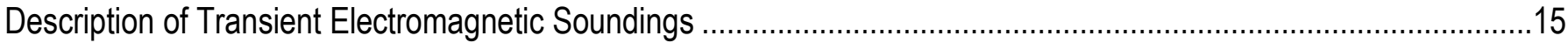

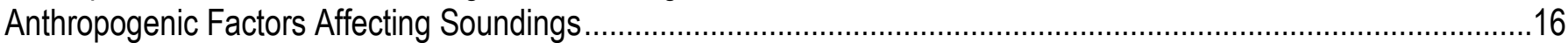

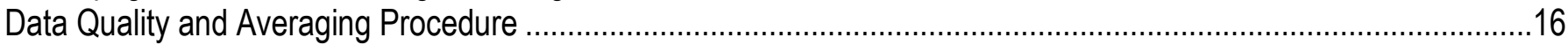

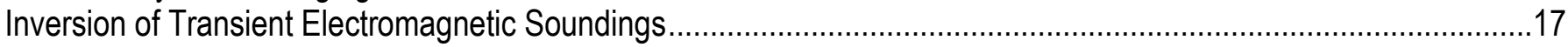

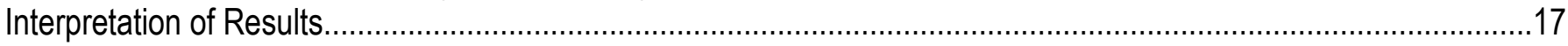

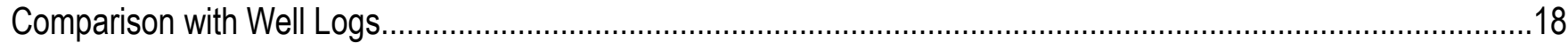

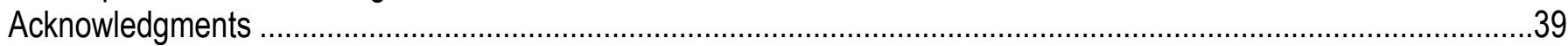

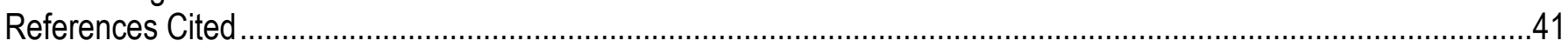

Appendix 1 Description of TEM Data Processing .........................................................................................4

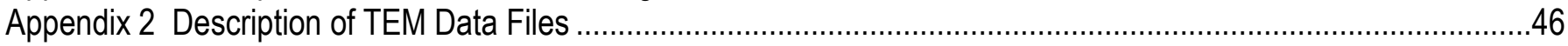

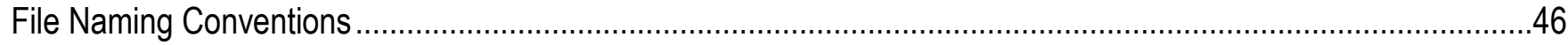

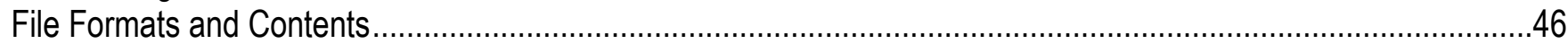

Appendix 3 Voltage Units and Apparent Resistivity ..................................................................................52

Appendix 4 TEM Sounding Report Files: 2008

Appendix 5 TEM Sounding Descriptions and Plots: 2008

Appendix 6 TEM Sounding Report Files: 2009

Appendix 7 TEM Sounding Descriptions and Plots: 2009 ……...................................................................218

\section{Figures}

1. Location of the study area, time-domain electromagnetic soundings, and the helicopter electromagnetic

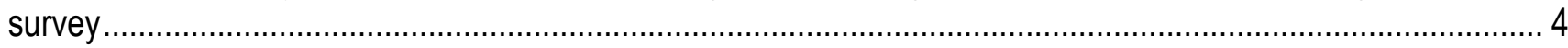

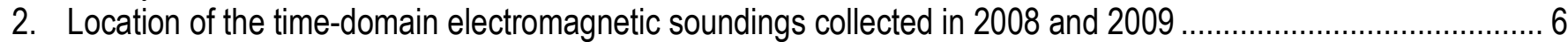

3. Location of the time-domain electromagnetic soundings collected in 2009 near the 2001 helicopter

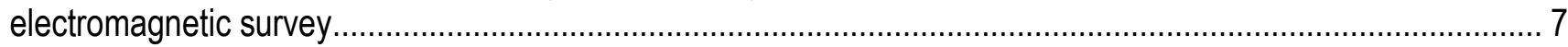

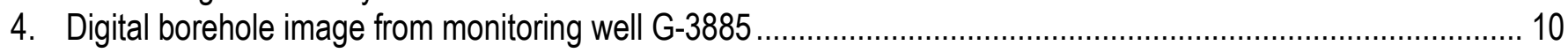

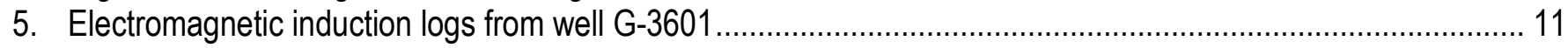

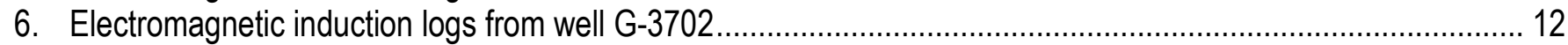

7. Relationship between the resistivity of water samples collected in monitoring wells in Miami-Dade County and the bulk aquifer resistivity measured by induction logs........................................................... 14

8. Relationship between resistivity and chloride content of water samples collected from monitoring wells

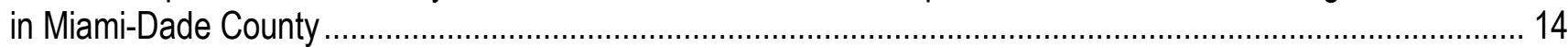

9. Comparison of TEM soundings MIA216, MIA217, and MIA235 with an induction log from well FKS $7 \ldots \ldots \ldots \ldots . . . .20$

10. Comparison of TEM soundings MIA222 and MIA232 with an induction log from well FKS 4 .......................... 21

11. Comparison of TEM soundings MIA212, MIA233, and MIA236 with an induction log from well FKS 8 ............. 23

12. Comparison of TEM soundings MIA225 and MIA226 with an induction log from well CEMEX-MW-03-FS ......... 25

13. Comparison of TEM soundings MIA213 and MIA214 with an induction log from well G-3946 …...................... 26

14. Comparison of TEM soundings MIA203 and MIA204 with an induction log from well Sec34-MW-03-FS ........... 28 
15. Comparison of TEM soundings MIA201 and MIA202 with an induction log from well Sec34-MW-02-FS

16. Comparison of TEM soundings MIA101, MIA102, MIA103, and MIA104 with induction logs from wells G-3699, G-3856, and G-3855.

17. Comparison of TEM soundings MIA105 and MIA106 with induction logs from wells G-3609 and G-3608 .

18. Comparison of TEM sounding MIA116 and MIA117 with an induction log from well G-3605 ......................... 34

19. Comparison of TEM soundings MIA118, MIA119, MIA120, and MIA121 with an induction log from well G-3604

20. Comparison of TEM sounding MIA114 and MIA115 with an induction log from well G-2903 ........................ 37

21. Comparison of TEM sounding MIA241 with an induction log from well G-2965 ....................................... 38

22. Comparison of TEM soundings MIA238 and MIA239 with an induction log from well G-2478 ........................ 40

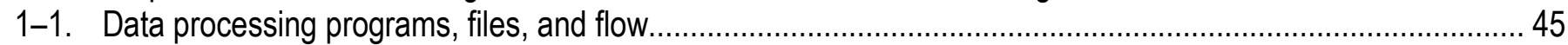

2-1. Diagram showing contents of the digital PROTEM-D receiver header fields............................................ 47

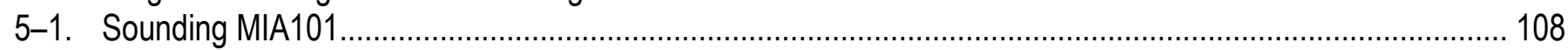

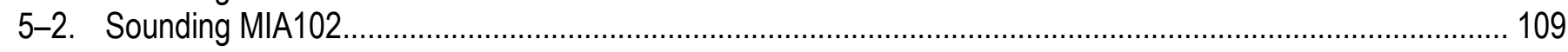

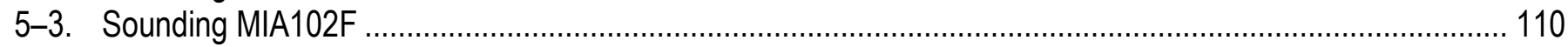

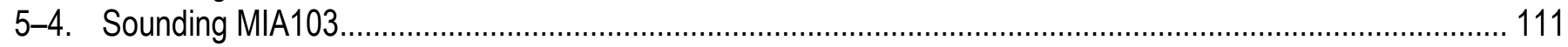

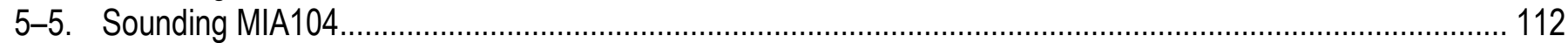

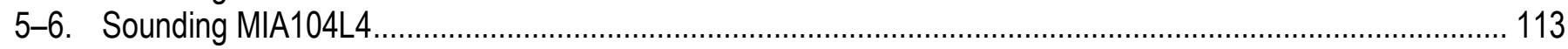

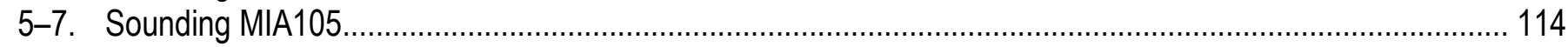

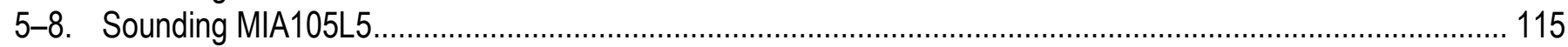

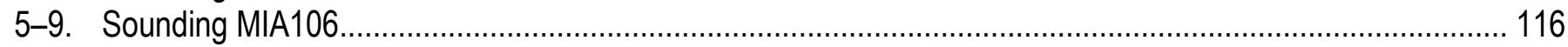

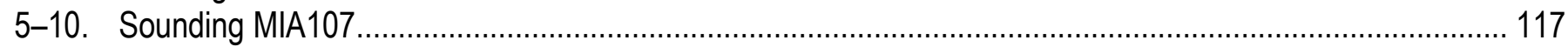

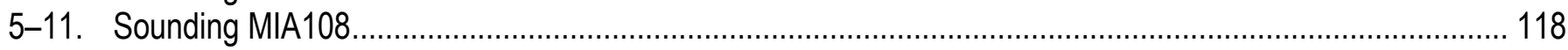

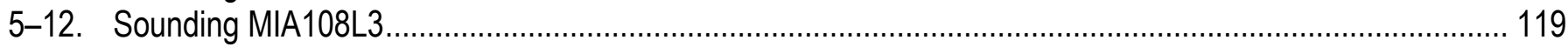

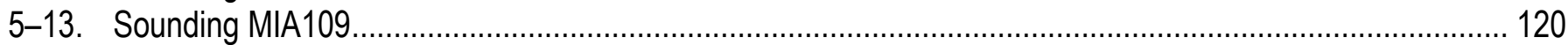

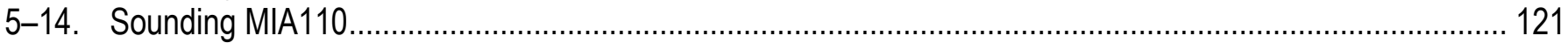

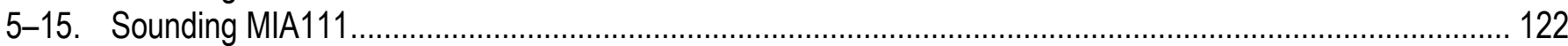

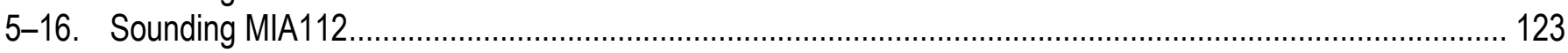

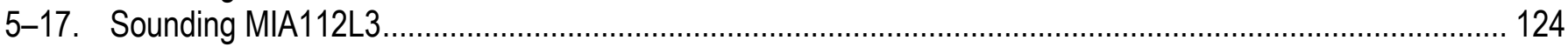

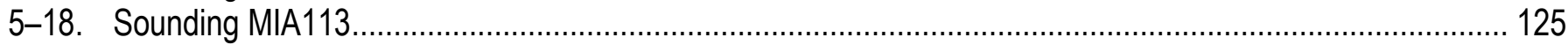

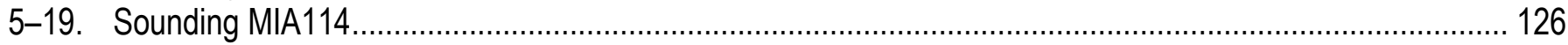

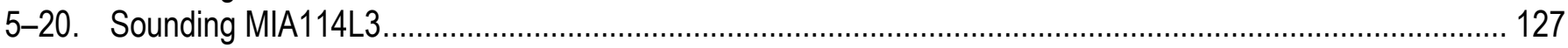

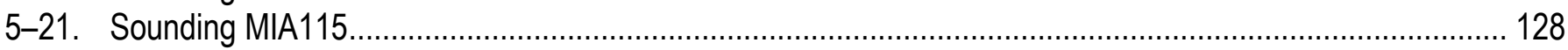

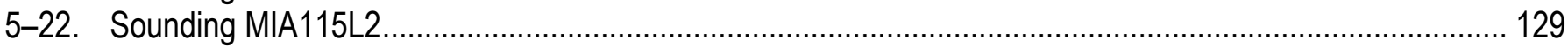

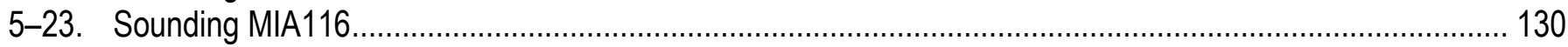

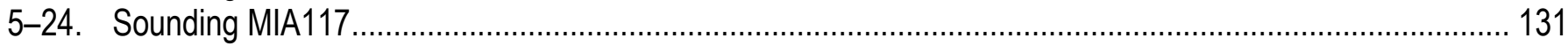

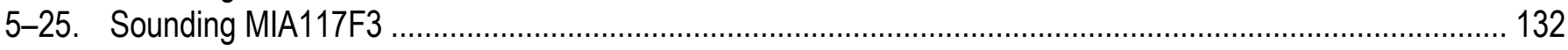

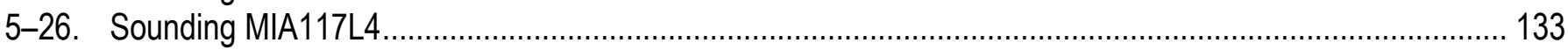

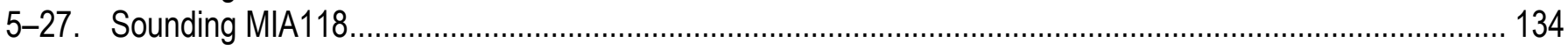

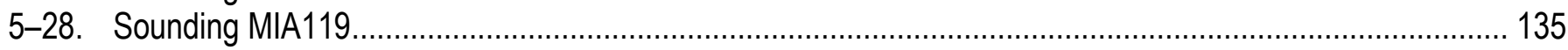

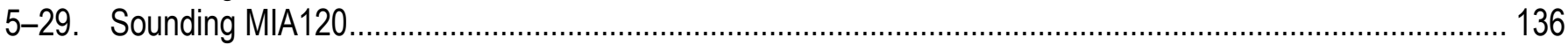

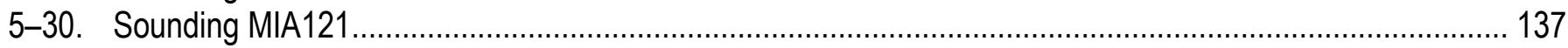

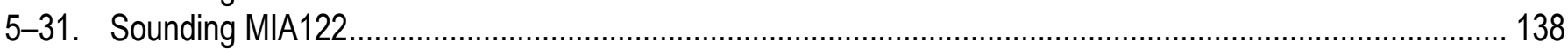

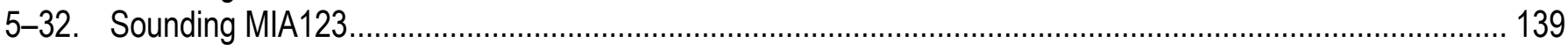

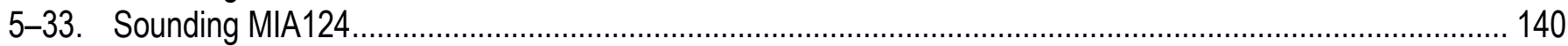

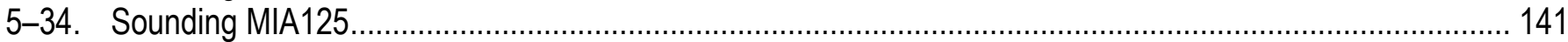

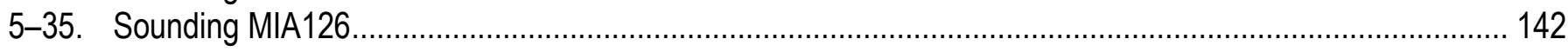




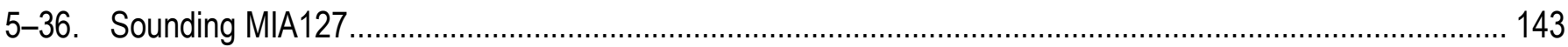

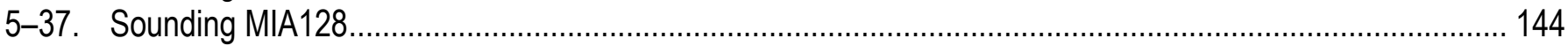

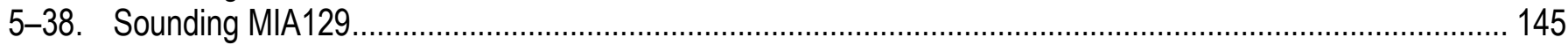

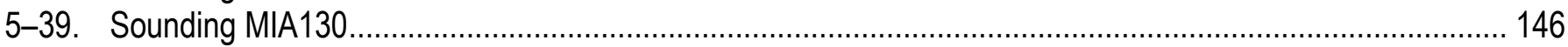

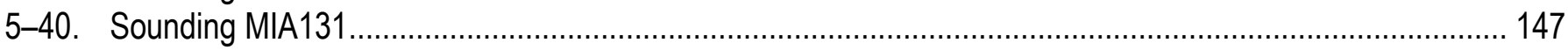

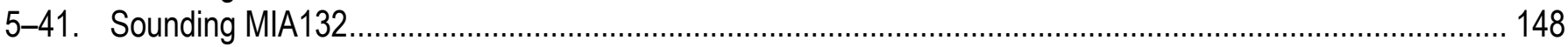

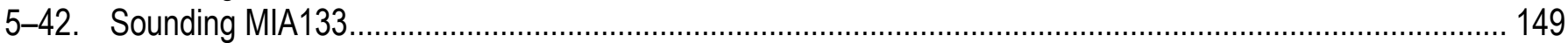

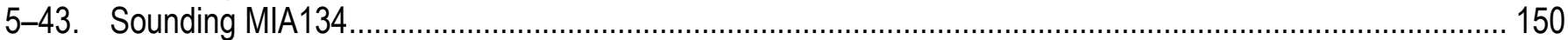

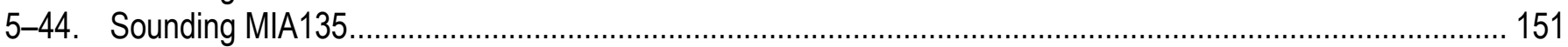

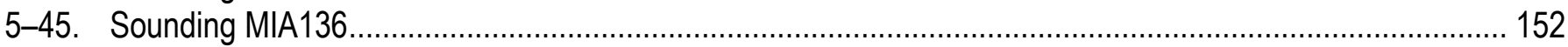

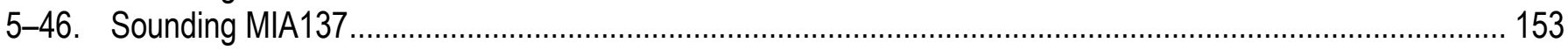

7-1. Sounding MIA201

7-2. Sounding MIA202

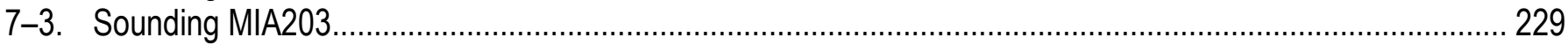

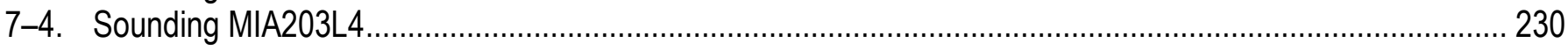

7-5. Sounding MIA204

7-6. Sounding MIA204L4

7-7. Sounding MIA205

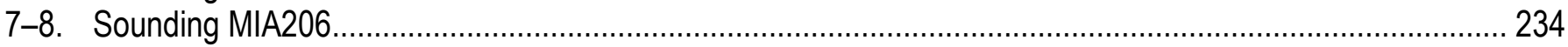

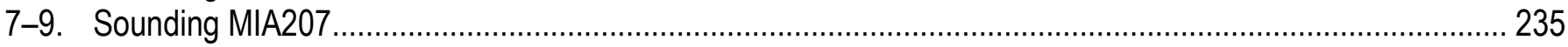

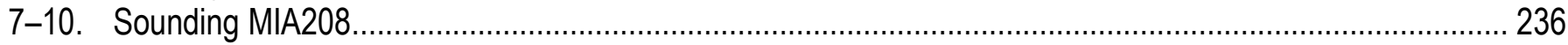

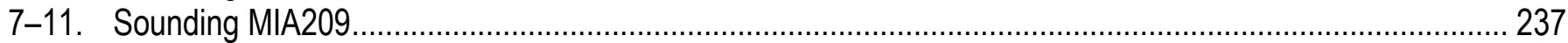

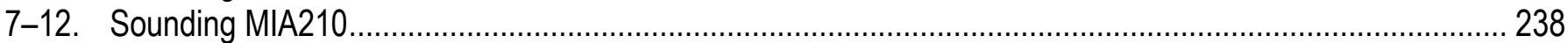

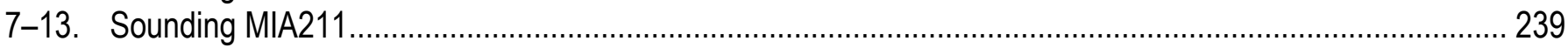

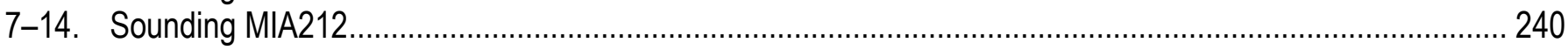

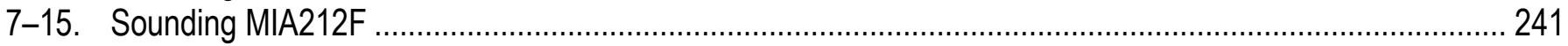

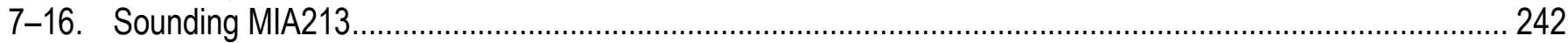

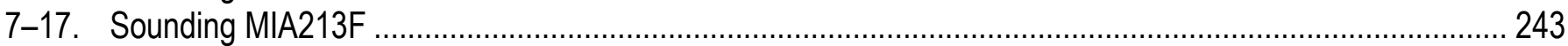

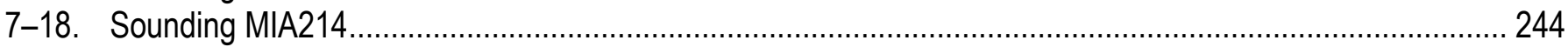

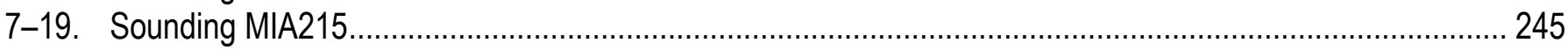

7-20. Sounding MIA216

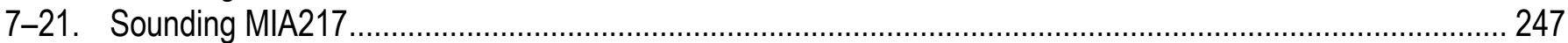

7-22. Sounding MIA218

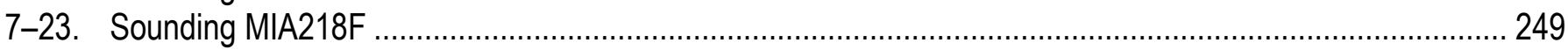

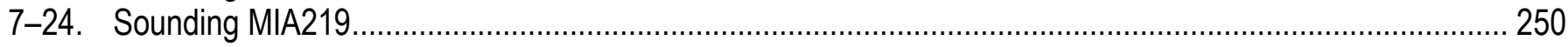

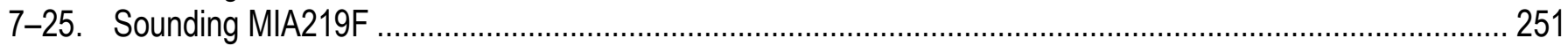

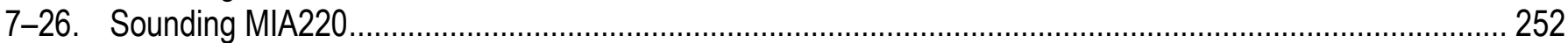

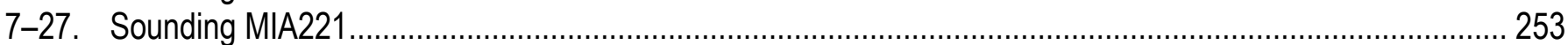

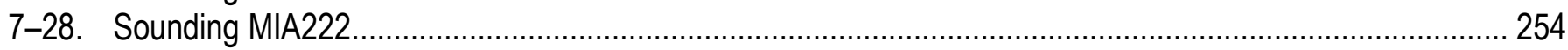

7-29. Sounding MIA222L4

7-30. Sounding MIA223

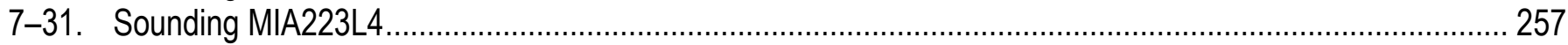

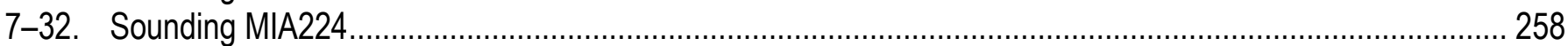

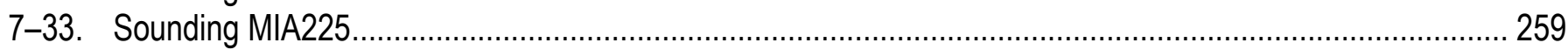

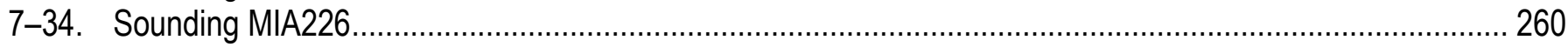

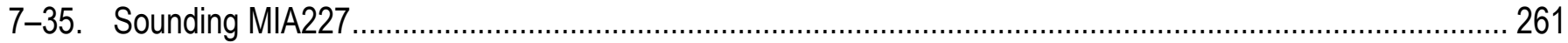

7-36. Sounding MIA228

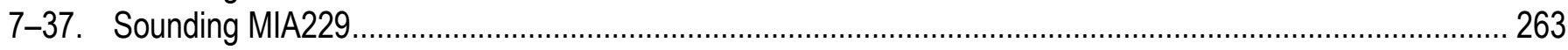


7-38. Sounding MIA230

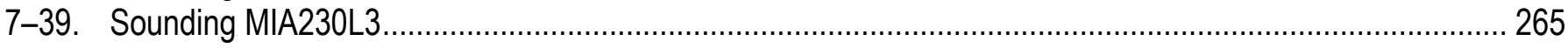

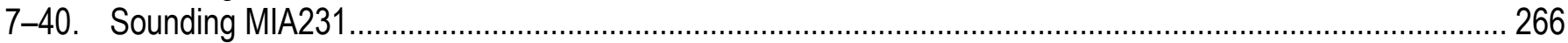

7-41. Sounding MIA232

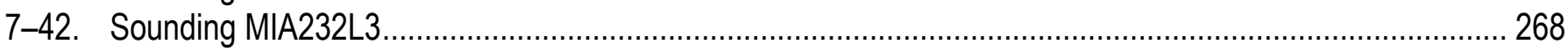

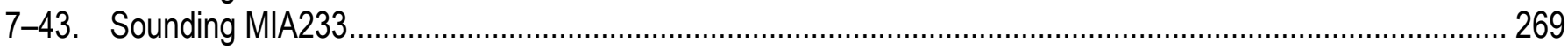

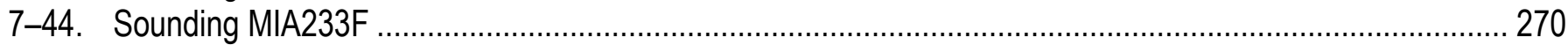

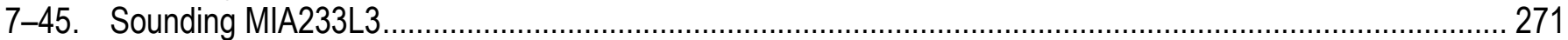

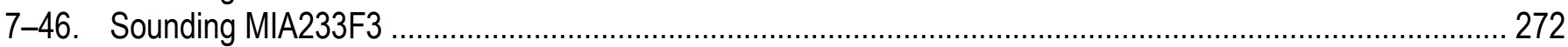

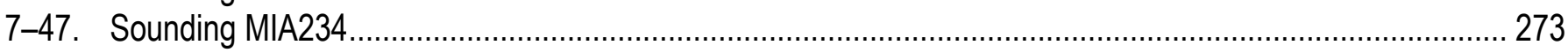

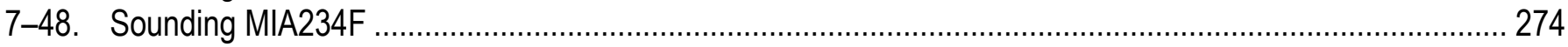

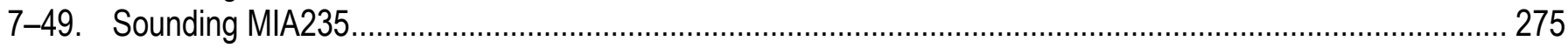

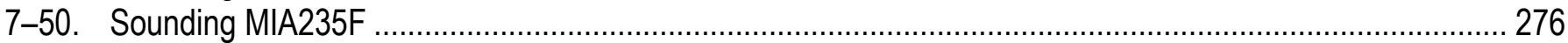

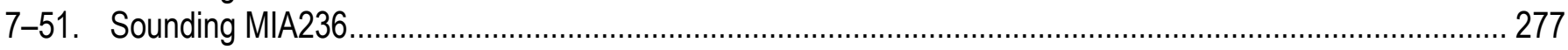

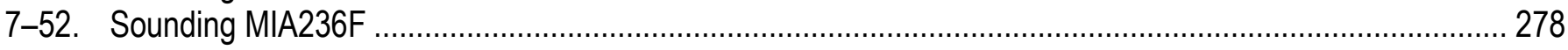

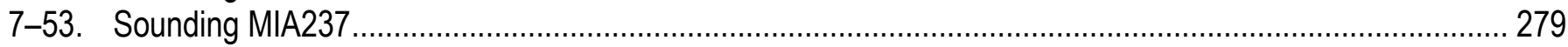

7-54. Sounding MIA238

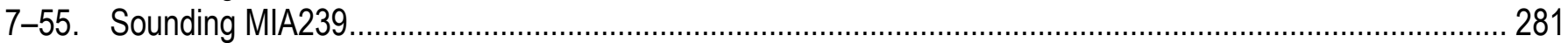

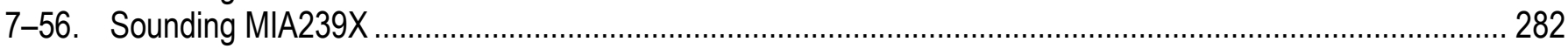

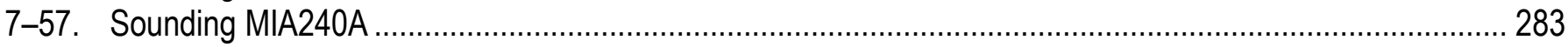

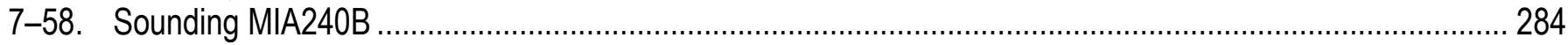

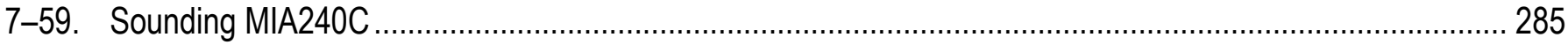

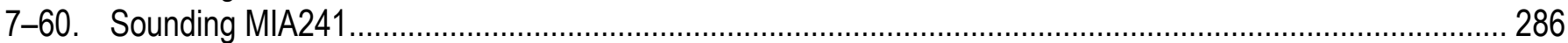

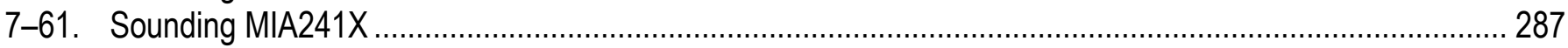

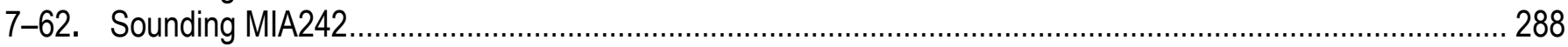

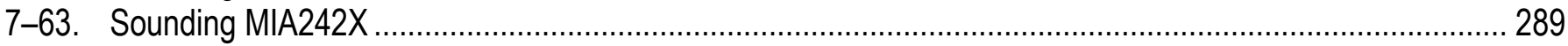

\section{Tables}

1. Location of TEM soundings made in 2008 and selected nearby wells ......................................................... 8

2. Location of TEM soundings made in 2009 and selected nearby wells .......................................................... 9

2-1. Description of the digital PROTEM-D receiver header record (HDR) header fields........................................ 47

2-2. Description of the digital PROTEM-D receiver data record (OPR) header fields ........................................... 48

2-3. Description of the digital PROTEM-D receiver header record (HDR) data fields (gates) ................................... 48

2-4. Description of the digital PROTEM-D data record (OPR) data fields (gates) .................................................. 48

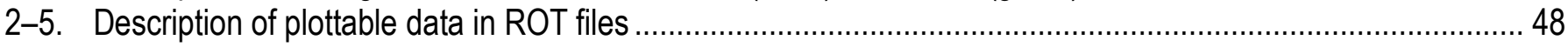




\section{Conversion Factors, Datum, Abbreviations, and Acronyms}

\section{SI to Inch/Pound}

\begin{tabular}{lll}
\hline \multicolumn{1}{c}{ Multiply } & \multicolumn{1}{c}{ By } & \multicolumn{1}{c}{ To obtain } \\
\hline & Length & \\
\hline centimeter $(\mathrm{cm})$ & 0.3937 & inch (in.) \\
millimeter (mm) & 0.03937 & inch (in.) \\
meter $(\mathrm{m})$ & 3.281 & foot (ft) \\
kilometer $(\mathrm{km})$ & 0.6214 & mile (mi) \\
kilometer $(\mathrm{km})$ & 0.5400 & mile, nautical (nmi) \\
meter $(\mathrm{m})$ & 1.094 & yard (yd) \\
\hline
\end{tabular}

Inch/Pound to SI

\begin{tabular}{ccc}
\hline Multiply & By & To obtain \\
\hline million gallons per day $(\mathrm{Mgal} / \mathrm{d})$ & 0.04381 & cubic meters per second \\
\hline
\end{tabular}

Electrical Conductivity and Electrical Resistivity

\begin{tabular}{lcl}
\hline Multiply & By & To obtain \\
\hline & Electrical conductivity & \\
\hline siemens per meter $(\mathrm{S} / \mathrm{m})$ & 1,000 & millisiemens per meter $(\mathrm{mS} / \mathrm{m})$ \\
\hline siemens per meter $(\mathrm{S} / \mathrm{m})$ & 10,000 & microsiemens per centimeter $(\mu \mathrm{S} / \mathrm{cm})$ \\
\hline \multicolumn{3}{c}{ Electrical resistivity } \\
\hline ohm-meters $($ ohm-m) & 0.001 & kiloohm-meters $($ kohm-m) \\
\hline
\end{tabular}

Electrical conductivity $\sigma$ in siemens per meter [S/m] can be converted to electrical resistivity $\rho$ in ohm-meters [ohm-m] as follows: $\rho=1 / \sigma$.

Electrical conductivity $\sigma$ in millisiemens per meter $[\mathrm{mS} / \mathrm{m}]$ can be converted to electrical resistivity $\rho$ in ohm-meters [ohm-m] as follows: $\rho=1,000 / \sigma$.

Electrical conductivity $\sigma$ in microsiemens per centimeter $[\mu \mathrm{S} / \mathrm{cm}]$ can be converted to electrical resistivity $\rho$ in ohmmeters [ohm-m] as follows: $\rho=10,000 / \sigma$.

Electrical resistivity $\rho$ in ohm-meters [ohm-m] can be converted to electrical conductivity $\sigma$ in siemens per meter $[\mathrm{S} / \mathrm{m}]$ as follows: $\sigma=1 / \rho$.

Electrical resistivity $\rho$ in ohm-meters [ohm-m] can be converted to electrical conductivity $\sigma$ in millisiemens per meter $[\mathrm{mS} / \mathrm{m}]$ as follows: $\sigma=1,000 / \rho$.

Electrical resistivity $\rho$ in ohm-meters [ohm-m] can be converted to electrical conductivity $\sigma$ in microsiemens per centimeter $[\mu \mathrm{S} / \mathrm{cm}]$ as follows: $\sigma=10,000 / \rho$.

Specific conductance SC refers to fluid electrical conductivity corrected to a temperature of $25^{\circ} \mathrm{C}$. The units of specific conductance are usually in microsiemens per centimeter $[\mu \mathrm{S} / \mathrm{cm}]$. The above conversions apply.

Horizontal coordinate information is referenced to the North American Datum of 1983 (NAD 83). 


\section{ABBREVIATIONS USED IN THIS REPORT:}

$\begin{array}{ll}\mathrm{A} & \text { ampere } \\ \mathrm{ft} & \text { feet } \\ \mathrm{H} / \mathrm{m} & \text { henry per meter } \\ \mathrm{Hz} & \text { hertz } \\ \mathrm{km} & \text { kilometer } \\ \mathrm{m} & \text { meter } \\ \mathrm{mi} & \text { mile } \\ \mathrm{mg} / \mathrm{L} & \text { milligram per liter } \\ \mathrm{m}^{2} & \text { meter squared } \\ \mathrm{msec} & \text { millisecond } \\ \mathrm{mV} & \text { millivolt } \\ \mathrm{nV} / \mathrm{m}^{2} & \text { nanovolt per meter squared } \\ \mathrm{ohm}-\mathrm{m} & \text { ohm-meter } \\ \mathrm{ppt} & \text { parts per thousand } \\ \mu \mathrm{S} & \text { microsecond } \\ \mu \mathrm{S} / \mathrm{cm} & \text { microsiemen per centimeter } \\ \mathrm{Wb} / \mathrm{m}^{2} & \text { weber per meter squared }\end{array}$

\section{ACRONYMS USED IN THIS REPORT:}

GPS global positioning system

HEM helicopter electromagnetic

$\mathrm{HI}$ high

NAD North American datum

SC specific conductance

TEM time-domain electromagnetic

UH ultra high

USGS U.S. Geological Survey 


\title{
Results of Time-Domain Electromagnetic Soundings in Miami-Dade and Southern Broward Counties, Florida
}

\author{
By David V. Fitterman ${ }^{1}$ and Scott T. Prinos ${ }^{2}$
}

\begin{abstract}
Time-domain electromagnetic (TEM) soundings were made in Miami-Dade and southern Broward Counties to aid in mapping the landward extent of saltwater in the Biscayne aquifer. A total of 79 soundings were collected in settings ranging from urban to undeveloped land, with some of the former posing problems of land access and interference from anthropogenic features. TEM soundings combined with monitoring-well data were used to determine if the saltwater front had moved since the last time it was mapped, to provide additional spatial coverage where existing monitoring wells were insufficient, and to help interpret a previously collected helicopter electromagnetic (HEM) survey flown in the southernmost portion of the study area.

TEM soundings were interpreted as layered resistivity-depth models. Using information from well logs and water-quality data, the resistivity of the freshwater saturated Biscayne aquifer is expected to be above $30 \mathrm{ohm}$-meters, and the saltwater-saturated aquifer will have resistivities of less than $10 \mathrm{ohm}$-meters allowing determination of water quality from the TEM interpretations. TEM models from 29 soundings were compared to electromagnetic induction logs collected in nearby monitoring wells. In general, the agreement of these results was very good, giving confidence in the use of the TEM data for mapping saltwater encroachment.
\end{abstract}

\section{Introduction}

The Biscayne aquifer in southeastern Florida is the principal source of drinking water for the residents of Miami-Dade (formerly Dade) and Broward Counties (Miller, 1990). In addition, the Biscayne aquifer is the sole source of potable water for all residents living in the Florida Keys, Monroe County delivered by an aqueduct that extends from a well field west of Florida City south to Key West. As the population of southeastern Florida has increased so have water withdrawals from the Biscayne aquifer (Prinos and others, 2002). As of 1990, $573 \mathrm{Mgal} / \mathrm{d}$ or 34 percent of all the groundwater withdrawn in Florida came from the Biscayne aquifer (Marella, 1993). Because of its proximity to the coast this aquifer is vulnerable to contamination by seawater.

Prior to widespread development of southeastern Florida, water levels in the Biscayne aquifer were generally sufficient to minimize the landward extent of saltwater ${ }^{3}$ in the Biscayne

\footnotetext{
${ }^{1}$ U.S. Geological Survey, emeritus

${ }^{2}$ U.S. Geological Survey

${ }^{3}$ The term seawater is used herein to refer only to water whose source is the ocean and has the same chemical composition as ocean water. Seawater that has mixed with freshwater in the canals or in the aquifer is considered to be saltwater because of dilution and chemical interaction with the rock matrix.
} 
aquifer. Wells 15 to $18 \mathrm{~m}$ (50 to $60 \mathrm{ft}$ ) deep near the mouth of the Miami River yielded freshwater, and freshwater springs flowed from the low ridges of Miami Limestone near Biscayne Bay (Parker and others, 1955). The installation of drainage canals in 1907 in the New River Basin at Ft.

Lauderdale and the Miami Canal in 1909 (Parker and others, 1955) began to allow saltwater (1) to encroach along the base of the aquifer, and (2) to flow long distances up these unregulated canals and into the aquifers, especially during periods of low rainfall and low canal flow.

Canal dredging operations were greatly expanded during the periods 1910 to 1918 and 1925 to 1927 as numerous major canals were added to aid in drainage of the area to create land for agricultural development (Parker, 1945). The addition of canals led to saltwater contamination in 1925 of the first well field of the city of Miami. This well field was $2.5 \mathrm{~km}$ (1.6 mi) inland from Biscayne Bay near the Miami River. By 1939, reduced aquifer water levels resulting from groundwater withdrawals combined with the effects of the drainage canals caused saltwater contamination of some of the wells at Miami's newer well field $10.5 \mathrm{~km}(6.5 \mathrm{mi})$ inland from Biscayne Bay near the Miami River.

To prevent the movement of saltwater up the canals during high tides and to reduce the drainage of the Biscayne aquifer, which was allowing saltwater to encroach at its base, water managers began to install salinity-control structures (Sonenshein, 1997). By 1946, steel sheet-pile dams had been installed in most of the canals in the Miami area (Leach and Grantham, 1966). Salinity-control structures helped to reduce saltwater encroachment in some areas.

In 1949, the Central and Southern Florida Flood Control Project and District was established. The District developed a comprehensive plan (1) to remove flood water rapidly, (2) to provide surface-water storage for regional water supply, (3) to prevent canal over drainage and saltwater encroachment, (4) to supply water to Everglades National Park, (5) to help preserve fish and wildlife, and (6) to serve recreational and navigational needs (Renken and others, 2005). During the 1950s and 1960s, an extensive drainage canal and water-control structure network was created under the auspices of this plan. Additional salinity-control structures were added throughout southeastern Florida. The original steel sheet-pile dams were eventually replaced by permanent automated control structures.

Despite these improvements, the drought of 1970 to 1971 reduced water levels in the aquifer resulting in increased saltwater encroachment (Sonenshein, 1997). As a result, additional changes to the water-management system were made. By 1995, Sonenshein (1997) found that the location of the saltwater interface in much of Miami-Dade County had remained approximately the same between 1984 and 1995.

Although there are now salinity-control structures on most canals in southeastern Florida that have proven useful in retarding or reversing saltwater encroachment, portions of some canals still remain uncontrolled for up to $8 \mathrm{~km}(5 \mathrm{mi})$ inland to allow navigation and dockage. As of December 2009 there was still no salinity-control structure on the Card Sound Road Canal south of Florida City.

To continually monitor saltwater encroachment in Miami-Dade and Broward Counties the U.S. Geological Survey (USGS) has operated a groundwater sampling network since 1939. Sampled chloride content is declining along the Miami River west of the salinity-control structure, which indicates that saltwater encroachment in this area has been halted and previous contamination is dissipating. In some areas chloride concentrations continue to increase indicating that encroachment continues. For this reason the extent of saltwater is periodically reevaluated.

Time-domain electromagnetic (TEM) sounding is a geophysical method that can provide subsurface electrical conductivity information relating to the occurrence and depth of conductive 
fluids, such as saltwater, in an aquifer. The advantage of this method is that it is not necessary to install monitoring wells in order to detect the presence of the saltwater interface and estimate its depth. These measurements were made in open fields large enough to accommodate a 40 by $40 \mathrm{~m}$ square transmitter loop. Smaller loops can be used, however, they usually do not provide the depth of exploration required. Combining TEM soundings with data from monitoring wells can greatly improve our understanding of the extent of saltwater encroachment.

Another geophysical method for mapping saltwater encroachment is helicopter electromagnetic (HEM) surveying. Where urban development is minimal this technique can provide very detailed information about the resistivity structure of the ground throughout the survey area. In 2001, an HEM survey was flown in southeastern Miami-Dade County south of Palm Drive and from U.S. Highway 1 eastward to Levee L31E. At the time the survey was flown there were insufficient resources to interpret the results and compare them to ground-based TEM soundings and well data. The HEM survey is the subject of a companion report (Fitterman and others $^{4}$, written commun., 2011).

The USGS in cooperation with Miami-Dade County recently undertook an investigation to (1) remap the landward extent of saltwater in the Biscayne aquifer, (2) augment the information provided by monitoring wells with time-domain electromagnetic (TEM) soundings, (3) collect additional TEM soundings in the vicinity of the 2001 HEM survey, which will aid in the interpretation of this survey and the evaluation of changes that have occurred since 2001, and (4) determine where additional monitoring would add to ongoing assessments of saltwater encroachment.

\section{Purpose and Scope}

This report (1) documents the collection, processing, and modeling procedures used to obtain TEM soundings in coastal Miami-Dade County and southern Broward County in southeastern Florida, (2) summarizes the results, and (3) describes the implications of these results for estimating the landward extent of saltwater in the Biscayne aquifer. The measurements and findings described in this report will be used in subsequent phases of an ongoing investigation.

\section{Description of Study Area}

The study area is primarily the of portion Miami-Dade County, Fla. within approximately $15 \mathrm{~km}$ of the coast in the southern part of the county and within approximately $5 \mathrm{~km}$ of the coast in the northern part (fig. 1). The study area also includes a portion of southern Broward County as far north as Hallandale Beach. The extension of this work into Broward County was done so that future saltwater-encroachment studies to the north can be seamlessly tied to the work done in Miami-Dade County, thereby avoiding artifacts associated with the boundary between the counties.

\footnotetext{
${ }^{4}$ This report is in preparation as a USGS Open-File Report. The tentative authorship and title are: Fitterman, D.V., Deszcz-Pan, M., and Prinos, S.T.: Helicopter electromagnetic survey of the Model Land area, southeastern Miami-Dade County, Florida.
} 


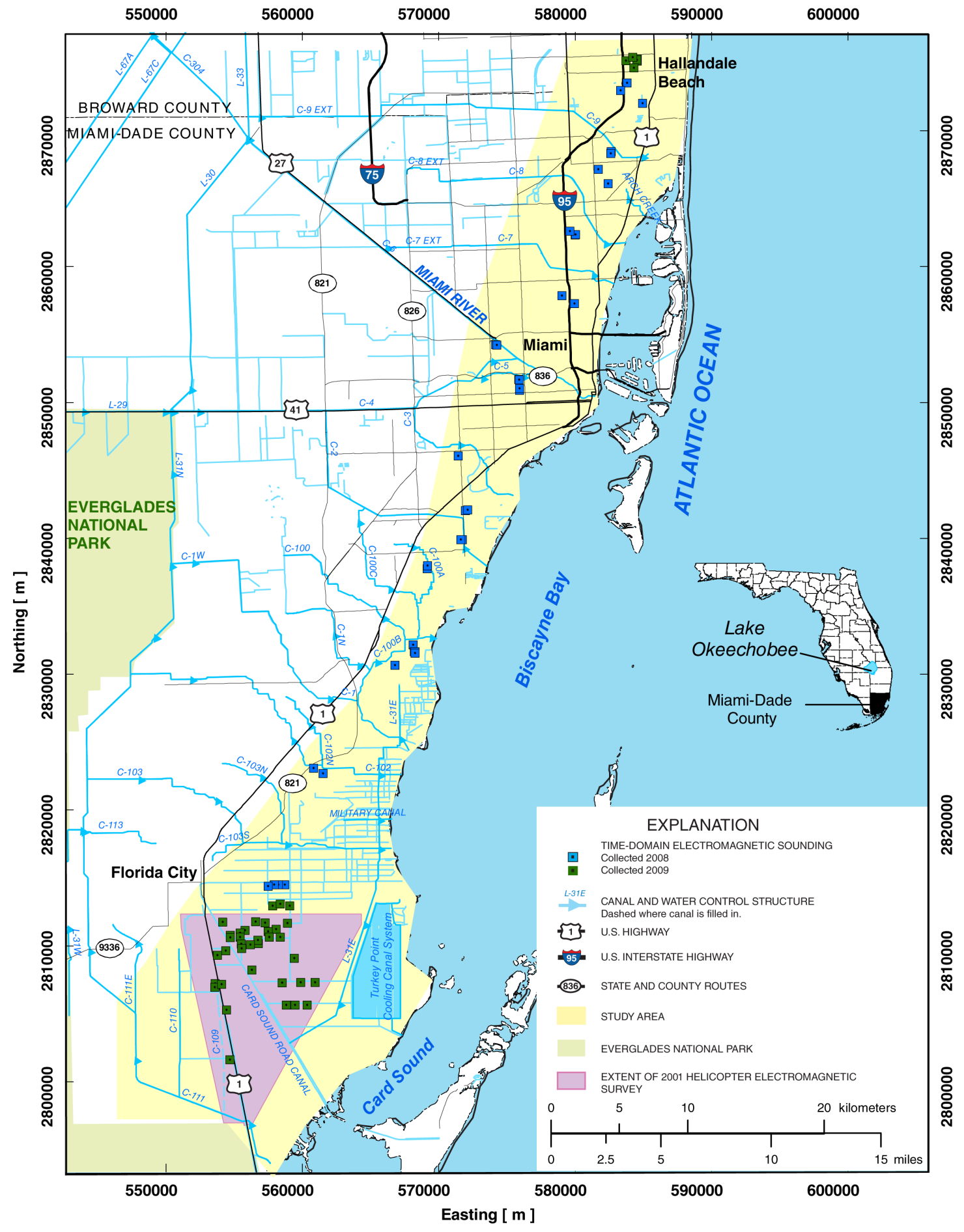

Figure 1. Location of the study area, time-domain electromagnetic soundings, and the helicopter electromagnetic survey. 


\section{Time-Domain Electromagnetic Sounding Locations}

A total of 79 TEM soundings were made during 2008 and 2009. The 2008 field season focused on gathering information from the Broward County line south to Florida City in southern Miami-Dade County to fill gaps in the spatial coverage of existing monitoring wells and to determine if the extent of saltwater encroachment had changed since the work of Sonenshein (1997). The locations of TEM soundings made in 2008 and 2009 are shown in figure 2. Most of these soundings are in Miami-Dade County. The 2009 field season extended the TEM coverage from the previous year further south in Miami-Dade County into the Model Land ${ }^{5}$ and north into Broward County near Hallandale Beach, Fla. (fig. 3). The soundings collected in the Model Land were located to help interpret the HEM survey flown in 2001 to map saltwater encroachment.

The locations of the TEM soundings were determined by global positioning system (GPS) measurements (tables 1 and 2). Horizontal positions are referenced to NAD 83. Positioning errors are 4 to $5 \mathrm{~m}$.

\section{Hydrogeologic Setting and Borehole Geophysical Information}

The surficial aquifer system in Miami-Dade and Broward Counties includes the Biscayne and the gray limestone aquifers. The Biscayne aquifer consists of the Pleistocene- and Holoceneage Satilla Formation, Pleistocene Miami and Key Largo Limestones, as well as the Anastasia and Fort Thompson Formations, and in some areas the Pliocene-age Tamiami formation (Fish and Stewart, 1991). The Biscayne aquifer is wedge-shaped and thinnest in western Miami-Dade and Broward Counties. It is approximately $98 \mathrm{~m}(320 \mathrm{ft})$ deep in eastern Broward County and approximately $55 \mathrm{~m}$ (180 ft) deep in northeastern Miami-Dade County (Fish and Stewart, 1991). The depth of the base of the Biscayne aquifer in the area of the HEM survey in southern MiamiDade County varies from about 17 to $30 \mathrm{~m}$ (55 to $100 \mathrm{ft}$ ) (Fish and Stewart, 1991).

Induction logs collected in Miami-Dade County indicate that the freshwater-saturated materials of the Biscayne aquifer have a resistivity of approximately $80 \mathrm{ohm}-\mathrm{m}$ on average and range from about 35 to $220 \mathrm{ohm}-\mathrm{m}$. Much of this variation is related to extreme changes in porosity. A digital borehole image showing the porosity variability observed in a core hole in the Fortt Thompson Formation of the Biscayne aquifer is shown in figure 4 (Cunningham and others, 2009). The image shows an approximately $0.4-\mathrm{m}$ thick solution cavity below a $0.2 \mathrm{~m}$ layer of very dense cycle-capping limestone. Variations in porosity like this are common in the Biscayne aquifer and are described in detail in previous investigations (Cunningham and others, 2006, 2009). Some of the changes in bulk conductivity with depth observed in inductions logs, such as those shown in figures 5 and 6 , are most likely the result of abrupt changes in porosity similar to those illustrated in figure 4.

Electromagnetic induction logs repeated annually or semiannually, like those depicted in figures 5 and 6 , are being used to evaluate changes in the bulk conductivity through time. The bulk conductivity measured is a function of the water conductivity and the porosity of the rocks and sediments that form the aquifer. Changes in formation resistivity are caused only by changes in the water conductivity. Because the induction log can measure the bulk conductivity through the PVC casing used in monitoring wells, changes in water conductivity can be detected without

\footnotetext{
${ }^{5}$ The term Model Land refers to all the wetlands in the area on both sides of Card Sound Road and south of SW 344 Street (Palm Drive) (C.K. Grossenbacher, Miami-Dade County DERM, written commun., 2010).
} 


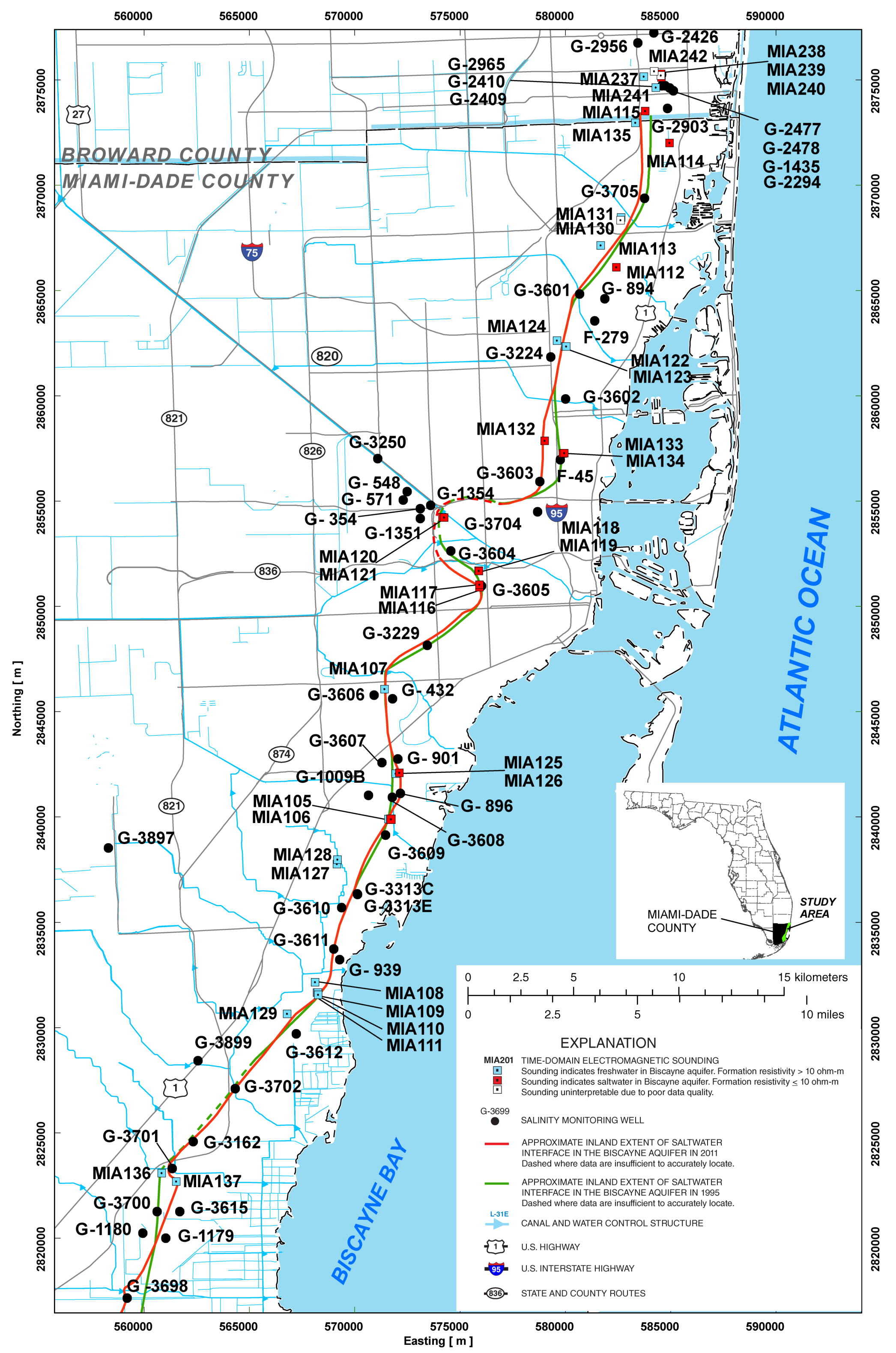

Figure 2. Location of the time-domain electromagnetic soundings collected in 2008 and 2009 to supplement the well network used to monitor saltwater encroachment. Extent of saltwater intrusion from Sonenshein (1997) and Prinos (2011). 


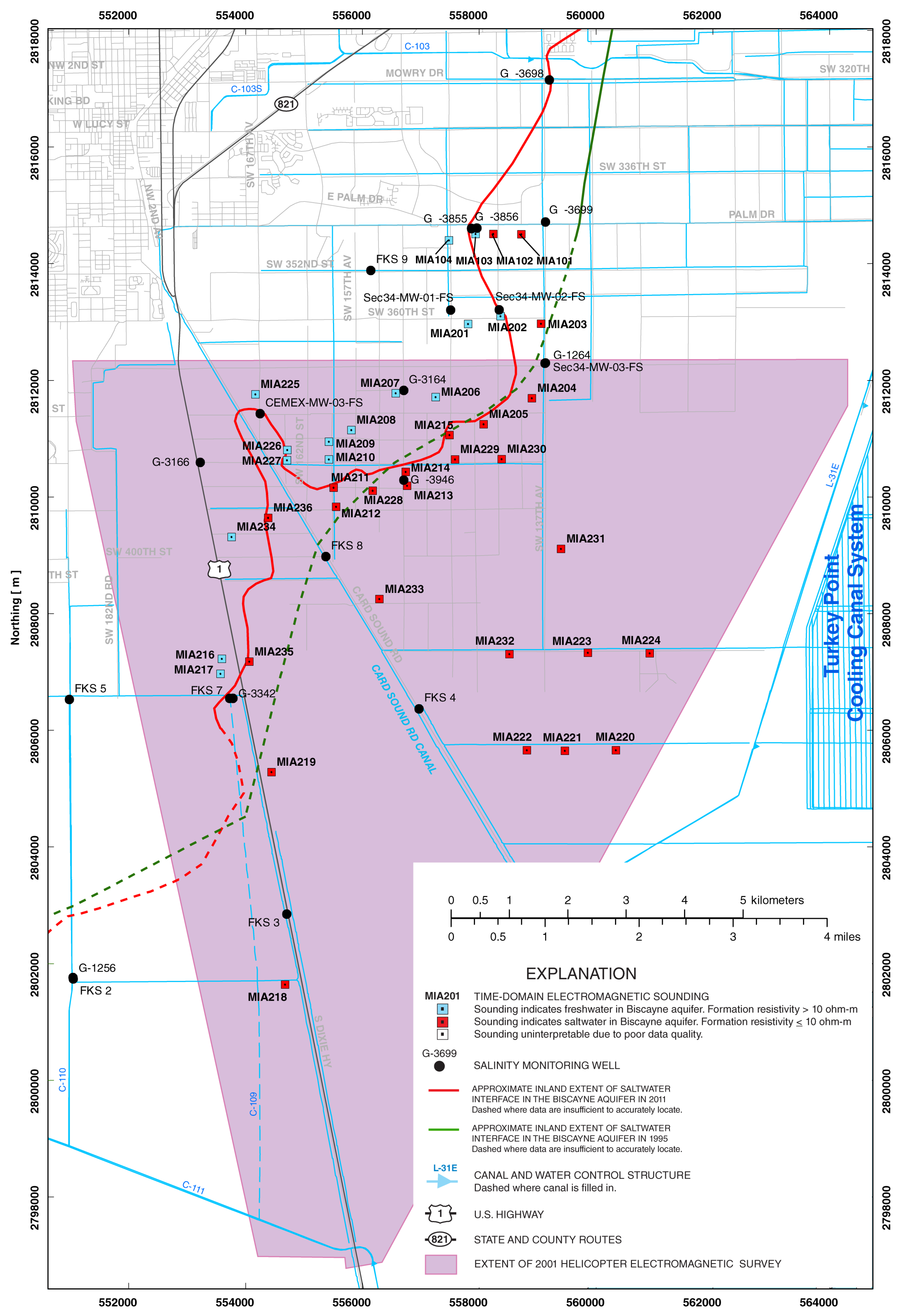

Figure 3. Location of the time-domain electromagnetic soundings collected in 2009 near the 2001 helicopter electromagnetic survey. Extent of saltwater intrusion from Sonenshein (1997) and Prinos (2011). 
Table 1. Location of TEM soundings made in 2008 and selected nearby wells

\begin{tabular}{|c|c|c|c|}
\hline Sounding $^{6}$ & Easting $^{7}[\mathrm{~m}]$ & Northing ${ }^{7}[\mathrm{~m}]$ & Nearby Wells ${ }^{8}$ \\
\hline MIA101 & 558727 & 2814504 & G-3699, G-3856 \\
\hline MIA102 & 558246 & 2814508 & G-3699, G-3856 \\
\hline MIA103 & 557942 & 2814507 & G-3699, G-3856 \\
\hline MIA104 & 557486 & 2814401 & G-3855, G-3856 \\
\hline MIA105 & 571727 & 2839900 & G3608, G-3609 \\
\hline MIA106 & 571608 & 2839907 & G3608, G-3609 \\
\hline MIA107 & 571420 & 2846074 & \\
\hline MIA108 & 568118 & 2832157 & \\
\hline MIA109 & 568218 & 2831666 & \\
\hline MIA110 & 568251 & 2831672 & \\
\hline MIA111 & 568268 & 2831566 & \\
\hline MIA112 & 582420 & 2866116 & \\
\hline MIA113 & 581685 & 2867153 & \\
\hline MIA114 & 584955 & 2872017 & G-2903 \\
\hline MIA115 & 583794 & 2873525 & G-2903 \\
\hline MIA116 & 575921 & 2850911 & G-3605 \\
\hline MIA117 & 575919 & 2851041 & G-3605 \\
\hline MIA118 & 575880 & 2851706 & G-3604 \\
\hline MIA119 & 575894 & 2851685 & G-3604 \\
\hline
\end{tabular}

\begin{tabular}{lclc}
\hline Sounding & Easting [m] & Northing [m] & Nearby Wells \\
\hline MIA120 & 574171 & 2854246 & G-3604 \\
MIA121 & 574249 & 2854232 & G-3604 \\
MIA122 & 580001 & 2862347 & \\
MIA123 & 580041 & 2862354 & \\
MIA124 & 579604 & 2862625 & \\
MIA125 & 571943 & 2842057 & \\
MIA126 & 572116 & 2842097 & \\
MIA127 & 569155 & 2837769 & \\
MIA128 & 569186 & 2837983 & \\
MIA129 & 566788 & 2830662 & \\
MIA130 u & 582639 & 2868352 & \\
MIA131 & 582649 & 2868479 & \\
MIA132 & 579026 & 2857869 & \\
MIA133 u & 579880 & 2857273 & \\
MIA134 & 579948 & 2857286 & \\
MIA135 & 583323 & 2872973 & \\
MIA136 & 560837 & 2823096 & \\
MIA137 & 561528 & 2822700 & \\
\hline
\end{tabular}

\footnotetext{
${ }^{6} \mathbf{F}$ indicates that the preferred model (MIAnnnF) had a conductive layer with resistivity fixed at $3.5 \mathrm{ohm}-\mathrm{m}$. $\mathbf{u}$ indicates that the sounding was not usable.

${ }^{7}$ Coordinates are in UTM Zone $17 \mathrm{R}$ and referenced to NAD83.

${ }^{8}$ Selected wells are within $2 \mathrm{~km}$ of a TEM sounding and have had an electromagnetic induction log run in them.
} 
Table 2. Location of TEM soundings made in 2009 and selected nearby wells.

\begin{tabular}{|c|c|c|c|c|}
\hline Sounding & & Easting $^{10}[\mathrm{~m}]$ & Northing $^{10}[\mathrm{~m}]$ & Nearby Wells ${ }^{11}$ \\
\hline MIA201 & & 557815 & 2812968 & Sec34-MW-02-FS \\
\hline MIA202 & & 558369 & 2813097 & Sec34-MW-02-FS \\
\hline MIA203 & & 559064 & 2812970 & Sec34-MW-03-FS \\
\hline MIA204 & & 558907 & 2811696 & Sec34-MW-03-FS \\
\hline MIA205 & & 558086 & 2811251 & \\
\hline MIA206 & & 557261 & 2811712 & \\
\hline MIA207 & & 556572 & 2811778 & \\
\hline MIA208 & & 555809 & 2811154 & \\
\hline MIA209 & & 555439 & 2810949 & \\
\hline MIA210 & & 555420 & 2810648 & \\
\hline MIA211 & & 555523 & 2810163 & \\
\hline MIA212 & $\mathbf{F}$ & 555558 & 2809832 & FKS 8 \\
\hline MIA213 & $\mathbf{F}$ & 556774 & 2810192 & G-3946 \\
\hline MIA214 & & 556751 & 2810422 & G-3946 \\
\hline MIA215 & & 557500 & 2811056 & \\
\hline MIA216 & & 553598 & 2807227 & FKS 7 \\
\hline MIA217 & & 553577 & 2806974 & FKS 7 \\
\hline MIA218 & $\mathbf{F}$ & 554678 & 2801646 & \\
\hline MIA219 & $\mathbf{F}$ & 554443 & 2805290 & \\
\hline MIA220 & & 560349 & 2805662 & \\
\hline MIA221 & & 559464 & 2805644 & \\
\hline
\end{tabular}

\begin{tabular}{|c|c|c|c|c|}
\hline \multicolumn{2}{|c|}{ Sounding } & Easting [m] & Northing [m] & Nearby Wells \\
\hline MIA222 & & 558824 & 2805659 & FKS 4 \\
\hline MIA223 & & 559876 & 2807328 & \\
\hline MIA224 & & 560925 & 2807316 & \\
\hline MIA225 & & 554172 & 2811757 & CEMEX-MW-03-FS \\
\hline MIA226 & & 554719 & 2810805 & CEMEX-MW-03-FS \\
\hline MIA227 & & 554717 & 2810623 & \\
\hline MIA228 & & 556179 & 2810102 & \\
\hline MIA229 & & 557588 & 2810641 & \\
\hline MIA230 & & 558393 & 2810648 & \\
\hline MIA231 & & 559404 & 2809111 & \\
\hline MIA232 & & 558515 & 2807305 & FKS 4 \\
\hline MIA233 & $\mathbf{F}$ & 556293 & 2808246 & FKS 8 \\
\hline MIA234 & & 553764 & 2809315 & \\
\hline MIA235 & $\mathbf{F}$ & 554067 & 2807176 & FKS 7 \\
\hline MIA236 & & 554391 & 2809645 & FKS 8 \\
\hline MIA237 & & 583733 & 2875167 & \\
\hline MIA238 & & 584560 & 2875121 & G-2478 \\
\hline MIA239 & & 584546 & 2875285 & G-2478 \\
\hline MIA240 & $\mathbf{u}$ & 584541 & 2875221 & \\
\hline MIA241 & & 584295 & 2874643 & G-2965 \\
\hline MIA242 & $\mathbf{u}$ & 584221 & 2875414 & \\
\hline
\end{tabular}

\footnotetext{
${ }^{9} \mathbf{F}$ indicates that the preferred model (MIAnnnF) had a conductive layer with resistivity fixed at $3.5 \mathrm{ohm}-\mathrm{m}$. $\mathbf{u}$ indicates that the sounding was not usable.

${ }^{10}$ Coordinates are in UTM Zone $17 \mathrm{R}$ and referenced to NAD83.

${ }^{11}$ Selected wells are within $2 \mathrm{~km}$ of a TEM sounding and have had an electromagnetic induction log run in them.
} 
open intervals of casing, which can potentially allow mixing of water from different depths. It is unlikely that the shallow influxes of conductive water observed in figures 5 and 6 could have been measured using a conventional open borehole because mixing of water from throughout an extensive open interval would have diminished the effect.

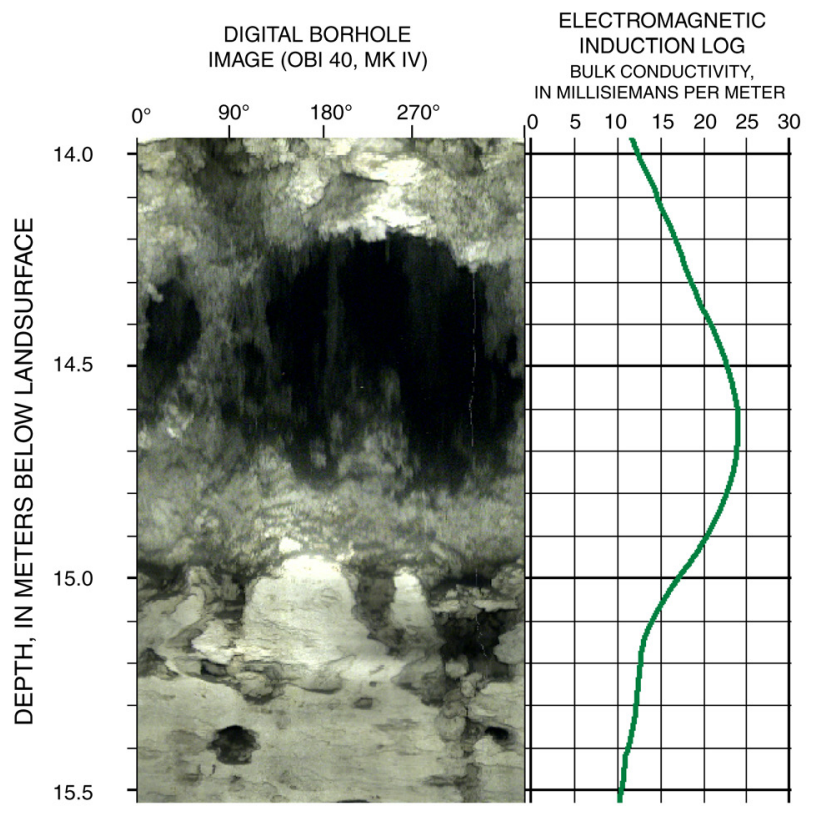

Figure 4. Digital borehole image from monitoring well G-3885 prior to its completion, and the electromagnetic induction log from the same depth interval. The water in the well was fresh from the surface to the bottom of the borehole. The increase in bulk conductivity in the depth interval from 14 to 15 $\mathrm{m}$ may be caused by the evident increase in porosity or a combination of changes in lithology and porosity. Cavities like the one shown here are evident in many of the borehole image logs collected. Recovery of materials enclosing these cavities is typically poor. The cavities may have been filled with sand prior to drilling.

The resistivity of the Biscayne aquifer decreases as the pore water is replaced by seawater. The relationship between aquifer resistivity and pore-water resistivity, called the formation factor (Archie, 1942), is determined by comparing induction-log data to water-sample resistivities as shown in figure 7. In conjunction with TEM-sounding results, this relation can be used to estimate aquifer-water resistivity. Although these estimates are coarse because of the previously discussed extreme variations in porosity, they nonetheless are quite valuable. The relation between water resistivity and chloride content determined from water samples collected in Miami-Dade County in the spring of 2009 is shown in figure 8 . Given these relations, the portions of the aquifer that are saturated with saltwater containing $1,000 \mathrm{mg} / \mathrm{L}$ of chloride are estimated to have a resistivity of approximately $10 \mathrm{ohm}-\mathrm{m}$. Accordingly, TEM-derived resistivities of $10 \mathrm{ohm}-\mathrm{m}$ or less are generally interpreted to be contaminated with saltwater.

The gray limestone aquifer, which is part of the Tamiami Formation, is separated from the Biscayne aquifer throughout most of southeastern Florida by the semi-confining upper clastic unit of the Tamiami Formation (Fish and Stewart, 1991). The gray limestone aquifer is generally not used for public water supply in Miami-Dade and Broward Counties. 


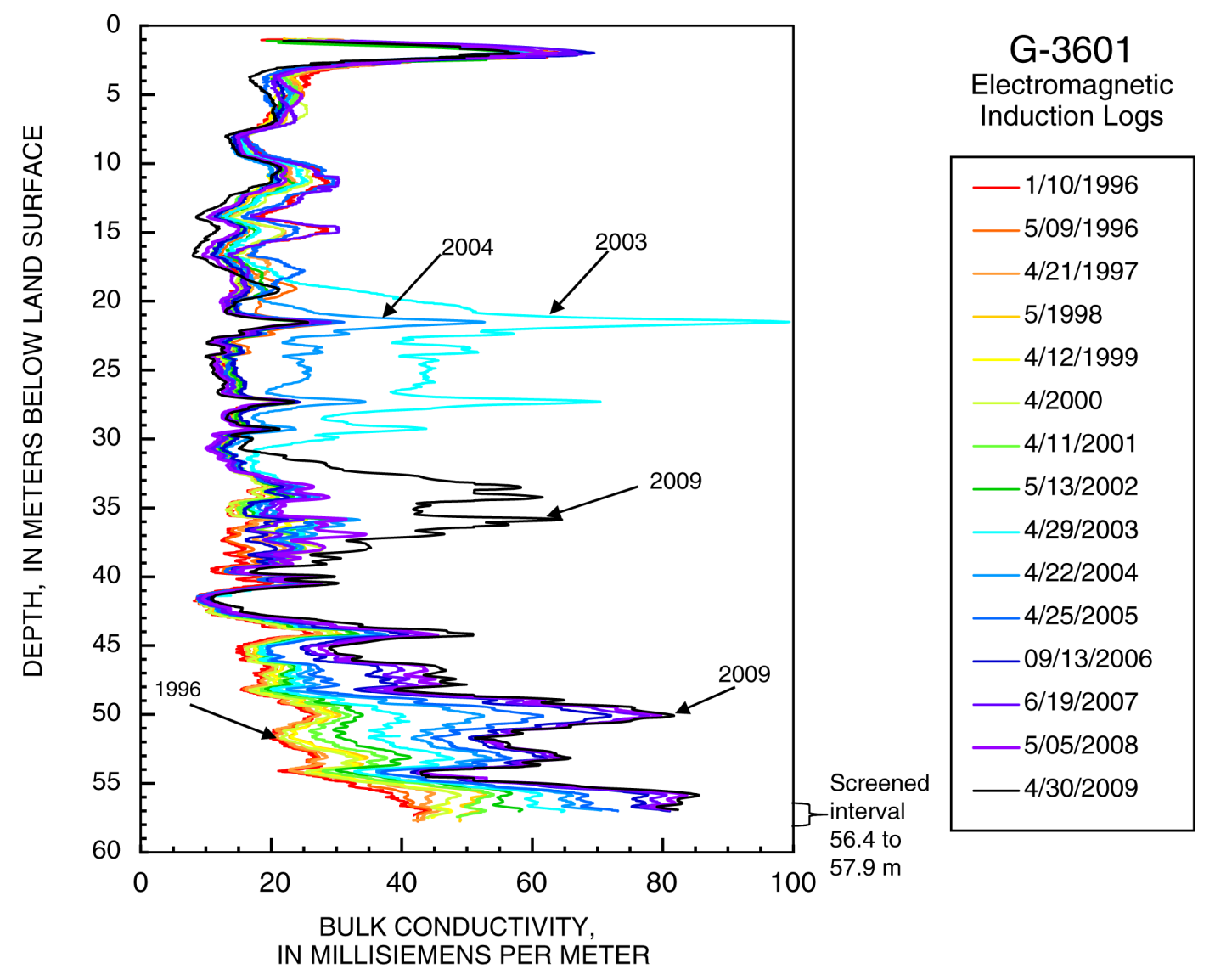

Figure 5. Electromagnetic induction logs from well G-3601 showing short-term changes in bulk conductivity occurring above the base of the Biscayne aquifer. The well is adjacent to a canal. There are temporary increases in bulk conductivity between the depths of $18.3 \mathrm{~m}$ and $40 \mathrm{~m}$ ( $60 \mathrm{ft}$ and $131 \mathrm{ft}$ ), that do not appear to be related to the steady bulk conductivity increases occurring below $42.7 \mathrm{~m}$ (140 ft). There is virtually no change in bulk conductivity at a depth of $41.1 \mathrm{~m}(135 \mathrm{ft})$. 


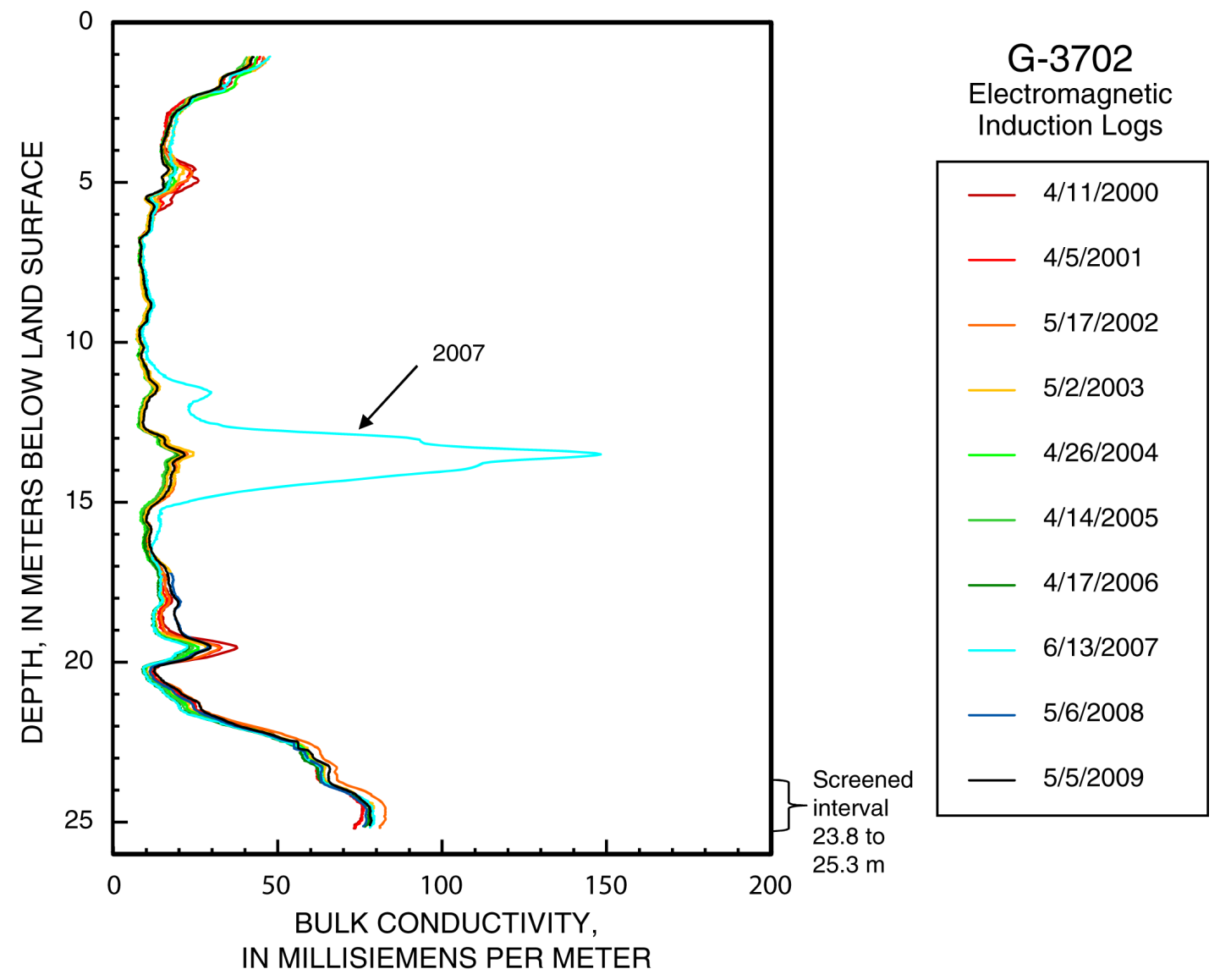

Figure 6. Electromagnetic induction logs from well G-3702, which is adjacent to a canal, showing short-term changes in bulk conductivity occurring above the base of the Biscayne aquifer. Some of the conductive water detected in 2007 between the depths of $10.7 \mathrm{~m}$ and $15.2 \mathrm{~m}(35 \mathrm{ft}$ and $50 \mathrm{ft}$ ) appears to be settling to the base of the aquifer. This is indictated by the increase in bulk conductivity occurring at $19.8 \mathrm{~m}(65 \mathrm{ft})$ in subsequent years. 


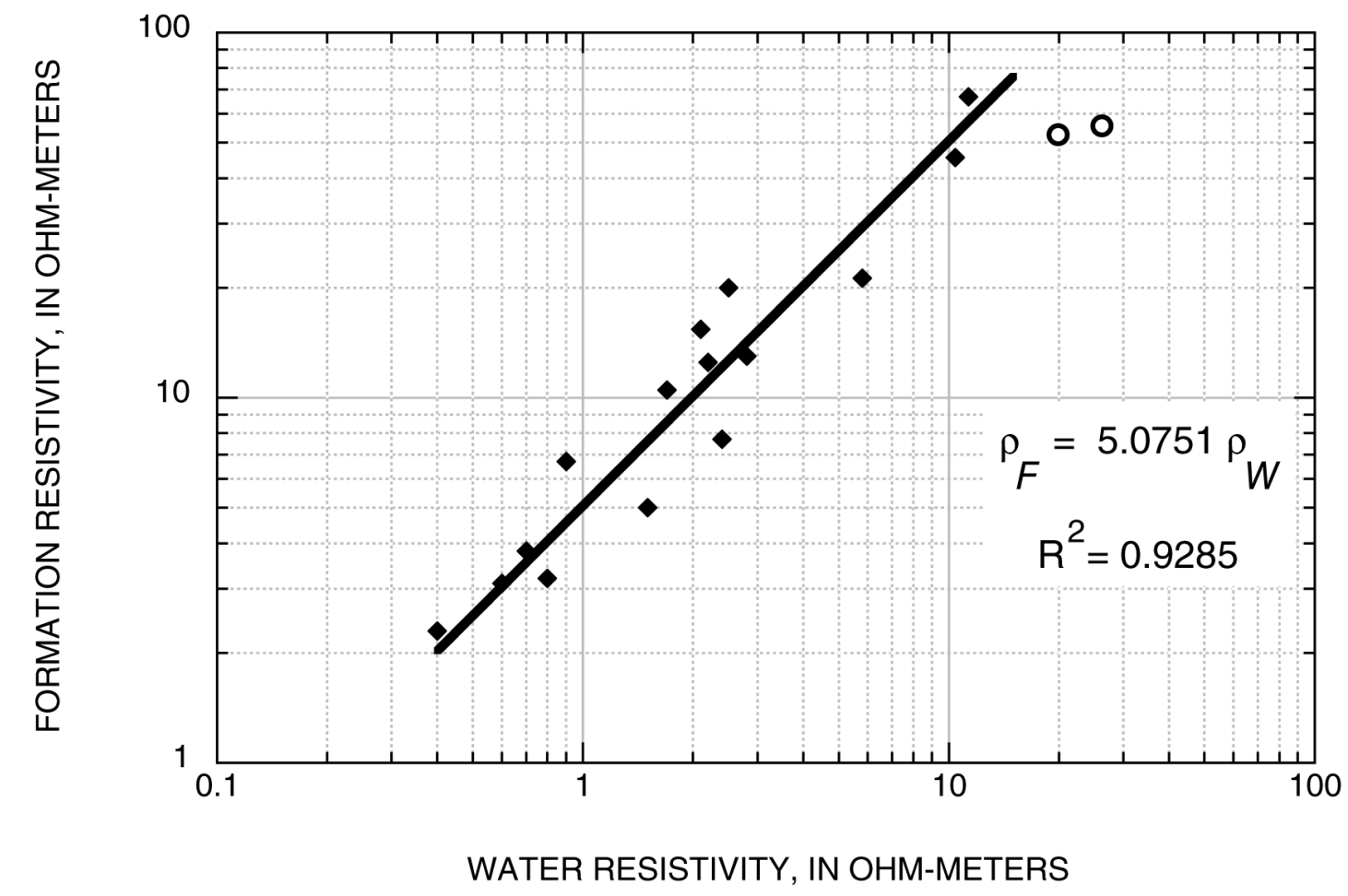

Figure 7. Relationship between the resistivity of water samples collected in monitoring wells in Miami-Dade County and the bulk aquifer resistivity measured by induction logs in the screened intervals of the same well. The two data points plotted as open circles were not used in the analysis. The equation describing the relationship is shown along with the coefficient of determination $\mathrm{R}^{2}$. 


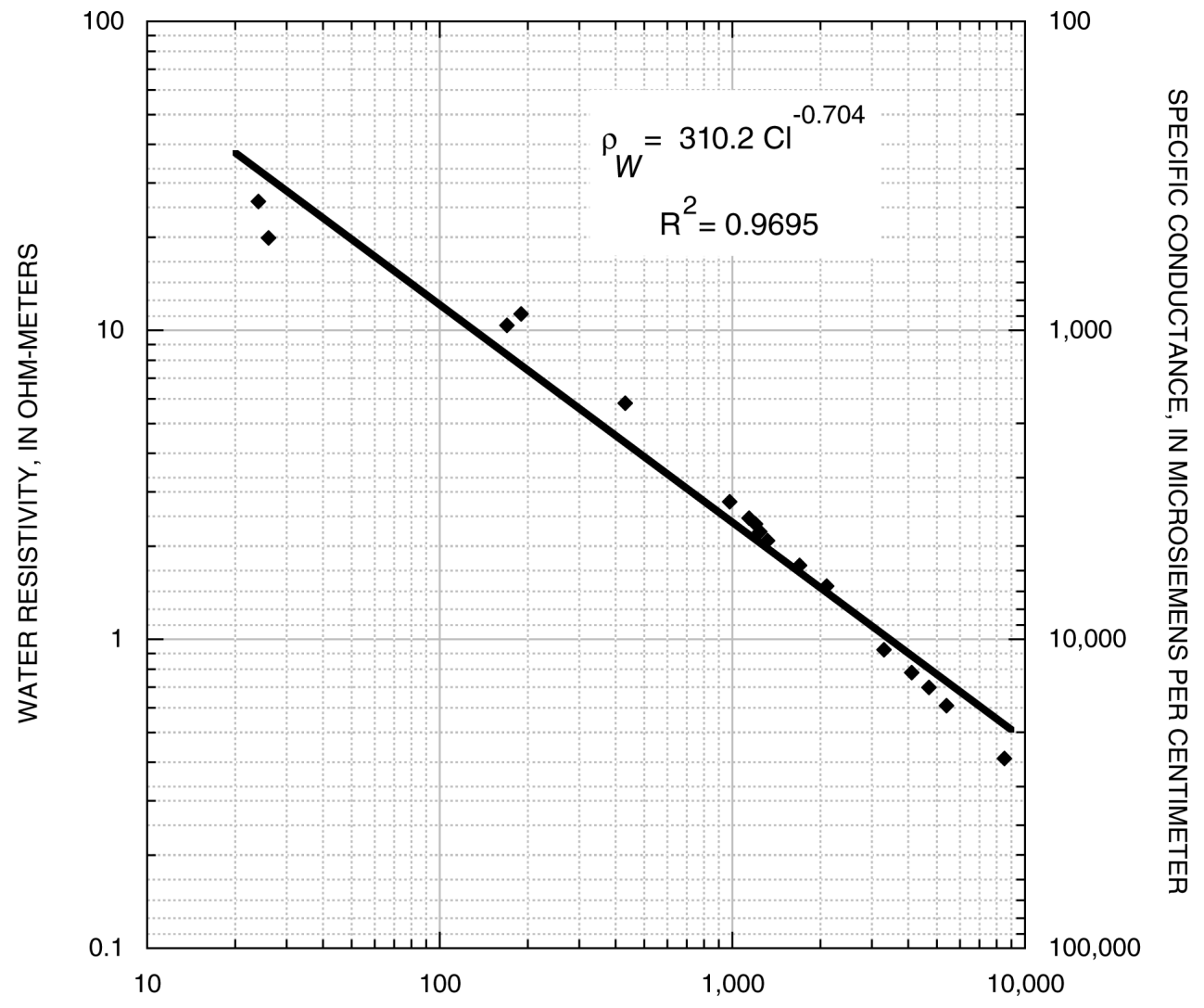

CHLORIDE CONTENT, IN MILLIGRAMS PER LITER

Figure 8. Relationship between resistivity and chloride content of water samples collected from monitoring wells in Miami-Dade County. The equation describing the relationship is shown along with the coefficient of determination $\mathrm{R}^{2}$.

\section{Previous Studies}

In 1939, the USGS in cooperation with the cities of Miami, Miami Beach, and Coral Gables, as well as Dade County, began to investigate the water resources of southeastern Florida with a special emphasis on the hydrogeology of the aquifer near the Miami River. This cooperative effort was expanded to Broward County in 1940. Brown and Parker (1945), Parker (1945), Hoy (1952), Parker and others (1955), and Klein (1957) evaluated the causes of saltwater encroachment and its extent in Miami-Dade County. Leach and Grantham (1966) evaluated the relation of saltwater encroachment to discharge in the Miami River and its tributaries.

The USGS mapped the extent of saltwater in the Biscayne aquifer in Miami-Dade County in 1951, 1978, 1984, and 1995 (Hoy, 1952; Swayze, 1980; Klein and Waller, 1985; Sonenshein, 1997) and in Broward County in 1990 (Koszalka, 1994). Klein and Ratzlaff (1989) and Sonenshein and Koszalka (1996) evaluated changes in the extent of saltwater intrusion in the Biscayne aquifer. At the time of Sonenshein's (1997) examination there was little information in extreme southeastern Miami-Dade County south of Florida City, so in this area the extent of encroachment was not well constrained. Sonenshein (1997) also used the TEM soundings to augment the information provided by monitoring wells. 
Kohout and Hoy (1963) evaluated some aspects of sampling salty groundwater in coastal aquifers. This work is crucial because the techniques described improved the quality of chloridecontent data collected in the Biscayne aquifer by the USGS. Because some of the data available from various organizations to evaluate saltwater encroachment were not collected using the methods described by Kohout and Hoy (1963), they were of limited value to the current investigation.

Fitterman and Deszcz-Pan (1998) and Fitterman and others (1999) collected and interpreted TEM soundings and an HEM survey to evaluate saltwater encroachment in southwestern MiamiDade County in Everglades National Park.

Prinos and others (2002) examined chloride content sampled from USGS monitoring wells during the period from 1974 to 1999 in the Biscayne aquifer. They identified statistically significant upward trends in chloride content in data from 40 percent of these wells. Downward trends in chloride content were identified in the data from 28 percent of these wells. Some USGS monitoring wells are farther inland than the current extent of saltwater in the aquifer. Chloride content in samples from these wells should only begin to change if saltwater continues to encroach. It is not surprising, therefore, that data from 32 percent of the monitoring wells exhibited no quantifiable trend for the period evaluated.

In 2008, the USGS monitoring network included 81 monitoring wells in the Biscayne aquifer that were sampled to evaluate chloride content. Thirty-five of these wells also had induction logs run annually to evaluate bulk formation conductivity of the aquifer. The results of this monitoring are provided to the public in several national and local websites and are used in the current investigation (USGS Annual Water Data Reports - http://wdr.water.usgs.gov, USGS Water Data for the Nation - http://waterdata.usgs.gov/nwis, Groundwater conditions in southern Florida http://www.sflorida.er.usgs.gov/edl_data/index_qw.html ).

\section{Description of Transient Electromagnetic Soundings}

TEM soundings, which are well suited for mapping conductive targets such as saltwater intrusion and clay zones, have been used for a number of groundwater studies (Fitterman and Stewart, 1986; Fitterman, 1989; Goldman and others, 1991; Fitterman and others, 1999). The TEM method is described in detail by Kaufman and Keller (1983), McNeill (1990), and Fitterman and Labson (2005).

Soundings are made by passing a current through a large, square transmitter loop. The current flow generates a steady magnetic field. Abruptly cutting off the current flow disrupts the magnetic field and induces a circulating current system in the ground below the transmitter loop. The diffusion of these induced currents is controlled by the electrical conductivity of the ground. Current attenuation is small in conductive regions, and the current passes slowly through such regions. Resistive regions (low conductivity), on the other hand, attenuate the current flow. Current traverses resistive regions more rapidly than conductive regions. The circulating induced current produces a secondary magnetic field that is sensed by a receiver coil located at the middle of the transmitter loop. Because of the relationship between the electrical conductivity structure of the ground, the current diffusion, and the secondary magnetic field, the voltage recorded by the receiver can be used to estimate the bulk conductivity of the subsurface materials. The measured voltage-time curves, or transients, are converted into resistivity-depth functions by a nonlinear parameter estimation process called inversion (Oldenburg and Li, 2005; Fitterman and Labson, 2005).

A Geonics PROTEM-D transient EM system with a digital receiver and a battery-powered EM-47 transmitter were used. The transmitter loop was set up as a square with a nominal side 
length of $38.1 \mathrm{~m}$. Transmitter current was typically $2.5 \mathrm{~A}$. The receiver coil was a rigid, multiturn loop about $1 \mathrm{~m}$ in diameter with an effective area of $31.4 \mathrm{~m}^{2}$. After adjusting receiver gain to an appropriate level, 10 measurements were made using base-frequency repetition rates of $285 \mathrm{~Hz}$ and $30 \mathrm{~Hz}$, referred to as ultra high (UH) and high (HI) frequency, respectively. All data from a single base frequency are commonly referred to as a sweep. The base frequency controls the time range over which the transient is recorded. There are 20 logarithmically spaced time gates in the measurement associated with each base frequency. Each measurement represents a stack of several hundred individual transients. Data processing procedures are discussed in appendix 1.

\section{Anthropogenic Factors Affecting Soundings}

TEM soundings are adversely affected by noise caused by man-made electromagnetic sources and nearby metallic objects. The most common cause of electromagnetic noise is power lines. Current flowing in power lines produces a time-varying magnetic field, which, in turn, induces a voltage in the receiver coil. Because the TEM receiver must be inherently broadband with virtually no filtering, 60-Hz signals from power lines pose a problem. The PROTEM-D TEM system uses repetition frequencies that are even subharmonics of the power-line frequency to reduce this type of noise. This noise-filtering strategy has limitations, so that measurements cannot be made closer than 300 to $400 \mathrm{~m}$ from large, power transmission lines. Power-line noise is recognized by very large variance in the measured data. Sounding MIA133 is a good example of this behavior (see figure 5-42). At times greater than $0.1 \mathrm{~ms}$ the measurement noise became very large for this sounding.

Nearby metallic objects, above ground or buried, will produce artifacts in the data. Metallic objects usually produce a very repeatable signal; however, they distort the apparent resistivity-time plots by introducing sharp bends and kinks that cannot be produced by a layered half-space. Small objects tend to influence the curves at the earlier times or in the middle of the curve. Sounding MIA1 19 (fig. 5-28) is an example of the former situation. The first eight data points have measurement error (not plotted) of 0.5 to 1.5 percent; however, the points do not form a smooth curve. Interpretation of the shallower portion of the resistivity structure is problematic. A similar situation is seen in sounding MIA104 (fig. 5-5). In this instance, the noise is at an intermediate time and only affects a few points, so the sounding can be interpreted with moderate confidence.

Buried conductors, such as metallic pipes, also cause problems. Sounding MIA121 (fig. 5-30) was made on a football field that had a lighting system. The data are very repeatable; however, the sounding curve is far from smooth. The field had conduits running to the light towers to provide electrical power. Specific information on the location and composition of the conduits was not available, but the conduits were most likely metallic. Lighting systems at municipal baseball fields also caused problems, so these sites were avoided.

Sounding MIA240 (figs. 7-57, 7-58, and 7-59) was made in Hallandale Cemetery where metal caskets presumably caused the interference. Moving the receiver coil around inside the transmitter loop did not alleviate the noise problem.

In general, anthropogenic noise was a bigger problem with the 2008 data than with the 2009 data. This is because the first field season was predominantly in an urban environment, and the second field season was in a much less developed part of the county.

\section{Data Quality and Averaging Procedure}

Data quality is highly dependent on anthropogenic factors. In areas where these factors were absent, the quality of the recorded data was excellent. At some locations the anthropogenic effects were so great that usable data could not be collected. This is the nature of working in an 
urban environment. Averaging of transients can be used to reduce noise from electromagnetic sources. At most sounding locations 10 or more TEM measurements were averaged for each base frequency using program NTEMAVG ${ }^{12}$ to reduce random noise and estimate measurement error. Some selective editing of the data was performed manually when it was obvious that a particular datum was significantly different from neighboring points. The average and standard deviation were calculated from the remaining measurements. The standard deviation of the apparent resistivity for the UH base frequency $(285 \mathrm{~Hz})$ was as low as 0.1 percent at early times. For most of the soundings the noise only increased to 1 to 3 percent at channel 20 . The HI base frequency $(30 \mathrm{~Hz})$ data had noise levels of 0.5 percent at channel 1, often increasing to very high levels (greater than 25 percent) at the later channels. The percent standard deviation usually increases with time after transmitter turnoff because the signal produced by the ground becomes small with respect to the background noise level. By the time the noise level reaches 10 percent in apparent resistivity the data are usually behaving discontinuously from one channel to the next and are not usable.

\section{Inversion of Transient Electromagnetic Soundings}

The averaged TEM soundings were individually inverted to the simplest model that fit the data using program TEMIXXL ${ }^{13}$. In general, a two- to five-layer model was adequate to provide a good fit. Most models show a decrease in resistivity with depth, sometimes followed by a more resistive layer at depth. After determining a preliminary model for each sounding, the result was compared with nearby soundings and well logs when available. Alternatives to the original model were tried in an attempt to improve the fit between the observed and computed apparent resistivities. Layers that were unresolvable because they were too thin or their resistivity was not significantly different from adjacent layers were eliminated from the final model. Some inversions resulted in a very thin conductive layer with resistivities that were considered to be unrealistically low, even for a saltwater intruded zone. The physics of TEM soundings does not allow both the thickness and resistivity of thin conductive zones to be determined. This phenomenon is termed equivalence. Only the ratio of layer thickness to resistivity can be estimated. In an attempt to resolve the thickness of thin, conductive layers, the resistivity of such layers usually was fixed at $3.5 \mathrm{ohm}-\mathrm{m}$, a value determined from well logs to be typical of saltwater intruded zones. This approach was tried when the conductive layer appeared to be unrealistically thin. A detailed description of each sounding is given in appendices 5 and 7 for the 2008 and 2009 data, respectively.

\section{Interpretation of Results}

As described previously, the analysis of water-quality data from the Biscayne aquifer and induction logs indicates that the resistivity of the saltwater-saturated Biscayne aquifer will be less than $10 \mathrm{ohm}-\mathrm{m}$. Using this criterion, the TEM location symbols shown in figures 2 and 3 are color coded to indicate the presence or absence of saltwater within the Biscayne aquifer. The location of the landward extent of saltwater as determined in 1995 (Sonenshein, 1997) and 2011 (Prinos, 2011)

\footnotetext{
${ }^{12}$ NTEMAVG is an unpublished program developed at the USGS.

${ }^{13}$ Program TEMIXXL is available from Interprex Limited, Golden, Colo.
} 
is also shown in these figures. The TEM results will be used along with other information in an ongoing study (Prinos and others ${ }^{14}$, written commun., 2011) to revise this boundary.

\section{Comparison with Well Logs}

Wherever possible TEM soundings were compared to induction logs collected in nearby monitoring wells to evaluate the quality of models and provide additional insights. In all, 33 TEM sounding models (tables 1 and 2) were compared with induction logs. The distance between the compared wells and TEM sites ranged from $36 \mathrm{~m}$ to $1.97 \mathrm{~km}$. Comparisons of results from other sites were also evaluated; however, they were not very informative and are not presented. Exact land-surface altitudes were not determined at TEM sounding locations. Land-surface altitudes had been determined at most monitoring-well locations where induction logs were collected. For comparison purposes the altitude of the base of the Biscayne aquifer was interpolated from the structural contour maps of Fish and Stewart (1991) and Fish (1988). These estimates were adjusted using the land-surface altitudes available at monitoring-well locations to approximate the depth to the base of Biscayne aquifer below land surface at TEM sounding and well locations. Because both the altitude of the base of the Biscayne aquifer and the altitude of the land surface vary from location to location, the depth comparisons depicted on these graphs must be considered approximate. In general, the areas where most of the TEM soundings and induction logs were collected tended to be relatively flat, so the comparisons provided are still informative.

Specific well and sounding comparisons are described below.

Well FKS 7 / Soundings MIA235, MIA216, and MIA217

An induction log was collected in monitoring well FKS 7 (fig. 9). This log is compared with the interpreted resistivity-depth functions from soundings MIA235, MIA216, and MIA217, which are $720 \mathrm{~m}$ northeast, $710 \mathrm{~m}$ north, and $470 \mathrm{~m}$ north of well FKS 7, respectively. The estimated depth to the base of the aquifer at the location of well FKS 7 is $22 \mathrm{~m}$; however, the base of the aquifer dips toward the northeast in this area. Monitoring well FKS 7 does not fully penetrate the aquifer. The bulk resistivity of the induction log decreases to $12.3 \mathrm{ohm}-\mathrm{m}$ at a depth of $17.4 \mathrm{~m}$; this would not be a full-tool response, however, because the resistivity was still decreasing when the logging tool encountered the bottom of the well, which is above the base of the Biscayne. It is reasonable to expect the resistivity to continue to decrease below the bottom of the well, so the maximum conductivity was probably not measured. This monitoring well is near the northern end of the C-109 Canal. An earthen plug at the southern end of this canal near the C-111 Canal was designed to prevent saltwater from flowing up the C-109 Canal. However, during the drought of 1971 salty water was detected 2 to $3 \mathrm{mi}$ north of this plug (Meyer, 1974). So it is possible the induction log collected at FKS 7 is either detecting saltwater that had once leaked out of this canal near FKS 7, or the reductions in freshwater head caused by this canal led to the encroachment of saltwater at the base of the aquifer near FKS 7. The interpreted TEM model for sounding MIA235F had the second layer resistivity fixed at $3.5 \mathrm{ohm}-\mathrm{m}$. This relatively thin layer is consistent with a thin layer of saltwater at the base of the aquifer. This layer is similar to that interpreted from sounding MIA236 (figs. 11 and 7-51) located $2.49 \mathrm{~km}$ (1.5 mi) north of sounding MIA235. Models for soundings MIA216 and MIA217 indicate a 13 to $17 \mathrm{ohm}-\mathrm{m}$ layer between the depths of approximately 25.6 and $29.6 \mathrm{~m}$, which is below the estimated base of the aquifer in this area. The

\footnotetext{
${ }^{14}$ This report is in preparation as a USGS Scientific Investigations Report. The tentative authorship and title are Prinos, S.T., Wacker, M.A., Cunningham, K.J., and Fitterman, D.V.: Examination of the landward extent and sources of saltwater in the Biscayne aquifer, Miami-Dade County, Florida.
} 
30-m depth slice of preliminary HEM data currently being analyzed (Fitterman and others, written commun., 2011) shows a finger of low resistivity material along U.S. Highway 1 and the C-109 Canal that extends farther inland than in the region to the west of the C-109 Canal. The western edge of this low resistivity zone is seen in the HEM data near well FKS 7 and at the approximate depth of the 13 to $17 \mathrm{ohm}-\mathrm{m}$ layer identified in soundings MIA216 and MIA217. The low resistivity zone identified in the HEM data and soundings MIA216 and MIA217 could contain residual saltwater that may have traveled inland along the ditch adjacent to U.S. Highway 1 and the C-109 Canal, or that encroached along the base of the aquifer as a result of lowered hydraulic head caused by the canal and ditch.

\section{Well FKS 4 / Soundings MIA222 and MIA232}

Interpreted resistivity-depth functions from soundings MIA222 and MIA232 are compared to an induction log from monitoring well FKS 4 (fig. 10). The soundings are approximately 1.97 $\mathrm{km}$ southeast and $1.79 \mathrm{~km}$ northeast, respectively, of the well (fig. 3). The first layer resistivities of the TEM models are in good agreement with those of the induction log. The induction-log resistivity starts to decrease at a depth of $7.3 \mathrm{~m}$ until it reaches a value of $3 \mathrm{ohm}-\mathrm{m}$ at the bottom of the well. The TEM models show a decrease in resistivity near a depth of $10 \mathrm{~m}$ to values of between 2 and $3 \mathrm{ohm}-\mathrm{m}$, which is in good agreement with the induction-log data. The induction-log resistivity was still decreasing at the bottom of the well, suggesting that the final induction-log resistivity was not the true resistivity of the conductive zone because the well was not deep enough to obtain a full-tool response of the conductive zone resistivity. The depth to the bottom of the second layer indicated by sounding MIA222 is in good agreement with the estimated base of the Biscayne aquifer at this location. 


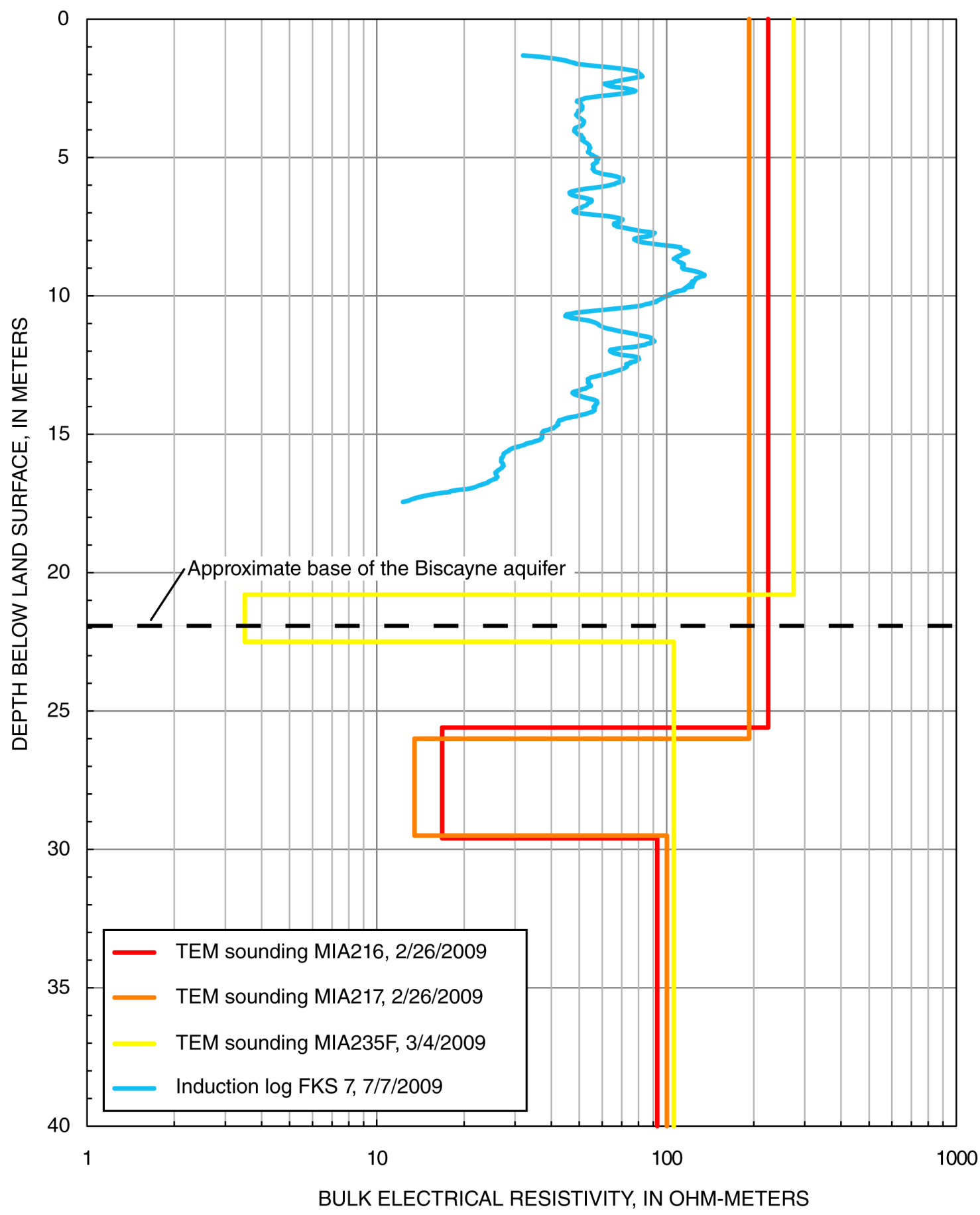

Figure 9. Comparison of TEM soundings MIA216, MIA217, and MIA235 with an induction log from well FKS 7. Well FKS 7 is approximately $720 \mathrm{~m}$ southwest of sounding MIA235, $710 \mathrm{~m}$ south of sounding MIA216, and $470 \mathrm{~m}$ south of sounding MIA217. The base of the aquifer was estimated from Fish and Stewart (1991) and adjusted for the land-surface elevation. The resistivity of the conductive layer that starts at about $20 \mathrm{~m}$ depth in sounding MIA235 was fixed in the inversion at $3.5 \mathrm{ohm}-\mathrm{m}$. 


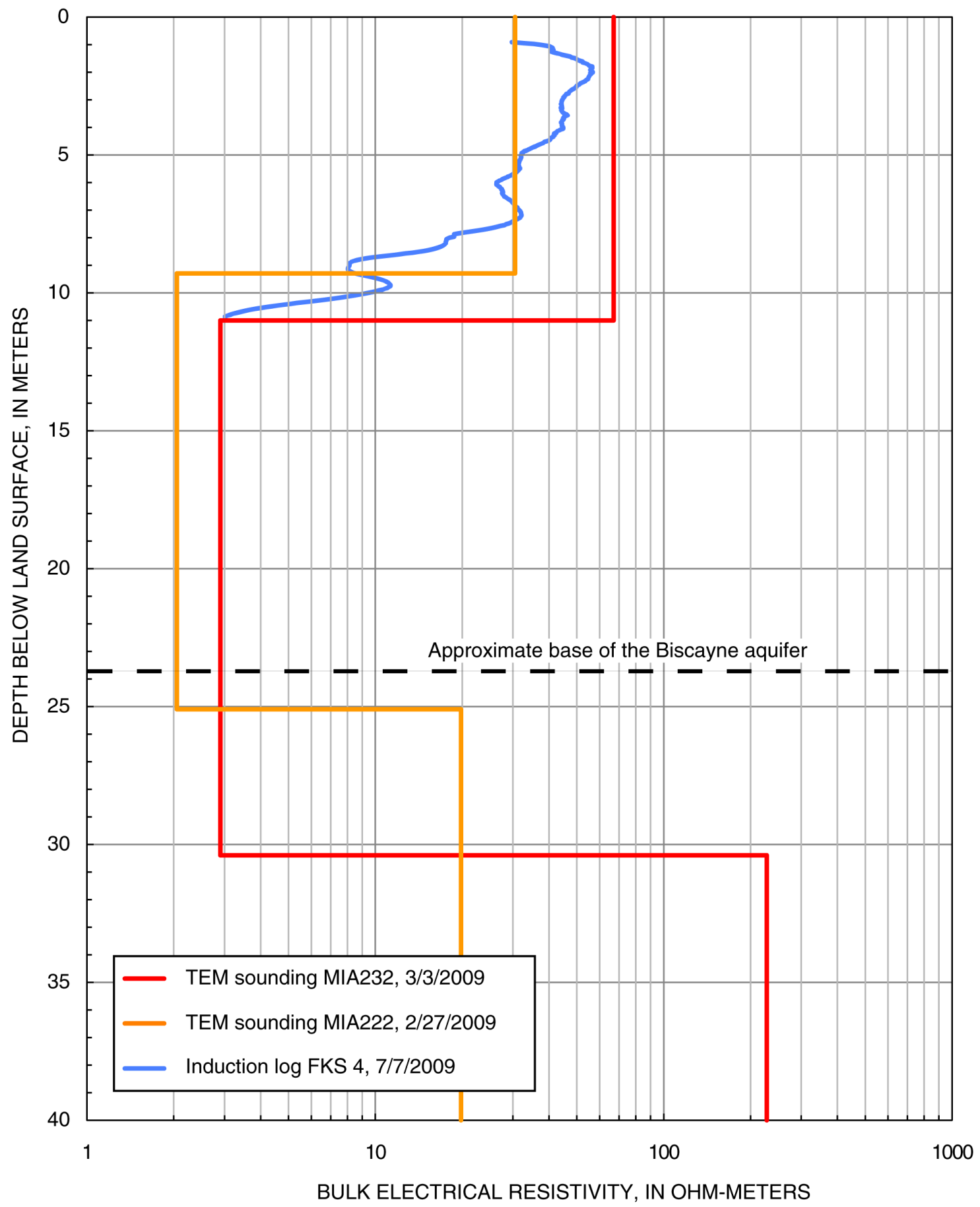

Figure 10. Comparison of TEM soundings MIA222 and MIA232 with an induction log from well FKS 4. Well FKS 4 is approximately $1.97 \mathrm{~km}$ northwest and $1.79 \mathrm{~km}$ southwest of soundings MIA222 and MIA232, respectively. The base of the aquifer was estimated from Fish and Stewart (1991) and adjusted for the land-surface elevation of the area. The base of the aquifer is slightly deeper at the locations of soundings MIA222 and MIA232 than at the well. 
Well FKS 8 / Soundings MIA212, MIA233, and MIA236

Models from TEM soundings MIA212, MIA233, and MIA236 are compared to an induction log collected in monitoring well FKS 8 (fig. 11). The soundings are located $880 \mathrm{~m}$ northnortheast, $1.17 \mathrm{~km}$ southeast, and $1.20 \mathrm{~km}$ northwest of the well, respectively (fig. 3). Well FKS 8 is adjacent to the Card Sound Road Canal. Water conductivity measured in the Card Sound Road Canal on December 5, 2008 indicated the canal water at the location of FKS 8 was fresh (salinity $0.3 \mathrm{ppt}$ ). Unusually dry conditions in this area during the winter of 2009 led to very low water levels in the Biscayne aquifer by May of 2009. On May 18, 2009, water-conductivity data collected in the Card Sound Road Canal near FKS 8 indicated an average salinity of the water of 38.6 ppt. Water samples collected by the Florida Keys Aqueduct Authority between March 31, 2009 and May 4, 2009 indicated the chloride content of water in well FKS 8 increased from 9,350 to 14,750 $\mathrm{mg} / \mathrm{L}$; on May 18, 2009 the chloride content of water in this well was 11,575 mg/L. Preliminary results of an HEM survey collected in this area in 2001 (Fitterman and others, written commun., 2011) depict a finger of low resistivity extending farther inland along Card Sound Road and U.S. Highway 1 than elsewhere in the general area. This finger of low resistivity material extends about $16 \mathrm{~km}(10 \mathrm{mi})$ inland and is also evidenced by the interpreted resistivity-depth functions of TEM soundings MIA211 (fig. 7-13), MIA225 (fig. 7-33), MIA226 (fig. 7-34), MIA227 (fig. 7-35), and the induction logs collected from monitoring well CEMEX-MW-03-FS (fig. 12). Given this information, the origin of the low resistivity zone is likely (1) leakage of saltwater into the aquifer from the Card Sound Road Canal during unusually dry periods, such as in May 2009, and (2) increased saltwater encroachment at the base of the Biscayne aquifer caused by unregulated drainage by the Card Sound Road Canal, which lowers the freshwater head in the aquifer.

The first layer resistivity of model MIA236 agrees moderately well with the peak resistivity of the induction log (fig. 11), whereas the first layer resistivities of models MIA212F and MIA233F are higher than the induction-log values. However, the uncertainty in the estimated first layer resistivity for the latter two models is large enough to explain this discrepancy. Some of the difference in resistivity values can also be explained by leakage and mixing of salty water from the canal with fresh groundwater in the aquifer at the location of the well, thereby lowering the nearsurface induction-log resistivity. The very low resistivity observed in the induction log near the land surface is probably caused by the metal surface casing. The resistivity of the second layer was fixed at $3.5 \mathrm{ohm}-\mathrm{m}$ in models MIA2 $12 \mathrm{~F}$ and MIA233F to reduce the effect of equivalence on the interpretation. This resistivity value is in good agreement with the resistivity measured in well FKS 8. The estimated depth to the top of the second layer is shallower for model MIA233F than for models MIA212F and MIA236. This is consistent with sounding MIA233 being the closest of the three soundings to the main mass of encroaching saltwater. Given that the TEM soundings are about $1 \mathrm{~km}(0.6 \mathrm{mi})$ from well 


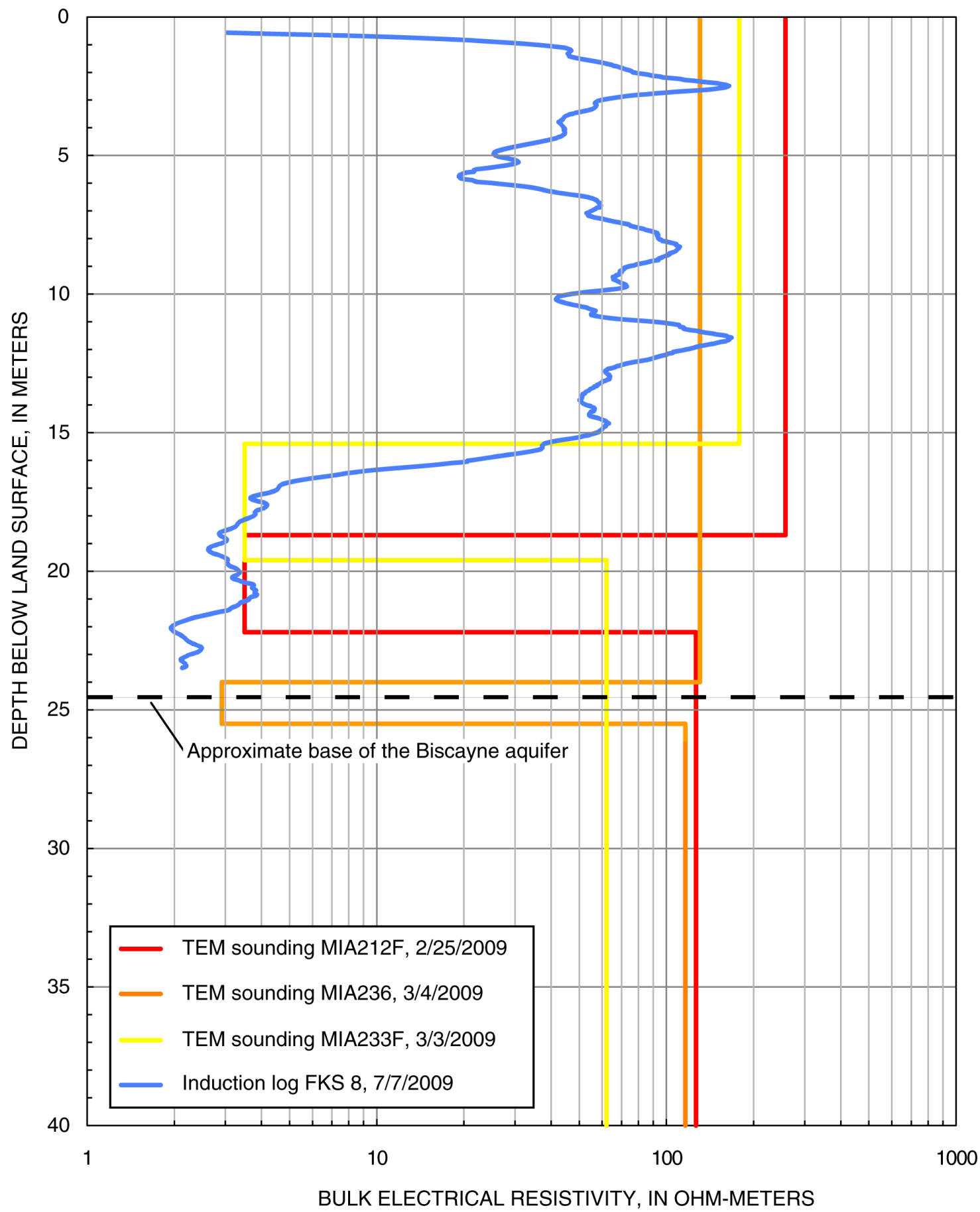

Figure 11. Comparison of TEM soundings MIA212, MIA233, and MIA236 with an induction log from well FKS 8 . Well FKS 8 is approximately $880 \mathrm{~m}$ south-southwest of sounding MIA212, $1.17 \mathrm{~km}$ northwest of sounding MIA233, and $1.20 \mathrm{~km}$ southeast of sounding MIA236. The base of the aquifer at the well was estimated from Fish and Stewart (1991) and adjusted for the land-surface elevation of the area. The resistivity of the second layer of models MIA212F and MIA233F was constrained at $3.5 \mathrm{ohm}-\mathrm{m}$. 
FKS 8 the discrepancy in the depth to the top of the conductive layer for soundings MIA212 and MIA233 is not unreasonable. The model for sounding MIA236, the most landward of these three soundings, suggests that the saltwater that occasionally leaks from the Card Sound Road Canal produces a thin layer of saltwater at the base of the Biscayne aquifer in this area. Sounding MIA236 is upgradient from the canal and the other soundings. Model MIA235F (fig. 7-50), which is also upgradient of the Card Sound Road Canal in this area, has an almost identical thin layer of saltwater at the base of the Biscayne aquifer.

\section{Well CEMEX-MW-03-FS / Soundings MIA225 and MIA226}

Comparison of models from TEM soundings MIA225 and MIA226 and an induction log collected in monitoring well CEMEX-MW-03-FS are shown in figure 12. The sounding sites are $360 \mathrm{~m}$ northwest and $760 \mathrm{~km}$ southeast of the well, respectively (fig. 3). The TEM models suggest a first layer resistivity higher than measured by the induction log. While there is some uncertainty in the interpreted TEM first layer resistivities, the interpretation does not support values as low as those seen in the induction log. The transition from the first to second layer in the TEM models agrees quite well with the decrease in resistivity between 18 and $22 \mathrm{~m}$ seen in the well $\log$. The resistivity of the second layer is in relatively good agreement with the induction log considering that the sounding and log are separated by several hundred meters. The top of the second layer of TEM model MIA226 is at a shallower depth than that of model MIA225. This is consistent with the location of sounding MIA226 being closer than sounding MIA225 to the main body of the encroaching seawater. The depth to the bottom of the second layer of sounding MIA226 is in good agreement with the estimated base of the Biscayne aquifer; for sounding MIA225 the bottom of the second layer is deeper than the base of the aquifer. This may be caused by seepage of moderately salty water into the basal confining unit or by variations in the depth to the top of the confining unit.

\section{Well G-3946 / Soundings MIA213 and MIA214}

Models from TEM soundings MIA213 and MIA214 are compared to an induction log collected in monitoring well G-3946 (fig. 13), which is $100 \mathrm{~m}$ northwest and $150 \mathrm{~m}$ southwest of the soundings, respectively (fig. 3). The interpreted first layer resistivity of the two sounding models is in good agreement with the measured resistivity from the induction log. The resistivities of the second layer bracket the induction-log values. The resistivity of the second layer for model MIA213F was fixed at $3.5 \mathrm{ohm}-\mathrm{m}$. The transition from the resistive surface layer to the saltwater interface evident in the induction log occurs at a shallower depth than determined by the TEM sounding. Sounding MIA213 has a thicker second layer than sounding MIA214, which is consistent with sounding MIA213 being closer to the main body of the encroaching saltwater than sounding MIA214. The bottom of the conductive zone determined by sounding MIA213 is in good agreement with the estimated base of the Biscayne aquifer. 


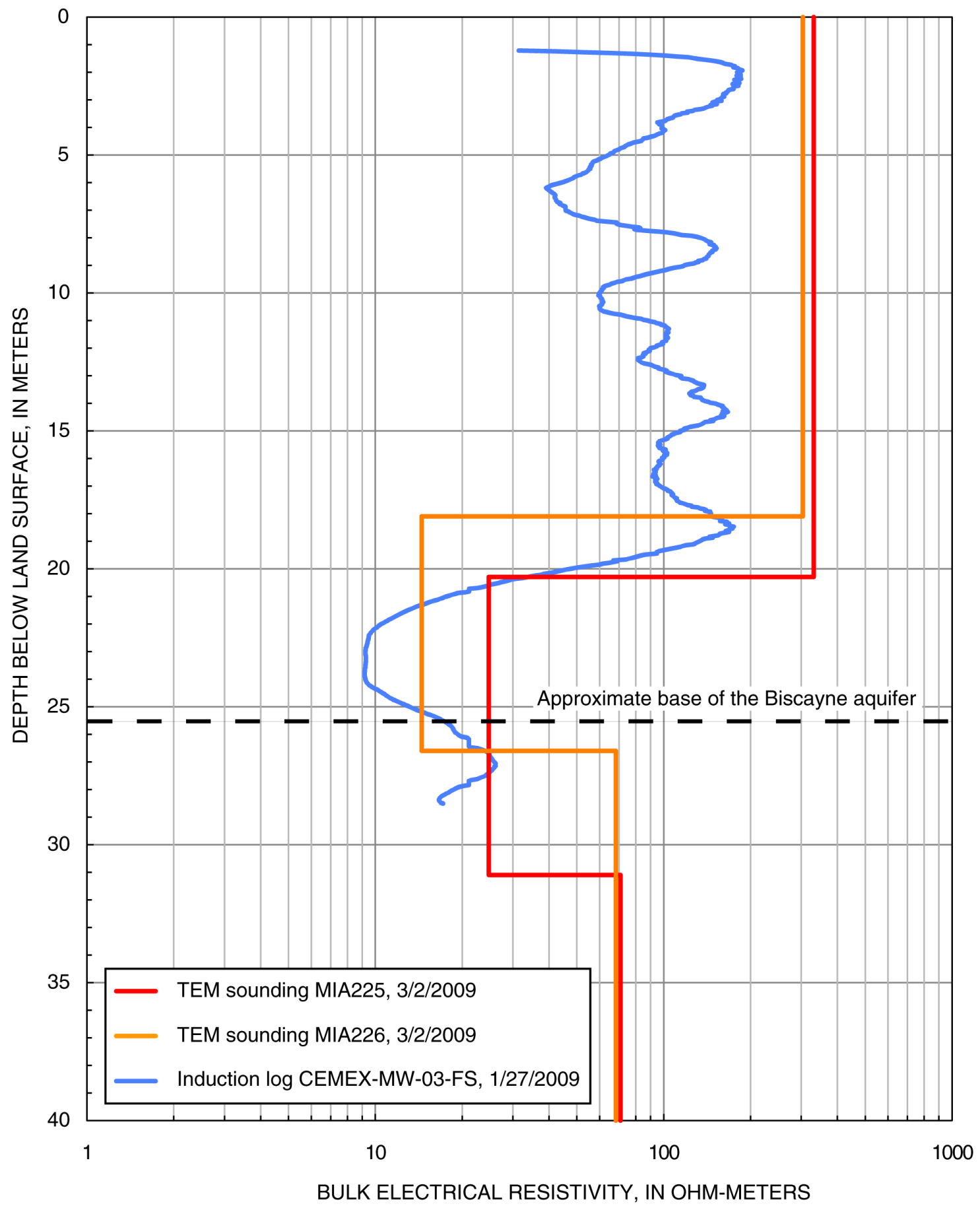

Figure 12. Comparison of TEM soundings MIA225 and MIA226 with an induction log from well CEMEX-MW03-FS. Well CEMEX-MW-03-FS is approximately $360 \mathrm{~m}$ south-southeast of sounding MIA225 and $760 \mathrm{~m}$ northwest of sounding MIA226. The base of the aquifer at the well was estimated from Fish and Stewart (1991) and adjusted for the land-surface elevation of the area. 


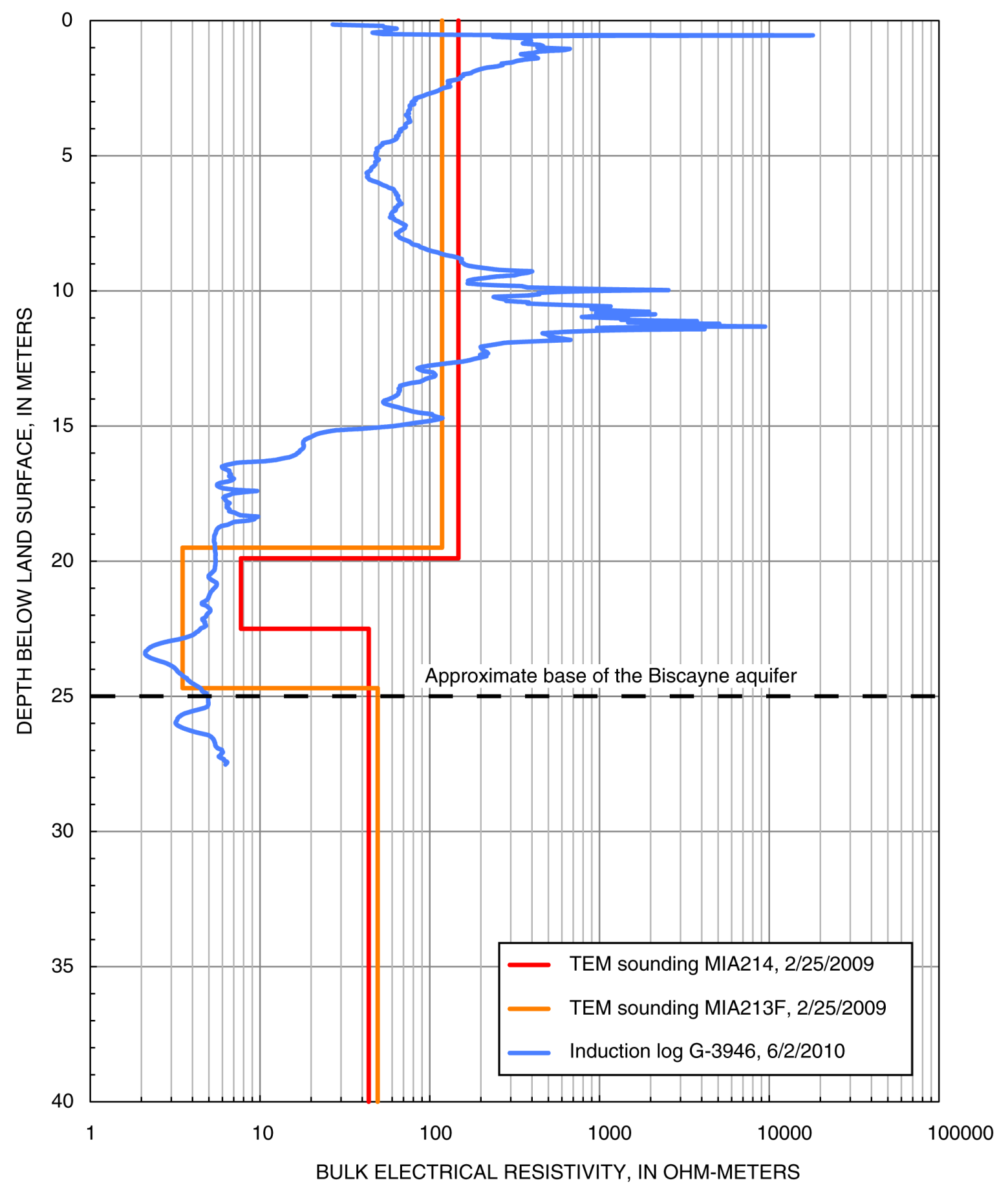

Figure 13. Comparison of TEM soundings MIA213 and MIA214 with an induction log from well G-3946. Well G-3946 is approximately $100 \mathrm{~m}$ northwest of sounding MIA213 and $150 \mathrm{~m}$ southwest of sounding MIA214. The base of the aquifer at the well was estimated from Fish and Stewart (1991) and adjusted for the land-surface elevation of the area. The resistivity of the second layer of model MIA213F was constrained at $3.5 \mathrm{ohm}-\mathrm{m}$. 
Well Sec34-MW-03-FS / Soundings MIA203 and MIA204

TEM models for soundings MIA203 and MIA204 are compared to an induction log collected in monitoring well Sec34-MW-03-FS (fig. 14), which is $670 \mathrm{~m}$ south and $630 \mathrm{~m}$ northnortheast of the soundings, respectively (fig. 3). The resistivity of the first three layers of model MIA204 agrees quite well with the induction log. The resistivity of the first two layers of model MIA203 are higher than the induction-log values. The resistivity minimum of the two models brackets the induction-log resistivity values. The depth to the bottom of the third layer is in fair agreement with the estimated depth of the base of the Biscayne aquifer in this area.

\section{Well Sec34-MW-02-FS / Soundings MIA201 and MIA202}

Models of data from TEM soundings MIA201 and MIA202 are compared to an induction log collected in monitoring well Sec34-MW-02-FS (fig. 15), which is 590 m northeast and $110 \mathrm{~m}$ north of the soundings, respectively (fig. 3). The first layer resistivity of both models agrees well with the induction log. The decrease in the induction-log resistivity starting around a depth of $20 \mathrm{~m}$ corresponds to the bottom of the first layer of model MIA202. Although the well was not deep enough for the induction tool to accurately determine the resistivity of the saltwater intruded zone, the resistivity of the second layer of model MIA202 is in relatively good agreement with the induction-log resistivity. It is reasonable to assume the conductive zone seen in the induction log extends to greater depth similar to the conductive second layer of TEM model MIA202. TEM sounding MIA 201, which is closer to the edge of the saltwater front, indicates that the water in the Biscayne aquifer is fresh. The decrease in interpreted resistivity of sounding MIA201 just below the estimated base of the Biscayne aquifer may be the result of lithologic, rather than water quality, changes.

Wells G-3699, G-3856, and G-3855 / Soundings MIA101, MIA102, MIA103, and MIA104

TEM soundings MIA101, MIA102, MIA103, and MIA104 were measured along an eastwest transect across the saltwater interface going from east to west (fig. 3). Wells G-3699, G-3856, and G-3855 are aligned along the same transect from east to west. Chloride content and specific conductance of samples from well G-3699 on April 30, 2008 were 8,500 mg/L and 24,300 $\mu \mathrm{S} / \mathrm{cm}$ respectively. Chloride content and bulk formation conductivity measured in induction logs have steadily increased since this well was installed in 2000. The induction logs and TEM interpretations indicate that the top of the saltwater interface deepens in the landward direction (westward).

The three-layer TEM models MIA101 and MIA102F indicate that the first layer consists of freshwater-saturated sediments underlain by a layer of saltwater-saturated sediments (fig. 16). Below the saltwater zone the resistivity is not as low, suggesting the deeper material is not saltwater saturated. TEM soundings MIA103 and MIA104 also indicate that the first layer consists of freshwater-saturated sediments. The resistivity of the second layer of these two soundings is lower than the surface layer, but not so low as to suggest the presence of saltwater. The interpreted TEM resistivities of the first layer for models MIA101, MIA102F, and MIA104 are similar to each other and correspond to the average induction-log resistivity in the first $15 \mathrm{~m}$ below land surface. Model MIA103 indicates a considerably more resistive first layer; however, the resistivity of this layer is not well constrained and a lower value would not appreciably change the misfit error. 


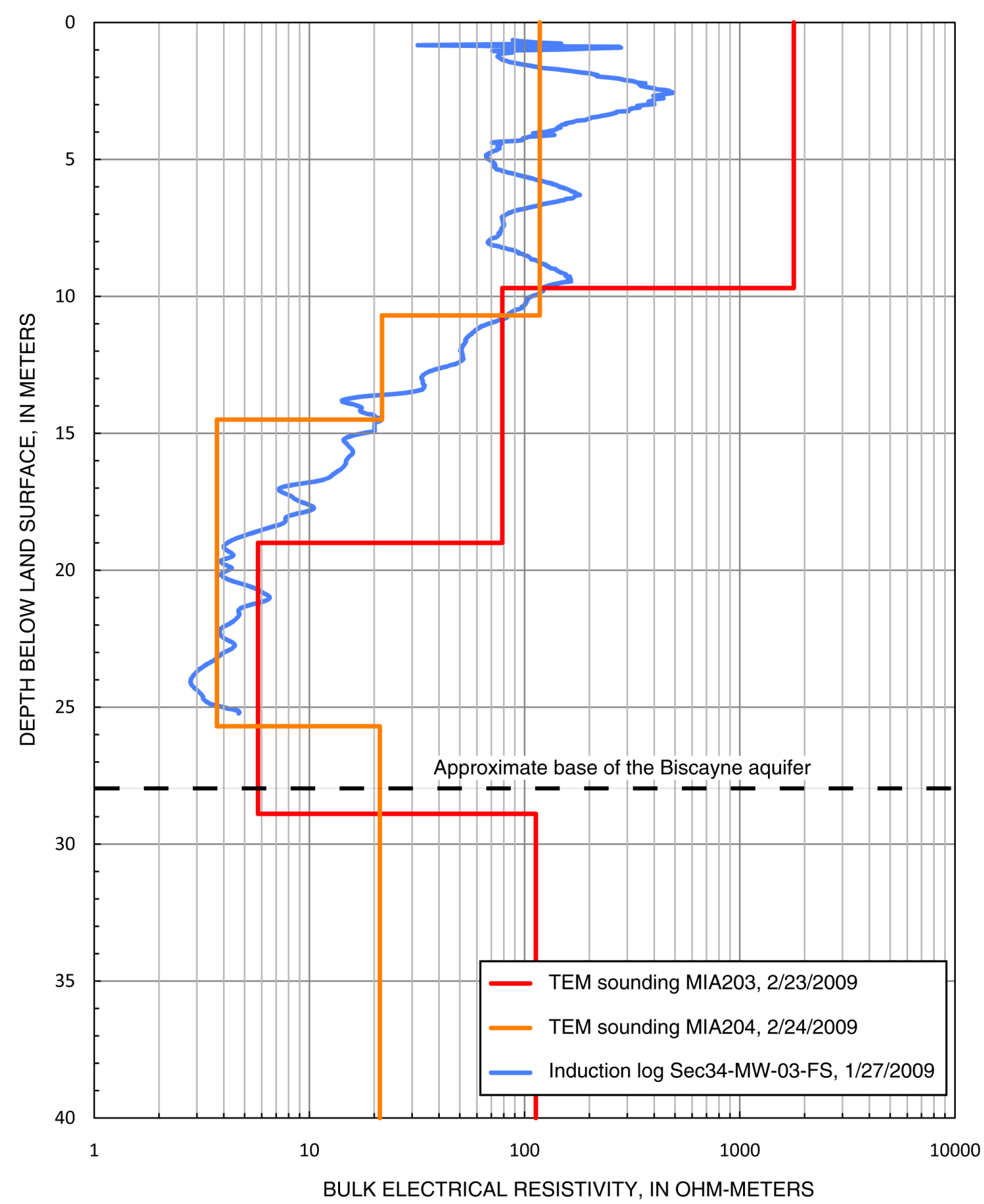

Figure 14. Comparison of TEM soundings MIA203 and MIA204 with an induction log from well Sec34-MW03-FS. Well Sec34-MW-03-FS is approximately $670 \mathrm{~m}$ south of sounding MIA203, and $630 \mathrm{~m}$ northnortheast of sounding MIA204. The base of the aquifer at the well was estimated from Fish and Stewart (1991) and adjusted for the land-surface elevation of the area. 


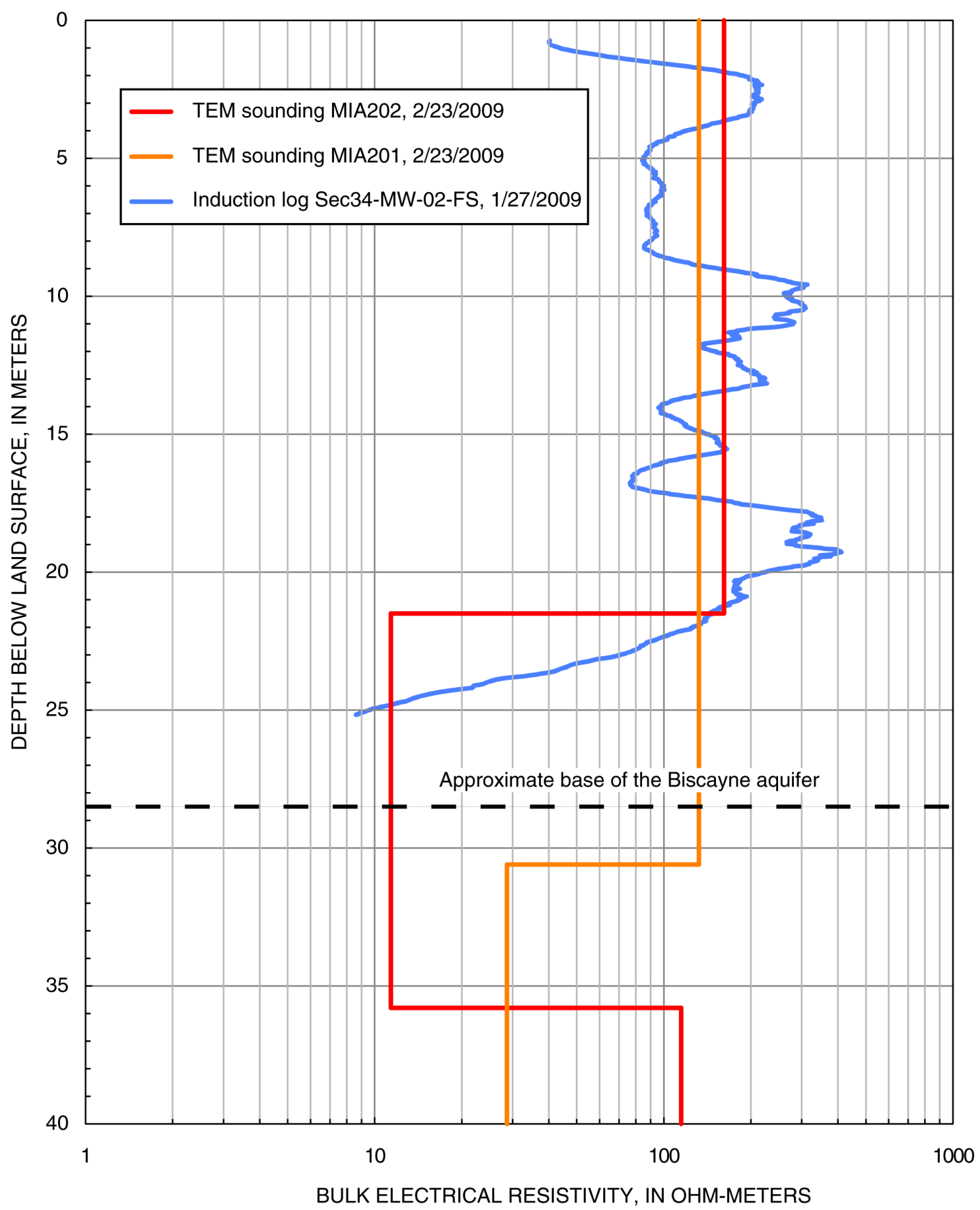

Figure 15. Comparison of TEM soundings MIA201 and MIA202 with an induction log from well Sec34-MW02-FS. Well Sec34-MW-02-FS is approximately $110 \mathrm{~m}$ north of sounding MIA202 and $590 \mathrm{~m}$ northnortheast of sounding MIA201. The base of the aquifer at the well was estimated from Fish and Stewart (1991) and adjusted for the land-surface elevation of the area. 


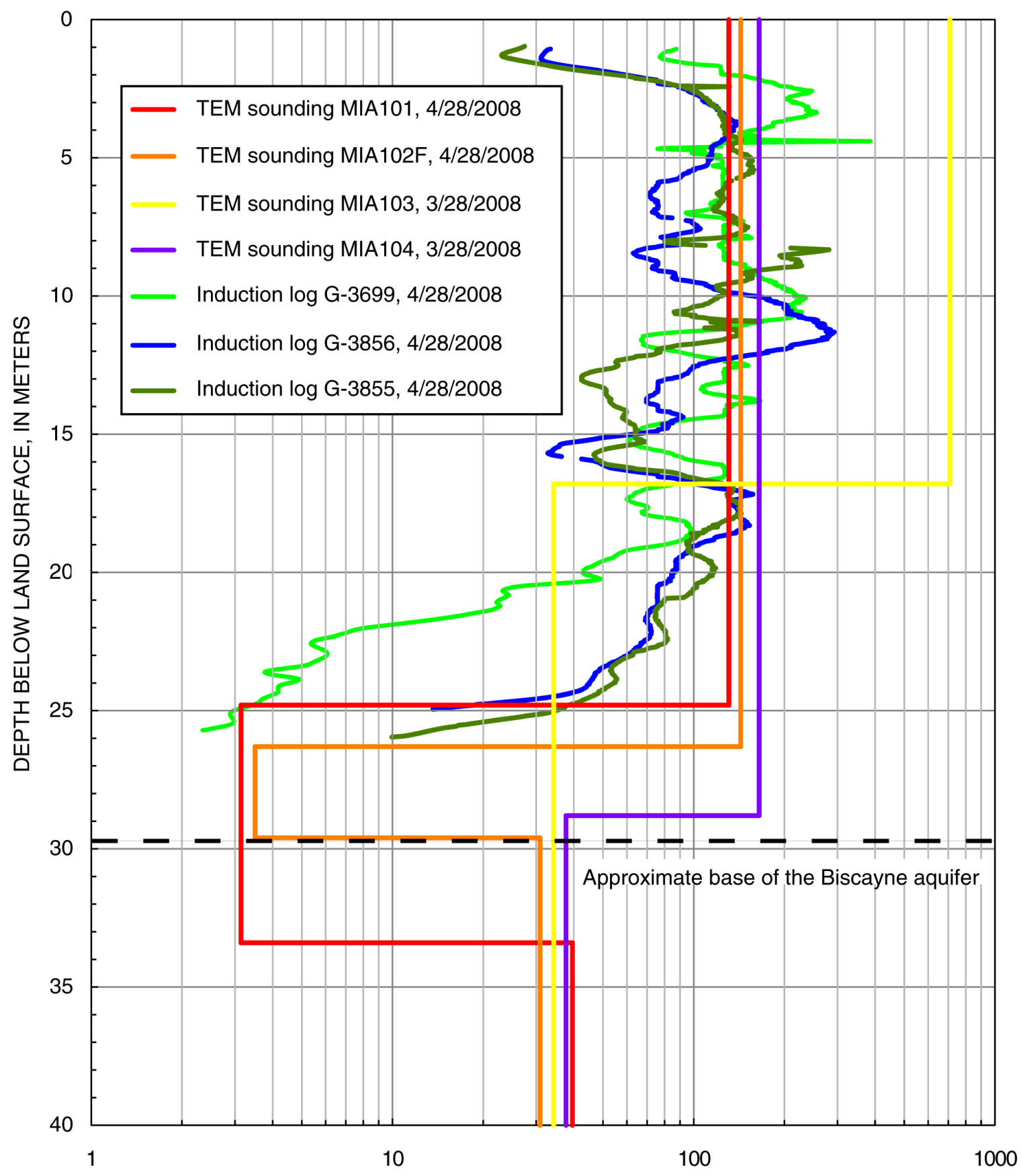

BULK ELECTRICAL RESISTIVITY, IN OHM-METERS

Figure 16. Comparison of TEM soundings MIA101, MIA102, MIA103, and MIA104 with induction logs from wells G-3699, G-3856, and G-3855. Well G-3699 is approximately $460 \mathrm{~m}$ east-northeast of sounding MIA101. Well G-3856 is $300 \mathrm{~m}$ west-northwest of sounding MIA102. Well G-3855 is $116 \mathrm{~m}$ northwest of sounding MIA103. The specific conductance of a water sample from well G-3699 on April 30, 2008 was $24,300 \mu \mathrm{S} / \mathrm{cm}$. The base of the aquifer at the well was estimated from Fish and Stewart (1991) and adjusted for the land-surface elevation of the area. The second layer resistivity of model MIA102F was constrained at $3.5 \mathrm{ohm}-\mathrm{m}$. 
The second layer resistivity of models MIA101 and MIA102 were 3.14 and 3.5 ohm-m, respectively, with the latter being fixed for the inversion. In the depth interval 21.9 to $25.7 \mathrm{~m}$, the bulk resistivity measured in well G-3699 decreased from 10.1 to $2.3 \mathrm{ohm}-\mathrm{m}$. The saltwatersaturated second layer of MIA101 begins at a depth of $24.8 \mathrm{~m}$, is $8.6 \mathrm{~m}$ thick, and extends below the estimated depth of the base of the Biscayne aquifer. The second layer of model MIA102 is saltwater saturated. It begins at a depth of $26.3 \mathrm{~m}$, has a thickness of $3.3 \mathrm{~m}$, and ends at almost the estimated depth of the Biscayne aquifer. These findings suggest that (1) the slope of the saltwater interface is approximately 3 to $6 \mathrm{~m} / \mathrm{km}$ deepening $\left(0.17\right.$ to $\left.0.34^{\circ}\right)$ to the west; and (2) either the base of the Biscayne aquifer at sounding MIA101 is deeper than estimated from the contours of Fish and Stewart (1991), or that saltwater is able to diffuse downward into the basal sediments.

Model MIA104 indicates the Biscayne aquifer at this location is freshwater saturated throughout its thickness, and the bulk resistivity below the Biscayne aquifer is lower, but not low enough to be considered saltwater saturated. The interpreted thickness of the first layer for sounding MIA104 is nearly identical to the estimated depth to the base of the Biscayne aquifer in this area.

The third layer resistivity of models MIA101 and MIA102F and the second layer resistivity of models MIA103 and MIA104 are all between 30 and $40 \mathrm{ohm}-\mathrm{m}$. These layers appear to represent the sediments below the base of the Biscayne aquifer. The resistivities are not low enough to suggest saltwater contamination. However, the top of the second layer for model MIA103 is about $12.2 \mathrm{~m}$ shallower than the estimated base of the Biscayne aquifer for this area. This finding suggests there may be a paleotopographic high in the base of the Biscayne aquifer near sounding MIA103.

The bulk formation resistivity measured in all three wells was still decreasing when the probe reached the bottom of the well. The modeled TEM results suggest all three wells penetrated only the uppermost portion of the saltwater invaded zone. Therefore, a full tool response could not be measured because the induction tool was only partially in the saltwater invaded zone, suggesting the formation resistivity near the bottom of these wells is lower than the deepest measured value.

\section{Well G-3609 and G-3608 / Soundings MIA105 and MIA106}

Models from TEM soundings MIA105 and MIA06 are compared to an induction log collected in monitoring well G-3609 (fig. 17), which is $780 \mathrm{~m}$ south of the sounding locations (fig. 2). Monitoring well G-3608 is $1.0 \mathrm{~km}$ north of soundings MIA105 and MIA106. Four-layer models MIA105 and MIA106 indicate decreasing resistivity with depth. The first two layers are freshwater saturated. Model MIA105 indicates the Biscayne aquifer is contaminated with saltwater from a depth of $23.4 \mathrm{~m}$ to at least its base at $39.5 \mathrm{~m}$. Sounding MIA106, however, which was collected only $120 \mathrm{~m}$ west of sounding MIA105, indicates the Biscayne aquifer is fresh throughout its thickness, and sediments below the base of the aquifer are saltwater saturated. These measurements suggest the saltwater interface is very steeply dipping $\left(146 \mathrm{~m} / \mathrm{km}\right.$ or about $\left.8^{\circ}\right)$ toward the west. This steep slope may be related to periodic leakage of saltwater from the Snapper Creek Canal (C-2) which is only 103 m east of the center of sounding MIA105.

Induction logs and geochemical sampling in groundwater monitoring well G-3608, which is adjacent to the Snapper Creek Canal, support the hypothesis of periodic saltwater leakage from Snapper Creek Canal. An induction log measured on April 30, 2003 in well G-3608 indicates an abrupt influx of saltwater in the depth interval of 8.5 to 


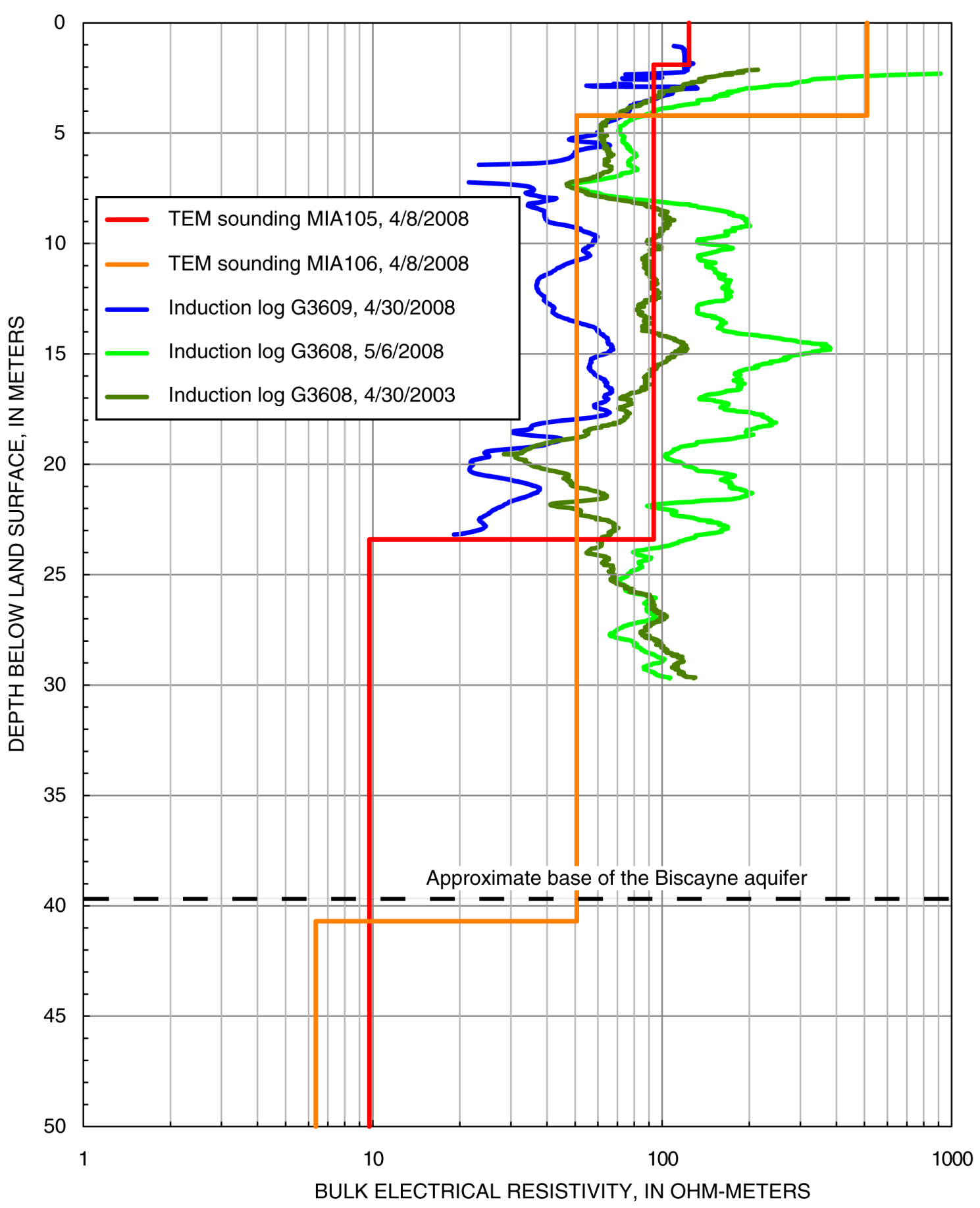

Figure 17. Comparison of TEM soundings MIA105 and MIA106 with induction logs from wells G-3609 and G-3608. Well G-3609 is approximately $780 \mathrm{~m}$ south of soundings MIA105 and MIA106. Well G-3608 is 1.0 $\mathrm{km}$ north of soundings MIA105 and MIA106. The specific conductance of water sampled from well G-3609 at a depth of $23 \mathrm{~m}$ on April 30, 2008 was $4,040 \mathrm{mS} / \mathrm{cm}$. The base of the aquifer at the well was estimated from Fish and Stewart (1991) and adjusted for the land-surface elevation of the area. 
$25 \mathrm{~m}$. The chloride content of water samples collected from this well at a depth of $30 \mathrm{~m}$ increased from $78 \mathrm{mg} / \mathrm{L}$ on July 25, 2003 to a maximum of $240 \mathrm{mg} / \mathrm{L}$ on August 4, 2004. An induction $\log$ measured on April 26, 2005 (not shown) revealed that the salty water had dissipated in the depth range of 8.5 to $25 \mathrm{~m}$, but the water conductivity had increased in the interval 26 to $30 \mathrm{~m}$. This information suggests at least some of the salty water first detected on April 30, 2003 sank to the base of the aquifer at this location. Samples of this water collected on July 29, 2009 indicated the $\delta^{18} \mathrm{O}$ and $\delta^{2} \mathrm{H}$ signatures of water sampled from well G-3608 more closely resembled that of the Snapper Creek Canal than other nearby groundwater monitoring wells (Prinos and others, written commun., 2011). This information suggests the Snapper Creek Canal may occasionally leak saltwater into the aquifer. This would also help explain why the slope of the saltwater interface detected by soundings MIA105 and MIA106 is so steep.

The second layer resistivities of models MIA105 and MIA106 are in good agreement with the resistivities measured at the corresponding depths of the induction logs from wells G-3608 and G-3609 (fig. 17). The induction $\log$ from G-3609 indicates that the top of the saltwater interface begins at a depth of about $19 \mathrm{~m}$. The water sampled from this well at a depth of $23 \mathrm{~m}$ on April 30, $2008 \mathrm{had}$ a specific conductance of $4,050 \mu \mathrm{S} / \mathrm{cm}$ and a chloride content of $1,140 \mathrm{mg} / \mathrm{L}$. Chloride content sampled from this well has gradually increased since 1995 when the well was installed. Well G-3609 does not fully penetrate the aquifer, and the bulk resistivity logged was still decreasing when the induction probe reached the bottom of the well. Sounding MIA105 indicates a deeper top of the saltwater interface than determined at the monitoring well. The depth to the top of the third layer of model MIA106 is in very good agreement with the estimated depth to the base of the Biscayne aquifer. Models MIA105 and MIA106 indicate the sediments are saturated with saltwater even below the base of the Biscayne aquifer.

\section{Well G-3605 / Soundings MIA116 and MIA117}

Models of soundings MIA116 and MIA117 are compared to an induction log collected in monitoring well G-3605 (fig. 18), which is $130 \mathrm{~m}$ northeast and $140 \mathrm{~m}$ southeast of the soundings, respectively (fig. 2). Two-layer model MIA116 consists of freshwater-saturated sediments underlain by sediments saturated with brackish water. The first layer of this model is more resistive than the uppermost $25 \mathrm{~m}$ of the induction log. The second layer resistivity of model MIA116 is in very good agreement with that of the induction $\log$ at a depth of $31 \mathrm{~m}$. Water samples collected from well G-3605 on May 2, 2008 indicated a specific conductance of 4,790 $\mu \mathrm{S} / \mathrm{cm}$ and a chloride content of $1,320 \mathrm{mg} / \mathrm{L}$. Chloride content sampled from this well has gradually increased since 1995 when the well was installed. Three-layer model MIA117 indicates freshwater sediments are underlain by saltwater-saturated sediments. At greater depth, the interpreted resistivity increases to a value intermediate between the first and second layer resistivities. The depth to the top of the second layer of model MIA117 is greater than for model MIA116.

Well G-3604 / Soundings MIA118, MIA119, MIA120, and MIA121

Models of soundings MIA118, MIA119, MIA120, and MIA121 are compared to an induction log collected in monitoring well G-3604 (fig. 19). Well G-3604 is $1.63 \mathrm{~km}$ northwest of soundings MIA118 and MIA119, and $1.63 \mathrm{~km}$ south of soundings MIA120 and MIA121 (fig. 2). Two-layer models MIA118, MIA119, and MIA121 indicate that freshwater-saturated sediments are underlain by saltwater-saturated sediments. Model MIA121 agrees quite well with 


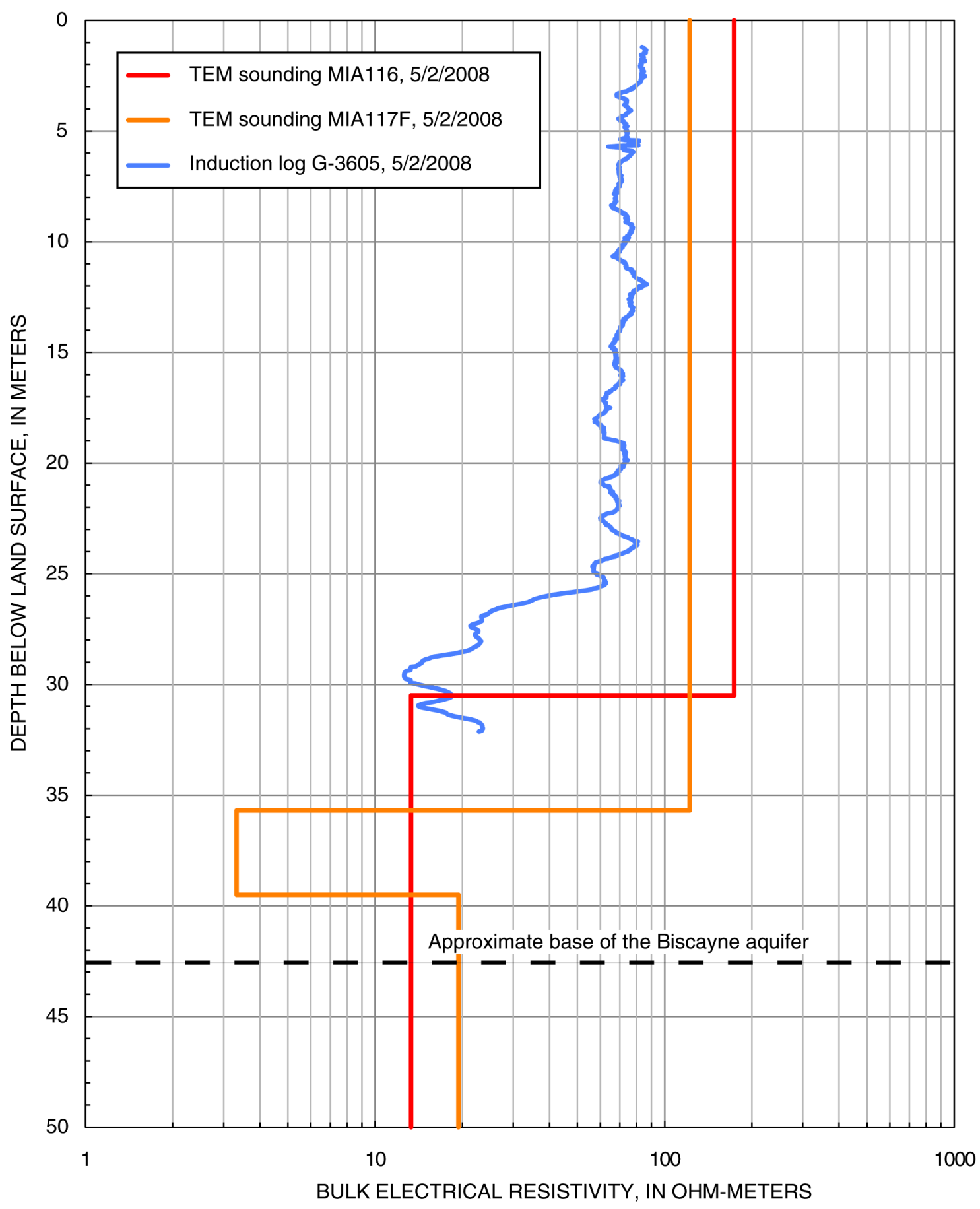

Figure 18. Comparison of TEM sounding MIA116 and MIA117 with an induction log from well G-3605. Well G-3605 is approximately $130 \mathrm{~m}$ northeast of sounding MIA116, and $140 \mathrm{~m}$ southeast of sounding MIA117. The specific conductance of water sampled from well G-3605 on May 2, 2008 was 4,790 $\mu \mathrm{S} / \mathrm{cm}$. The base of the aquifer at the well was estimated from Fish and Stewart (1991) and adjusted for the landsurface elevation of the area. 


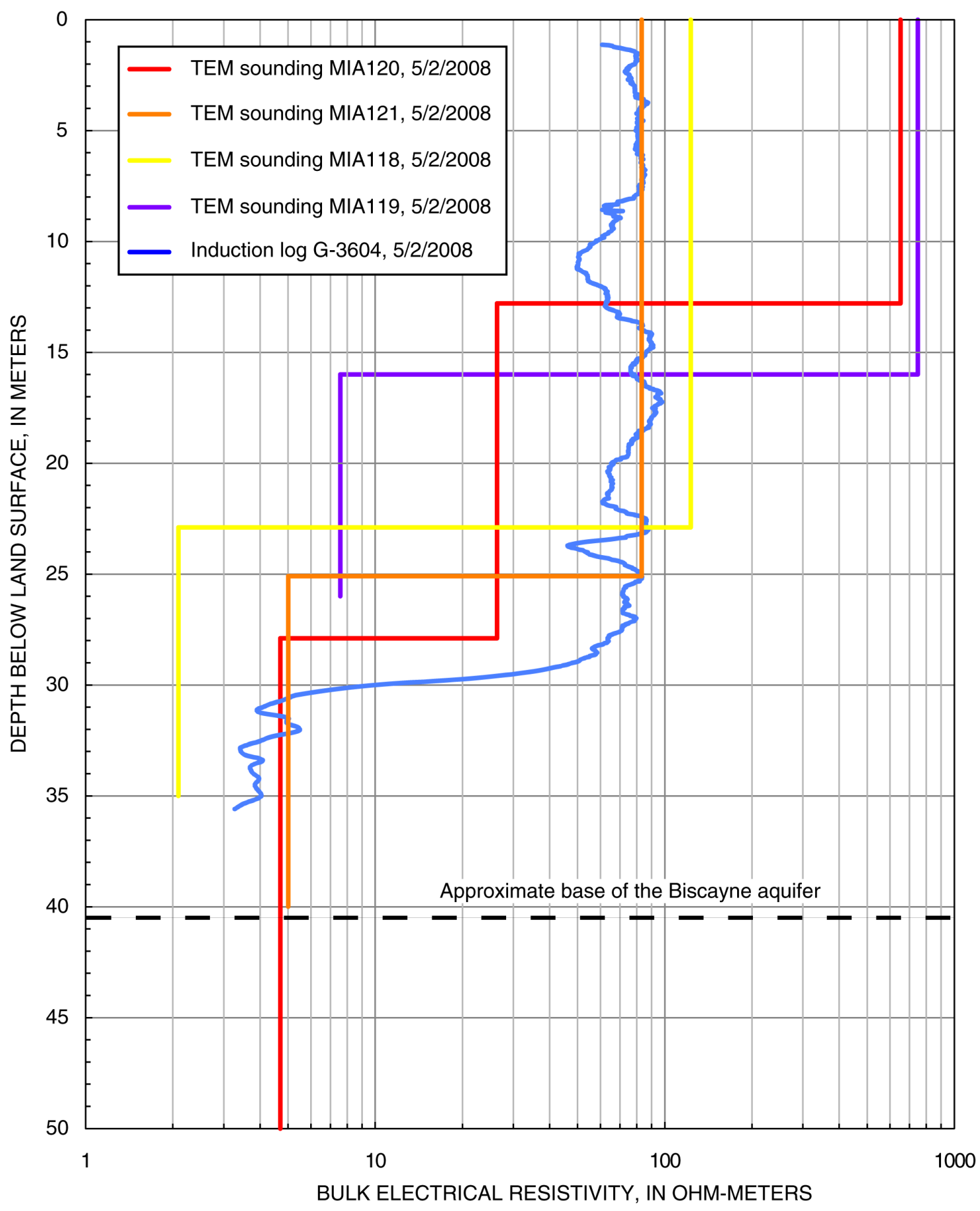

Figure 19. Comparison of TEM soundings MIA118, MIA119, MIA120, and MIA121 with an induction log from well G-3604. Well G-3604 is approximately $1.63 \mathrm{~km}$ northwest of soundings MIA118 and MIA119, and $1.63 \mathrm{~km}$ south of soundings MIA120 and MIA121. The specific conductance of water sampled from well G-3604 on May 2, 2008 was 12,800 $\mu \mathrm{S} / \mathrm{cm}$. The base of the aquifer at the well was estimated from Fish and Stewart (1991) and adjusted for the land-surface elevation of the area. 
the induction log from well G-3604 even though the sites are separated by $1.63 \mathrm{~km}$. The only significant difference between the model MIA121 and the G-3604 induction log is that the modeled depth to the saltwater interface is $5 \mathrm{~m}$ shallower than in the well log. This is reasonable because sounding MIA121 is much closer to the Miami Canal (C-8), which is one of the main sources of the saltwater found in the aquifer in this area. Model MIA120 has three layers, of which the second and third layers show good agreement with the induction log in terms of locating the top of the saltwater zone. The third layer resistivity of model MIA120 agrees quite well with the induction$\log$ values below $31 \mathrm{~m}$ depth. The first layers of models MIA118, MIA119, and MIA120 are more resistive than the uppermost $29 \mathrm{~m}$ of the induction log. For models MIA119 and MIA120, the difference is close to an order of magnitude. Water samples collected from well G-3604 on May 2, 2008 indicated a specific conductance of $12,800 \mu \mathrm{S} / \mathrm{cm}$ and a chloride content of $4,100 \mathrm{mg} / \mathrm{L}$.

Well G-2903 / Soundings MIA114 and MIA115

Models of TEM soundings MIA114 and MIA115 are compared to an induction log collected in monitoring well G-2903 (fig. 20), which is $1.6 \mathrm{~km}$ north and $1.1 \mathrm{~km}$ east, respectively, of the sounding locations (fig. 2). Models MIA114 and MIA115 indicate freshwater-saturated sediments underlain by saltwater-saturated sediments. For model MIA114 the transition to saltwater saturation is more gradual requiring an intermediate layer; for model MIA115 the transition is made in one step. The TEM models do not agree very well with the G-2903 induction log; however, the distance between them is rather large. The shallow conductive zone seen in model MIA114 may be the result of saltwater leakage from the Oleta River, which is only $36 \mathrm{~m}$ away. The minimum resistivity of both models is similar to the resistivity seen near the bottom of the well log. Water samples collected from well G-2903 on May 15, 2008 indicated a specific conductance of $26,500 \mu \mathrm{S} / \mathrm{cm}$ and a chloride content of $9,100 \mathrm{mg} / \mathrm{L}$.

The conductive water inferred from the second layer of model MIA115 is much shallower and farther inland than would be expected on the basis of the G-2903 induction log, which is closer to the saltwater front. The source of this shallow conductive water is unknown. TEM sounding MIA135, which is $450 \mathrm{~m}$ west of sounding MIA115, indicates freshwater for the full thickness of the Biscayne aquifer.

\section{Well G-2965 / Sounding MIA241}

The model for TEM sounding MIA241 is compared to the induction log collected in monitoring well G-2965 (fig. 21), which is 310 m east-northeast of sounding MIA241 (fig. 2). Between the year 2005, when well G-2965 was installed, and 2010, the sampled chloride content in the well increased from 410 to $1,190 \mathrm{mg} / \mathrm{L}$. The saltwater front is currently passing under the local, water-supply well field immediately east of sounding MIA241. On May 1, 2009, when the induction log was collected in well G-2965, the sampled specific conductance in the well was $2,440 \mu \mathrm{S} / \mathrm{cm}$, and the chloride content was $620 \mathrm{mg} / \mathrm{L}$. Sounding MIA241 was collected in a field just west of the well field. The high resistivity of the first two layers of model MIA241 indicates the Biscayne aquifer is fresh throughout its full thickness. The resistivity of the second of these layers is in pretty good agreement with the well log. The low resistivity third layer indicates saltwater-saturated sediments are present about $12.1 \mathrm{~m}$ below the base of the Biscayne aquifer. 


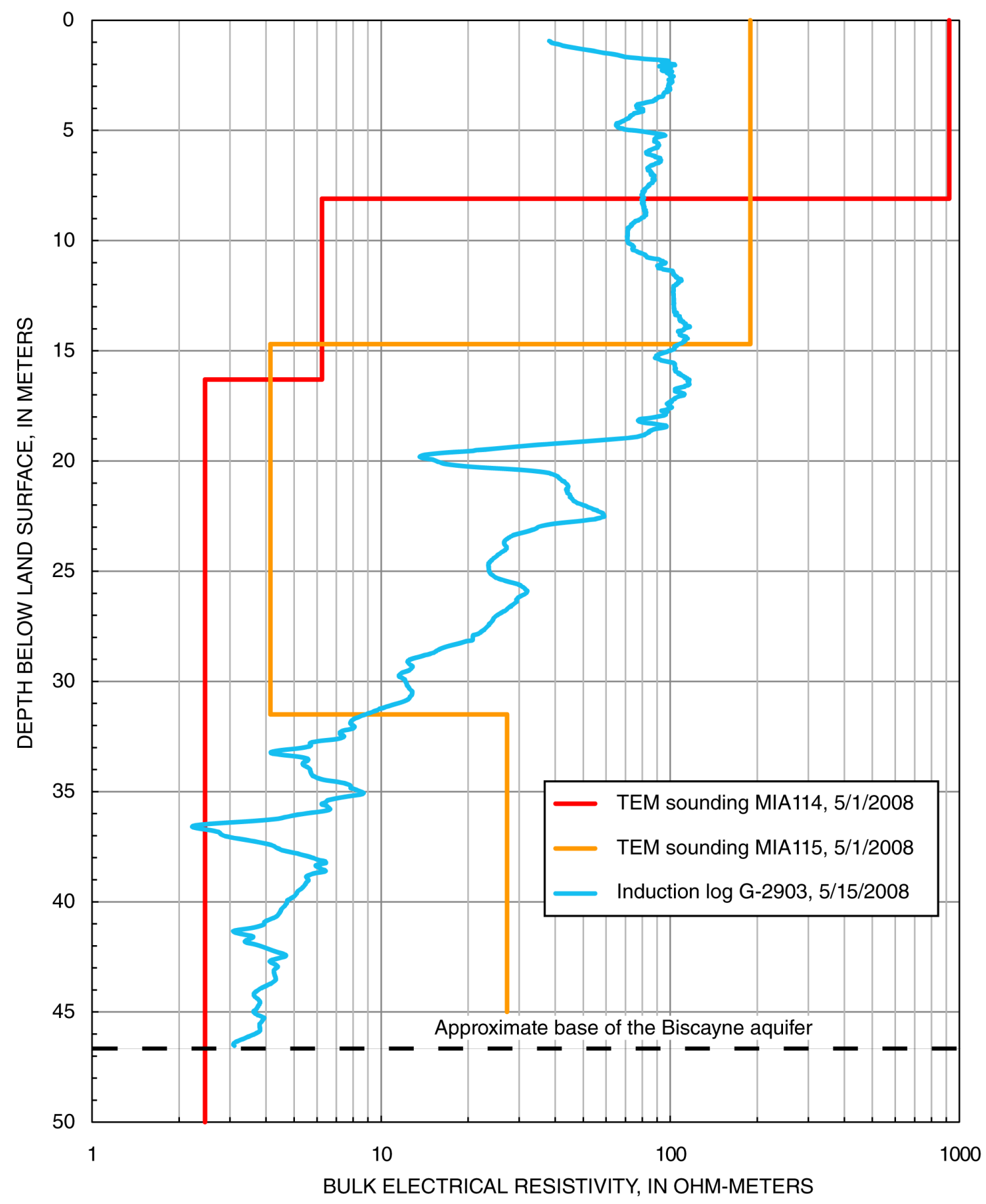

Figure 20. Comparison of TEM sounding MIA114 and MIA115 with an induction log from well G-2903. Well G-2903 is approximately $1.6 \mathrm{~km}$ north of sounding MIA114, and $1.1 \mathrm{~km}$ east of MIA115. The specific conductance of water sampled from well G-2903 on May 2, 2008 was 26,500 $\mu \mathrm{S} / \mathrm{cm}$. The base of the aquifer at the well was estimated from Fish and Stewart (1991) and adjusted for the land-surface elevation of the area. 


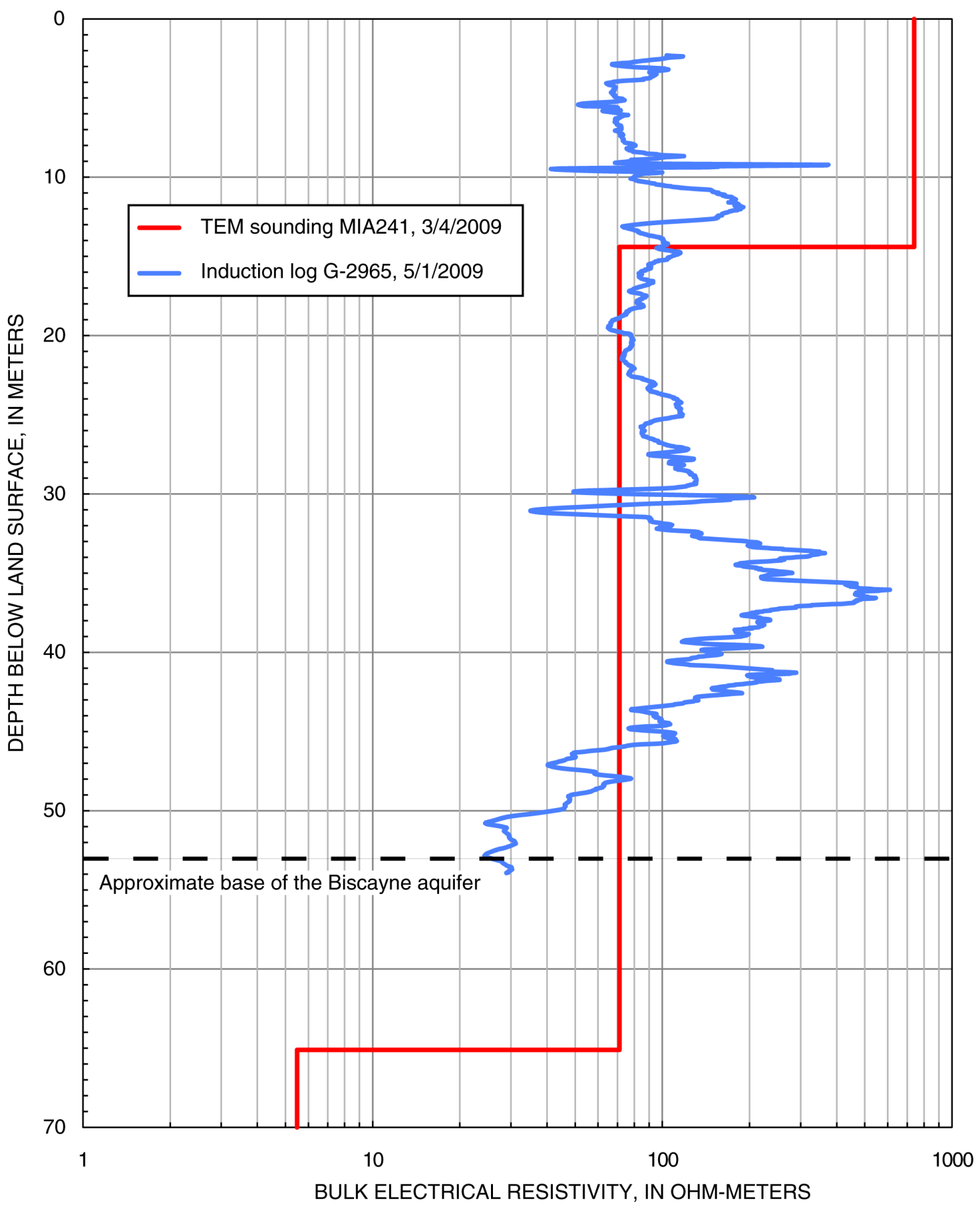

Figure 21. Comparison of TEM sounding MIA241 with an induction log from well G-2965. Well G-2965 is approximately $310 \mathrm{~m}$ east-northeast of sounding MIA241. The specific conductance of water sampled from well G-2965 on May 1, 2009 was 2,440 mS/cm. The base of the aquifer at the well was estimated from Fish (1988) and adjusted for land-surface elevation of the area. 
Well G-2478 / Soundings MIA238 and MIA239

The modeled soundings MIA238, MIA239, and MIA239X are compared to an induction log collected in monitoring well G-2478 (fig. 22), which is $142 \mathrm{~m}$ north of sounding MIA238 and $36 \mathrm{~m}$ southeast of sounding MIA239. Two models are shown for sounding MIA239: The first is a three-layer model (MIA239), for which the last nine data points were masked, and the second is a four-layer model (MIA239X) that used all data points. The resistivities of the first layer of models MIA239 and MIA239X are higher than the induction-log values. The resistivity of the second layer of model MIA239X is a bit lower than the average induction-log resistivity in the depth range of 48 to $57 \mathrm{~m}$; it is also lower than the second layer resistivity for model MIA239. The depth to the top of the third layer of model MIA239X is very close to the estimated depth of the Biscayne aquifer in this area, while the third layer of model MIA239 is a few meters below the base of the aquifer. Given these comparisons either model is reasonable. One of the reasons for preferring model MIA239 is that the resistivity of the fourth layer of model MIA239X is unusually conductive and could possibly be caused by interference from a metallic object, such as a pipe or a nearby fence. The fourth layer of model MIA241X, which is nearby, was also very conductive. The MIA241 site appeared to be free of fences and buried objects. The depths to the top of the conductive fourth layer for both models MIA239X and MIA241X are in close agreement with the depth of the green clay, silt, limestone, and fine sand sediments of the undifferentiated Tamiami and Hawthorn Formations (Fish, 1988). These dense marine clays may also account for the unusually low resistivities at depth at this location.

Sounding MIA238 was farther away from well G-2478 than sounding MIA239. The depth to the top of the third layer of model MIA238 agrees fairly well with the depth at which the bulk resistivity in well G-2478 decreases from 90 to about $30 \mathrm{ohm}-\mathrm{m}$. The resistivities of the first and second layer of model MIA238 are higher and lower, respectively, than the corresponding induction-log values.

\section{Acknowledgments}

The authors would like to acknowledge the contributions of Brian Banks, Adrian Castillo, Eric Carlson, and Jeff Robinson of the USGS and Jorge Corrales of the Miami-Dade County Public Schools who helped collect the TEM measurements. Bob Brown of the Archdiocese of Miami; Edward Swankon of EAS Engineering; Michael Alexander of the Homestead-Miami Speedway; Dr. Claudius Carnegie, Jorge Corrales, and Greg Mohr of the Miami-Dade County Public Schools; Maria Macfarlane, Sonia Villamil, and Virginia Walsh of the Miami-Dade County Water and Sewer Department; and Steve Krupa of the South Florida Water Management District were instrumental in helping us gain access to various properties where the TEM measurements were conducted. The authors greatly appreciate the cooperation of the property owners without whose consent none of these measurements could have been made, including the AA Baker Group LTD; the Archdiocese of Miami; Alamo Rent A Car; Barry University; FRS Holdings Inc.; the City of Homestead; Homestead-Miami Speedway; Hallandale Beach Elementary School; Miami-Dade County Department of Parks, Recreation, and Open Spaces; Miami-Dade County Public Schools; Palmer Trinity School; and the South Florida Water Management District. The authors also appreciate the technical reviews of Maria Deszcz-Pan and Carole Johnson of the USGS who improved this document by providing substantive comments and suggestions. 


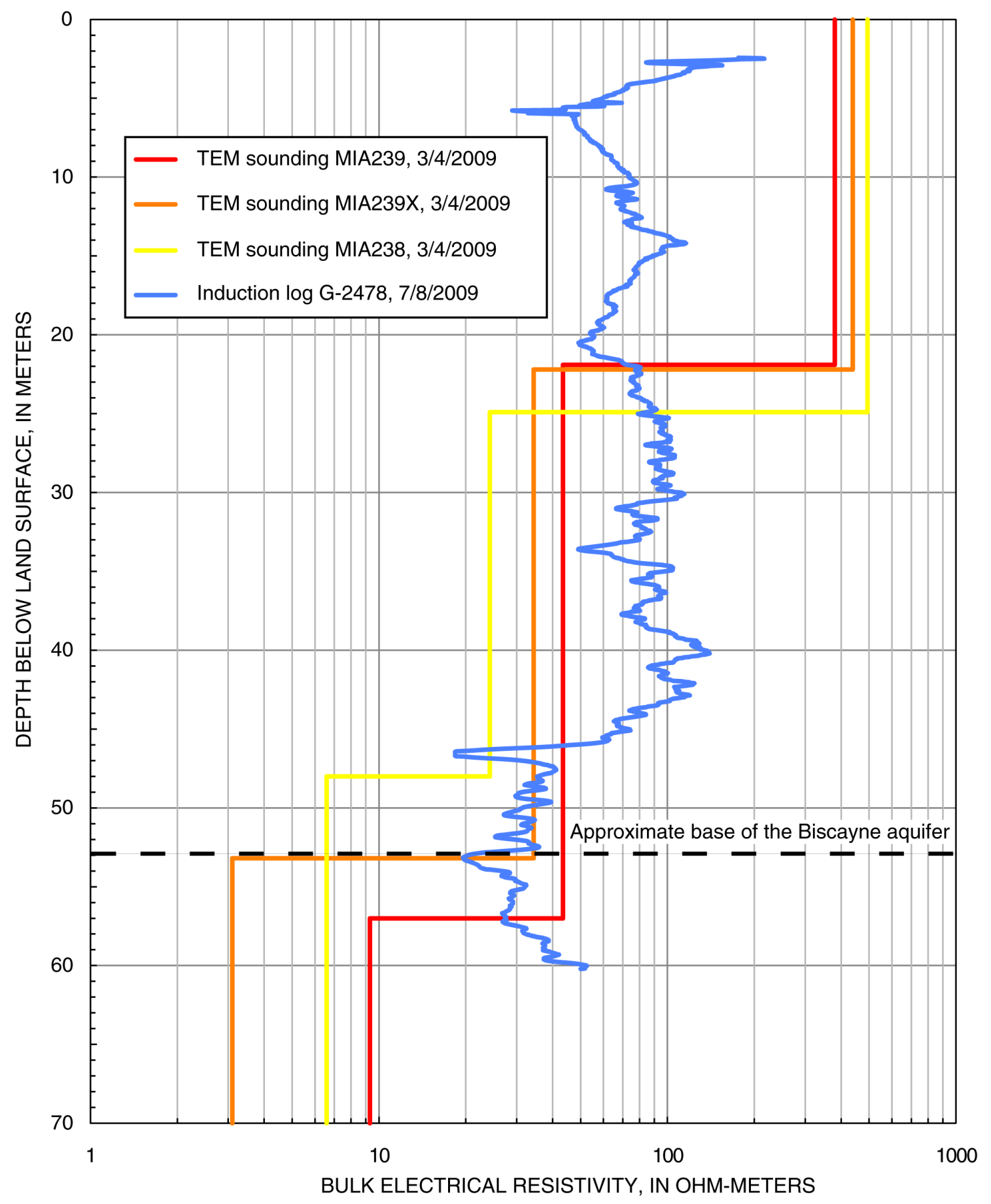

Figure 22. Comparison of TEM soundings MIA238 and MIA239 with an induction log from well G-2478. Well G-2478 is approximately $142 \mathrm{~m}$ north of sounding MIA238, and $36 \mathrm{~m}$ southeast of MIA239. Model MIA239X is an alternative model which uses all later time data that were masked for model MIA239. The specific conductance of water sampled from well G-2478 on July 8, 2009 was $2,640 \mathrm{mS} / \mathrm{cm}$. The base of the aquifer at the well was estimated from Fish (1988) and adjusted for land-surface elevation of the area. 


\section{References Cited}

Archie, G.E., 1942, The electrical resistivity log as an aid to determining some reservoir characteristics: Transactions of the AIME, v. 146, p. 54-62.

Brown, R.H., and Parker, G.G., 1945, Salt water encroachment in limestone at Silver Bluff, Miami, Florida: Economic Geology, v. 60, no. 4, p. 235-262.

Cunningham K.J., Sukop M.C., Huang, H., Alvarez, P.F., Curran, H.A., Renken, R.A., and Dixon, J.F, 2009, Prominence of ichnologically influenced macroporosity in the karst Biscayne aquifer-Stratiform "super K" zones: Geological Society of America Bulletin, v. 121, no. 1/2, p. 164-180.

Cunningham, K.J., Wacker, M.A., Robinson, E., Dixon, J.F., and Wingard, G.L. 2006, A cyclostratigraphic and borehole-geophysical approach to development of a three-dimensional conceptual hydrogeologic model of the karstic Biscayne aquifer, southeastern Florida: U.S. Geological Survey Scientific Investigations Report 2005-5235, 75 p., 4 sheets.

Fish, J.E., 1988, Hydrogeology, aquifer characteristics, and groundwater flow of the surficial aquifer system, Broward County, Florida: U.S. Geological Survey Water-Resources Investigations Report 87-4034, 80 p.

Fish, J.E., and Stewart, M., 1991, Hydrogeology of the surficial aquifer system, Dade County, Florida: U.S. Geological Survey Water-Resources Investigations Report 90-4108, 50 p, 11 sheets.

Fitterman, D.V., 1989, Detectability levels for central induction transient soundings: Geophysics, v. 54, p. 127-129.

Fitterman, D.V., and Deszcz-Pan, M., 1998, Helicopter EM mapping of saltwater intrusion in Everglades National Park, Florida: Exploration Geophysics, v. 29, p. 240-243.

Fitterman, D.V., Deszcz-Pan, M., and Stoddard, C.E., 1999, Results of time-domain electromagnetic soundings in Everglades National Park, Florida (on CD-ROM): U.S. Geological Survey Open-File Report 99-426, 152 p.

Fitterman, D., and Labson, V.F., 2005, Electromagnetic induction methods for environmental problems, in Butler, D.K., ed., Near surface geophysics, part 1-Concepts and fundamentals: Tulsa, Society of Exploration Geophysicists, p. 295-349.

Fitterman, D.V., and Stewart, M.T., 1986, Transient electromagnetic sounding for groundwater: Geophysics, v. 51, p. 995-1005.

Goldman, M., Gilad, D., Ronen, A., and Melloul, A., 1991, Mapping seawater intrusion into the coastal aquifer of Israel by the time domain electromagnetic method: Geoexploration, v. 28, p. 153-174.

Hoy, N.D., 1952, Two maps of Dade County, Florida, showing approximate position of the 1,000ppm isochlor as of 1951 and approximate area of potential salt-water penetration under 1945 conditions: U.S. Geological Survey Open-File Report 52-78.

Kaufman, A.A., and Keller, G.V., 1983, Frequency and transient sounding: Amsterdam, Elsevier, $685 \mathrm{p}$.

Keller, G.V., and Frischknecht, F.C., 1966, Electrical methods in geophysical prospectingInternational Series of Monographs in Electromagnetic Waves: Oxford, Pergamon, 519 p.

Klein, H., 1957, Salt-water encroachment in Dade County, Florida: Florida Geological Survey Information Circular 9, $5 \mathrm{p}$. 
Klein, H., and Ratzlaff, K.W., 1989, Changes in saltwater intrusion in the Biscayne aquifer, Hialeah-Miami Springs area, Dade County, Florida: U.S. Geological Survey Water-Resources Investigations Report 87-4249, 1 sheet.

Klein, H., and Waller, B.G., 1985, Synopsis of saltwater intrusion in Dade County, Florida, through 1984: U.S. Geological Survey Water-Resources Investigations Report 85-4101, 1 sheet.

Kohout, F.A., and Hoy, N.D., 1963, Some aspects of sampling salty ground water in coastal aquifers: Ground Water, v. 1, no. 1, p 28-32.

Koszalka, E.J., 1994, Delineation of saltwater intrusion in the Biscayne aquifer, eastern Broward County Florida, 1990: U.S. Geological Survey Water-Resources Investigations Report 93-4164, 1 sheet.

Leach S.D., and Grantham, R.G., 1966, Salt-water study of the Miami River and its tributaries, Dade County, Florida: Florida Geological Survey Report of Investigations No. 45, 35 p.

Marella, R.L., 1993, Public-supply water use in Florida, 1990: U.S. Geological Survey Open-File Report 93-134, 46 p.

McNeill, J.D., 1990, Use of electromagnetic methods for groundwater studies, in Ward, S.H., ed., Geotechnical and environmental geophysics: Tulsa, Society of Exploration Geophysicists, p. 191-218.

Meyer, F.W., 1974, Availability of ground water for the U.S. Navy well field near Florida City, Dade County, Florida: U.S. Geological Survey Open-File Report 74-1072, 50 p.

Miller, J.A., 1990, Ground water atlas of the United States, Segment 6 -Alabama, Florida, Georgia, and South Carolina: U.S. Geological Survey Hydrologic Investigations Atlas 730-G, 28 p.

Oldenburg, D.W., and Li, Y., 2005, Inversion for applied geophysics-A tutorial, in Butler, D.K., ed., Near surface geophysics, Part 1 - Concepts and fundamentals: Tulsa, Society of Exploration Geophysicists, p. 89-150.

Parker, G.G., 1945, Salt water encroachment in southern Florida: Journal of the American Water Works Association, v. 37, no. 6, p. 526-542.

Parker, G.G., Ferguson, G.E., Love, S.K., and others, 1955, Water resources of southeastern Florida, with special reference to the geology and ground water of the Miami area: U.S. Geological Survey Water-Supply Paper 1255, 965 p.

Prinos, S.T., ed., 2011, Approximate inland extent of the saltwater interface in the Biscayne aquifer in 2011, Miami-Dade County, FL: U.S. Geological Survey Arc-GIS shapefile available through World Wide Web: <http://www.envirobase.usgs.gov/FLIMS/SaltFront/viewer.htm>

Prinos, S.T., Lietz, A.C., and Irvin, R.B., 2002, Design of a real-time ground-water level monitoring network and portrayal of hydrologic data in southern Florida: U.S. Geological Survey Water-Resources Investigations Report 01-4275, 108 p.

Renken, R.A., Dixon, J., Koehmstedt, J., Ishman, S., Lietz, A.C., Marella, R.L., Telis, P., Rodgers, J., and Memberg, R., 2005, Impact of anthropogenic development on coastal ground-water hydrology in southeastern Florida, 1900-2000: U.S. Geological Survey Circular 1275, 77 p.

Sonenshein, R.S., 1997, Delineation and extent of saltwater intrusion in the Biscayne aquifer, eastern Dade County, Florida 1995: U.S. Geological Survey Water-Resources Investigation Report 96-4285, 1 sheet.

Sonenshein, R.S., and Koszalka, E.J., 1996, Trends in water-table altitude (1984-1993) and saltwater intrusion (1974-1993) in the Biscayne aquifer, Dade County, Florida: U.S. Geological Survey Open-File Report 95-705, 2 sheets.

Swayze, L.J., 1980, Water-level contour and salt front map, Hialeah-Miami Springs well field area, Dade County, Florida, October 13, 1978: U.S. Geological Survey Open-File Report 80-8, 1 sheet. 
THIS PAGE INTENTIONALLY LEFT BLANK. 


\section{Appendix 1 Description of TEM Data Processing}

Processing TEM data includes the following steps: (1) downloading, (2) averaging, (3) inversion, (4) extracting results, (5) plotting results, and (6) report generation. Figure 1-1 summarizes the data flow and processing programs used.

The data in the Geonics PROTEM receiver are downloaded to a PC using program PROTEM, which is supplied by Geonics. Typically, data from one sounding location are downloaded into a single raw data file (filename extension .TEM). The format of this file is referred to as Geonics TEM File (GTF) format. Following downloading, selected data records from the raw data file are averaged using program NTEMAVG ${ }^{15}$. Data are usually selected to include measurements made with the same receiver gain and integration time, though this is not mandatory. The averaged data files (filename extension .TAV) are saved in GTF format. In addition to the averaged data file, a file containing a summary of the averaging process is saved (filename extension .PRV).

Both the raw and averaged data files can be read into TEMIXXL, a program commercially available from Interpex Ltd., that was used for data interpretation. TEMIXXL stores a copy of the data, the model, and the calculated response of the model in a proprietary, binary database file (filename extension .TX3). The database can hold a large number of soundings, so one database is usually enough for an entire survey. Sometimes it is helpful to retain several alternative models for a given data set. These are stored as separate soundings in the TEMIXXL database. Alternative models are given the original sounding name with up to three characters added at the end. For example a sounding called ABC101 might have variants ABC101L4 and ABC101L5 to indicate models with four and five layers, respectively. Soundings that had a layer resistivity fixed were given names with an $\mathrm{F}$ in them, such as $\mathrm{ABC} 101 \mathrm{~F} 3$. There are no restrictions on the extra characters added to the sounding name other than that the total number of characters in the sounding name cannot exceed eight. The results of the inversion are reported in an inversion output file (filename extension .INV).

Program TEM_EXTRACT is used to extract apparent-resistivity-time (filename extension .ROT) and interpreted-resistivity-depth (filename extension .ROZ) files for plotting. After the interpretation process is completed, a report file (filename extension .RPT) is generated using program TEM_REPORT. This file contains all of the information about the sounding parameters, the data values, the model parameters, and the model response. There is adequate information in this file to replicate and verify the results using another TEM modeling program.

${ }^{15}$ Unpublished programs NTEMAVG, TEM_EXTRACT, and TEM_REPORT were developed at the USGS. 


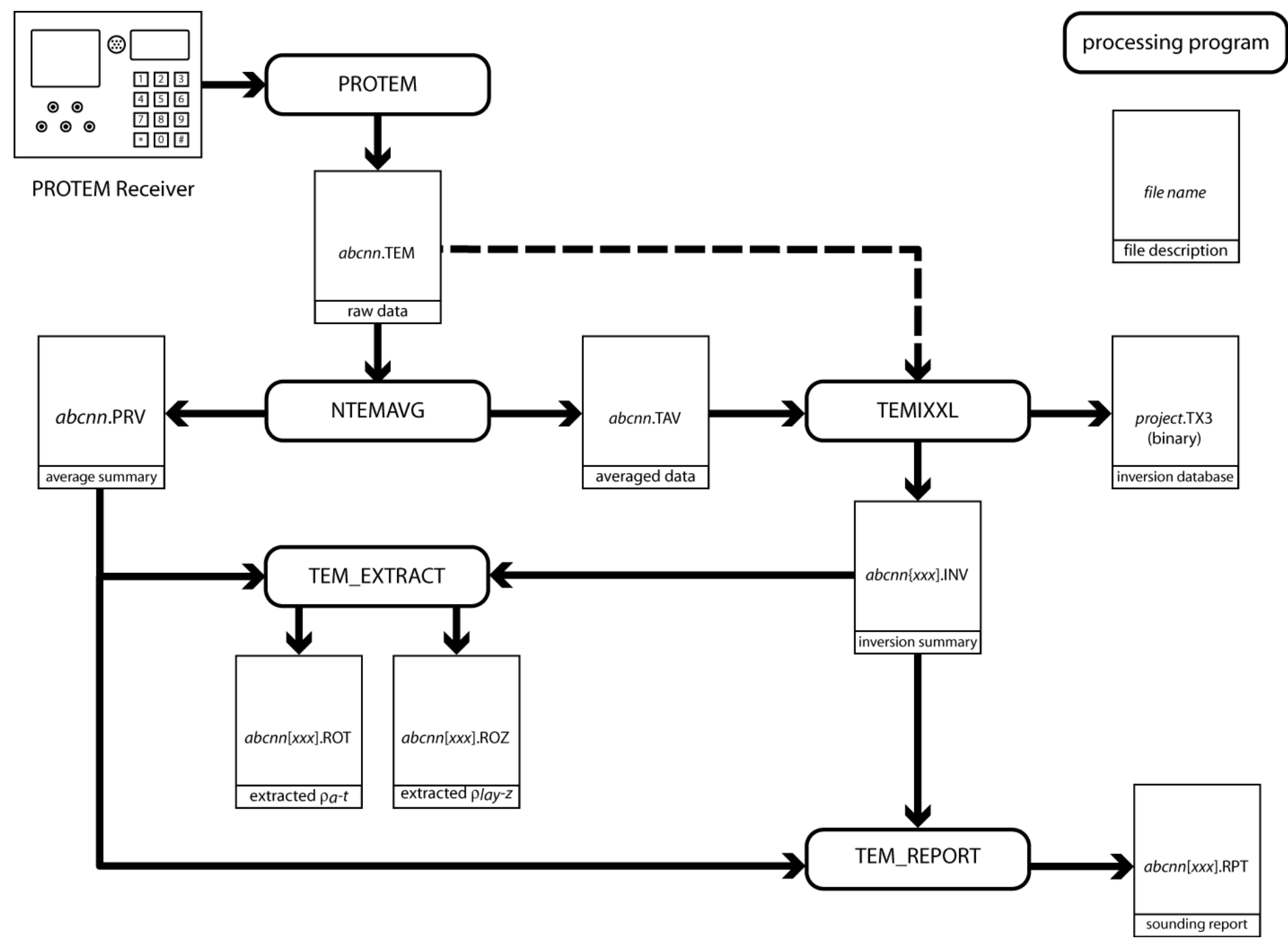

Figure 1-1. Data processing programs, files, and flow. Functions of the programs are described above. A description of the file formats in given in Appendix 2. Solid lines show processing flow. The dashed line shows an alternative processing path. Program PROTEM is available from Geonics Limited. Program TEMIXXL is available from Interpex Limited. Programs NTEMAVG, TEM_EXTRACT, and TEM_REPORT were developed at the USGS. 


\section{Appendix 2 Description of TEM Data Files}

The file naming conventions and formats of files generated during downloading, processing, inversion, and extraction are described below.

\section{File Naming Conventions}

The downloaded GTF format files are typically given a name of the form aaannn.TEM, where $a a a$ is a three-character identifier, typically associated the survey and $n n n$ is a three-digit number. The sounding "name" would be considered aaannn. Geonics and some software vendors give the GTF files an extension of .RED.

Averaged data files are named aaannn.TAV. These files are also in GTF format. The printed summary of the averaging process is written to a file named aaannn.PRV

TEMIXXL database files are binary files in a proprietary format. These files have a filename extension of .TX3. Data sets include observed data, measurement parameters, calculated model response, and inversion model parameters. A data set is identified by a name of up to 8 characters. The data-set identifiers are the same as the sounding names, unless it is an alternative model, in which case additional letters are added after the sounding name. The additional letters usually indicate the number of layers in the model (for example, L3, for a three-layer model, or F4 for a four-layer model with a layer parameter fixed).

A summary of the inversion is written to a file name aaannn $[x x]$.INV, where $[x x]$ represents optional characters added to the sounding name in TEMIXXL to identify alternative models.

Extracted apparent-resistivity-time and interpreted-resistivity-depth files suitable for plotting are named aaannn $[x x]$.ROT and aaannn $[x x]$.ROZ, respectively.

\section{File Formats and Contents}

\section{Geonics TEM File Format}

The raw data collected in the field are written in Geonics TEM file (GTF) format. GTF files consist of 256-character long records containing a 50-character header field followed by 25 eightcharacter data fields. The last two characters of the record are a carriage return and a line feed. Two types of records are created during PROTEM receiver downloading. The record type is indicated by characters 19-21 of the header field: "HDR" for a header record and "OPR" for a data record. A header record is created every time a change is made in certain measurement parameters in the PROTEM-D receiver. For example, changes in the sounding name, transmitter current, transmitter loop size, turnoff time, or receiver moment will produce a new header record.

The raw data files from this survey can be found in directory raw_data_files with the accompanying data.

\section{Digital PROTEM-D File Format}

Figure 2-1 shows the structure of the digital PROTEM-D receiver header fields for header (HDR) and data (OPR) records; descriptions for these two types of header fields are given in tables $2-1$ and $2-2$, respectively.

The data fields of header and data records contain 25 eight-character data fields. Geonics' documentation refers to these data fields as gates. The contents of the gates is given in tables $2-3$ and 2-4 for header and data records, respectively. 


\section{Column number}

$1 \quad 2 \quad 3 \quad 4$

123456789012345678901234567890123456789012345678

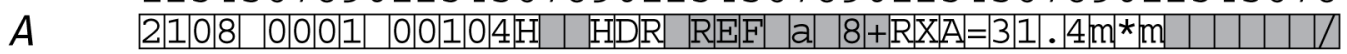

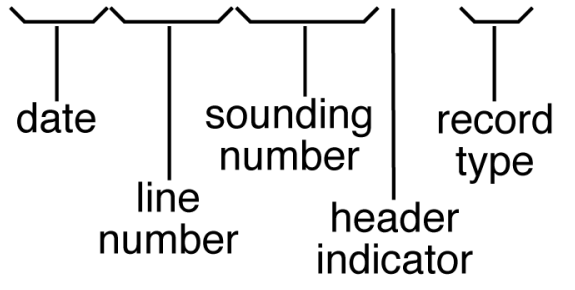

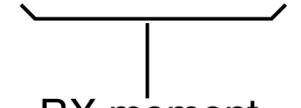

RX moment

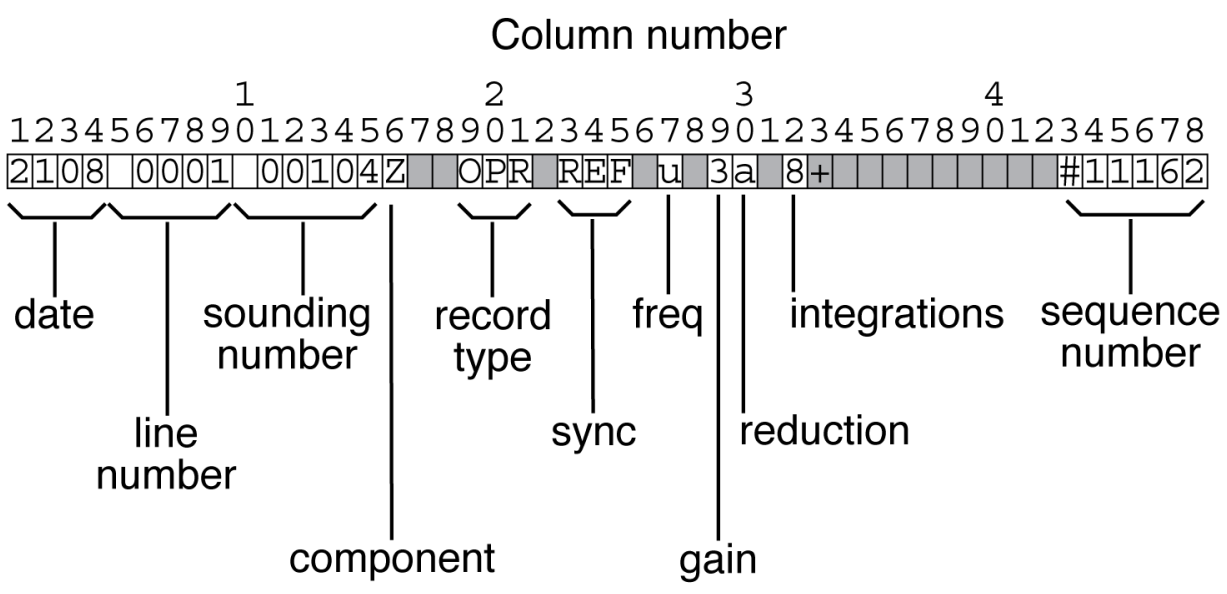

Figure 2-1. Diagram showing contents of the digital PROTEM-D receiver header fields. A, Header record (HDR) header fields. B, Data record (OPR) header fields. The contents of shaded cells are ignored in further processing.

Table 2-1. Description of the digital PROTEM-D receiver header record (HDR) header fields.

\begin{tabular}{|c|c|c|}
\hline Field & Columns & Description \\
\hline date & $1-4$ & $\begin{array}{l}\text { date of measurement; usually [ddmm], but } \\
\text { format depends on particular receiver used }\end{array}$ \\
\hline line number & $5-9$ & $\begin{array}{l}\text { set by operator; often equal to the } \\
\text { sounding number }\end{array}$ \\
\hline sounding number & $10-15$ & set by operator to identify the sounding \\
\hline header indicator & & $H$ to indicate header record \\
\hline record type & $19-21$ & $H D R$ to indicate header record \\
\hline RX moment & $31-41$ & RX coil moment [turn- $\mathrm{m}^{2}$ ] \\
\hline
\end{tabular}


Table 2-2. Description of the digital PROTEM-D receiver data record (OPR) header fields.

\begin{tabular}{|c|c|c|}
\hline Field & Columns & Description \\
\hline date & $1-4$ & $\begin{array}{l}\text { date of measurement; usually [ddmm], but } \\
\text { format depends on particular receiver used }\end{array}$ \\
\hline line number & $5-9$ & $\begin{array}{l}\text { set by operator; often equal to the sounding } \\
\text { number }\end{array}$ \\
\hline sounding number & $10-15$ & $\begin{array}{l}\text { sequence number of sounding; requires } \\
\text { operator to manually update otherwise values } \\
\text { will not be significant }\end{array}$ \\
\hline component & 16 & indicates measured component: $X, Y$, or $Z$ \\
\hline record type & $19-21$ & $O P R$ to indicate data record \\
\hline sync & $23-25$ & $\begin{array}{l}\text { synchronization method: } R E F \text { indicates } \\
\text { reference cable, } X T L \text { indicates crystal } \\
\text { oscillators }\end{array}$ \\
\hline freq & 27 & $\begin{array}{l}\text { transmitter repetition frequency; determines } \\
\text { measurement times: } u=\text { ultra high, } v=\text { very } \\
\text { high, } h=\text { high, } m=\text { medium, } l=\text { low }\end{array}$ \\
\hline gain & 29 & $\begin{array}{l}\text { coded PROTEM adjustable gain } G \text { (gain } \\
\text { factor is } 2^{\mathrm{G}} \text { ) }\end{array}$ \\
\hline reduction & 30 & $\begin{array}{l}\text { value of } a \text { indicates that receiver } 4 \mathrm{x} \text { and } 10 \mathrm{x} \\
\text { gain factors have been removed }\end{array}$ \\
\hline integrations & & PROTEM coded integration time setting \\
\hline sequence number & $43-48$ & sequence number of data record [\#nnnnn] \\
\hline
\end{tabular}

Table 2-3. Description of the digital PROTEM-D receiver header record (HDR) data fields (gates).

\begin{tabular}{|c|c|}
\hline Field (Gate) & Contents \\
\hline 0 & date of measurement [mmdd] \\
\hline 2 & time of measurement [hhmm] \\
\hline 4 & TX current $[\mathrm{A}]$ \\
\hline 5 & TX turnoff time $[\mu \mathrm{s}]$ \\
\hline 6,7 & TX loop side length $L X[\mathrm{~m}]$ and $L Y[\mathrm{~m}]$ \\
\hline 8,9 & $\begin{array}{l}\mathrm{RX} \text { coil position } X R[\mathrm{~m}] \text { and } Y R[\mathrm{~m}] \text { relative to center of } \mathrm{TX} \\
\quad \text { loop }\end{array}$ \\
\hline 10 & $\mathrm{RX}$ coil moment $R X A$ [turn- $\left.{ }^{2}\right]$ \\
\hline 11 & TX number $(37,47$, or 57$)$ \\
\hline 22 & logger record number for this header record \\
\hline
\end{tabular}

Table 2-4. Description of the digital PROTEM-D data record (OPR) data fields (gates).

\begin{tabular}{ll}
\hline \multicolumn{1}{c}{ Field (Gate) } & \multicolumn{1}{c}{ Contents } \\
\hline 0 & primary field value \\
$1-20$ & channels 1-20 TEM data [mV] \\
21 & TX turnoff time [ms] \\
22 & first RX gate time [ms] \\
23 & TX current [A] \\
24 & TX moment [turn- $\left.\mathrm{m}^{2}\right]$, may include trailing "/" \\
25 & time of measurement [hhmm] \\
\hline
\end{tabular}




\section{PRV Files}

Printed voltage summaries from the data averaging process are found in the PRV files. The first page of the file consists of a summary of all of the header record (HDR) and data record (OPR) header fields. The first line is a descriptive header indicating that the data came from a PROTEM logger ("Data from Geonics TEM58 RX"). The second line usually contains HDR header fields. Subsequent lines are OPR header fields associated with the recorded data. Additional HDR header fields will be present whenever the operator made a change in the header information on the PROTEM receiver. The output concludes with a record whose header field starts with " $X X X X X X$ " to indicate the end of the data processing.

Following the summary page, a separate page is devoted to data records that were averaged together. There is usually one page for each transmitter repetition frequency used for the sounding. The first line of these pages indicates the program version used to average the data, the averagedoutput file name, the record number of the averaged-output file to which this page corresponds, and the GTF format input file name.

Each data page of the PRV file contains a summary of all of the data recorded by the PROTEM-D receiver for a single frequency. For each measurement there is a data record number $(D R N)$, frequency $(F R E Q)$, transmitter current $(C U R)$, receiver gain $(G A I N)$, integrations value (NSTK), transmitter turnoff time $(T / O)$, and a time shift (SHIFT). The time shift is applied during data averaging to adjust for incorrect turnoff time settings during data recording. If no adjustment was made this value will be zero. This part of the output is followed by a list of the SI units of the various reported quantities, the TEM system used, the receiver coil moment $(R X A)$, the transmitter loop dimensions ( $L X$ and $L Y$ ), and the location of the receiver coil with respect to the center of the transmitter loop $(X R$ and $Y R)$.

The next section of the data page gives the channel number and the voltage induced in the receiver coil in units of $\left[\mu \mathrm{V} / \mathrm{m}^{2}\right]$. The average of these voltages is computed and expressed as PROTEM-D receiver units $[\mathrm{mV}]$. The standard deviation as a percentage of the average data value is also reported.

The last section of the data page presents the voltages after they have been transformed to apparent resistivity. The printout includes the channel number, the receiver channel time, the square root of the time in seconds $\left[\mathrm{s}^{1 / 2}\right]$, the late stage apparent resistivity, the average of the resistivity values, and the percentage deviation of the apparent resistivity.

The PRV files from this survey can be found in directory averaged_report_files with the accompanying data.

\section{TAV Files}

The averaged data are contained in TAV files and use the GTF format described above. The header of the first record indicates the type of instrument the data came from and whether the data have been averaged ("Data from Geonics TEM58 RX. -- AVERAGED"). The data fields of this record are all set to zero. The second record is an HDR record with header fields and data fields as described in tables 2-1 and 2-3. Next is an OPR record for each frequency average computed by program NTEMAVG. If different groups of data records were averaged, there will be multiple output records — one for each group. A record whose header field starts with " $X X X X X$ " follows the last OPR record. The numbers in the data fields are meaningless.

The averaged data files from this survey can be found in directory averaged_data_files with the accompanying data. 


\section{INV Files}

This file is a report generated by TEM inversion program TEMIXXL. It provides information on the sounding location, the measurement geometry, the model misfit error, the model parameter estimates, the measured apparent resistivity and calculated model response, and usually a resolution matrix.

Misfit error reported by the TEM inversion program (TEMIXXL) is given as the percentage RMS misfit for voltage data. The apparent resistivity misfit is approximately $2 / 3$ of this value.

The inversion output files from this survey can be found in directory inversion_files with the accompanying data.

\section{ROT and ROZ Files}

The ROZ and ROT files are text files that contain data and models in a form for plotting. The ROT file contains the following information:

The sounding name.

Tab delimited column headings for the data that follow.

Tab delimited time and apparent resistivity data consisting of 6, 9, or 12 columns corresponding to 1,2 , or 3 transmitter repetition frequencies, respectively. Typically the data are given in order of decreasing repetition frequency corresponding to later time. The contents of the columns are specified in table 2-5.

The ROZ files contain the following information:

1. The sounding name.

2. Tab delimited titles for the data that follow: "Depth $(\mathrm{m}) \quad$ Resistivity (ohm-m)".

3. Tab delimited data for plotting a resistivity-depth plot. Depths are given as positive values below the surface. The depth sequence will be: $0, z_{1}, z_{1}, z_{2}, z_{2}, \ldots, z_{n-1}, z_{n-1}, z_{n-1}+$ $\max \left(10,1.2^{*} z_{n-1}\right)$. The resistivity sequence will be $\rho_{1}, \rho_{1}, \rho_{2}, \rho_{2}, \ldots \rho_{n-1}, \rho_{n-1}, \rho_{n}, \rho_{n}$.

The extracted data files (ROT and ROZ) from this survey can be found in directory extracted_data_files with the accompanying data.

Table 2-5. Description of plottable data in ROT files.

\begin{tabular}{lll}
\hline Column $^{16}$ & \multicolumn{1}{c}{ Contents } & \multicolumn{1}{c}{ Description } \\
\hline 1 & time & time of data point after TX turnoff [msec] \\
2 & freq1_avg & averaged apparent resistivity [ohm-m] for FREQ1 \\
3 & freq1_std & standard error in FREQ1 averaged apparent resistivity \\
4 & freq1_cal & calculated apparent resistivity [ohm-m] for FREQ1 \\
5 & freq2_avg & averaged apparent resistivity [ohm-m] for FREQ2 \\
6 & freq2_std & standard error in FREQ2 averaged apparent resistivity \\
7 & freq2_cal & calculated apparent resistivity [ohm-m] for FREQ2 \\
8 & freq3_avg & averaged apparent resistivity [ohm-m] for FREQ3 \\
9 & freq3_std & standard error in FREQ3 averaged apparent resistivity \\
10 & freq3_cal & calculated apparent resistivity [ohm-m] for FREQ3 \\
11 & masked & apparent resistivity of a masked data point \\
12 & m_err & standard error of a masked data point \\
\hline
\end{tabular}


${ }^{16}$ When a sounding has fewer than three base frequencies (or sweeps), the unnecessary columns are removed. The masked and $\mathrm{m}_{-}$err data follow immediately after the last sweep.

RPT Files

The sounding report files are presented in Appendices D and F. The report file contains all relevant information for the sounding and its interpretation summarized on a single sheet. The report files can be found in directory sounding_report_files with the accompanying data.

\section{TEMIXXL Database File}

The TEMIXXL database files MIAMI08.TX3 and MIAMI09.TX3 used for interpretation of the 2008 and 2009 TEM soundings, respectively, can be found in directory TEMIXXL_database_files with the accompanying data. 


\section{Appendix 3 Voltage Units and Apparent Resistivity}

Measured electromagnetic transients can be reported in two different ways: either as the voltage recorded in the receiver itself, or the voltage at the receiver coil normalized by the receivercoil moment (number of coil turns times the coil area). The voltage at the receiver coil can be determined using Faraday's Law

$$
V_{c o i l}=-M_{R X} \frac{d \mathbf{B} \bullet \hat{\mathbf{n}}}{d t}=-M_{R X} \frac{d B}{d t},
$$

where $\mathbf{B}$ is the magnetic induction in units of weber per meter squared $\left[\mathrm{Wb} / \mathrm{m}^{2}\right], \hat{\mathbf{n}}$ is the unit normal to the receiver coil (assumed to be vertical), and $M_{R X}$ is the receiver-coil moment. Rearranging (3-1) gives

$$
V_{c o i l} / M_{R X}=-\frac{d B}{d t}
$$

This representation of the data is convenient for comparison against background electromagnetic noise levels, which are typically $0.1-1 \mathrm{nV} / \mathrm{m}^{2}$ (Fitterman, 1989).

For the PROTEM receiver used in this study the voltage at the coil $V_{c o i l}$ and the voltage recorded at the receiver $V_{R X}$ are related by

$$
V_{\text {coil }}[\mathrm{nV}]=\frac{10^{-6} V_{R X}[\mathrm{mV}]}{52.1 \cdot 2^{G}},
$$

where $G$ is the receiver gain setting (see table 2-2), and the factor 52.1 accounts for additional fixed receiver gain. The magnitude of the time derivative of the magnetic induction is then

$$
\frac{d B}{d t}\left[\mathrm{nV} / \mathrm{m}^{2}\right]=\frac{V_{c o i l}[\mathrm{nV}]}{M_{R X}\left[\mathrm{~m}^{2}\right]}=\frac{10^{-6} V_{R X}[\mathrm{mV}]}{52.1 \cdot 2^{G} M_{R X}\left[\mathrm{~m}^{2}\right]}
$$

The late stage apparent resistivity (Kaufman and Keller, 1983; Fitterman and Labson, 2005) is computed from the voltage induced in the receiver coil

$$
\rho_{a}^{L S}=\frac{\mu_{o}}{4 \pi t}\left[\frac{2 \mu_{o} L^{2} M_{R X} I_{T X}}{5 t V_{\text {coil }}}\right]^{2 / 3},
$$

where $t$ is the time after current turn off, $L$ is the square transmitter loop side length, $I_{T X}$ is the transmitter current, $\mu_{o}=4 \pi \cdot 10^{-7}[\mathrm{H} / \mathrm{m}]$ is the magnetic permeability of free space, and all units are SI. Recasting equation (3-5) into typical field units and incorporating the PROTEM gain constants gives

$$
\rho_{a}^{L S}[\mathrm{ohm}-\mathrm{m}]=\frac{10^{-4}}{t[\mathrm{~ms}]}\left\{\frac{0.16 \cdot \pi \cdot 52.1 \cdot 2^{G} L^{2}\left[\mathrm{~m}^{2}\right] M_{R X}\left[\mathrm{~m}^{2}\right] I_{T X}[\mathrm{~A}]}{t[\mathrm{~ms}] V_{R X}[\mathrm{mV}]}\right\}^{2 / 3}
$$

and

$$
\rho_{a}^{L S}[\mathrm{ohm}-\mathrm{m}]=\frac{10}{t[\mu \mathrm{s}]}\left\{\frac{0.16 \cdot \pi \cdot 52.1 \cdot 2^{G} L^{2}\left[\mathrm{~m}^{2}\right] M_{R X}\left[\mathrm{~m}^{2}\right] I_{T X}[\mathrm{~A}]}{t[\mu \mathrm{s}] V_{R X}[\mathrm{mV}]}\right\}^{2 / 3} .
$$


Appendix 4 TEM Sounding Report Files: 2008 
Sounding: MIA101

Client: US Geological Survey Location: Homestead Speedway ovrflow lot

Project: Saltwater Intrusion

County: Miami-Dade County

TX loop size: X $[\mathrm{m}] \quad 38.1 \quad \mathrm{Y}[\mathrm{m}] \quad 38.1$

$\begin{array}{cccc}\text { Model } & \text { Resistivity } & \text { Thickness } & \text { Elevation } \\ \text { Layer } & {[\text { ohm-m] }} & {[\mathrm{m}]} & {[\mathrm{m}]} \\ 1 & 130.800 & 24.8 & 0.0 \\ 2 & 3.140 & 8.6 & -24.8 \\ 3 & 39.560 & -- & -33.4\end{array}$

System: EM-58 Freq[Hz]: 285 Data Set Code: uh TX $\operatorname{Cur}[A]: \quad 1.40$ Turn Off[usec]: 2.5

RX Moment turns-m^2]: 31.4 Gain Setting:

$\begin{array}{rrrrrr}\text { Time } & \begin{array}{c}\text { rhoa_obs } \\ \text { [ohm-m] }\end{array} & \begin{array}{c}\text { obs_err } \\ {\left[\frac{\mathrm{o}}{0}\right]}\end{array} & \text { mask } & \begin{array}{r}\text { rhoa_cal } \\ \text { [ohm-m] }\end{array} \\ 1 & 0.00680 & 165.7 & 0.4 & \mathrm{u} & 163.3 \\ 2 & 0.00860 & 144.5 & 0.4 & \mathrm{u} & 148.0 \\ 3 & 0.01110 & 123.1 & 0.5 & \mathrm{u} & 125.2 \\ 4 & 0.01410 & 100.0 & 0.4 & \mathrm{u} & 99.9 \\ 5 & 0.01800 & 78.5 & 0.4 & \mathrm{u} & 77.2 \\ 6 & 0.02300 & 60.4 & 0.4 & \mathrm{u} & 59.2 \\ 7 & 0.02940 & 46.6 & 0.4 & \mathrm{u} & 45.7 \\ 8 & 0.03750 & 36.5 & 0.4 & \mathrm{u} & 36.1 \\ 9 & 0.04790 & 29.3 & 0.4 & \mathrm{u} & 29.2 \\ 10 & 0.06110 & 24.3 & 0.4 & \mathrm{u} & 24.4 \\ 11 & 0.07790 & 20.8 & 0.3 & \mathrm{u} & 21.1 \\ 12 & 0.09930 & 18.4 & 0.4 & \mathrm{u} & 18.8 \\ 13 & 0.12670 & 16.8 & 0.4 & \mathrm{u} & 17.2 \\ 14 & 0.16640 & 16.0 & 0.3 & \mathrm{u} & 16.2 \\ 15 & 0.20600 & 15.6 & 0.5 & \mathrm{u} & 15.9 \\ 16 & 0.26280 & 15.8 & 0.7 & \mathrm{u} & 15.9 \\ 17 & 0.33520 & 16.2 & 1.1 & \mathrm{u} & 16.2 \\ 18 & 0.42770 & 17.2 & 2.1 & \mathrm{u} & 17.0 \\ 19 & 0.54560 & 18.2 & 4.0 & \mathrm{u} & 18.1 \\ 20 & 0.69590 & 19.8 & 8.5 & \mathrm{u} & 19.6\end{array}$

Date: 28-APR-08

UTM Coord: $\mathrm{E}[\mathrm{m}]$

Elevation $[\mathrm{m}]$ :

$558727.0 \quad \mathrm{~N}[\mathrm{~m}]$

2814504.0

0.0

RX location: $\mathrm{X}[\mathrm{m}]$

$0.0 \quad \mathrm{Y}[\mathrm{m}]$

0.0

Fit Error $\left[\frac{\circ}{0}\right]: 3.095$

System: EM-58 Freq[Hz]: 30 Data Set Code: hi TX Cur $[A]: \quad 1.40$ Turn Off[usec]: 2.5

RX Moment[turns-m^2]: 31.4 Gain Setting: 7

Time
$[\mathrm{ms}]$
0.08800
0.10600
0.13100
0.16100
0.20000
0.25000
0.31400
0.39500
0.49900
0.63100
0.79900
1.01000
1.28000
1.63000
2.08000
2.64000
3.37000
4.29000
5.47000
6.97000

rhoa_obs
[ohm-m]
19.4
18.0
16.9
16.1
15.8
15.8
16.1
16.8
17.5
18.4
18.7
18.6
21.2
72.4
17.2
23.0
13.7
17.7
12.8
4.4

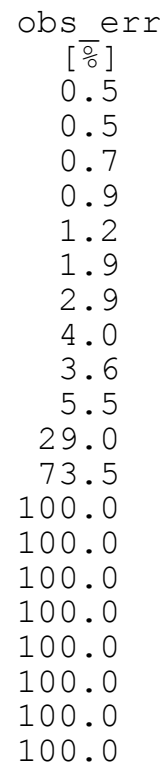

$\begin{array}{lr} & {[\mathrm{ohm}-\mathrm{m}]} \\ \mathrm{u} & 19.7 \\ \mathrm{u} & 18.2 \\ \mathrm{u} & 17.0 \\ \mathrm{u} & 16.2 \\ \mathrm{u} & 15.8 \\ \mathrm{u} & 15.6 \\ \mathrm{u} & 15.8 \\ \mathrm{u} & 16.2 \\ \mathrm{u} & 16.9 \\ \mathrm{u} & 17.7 \\ \mathrm{u} & 18.8 \\ \mathrm{u} & 19.9 \\ \mathrm{u} & 21.2 \\ \mathrm{~d} & - \\ \mathrm{d} & - \\ \mathrm{d} & - \\ \mathrm{d} & - \\ \mathrm{d} & - \\ \mathrm{d} & - \\ \mathrm{d} & -\end{array}$


Sounding: MIA102

Location: Homestead Speedway ovrflow lot

Project: Saltwater Intrusion

County: Miami-Dade County

TX loop size: X $[\mathrm{m}] \quad 38.1 \quad \mathrm{Y}[\mathrm{m}] \quad 38.1$

$\begin{array}{cccc}\text { Model } & \text { Resistivity } & \text { Thickness } & \text { Elevation } \\ \text { Layer } & {[\text { ohm-m] }} & {[\mathrm{m}]} & {[\mathrm{m}]} \\ 1 & 141.400 & 27.0 & 0.0 \\ 2 & 1.570 & 1.3 & -27.0 \\ 3 & 29.810 & -- & -28.4\end{array}$

System: EM-58 Freq[Hz]: 285 Data Set Code: uh TX Cur $[A]: \quad 1.40$ Turn Off[usec]: 2.5

RX Moment[turns-m^2]: 31.4 Gain Setting: 2

$\begin{array}{rrrrrr}\text { Time } & \begin{array}{c}\text { rhoa_obs } \\ \text { [ohm-m] }\end{array} & \begin{array}{c}\text { obs_err } \\ {\left[\frac{\mathrm{o}}{0}\right]}\end{array} & \text { mask } & \begin{array}{r}\text { rhoa_cal } \\ {[\text { ohm-m] }}\end{array} \\ 1 & 0.00680 & 161.0 & 0.3 & \mathrm{u} & 160.2 \\ 2 & 0.00860 & 134.0 & 0.2 & \mathrm{u} & 136.3 \\ 3 & 0.01110 & 110.7 & 0.3 & \mathrm{u} & 110.0 \\ 4 & 0.01410 & 89.3 & 0.2 & \mathrm{u} & 87.9 \\ 5 & 0.01800 & 72.4 & 0.2 & \mathrm{u} & 70.9 \\ 6 & 0.02300 & 59.2 & 0.2 & \mathrm{u} & 58.5 \\ 7 & 0.02940 & 49.4 & 0.2 & \mathrm{u} & 49.5 \\ 8 & 0.03750 & 42.4 & 0.2 & \mathrm{u} & 43.1 \\ 9 & 0.04790 & 37.5 & 0.2 & \mathrm{u} & 38.5 \\ 10 & 0.06110 & 34.2 & 0.2 & \mathrm{u} & 35.2 \\ 11 & 0.07790 & 32.2 & 0.2 & \mathrm{u} & 32.9 \\ 12 & 0.09930 & 30.9 & 0.2 & \mathrm{u} & 31.3 \\ 13 & 0.12670 & 30.4 & 0.3 & \mathrm{u} & 30.3 \\ 14 & 0.16640 & 30.4 & 0.4 & \mathrm{u} & 29.6 \\ 15 & 0.20600 & 30.4 & 0.4 & \mathrm{u} & 29.3 \\ 16 & 0.26280 & 30.7 & 1.0 & \mathrm{u} & 29.3 \\ 17 & 0.33520 & 30.6 & 1.6 & \mathrm{u} & 29.5 \\ 18 & 0.42770 & 30.2 & 2.6 & \mathrm{u} & 29.9 \\ 19 & 0.54560 & 30.0 & 5.1 & \mathrm{u} & 30.7 \\ 20 & 0.69590 & 30.6 & 7.5 & \mathrm{u} & 31.8\end{array}$

Date: $28-\mathrm{APR}-08$

UTM Coord: $E[\mathrm{~m}]$

Elevation $[\mathrm{m}]$ :

$558246.0 \quad \mathrm{~N}[\mathrm{~m}] \quad 2814508.0$ 0.0

RX location: $\mathrm{X}[\mathrm{m}]$

$0.0 \quad \mathrm{Y}[\mathrm{m}] \quad 0.0$

Fit Error [\%]: 3.659

System: EM-58 Freq[Hz]: 30 Data Set Code: hi TX Cur $[A]: \quad 1.40$ Turn Off[usec]: 2.5

RX Moment [turns-m^2]: 31.4 Gain Setting: 7

$\begin{array}{rrrrrr}\text { Time } & \begin{array}{c}\text { rhoa_obs } \\ \text { [ohm-m] }\end{array} & \begin{array}{c}\text { obserr } \\ {\left[\begin{array}{c}\text { o }] \\ \text { [ms }\end{array}\right.}\end{array} & \text { mask } & \begin{array}{c}\text { rhoa_cal } \\ {[\text { ohm-m] }}\end{array} \\ 1 & 0.08800 & 30.7 & 1.0 & \mathrm{~m} & 32.0 \\ 2 & 0.10600 & 29.8 & 0.5 & \mathrm{~m} & 30.9 \\ 3 & 0.13100 & 30.9 & 0.3 & \mathrm{~m} & 30.0 \\ 4 & 0.16100 & 32.0 & 0.8 & \mathrm{~m} & 29.4 \\ 5 & 0.20000 & 29.8 & 0.7 & \mathrm{~m} & 29.0 \\ 6 & 0.25000 & 30.1 & 1.7 & \mathrm{~m} & 28.7 \\ 7 & 0.31400 & 29.4 & 1.7 & \mathrm{~m} & 28.6 \\ 8 & 0.39500 & 28.6 & 2.2 & \mathrm{~m} & 28.5 \\ 9 & 0.49900 & 27.9 & 4.6 & \mathrm{~m} & 28.5 \\ 10 & 0.63100 & 26.4 & 4.9 & \mathrm{~m} & 28.5 \\ 11 & 0.79900 & 25.5 & 9.6 & \mathrm{~m} & 28.6 \\ 12 & 1.01000 & 23.4 & 14.5 & \mathrm{~m} & 28.7 \\ 13 & 1.28000 & 24.3 & 39.8 & \mathrm{~m} & 28.8 \\ 14 & 1.63000 & 31.4 & 70.1 & \mathrm{~m} & 29.0 \\ 15 & 2.08000 & 30.0 & 100.0 & \mathrm{~m} & 29.2 \\ 16 & 2.64000 & 12.7 & 77.8 & \mathrm{~d} & - \\ 17 & 3.37000 & 36.1 & 100.0 & \mathrm{~d} & - \\ 18 & 4.29000 & 16.0 & 100.0 & \mathrm{~d} & - \\ 19 & 5.47000 & 34.8 & 100.0 & \mathrm{~d} & - \\ 20 & 6.97000 & 17.2 & 100.0 & \mathrm{~d} & -\end{array}$


Sounding: MIA102F

Location: Homestead Speedway ovrflow lot

Project: Saltwater Intrusion

County: Miami-Dade County

TX loop size: X[m] $38.1 \quad \mathrm{Y}[\mathrm{m}] \quad 38.1$

$\begin{array}{cccc}\text { Model } & \text { Resistivity } & \text { Thickness } & \text { Elevation } \\ \text { Layer } & {[\text { ohm-m] }} & {[\mathrm{m}]} & {[\mathrm{m}]} \\ 1 & 143.200 & 26.3 & 0.0 \\ 2 & 3.500 & 3.2 & -26.3 \\ 3 & 30.910 & -- & -29.6\end{array}$

System: EM-58 Freq[Hz]: 285 Data Set Code: uh TX $\operatorname{Cur}[\mathrm{A}]: \quad 1.40$ Turn Off [usec]: 2.5

RX Moment[turns-m^2]: 31.4 Gain Setting: 2

$\begin{array}{rrrrrr}\text { Time } & \text { [ms }] & \begin{array}{c}\text { rhoa_obs } \\ \text { [ohm-m] }\end{array} & \begin{array}{c}\text { obserr } \\ {\left[\frac{\mathrm{o}}{0}\right]}\end{array} & \text { mask } & \begin{array}{r}\text { rhoa_cal } \\ \text { [ohm-m] }\end{array} \\ 1 & 0.00680 & 161.0 & 0.3 & \mathrm{u} & 160.0 \\ 2 & 0.00860 & 134.0 & 0.2 & \mathrm{u} & 136.0 \\ 3 & 0.01110 & 110.7 & 0.3 & \mathrm{u} & 110.1 \\ 4 & 0.01410 & 89.3 & 0.2 & \mathrm{u} & 88.0 \\ 5 & 0.01800 & 72.4 & 0.2 & \mathrm{u} & 71.0 \\ 6 & 0.02300 & 59.2 & 0.2 & \mathrm{u} & 58.6 \\ 7 & 0.02940 & 49.4 & 0.2 & \mathrm{u} & 49.5 \\ 8 & 0.03750 & 42.4 & 0.2 & \mathrm{u} & 43.1 \\ 9 & 0.04790 & 37.5 & 0.2 & \mathrm{u} & 38.4 \\ 10 & 0.06110 & 34.2 & 0.2 & \mathrm{u} & 35.1 \\ 11 & 0.07790 & 32.2 & 0.2 & \mathrm{u} & 32.8 \\ 12 & 0.09930 & 30.9 & 0.2 & \mathrm{u} & 31.3 \\ 13 & 0.12670 & 30.4 & 0.3 & \mathrm{u} & 30.2 \\ 14 & 0.16640 & 30.4 & 0.4 & \mathrm{u} & 29.6 \\ 15 & 0.20600 & 30.4 & 0.4 & \mathrm{u} & 29.4 \\ 16 & 0.26280 & 30.7 & 1.0 & \mathrm{u} & 29.4 \\ 17 & 0.33520 & 30.6 & 1.6 & \mathrm{u} & 29.6 \\ 18 & 0.42770 & 30.2 & 2.6 & \mathrm{u} & 30.2 \\ 19 & 0.54560 & 30.0 & 5.1 & \mathrm{u} & 31.0 \\ 20 & 0.69590 & 30.6 & 7.5 & \mathrm{u} & 32.2\end{array}$

Date: $28-\mathrm{APR}-08$

UTM Coord: $\mathrm{E}[\mathrm{m}]$

Elevation $[\mathrm{m}]$ :

$558246.0 \quad \mathrm{~N}[\mathrm{~m}] \quad 2814508.0$ 0.0

RX location: $\mathrm{X}[\mathrm{m}]$

$0.0 Y[\mathrm{~m}] \quad 0.0$
Fit Error $\left[\frac{\circ}{0}\right]: 3.701$

System: EM-58 Freq[Hz]: 30 Data Set Code: hi TX Cur $[A]: \quad 1.40$ Turn Off[usec]: 2.5

RX Moment [turns-m^2]: 31.4 Gain Setting: 7

$\begin{array}{rrrrrr}\text { Time } & \begin{array}{c}\text { rhoa_obs } \\ \text { [ohm-m] }\end{array} & \begin{array}{c}\text { obs_err } \\ {\left[\frac{\text { o }}{0}\right]}\end{array} & \text { mask } & \begin{array}{c}\text { rhoa_cal } \\ {[\text { ohm-m] }}\end{array} \\ 1 & 0.08800 & 30.7 & 1.0 & \mathrm{~m} & 31.9 \\ 2 & 0.10600 & 29.8 & 0.5 & \mathrm{~m} & 30.8 \\ 3 & 0.13100 & 30.9 & 0.3 & \mathrm{~m} & 30.0 \\ 4 & 0.16100 & 32.0 & 0.8 & \mathrm{~m} & 29.4 \\ 5 & 0.20000 & 29.8 & 0.7 & \mathrm{~m} & 29.0 \\ 6 & 0.25000 & 30.1 & 1.7 & \mathrm{~m} & 28.8 \\ 7 & 0.31400 & 29.4 & 1.7 & \mathrm{~m} & 28.7 \\ 8 & 0.39500 & 28.6 & 2.2 & \mathrm{~m} & 28.8 \\ 9 & 0.49900 & 27.9 & 4.6 & \mathrm{~m} & 28.8 \\ 10 & 0.63100 & 26.4 & 4.9 & \mathrm{~m} & 28.9 \\ 11 & 0.79900 & 25.5 & 9.6 & \mathrm{~m} & 29.0 \\ 12 & 1.01000 & 23.4 & 14.5 & \mathrm{~m} & 29.2 \\ 13 & 1.28000 & 24.3 & 39.8 & \mathrm{~m} & 29.4 \\ 14 & 1.63000 & 31.4 & 70.1 & \mathrm{~m} & 29.6 \\ 15 & 2.08000 & 30.0 & 100.0 & \mathrm{~m} & 29.8 \\ 16 & 2.64000 & 12.7 & 77.8 & \mathrm{~d} & - \\ 17 & 3.37000 & 36.1 & 100.0 & \mathrm{~d} & - \\ 18 & 4.29000 & 16.0 & 100.0 & \mathrm{~d} & - \\ 19 & 5.47000 & 34.8 & 100.0 & \mathrm{~d} & - \\ 20 & 6.97000 & 17.2 & 100.0 & \mathrm{~d} & -\end{array}$


Sounding: MIA103

Location: Homestead Speedway ovrflow lot

Project: Saltwater Intrusion

County: Miami-Dade County

TX loop size: X[m] $38.1 \quad \mathrm{Y}[\mathrm{m}] \quad 38.1$

$\begin{array}{cccc}\text { Model } & \text { Resistivity } & \text { Thickness } & \text { Elevation } \\ \text { Layer } & {[\text { ohm-m] }} & {[\mathrm{m}]} & {[\mathrm{m}]} \\ 1 & 708.800 & 16.8 & 0.0 \\ 2 & 34.280 & 68.7 & -16.8 \\ 3 & 8.390 & -- & -85.5\end{array}$

System: EM-58 Freq[Hz]: 285 Data Set Code: uh TX $\operatorname{Cur}[A]: \quad 1.40$ Turn Off[usec] : 2.5

RX Moment [turns-m^2]: 31.4 Gain Setting: 3

$\begin{array}{rrrrrr}\text { Time } & \begin{array}{c}\text { rhoa_obs } \\ \text { [ohm-m] }\end{array} & \begin{array}{c}\text { obs_err } \\ {\left[\frac{\mathrm{o}}{0}\right]}\end{array} & \text { mask } & \begin{array}{r}\text { rhoa_cal } \\ \text { [ohm-m] }\end{array} \\ 1 & 0.00680 & 149.3 & 0.4 & \mathrm{u} & 148.0 \\ 2 & 0.00860 & 128.2 & 0.3 & \mathrm{u} & 126.1 \\ 3 & 0.01110 & 113.4 & 0.4 & \mathrm{u} & 111.0 \\ 4 & 0.01410 & 99.4 & 0.4 & \mathrm{u} & 99.2 \\ 5 & 0.01800 & 88.5 & 0.3 & \mathrm{u} & 89.2 \\ 6 & 0.02300 & 79.4 & 0.3 & \mathrm{u} & 80.9 \\ 7 & 0.02940 & 72.1 & 0.3 & \mathrm{u} & 74.0 \\ 8 & 0.03750 & 66.6 & 0.3 & \mathrm{u} & 68.5 \\ 9 & 0.04790 & 62.7 & 0.3 & \mathrm{u} & 64.2 \\ 10 & 0.06110 & 60.2 & 0.3 & \mathrm{u} & 61.1 \\ 11 & 0.07790 & 59.1 & 0.3 & \mathrm{u} & 58.9 \\ 12 & 0.09930 & 56.7 & 0.3 & \mathrm{u} & 57.1 \\ 13 & 0.12670 & 55.1 & 0.4 & \mathrm{u} & 55.2 \\ 14 & 0.16640 & 52.0 & 0.6 & \mathrm{u} & 52.5 \\ 15 & 0.20600 & 49.1 & 1.1 & \mathrm{u} & 49.7 \\ 16 & 0.26280 & 45.8 & 2.3 & \mathrm{u} & 46.2 \\ 17 & 0.33520 & 42.1 & 3.5 & \mathrm{u} & 42.5 \\ 18 & 0.42770 & 38.2 & 6.0 & \mathrm{u} & 39.1 \\ 19 & 0.54560 & 35.9 & 7.5 & \mathrm{u} & 36.3 \\ 20 & 0.69590 & 35.1 & 8.7 & \mathrm{u} & 34.0\end{array}$

Date: $28-A P R-08$

UTM Coord: $E[\mathrm{~m}]$

Elevation $[\mathrm{m}]$ :

$557942.0 \quad \mathrm{~N}[\mathrm{~m}] \quad 2814507.0$ 0.0

RX location: X [m]

$0.0 Y[\mathrm{~m}] \quad 0.0$
Fit Error [응: 2.645

System: EM-58 Freq[Hz]: 30 Data Set Code: hi TX Cur $[\mathrm{A}]: \quad 1.40$ Turn Off[usec]: 2.5

RX Moment [turns-m^2] : 31.4 Gain Setting: 7

$\begin{array}{crrcc}\begin{array}{c}\text { Time } \\ \text { [ms] }\end{array} & \begin{array}{r}\text { rhoa_obs } \\ \text { [ohm-m] }\end{array} & \begin{array}{c}\text { obs_err } \\ {\left[\begin{array}{c}\overline{\%} \\ \mathrm{o}\end{array}\right.}\end{array} & \text { mask } & \begin{array}{c}\text { rhoa_cal } \\ \text { [ohm-m] }\end{array} \\ 0.08800 & 58.3 & 1.5 & \mathrm{u} & 57.5 \\ 0.10600 & 57.4 & 1.4 & \mathrm{u} & 55.9 \\ 0.13100 & 55.6 & 1.7 & \mathrm{u} & 54.0 \\ 0.16100 & 53.0 & 3.4 & \mathrm{u} & 51.5 \\ 0.20000 & 48.9 & 2.9 & \mathrm{u} & 48.3 \\ 0.25000 & 44.8 & 4.7 & \mathrm{u} & 44.5 \\ 0.31400 & 40.4 & 4.6 & \mathrm{u} & 40.4 \\ 0.39500 & 36.7 & 6.5 & \mathrm{u} & 36.4 \\ 0.49900 & 32.4 & 14.5 & \mathrm{~m} & 32.6 \\ 0.63100 & 30.6 & 23.8 & \mathrm{~m} & 29.2 \\ 0.79900 & 29.4 & 57.1 & \mathrm{~m} & 26.3 \\ 1.01000 & 31.9 & 83.6 & \mathrm{~m} & 23.8 \\ 1.28000 & 31.3 & 100.0 & \mathrm{~m} & 21.7 \\ 1.63000 & 18.0 & 100.0 & \mathrm{~d} & - \\ 2.08000 & 17.3 & 69.5 & \mathrm{~d} & - \\ 2.64000 & 10.7 & 100.0 & \mathrm{~d} & - \\ 3.37000 & 6.6 & 76.6 & \mathrm{~d} & - \\ 4.29000 & 6.4 & 100.0 & \mathrm{~d} & - \\ 5.47000 & 15.7 & 100.0 & \mathrm{~d} & - \\ 6.97000 & 15.5 & 100.0 & \mathrm{~d} & -\end{array}$


Sounding: MIA104

Location: Homestead Speedway ovrflow lot

Project: Saltwater Intrusion

County: Miami-Dade County

TX loop size: X[m] $38.1 \quad \mathrm{Y}[\mathrm{m}] \quad 38.1$

$\begin{array}{cccc}\text { Model } & \text { Resistivity } & \text { Thickness } & \text { Elevation } \\ \text { Layer } & {[\text { ohm-m] }} & {[\mathrm{m}]} & {[\mathrm{m}]} \\ 1 & 164.900 & 28.8 & 0.0 \\ 2 & 37.670 & 45.9 & -28.8 \\ 3 & 10.800 & -- & -74.8\end{array}$

System: EM-58 Freq[Hz]: 285 Data Set Code: uh TX Cur[A]: 1.40 Turn Off[usec]: 2.5

RX Moment[turns-m^2]: 31.4 Gain Setting: 4

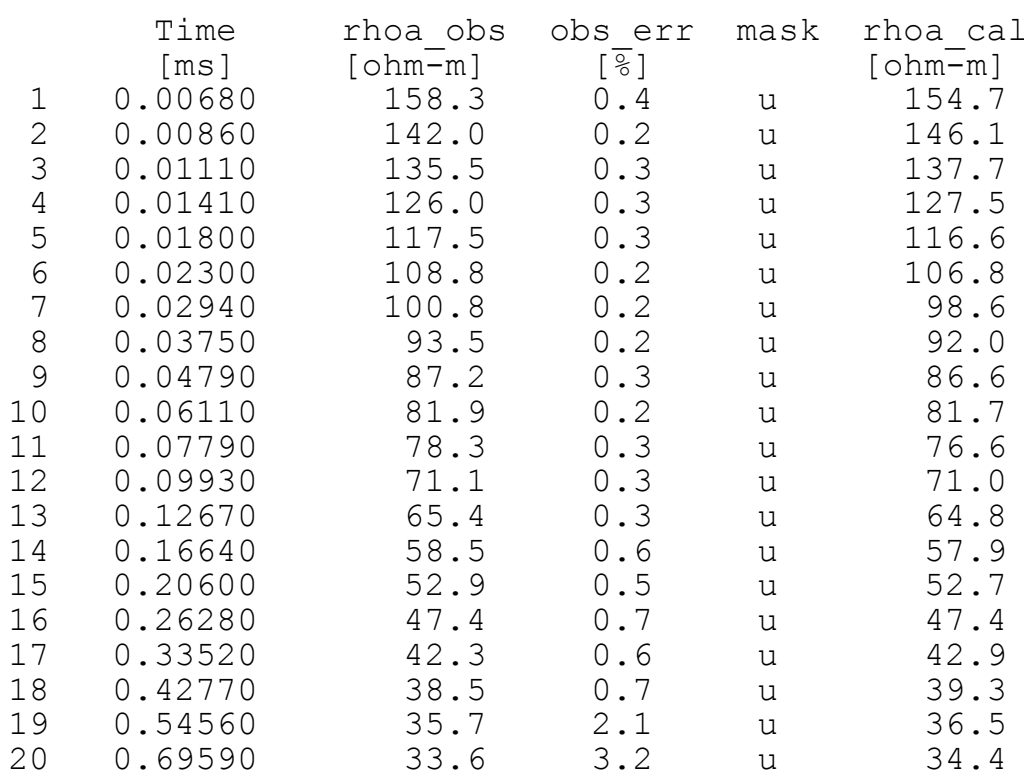

Date: $28-\mathrm{APR}-08$

UTM Coord: $\mathrm{E}[\mathrm{m}]$

Elevation $[\mathrm{m}]$ :

$557486.0 \quad \mathrm{~N}[\mathrm{~m}] \quad 2814401.0$ 0.0

RX location: $\mathrm{X}[\mathrm{m}]$

$0.0 \quad \mathrm{Y}[\mathrm{m}] \quad 0.0$
Fit Error [\%]: 7.260

System: EM-58 Freq[Hz]: 30 Data Set Code: hi TX $\operatorname{Cur}[A]: \quad 1.40$ Turn Off[usec]: 2.5

RX Moment[turns-m^2]: 31.4 Gain Setting: 7

$\begin{array}{ccccc}\begin{array}{c}\text { Time } \\ \text { [ms] }\end{array} & \begin{array}{c}\text { rhoa_obs } \\ \text { [ohm-m] }\end{array} & \begin{array}{c}\text { obs_err } \\ {\left[\begin{array}{c}\bar{o} \\ \mathrm{o}\end{array}\right.}\end{array} & \text { mask } & \begin{array}{c}\text { rhoa_cal } \\ \text { [ohm-m] }\end{array} \\ 0.08800 & 65.7 & 2.6 & \mathrm{u} & 73.1 \\ 0.10600 & 61.1 & 0.8 & \mathrm{u} & 68.2 \\ 0.13100 & 66.4 & 0.7 & \mathrm{u} & 62.7 \\ 0.16100 & 68.4 & 2.1 & \mathrm{u} & 57.0 \\ 0.20000 & 50.5 & 1.0 & \mathrm{u} & 51.4 \\ 0.25000 & 46.8 & 6.2 & \mathrm{u} & 46.0 \\ 0.31400 & 40.9 & 3.0 & \mathrm{u} & 41.1 \\ 0.39500 & 36.3 & 2.5 & \mathrm{u} & 36.9 \\ 0.49900 & 32.4 & 3.1 & \mathrm{u} & 33.1 \\ 0.63100 & 28.7 & 4.7 & \mathrm{u} & 29.9 \\ 0.79900 & 26.4 & 4.9 & \mathrm{u} & 27.2 \\ 1.01000 & 25.0 & 12.2 & \mathrm{u} & 24.9 \\ 1.28000 & 24.5 & 28.1 & \mathrm{u} & 23.0 \\ 1.63000 & 22.0 & 28.4 & \mathrm{u} & 21.4 \\ 2.08000 & 29.2 & 77.2 & \mathrm{~d} & - \\ 2.64000 & 68.1 & 100.0 & \mathrm{~d} & - \\ 3.37000 & 23.2 & 100.0 & \mathrm{~d} & - \\ 4.29000 & 54.7 & 100.0 & \mathrm{~d} & - \\ 5.47000 & 39.7 & 100.0 & \mathrm{~d} & - \\ 6.97000 & 24.5 & 100.0 & \mathrm{~d} & -\end{array}$


Sounding: MIA104L4

Client: US Geological Survey Location: Homestead Speedway ovrflow lot

Project: Saltwater Intrusion

County: Miami-Dade County

TX loop size: X $[\mathrm{m}] \quad 38.1 \quad \mathrm{Y}[\mathrm{m}] \quad 38.1$

$\begin{array}{cccc}\text { Model } & \text { Resistivity } & \text { Thickness } & \text { Elevation } \\ \text { Layer } & {[\text { ohm-m] }} & {[\mathrm{m}]} & {[\mathrm{m}]} \\ 1 & 563.200 & 9.8 & 0.0 \\ 2 & 92.960 & 23.9 & -9.8 \\ 3 & 35.430 & 42.1 & -33.7 \\ 4 & 10.690 & -- & -75.8\end{array}$

System: EM-58 Freq[Hz]: 285 Data Set Code: uh TX Cur[A]: 1.40 Turn Off[usec]: 2.5

RX Moment[turns-m^2]: 31.4 Gain Setting: 4

$\begin{array}{rrrrrr}\text { Time } & \begin{array}{c}\text { rhoa_obs } \\ {[\text { ohm-m] }}\end{array} & \begin{array}{c}\text { obs_err } \\ {\left[\frac{\mathrm{o}}{0}\right]}\end{array} & \text { mask } & \begin{array}{r}\text { rhoa_cal } \\ {[\text { ohm-m] }}\end{array} \\ 1 & 0.00680 & 158.3 & 0.4 & \mathrm{u} & 155.0 \\ 2 & 0.00860 & 142.0 & 0.2 & \mathrm{u} & 144.5 \\ 3 & 0.01110 & 135.5 & 0.3 & \mathrm{u} & 136.6 \\ 4 & 0.01410 & 126.0 & 0.3 & \mathrm{u} & 127.5 \\ 5 & 0.01800 & 117.5 & 0.3 & \mathrm{u} & 117.4 \\ 6 & 0.02300 & 108.8 & 0.2 & \mathrm{u} & 107.8 \\ 7 & 0.02940 & 100.8 & 0.2 & \mathrm{u} & 99.4 \\ 8 & 0.03750 & 93.5 & 0.2 & \mathrm{u} & 92.4 \\ 9 & 0.04790 & 87.2 & 0.3 & \mathrm{u} & 86.7 \\ 10 & 0.06110 & 81.9 & 0.2 & \mathrm{u} & 81.5 \\ 11 & 0.07790 & 78.3 & 0.3 & \mathrm{u} & 76.3 \\ 12 & 0.09930 & 71.1 & 0.3 & \mathrm{u} & 70.7 \\ 13 & 0.12670 & 65.4 & 0.3 & \mathrm{u} & 64.7 \\ 14 & 0.16640 & 58.5 & 0.6 & \mathrm{u} & 57.9 \\ 15 & 0.20600 & 52.9 & 0.5 & \mathrm{u} & 52.7 \\ 16 & 0.26280 & 47.4 & 0.7 & \mathrm{u} & 47.5 \\ 17 & 0.33520 & 42.3 & 0.6 & \mathrm{u} & 43.0 \\ 18 & 0.42770 & 38.5 & 0.7 & \mathrm{u} & 39.4 \\ 19 & 0.54560 & 35.7 & 2.1 & \mathrm{u} & 36.5 \\ 20 & 0.69590 & 33.6 & 3.2 & \mathrm{u} & 34.4\end{array}$

Date: $28-\mathrm{APR}-08$

UTM Coord: $\mathrm{E}[\mathrm{m}]$

Elevation $[\mathrm{m}]$ :

$557486.0 \quad \mathrm{~N}[\mathrm{~m}] \quad 2814401.0$

0.0

RX location: X $[\mathrm{m}]$

$0.0 \quad Y[m]$

0.0
Fit Error $\left[\frac{\circ}{0}\right]: 7.177$

System: EM-58 Freq[Hz]: 30 Data Set Code: hi TX Cur $[\mathrm{A}]: \quad 1.40$ Turn Off[usec]: 2.5

RX Moment [turns-m^2] : $31.4 \quad$ Gain Setting: 7

Time
$[\mathrm{ms}]$
0.08800
0.10600
0.13100
0.16100
0.20000
0.25000
0.31400
0.39500
0.49900
0.63100
0.79900
1.01000
1.28000
1.63000
2.08000
2.64000
3.37000
4.29000
5.47000
6.97000

rhoa_cal

[ohm-m]

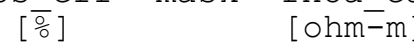

2.6 u 72.8

0.8 u 68.0

62.6

57.0

51.4

46.1

41.2

36.9

33.1

29.9

27.2

24.9

23.0

21.4

$-$ 
Project: Saltwater Intrusion

County: Miami-Dade County

TX loop size: X[m] $38.1 \quad Y[m] \quad 38.1$

RX location: X $[\mathrm{m}] \quad 0.0 \quad \mathrm{Y}[\mathrm{m}] \quad 0.0$

$\begin{array}{cccc}\text { Model } & \text { Resistivity } & \text { Thickness } & \text { Elevation } \\ \text { Layer } & {[\text { ohm-m] }} & {[\mathrm{m}]} & {[\mathrm{m}]} \\ 1 & 123.900 & 1.9 & 0.0 \\ 2 & 93.430 & 21.5 & -1.9 \\ 3 & 9.740 & 32.7 & -23.4 \\ 4 & 1.390 & -- & -56.2\end{array}$

System: EM-58 Freq[Hz]: 285 Data Set Code: uh TX Cur $[A]: \quad 1.40$ Turn Off [usec]: 2.5

RX Moment[turns-m^2]: 31.4 Gain Setting: 4

\begin{tabular}{|c|c|c|c|c|}
\hline $\begin{array}{l}\text { Time } \\
\text { [ms ] }\end{array}$ & $\begin{array}{l}\text { rhoa_obs } \\
\text { [ohm-m] }\end{array}$ & $\begin{array}{c}\text { obs err } \\
{\left[\frac{\overline{0}}{0}\right]}\end{array}$ & mask & $\begin{array}{l}\text { rhoa_ca] } \\
\text { [ohm-m] }\end{array}$ \\
\hline 0.00680 & 107.3 & 0.3 & $u$ & 105.7 \\
\hline 0.00860 & 102.4 & 0.3 & $u$ & 99.3 \\
\hline 0.01110 & 87.6 & 0.4 & $\mathrm{u}$ & 91.1 \\
\hline 0.01410 & 75.6 & 0.3 & $u$ & 80.4 \\
\hline 0.01800 & 65.2 & 0.4 & u & 59. \\
\hline 0.02300 & 60.4 & 0.4 & u & 59.6 \\
\hline 0.02940 & 53.8 & 0.3 & u & 1 . \\
\hline 0.03750 & 47.6 & 0.4 & u & . \\
\hline 0.04790 & 41.9 & 0.4 & u & 39. \\
\hline 0.06110 & 38.8 & 0.5 & u & 35 . \\
\hline 0.07790 & 33.0 & 0.4 & $\mathrm{u}$ & 33. \\
\hline 0.09930 & 29.2 & 0.9 & $\mathrm{u}$ & 31.2 \\
\hline 0.12670 & 30.1 & 1.1 & $\mathrm{u}$ & 29. \\
\hline 0.16640 & 25.5 & 1.6 & $\mathrm{u}$ & 27. \\
\hline 0.20600 & 24.5 & 2.3 & $\mathrm{u}$ & 24. \\
\hline 0.26280 & 21.8 & 3.6 & $u$ & 22 . \\
\hline 0.33520 & 19.7 & 5.8 & u & 19. \\
\hline 0.42770 & 17.4 & 8.2 & u & 1. \\
\hline 0 . & 15.7 & 8.8 & u & \\
\hline 2 & 3.3 & 11.2 & $\mathrm{u}$ & \\
\hline
\end{tabular}

\section{Fit Error $\left[\frac{\circ}{0}\right]: \quad 6.526$}

System: EM-58 Freq[Hz]: 30 Data Set Code: hi TX Cur $[A]: \quad 1.40$ Turn Off[usec]: 2.5

RX Moment [turns-m^2] : 31.4 Gain Setting: 7

$\begin{array}{crrcr}\begin{array}{c}\text { Time } \\ \text { [ms] }\end{array} & \begin{array}{r}\text { rhoa_obs } \\ \text { [ohm-m] }\end{array} & \begin{array}{c}\text { obs_err } \\ {\left[\begin{array}{c}\overline{\%} \\ \text { o }]\end{array}\right.}\end{array} & \text { mask } & \begin{array}{c}\text { rhoa_cal } \\ \text { [ohm-m] }\end{array} \\ 0.08800 & 29.0 & 2.0 & \mathrm{u} & 31.6 \\ 0.10600 & 29.7 & 1.4 & \mathrm{u} & 30.0 \\ 0.13100 & 30.0 & 1.8 & \mathrm{u} & 28.1 \\ 0.16100 & 25.7 & 3.7 & \mathrm{u} & 26.0 \\ 0.20000 & 24.4 & 4.3 & \mathrm{u} & 23.5 \\ 0.25000 & 20.4 & 2.7 & \mathrm{u} & 20.7 \\ 0.31400 & 18.4 & 6.7 & \mathrm{u} & 17.9 \\ 0.39500 & 15.2 & 5.0 & \mathrm{u} & 15.3 \\ 0.49900 & 12.8 & 8.1 & \mathrm{u} & 13.0 \\ 0.63100 & 10.6 & 11.1 & \mathrm{u} & 11.0 \\ 0.79900 & 8.9 & 18.6 & \mathrm{~m} & 9.4 \\ 1.01000 & 7.0 & 16.2 & \mathrm{~m} & 8.0 \\ 1.28000 & 6.9 & 15.7 & \mathrm{~m} & 6.9 \\ 1.63000 & 4.5 & 37.0 & \mathrm{~m} & 6.1 \\ 2.08000 & 3.2 & 40.1 & \mathrm{~m} & 5.3 \\ 2.64000 & 3.0 & 32.0 & \mathrm{~m} & 4.8 \\ 3.37000 & 6.3 & 100.0 & \mathrm{~d} & - \\ 4.29000 & 2.0 & 100.0 & \mathrm{~d} & - \\ 5.47000 & 5.0 & 100.0 & \mathrm{~d} & - \\ 6.97000 & 1.8 & 100.0 & \mathrm{~d} & -\end{array}$


Sounding: MIA105L5

Client: US Geological Survey

Pcation: Pine Crest School East

Project: Saltwater Intrusion

County: Miami-Dade County

TX loop size: X[m] $38.1 \quad Y[m] \quad 38.1$

$\begin{array}{cccc}\text { Model } & \text { Resistivity } & \text { Thickness } & \text { Elevation } \\ \text { Layer } & {[\text { ohm-m] }} & {[\mathrm{m}]} & {[\mathrm{m}]} \\ 1 & 124.700 & 2.1 & 0.0 \\ 2 & 105.900 & 19.1 & -2.1 \\ 3 & 12.590 & 16.4 & -21.2 \\ 4 & 5.850 & 20.1 & -37.6 \\ 5 & .930 & -- & -57.8\end{array}$

System: EM-58 Freq[Hz]: 285 Data Set Code: uh TX Cur $[A]: 1.40$ Turn Off [usec]: 2.5

RX Moment[turns-m^2]: 31.4 Gain Setting:

$\begin{array}{rrrrrr}\text { Time } & \begin{array}{c}\text { rhoa_obs } \\ \text { [ohm-m] }\end{array} & \begin{array}{c}\text { obs_err } \\ {[\overline{0}]}\end{array} & \text { mask } & \begin{array}{r}\text { rhoa_cal } \\ \text { [ohm-m] }\end{array} \\ 1 & 0.00680 & 107.3 & 0.3 & \mathrm{u} & 109.5 \\ 2 & 0.00860 & 102.4 & 0.3 & \mathrm{u} & 98.0 \\ 3 & 0.01110 & 87.6 & 0.4 & \mathrm{u} & 87.3 \\ 4 & 0.01410 & 75.6 & 0.3 & \mathrm{u} & 77.0 \\ 5 & 0.01800 & 65.2 & 0.4 & \mathrm{u} & 67.8 \\ 6 & 0.02300 & 60.4 & 0.4 & \mathrm{u} & 59.9 \\ 7 & 0.02940 & 53.8 & 0.3 & \mathrm{u} & 53.0 \\ 8 & 0.03750 & 47.6 & 0.4 & \mathrm{u} & 46.9 \\ 9 & 0.04790 & 41.9 & 0.4 & \mathrm{u} & 41.5 \\ 10 & 0.06110 & 38.8 & 0.5 & \mathrm{u} & 37.1 \\ 11 & 0.07790 & 33.0 & 0.4 & \mathrm{u} & 33.6 \\ 12 & 0.09930 & 29.2 & 0.9 & \mathrm{u} & 30.9 \\ 13 & 0.12670 & 30.1 & 1.1 & \mathrm{u} & 28.8 \\ 14 & 0.16640 & 25.5 & 1.6 & \mathrm{u} & 26.6 \\ 15 & 0.20600 & 24.5 & 2.3 & \mathrm{u} & 24.7 \\ 16 & 0.26280 & 21.8 & 3.6 & \mathrm{u} & 22.3 \\ 17 & 0.33520 & 19.7 & 5.8 & \mathrm{u} & 19.8 \\ 18 & 0.42770 & 17.4 & 8.2 & \mathrm{u} & 17.4 \\ 19 & 0.54560 & 15.7 & 8.8 & \mathrm{u} & 15.3 \\ 20 & 0.69590 & 13.3 & 11.2 & \mathrm{u} & 13.5\end{array}$

Date: 29-APR-08

UTM Coord: $E[\mathrm{~m}]$

Elevation $[\mathrm{m}]$ :

$571727.0 \quad \mathrm{~N}[\mathrm{~m}] \quad 2839900.0$ 0.0

RX location: $\mathrm{X}[\mathrm{m}]$

$0.0 Y[\mathrm{~m}] \quad 0.0$
Fit Error $\left[\frac{\circ}{0}\right]: 5.187$

System: EM-58 Freq[Hz]: 30 Data Set Code: hi TX Cur $[A]: \quad 1.40$ Turn Off[usec]: 2.5

RX Moment[turns-m^2] : 31.4 Gain Setting: 7

Time
$[\mathrm{ms}]$
0.08800
0.10600
0.13100
0.16100
0.20000
0.25000
0.31400
0.39500
0.49900
0.63100
0.79900
1.01000
1.28000
1.63000
2.08000
2.64000
3.37000
4.29000
5.47000
6.97000

.

$\begin{array}{rr}\text { rhoa_obs } & \text { obs err } \\ \text { [ohm-m] } & {[\bar{\circ} \text { ] }]} \\ 29.0 & 2.0 \\ 29.7 & 1.4 \\ 30.0 & 1.8 \\ 25.7 & 3.7 \\ 24.4 & 4.3 \\ 20.4 & 2.7 \\ 18.4 & 6.7 \\ 15.2 & 5.0 \\ 12.8 & 8.1 \\ 10.6 & 11.1 \\ 8.9 & 18.6 \\ 7.0 & 16.2 \\ 6.9 & 15.7 \\ 4.5 & 37.0 \\ 3.2 & 40.1 \\ 3.0 & 32.0 \\ 6.3 & 100.0 \\ 2.0 & 100.0 \\ 5.0 & 100.0 \\ 1.8 & 100.0\end{array}$

rhoa_cal

[ohm-m]

31.6

29.6

27.6

25.5

23.3

20.

17.9

15.3

12.8

10.8

9.0

7.6

6.5

5.5

4.8

4. 2

$-$ 
Sounding: MIA106

Location: Pine Crest School West

Project: Saltwater Intrusion

County: Miami-Dade County

TX loop size: X $[\mathrm{m}] \quad 38.1 \quad \mathrm{Y}[\mathrm{m}] \quad 38.1$

$\begin{array}{cccc}\text { Model } & \text { Resistivity } & \text { Thickness } & \text { Elevation } \\ \text { Layer } & {[\text { ohm-m] }} & {[\mathrm{m}]} & {[\mathrm{m}]} \\ 1 & 510.400 & 4.2 & 0.0 \\ 2 & 50.700 & 36.5 & -4.2 \\ 3 & 6.360 & 23.2 & -40.7 \\ 4 & .656 & -- & -64.0\end{array}$

System: EM-58 Freq[Hz]: 285 Data Set Code: uh TX Cur[A]: 1.40 Turn Off[usec]: 2.5

RX Moment[turns-m^2]: 31.4 Gain Setting: 3

$\begin{array}{rrrrrr}\text { Time } & \begin{array}{c}\text { rhoa_obs } \\ \text { [ohm-m] }\end{array} & \begin{array}{c}\text { obs_err } \\ {\left[\frac{\mathrm{o}}{0}\right]}\end{array} & \text { mask } & \begin{array}{r}\text { rhoa_cal } \\ \text { [ohm-m] }\end{array} \\ 1 & 0.00680 & 75.9 & 0.2 & \mathrm{u} & 73.1 \\ 2 & 0.00860 & 69.6 & 0.1 & \mathrm{u} & 68.8 \\ 3 & 0.01110 & 64.8 & 0.1 & \mathrm{u} & 68.2 \\ 4 & 0.01410 & 65.2 & 0.1 & \mathrm{u} & 68.5 \\ 5 & 0.01800 & 67.7 & 0.1 & \mathrm{u} & 69.2 \\ 6 & 0.02300 & 72.2 & 0.1 & \mathrm{u} & 69.2 \\ 7 & 0.02940 & 73.6 & 0.1 & \mathrm{u} & 67.6 \\ 8 & 0.03750 & 64.0 & 0.1 & \mathrm{u} & 63.8 \\ 9 & 0.04790 & 52.5 & 0.2 & \mathrm{u} & 58.7 \\ 10 & 0.06110 & 59.4 & 0.1 & \mathrm{u} & 53.4 \\ 11 & 0.07790 & 45.1 & 0.2 & \mathrm{u} & 49.1 \\ 12 & 0.09930 & 47.1 & 0.5 & \mathrm{u} & 45.8 \\ 13 & 0.12670 & 43.3 & 1.1 & \mathrm{u} & 43.1 \\ 14 & 0.16640 & 38.3 & 1.3 & \mathrm{u} & 40.0 \\ 15 & 0.20600 & 35.6 & 2.6 & \mathrm{u} & 36.9 \\ 16 & 0.26280 & 31.9 & 2.5 & \mathrm{u} & 32.8 \\ 17 & 0.33520 & 27.7 & 4.3 & \mathrm{u} & 28.5 \\ 18 & 0.42770 & 24.1 & 7.2 & \mathrm{u} & 24.4 \\ 19 & 0.54560 & 20.7 & 13.6 & \mathrm{u} & 20.9 \\ 20 & 0.69590 & 17.6 & 19.8 & \mathrm{u} & 18.1\end{array}$

Date: $29-\mathrm{APR}-08$

UTM Coord: $\mathrm{E}[\mathrm{m}]$

Elevation $[\mathrm{m}]$ :

$571608.0 \quad \mathrm{~N}[\mathrm{~m}] \quad 2839907.0$

0.0

RX location: X $[\mathrm{m}]$

$0.0 \quad Y[m]$

0.0
Fit Error $[\%]: 8.598$

System: EM-58 Freq[Hz]: 30 Data Set Code: hi TX Cur $[A]: \quad 1.40$ Turn Off[usec]: 2.5

RX Moment [turns-m^2]: $31.4 \quad$ Gain Setting: 7

Time
$[\mathrm{ms}]$
0.08800
0.10600
0.13100
0.16100
0.20000
0.25000
0.31400
0.39500
0.49900
0.63100
0.79900
1.01000
1.28000
1.63000
2.08000
2.64000
3.37000
4.29000
5.47000
6.97000

obs err mask

$\left[\frac{\mathrm{o}}{0}\right]$

2. 2

2.2

3.9

2.8

5.6

4.6

11.4

$9 \cdot 3$

19.3

13.4

23.1

29.6

42.1

52.9

64.7

56.6

100.0

100.0

100.0

100.0 rhoa_cal

[ohm-m]

46.3

43.6

40.8

37.7

33.9

29.5

24.9

20.6

16.9

13.8

11.2

9.2

6.3

5.3

4.5

4.0

3. 5

3. 1 
Sounding: MIA107

Location: Fairchild School

Project: Saltwater Intrusion

County: Miami-Dade County

TX loop size: X[m] $38.1 \quad \mathrm{Y}[\mathrm{m}] \quad 38.1$

$\begin{array}{cccc}\text { Model } & \text { Resistivity } & \text { Thickness } & \text { Elevation } \\ \text { Layer } & {[\text { ohm-m] }} & {[\mathrm{m}]} & {[\mathrm{m}]} \\ 1 & 443.600 & 7.3 & 0.0 \\ 2 & 39.120 & 34.4 & -7.3 \\ 3 & 1.130 & -- & -41.7\end{array}$

System: EM-58 Freq[Hz]: 285 Data Set Code: uh TX Cur[A]: 1.40 Turn Off [usec]: 2.5

RX Moment[turns-m^2]: 31.4 Gain Setting: 4

$\begin{array}{cccccc}\text { Time } & \text { [ms] } & \begin{array}{c}\text { rhoa_obs } \\ \text { [ohm-m] }\end{array} & \begin{array}{c}\text { obserr } \\ {\left[\begin{array}{c}o \\ 0\end{array}\right.}\end{array} & \text { mask } & \begin{array}{c}\text { rhoa_cal } \\ \text { [ohm-m] }\end{array} \\ 1 & 0.00680 & 77.3 & 0.4 & \mathrm{u} & 78.6 \\ 2 & 0.00860 & 73.7 & 0.3 & \mathrm{u} & 71.6 \\ 3 & 0.01110 & 68.9 & 0.6 & \mathrm{u} & 68.6 \\ 4 & 0.01410 & 68.1 & 0.6 & \mathrm{u} & 67.9 \\ 5 & 0.01800 & 66.8 & 0.7 & \mathrm{u} & 68.9 \\ 6 & 0.02300 & 72.2 & 0.9 & \mathrm{u} & 71.3 \\ 7 & 0.02940 & 75.9 & 1.8 & \mathrm{u} & 73.7 \\ 8 & 0.03750 & 73.8 & 1.1 & \mathrm{u} & 73.6 \\ 9 & 0.04790 & 65.8 & 3.0 & \mathrm{u} & 68.8 \\ 10 & 0.06110 & 58.7 & 3.2 & \mathrm{u} & 60.2 \\ 11 & 0.07790 & 52.1 & 4.6 & \mathrm{u} & 50.1 \\ 12 & 0.09930 & 41.4 & 8.4 & \mathrm{u} & 40.7 \\ 13 & 0.12670 & 31.6 & 5.2 & \mathrm{u} & 32.9 \\ 14 & 0.16640 & 26.5 & 8.8 & \mathrm{u} & 26.0 \\ 15 & 0.20600 & 21.9 & 14.2 & \mathrm{u} & 21.7 \\ 16 & 0.26280 & 18.4 & 20.0 & \mathrm{u} & 17.9 \\ 17 & 0.33520 & 15.4 & 22.8 & \mathrm{u} & 14.9 \\ 18 & 0.42770 & 12.2 & 30.7 & \mathrm{u} & 12.7 \\ 19 & 0.54560 & 10.7 & 31.5 & \mathrm{u} & 10.9 \\ 20 & 0.69590 & 24.7 & 100.0 & \mathrm{~d} & -\end{array}$

Date: $29-\mathrm{APR}-08$

UTM Coord: $\mathrm{E}[\mathrm{m}]$

Elevation $[\mathrm{m}]$ :

$571420.0 \quad \mathrm{~N}[\mathrm{~m}] \quad 2846074.0$ 0.0

RX location: $\mathrm{X}[\mathrm{m}]$

$0.0 \quad \mathrm{Y}[\mathrm{m}] \quad 0.0$

Fit Error [으: 4.096 
Sounding: MIA108

Client: US Geological Survey

Location: Palmer Trinity School Ball Field

Project: Saltwater Intrusion

County: Miami-Dade County

TX loop size: X [m] $38.1 \quad \mathrm{Y}[\mathrm{m}] \quad 38.1$

$\begin{array}{cccc}\text { Model } & \text { Resistivity } & \text { Thickness } & \text { Elevation } \\ \text { Layer } & {[\text { ohm-m] }} & {[\mathrm{m}]} & {[\mathrm{m}]} \\ 1 & 679.000 & 5.6 & 0.0 \\ 2 & 87.160 & 28.3 & -5.6 \\ 3 & 42.800 & 53.5 & -34.0 \\ 4 & 480.800 & -- & -87.5\end{array}$

System: EM-58 Freq[Hz]: 285 Data Set Code: uh TX Cur[A]: 1.40 Turn Off[usec]: 2.5

RX Moment[turns-m^2]: 31.4 Gain Setting: 4

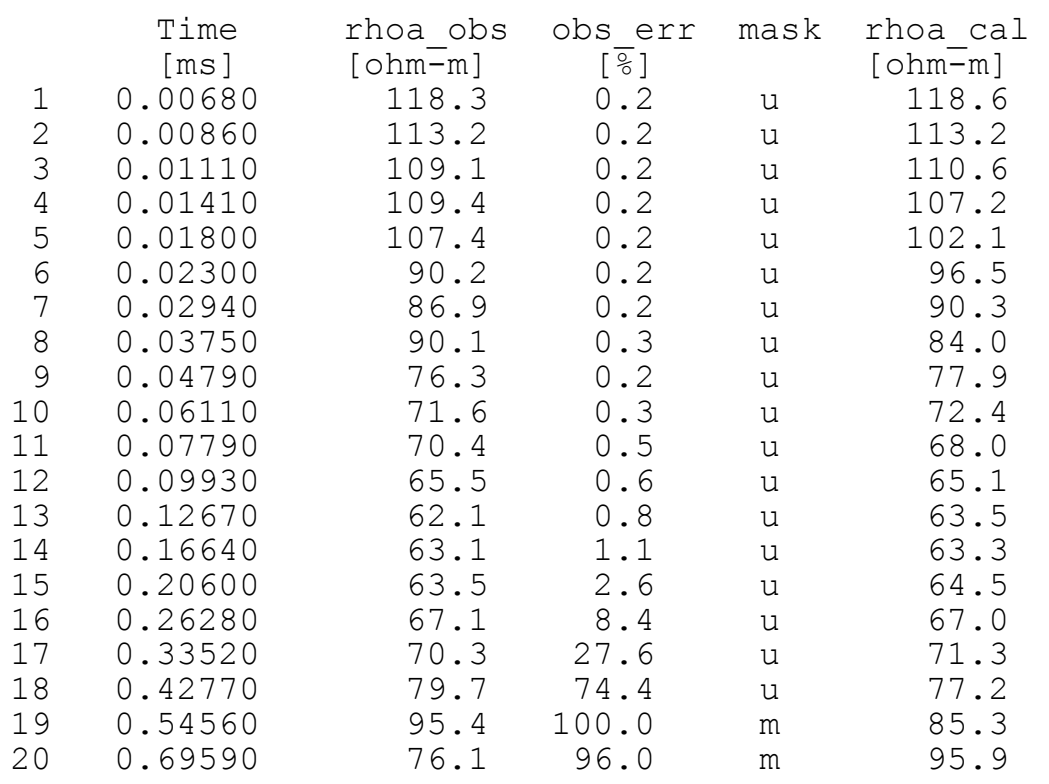

Date: $30-A P R-08$

UTM Coord: $\mathrm{E}[\mathrm{m}]$

Elevation $[\mathrm{m}]$ :

$568118.0 \quad \mathrm{~N}[\mathrm{~m}] \quad 2832157.0$

0.0

RX location: $\mathrm{X}[\mathrm{m}]$

$0.0 \quad \mathrm{Y}[\mathrm{m}]$

0.0

Fit Error $\left[\frac{\circ}{0}\right]: 4.802$ 
Sounding: MIA108L3

Location: Palmer Trinity School Ball Field

County: Miami-Dade County

TX loop size: X[m] $38.1 \quad \mathrm{Y}[\mathrm{m}] \quad 38.1$

$\begin{array}{cccc}\text { Model } & \text { Resistivity } & \text { Thickness } & \text { Elevation } \\ \text { Layer } & {[\text { ohm-m] }} & {[\mathrm{m}]} & {[\mathrm{m}]} \\ 1 & 581.900 & 4.3 & 0.0 \\ 2 & 98.330 & 23.6 & -4.3 \\ 3 & 47.430 & -- & -28.0\end{array}$

System: EM-58 Freq[Hz]: 285 Data Set Code: uh TX Cur $[A]: \quad 1.40$ Turn Off[usec]: 2.5

RX Moment[turns-m^2]: 31.4 Gain Setting: 4

\begin{tabular}{|c|c|c|c|c|c|}
\hline & $\begin{array}{l}\text { Time } \\
{[\mathrm{ms}]}\end{array}$ & $\begin{array}{l}\text { rhoa_obs } \\
\text { [ohm-m ] }\end{array}$ & $\begin{array}{c}\text { obs err } \\
{\left[\frac{\overline{0}}{0}\right]}\end{array}$ & mask & $\begin{array}{l}\text { rhoa_cal } \\
\text { [ohm-m] }\end{array}$ \\
\hline 1 & 0.00680 & 118.3 & 0.2 & $u$ & 120.3 \\
\hline 2 & 0.00860 & 113.2 & 0.2 & $u$ & 114.2 \\
\hline 3 & 0.01110 & 109.1 & 0.2 & $u$ & 110.2 \\
\hline 4 & 0.01410 & 109.4 & 0.2 & $u$ & 105.5 \\
\hline 5 & 0.01800 & 107.4 & 0.2 & $u$ & 99.8 \\
\hline 6 & 0.02300 & 90.2 & 0.2 & $\mathrm{u}$ & 94.0 \\
\hline 7 & 0.02940 & 86.9 & 0.2 & $\mathrm{u}$ & 88.5 \\
\hline 8 & 0.03750 & 90.1 & 0.3 & u & 83.4 \\
\hline 9 & 0.04790 & 76.3 & 0.2 & $u$ & 78.9 \\
\hline 10 & 0.06110 & 71.6 & 0.3 & $u$ & 75.0 \\
\hline 11 & 0.07790 & 70.4 & 0.5 & $u$ & 71.5 \\
\hline 12 & 0.09930 & 65.5 & 0.6 & $u$ & 68.5 \\
\hline 13 & 0.12670 & 62.1 & 0.8 & $u$ & 65.9 \\
\hline 14 & 0.16640 & 63.1 & 1.1 & $u$ & 63.6 \\
\hline 15 & 0.20600 & 63.5 & 2.6 & $u$ & 62.1 \\
\hline 16 & 0.26280 & 67.1 & 8.4 & $u$ & 60.9 \\
\hline 17 & 0.33520 & 70.3 & 27.6 & $\mathrm{~m}$ & 60.1 \\
\hline 18 & 0.42770 & 79.7 & 74.4 & $\mathrm{~m}$ & 59.7 \\
\hline 19 & 0.54560 & 95.4 & 100.0 & $\mathrm{~m}$ & 60.1 \\
\hline 20 & 0.69590 & 76.1 & 96.0 & $\mathrm{~m}$ & 61.3 \\
\hline
\end{tabular}

Date: $30-A P R-08$

UTM Coord: $\mathrm{E}[\mathrm{m}]$

Elevation $[\mathrm{m}]$ :

$568118.0 \quad \mathrm{~N}[\mathrm{~m}] \quad 2832157.0$

0.0

RX location: $\mathrm{X}[\mathrm{m}]$

$0.0 \quad Y[m]$

0.0

Fit Error $[\%]: 7.174$ 
Sounding: MIA109

Client: US Geological Survey Location: Palmer Trinity School Orchard West

Project: Saltwater Intrusion

County: Miami-Dade County

TX loop size: X[m] $38.1 \quad \mathrm{Y}[\mathrm{m}] \quad 38.1$

$\begin{array}{cccc}\text { Model } & \text { Resistivity } & \text { Thickness } & \text { Elevation } \\ \text { Layer } & {[\text { ohm-m] }} & {[\mathrm{m}]} & {[\mathrm{m}]} \\ 1 & 651.400 & 7.0 & 0.0 \\ 2 & 123.700 & 29.0 & -7.0 \\ 3 & 48.350 & -- & -36.1\end{array}$

System: EM-58 Freq[Hz]: 285 Data Set Code: uh TX Cur $[A]: \quad 1.40$ Turn Off[usec]: 2.5

RX Moment [turns-m^2] : 31.4 Gain Setting: 4

$\begin{array}{rrrrrr}\text { Time } & \text { [ms] } & \begin{array}{c}\text { rhoa_obs } \\ \text { [ohm-m] } \\ 166.3\end{array} & \begin{array}{c}\text { obserr } \\ {\left[\begin{array}{c}\text { o } \\ 0\end{array}\right.}\end{array} & \text { mask } & \begin{array}{r}\text { rhoa_cal } \\ \text { [ohm-m] }\end{array} \\ 1 & 0.00680 & 165.1 & \mathrm{u} & 164.6 \\ 2 & 0.00860 & 155.8 & 0.3 & \mathrm{u} & 157.1 \\ 3 & 0.01110 & 149.9 & 0.2 & \mathrm{u} & 151.8 \\ 4 & 0.01410 & 144.1 & 0.4 & \mathrm{u} & 144.6 \\ 5 & 0.01800 & 136.4 & 0.3 & \mathrm{u} & 135.3 \\ 6 & 0.02300 & 127.6 & 0.3 & \mathrm{u} & 125.5 \\ 7 & 0.02940 & 117.7 & 0.3 & \mathrm{u} & 116.1 \\ 8 & 0.03750 & 108.3 & 0.3 & \mathrm{u} & 107.5 \\ 9 & 0.04790 & 99.7 & 0.4 & \mathrm{u} & 99.8 \\ 10 & 0.06110 & 92.3 & 0.4 & \mathrm{u} & 93.0 \\ 11 & 0.07790 & 87.7 & 0.7 & \mathrm{u} & 87.2 \\ 12 & 0.09930 & 80.0 & 0.7 & \mathrm{u} & 82.2 \\ 13 & 0.12670 & 76.4 & 1.3 & \mathrm{u} & 77.9 \\ 14 & 0.16640 & 73.1 & 1.9 & \mathrm{u} & 74.0 \\ 15 & 0.20600 & 70.3 & 3.6 & \mathrm{u} & 71.4 \\ 16 & 0.26280 & 69.8 & 8.8 & \mathrm{u} & 69.2 \\ 17 & 0.33520 & 70.2 & 17.9 & \mathrm{u} & 67.5 \\ 18 & 0.42770 & 72.1 & 31.8 & \mathrm{~m} & 66.6 \\ 19 & 0.54560 & 68.7 & 56.3 & \mathrm{~m} & 66.4 \\ 20 & 0.69590 & 57.5 & 57.8 & \mathrm{~m} & 67.2\end{array}$

Date: $30-A P R-08$

UTM Coord: $\mathrm{E}[\mathrm{m}]$

Elevation $[\mathrm{m}]$ :

$568218.0 \quad \mathrm{~N}[\mathrm{~m}] \quad 2831666.0$

0.0

RX location: $\mathrm{X}[\mathrm{m}]$

$0.0 \quad Y[m]$

0.0

Fit Error $[\%]: 2.367$ 
Sounding: MIA110

Client: US Geological Survey

Location: Palmer Trinity School Orchard East

Project: Saltwater Intrusion

County: Miami-Dade County

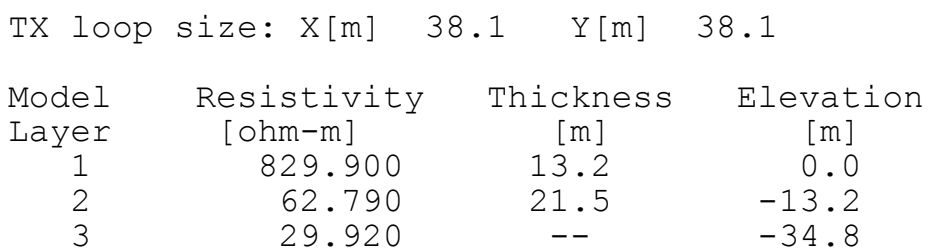

System: EM-58 Freq[Hz]:285 Data Set Code: uh TX Cur $[A]: \quad 1.30$ Turn Off [usec] : 2.5

RX Moment[turns-m^2]: 31.4 Gain Setting: 4

$\begin{array}{rrrrrr}\text { Time } & \begin{array}{c}\text { rhoa_obs } \\ \text { [ohm-m] }\end{array} & \begin{array}{r}\text { obs_err } \\ {\left[\frac{\mathrm{o}}{0}\right]}\end{array} & \text { mask } & \begin{array}{r}\text { rhoa_cal } \\ {[\text { ohm-m] }}\end{array} \\ 1 & 0.00680 & 157.3 & 0.8 & \mathrm{u} & 155.2 \\ 2 & 0.00860 & 137.5 & 0.3 & \mathrm{u} & 140.2 \\ 3 & 0.01110 & 129.5 & 0.4 & \mathrm{u} & 129.4 \\ 4 & 0.01410 & 118.8 & 0.5 & \mathrm{u} & 119.2 \\ 5 & 0.01800 & 108.8 & 0.5 & \mathrm{u} & 108.7 \\ 6 & 0.02300 & 99.7 & 0.5 & \mathrm{u} & 98.7 \\ 7 & 0.02940 & 90.4 & 0.5 & \mathrm{u} & 89.4 \\ 8 & 0.03750 & 82.0 & 0.5 & \mathrm{u} & 81.2 \\ 9 & 0.04790 & 74.8 & 0.6 & \mathrm{u} & 74.0 \\ 10 & 0.06110 & 68.0 & 0.6 & \mathrm{u} & 67.9 \\ 11 & 0.07790 & 62.6 & 0.6 & \mathrm{u} & 62.6 \\ 12 & 0.09930 & 56.5 & 0.7 & \mathrm{u} & 58.2 \\ 13 & 0.12670 & 53.2 & 0.8 & \mathrm{u} & 54.5 \\ 14 & 0.16640 & 50.5 & 1.2 & \mathrm{u} & 51.0 \\ 15 & 0.20600 & 48.5 & 1.6 & \mathrm{u} & 48.8 \\ 16 & 0.26280 & 47.5 & 2.7 & \mathrm{u} & 46.8 \\ 17 & 0.33520 & 45.1 & 2.8 & \mathrm{u} & 45.3 \\ 18 & 0.42770 & 45.4 & 5.4 & \mathrm{u} & 44.3 \\ 19 & 0.54560 & 43.8 & 15.1 & \mathrm{u} & 43.9 \\ 20 & 0.69590 & 44.4 & 29.9 & \mathrm{u} & 44.1\end{array}$

Date: $30-A P R-08$

UTM Coord: $\mathrm{E}[\mathrm{m}]$

Elevation $[\mathrm{m}]$ :

$568251.0 \quad \mathrm{~N}[\mathrm{~m}] \quad 2831672.0$

0.0

RX location: $\mathrm{X}[\mathrm{m}]$

$0.0 \quad Y[m]$

0.0

Fit Error [\%]: 1.954 
Sounding: MIA111

Client: US Geological Survey

Location: Palmer Trinity School Orchard SE

Project: Saltwater Intrusion

County: Miami-Dade County

TX loop size: X[m] $38.1 \quad \mathrm{Y}[\mathrm{m}] \quad 38.1$

$\begin{array}{cccc}\text { Model } & \text { Resistivity } & \text { Thickness } & \text { Elevation } \\ \text { Layer } & {[\text { ohm-m] }} & {[\mathrm{m}]} & {[\mathrm{m}]} \\ 1 & 271.600 & 21.9 & 0.0 \\ 2 & 28.490 & -- & -21.9\end{array}$

System: EM-58 Freq[Hz]: 285 Data set Code: uh TX $\operatorname{Cur}[A]: \quad 1.30$ Turn Off[usec] : 2.5 RX Moment[turns-m^2]: 31.4 Gain Setting:

$\begin{array}{rrrrrr}\text { Time } & \begin{array}{c}\text { rhoa_obs } \\ \text { [ohm-m] }\end{array} & \begin{array}{c}\text { obs_err } \\ {\left[\frac{\text { o }}{0}\right]}\end{array} & \text { mask } & \begin{array}{r}\text { rhoa_cal } \\ \text { [ohm-m] }\end{array} \\ 1 & 0.00680 & 168.3 & 0.2 & \mathrm{u} & 167.2 \\ 2 & 0.00860 & 140.5 & 0.9 & \mathrm{u} & 142.3 \\ 3 & 0.01110 & 124.8 & 0.7 & \mathrm{u} & 124.0 \\ 4 & 0.01410 & 109.2 & 0.7 & \mathrm{u} & 109.1 \\ 5 & 0.01800 & 96.1 & 0.8 & \mathrm{u} & 96.4 \\ 6 & 0.02300 & 85.3 & 0.8 & \mathrm{u} & 85.8 \\ 7 & 0.02940 & 76.7 & 0.8 & \mathrm{u} & 76.9 \\ 8 & 0.03750 & 69.5 & 0.8 & \mathrm{u} & 69.6 \\ 9 & 0.04790 & 63.2 & 0.8 & \mathrm{u} & 63.4 \\ 10 & 0.06110 & 58.4 & 1.0 & \mathrm{u} & 58.3 \\ 11 & 0.07790 & 56.1 & 1.4 & \mathrm{u} & 54.1 \\ 12 & 0.09930 & 51.2 & 2.2 & \mathrm{u} & 50.6 \\ 13 & 0.12670 & 48.2 & 4.0 & \mathrm{u} & 47.6 \\ 14 & 0.16640 & 44.4 & 7.4 & \mathrm{u} & 44.9 \\ 15 & 0.20600 & 41.9 & 10.1 & \mathrm{u} & 43.2 \\ 16 & 0.26280 & 41.7 & 15.0 & \mathrm{u} & 41.7 \\ 17 & 0.33520 & 46.0 & 19.0 & \mathrm{~m} & 40.6 \\ 18 & 0.42770 & 45.8 & 60.2 & \mathrm{~m} & 39.9 \\ 19 & 0.54560 & 38.6 & 98.0 & \mathrm{~m} & 39.7 \\ 20 & 0.69590 & 26.7 & 58.1 & \mathrm{~m} & 40.1\end{array}$

Date: $30-\mathrm{APR}-08$

UTM Coord: $\mathrm{E}[\mathrm{m}]$

Elevation $[\mathrm{m}]$ :

$568268.0 \mathrm{~N}[\mathrm{~m}] \quad 2831566.0$

0.0

RX location: X $[\mathrm{m}]$

$0.0 Y[\mathrm{~m}]$

0.0

Fit Error $[\%]: 2.118$ 
Sounding: MIA112

Client: US Geological Survey

Location: Holy Family Church

Project: Saltwater Intrusion

County: Miami-Dade County

TX loop size: X $[\mathrm{m}] \quad 38.1 \quad \mathrm{Y}[\mathrm{m}] \quad 38.1$

$\begin{array}{cccc}\text { Model } & \text { Resistivity } & \text { Thickness } & \text { Elevation } \\ \text { Layer } & {[\text { ohm-m] }} & {[\mathrm{m}]} & {[\mathrm{m}]} \\ 1 & 85.910 & 32.1 & 0.0 \\ 2 & 5.410 & -- & -32.1\end{array}$

System: EM-58 Freq[Hz]: 285 Data set Code: uh TX Cur $[A]: \quad 1.40$ Turn Off[usec]: 2.5

RX Moment [turns-m^2]: 31.4 Gain Setting:

$\begin{array}{rrrrrr}\text { Time } & \begin{array}{r}\text { rhoa_obs } \\ \text { [ohm-m] }\end{array} & \begin{array}{c}\text { obs_err } \\ {\left[\frac{\mathrm{o}}{0}\right]}\end{array} & \text { mask } & \begin{array}{r}\text { rhoa_cal } \\ \text { [ohm-m] }\end{array} \\ 1 & 0.00680 & 97.9 & 0.3 & \mathrm{u} & 90.1 \\ 2 & 0.00860 & 95.9 & 0.2 & \mathrm{u} & 93.0 \\ 3 & 0.01110 & 96.1 & 0.2 & \mathrm{u} & 98.7 \\ 4 & 0.01410 & 100.3 & 0.2 & \mathrm{u} & 99.3 \\ 5 & 0.01800 & 92.0 & 0.3 & \mathrm{u} & 93.8 \\ 6 & 0.02300 & 69.9 & 0.3 & \mathrm{u} & 83.1 \\ 7 & 0.02940 & 60.7 & 0.2 & \mathrm{u} & 71.1 \\ 8 & 0.03750 & 59.5 & 0.3 & \mathrm{u} & 59.7 \\ 9 & 0.04790 & 46.9 & 0.3 & \mathrm{u} & 50.0 \\ 10 & 0.06110 & 42.1 & 0.3 & \mathrm{u} & 41.9 \\ 11 & 0.07790 & 36.3 & 0.3 & \mathrm{u} & 35.4 \\ 12 & 0.09930 & 31.5 & 0.3 & \mathrm{u} & 30.2 \\ 13 & 0.12670 & 27.4 & 0.3 & \mathrm{u} & 26.0 \\ 14 & 0.16640 & 23.6 & 0.2 & \mathrm{u} & 22.3 \\ 15 & 0.20600 & 20.8 & 0.3 & \mathrm{u} & 19.9 \\ 16 & 0.26280 & 18.3 & 0.4 & \mathrm{u} & 17.8 \\ 17 & 0.33520 & 16.2 & 0.3 & \mathrm{u} & 16.2 \\ 18 & 0.42770 & 14.5 & 0.4 & \mathrm{u} & 14.9 \\ 19 & 0.54560 & 13.5 & 0.5 & \mathrm{u} & 14.0 \\ 20 & 0.69590 & 12.8 & 0.6 & \mathrm{u} & 13.4\end{array}$

Date: 01-MAY-08

UTM Coord: $\mathrm{E}[\mathrm{m}]$

Elevation $[\mathrm{m}]$ :

$582420.0 \quad \mathrm{~N}[\mathrm{~m}] \quad 2866116.0$

0.0

RX location: X $[\mathrm{m}]$

$0.0 \quad Y[m]$

0.0

Fit Error $\left[\frac{\circ}{0}\right]: 9.278$

System: EM-58 Freq[Hz]: 30 Data Set Code: hi TX Cur $[A]: \quad 1.40$ Turn Off[usec]: 2.5

RX Moment[turns-m^2]: 31.4 Gain Setting: 6

Time
$[\mathrm{ms}]$
0.08800
0.10600
0.13100
0.16100
0.20000
0.25000
0.31400
0.39500
0.49900
0.63100
0.79900
1.01000
1.28000
1.63000
2.08000
2.64000
3.37000
4.29000
5.47000
6.97000

rhoa_obs
[ohm-m]
34.0
30.4
27.0
23.8
20.7
18.1
15.9
14.0
12.4
11.3
10.4
9.7
9.2
8.5
10.9
13.2
7.0
5.3
5.3
8.0

$\begin{array}{llr}\text { err } & \text { mask } & \begin{array}{r}\text { rhoa_cal } \\ \text { [ohm-m] }\end{array} \\ 0.4 & \text { u } & 32.2 \\ 0.2 & \mathrm{u} & 28.4 \\ 0.3 & \mathrm{u} & 25.0 \\ 0.4 & \mathrm{u} & 22.0 \\ 0.3 & \mathrm{u} & 19.6 \\ 0.5 & \mathrm{u} & 17.4 \\ 0.4 & \mathrm{u} & 15.6 \\ 0.5 & \mathrm{u} & 14.1 \\ 0.8 & \mathrm{u} & 12.8 \\ 0.8 & \mathrm{u} & 11.8 \\ 1.5 & \mathrm{u} & 10.9 \\ 1.9 & \mathrm{u} & 10.1 \\ 4.7 & \mathrm{u} & 9.5 \\ 4.3 & \mathrm{u} & 9.0 \\ 6.4 & \mathrm{~m} & 8.6 \\ 3.0 & \mathrm{~m} & 8.2 \\ 25.8 & \mathrm{~m} & 8.0 \\ 27.6 & \mathrm{~m} & 7.8 \\ 5.1 & \mathrm{~m} & 7.8 \\ 0.0 & \mathrm{~m} & 7.9\end{array}$


Sounding: MIA112L3

Client: US Geological Survey

Soution: Holy Family Church

Project: Saltwater Intrusion

County: Miami-Dade County

TX loop size: X $[\mathrm{m}] \quad 38.1 \quad \mathrm{Y}[\mathrm{m}] \quad 38.1$

$\begin{array}{cccc}\text { Model } & \text { Resistivity } & \text { Thickness } & \text { Elevation } \\ \text { Layer } & {[\text { ohm }-\mathrm{m}]} & {[\mathrm{m}]} & {[\mathrm{m}]} \\ 1 & 1105.900 & 7.7 & 0.0 \\ 2 & 49.540 & 26.1 & -7.7 \\ 3 & 5.110 & -- & -33.9\end{array}$

System: EM-58 Freq[Hz]: 285 Data Set Code: uh TX $\operatorname{Cur}[A]: \quad 1.40$ Turn Off[usec] : 2.5

RX Moment[turns-m^2]: 31.4 Gain Setting: 2

$\begin{array}{rrrrrr}\text { Time } & \begin{array}{r}\text { rhoa_obs } \\ {[\text { ohm-m] }}\end{array} & \begin{array}{c}\text { obs_err } \\ {\left[\frac{\mathrm{o}}{0}\right]}\end{array} & \text { mask } & \begin{array}{r}\text { rhoa_cal } \\ {[\text { ohm-m] }}\end{array} \\ 1 & 0.00680 & 97.9 & 0.3 & \mathrm{u} & 99.8 \\ 2 & 0.00860 & 95.9 & 0.2 & \mathrm{u} & 94.3 \\ 3 & 0.01110 & 96.1 & 0.2 & \mathrm{u} & 93.7 \\ 4 & 0.01410 & 100.3 & 0.2 & \mathrm{u} & 92.2 \\ 5 & 0.01800 & 92.0 & 0.3 & \mathrm{u} & 87.8 \\ 6 & 0.02300 & 69.9 & 0.3 & \mathrm{u} & 80.2 \\ 7 & 0.02940 & 60.7 & 0.2 & \mathrm{u} & 70.4 \\ 8 & 0.03750 & 59.5 & 0.3 & \mathrm{u} & 60.2 \\ 9 & 0.04790 & 46.9 & 0.3 & \mathrm{u} & 50.8 \\ 10 & 0.06110 & 42.1 & 0.3 & \mathrm{u} & 42.8 \\ 11 & 0.07790 & 36.3 & 0.3 & \mathrm{u} & 36.2 \\ 12 & 0.09930 & 31.5 & 0.3 & \mathrm{u} & 30.8 \\ 13 & 0.12670 & 27.4 & 0.3 & \mathrm{u} & 26.4 \\ 14 & 0.16640 & 23.6 & 0.2 & \mathrm{u} & 22.6 \\ 15 & 0.20600 & 20.8 & 0.3 & \mathrm{u} & 20.1 \\ 16 & 0.26280 & 18.3 & 0.4 & \mathrm{u} & 17.9 \\ 17 & 0.33520 & 16.2 & 0.3 & \mathrm{u} & 16.2 \\ 18 & 0.42770 & 14.5 & 0.4 & \mathrm{u} & 14.9 \\ 19 & 0.54560 & 13.5 & 0.5 & \mathrm{u} & 13.9 \\ 20 & 0.69590 & 12.8 & 0.6 & \mathrm{u} & 13.3\end{array}$

Date: 01-MAY-08

UTM Coord: $E[\mathrm{~m}]$

Elevation $[\mathrm{m}]$ :

$582420.0 \quad \mathrm{~N}[\mathrm{~m}] \quad 2866116.0$ 0.0

RX location: $\mathrm{X}[\mathrm{m}]$

$0.0 \quad \mathrm{Y}[\mathrm{m}] \quad 0.0$

Fit Error [\%]: 7.815

System: EM-58 Freq[Hz]: 30 Data Set Code: hi TX Cur $[A]: \quad 1.40$ Turn Off [usec] : 2.5

RX Moment [turns-m^2]: 31.4 Gain Setting: 6

$\begin{array}{rrrrrr}\text { Time } & \begin{array}{c}\text { rhoa_obs } \\ \text { [ohm-m] }\end{array} & \begin{array}{c}\text { obs_err } \\ {\left[\begin{array}{c}\text { o }] \\ 0\end{array}\right.}\end{array} & \text { mask } & \begin{array}{r}\text { rhoa_cal } \\ {[\text { ohm-m] }}\end{array} \\ 1 & 0.08800 & 34.0 & 0.4 & \mathrm{u} & 32.9 \\ 2 & 0.10600 & 30.4 & 0.2 & \mathrm{u} & 28.9 \\ 3 & 0.13100 & 27.0 & 0.3 & \mathrm{u} & 25.4 \\ 4 & 0.16100 & 23.8 & 0.4 & \mathrm{u} & 22.3 \\ 5 & 0.20000 & 20.7 & 0.3 & \mathrm{u} & 19.7 \\ 6 & 0.25000 & 18.1 & 0.5 & \mathrm{u} & 17.5 \\ 7 & 0.31400 & 15.9 & 0.4 & \mathrm{u} & 15.6 \\ 8 & 0.39500 & 14.0 & 0.5 & \mathrm{u} & 14.1 \\ 9 & 0.49900 & 12.4 & 0.8 & \mathrm{u} & 12.7 \\ 10 & 0.63100 & 11.3 & 0.8 & \mathrm{u} & 11.6 \\ 11 & 0.79900 & 10.4 & 1.5 & \mathrm{u} & 10.7 \\ 12 & 1.01000 & 9.7 & 1.9 & \mathrm{u} & 9.9 \\ 13 & 1.28000 & 9.2 & 4.7 & \mathrm{u} & 9.3 \\ 14 & 1.63000 & 8.5 & 4.3 & \mathrm{u} & 8.8 \\ 15 & 2.08000 & 10.9 & 16.4 & \mathrm{~m} & 8.3 \\ 16 & 2.64000 & 13.2 & 33.0 & \mathrm{~m} & 8.0 \\ 17 & 3.37000 & 7.0 & 25.8 & \mathrm{~m} & 7.7 \\ 18 & 4.29000 & 5.3 & 27.6 & \mathrm{~m} & 7.6 \\ 19 & 5.47000 & 5.3 & 35.1 & \mathrm{~m} & 7.5 \\ 20 & 6.97000 & 8.0 & 100.0 & \mathrm{~m} & 7.5\end{array}$


Sounding: MIA113

Location: Oak Grove Park

Project: Saltwater Intrusion

County: Miami-Dade County

TX loop size: X $[\mathrm{m}] \quad 38.1 \quad \mathrm{Y}[\mathrm{m}] \quad 38.1$

$\begin{array}{cccc}\text { Model } & \text { Resistivity } & \text { Thickness } & \text { Elevation } \\ \text { Layer } & {[\text { ohm-m] }} & {[\mathrm{m}]} & {[\mathrm{m}]} \\ 1 & 790.200 & 7.7 & 0.0 \\ 2 & 73.980 & 50.5 & -7.7 \\ 3 & 5.300 & -- & -58.2\end{array}$

System: EM-58 Freq[Hz]: 285 Data set Code: uh TX Cur $[A]: \quad 1.40$ Turn Off [usec] : 2.5

RX Moment[turns-m^2]: 31.4 Gain Setting:

\begin{tabular}{|c|c|c|c|c|c|}
\hline & $\begin{array}{l}\text { Time } \\
{[\mathrm{ms}]}\end{array}$ & $\begin{array}{l}\text { rhoa_obs } \\
\text { [ohm-m] }\end{array}$ & $\begin{array}{c}\text { obs err } \\
{\left[\frac{\mathrm{o}}{0}\right]}\end{array}$ & mask & $\begin{array}{l}\text { rhoa_cal } \\
{[\mathrm{ohm}-\mathrm{m}]}\end{array}$ \\
\hline 1 & 0.00680 & 116.8 & 0.3 & u & 117.6 \\
\hline 2 & 0.00860 & 107.9 & 0.3 & $\mathrm{u}$ & 109.6 \\
\hline 3 & 0.01110 & 107.2 & 0.3 & $\mathrm{u}$ & 107.2 \\
\hline 4 & 0.01410 & 107.5 & 0.3 & $u$ & 106.5 \\
\hline 5 & 0.01800 & 110.4 & 0.3 & $u$ & 107.4 \\
\hline 6 & 0.02300 & 126.4 & 0.3 & $\mathrm{u}$ & 109.4 \\
\hline 7 & 0.02940 & 102.0 & 0.3 & $\mathrm{u}$ & 111.1 \\
\hline 8 & 0.03750 & 100.7 & 0.3 & $\mathrm{u}$ & 109.9 \\
\hline 9 & 0.04790 & 104.3 & 0.4 & $u$ & 103.9 \\
\hline 10 & 0.06110 & 90.6 & 0.3 & $u$ & 93.5 \\
\hline 11 & 0.07790 & 82.0 & 0.4 & $u$ & 81.1 \\
\hline 12 & 0.09930 & 68.8 & 0.4 & $u$ & 68.8 \\
\hline 13 & 0.12670 & 58.5 & 0.5 & $u$ & 57.9 \\
\hline 14 & 0.16640 & 48.5 & 0.4 & $\mathrm{u}$ & 47.9 \\
\hline 15 & 0.20600 & 41.9 & 0.6 & $\mathrm{u}$ & 41.6 \\
\hline 16 & 0.26280 & 36.9 & 0.4 & $\mathrm{u}$ & 35.7 \\
\hline 17 & 0.33520 & 31.4 & 1.0 & $u$ & 31.1 \\
\hline 18 & 0.42770 & 27.6 & 2.3 & $u$ & 27.5 \\
\hline 19 & 0.5 & 24.4 & 3.2 & $\mathrm{u}$ & 24.8 \\
\hline 20 & 0.6 & 22.2 & 3.1 & $u$ & 22 . \\
\hline
\end{tabular}

Date: 01-MAY-08

UTM Coord: $\mathrm{E}[\mathrm{m}]$

Elevation $[\mathrm{m}]$ :

$581685.0 \quad \mathrm{~N}[\mathrm{~m}] \quad 2867153.0$

0.0

RX location: X $[\mathrm{m}]$

$0.0 \quad Y[m]$

0.0

\section{Fit Error [] : 6.967}

System: EM-58 Freq[Hz]: 30 Data Set Code: hi TX $\operatorname{Cur}[\mathrm{A}]: \quad 1.40$ Turn Off[usec]: 2.5

RX Moment [turns-m^2] : $31.4 \quad$ Gain Setting: 7

Time
$[\mathrm{ms}]$
0.08800
0.10600
0.13100
0.16100
0.20000
0.25000
0.31400
0.39500
0.49900
0.63100
0.79900
1.01000
1.28000
1.63000
2.08000
2.64000
3.37000
4.29000
5.47000
6.97000

rhoa_obs
[ohm-m]
68.3
57.2
49.0
47.0
36.6
29.2
22.2
18.0
14.6
15.4
9.5
12.4
4.2
2.7
2.3
3.3
2.2
1.4
1.6
.6

obs_err mask [응]

$$
0.0
$$$$
0.0
$$$$
0.0
$$$$
0.0
$$$$
0.0
$$$$
0.0
$$$$
0.0
$$$$
0.0
$$$$
0.0
$$$$
0.0
$$$$
0.0
$$$$
0.0
$$$$
0.0
$$$$
0.0
$$$$
0.0
$$$$
0.0
$$$$
0.0
$$$$
0.0
$$$$
0.0
$$

0.0
0.0

$\mathrm{m}$
$\mathrm{m}$
$\mathrm{m}$
$\mathrm{m}$
$\mathrm{m}$
$\mathrm{m}$
$\mathrm{m}$
$\mathrm{m}$
$\mathrm{m}$
$\mathrm{m}$
$\mathrm{m}$
$\mathrm{m}$
$\mathrm{m}$
$\mathrm{m}$
$\mathrm{m}$
$\mathrm{m}$
$\mathrm{m}$
$\mathrm{m}$
$\mathrm{m}$
$\mathrm{m}$

rhoa_cal [ohm-m]

73.2

63.7

54.7

46.8

40.1

34.3

29.5

25.5

22.2

19.5

17.3

15.5

14.0

12.7

11.8

11.0

10.4

9.9

9.6 
Sounding: MIA114

Client: US Geological Survey

Location: Highland Oaks Park

Project: Saltwater Intrusion

County: Miami-Dade County

TX loop size: X $[\mathrm{m}] \quad 38.1 \quad \mathrm{Y}[\mathrm{m}] \quad 38.1$

$\begin{array}{cccc}\text { Model } & \text { Resistivity } & \text { Thickness } & \text { Elevation } \\ \text { Layer } & {[\text { ohm-m] }} & {[\mathrm{m}]} & {[\mathrm{m}]} \\ 1 & 921.900 & 8.1 & 0.0 \\ 2 & 6.240 & 8.2 & -8.1 \\ 3 & 2.460 & 59.3 & -16.3 \\ 4 & 27.000 & -- & -75.6\end{array}$

System: EM-58 Freq[Hz]: 285 Data Set Code: uh TX Cur $[\mathrm{A}]: \quad 1.40$ Turn Off[usec]: 2.5

RX Moment[turns-m^2]: 31.4 Gain Setting: 2

$\begin{array}{rrrrrr}\text { Time } & \begin{array}{c}\text { rhoa_obs } \\ \text { [ohm-m] }\end{array} & \begin{array}{c}\text { obs_err } \\ {\left[\frac{\mathrm{o}}{0}\right]}\end{array} & \text { mask } & \begin{array}{c}\text { rhoa_cal } \\ \text { [ohm-m] }\end{array} \\ 1 & 0.00680 & 55.6 & 0.1 & \mathrm{u} & 57.0 \\ 2 & 0.00860 & 47.1 & 0.1 & \mathrm{u} & 45.8 \\ 3 & 0.01110 & 39.1 & 0.1 & \mathrm{u} & 38.3 \\ 4 & 0.01410 & 32.6 & 0.1 & \mathrm{u} & 32.5 \\ 5 & 0.01800 & 27.2 & 0.1 & \mathrm{u} & 27.5 \\ 6 & 0.02300 & 22.9 & 0.1 & \mathrm{u} & 23.3 \\ 7 & 0.02940 & 19.3 & 0.1 & \mathrm{u} & 19.6 \\ 8 & 0.03750 & 16.4 & 0.1 & \mathrm{u} & 16.6 \\ 9 & 0.04790 & 14.0 & 0.1 & \mathrm{u} & 14.1 \\ 10 & 0.06110 & 12.0 & 0.1 & \mathrm{u} & 12.0 \\ 11 & 0.07790 & 10.4 & 0.1 & \mathrm{u} & 10.3 \\ 12 & 0.09930 & 9.0 & 0.1 & \mathrm{u} & 9.0 \\ 13 & 0.12670 & 7.9 & 0.1 & \mathrm{u} & 7.9 \\ 14 & 0.16640 & 6.9 & 0.1 & \mathrm{u} & 7.0 \\ 15 & 0.20600 & 6.3 & 0.1 & \mathrm{u} & 6.4 \\ 16 & 0.26280 & 5.7 & 0.1 & \mathrm{u} & 5.8 \\ 17 & 0.33520 & 5.3 & 0.1 & \mathrm{u} & 5.4 \\ 18 & 0.42770 & 4.9 & 0.1 & \mathrm{u} & 5.1 \\ 19 & 0.54560 & 4.7 & 0.6 & \mathrm{u} & 4.8 \\ 20 & 0.69590 & 4.6 & 1.1 & \mathrm{u} & 4.6\end{array}$

Date: 01-MAY-08

UTM Coord: $\mathrm{E}[\mathrm{m}]$

Elevation $[\mathrm{m}]$ :

$584955.0 \quad \mathrm{~N}[\mathrm{~m}] \quad 2872017.0$

0.0

RX location: X $[\mathrm{m}]$

$0.0 \quad Y[m]$

0.0
Fit Error $[\%]: 3.249$

System: EM-58 Freq[Hz]: 30 Data Set Code: hi TX Cur $[A]: \quad 1.40$ Turn Off[usec]: 2.5

RX Moment [turns-m^2] : 31.4 Gain Setting: 7

Time
$[\mathrm{ms}]$
0.08800
0.10600
0.13100
0.16100
0.20000
0.25000
0.31400
0.39500
0.49900
0.63100
0.79900
1.01000
1.28000
1.63000
2.08000
2.64000
3.37000
4.29000
5.47000
6.97000

$\begin{array}{rr}\text { rhoa_obs } & \text { obs err } \\ \text { [ohm-m] } & {[\%]} \\ 9.7 & 0.3 \\ 8.8 & 0.3 \\ 7.9 & 0.4 \\ 7.1 & 0.3 \\ 6.4 & 0.3 \\ 5.8 & 0.3 \\ 5.2 & 0.3 \\ 4.8 & 0.4 \\ 4.4 & 0.5 \\ 4.1 & 0.6 \\ 3.9 & 1.6 \\ 3.6 & 2.2 \\ 3.4 & 4.2 \\ 3.4 & 4.3 \\ 3.4 & 6.1 \\ 3.6 & 12.0 \\ 3.7 & 35.9 \\ 6.2 & 71.7 \\ 3.9 & 84.2 \\ 8.7 & 100.0\end{array}$

mask rhoa cal

[ohm-m]

9.5

8.6

7.7

6.9

6.3

5.7

5.2

4.8

4.4

4.1

3.8

3.6

3.5

3.4

3.4

3.4

3.5

3. 8

4.1
4.5 
Sounding: MIA114L3

Location: Highland Oaks Park

Project: Saltwater Intrusion

County: Miami-Dade County

TX loop size: X[m] $38.1 \quad Y[m] \quad 38.1$

$\begin{array}{cccc}\text { Model } & \text { Resistivity } & \text { Thickness } & \text { Elevation } \\ \text { Layer } & {[\text { ohm-m] }} & {[\mathrm{m}]} & {[\mathrm{m}]} \\ 1 & 34.750 & 13.0 & 0.0 \\ 2 & 2.610 & 68.3 & -13.0 \\ 3 & 29.230 & -- & -81.3\end{array}$

System: EM-58 Freq[Hz]: 285 Data Set Code: uh TX Cur[A]: 1.40 Turn Off[usec]: 2.5

RX Moment[turns-m^2]: 31.4 Gain Setting: 2

$\begin{array}{rrrrrr}\text { Time } & \begin{array}{c}\text { rhoa_obs } \\ \text { [ohm-m] }\end{array} & \begin{array}{c}\text { obserr } \\ {\left[\frac{o}{0}\right]}\end{array} & \text { mask } & \begin{array}{r}\text { rhoa_cal } \\ \text { [ohm-m] }\end{array} \\ 1 & 0.00680 & 55.6 & 0.1 & u & 52.3 \\ 2 & 0.00860 & 47.1 & 0.1 & u & 47.2 \\ 3 & 0.01110 & 39.1 & 0.1 & u & 41.5 \\ 4 & 0.01410 & 32.6 & 0.1 & u & 35.0 \\ 5 & 0.01800 & 27.2 & 0.1 & u & 28.9 \\ 6 & 0.02300 & 22.9 & 0.1 & u & 23.8 \\ 7 & 0.02940 & 19.3 & 0.1 & u & 19.6 \\ 8 & 0.03750 & 16.4 & 0.1 & u & 16.3 \\ 9 & 0.04790 & 14.0 & 0.1 & u & 13.7 \\ 10 & 0.06110 & 12.0 & 0.1 & u & 11.7 \\ 11 & 0.07790 & 10.4 & 0.1 & u & 10.0 \\ 12 & 0.09930 & 9.0 & 0.1 & u & 8.8 \\ 13 & 0.12670 & 7.9 & 0.1 & u & 7.7 \\ 14 & 0.16640 & 6.9 & 0.1 & u & 6.8 \\ 15 & 0.20600 & 6.3 & 0.1 & u & 6.3 \\ 16 & 0.26280 & 5.7 & 0.1 & u & 5.8 \\ 17 & 0.33520 & 5.3 & 0.1 & u & 5.4 \\ 18 & 0.42770 & 4.9 & 0.1 & u & 5.1 \\ 19 & 0.54560 & 4.7 & 0.6 & u & 4.9 \\ 20 & 0.69590 & 4.6 & 1.1 & u & 4.7\end{array}$

Date: 01-MAY-08

UTM Coord: $\mathrm{E}[\mathrm{m}]$

Elevation $[\mathrm{m}]$ :

$584955.0 \quad \mathrm{~N}[\mathrm{~m}] \quad 2872017.0$ 0.0

RX location: $\mathrm{X}[\mathrm{m}]$

$0.0 \quad \mathrm{Y}[\mathrm{m}] \quad 0.0$
Fit Error $[\%]: 5.378$

System: EM-58 Freq[Hz]: 30 Data Set Code: hi TX $\operatorname{Cur}[A]: \quad 1.40$ Turn Off[usec]: 2.5

RX Moment [turns-m^2] : 31.4 Gain Setting: 7

Time
$[\mathrm{ms}]$
0.08800
0.10600
0.13100
0.16100
0.20000
0.25000
0.31400
0.39500
0.49900
0.63100
0.79900
1.01000
1.28000
1.63000
2.08000
2.64000
3.37000
4.29000
5.47000
6.97000

$\begin{array}{rr}\text { rhoa_obs } & \text { obs_err } \\ \text { [ohm-m] } & {[\bar{\circ} \text { ] }} \\ 9.7 & 0.3 \\ 8.8 & 0.3 \\ 7.9 & 0.4 \\ 7.1 & 0.3 \\ 6.4 & 0.3 \\ 5.8 & 0.3 \\ 5.2 & 0.3 \\ 4.8 & 0.4 \\ 4.4 & 0.5 \\ 4.1 & 0.6 \\ 3.9 & 1.6 \\ 3.6 & 2.2 \\ 3.4 & 4.2 \\ 3.4 & 4.3 \\ 3.4 & 6.1 \\ 3.6 & 12.0 \\ 3.7 & 35.9 \\ 6.2 & 71.7 \\ 3.9 & 84.2 \\ 8.7 & 100.0\end{array}$

mask rhoa cal

[ohm-m]

9.3

8.3

7.5

6.8

6.2

5.7

5.2

4.8

4.5

4.2

3.9

3.7

3.5

3.4

3.4

3.4

3.6

3. 8

4. 1

4.5 
Sounding: MIA115

Client: US Geological Survey

Date: 01-MAY-08

UTM Coord: $\mathrm{E}[\mathrm{m}]$

ocation: Hallandale Elementary School

Project: Saltwater Intrusion

County: Broward County

TX loop size: X $[\mathrm{m}] \quad 38.1 \quad \mathrm{Y}[\mathrm{m}] \quad 38.1$

$\begin{array}{cccc}\text { Model } & \text { Resistivity } & \text { Thickness } & \text { Elevation } \\ \text { Layer } & \text { [ohm-m] } & {[\mathrm{m}]} & {[\mathrm{m}]} \\ 1 & 189.200 & 14.7 & 0.0 \\ 2 & 4.140 & 16.8 & -14.7 \\ 3 & 27.270 & -- & -31.5\end{array}$

System: EM-58 Freq[Hz]: 285 Data Set Code: uh TX Cur $[A]: \quad 1.40$ Turn Off [usec]: 2.5

RX Moment[turns-m^2]: 31.4 Gain Setting: 2

\begin{tabular}{|c|c|c|c|c|}
\hline $\begin{array}{l}\text { Time } \\
{[\mathrm{ms}]}\end{array}$ & $\begin{array}{l}\text { rhoa_obs } \\
\text { [ohm-m] }\end{array}$ & $\begin{array}{c}\text { obs err } \\
{\left[\frac{\overline{0}}{0}\right]}\end{array}$ & mask & $\begin{array}{l}\text { rhoa_ca } \\
{[\text { ohm-m] }}\end{array}$ \\
\hline 0.00680 & 100.8 & 0.4 & $\mathrm{u}$ & 100.7 \\
\hline 0.00860 & 78.7 & 0.2 & u & 78. \\
\hline 0.01110 & 62.3 & 0.2 & u & 61. \\
\hline 0.01410 & 49.3 & 0.2 & u & 49. \\
\hline 0.01800 & 39.5 & 0.2 & u & 40 \\
\hline 0.02300 & 31.6 & 0.2 & u & 32. \\
\hline 0.02940 & 26.3 & 0.2 & u & 26. \\
\hline 0.03750 & 22.4 & 0.2 & u & 21. \\
\hline 0.04790 & 18.6 & 0.2 & $\mathrm{u}$ & 17. \\
\hline 0.06110 & 16.0 & 0.2 & u & 15. \\
\hline 0.07790 & 13.7 & 0.3 & u & 13. \\
\hline 0.09930 & 11.9 & 0.2 & $\mathrm{u}$ & 11. \\
\hline 0.12670 & 10.8 & 0.3 & u & 10. \\
\hline 0.16640 & 10.5 & 0.6 & u & 10. \\
\hline 0.20600 & 10.8 & 0.8 & u & 9. \\
\hline 0.26280 & 12.3 & 1.2 & $\mathrm{~m}$ & 9. \\
\hline 0.33520 & 15.9 & 1.3 & $\mathrm{~m}$ & 10 \\
\hline 0.4 & 23.5 & 2.3 & $\mathrm{~m}$ & 10 \\
\hline & 42.2 & 10.6 & $\mathrm{~m}$ & 11 \\
\hline & 35 & 100.0 & $\mathrm{~m}$ & \\
\hline
\end{tabular}

\section{Fit Error $\left[\frac{\circ}{0}\right]: \quad 6.620$}

System: EM-58 Freq[Hz]: 30 Data Set Code: hi TX Cur $[A]: \quad 1.40$ Turn off[usec] : 2.5

RX Moment [turns-m^2] : 31.4 Gain Setting: 5

$\begin{array}{rrrrrr}\text { Time } & \begin{array}{c}\text { rhoa_obs } \\ \text { [ohm-m] }\end{array} & \begin{array}{r}\text { obs_err } \\ {\left[\frac{\text { o }}{0}\right]}\end{array} & \text { mask } & \begin{array}{r}\text { rhoa_cal } \\ {[\text { ohm-m] }}\end{array} \\ 1 & 0.08800 & 11.6 & 1.5 & \mathrm{u} & 12.4 \\ 2 & 0.10600 & 10.6 & 1.1 & \mathrm{u} & 11.4 \\ 3 & 0.13100 & 9.9 & 2.5 & \mathrm{u} & 10.7 \\ 4 & 0.16100 & 9.7 & 5.0 & \mathrm{u} & 10.2 \\ 5 & 0.20000 & 10.4 & 11.3 & \mathrm{u} & 9.9 \\ 6 & 0.25000 & 12.0 & 23.2 & \mathrm{~m} & 9.8 \\ 7 & 0.31400 & 19.0 & 100.0 & \mathrm{~m} & 9.8 \\ 8 & 0.39500 & 55.3 & 100.0 & \mathrm{~m} & 10.1 \\ 9 & 0.49900 & 18.8 & 100.0 & \mathrm{~m} & 10.4 \\ 10 & 0.63100 & 10.9 & 100.0 & \mathrm{~m} & 10.9 \\ 11 & 0.79900 & 6.9 & 100.0 & \mathrm{~m} & 11.6 \\ 12 & 1.01000 & 8.1 & 100.0 & \mathrm{~m} & 12.3 \\ 13 & 1.28000 & 4.0 & 100.0 & \mathrm{~m} & 13.2 \\ 14 & 1.63000 & 2.6 & 100.0 & \mathrm{~m} & 14.1 \\ 15 & 2.08000 & 49.2 & 100.0 & \mathrm{~m} & 15.1 \\ 16 & 2.64000 & .9 & 100.0 & \mathrm{~m} & 16.1 \\ 17 & 3.37000 & 1.3 & 100.0 & \mathrm{~m} & 17.2 \\ 18 & 4.29000 & 4.1 & 100.0 & \mathrm{~m} & 18.3 \\ 19 & 5.47000 & 1.0 & 100.0 & \mathrm{~m} & 19.9 \\ 20 & 6.97000 & .2 & 79.8 & \mathrm{~m} & 21.5\end{array}$


Sounding: MIA115L2

Location: Hallandale Elementary School

Project: Saltwater Intrusion

County: Broward County

TX loop size: X[m] $38.1 \quad \mathrm{Y}[\mathrm{m}] \quad 38.1$

$\begin{array}{cccc}\text { Model } & \text { Resistivity } & \text { Thickness } & \text { Elevation } \\ \text { Layer } & {[\text { ohm-m] }} & {[\mathrm{m}]} & {[\mathrm{m}]} \\ 1 & 707.300 & 13.9 & 0.0 \\ 2 & 4.240 & -- & -13.9\end{array}$

System: EM-58 Freq[Hz]: 285 Data Set Code: uh TX Cur $[\mathrm{A}]: \quad 1.40$ Turn Off[usec] : 2.5

RX Moment[turns-m^2]: 31.4 Gain Setting: 2

\begin{tabular}{|c|c|c|c|c|c|}
\hline & $\begin{array}{l}\text { Time } \\
{[\mathrm{ms}]}\end{array}$ & $\begin{array}{l}\text { rhoa_obs } \\
\text { [ohm-m ] }\end{array}$ & $\begin{array}{c}\text { obs err } \\
{\left[\frac{\overline{0}}{0}\right]}\end{array}$ & mask & $\begin{array}{l}\text { rhoa_cal } \\
\text { [ohm-m] }\end{array}$ \\
\hline 1 & 0.00680 & 100.8 & 0.4 & $u$ & 100.1 \\
\hline 2 & 0.00860 & 78.7 & 0.2 & $u$ & 76.7 \\
\hline 3 & 0.01110 & 62.3 & 0.2 & $u$ & 60.5 \\
\hline 4 & 0.01410 & 49.3 & 0.2 & $u$ & 48.4 \\
\hline 5 & 0.01800 & 39.5 & 0.2 & $u$ & 39.1 \\
\hline 6 & 0.02300 & 31.6 & 0.2 & $u$ & 31.9 \\
\hline 7 & 0.02940 & 26.3 & 0.2 & $u$ & 26.4 \\
\hline 8 & 0.03750 & 22.4 & 0.2 & u & 22.1 \\
\hline 9 & 0.04790 & 18.6 & 0.2 & u & 18.7 \\
\hline 10 & 0.06110 & 16.0 & 0.2 & $\mathrm{u}$ & 16.1 \\
\hline 11 & 0.07790 & 13.7 & 0.3 & $u$ & 14.0 \\
\hline 12 & 0.09930 & 11.9 & 0.2 & $u$ & 12.4 \\
\hline 13 & 0.12670 & 10.8 & 0.3 & $u$ & 11.1 \\
\hline 14 & 0.16640 & 10.5 & 0.6 & $u$ & 9.9 \\
\hline 15 & 0.20600 & 10.8 & 0.8 & $u$ & 9.2 \\
\hline 16 & 0.26280 & 12.3 & 1.2 & $\mathrm{~m}$ & 8.5 \\
\hline 17 & 0.33520 & 15.9 & 1.3 & $\mathrm{~m}$ & 8.0 \\
\hline 18 & 0.42770 & 23.5 & 2.3 & $\mathrm{~m}$ & 7.7 \\
\hline 19 & 0.54560 & 42.2 & 10.6 & $\mathrm{~m}$ & 7.5 \\
\hline 20 & 0.69590 & 353.1 & 100.0 & $\mathrm{~m}$ & 7.3 \\
\hline
\end{tabular}

Date: 01-MAY-08

UTM Coord: $\mathrm{E}[\mathrm{m}]$

Elevation $[\mathrm{m}]$ :

$583794.0 \quad \mathrm{~N}[\mathrm{~m}] \quad 2873525.0$ 0.0

RX location: $\mathrm{X}[\mathrm{m}]$

$0.0 \quad \mathrm{Y}[\mathrm{m}] \quad 0.0$

\section{Fit Error [\%] : 10.254}

System: EM-58 Freq[Hz]: 30 Data Set Code: hi TX Cur $[A]: \quad 1.40$ Turn Off[usec] : 2.5

RX Moment [turns-m^2]: 31.4 Gain Setting: 5

$\begin{array}{rrrrrr}\text { Time } & \begin{array}{c}\text { rhoa_obs } \\ {[\text { ohm-m] }}\end{array} & \begin{array}{r}\text { obserr } \\ {\left[\begin{array}{c}\text { o }] \\ \text { [ms }\end{array}\right.}\end{array} & \text { mask } & \begin{array}{r}\text { rhoa_cal } \\ \text { [ohm-m] }\end{array} \\ 1 & 0.08800 & 11.6 & 1.5 & \mathrm{u} & 13.0 \\ 2 & 0.10600 & 10.6 & 1.1 & \mathrm{u} & 11.8 \\ 3 & 0.13100 & 9.9 & 2.5 & \mathrm{u} & 10.8 \\ 4 & 0.16100 & 9.7 & 5.0 & \mathrm{u} & 9.8 \\ 5 & 0.20000 & 10.4 & 11.3 & \mathrm{u} & 9.1 \\ 6 & 0.25000 & 12.0 & 23.2 & \mathrm{~m} & 8.4 \\ 7 & 0.31400 & 19.0 & 100.0 & \mathrm{~m} & 7.8 \\ 8 & 0.39500 & 55.3 & 100.0 & \mathrm{~m} & 7.3 \\ 9 & 0.49900 & 18.8 & 100.0 & \mathrm{~m} & 6.9 \\ 10 & 0.63100 & 10.9 & 100.0 & \mathrm{~m} & 6.5 \\ 11 & 0.79900 & 6.9 & 100.0 & \mathrm{~m} & 6.2 \\ 12 & 1.01000 & 8.1 & 100.0 & \mathrm{~m} & 6.0 \\ 13 & 1.28000 & 4.0 & 100.0 & \mathrm{~m} & 5.8 \\ 14 & 1.63000 & 2.6 & 100.0 & \mathrm{~m} & 5.6 \\ 15 & 2.08000 & 49.2 & 100.0 & \mathrm{~m} & 5.4 \\ 16 & 2.64000 & .9 & 100.0 & \mathrm{~m} & 5.3 \\ 17 & 3.37000 & 1.3 & 100.0 & \mathrm{~m} & 5.3 \\ 18 & 4.29000 & 4.1 & 100.0 & \mathrm{~m} & 5.3 \\ 19 & 5.47000 & 1.0 & 100.0 & \mathrm{~m} & 5.3 \\ 20 & 6.97000 & .2 & 79.8 & \mathrm{~m} & 5.4\end{array}$


Sounding: MIA116

Location: St. Michael School South

Project: Saltwater Intrusion

County: Miami-Dade County

TX loop size: X[m] $38.1 \quad Y[m] \quad 38.1$

$\begin{array}{cccc}\text { Model } & \text { Resistivity } & \text { Thickness } & \text { Elevation } \\ \text { Layer } & {[\text { ohm-m] }} & {[\mathrm{m}]} & {[\mathrm{m}]} \\ 1 & 173.600 & 30.5 & 0.0 \\ 2 & 13.310 & -- & -30.5\end{array}$

System: EM-58 Freq[Hz]: 285 Data Set Code: uh TX Cur $[A]: \quad 1.40$ Turn Off[usec]: 2.5

RX Moment[turns-m^2]: 31.4 Gain Setting: 5

\begin{tabular}{|c|c|c|c|c|c|}
\hline & $\begin{array}{l}\text { Time } \\
{[\mathrm{ms}]}\end{array}$ & $\begin{array}{l}\text { rhoa_obs } \\
\text { [ohm-m] }\end{array}$ & $\begin{array}{c}\text { obs err } \\
{\left[\frac{\mathrm{o}}{0}\right]}\end{array}$ & mask & $\begin{array}{l}\text { rhoa_cal } \\
\text { [ohm-m] }\end{array}$ \\
\hline 1 & 0.00680 & 184.3 & 0.4 & $u$ & 180.6 \\
\hline 2 & 0.00860 & 163.2 & 0.2 & $u$ & 168.5 \\
\hline 3 & 0.01110 & 151.4 & 0.3 & u & 152.6 \\
\hline 4 & 0.01410 & 132.1 & 0.3 & $u$ & 132.8 \\
\hline 5 & 0.01800 & 115.6 & 0.3 & $u$ & 113.2 \\
\hline 6 & 0.02300 & 102.5 & 0.3 & $\mathrm{u}$ & 96.1 \\
\hline 7 & 0.02940 & 87.8 & 0.3 & u & 81.6 \\
\hline 8 & 0.03750 & 70.9 & 0.2 & u & 69.8 \\
\hline 9 & 0.04790 & 53.5 & 0.2 & u & 60.2 \\
\hline 10 & 0.06110 & 49.4 & 0.2 & u & 52.3 \\
\hline 11 & 0.07790 & 44.8 & 0.2 & u & 46.0 \\
\hline 12 & 0.09930 & 36.4 & 0.2 & u & 40.8 \\
\hline 13 & 0.12670 & 38.0 & 0.5 & u & 36.7 \\
\hline 14 & 0.16640 & 34.5 & 0.8 & $\mathrm{u}$ & 32.9 \\
\hline 15 & 0.20600 & 31.1 & 1.2 & $\mathrm{u}$ & 30.5 \\
\hline 16 & 0.26280 & 28.3 & 2.1 & u & 28.3 \\
\hline 17 & 0.33520 & 27.4 & 5.0 & $u$ & 26.6 \\
\hline 18 & 0.42770 & 24.0 & 8.2 & $u$ & 25.4 \\
\hline 19 & 0. & 24.8 & 16.1 & $u$ & 24.6 \\
\hline 20 & 0.69 & 23.2 & 24.6 & $u$ & 24.2 \\
\hline
\end{tabular}

Date: 02-MAY-08

UTM Coord: $\mathrm{E}[\mathrm{m}]$

Elevation $[\mathrm{m}]$ :

$575921.0 \quad \mathrm{~N}[\mathrm{~m}] \quad 2850911.0$ 0.0

RX location: $\mathrm{X}[\mathrm{m}]$

$0.0 Y[\mathrm{~m}] \quad 0.0$

\section{Fit Error $\left[\frac{\circ}{0}\right]: 8.888$}

System: EM-58 Freq[Hz]: 30 Data Set Code: hi TX Cur $[\mathrm{A}]: \quad 1.40$ Turn Off[usec]: 2.5

RX Moment [turns-m^2] : $31.4 \quad$ Gain Setting: 7

Time
$[\mathrm{ms}]$
0.08800
0.10600
0.13100
0.16100
0.20000
0.25000
0.31400
0.39500
0.49900
0.63100
0.79900
1.01000
1.28000
1.63000
2.08000
2.64000
3.37000
4.29000
5.47000
6.97000

rhoa_obs
[ohm-m]
44.4
35.2
37.5
34.0
33.5
35.5
47.5
31.7
45.7
42.3
23.3
11.8
9.0
16.3
5.4
1.9
1.7
2.1
.7
.7

mask

rhoa cal

[흥]

[ohm-m]

0.0 u 43.0

$\begin{array}{lll}0.0 & u & 39.2\end{array}$

0.0 u $\quad 35.7$

$\begin{array}{lll}0.0 & u & 32.7\end{array}$

0.0 u 30.1

$0.0 \mathrm{~m} \quad 27.8$

$0.0 \mathrm{~m} \quad 25.8$

$0.0 \quad \mathrm{~m} \quad 24.1$

$0.0 \mathrm{~m} \quad 22.7$

$0.0 \mathrm{~m} \quad 21.4$

$0.0 \quad m \quad 20.4$

$\begin{array}{lll}0.0 & m & 19.5\end{array}$

18.

18.1

17.6

17.2

$\begin{array}{lll}0.0 & \mathrm{~m} & 17.0 \\ 0.0 & \mathrm{~m} & 16.9 \\ 0.0 & \mathrm{~m} & 17.0\end{array}$

$\begin{array}{lll}0.0 & \mathrm{~m} & 17.0 \\ 0.0 & \mathrm{~m} & 16.9 \\ 0.0 & \mathrm{~m} & 17.0\end{array}$

$\begin{array}{lll}0.0 & \mathrm{~m} & 17.0 \\ 0.0 & \mathrm{~m} & 16.9 \\ 0.0 & \mathrm{~m} & 17.0\end{array}$

0.0

17.4 
Sounding: MIA117

Location: St. Michael School North

Project: Saltwater Intrusion

County: Miami-Dade County

TX loop size: X[m] $38.1 \quad Y[m] \quad 38.1$

$\begin{array}{cccc}\text { Model } & \text { Resistivity } & \text { Thickness } & \text { Elevation } \\ \text { Layer } & {[\text { ohm-m] }} & {[\mathrm{m}]} & {[\mathrm{m}]} \\ 1 & 122.100 & 35.7 & 0.0 \\ 2 & 3.320 & 3.7 & -35.7 \\ 3 & 19.390 & -- & -39.5\end{array}$

System: EM-58 Freq[Hz]: 285 Data Set Code: uh TX Cur[A]: 1.40 Turn Off[usec]: 2.5

RX Moment[turns-m^2]: 31.4 Gain Setting: 5

$\begin{array}{rrrrrr}\text { Time } & \begin{array}{c}\text { rhoa_obs } \\ \text { [ohm-m] }\end{array} & \begin{array}{c}\text { obs_err } \\ {\left[\frac{\mathrm{o}}{0}\right]}\end{array} & \text { mask } & \begin{array}{r}\text { rhoa_cal } \\ \text { [ohm-m] }\end{array} \\ 1 & 0.00680 & 135.3 & 0.5 & \mathrm{u} & 130.5 \\ 2 & 0.00860 & 132.4 & 0.2 & \mathrm{u} & 136.9 \\ 3 & 0.01110 & 140.1 & 0.3 & \mathrm{u} & 141.9 \\ 4 & 0.01410 & 133.6 & 0.4 & \mathrm{u} & 132.7 \\ 5 & 0.01800 & 114.7 & 0.2 & \mathrm{u} & 113.5 \\ 6 & 0.02300 & 94.8 & 0.2 & \mathrm{u} & 92.9 \\ 7 & 0.02940 & 76.8 & 0.2 & \mathrm{u} & 75.6 \\ 8 & 0.03750 & 62.1 & 0.2 & \mathrm{u} & 62.3 \\ 9 & 0.04790 & 51.2 & 0.1 & \mathrm{u} & 52.4 \\ 10 & 0.06110 & 43.8 & 0.2 & \mathrm{u} & 45.0 \\ 11 & 0.07790 & 38.8 & 0.1 & \mathrm{u} & 39.5 \\ 12 & 0.09930 & 34.3 & 0.2 & \mathrm{u} & 35.4 \\ 13 & 0.12670 & 32.2 & 0.1 & \mathrm{u} & 32.3 \\ 14 & 0.16640 & 31.3 & 0.2 & \mathrm{u} & 29.7 \\ 15 & 0.20600 & 29.0 & 0.4 & \mathrm{u} & 28.2 \\ 16 & 0.26280 & 28.0 & 0.9 & \mathrm{u} & 27.0 \\ 17 & 0.33520 & 26.5 & 1.6 & \mathrm{u} & 26.2 \\ 18 & 0.42770 & 25.4 & 2.9 & \mathrm{u} & 25.7 \\ 19 & 0.54560 & 25.2 & 4.7 & \mathrm{u} & 25.6 \\ 20 & 0.69590 & 25.7 & 9.1 & \mathrm{u} & 25.8\end{array}$

Date: 02-MAY-08

UTM Coord: $\mathrm{E}[\mathrm{m}]$

Elevation $[\mathrm{m}]$ :

$575919.0 \quad \mathrm{~N}[\mathrm{~m}] \quad 2851041.0$ 0.0

RX location: $\mathrm{X}[\mathrm{m}]$

$0.0 \quad \mathrm{Y}[\mathrm{m}] \quad 0.0$
Fit Error [응 $\quad 3.520$

System: EM-58 Freq[Hz]: 30 Data Set Code: hi TX Cur[A]: 1.40 Turn Off[usec]: 2.5

RX Moment [turns-m^2]: 31.4 Gain Setting: 7

$\begin{array}{rrrrrr}\text { Time } & \text { rhs] } & \begin{array}{c}\text { rhoa_obs } \\ \text { [ohm-m] }\end{array} & \begin{array}{c}\text { obs_err } \\ {\left[\frac{\mathrm{o}}{0}\right]}\end{array} & \text { mask } & \begin{array}{c}\text { rhoa_cal } \\ {[\text { ohm-m] }}\end{array} \\ 1 & 0.08800 & 36.9 & 1.0 & \mathrm{u} & 37.1 \\ 2 & 0.10600 & 33.2 & 1.0 & \mathrm{u} & 34.1 \\ 3 & 0.13100 & 31.7 & 1.2 & \mathrm{u} & 31.6 \\ 4 & 0.16100 & 30.5 & 2.0 & \mathrm{u} & 29.6 \\ 5 & 0.20000 & 28.3 & 2.6 & \mathrm{u} & 27.9 \\ 6 & 0.25000 & 26.6 & 4.8 & \mathrm{u} & 26.5 \\ 7 & 0.31400 & 24.4 & 5.9 & \mathrm{u} & 25.4 \\ 8 & 0.39500 & 22.5 & 10.1 & \mathrm{~m} & 24.5 \\ 9 & 0.49900 & 20.1 & 18.2 & \mathrm{~m} & 23.7 \\ 10 & 0.63100 & 18.4 & 25.7 & \mathrm{~m} & 23.1 \\ 11 & 0.79900 & 14.7 & 24.7 & \mathrm{~m} & 22.6 \\ 12 & 1.01000 & 11.3 & 60.0 & \mathrm{~m} & 22.2 \\ 13 & 1.28000 & 8.4 & 73.6 & \mathrm{~m} & 21.9 \\ 14 & 1.63000 & 7.9 & 71.8 & \mathrm{~m} & 21.6 \\ 15 & 2.08000 & 7.1 & 74.0 & \mathrm{~m} & 21.4 \\ 16 & 2.64000 & 4.7 & 100.0 & \mathrm{~m} & 21.4 \\ 17 & 3.37000 & 3.0 & 59.3 & \mathrm{~m} & 21.4 \\ 18 & 4.29000 & 2.2 & 82.0 & \mathrm{~m} & 21.6 \\ 19 & 5.47000 & 2.2 & 100.0 & \mathrm{~m} & 22.1 \\ 20 & 6.97000 & 1.0 & 20.3 & \mathrm{~m} & 22.8\end{array}$


Sounding: MIA117F3

Client: US Geological Survey

Lcation: St. Michael School North

Project: Saltwater Intrusion

County: Miami-Dade County

TX loop size: X[m] $38.1 \quad Y[m] \quad 38.1$

$\begin{array}{cccc}\text { Model } & \text { Resistivity } & \text { Thickness } & \text { Elevation } \\ \text { Layer } & {[\text { ohm-m] }} & {[\mathrm{m}]} & {[\mathrm{m}]} \\ 1 & 122.200 & 35.7 & 0.0 \\ 2 & 3.500 & 4.0 & -35.7 \\ 3 & 19.660 & -- & -39.7\end{array}$

System: EM-58 Freq[Hz]: 285 Data Set Code: uh TX Cur[A]: 1.40 Turn Off[usec]: 2.5

RX Moment[turns-m^2]: 31.4 Gain Setting: 5

$\begin{array}{rrrrrr}\text { Time } & \text { [ms }] & \begin{array}{c}\text { rhoa_obs } \\ \text { [ohm-m] }\end{array} & \begin{array}{c}\text { obs_err } \\ {\left[\frac{\mathrm{o}}{0}\right]}\end{array} & \text { mask } & \begin{array}{r}\text { rhoa_cal } \\ \text { [ohm-m] }\end{array} \\ 1 & 0.00680 & 135.3 & 0.5 & \mathrm{u} & 130.5 \\ 2 & 0.00860 & 132.4 & 0.2 & \mathrm{u} & 136.8 \\ 3 & 0.01110 & 140.1 & 0.3 & \mathrm{u} & 141.9 \\ 4 & 0.01410 & 133.6 & 0.4 & \mathrm{u} & 132.8 \\ 5 & 0.01800 & 114.7 & 0.2 & \mathrm{u} & 113.6 \\ 6 & 0.02300 & 94.8 & 0.2 & \mathrm{u} & 92.9 \\ 7 & 0.02940 & 76.8 & 0.2 & \mathrm{u} & 75.6 \\ 8 & 0.03750 & 62.1 & 0.2 & \mathrm{u} & 62.2 \\ 9 & 0.04790 & 51.2 & 0.1 & \mathrm{u} & 52.3 \\ 10 & 0.06110 & 43.8 & 0.2 & \mathrm{u} & 44.9 \\ 11 & 0.07790 & 38.8 & 0.1 & \mathrm{u} & 39.5 \\ 12 & 0.09930 & 34.3 & 0.2 & \mathrm{u} & 35.3 \\ 13 & 0.12670 & 32.2 & 0.1 & \mathrm{u} & 32.2 \\ 14 & 0.16640 & 31.3 & 0.2 & \mathrm{u} & 29.7 \\ 15 & 0.20600 & 29.0 & 0.4 & \mathrm{u} & 28.2 \\ 16 & 0.26280 & 28.0 & 0.9 & \mathrm{u} & 27.0 \\ 17 & 0.33520 & 26.5 & 1.6 & \mathrm{u} & 26.2 \\ 18 & 0.42770 & 25.4 & 2.9 & \mathrm{u} & 25.8 \\ 19 & 0.54560 & 25.2 & 4.7 & \mathrm{u} & 25.7 \\ 20 & 0.69590 & 25.7 & 9.1 & \mathrm{u} & 26.0\end{array}$

Date: 02-MAY-08

UTM Coord: $\mathrm{E}[\mathrm{m}]$

Elevation $[\mathrm{m}]$ :

$575919.0 \quad \mathrm{~N}[\mathrm{~m}] \quad 2851041.0$ 0.0

RX location: $\mathrm{X}[\mathrm{m}]$

$0.0 Y[\mathrm{~m}] \quad 0.0$

Fit Error [\%]: 3.517

System: EM-58 Freq[Hz]: 30 Data Set Code: hi TX Cur $[\mathrm{A}]: \quad 1.40$ Turn Off[usec]: 2.5

RX Moment [turns-m^2]: $31.4 \quad$ Gain Setting: 7

$\begin{array}{rrrrrr}\text { Time } & \text { [ms] } & \begin{array}{c}\text { rhoa_obs } \\ \text { [ohm-m] }\end{array} & \begin{array}{c}\text { obs_err } \\ {\left[\frac{\mathrm{o}}{0}\right]}\end{array} & \text { mask } & \begin{array}{c}\text { rhoa_cal } \\ {[\text { ohm-m] }}\end{array} \\ 1 & 0.08800 & 36.9 & 1.0 & \mathrm{u} & 37.0 \\ 2 & 0.10600 & 33.2 & 1.0 & \mathrm{u} & 34.1 \\ 3 & 0.13100 & 31.7 & 1.2 & \mathrm{u} & 31.6 \\ 4 & 0.16100 & 30.5 & 2.0 & \mathrm{u} & 29.6 \\ 5 & 0.20000 & 28.3 & 2.6 & \mathrm{u} & 27.9 \\ 6 & 0.25000 & 26.6 & 4.8 & \mathrm{u} & 26.6 \\ 7 & 0.31400 & 24.4 & 5.9 & \mathrm{u} & 25.5 \\ 8 & 0.39500 & 22.5 & 10.1 & \mathrm{~m} & 24.6 \\ 9 & 0.49900 & 20.1 & 18.2 & \mathrm{~m} & 23.8 \\ 10 & 0.63100 & 18.4 & 25.7 & \mathrm{~m} & 23.2 \\ 11 & 0.79900 & 14.7 & 24.7 & \mathrm{~m} & 22.7 \\ 12 & 1.01000 & 11.3 & 60.0 & \mathrm{~m} & 22.3 \\ 13 & 1.28000 & 8.4 & 73.6 & \mathrm{~m} & 22.0 \\ 14 & 1.63000 & 7.9 & 71.8 & \mathrm{~m} & 21.8 \\ 15 & 2.08000 & 7.1 & 74.0 & \mathrm{~m} & 21.6 \\ 16 & 2.64000 & 4.7 & 100.0 & \mathrm{~m} & 21.5 \\ 17 & 3.37000 & 3.0 & 59.3 & \mathrm{~m} & 21.6 \\ 18 & 4.29000 & 2.2 & 82.0 & \mathrm{~m} & 21.8 \\ 19 & 5.47000 & 2.2 & 100.0 & \mathrm{~m} & 22.3 \\ 20 & 6.97000 & 1.0 & 20.3 & \mathrm{~m} & 23.0\end{array}$


Sounding: MIA117L4

Client: US Geological Survey

Lcation: St Michael School North

Project: Saltwater Intrusion

County: Miami-Dade County

TX loop size: X[m] $38.1 \quad Y[m] \quad 38.1$

$\begin{array}{cccc}\text { Model } & \text { Resistivity } & \text { Thickness } & \text { Elevation } \\ \text { Layer } & {[\text { ohm-m] }} & {[\mathrm{m}]} & {[\mathrm{m}]} \\ 1 & 125.300 & 28.1 & 0.0 \\ 2 & 49.760 & 8.9 & -28.1 \\ 3 & 1.970 & 1.9 & -37.0 \\ 4 & 19.050 & -- & -39.0\end{array}$

System: EM-58 Freq[Hz]: 285 Data Set Code: uh TX Cur $[\mathrm{A}]: \quad 1.40$ Turn Off[usec]: 2.5 RX Moment [turns-m^2]: 31.4 Gain Setting: 5

$\begin{array}{rrrrrr}\text { Time } & \begin{array}{c}\text { rhoa_obs } \\ \text { [ohm-m] }\end{array} & \begin{array}{c}\text { obs_err } \\ {\left[\frac{\mathrm{o}}{0}\right]}\end{array} & \text { mask } & \begin{array}{r}\text { rhoa_cal } \\ \text { [ohm-m] }\end{array} \\ 1 & 0.00680 & 135.3 & 0.5 & \mathrm{u} & 131.7 \\ 2 & 0.00860 & 132.4 & 0.2 & \mathrm{u} & 136.6 \\ 3 & 0.01110 & 140.1 & 0.3 & \mathrm{u} & 141.0 \\ 4 & 0.01410 & 133.6 & 0.4 & \mathrm{u} & 132.0 \\ 5 & 0.01800 & 114.7 & 0.2 & \mathrm{u} & 113.2 \\ 6 & 0.02300 & 94.8 & 0.2 & \mathrm{u} & 93.0 \\ 7 & 0.02940 & 76.8 & 0.2 & \mathrm{u} & 75.7 \\ 8 & 0.03750 & 62.1 & 0.2 & \mathrm{u} & 62.4 \\ 9 & 0.04790 & 51.2 & 0.1 & \mathrm{u} & 52.5 \\ 10 & 0.06110 & 43.8 & 0.2 & \mathrm{u} & 45.1 \\ 11 & 0.07790 & 38.8 & 0.1 & \mathrm{u} & 39.6 \\ 12 & 0.09930 & 34.3 & 0.2 & \mathrm{u} & 35.4 \\ 13 & 0.12670 & 32.2 & 0.1 & \mathrm{u} & 32.3 \\ 14 & 0.16640 & 31.3 & 0.2 & \mathrm{u} & 29.7 \\ 15 & 0.20600 & 29.0 & 0.4 & \mathrm{u} & 28.2 \\ 16 & 0.26280 & 28.0 & 0.9 & \mathrm{u} & 26.9 \\ 17 & 0.33520 & 26.5 & 1.6 & \mathrm{u} & 26.1 \\ 18 & 0.42770 & 25.4 & 2.9 & \mathrm{u} & 25.6 \\ 19 & 0.54560 & 25.2 & 4.7 & \mathrm{u} & 25.4 \\ 20 & 0.69590 & 25.7 & 9.1 & \mathrm{u} & 25.7\end{array}$

Date: 02-MAY-08

UTM Coord: $\mathrm{E}[\mathrm{m}]$

Elevation $[\mathrm{m}]$ :

$575919.0 \quad \mathrm{~N}[\mathrm{~m}] \quad 2851041.0$ 0.0

RX location: $\mathrm{X}[\mathrm{m}]$

$0.0 Y[\mathrm{~m}]$

0.0

Fit Error [\%]: 3.478

System: EM-58 Freq[Hz]: 30 Data Set Code: hi TX Cur $[\mathrm{A}]: \quad 1.40$ Turn Off[usec]: 2.5 RX Moment[turns-m^2]: $31.4 \quad$ Gain Setting: 7

$\begin{array}{rrrrrr}\text { Time } & \begin{array}{c}\text { rhoa_obs } \\ \text { [ohm-m] }\end{array} & \begin{array}{c}\text { obserr } \\ {\left[\frac{\text { o }}{0}\right]}\end{array} & \text { mask } & \begin{array}{c}\text { rhoa_cal } \\ {[\text { ohm-m] }}\end{array} \\ 1 & 0.08800 & 36.9 & 1.0 & \mathrm{u} & 37.1 \\ 2 & 0.10600 & 33.2 & 1.0 & \mathrm{u} & 34.2 \\ 3 & 0.13100 & 31.7 & 1.2 & \mathrm{u} & 31.6 \\ 4 & 0.16100 & 30.5 & 2.0 & \mathrm{u} & 29.6 \\ 5 & 0.20000 & 28.3 & 2.6 & \mathrm{u} & 27.9 \\ 6 & 0.25000 & 26.6 & 4.8 & \mathrm{u} & 26.5 \\ 7 & 0.31400 & 24.4 & 5.9 & \mathrm{u} & 25.3 \\ 8 & 0.39500 & 22.5 & 10.1 & \mathrm{~m} & 24.4 \\ 9 & 0.49900 & 20.1 & 18.2 & \mathrm{~m} & 23.6 \\ 10 & 0.63100 & 18.4 & 25.7 & \mathrm{~m} & 22.9 \\ 11 & 0.79900 & 14.7 & 24.7 & \mathrm{~m} & 22.4 \\ 12 & 1.01000 & 11.3 & 60.0 & \mathrm{~m} & 22.0 \\ 13 & 1.28000 & 8.4 & 73.6 & \mathrm{~m} & 21.6 \\ 14 & 1.63000 & 7.9 & 71.8 & \mathrm{~m} & 21.4 \\ 15 & 2.08000 & 7.1 & 74.0 & \mathrm{~m} & 21.2 \\ 16 & 2.64000 & 4.7 & 100.0 & \mathrm{~m} & 21.1 \\ 17 & 3.37000 & 3.0 & 59.3 & \mathrm{~m} & 21.1 \\ 18 & 4.29000 & 2.2 & 82.0 & \mathrm{~m} & 21.4 \\ 19 & 5.47000 & 2.2 & 100.0 & \mathrm{~m} & 21.8 \\ 20 & 6.97000 & 1.0 & 20.3 & \mathrm{~m} & 22.4\end{array}$


Sounding: MIA118

Location: Kensington Park School NW

Project: Saltwater Intrusion

County: Miami-Dade County

TX loop size: X[m] $38.1 \quad Y[m] \quad 38.1$

$\begin{array}{cccc}\text { Model } & \text { Resistivity } & \text { Thickness } & \text { Elevation } \\ \text { Layer } & {[\text { ohm-m] }} & {[\mathrm{m}]} & {[\mathrm{m}]} \\ 1 & 123.000 & 22.9 & 0.0 \\ 2 & 2.090 & -- & -22.9\end{array}$

System: EM-58 Freq[Hz]: 285 Data Set Code: uh TX Cur $[A]: \quad 1.40$ Turn Off[usec]: 2.5

RX Moment[turns-m^2]: 31.4 Gain Setting: 4

\begin{tabular}{|c|c|c|c|c|c|}
\hline & $\begin{array}{l}\text { Time } \\
\text { [ms ] }\end{array}$ & $\begin{array}{l}\text { rhoa_obs } \\
\text { [ohm-m] }\end{array}$ & obs err & mask & $\begin{array}{l}\text { rhoa_cal } \\
\text { [ohm-m] }\end{array}$ \\
\hline 1 & 0.00680 & 102.6 & 0.9 & $\mathrm{~m}$ & 163.0 \\
\hline 2 & 0.00860 & 104.2 & 0.9 & $\mathrm{~m}$ & 144.6 \\
\hline 3 & 0.01110 & 178.8 & 1.6 & $\mathrm{~m}$ & 120.8 \\
\hline 4 & 0.01410 & 2323.0 & 29.8 & $\mathrm{~m}$ & 97.0 \\
\hline 5 & 0.01800 & 90.2 & 0.7 & $\mathrm{~m}$ & 76.8 \\
\hline 6 & 0.02300 & 39.5 & 0.6 & $\mathrm{~m}$ & 60.5 \\
\hline 7 & 0.02940 & 54.0 & 0.9 & $\mathrm{u}$ & 47.7 \\
\hline 8 & 0.03750 & 42.6 & 0.5 & u & 37.9 \\
\hline 9 & 0.04790 & 28.1 & 0.6 & u & 30.3 \\
\hline 10 & 0.06110 & 22.5 & 0.6 & $\mathrm{u}$ & 24.5 \\
\hline 11 & 0.07790 & 19.6 & 0.5 & $u$ & 20.0 \\
\hline 12 & 0.09930 & 15.9 & 0.5 & $u$ & 16.5 \\
\hline 13 & 0.12670 & 13.2 & 0.6 & $u$ & 13.8 \\
\hline 14 & 0.16640 & 11.1 & 1.1 & $u$ & 11.5 \\
\hline 15 & 0.20600 & 9.8 & 0.9 & $u$ & 10.1 \\
\hline 16 & 0.26280 & 8.7 & 1.3 & $u$ & 8.8 \\
\hline 17 & 0.33520 & 7.9 & 1.3 & $u$ & 7.8 \\
\hline 18 & 0.42770 & 7.3 & 3.2 & $u$ & 7.1 \\
\hline 19 & 0.54560 & 6.8 & 4.4 & $\mathrm{u}$ & 6.5 \\
\hline 20 & 0.69590 & 6.3 & 6.2 & $\mathrm{u}$ & 6.1 \\
\hline
\end{tabular}

Date: 02-MAY-08

UTM Coord: $\mathrm{E}[\mathrm{m}]$

Elevation $[\mathrm{m}]$ :

$575880.0 \quad \mathrm{~N}[\mathrm{~m}] \quad 2851706.0$ 0.0

$0.0 Y[\mathrm{~m}] \quad 0.0$

Fit Error $[\%]: 9.609$

0.0


Sounding: MIA119

Location: Kensington Park School SE

Project: Saltwater Intrusion

County: Miami-Dade County

TX loop size: X[m] $38.1 \quad \mathrm{Y}[\mathrm{m}] \quad 38.1$

$\begin{array}{cccc}\text { Model } & \text { Resistivity } & \text { Thickness } & \text { Elevation } \\ \text { Layer } & {[\text { ohm-m] }} & {[\mathrm{m}]} & {[\mathrm{m}]} \\ 1 & 748.900 & 16.0 & 0.0 \\ 2 & 7.580 & -- & -16.0\end{array}$

System: EM-58 Freq[Hz]: 285 Data Set Code: uh TX Cur $[A]: \quad 1.40$ Turn Off[usec]: 2.5

RX Moment[turns-m^2]: 31.4 Gain Setting: 4

$\begin{array}{rrrrrr}\text { Time } & \text { rhs } & \begin{array}{c}\text { rhoa_obs } \\ \text { [ohm-m] }\end{array} & \begin{array}{c}\text { obs_err } \\ {\left[\frac{\mathrm{o}}{0}\right]}\end{array} & \text { mask } & \begin{array}{c}\text { rhoa_cal } \\ \text { [ohm-m] }\end{array} \\ 1 & 0.00680 & 167.8 & 1.2 & \mathrm{~m} & 118.4 \\ 2 & 0.00860 & 174.6 & 1.6 & \mathrm{~m} & 92.9 \\ 3 & 0.01110 & 334.7 & 1.6 & \mathrm{~m} & 74.8 \\ 4 & 0.01410 & 127.6 & 0.6 & \mathrm{~m} & 61.4 \\ 5 & 0.01800 & 145.3 & 0.9 & \mathrm{~m} & 50.8 \\ 6 & 0.02300 & 29.5 & 0.6 & \mathrm{~m} & 42.5 \\ 7 & 0.02940 & 70.6 & 0.8 & \mathrm{~m} & 35.9 \\ 8 & 0.03750 & 44.5 & 0.8 & \mathrm{~m} & 30.8 \\ 9 & 0.04790 & 27.7 & 0.5 & \mathrm{u} & 26.7 \\ 10 & 0.06110 & 22.0 & 0.6 & \mathrm{u} & 23.5 \\ 11 & 0.07790 & 22.0 & 0.6 & \mathrm{u} & 20.9 \\ 12 & 0.09930 & 19.1 & 0.6 & \mathrm{u} & 18.8 \\ 13 & 0.12670 & 16.7 & 0.7 & \mathrm{u} & 17.1 \\ 14 & 0.16640 & 15.3 & 1.0 & \mathrm{u} & 15.6 \\ 15 & 0.20600 & 14.3 & 1.3 & \mathrm{u} & 14.6 \\ 16 & 0.26280 & 13.5 & 2.0 & \mathrm{u} & 13.8 \\ 17 & 0.33520 & 12.9 & 3.2 & \mathrm{u} & 13.1 \\ 18 & 0.42770 & 12.7 & 3.7 & \mathrm{u} & 12.6 \\ 19 & 0.54560 & 12.8 & 4.3 & \mathrm{u} & 12.4 \\ 20 & 0.69590 & 12.5 & 15.4 & \mathrm{u} & 12.3\end{array}$

Date: 02-MAY-08

UTM Coord: $\mathrm{E}[\mathrm{m}]$

Elevation $[\mathrm{m}]$ :

$575894.0 \quad \mathrm{~N}[\mathrm{~m}] \quad 2851685.0$ 0.0

$0.0 Y[\mathrm{~m}] \quad 0.0$

Fit Error $[\%]: 4.860$

0.0


Sounding: MIA120

Client: US Geological Survey

(vacant lot) West

Project: Saltwater Intrusion

County: Miami-Dade County

TX loop size: X $[\mathrm{m}] \quad 38.1 \quad \mathrm{Y}[\mathrm{m}] \quad 38.1$

$\begin{array}{cccc}\text { Model } & \text { Resistivity } & \text { Thickness } & \text { Elevation } \\ \text { Layer } & {[\text { ohm }-\mathrm{m}]} & {[\mathrm{m}]} & {[\mathrm{m}]} \\ 1 & 651.800 & 12.8 & 0.0 \\ 2 & 26.370 & 15.0 & -12.8 \\ 3 & 4.700 & -- & -27.9\end{array}$

System: EM-58 Freq[Hz]: 285 Data Set Code: uh TX Cur[A]: 1.40 Turn Off[usec]: 2.5

RX Moment[turns-m^2]: 31.4 Gain Setting: 4

$\begin{array}{rrrrrr}\text { Time } & \begin{array}{c}\text { rhoa_obs } \\ \text { [ohm-m] }\end{array} & \begin{array}{c}\text { obs_err } \\ {\left[\overline{\frac{o}{0}}\right]}\end{array} & \text { mask } & \begin{array}{c}\text { rhoa_cal } \\ \text { [ohm-m] }\end{array} \\ 1 & 0.00680 & 115.4 & 0.6 & \mathrm{u} & 116.1 \\ 2 & 0.00860 & 104.8 & 0.4 & \mathrm{u} & 103.0 \\ 3 & 0.01110 & 95.3 & 0.4 & \mathrm{u} & 94.1 \\ 4 & 0.01410 & 82.5 & 0.4 & \mathrm{u} & 84.5 \\ 5 & 0.01800 & 71.7 & 0.4 & \mathrm{u} & 74.0 \\ 6 & 0.02300 & 62.1 & 0.4 & \mathrm{u} & 63.3 \\ 7 & 0.02940 & 54.1 & 0.4 & \mathrm{u} & 53.3 \\ 8 & 0.03750 & 47.5 & 0.4 & \mathrm{u} & 44.7 \\ 9 & 0.04790 & 40.8 & 0.4 & \mathrm{u} & 37.5 \\ 10 & 0.06110 & 33.0 & 0.4 & \mathrm{u} & 31.6 \\ 11 & 0.07790 & 26.5 & 0.3 & \mathrm{u} & 26.9 \\ 12 & 0.09930 & 21.8 & 0.5 & \mathrm{u} & 23.1 \\ 13 & 0.12670 & 20.0 & 0.3 & \mathrm{u} & 20.0 \\ 14 & 0.16640 & 16.7 & 0.6 & \mathrm{u} & 17.3 \\ 15 & 0.20600 & 14.6 & 0.8 & \mathrm{u} & 15.6 \\ 16 & 0.26280 & 13.3 & 1.1 & \mathrm{u} & 14.0 \\ 17 & 0.33520 & 12.7 & 1.7 & \mathrm{u} & 12.8 \\ 18 & 0.42770 & 12.3 & 3.1 & \mathrm{u} & 11.9 \\ 19 & 0.54560 & 11.1 & 4.1 & \mathrm{u} & 11.2 \\ 20 & 0.69590 & 11.2 & 5.4 & \mathrm{u} & 10.8\end{array}$

Date: 02-MAY-08

UTM Coord: $\mathrm{E}[\mathrm{m}]$

Elevation $[\mathrm{m}]$ :

$574171.0 \quad \mathrm{~N}[\mathrm{~m}] \quad 2854246.0$ 0.0

RX location: $\mathrm{X}[\mathrm{m}]$

$0.0 Y[\mathrm{~m}] \quad 0.0$
Fit Error [응: 5.453

System: EM-58 Freq[Hz]: 30 Data Set Code: hi TX $\operatorname{Cur}[A]: \quad 1.40$ Turn Off[usec]: 2.5

RX Moment [turns-m^2]: 31.4 Gain Setting: 7

Time
$[\mathrm{ms}]$
0.08800
0.10600
0.13100
0.16100
0.20000
0.25000
0.31400
0.39500
0.49900
0.63100
0.79900
1.01000
1.28000
1.63000
2.08000
2.64000
3.37000
4.29000
5.47000
6.97000

rhoa_obs
[ohm-m]
23.8
22.2
19.5
16.7
14.7
14.2
12.4
11.1
10.9
9.8
11.6
9.5
3.8
3.5
7.8
2.5
31.2
1.8
.7
.6

rhoa_cal

$\left[\overline{\left.\frac{0}{0}\right]}\right]$
0.0
0.0
0.0
0.0
0.0
0.0
0.0
0.0
0.0
0.0
0.0
0.0
0.0
0.0
0.0
0.0
0.0
0.0
0.0
0.0

[ohm-m]

24.6

21.8

19.3

17.1

15.3

13.7

12.4

11.3

10.3

9.5

8.8

8.3

7.8

7.4

7.1

6.9

6.7

6.6

6.6

6.6 
Sounding: MIA121

Location: Alamo Rental (vacant lot) East

Project: Saltwater Intrusion

County: Miami-Dade County

TX loop size: X[m] $38.1 \quad \mathrm{Y}[\mathrm{m}] \quad 38.1$

$\begin{array}{cccc}\text { Model } & \text { Resistivity } & \text { Thickness } & \text { Elevation } \\ \text { Layer } & {[\text { ohm-m] }} & {[\mathrm{m}]} & {[\mathrm{m}]} \\ 1 & 83.790 & 25.1 & 0.0 \\ 2 & 5.020 & -- & -25.1\end{array}$

System: EM-58 Freq[Hz]: 285 Data Set Code: uh TX Cur $[A]: \quad 1.40$ Turn Off[usec]: 2.5

RX Moment[turns-m^2]: $31.4 \quad$ Gain Setting: 4

\begin{tabular}{|c|c|c|c|c|c|}
\hline & $\begin{array}{l}\text { Time } \\
{[\mathrm{ms}]}\end{array}$ & $\begin{array}{l}\text { rhoa_obs } \\
\text { [ohm-m] }\end{array}$ & obs err & mask & $\begin{array}{l}\text { rhoa_cal } \\
\text { [ohm-m] }\end{array}$ \\
\hline 1 & 0.00680 & 103.4 & 1.2 & $u$ & 96.7 \\
\hline 2 & 0.00860 & 104.4 & 0.5 & $u$ & 96.8 \\
\hline 3 & 0.01110 & 82.6 & 0.6 & $u$ & 94.0 \\
\hline 4 & 0.01410 & 77.9 & 0.6 & $u$ & 85.2 \\
\hline 5 & 0.01800 & 63.1 & 0.6 & $u$ & 73.3 \\
\hline 6 & 0.02300 & 57.0 & 0.5 & $u$ & 61.4 \\
\hline 7 & 0.02940 & 55.4 & 0.5 & $u$ & 51.0 \\
\hline 8 & 0.03750 & 54.8 & 0.6 & u & 42.5 \\
\hline 9 & 0.04790 & 45.1 & 0.4 & $u$ & 35.6 \\
\hline 10 & 0.06110 & 28.7 & 0.6 & $\mathrm{u}$ & 30.1 \\
\hline 11 & 0.07790 & 22.7 & 0.6 & $u$ & 25.6 \\
\hline 12 & 0.09930 & 19.0 & 0.9 & $u$ & 22.1 \\
\hline 13 & 0.12670 & 20.6 & 1.6 & $u$ & 19.3 \\
\hline 14 & 0.16640 & 21.4 & 2.6 & $u$ & 16.8 \\
\hline 15 & 0.20600 & 12.0 & 1.6 & $u$ & 15.2 \\
\hline 16 & 0.26280 & 11.1 & 2.4 & $u$ & 13.8 \\
\hline 17 & 0.33520 & 12.8 & 6.5 & u & 12.7 \\
\hline 18 & 0.42770 & 14.4 & 15.8 & $u$ & 11.8 \\
\hline 19 & 0.54560 & 10.7 & 10.7 & $u$ & 11.2 \\
\hline 20 & 0.69590 & 10.9 & 29.4 & u & 10.8 \\
\hline
\end{tabular}

Date: 02-MAY-08

UTM Coord: $\mathrm{E}[\mathrm{m}]$

Elevation $[\mathrm{m}]$ :

$574249.0 \quad \mathrm{~N}[\mathrm{~m}] \quad 2854232.0$ 0.0

$0.0 Y[\mathrm{~m}] \quad 0.0$

Fit Error [응: 24.840 
Sounding: MIA122

Client: US Geological Survey

Location: Barry University Field SW

Project: Saltwater Intrusion

County: Miami-Dade County

TX loop size: X[m] $38.1 \quad \mathrm{Y}[\mathrm{m}] \quad 38.1$

Model
Layer
1
2
3
4

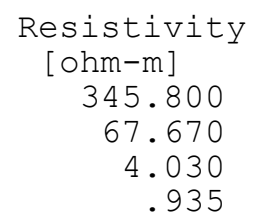

System: EM-58 Freq[Hz]: 285 Data Set Code: uh TX $\operatorname{Cur}[A]: \quad 1.40$ Turn Off[usec]: 2.5 RX Moment[turns-m^2]: 31.4 Gain Setting: 4

$\begin{array}{rrrrrr}\text { Time } & \begin{array}{c}\text { rhoa_obs } \\ \text { [ohm-m] }\end{array} & \begin{array}{c}\text { obs_err } \\ {\left[\frac{\mathrm{o}}{0}\right]}\end{array} & \text { mask } & \begin{array}{r}\text { rhoa_cal } \\ \text { [ohm-m] }\end{array} \\ 1 & 0.00680 & 95.7 & 1.1 & \mathrm{u} & 99.3 \\ 2 & 0.00860 & 95.3 & 0.3 & \mathrm{u} & 93.8 \\ 3 & 0.01110 & 96.3 & 0.2 & \mathrm{u} & 92.7 \\ 4 & 0.01410 & 96.2 & 0.2 & \mathrm{u} & 93.7 \\ 5 & 0.01800 & 96.6 & 0.4 & \mathrm{u} & 95.8 \\ 6 & 0.02300 & 96.5 & 0.4 & \mathrm{u} & 98.7 \\ 7 & 0.02940 & 96.7 & 0.5 & \mathrm{u} & 100.5 \\ 8 & 0.03750 & 97.2 & 0.8 & \mathrm{u} & 99.3 \\ 9 & 0.04790 & 98.1 & 1.4 & \mathrm{u} & 94.5 \\ 10 & 0.06110 & 91.7 & 1.3 & \mathrm{u} & 87.1 \\ 11 & 0.07790 & 75.8 & 1.9 & \mathrm{u} & 78.7 \\ 12 & 0.09930 & 67.5 & 3.2 & \mathrm{u} & 70.0 \\ 13 & 0.12670 & 60.9 & 3.6 & \mathrm{u} & 61.2 \\ 14 & 0.16640 & 53.6 & 4.8 & \mathrm{u} & 51.5 \\ 15 & 0.20600 & 43.5 & 3.8 & \mathrm{u} & 44.3 \\ 16 & 0.26280 & 38.5 & 9.4 & \mathrm{u} & 37.1 \\ 17 & 0.33520 & 30.8 & 10.4 & \mathrm{u} & 31.0 \\ 18 & 0.42770 & 25.5 & 21.0 & \mathrm{u} & 26.1 \\ 19 & 0.54560 & 22.8 & 21.7 & \mathrm{u} & 22.1 \\ 20 & 0.69590 & 18.4 & 35.8 & \mathrm{u} & 19.0\end{array}$

Date: 05-MAY-08

UTM Coord: E[m]

Elevation [m] :

$580001.0 \quad \mathrm{~N}[\mathrm{~m}]$

2862347.0

RX location: X [m]

$0.0 Y[\mathrm{~m}] \quad 0.0$

Fit Error [응 : 4.756 
Sounding: MIA123

Location: Barry University Field SE

Project: Saltwater Intrusion

County: Miami-Dade County

TX loop size: X[m] $38.1 \quad Y[m] \quad 38.1$

$\begin{array}{cccc}\text { Model } & \text { Resistivity } & \text { Thickness } & \text { Elevation } \\ \text { Layer } & {[\text { ohm-m] }} & {[\mathrm{m}]} & {[\mathrm{m}]} \\ 1 & 312.600 & 7.4 & 0.0 \\ 2 & 69.080 & 75.8 & -7.4 \\ 3 & 15.760 & -- & -83.3\end{array}$

System: EM-58 Freq[Hz]: 285 Data Set Code: uh TX Cur $[\mathrm{A}]: \quad 1.40$ Turn Off[usec]: 2.5

RX Moment [turns-m^2]: 31.4 Gain Setting: 4

$\begin{array}{rrrrrr}\text { Time } & \begin{array}{c}\text { rhoa_obs } \\ {[\text { ohm-m] }}\end{array} & \begin{array}{c}\text { obs_err } \\ {\left[\frac{\overline{0}}{0}\right]}\end{array} & \text { mask } & \begin{array}{c}\text { rhoa_cal } \\ \text { [ohm-m] }\end{array} \\ 1 & 0.00680 & 97.6 & 1.0 & \mathrm{u} & 103.0 \\ 2 & 0.00860 & 95.8 & 0.6 & \mathrm{u} & 96.5 \\ 3 & 0.01110 & 95.5 & 0.2 & \mathrm{u} & 93.4 \\ 4 & 0.01410 & 96.4 & 0.2 & \mathrm{u} & 91.0 \\ 5 & 0.01800 & 91.4 & 0.2 & \mathrm{u} & 88.5 \\ 6 & 0.02300 & 85.4 & 0.1 & \mathrm{u} & 86.6 \\ 7 & 0.02940 & 86.4 & 0.2 & \mathrm{u} & 85.4 \\ 8 & 0.03750 & 84.8 & 0.2 & \mathrm{u} & 84.8 \\ 9 & 0.04790 & 87.3 & 0.2 & \mathrm{u} & 84.8 \\ 10 & 0.06110 & 85.6 & 0.3 & \mathrm{u} & 84.8 \\ 11 & 0.07790 & 78.9 & 0.2 & \mathrm{u} & 83.8 \\ 12 & 0.09930 & 77.6 & 0.3 & \mathrm{u} & 81.3 \\ 13 & 0.12670 & 75.6 & 0.4 & \mathrm{u} & 77.2 \\ 14 & 0.16640 & 73.7 & 5.2 & \mathrm{u} & 71.2 \\ 15 & 0.20600 & 66.0 & 4.7 & \mathrm{u} & 66.0 \\ 16 & 0.26280 & 62.5 & 1.6 & \mathrm{u} & 60.5 \\ 17 & 0.33520 & 56.2 & 3.3 & \mathrm{u} & 55.5 \\ 18 & 0.42770 & 51.5 & 8.5 & \mathrm{u} & 51.4 \\ 19 & 0.54560 & 46.7 & 6.3 & \mathrm{u} & 48.1 \\ 20 & 0.69590 & 44.9 & 5.3 & \mathrm{u} & 45.8\end{array}$

Date: 05-MAY-08

UTM Coord: $E[\mathrm{~m}]$

Elevation $[\mathrm{m}]$ :

$580041.0 \quad \mathrm{~N}[\mathrm{~m}]$

2862354.0

RX location: $\mathrm{X}[\mathrm{m}]$

$0.0 \quad \mathrm{Y}[\mathrm{m}] \quad 0.0$

Fit Error $[\%]: 4.726$ 
Sounding: MIA124

Client: US Geological Survey

Location: Barry University Vacant Lot NW

Project: Saltwater Intrusion

County: Miami-Dade County

TX loop size: $X[\mathrm{~m}] \quad 38.1 \quad \mathrm{Y}[\mathrm{m}] \quad 38.1$

$\begin{array}{cccc}\text { Model } & \text { Resistivity } & \text { Thickness } & \text { Elevation } \\ \text { Layer } & {[\text { ohm-m] }} & {[\mathrm{m}]} & {[\mathrm{m}]} \\ 1 & 327.000 & 12.0 & 0.0 \\ 2 & 48.990 & 19.4 & -12.0 \\ 3 & 261.000 & 40.7 & -31.5 \\ 4 & 6.070 & 22.7 & -72.3 \\ 5 & 1.470 & -- & -95.0\end{array}$

System: EM-58 Freq[Hz]: 285 Data Set Code: uh TX Cur $[\mathrm{A}]:$ 1.40 Turn Off[usec]: 2.5

RX Moment[turns-m^2]: 31.4 Gain Setting: 4

$\begin{array}{crrrr}\begin{array}{c}\text { Time } \\ \text { [ms] }\end{array} & \begin{array}{c}\text { rhoa_obs } \\ \text { [ohm-m] }\end{array} & \begin{array}{c}\text { obs err } \\ {\left[\frac{\bar{o}}{0}\right]}\end{array} & \text { mask } & \begin{array}{r}\text { rhoa_cal } \\ \text { [ohm-m] }\end{array} \\ 0.00680 & 107.4 & 0.4 & u & 108.4 \\ 0.00860 & 97.0 & 0.4 & u & 97.0 \\ 0.01110 & 93.1 & 0.4 & u & 91.4 \\ 0.01410 & 89.3 & 0.4 & u & 89.1 \\ 0.01800 & 89.4 & 0.3 & u & 89.0 \\ 0.02300 & 90.8 & 0.4 & u & 91.6 \\ 0.02940 & 96.2 & 0.4 & u & 96.4 \\ 0.03750 & 102.9 & 0.4 & u & 102.7 \\ 0.04790 & 108.3 & 0.4 & u & 108.8 \\ 0.06110 & 111.1 & 0.3 & u & 111.9 \\ 0.07790 & 113.5 & 0.7 & u & 110.5 \\ 0.09930 & 103.3 & 0.3 & u & 104.7 \\ 0.12670 & 95.8 & 1.1 & u & 96.6 \\ 0.16640 & 86.3 & 0.8 & u & 86.1 \\ 0.20600 & 76.4 & 1.0 & u & 77.3 \\ 0.26280 & 68.3 & 1.7 & u & 67.5 \\ 0.33520 & 58.8 & 1.8 & u & 58.1 \\ 0.42770 & 50.1 & 3.1 & u & 49.8 \\ 0.54560 & 42.6 & 4.4 & u & 42.7 \\ 0.69590 & 36.5 & 4.8 & u & 37.0\end{array}$

Date: 05-MAY-08

UTM Coord: $\mathrm{E}[\mathrm{m}]$

Elevation $[\mathrm{m}]$ :

$579604.0 \quad \mathrm{~N}[\mathrm{~m}] \quad 2862625.0$

0.0

RX location: $\mathrm{X}[\mathrm{m}]$

$0.0 \quad Y[m]$

0.0

Fit Error $\left[\frac{\circ}{0}\right]: \quad 1.587$ 
Sounding: MIA125

Client: US Geological Survey

Location: Epiphany Parish Baseball Field

Project: Saltwater Intrusion

County: Miami-Dade County

TX loop size: X[m] $38.1 \quad Y[m] \quad 38.1$

$\begin{array}{cccc}\text { Model } & \text { Resistivity } & \text { Thickness } & \text { Elevation } \\ \text { Layer } & {[\text { ohm-m] }} & {[\mathrm{m}]} & {[\mathrm{m}]} \\ 1 & 279.800 & 12.0 & 0.0 \\ 2 & 50.510 & 37.6 & -12.0 \\ 3 & 3.300 & -- & -49.6\end{array}$

System: EM-58 Freq[Hz]: 285 Data Set Code: uh TX Cur $[A]: \quad 1.40$ Turn Off[usec] : 2.5

RX Moment[turns-m^2]: 31.4 Gain Setting: 4

\begin{tabular}{|c|c|c|c|c|}
\hline $\begin{array}{l}\text { Time } \\
{[\mathrm{ms}]}\end{array}$ & $\begin{array}{l}\text { rhoa_obs } \\
\text { [ohm-m ] }\end{array}$ & $\begin{array}{c}\text { obs err } \\
{\left[\frac{\overline{0}}{0}\right]}\end{array}$ & mask & $\begin{array}{l}\text { rhoa_ca } \\
\text { [ohm-m] }\end{array}$ \\
\hline 0.00680 & 108.3 & 0.3 & $u$ & 112.3 \\
\hline 0.00860 & 102.8 & 0.1 & $u$ & 102.0 \\
\hline 0.01110 & 99.9 & 0.1 & $u$ & 97.0 \\
\hline 0.01410 & 96.8 & 0.1 & $u$ & 94.6 \\
\hline 0.01800 & 96.5 & 0.1 & $u$ & 94. \\
\hline 0.02300 & 95.4 & 0.0 & $u$ & 94. \\
\hline 0.02940 & 91.7 & 0.1 & $u$ & 94. \\
\hline 0.03750 & 87.8 & 0.1 & $u$ & 90. \\
\hline 0.04790 & 80.7 & 0.2 & $u$ & 83. \\
\hline 0.06110 & 72.6 & 0.2 & $u$ & 73. \\
\hline 0.07790 & 63.4 & 0.3 & $u$ & 62. \\
\hline 9930 & 53.4 & 0.3 & $u$ & 51. \\
\hline 70 & 44.4 & 0.4 & u & 43. \\
\hline & 35.8 & 0.6 & $u$ & 35 . \\
\hline & 30.0 & 0.5 & $u$ & 30 . \\
\hline 80 & 25.3 & 0.8 & $u$ & 25 . \\
\hline & 21.8 & 1.1 & $u$ & 22 . \\
\hline & 19.2 & 1.9 & $u$ & 19. \\
\hline & 17.3 & 3.3 & $u$ & 17. \\
\hline 90 & 16.0 & 4.6 & $u$ & 15. \\
\hline
\end{tabular}

Date: 05-MAY-08

UTM Coord: $\mathrm{E}[\mathrm{m}]$

Elevation $[\mathrm{m}]$ :

$571943.0 \quad \mathrm{~N}[\mathrm{~m}] \quad 2842057.0$

0.0

RX location: X $[\mathrm{m}]$

$0.0 \quad Y[m]$

0.0

Fit Error [\%]: 3.469 
Sounding: MIA126

Client: US Geological Survey

Location: Lourdes Academy Baseball Field

Project: Saltwater Intrusion

County: Miami-Dade County

TX loop size: X[m] $38.1 \quad Y[m] \quad 38.1$

$\begin{array}{cccc}\text { Model } & \text { Resistivity } & \text { Thickness } & \text { Elevation } \\ \text { Layer } & \text { [ohm-m] } & {[\mathrm{m}]} & {[\mathrm{m}]} \\ 1 & 118.100 & 31.9 & 0.0 \\ 2 & 11.140 & 5.3 & -31.9 \\ 3 & 390.300 & 47.6 & -37.2 \\ 4 & 11.060 & -- & -84.9\end{array}$

Date: 05-MAY-08

UTM Coord: $E[\mathrm{~m}]$

Elevation $[\mathrm{m}]$ :

$572116.0 \quad \mathrm{~N}[\mathrm{~m}] \quad 2842097.0$ 0.0

RX location: $\mathrm{X}[\mathrm{m}]$

$0.0 \quad \mathrm{Y}[\mathrm{m}]$

0.0

System: EM-58 Freq[Hz]: 285 Data Set Code: uh TX Cur $[\mathrm{A}]: 1.40$ Turn Off [usec]: 2.5

RX Moment[turns-m^2]: 31.4 Gain Setting: 4

$\begin{array}{rrrrrr}\text { Time } & \begin{array}{c}\text { rhoa_obs } \\ \text { [ohm-m] }\end{array} & \begin{array}{c}\text { obs_err } \\ {\left[\frac{\text { o }}{0}\right]}\end{array} & \text { mask } & \begin{array}{r}\text { rhoa_cal } \\ \text { [ohm-m] }\end{array} \\ 1 & 0.00680 & 119.2 & 0.2 & \mathrm{u} & 118.8 \\ 2 & 0.00860 & 114.4 & 0.2 & \mathrm{u} & 114.5 \\ 3 & 0.01110 & 106.5 & 0.2 & \mathrm{u} & 107.2 \\ 4 & 0.01410 & 97.1 & 0.2 & \mathrm{u} & 97.0 \\ 5 & 0.01800 & 88.3 & 0.2 & \mathrm{u} & 87.0 \\ 6 & 0.02300 & 80.4 & 0.2 & \mathrm{u} & 79.8 \\ 7 & 0.02940 & 75.0 & 0.2 & \mathrm{u} & 75.3 \\ 8 & 0.03750 & 72.2 & 0.1 & \mathrm{u} & 73.6 \\ 9 & 0.04790 & 72.3 & 0.1 & \mathrm{u} & 73.8 \\ 10 & 0.06110 & 75.1 & 0.1 & \mathrm{u} & 75.6 \\ 11 & 0.07790 & 80.4 & 0.1 & \mathrm{u} & 78.0 \\ 12 & 0.09930 & 80.3 & 0.3 & \mathrm{u} & 79.3 \\ 13 & 0.12670 & 78.7 & 0.4 & \mathrm{u} & 78.5 \\ 14 & 0.16640 & 73.5 & 0.8 & \mathrm{u} & 74.1 \\ 15 & 0.20600 & 68.0 & 1.0 & \mathrm{u} & 69.1 \\ 16 & 0.26280 & 62.6 & 1.7 & \mathrm{u} & 62.4 \\ 17 & 0.33520 & 56.6 & 3.6 & \mathrm{u} & 56.5 \\ 18 & 0.42770 & 51.5 & 9.9 & \mathrm{u} & 51.1 \\ 19 & 0.54560 & 49.0 & 23.5 & \mathrm{~m} & 46.9 \\ 20 & 0.69590 & 51.8 & 64.1 & \mathrm{~m} & 43.7\end{array}$


Sounding: MIA127

Location: Morning Star Renewal Center

Project: Saltwater Intrusion

County: Miami-Dade County

TX loop size: X[m] $38.1 \quad \mathrm{Y}[\mathrm{m}] \quad 38.1$

$\begin{array}{cccc}\text { Model } & \text { Resistivity } & \text { Thickness } & \text { Elevation } \\ \text { Layer } & {[\text { ohm-m] }} & {[\mathrm{m}]} & {[\mathrm{m}]} \\ 1 & 133.400 & 42.6 & 0.0 \\ 2 & 10.230 & 28.7 & -42.6 \\ 3 & .711 & -- & -71.4\end{array}$

System: EM-58 Freq[Hz]: 285 Data Set Code: uh TX $\operatorname{Cur}[\mathrm{A}]: \quad 1.40$ Turn Off[usec]: 2.5

RX Moment[turns-m^2]: 31.4 Gain Setting: 5

$\begin{array}{rrrrrr}\text { Time } & \begin{array}{c}\text { rhoa_obs } \\ \text { [ohm-m] }\end{array} & \begin{array}{c}\text { obs_err } \\ {\left[\frac{\bar{o}}{0}\right]}\end{array} & \text { mask } & \begin{array}{c}\text { rhoa_cal } \\ \text { [ohm-m] }\end{array} \\ 1 & 0.00680 & 126.7 & 0.2 & \mathrm{u} & 129.5 \\ 2 & 0.00860 & 120.4 & 0.1 & \mathrm{u} & 134.5 \\ 3 & 0.01110 & 132.8 & 0.1 & \mathrm{u} & 143.5 \\ 4 & 0.01410 & 168.6 & 0.1 & \mathrm{u} & 147.9 \\ 5 & 0.01800 & 201.2 & 0.2 & \mathrm{u} & 143.4 \\ 6 & 0.02300 & 112.3 & 0.3 & \mathrm{u} & 131.5 \\ 7 & 0.02940 & 84.8 & 0.2 & \mathrm{u} & 115.8 \\ 8 & 0.03750 & 121.8 & 0.1 & \mathrm{u} & 101.0 \\ 9 & 0.04790 & 86.9 & 0.3 & \mathrm{u} & 88.9 \\ 10 & 0.06110 & 85.6 & 0.1 & \mathrm{u} & 80.4 \\ 11 & 0.07790 & 77.7 & 0.4 & \mathrm{u} & 74.5 \\ 12 & 0.09930 & 68.4 & 0.5 & \mathrm{u} & 70.3 \\ 13 & 0.12670 & 65.1 & 1.3 & \mathrm{u} & 66.2 \\ 14 & 0.16640 & 57.9 & 2.8 & \mathrm{u} & 60.3 \\ 15 & 0.20600 & 56.0 & 5.0 & \mathrm{u} & 54.2 \\ 16 & 0.26280 & 45.1 & 7.4 & \mathrm{u} & 46.8 \\ 17 & 0.33520 & 40.3 & 11.1 & \mathrm{u} & 39.5 \\ 18 & 0.42770 & 34.0 & 11.1 & \mathrm{u} & 33.3 \\ 19 & 0.54560 & 28.5 & 4.9 & \mathrm{u} & 28.0 \\ 20 & 0.69590 & 23.7 & 24.3 & \mathrm{u} & 23.9\end{array}$

Date: 06-MAY-08

UTM Coord: $\mathrm{E}[\mathrm{m}]$

Elevation $[\mathrm{m}]$ :

$569155.0 \quad \mathrm{~N}[\mathrm{~m}] \quad 2837769.0$ 0.0

RX location: $\mathrm{X}[\mathrm{m}]$

$0.0 Y[\mathrm{~m}] \quad 0.0$

Fit Error [을 : 20.904 
Sounding: MIA128

Location: St. Luke School

Project: Saltwater Intrusion

County: Miami-Dade County

TX loop size: X[m] $38.1 \quad \mathrm{Y}[\mathrm{m}] \quad 38.1$

$\begin{array}{cccc}\text { Model } & \text { Resistivity } & \text { Thickness } & \text { Elevation } \\ \text { Layer } & {[\text { ohm-m] }} & {[\mathrm{m}]} & {[\mathrm{m}]} \\ 1 & 123.000 & 44.9 & 0.0 \\ 2 & 24.500 & 53.2 & -44.9 \\ 3 & 4.300 & -- & -98.1\end{array}$

System: EM-58 Freq[Hz]: 285 Data Set Code: uh TX Cur $[A]: \quad 1.40$ Turn Off[usec]: 2.5

RX Moment[turns-m^2]: 31.4 Gain Setting: 5

$\begin{array}{rrrrrr}\text { Time } & \begin{array}{c}\text { rhoa_obs } \\ \text { [ohm-m] }\end{array} & \begin{array}{c}\text { obs_err } \\ {\left[\frac{\bar{o}}{0}\right]}\end{array} & \text { mask } & \begin{array}{c}\text { rhoa_cal } \\ \text { [ohm-m] }\end{array} \\ 1 & 0.00680 & 119.7 & 0.2 & \mathrm{u} & 115.7 \\ 2 & 0.00860 & 110.0 & 0.5 & \mathrm{u} & 117.2 \\ 3 & 0.01110 & 106.1 & 0.3 & \mathrm{u} & 122.5 \\ 4 & 0.01410 & 125.4 & 0.6 & \mathrm{u} & 126.3 \\ 5 & 0.01800 & 154.5 & 1.3 & \mathrm{u} & 125.6 \\ 6 & 0.02300 & 146.0 & 1.4 & \mathrm{u} & 121.0 \\ 7 & 0.02940 & 98.3 & 1.5 & \mathrm{u} & 112.9 \\ 8 & 0.03750 & 90.7 & 0.5 & \mathrm{u} & 103.3 \\ 9 & 0.04790 & 95.8 & 1.5 & \mathrm{u} & 94.0 \\ 10 & 0.06110 & 85.2 & 2.4 & \mathrm{u} & 86.0 \\ 11 & 0.07790 & 81.8 & 1.4 & \mathrm{u} & 79.8 \\ 12 & 0.09930 & 76.3 & 0.3 & \mathrm{u} & 75.0 \\ 13 & 0.12670 & 72.2 & 0.7 & \mathrm{u} & 71.3 \\ 14 & 0.16640 & 67.2 & 0.7 & \mathrm{u} & 67.0 \\ 15 & 0.20600 & 62.7 & 1.2 & \mathrm{u} & 63.2 \\ 16 & 0.26280 & 58.0 & 1.8 & \mathrm{u} & 57.9 \\ 17 & 0.33520 & 51.9 & 2.4 & \mathrm{u} & 52.4 \\ 18 & 0.42770 & 46.3 & 4.0 & \mathrm{u} & 46.9 \\ 19 & 0.54560 & 42.3 & 5.8 & \mathrm{u} & 42.0 \\ 20 & 0.69590 & 38.8 & 9.3 & \mathrm{u} & 37.9\end{array}$

Date: 06-MAY-08

UTM Coord: $\mathrm{E}[\mathrm{m}]$

Elevation $[\mathrm{m}]$ :

$569186.0 \quad \mathrm{~N}[\mathrm{~m}] \quad 2837983.0$ 0.0

RX location: $\mathrm{X}[\mathrm{m}]$

$0.0 \quad Y[m] \quad 0.0$

Fit Error [\%] : 13.520 
Sounding: MIA129

Client: US Geological Survey

Location: Whispering Pines School

Project: Saltwater Intrusion

County: Miami-Dade County

TX loop size: X[m] $38.1 \quad Y[m] \quad 38.1$

$\begin{array}{cccc}\text { Model } & \text { Resistivity } & \text { Thickness } & \text { Elevation } \\ \text { Layer } & {[\text { ohm-m] }} & {[\mathrm{m}]} & {[\mathrm{m}]} \\ 1 & 133.000 & 34.4 & 0.0 \\ 2 & 36.330 & -- & -34.4\end{array}$

System: EM-58 Freq[Hz]: 285 Data set Code: uh TX Cur $[\mathrm{A}]: \quad 1.40$ Turn Off [usec] : 2.5

RX Moment[turns-m^2]: 31.4 Gain Setting: 5

\begin{tabular}{|c|c|c|c|c|c|}
\hline & $\begin{array}{l}\text { Time } \\
{[\mathrm{ms}]}\end{array}$ & $\begin{array}{l}\text { rhoa_obs } \\
\text { [ohm-m ] }\end{array}$ & $\begin{array}{c}\text { obs err } \\
{\left[\frac{\overline{0}}{0}\right]}\end{array}$ & mask & $\begin{array}{l}\text { rhoa_cal } \\
\text { [ohm-m] }\end{array}$ \\
\hline 1 & 0.00680 & 130.4 & 0.4 & $u$ & 127.9 \\
\hline 2 & 0.00860 & 129.0 & 0.2 & $u$ & 127.5 \\
\hline 3 & 0.01110 & 126.9 & 0.3 & $u$ & 127.7 \\
\hline 4 & 0.01410 & 121.1 & 0.4 & $u$ & 124.5 \\
\hline 5 & 0.01800 & 114.5 & 0.4 & $u$ & 117.4 \\
\hline 6 & 0.02300 & 108.0 & 0.3 & $u$ & 109.1 \\
\hline 7 & 0.02940 & 101.3 & 0.4 & u & 100.4 \\
\hline 8 & 0.03750 & 93.4 & 0.4 & u & 92.3 \\
\hline 9 & 0.04790 & 86.5 & 1.2 & u & 84.9 \\
\hline 10 & 0.06110 & 80.2 & 0.9 & $\mathrm{u}$ & 78.4 \\
\hline 11 & 0.07790 & 74.6 & 1.9 & $u$ & 72.8 \\
\hline 12 & 0.09930 & 67.6 & 2.7 & $u$ & 68.0 \\
\hline 13 & 0.12670 & 63.8 & 3.4 & $u$ & 63.9 \\
\hline 14 & 0.16640 & 59.0 & 8.9 & $u$ & 60.1 \\
\hline 15 & 0.20600 & 56.7 & 17.6 & $u$ & 57.7 \\
\hline 16 & 0.26280 & 47.5 & 21.2 & $\mathrm{~m}$ & 55.4 \\
\hline 17 & 0.33520 & 46.3 & 40.8 & $\mathrm{~m}$ & 53.8 \\
\hline 18 & 0.42770 & 47.1 & 62.6 & $\mathrm{~m}$ & 52.8 \\
\hline 19 & 0.54560 & 53.4 & 100.0 & $\mathrm{~m}$ & 52.3 \\
\hline 20 & 0.69590 & 49.8 & 100.0 & $\mathrm{~m}$ & 52.7 \\
\hline
\end{tabular}
5
Date: $06-\mathrm{MAY}-08$

UTM Coord: $E[\mathrm{~m}]$

Elevation $[\mathrm{m}]$ :

$566788.0 \quad \mathrm{~N}[\mathrm{~m}] \quad 2830662.0$ 0.0

RX location: $\mathrm{X}[\mathrm{m}]$

$0.0 \quad \mathrm{Y}[\mathrm{m}] \quad 0.0$

Fit Error [\%] : 2.594 
Sounding: MIA130

Location: N Miami Beach HS East Field S

Project: Saltwater Intrusion

County: Miami-Dade County

TX loop size: X[m] $38.1 \quad \mathrm{Y}[\mathrm{m}] \quad 38.1$

$\begin{array}{cccc}\text { Model } & \text { Resistivity } & \text { Thickness } & \text { Elevation } \\ \text { Layer } & {[\text { ohm-m] }} & {[\mathrm{m}]} & {[\mathrm{m}]} \\ 1 & 668.900 & 6.7 & 0.0 \\ 2 & 137.600 & 8.6 & -6.7 \\ 3 & .032 & .1 & -15.3 \\ 4 & 95.210 & 8.5 & -15.4 \\ 5 & .004 & -- & -24.0\end{array}$

System: EM-58 Freq[Hz]: 285 Data Set Code: uh TX Cur $[A]: \quad 1.40$ Turn Off[usec] : 2.5 RX Moment[turns-m^2]: 31.4 Gain Setting:

$\begin{array}{rrrrrr}\text { Time } & \begin{array}{c}\text { rhoa_obs } \\ \text { [ohm-m] }\end{array} & \begin{array}{c}\text { obs_err } \\ {\left[\frac{\overline{0}}{0}\right]}\end{array} & \text { mask } & \begin{array}{r}\text { rhoa_cal } \\ \text { [ohm-m] }\end{array} \\ 1 & 0.00680 & 72.7 & 0.2 & \mathrm{~m} & 144.8 \\ 2 & 0.00860 & 71.4 & 0.4 & \mathrm{~m} & 99.5 \\ 3 & 0.01110 & 71.1 & 0.9 & \mathrm{u} & 70.1 \\ 4 & 0.01410 & 52.4 & 0.6 & \mathrm{u} & 50.8 \\ 5 & 0.01800 & 37.7 & 0.4 & \mathrm{u} & 37.6 \\ 6 & 0.02300 & 29.2 & 0.7 & \mathrm{u} & 28.6 \\ 7 & 0.02940 & 22.6 & 0.6 & \mathrm{u} & 22.5 \\ 8 & 0.03750 & 18.1 & 0.4 & \mathrm{u} & 18.4 \\ 9 & 0.04790 & 15.5 & 0.9 & \mathrm{u} & 15.9 \\ 10 & 0.06110 & 13.9 & 0.7 & \mathrm{u} & 14.6 \\ 11 & 0.07790 & 13.4 & 1.9 & \mathrm{u} & 14.3 \\ 12 & 0.09930 & 14.1 & 2.0 & \mathrm{u} & 15.1 \\ 13 & 0.12670 & 16.7 & 6.9 & \mathrm{u} & 16.8 \\ 14 & 0.16640 & 21.0 & 21.2 & \mathrm{u} & 19.2 \\ 15 & 0.20600 & 22.6 & 51.6 & \mathrm{u} & 19.9 \\ 16 & 0.26280 & 18.6 & 53.1 & \mathrm{u} & 18.3 \\ 17 & 0.33520 & 14.3 & 58.7 & \mathrm{u} & 15.3 \\ 18 & 0.42770 & 12.4 & 65.2 & \mathrm{u} & 12.5 \\ 19 & 0.54560 & 7.8 & 27.4 & \mathrm{~m} & 10.2 \\ 20 & 0.69590 & 5.9 & 100.0 & \mathrm{~m} & 8.3\end{array}$

Date: 07-MAY-08

UTM Coord: $\mathrm{E}[\mathrm{m}]$

Elevation $[\mathrm{m}]$ :

$58639.0 \mathrm{~N}[\mathrm{~m}]$

2868352.0

RX location: $\mathrm{X}[\mathrm{m}]$
$0.0 \quad \mathrm{Y}[\mathrm{m}] \quad 0.0$

$0.0 \quad \mathrm{Y}[\mathrm{m}] \quad 0.0$

0.0

Fit Error $\left[\frac{\circ}{0}\right]: \quad 8.211$ 
Sounding: MIA131

Client: US Geological Survey

Location: N Miami Beach HS East Field N

Project: Saltwater Intrusion

County: Miami-Dade County

TX loop size: X $[\mathrm{m}] \quad 38.1 \quad \mathrm{Y}[\mathrm{m}] \quad 38.1$

$\begin{array}{cccc}\text { Model } & \text { Resistivity } & \text { Thickness } & \text { Elevation } \\ \text { Layer } & {[\text { ohm-m] }} & {[\mathrm{m}]} & {[\mathrm{m}]} \\ 1 & 91.360 & 25.4 & 0.0 \\ 2 & 26.900 & -- & -25.4\end{array}$

System: EM-58 Freg[Hz]: 285 Data Set Code: uh TX Cur $[A]: 1.40$ Turn Off[usec]: 2.5

RX Moment[turns-m^2]: 31.4 Gain Setting: 4

$\begin{array}{lrrrc}\begin{array}{c}\text { Time } \\ \text { [ms }\end{array} & \begin{array}{c}\text { rhoa_obs } \\ \text { [ohm-m] }\end{array} & \begin{array}{c}\text { obs_err } \\ {\left[\frac{\mathrm{o}}{\mathrm{o}}\right]}\end{array} & \text { mask } & \begin{array}{c}\text { rhoa_cal } \\ \text { [ohm-m] }\end{array} \\ 0.00680 & 96.1 & 0.3 & \mathrm{~d} & - \\ 0.00860 & 91.1 & 0.3 & \mathrm{u} & 89.1 \\ 0.01110 & 85.7 & 0.4 & \mathrm{u} & 87.0 \\ 0.01410 & 80.3 & 0.4 & \mathrm{u} & 82.9 \\ 0.01800 & 77.6 & 0.6 & \mathrm{u} & 77.4 \\ 0.02300 & 72.5 & 1.0 & \mathrm{u} & 71.5 \\ 0.02940 & 66.7 & 1.3 & \mathrm{u} & 65.8 \\ 0.03750 & 60.0 & 1.1 & \mathrm{u} & 60.7 \\ 0.04790 & 58.4 & 1.3 & \mathrm{u} & 56.1 \\ 0.06110 & 50.9 & 2.4 & \mathrm{u} & 52.2 \\ 0.07790 & 48.5 & 1.2 & \mathrm{u} & 48.8 \\ 0.09930 & 51.6 & 2.8 & \mathrm{~m} & 46.0 \\ 0.12670 & 65.6 & 10.1 & \mathrm{~m} & 43.5 \\ 0.16640 & 69.4 & 31.4 & \mathrm{~m} & 41.2 \\ 0.20600 & 79.4 & 53.2 & \mathrm{~m} & 39.8 \\ 0.26280 & 80.5 & 100.0 & \mathrm{~m} & 38.5 \\ 0.33520 & 48.7 & 70.2 & \mathrm{~m} & 37.6 \\ 0.42770 & 19.7 & 29.3 & \mathrm{~m} & 37.0 \\ 0.54560 & 12.2 & 24.2 & \mathrm{~m} & 37.0 \\ 0.69590 & 7.1 & 23.0 & \mathrm{~m} & 37.3\end{array}$

Date: 07-MAY-08

UTM Coord: $\mathrm{E}[\mathrm{m}]$

Elevation $[\mathrm{m}]$ :

$582649.0 \mathrm{~N}[\mathrm{~m}]$

2868479.0

0.0

RX location: X [m]

$0.0 \quad Y[m]$

0.0

Fit Error [응 $\quad 3.234$ 
Sounding: MIA132

Client: US Geological Survey

Lation: Miami-Dade WASA Water Tank 68

Project: Saltwater Intrusion

County: Miami-Dade County

TX loop size: X[m] $38.1 \quad \mathrm{Y}[\mathrm{m}] \quad 38.1$

Model
Layer
1
2
3
4

$$
\begin{gathered}
\text { Resistivity } \\
\text { [ohm-m] } \\
187.400 \\
61.520 \\
5.380 \\
35.240
\end{gathered}
$$$$
\text { Thickness }
$$$$
[\mathrm{m}]
$$$$
14.7
$$$$
36.4
$$$$
22.4
$$

\section{Elevation}

$[\mathrm{m}]$

$$
\begin{array}{r}
0.0 \\
-14.7 \\
-51.1 \\
-73.6
\end{array}
$$

$\begin{array}{llll}\text { System: EM-58 Freq[Hz]: } 285 \text { Data } & \text { Set Code: uh } \\ \text { TX Cur }[A]: \quad 1.40 \text { Turn Off[usec] : } & 2.5\end{array}$

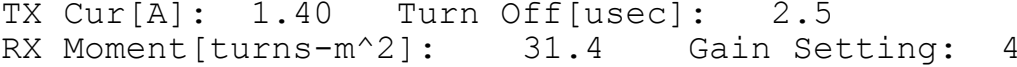

$\begin{array}{rrrrrr}\text { Time } & \begin{array}{c}\text { rhoa_obs } \\ \text { [ohm-m] }\end{array} & \begin{array}{c}\text { obs_err } \\ {\left[\frac{\bar{o}}{0}\right]}\end{array} & \text { mask } & \begin{array}{c}\text { rhoa_cal } \\ \text { [ohm-m] }\end{array} \\ 1 & 0.00680 & 121.8 & 0.4 & \mathrm{u} & 122.1 \\ 2 & 0.00860 & 114.6 & 0.1 & \mathrm{u} & 113.5 \\ 3 & 0.01110 & 109.2 & 0.1 & \mathrm{u} & 110.1 \\ 4 & 0.01410 & 109.6 & 0.1 & \mathrm{u} & 109.2 \\ 5 & 0.01800 & 128.2 & 0.1 & \mathrm{~m} & 109.2 \\ 6 & 0.02300 & 158.2 & 0.2 & \mathrm{~m} & 109.1 \\ 7 & 0.02940 & 161.0 & 0.5 & \mathrm{~m} & 106.6 \\ 8 & 0.03750 & 101.1 & 0.3 & \mathrm{u} & 100.0 \\ 9 & 0.04790 & 86.8 & 0.2 & \mathrm{u} & 89.1 \\ 10 & 0.06110 & 76.1 & 0.4 & \mathrm{u} & 75.8 \\ 11 & 0.07790 & 63.2 & 0.5 & \mathrm{u} & 62.5 \\ 12 & 0.09930 & 52.7 & 0.5 & \mathrm{u} & 51.0 \\ 13 & 0.12670 & 41.0 & 0.4 & \mathrm{u} & 41.9 \\ 14 & 0.16640 & 33.6 & 0.9 & \mathrm{u} & 34.3 \\ 15 & 0.20600 & 29.7 & 1.1 & \mathrm{u} & 30.0 \\ 16 & 0.26280 & 27.3 & 1.8 & \mathrm{u} & 26.5 \\ 17 & 0.33520 & 24.1 & 2.9 & \mathrm{u} & 24.1 \\ 18 & 0.42770 & 22.6 & 4.7 & \mathrm{u} & 22.6 \\ 19 & 0.54560 & 21.8 & 7.8 & \mathrm{u} & 21.8 \\ 20 & 0.69590 & 21.7 & 15.1 & \mathrm{u} & 21.7\end{array}$

Date: 07-MAY-08

UTM Coord:

Elevation $[\mathrm{m}]$ :

$579026.0 \quad \mathrm{~N}[\mathrm{~m}] \quad 2857869.0$ 0.0

$0.0 Y[\mathrm{~m}] \quad 0.0$

Fit Error $[\circ]: 2.355$ 
Sounding: MIA133

Client: US Geological Survey

Location: Edison HS Football Field West

Project: Saltwater Intrusion

County: Miami-Dade County

TX loop size: X[m] $38.1 \quad Y[m] \quad 38.1$

$\begin{array}{cccc}\text { Model } & \text { Resistivity } & \text { Thickness } & \text { Elevation } \\ \text { Layer } & {[\text { ohm-m] }} & {[\mathrm{m}]} & {[\mathrm{m}]} \\ 1 & 39.810 & 28.1 & 0.0 \\ 2 & .077 & -- & -28.1\end{array}$

System: EM-58 Freq[Hz]: 285 Data Set Code: uh TX Cur $[\mathrm{A}]: \quad 1.40$ Turn Off [usec] : 2.5

RX Moment[turns-m^2]: 31.4 Gain Setting: 3

$\begin{array}{rrrrrr}\text { Time } & \text { [ms] } & \begin{array}{c}\text { rhoa_obs } \\ \text { [ohm-m] }\end{array} & \begin{array}{c}\text { obserr } \\ {\left[\begin{array}{c}\text { oo } \\ 0\end{array}\right.}\end{array} & \text { mask } & \begin{array}{r}\text { rhoa_cal } \\ \text { [ohm-m] }\end{array} \\ 1 & 0.00680 & 44.5 & 0.1 & \mathrm{u} & 46.1 \\ 2 & 0.00860 & 47.1 & 0.4 & \mathrm{u} & 46.2 \\ 3 & 0.01110 & 50.2 & 0.3 & \mathrm{u} & 50.3 \\ 4 & 0.01410 & 53.4 & 0.6 & \mathrm{u} & 57.9 \\ 5 & 0.01800 & 72.1 & 1.3 & \mathrm{u} & 68.6 \\ 6 & 0.02300 & 87.8 & 2.4 & \mathrm{u} & 81.3 \\ 7 & 0.02940 & 71.1 & 3.3 & \mathrm{u} & 90.5 \\ 8 & 0.03750 & 134.6 & 13.8 & \mathrm{u} & 86.1 \\ 9 & 0.04790 & 55.6 & 5.0 & \mathrm{u} & 73.0 \\ 10 & 0.06110 & 52.3 & 11.6 & \mathrm{u} & 57.3 \\ 11 & 0.07790 & 41.4 & 21.1 & \mathrm{u} & 44.2 \\ 12 & 0.09930 & 35.1 & 26.1 & \mathrm{u} & 34.0 \\ 13 & 0.12670 & 29.8 & 39.7 & \mathrm{u} & 26.1 \\ 14 & 0.16640 & 20.1 & 32.6 & \mathrm{~m} & 19.6 \\ 15 & 0.20600 & 22.6 & 75.9 & \mathrm{~m} & 15.7 \\ 16 & 0.26280 & 45.9 & 100.0 & \mathrm{~m} & 12.3 \\ 17 & 0.33520 & 67.0 & 100.0 & \mathrm{~m} & 9.7 \\ 18 & 0.42770 & 81.8 & 100.0 & \mathrm{~m} & 7.8 \\ 19 & 0.54560 & 17.9 & 100.0 & \mathrm{~m} & 6.3 \\ 20 & 0.69590 & 6.8 & 100.0 & \mathrm{~m} & 5.2\end{array}$

Date: 07-MAY-08

UTM Coord:

Elevation $[\mathrm{m}]$ :

$579880.0 \quad \mathrm{~N}[\mathrm{~m}] \quad 2857273.0$ 0.0

$0.0 Y[\mathrm{~m}] \quad 0.0$

Fit Error [응: 29.140 
Sounding: MIA134

Client: US Geological Survey

Date: 07-MAY-08

UTM Coord: $\mathrm{E}[\mathrm{m}]$

Elevation $[\mathrm{m}]$ :

$579948.0 \quad \mathrm{~N}[\mathrm{~m}] \quad 2857286.0$ 0.0

RX location: $\mathrm{X}[\mathrm{m}]$

$0.0 Y[\mathrm{~m}] \quad 0.0$

Fit Error [응: 9.724

System: EM-58 Freq[Hz]: 285 Data Set Code: uh TX Cur $[A]: \quad 1.40$ Turn Off[usec]: 2.5

RX Moment [turns-m^2]: 31.4 Gain Setting: 3

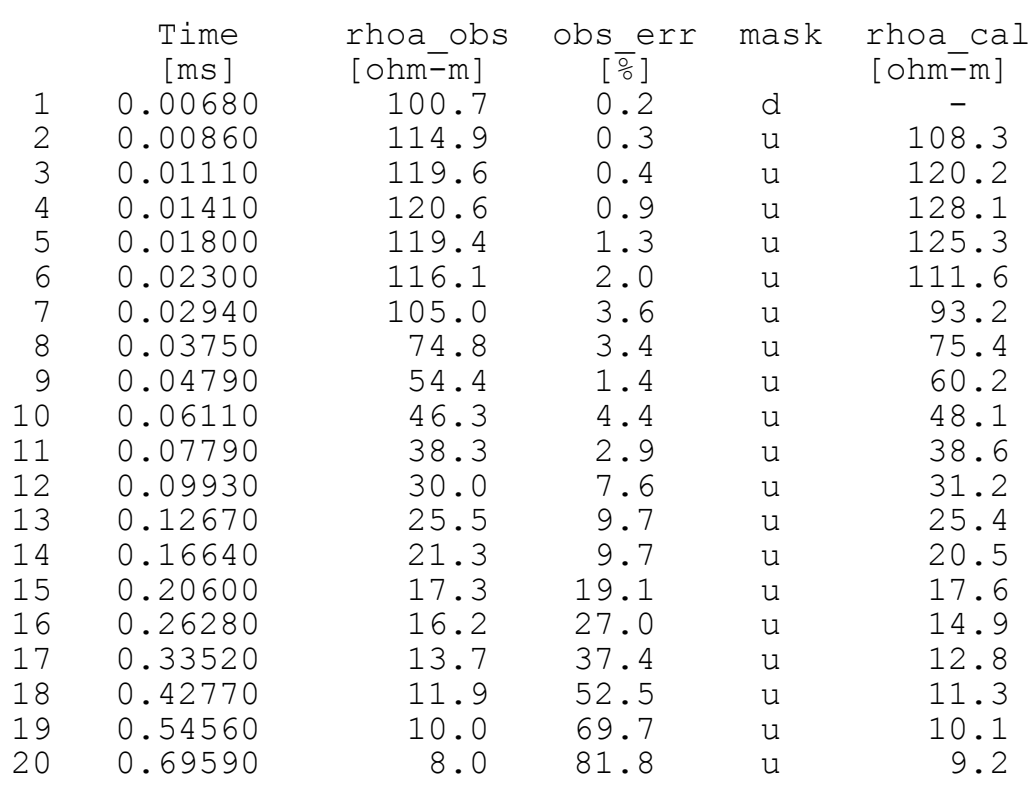


Sounding: MIA135

Location: Ives Estates Park

Project: Saltwater Intrusion

County: Miami-Dade County

TX loop size: X[m] $38.1 \quad \mathrm{Y}[\mathrm{m}] \quad 38.1$

$\begin{array}{cccc}\text { Model } & \text { Resistivity } & \text { Thickness } & \text { Elevation } \\ \text { Layer } & {[\text { ohm }-\mathrm{m}]} & {[\mathrm{m}]} & {[\mathrm{m}]} \\ 1 & 353.700 & 8.7 & 0.0 \\ 2 & 63.860 & 72.8 & -8.7 \\ 3 & 13.850 & -- & -81.5\end{array}$

System: EM-58 Freq[Hz]: 285 Data Set Code: uh TX Cur $[A]: \quad 1.40$ Turn Off[usec]: 2.5

RX Moment[turns-m^2]: 31.4 Gain Setting: 3

$\begin{array}{rrrrrr}\text { Time } & \begin{array}{c}\text { rhoa_obs } \\ \text { [ohm-m] }\end{array} & \begin{array}{c}\text { obs_err } \\ {\left[\frac{\bar{o}}{0}\right]}\end{array} & \text { mask } & \begin{array}{r}\text { rhoa_cal } \\ \text { [ohm-m] }\end{array} \\ 1 & 0.00680 & 109.0 & 0.1 & \mathrm{u} & 107.3 \\ 2 & 0.00860 & 99.7 & 0.1 & \mathrm{u} & 99.3 \\ 3 & 0.01110 & 93.8 & 0.2 & \mathrm{u} & 94.9 \\ 4 & 0.01410 & 90.3 & 0.2 & \mathrm{u} & 91.5 \\ 5 & 0.01800 & 87.3 & 0.2 & \mathrm{u} & 88.3 \\ 6 & 0.02300 & 86.0 & 0.2 & \mathrm{u} & 85.6 \\ 7 & 0.02940 & 84.7 & 0.2 & \mathrm{u} & 83.8 \\ 8 & 0.03750 & 81.8 & 0.2 & \mathrm{u} & 82.8 \\ 9 & 0.04790 & 83.5 & 0.3 & \mathrm{u} & 82.5 \\ 10 & 0.06110 & 80.8 & 0.5 & \mathrm{u} & 82.1 \\ 11 & 0.07790 & 83.0 & 0.6 & \mathrm{u} & 80.9 \\ 12 & 0.09930 & 76.9 & 0.9 & \mathrm{u} & 78.3 \\ 13 & 0.12670 & 75.7 & 2.1 & \mathrm{u} & 74.1 \\ 14 & 0.16640 & 87.8 & 4.0 & \mathrm{~m} & 67.9 \\ 15 & 0.20600 & 62.0 & 5.9 & \mathrm{u} & 62.8 \\ 16 & 0.26280 & 57.1 & 4.8 & \mathrm{u} & 57.1 \\ 17 & 0.33520 & 52.3 & 10.3 & \mathrm{u} & 52.2 \\ 18 & 0.42770 & 41.0 & 8.8 & \mathrm{~m} & 48.1 \\ 19 & 0.54560 & 41.7 & 20.3 & \mathrm{~m} & 44.8 \\ 20 & 0.69590 & 33.5 & 17.9 & \mathrm{~m} & 42.5\end{array}$

Date: 08-MAY-08

UTM Coord: $\mathrm{E}[\mathrm{m}]$

Elevation $[\mathrm{m}]$ :

$583323.0 \quad \mathrm{~N}[\mathrm{~m}] \quad 2872973.0$ 0.0

RX location: $\mathrm{X}[\mathrm{m}]$

$0.0 \quad \mathrm{Y}[\mathrm{m}] \quad 0.0$

Fit Error [] : 2.055 
Sounding: MIA136

Location: Kevin Broils Park

Project: Saltwater Intrusion

County: Miami-Dade County

TX loop size: X[m] $38.1 \quad Y[m] \quad 38.1$

$\begin{array}{cccc}\text { Model } & \text { Resistivity } & \text { Thickness } & \text { Elevation } \\ \text { Layer } & {[\text { ohm-m] }} & {[\mathrm{m}]} & {[\mathrm{m}]} \\ 1 & 492.200 & 14.1 & 0.0 \\ 2 & 79.410 & 69.9 & -14.1 \\ 3 & 21.800 & -- & -84.0\end{array}$

System: EM-58 Freq[Hz]: 285 Data Set Code: uh TX Cur $[A]: \quad 1.40$ Turn Off[usec]: 2.5

RX Moment[turns-m^2]: 31.4 Gain Setting: 3

$\begin{array}{rrrrrr}\text { Time } & \begin{array}{c}\text { rhoa_obs } \\ \text { [ohm-m] }\end{array} & \begin{array}{c}\text { obs_err } \\ {\left[\frac{\bar{o}}{0}\right]}\end{array} & \text { mask } & \begin{array}{c}\text { rhoa_cal } \\ \text { [ohm-m] }\end{array} \\ 1 & 0.00680 & 171.0 & 0.5 & \mathrm{u} & 163.4 \\ 2 & 0.00860 & 146.2 & 0.4 & \mathrm{u} & 148.9 \\ 3 & 0.01110 & 139.4 & 0.3 & \mathrm{u} & 140.2 \\ 4 & 0.01410 & 131.2 & 0.3 & \mathrm{u} & 133.3 \\ 5 & 0.01800 & 125.7 & 0.3 & \mathrm{u} & 127.0 \\ 6 & 0.02300 & 120.7 & 0.3 & \mathrm{u} & 122.2 \\ 7 & 0.02940 & 115.4 & 0.4 & \mathrm{u} & 118.7 \\ 8 & 0.03750 & 111.8 & 0.5 & \mathrm{u} & 116.1 \\ 9 & 0.04790 & 107.8 & 0.7 & \mathrm{u} & 113.6 \\ 10 & 0.06110 & 106.9 & 1.1 & \mathrm{u} & 110.3 \\ 11 & 0.07790 & 123.1 & 2.5 & \mathrm{u} & 105.6 \\ 12 & 0.09930 & 118.9 & 4.5 & \mathrm{u} & 99.3 \\ 13 & 0.12670 & 83.6 & 3.9 & \mathrm{u} & 91.9 \\ 14 & 0.16640 & 73.4 & 5.7 & \mathrm{u} & 83.3 \\ 15 & 0.20600 & 95.1 & 12.0 & \mathrm{u} & 77.0 \\ 16 & 0.26280 & 57.0 & 6.5 & \mathrm{u} & 70.4 \\ 17 & 0.33520 & 61.6 & 12.6 & \mathrm{u} & 65.0 \\ 18 & 0.42770 & 56.9 & 37.1 & \mathrm{u} & 60.5 \\ 19 & 0.54560 & 66.3 & 100.0 & \mathrm{u} & 57.3 \\ 20 & 0.69590 & 56.4 & 100.0 & \mathrm{u} & 54.9\end{array}$

Date: 08-MAY-08

UTM Coord: $\mathrm{E}[\mathrm{m}]$

Elevation $[\mathrm{m}]$ :

$560837.0 \quad \mathrm{~N}[\mathrm{~m}] \quad 2823096.0$ 0.0

RX location: $\mathrm{X}[\mathrm{m}]$

$0.0 \quad Y[m] \quad 0.0$

Fit Error[\%] : 16.531 
Sounding: MIA137

Client: US Geological Survey Location: SW 122 Ave \& Moody Drive

Project: Saltwater Intrusion

County: Miami-Dade County

TX loop size: X [m] $38.1 \quad \mathrm{Y}[\mathrm{m}] \quad 38.1$

$\begin{array}{cccc}\text { Model } & \text { Resistivity } & \text { Thickness } & \text { Elevation } \\ \text { Layer } & {[\text { ohm-m] }} & {[\mathrm{m}]} & {[\mathrm{m}]} \\ 1 & 529.500 & 15.4 & 0.0 \\ 2 & 58.820 & 77.6 & -15.4 \\ 3 & 26.050 & -- & -93.1\end{array}$

System: EM-58 Freq[Hz]: 285 Data Set Code: uh TX Cur $[\mathrm{A}]: \quad 1.40$ Turn Off[usec]: 2.5

RX Moment[turns-m^2]: 31.4 Gain Setting: 4

$\begin{array}{crrcc}\begin{array}{c}\text { Time } \\ \text { [ms }]\end{array} & \begin{array}{c}\text { rhoa_obs } \\ \text { [ohm-m] }\end{array} & \begin{array}{c}\text { obserr } \\ {\left[\begin{array}{c}\text { o }] \\ 0.00680\end{array}\right.}\end{array} & \begin{array}{c}\text { mask } \\ 161.7\end{array} & \begin{array}{c}\text { rhoa_cal } \\ \text { [ohm-m] }\end{array} \\ 0.00860 & 136.1 & 0.3 & \mathrm{u} & 156.6 \\ 0.01110 & 127.7 & 0.3 & \mathrm{u} & 139.2 \\ 0.01410 & 118.1 & 0.3 & \mathrm{u} & 127.9 \\ 0.01800 & 109.2 & 0.3 & \mathrm{u} & 118.8 \\ 0.02300 & 102.9 & 0.2 & \mathrm{u} & 103.6 \\ 0.02940 & 97.7 & 0.3 & \mathrm{u} & 97.9 \\ 0.03750 & 93.8 & 0.2 & \mathrm{u} & 93.2 \\ 0.04790 & 90.9 & 0.3 & \mathrm{u} & 89.5 \\ 0.06110 & 87.3 & 0.4 & \mathrm{u} & 86.5 \\ 0.07790 & 86.2 & 0.4 & \mathrm{u} & 83.8 \\ 0.09930 & 79.7 & 0.4 & \mathrm{u} & 81.0 \\ 0.12670 & 75.5 & 0.7 & \mathrm{u} & 77.9 \\ 0.16640 & 72.6 & 0.9 & \mathrm{u} & 73.8 \\ 0.20600 & 70.1 & 1.1 & \mathrm{u} & 70.4 \\ 0.26280 & 67.1 & 2.0 & \mathrm{u} & 66.6 \\ 0.33520 & 64.9 & 5.1 & \mathrm{u} & 63.2 \\ 0.42770 & 62.0 & 13.1 & \mathrm{u} & 60.3 \\ 0.54560 & 58.0 & 25.2 & \mathrm{u} & 58.0 \\ 0.69590 & 54.8 & 24.6 & \mathrm{u} & 56.7\end{array}$

Date: $08-\mathrm{MAY}-08$

UTM Coord: $\mathrm{E}[\mathrm{m}]$

Elevation $[\mathrm{m}]$ :

$561528.0 \quad \mathrm{~N}[\mathrm{~m}] \quad 2822700.0$

0.0

RX location: X $[\mathrm{m}]$

$0.0 \quad \mathrm{Y}[\mathrm{m}]$

0.0

Fit Error []ㅜ: 2.841 
THIS PAGE INTENTIONALLY LEFT BLANK. 


\section{Appendix 5 TEM Sounding Descriptions and Plots: 2008}

Descriptions of the sounding results are given below, along with plots of the measured apparent resistivity, calculated apparent resistivity for the inversion model, and the inversion estimated resistivity-depth function. The TEM data were measured at two transmitter repetition frequencies. The $285-\mathrm{Hz}$ data $(\mathrm{UH})$ are plotted as open circles $(\mathrm{O})$, and the $30-\mathrm{Hz}$ data $(\mathrm{HI})$ are plotted as open squares $(\square)$. The apparent resistivity measurement error bars are plotted as vertical lines through the data points. In most cases, the error bars are smaller than the plotting symbol. The calculated $285-\mathrm{Hz}$ model response is plotted as a solid line, and the $30-\mathrm{Hz}$ model response is plotted as a dashed line. Some plots show only the $285-\mathrm{Hz}$ data because the lower repetition rate data were too noisy to be of value. Masked data points are plotted as solid diamonds $(\checkmark)$ and were not used in the inversion. All soundings were made with a square transmitter loop with a side length of $38.1 \mathrm{~m}$. Unusable soundings are noted in table 1.

Geoelectrical models can be classified by the variation of resistivity with depth (Keller and Frischknecht, 1966, p. 135). A three-layer model with a resistivity minimum $\left(\rho_{1}>\rho_{2}<\rho_{3}\right)$ is called a type-H curve. A model with a resistivity maximum $\left(\rho_{1}<\rho_{2}>\rho_{3}\right)$ is called a type-K curve. A model with a monotonic increase in resistivity $\left(\rho_{1}<\rho_{2}<\rho_{3}\right)$ is called a type-A curve. A model with a monotonic decrease in resistivity $\left(\rho_{1}>\rho_{2}>\rho_{3}\right)$ is called a type-Q curve. For curves with more than three layers, this classification is applied to the first through third layer, the second through fourth layer, and so on. For example, a curve with $\left(\rho_{1}>\rho_{2}<\rho_{3}>\rho_{4}\right)$ is classified as type HK.

The resistivity-depth models determined by inversion are usually not unique; that is, there is uncertainty in the estimated model parameters caused by observation and parameterization errors. The latter is due to naturally occurring variations in geology that are not describable by the onedimensional (layered earth) model used for the inversion. A qualitative estimate of the parameter uncertainty is given in the descriptions below. Another issue in the inversion is the resolution of the model parameters. To understand model resolution, assume that there is an actual set of model parameters that we can only view through a set of glasses. If the glasses give a good image of the actual parameters, then the resolution is good. If, on the other hand, the glasses distort the viewing of the actual parameters, then the resolution is poor. When a descriptive resolution is given for a sequence of layer resistivities or thicknesses, such as "good, very good, poor," the order is from the first layer downward.

\section{Sounding MIA101}

A three-layer model with a less resistive (more conductive) second layer (type $\mathrm{H}$ ) fits the data with a misfit error of 3.10 percent. The first layer resistivity and thickness are well constrained. The conductive second and third layer resistivities are moderately well constrained. The second layer thickness is not as well constrained as the first layer thickness.

\section{Sounding MIA102}

A three-layer model with a less resistive second layer (type $\mathrm{H}$ ) fits the data with a misfit error of 3.66 percent. The conductive second layer is thin compared to the depth of burial resulting in an S-equivalence. This means that the measurement can only resolve the layer conductance (the 
ratio of the layer thickness to layer resistivity), not the individual layer parameters. The first and third layer resistivities are well constrained, as is the thickness of the first layer.

An alternative model using a fixed resistivity for the conductive second layer was investigated (MIA102F). The resistivity of the thin, second layer, which is a saltwater-intruded zone, was set to $3.5 \mathrm{ohm}-\mathrm{m}$. This value was determined from induction logs. This model has a misfit error of 3.70 percent. The resolutions of the unconstrained parameters are similar to model MIA102. The constrained model MIA102F is the preferred one.

\section{Sounding MIA103}

The data are well fit with a three-layer model whose resistivities decrease with depth (type Q). The resistive surface layer is not well constrained, while the second layer resistivity and depth are well resolved. The resistivity and thickness of the third layer are moderately well resolved. Misfit error is 2.65 percent.

\section{Sounding MIA104}

The data quality is pretty good, except around $0.70 \mathrm{~ms}$ where there are some sharp breaks in the apparent resistivity curve, which are mostly likely due to metallic objects near the sounding site. A three-layer model with decreasing resistivity with depth (type Q) does a reasonable job of fitting the data. Resolutions of all layer resistivities are moderately good, while the resolution of the layer thicknesses is not as good. Resolution of the first two layer resistivities is very poor, while the third layer resistivity resolution is poor to good. Misfit error is 7.26 percent. An alternative fourlayer model (MIA104L4) was also tried. While the misfit error is slightly smaller (7.18 percent), the parameter resolution is worse. The three-layer model is preferred.

\section{Sounding MIA105}

A four-layer model fits the data with a misfit of 6.53 percent. There is some noise in the data near $0.5 \mathrm{~ms}$, possibly due to nearby metallic objects that contribute to the misfit error. The resolution of the layer resistivities is good for all but the first layer. The resolution of layer thicknesses is good. A five-layer model (MIA105L5) does a slightly better job of fitting the data in the $0.01-0.08 \mathrm{~ms}$ time range, however, the resolution of layers three and four is not good. The fivelayer model misfit is 5.19 percent. The four-layer model is preferred.

\section{Sounding MIA106}

The data are moderately noisy, particularly in the time range of 0.03 to $0.1 \mathrm{~ms}$ due to the presence of a non-geologic conductor. Nonetheless, a four-layer model with resistivity decreasing with depth (type QQ) does a reasonable job of fitting the data. Because of the noisy data, resolution of some model parameters is not very good. The first layer thickness and resistivity are very poorly resolved. The resistive second layer is well resolved, while its thickness is only moderately well determined. Deeper layer resistivities and thicknesses are even more poorly resolved. Misfit error is 8.60 percent.

\section{Sounding MIA107}

The UH frequency data are of very good quality. Using only the UH data, a very good inversion is obtained with a misfit error of 4.10 percent for a three-layer type-Q model. All model 
parameters, with the exception of the first layer resistivity, are well resolved, especially the second layer resistivity.

The HI frequency data (not shown) were offset from the UH data in the region where their times overlap, suggesting a gain, current, or turnoff time problem. The cause could not be determined, so the HI data were not used.

\section{Sounding MIA108}

These data are fit with a four-layer model that has a more conductive zone separating two resistive zones (type $\mathrm{QH}$ ). The misfit error is 4.80 percent. The bottom of the conductive zone and the deepest layer resistivity are not well resolved.

A three-layer model (MIA108L3) does a very poor job of fitting the data. This model has a monotonically decreasing resistivity with depth (type Q). Misfit error is 7.17 percent.

\section{Sounding MIA109}

The data are modeled with a three-layer model with decreasing resistivity with depth (type Q). The high first layer resistivity is not well resolved. The second layer resistivity is well resolved. The third layer resistivity is moderately well resolved. All layer thicknesses are moderately well resolved. Misfit error is 2.37 percent.

\section{Sounding MIA110}

The three-layer model has decreasing layer resistivity with depth (type Q). The very resistive first layer parameters are not well resolved. Successively deeper layers have improved resolution. The misfit error is 1.95 percent.

\section{Sounding MIA111}

A two-layer model does a very good job of fitting the data. The layer resistivity decreases with depth. The first layer resistivity and thickness are moderately well determined. The second layer resistivity is very well constrained. The misfit error is 2.12 percent. A three-model (not shown) did not improve the misfit error, and none of the resistivities and thicknesses of the first two layers were resolvable.

\section{Sounding MIA112}

The data exhibit cultural noise in the 0.014-0.048 ms time range. A three-layer, type-Q model (MIA112L3) has poor resolution of the first layer parameters. The second and third layer resistivities are pretty well constrained, and the second layer thickness is moderately well constrained. The misfit error is 4.29 percent.

Even though the two-layer model (MIA112) has a misfit error of 9.28 percent, the resolution of all of the layers was very good. Considering that most of the misfit is in the time range where the data are noisy, the two-layer model is a better choice.

\section{Sounding MIA113}

Only the UH data were used in the inversion of this sounding because of the noise in the HI data and an offset between the two sweeps. A three-layer, type-Q model produces a very good fit (6.97 percent misfit error), and the resolution of all but the first layer resistivity is very good. 


\section{Sounding MIA114}

A four-layer model with a low resistivity third layer (type $\mathrm{QH}$ ) does the best job of fitting the data. The high-resistivity first layer is not well resolved, while the conductive third layer is well resolved. A moderately good job is done in resolving the transition between the first and third layer. The transition to the slightly more resistive fourth layer is not well resolved. The misfit error is 3.25 percent.

A three-layer, type-H model (MIA114L3) appears to do a better job of resolving the resistive first layer. However, looking in detail at the model response and data values in the time range $0.0068-0.099 \mathrm{~ms}$ it is apparent that the three-layer model does not adequately conform to the shape of the data. The misfit error of the three-layer model is larger (5.39 percent). The four-layer model is preferred

\section{Sounding MIA115}

The data suffer from noise produced by nearby buried metallic objects. The initial $0.1 \mathrm{~ms}$ of the data look good, but then there are obvious departures from the usual shape of a sounding with a maximum in the data around $0.7 \mathrm{~ms}$. Using the data up to this point, the resistive first layer is moderately well resolved, and the conductive second-layer resistivity is well resolved. The depth to the bottom of the conductive layer is not well constrained. The transition to a more resistive third layer is suggested by the minimum in the apparent resistivity data. The resistivity of the third layer is not well constrained. The misfit error is 6.62 percent.

A two-layer model (MIA115L2) gave a similar fit to the first 13 points. The total misfit error was 10.3 percent. Both the two- and three-layer models require a resistive first layer that is about $14 \mathrm{~m}$ thick which is underlain by a $4 \mathrm{ohm}-\mathrm{m}$ conductor. The conductive second layer is again well resolved. The three-layer model is preferred.

\section{Sounding MIA116}

The data quality is not very good after the first eight channels (at times greater than 0.04 $\mathrm{ms}$ ) probably because of a buried metallic object. A two-layer model with decreasing resistivity does a reasonable job of fitting the data in spite of their noisy character. The layer resistivities are well constrained, while the thickness of the first layer is only moderately well constrained. The misfit error is 8.89 percent.

\section{Sounding MIA117}

The data quality of the UH sweep is very good, while the quality of the HI sweep quality is poorer at later times. A three-layer model (MIA117) with a conductive middle layer (type H) results in a misfit of 3.52 percent; however, the thin, conductive second layer means that there is an S-equivalence. To assess the equivalence, the data were reinverted with a model having a fixed second layer resistivity (MIA117F3). The second layer, which is saltwater intruded, was assigned a resistivity of $3.5 \mathrm{ohm}-\mathrm{m}$ based on well logs. The misfit of this model is also 3.52 percent. Comparison of the models shows that they are essentially the same. A four-layer, type-QH model (MIA117L4) has only a moderately better fit (3.48 percent), however, the resolution of the transition layers from the surface resistor to the conductive layer is much worse. In addition, the conductive layer becomes much thinner making the equivalence even more severe. The initial unconstrained model MIA117 is preferred. 


\section{Sounding MIA118}

The early time data are very noisy. The best fit was achieved with a two-layer model with decreasing resistivity. All model parameters are moderately well constrained. The misfit error is 9.61 percent.

\section{Sounding MIA119}

The early time data are very noisy. A two-layer model with decreasing resistivity does the best job of fitting the data. The first layer resistivity is very poorly constrained because the early time channels are not usable. The second layer resistivity is well constrained, while the depth to the second layer is moderately well constrained. The misfit error is 4.86 percent.

\section{Sounding MIA120}

The best fit model has three layers with resistivity decreasing with depth (type Q). The first layer resistivity and thickness are not well resolved. The second layer resistivity and thickness are resolved better. The misfit error is 5.45 percent.

\section{Sounding MIA121}

The data quality is very poor because of noise from nearby conductors. A two-layer model with decreasing layer resistivity fits the general trend of the data. While the first and second layer resistivities are moderately well determined, the first layer thickness is poorly determined. For the purposes of this study, the sounding is adequate to determine that saltwater is present. The misfit error is 24.8 percent.

\section{Sounding MIA122}

A four-layer model provides the best fit of the data. Layer resistivities decrease with depth (type QQ). The very resistive first layer is not well constrained, while the resistive second layer is. The depth to the bottom of the second layer is well constrained. The conductive third layer is reasonable well constrained, while the resolution of the very conductive fourth layer is not as well constrained. The misfit error is 4.76 percent.

\section{Sounding MIA123}

While the data are very repeatable up to times of $0.3335 \mathrm{~ms}$, there are channel-to-channel undulations that cannot be fit with a layered earth model suggesting that there are buried anthropogenic conductors nearby. The model is type Q. In spite of the anthropogenic effects, the resistivity of the second layer is very well constrained. The very resistive surface layer is the least well constrained resistivity of the model. The transition to the third layer is moderately well constrained. The third layer resistivity is the second best determined resistivity. The misfit error is 4.73 percent.

\section{Sounding MIA124}

This sounding is very different from the other soundings in that the second layer is a moderate conductor sandwiched between two high-resistivity layers, all of which is underlain by two increasingly conductive layers (type HKQ). The resolution of the resistive first and third layers 
is poor. The conductive fourth layer is well constrained, while the last layer is not as well constrained. The misfit error is 1.59 percent.

\section{Sounding MIA125}

This is a very high quality three-layer inversion with layer resistivities decreasing with depth (type Q). The first layer resistivity and thickness are poorly determined, while the more conductive second and third layer parameters are well determined. The misfit error is 3.47 percent.

\section{Sounding MIA126}

A four-layer, type-HK model does a very good job of fitting the data. The model consists of a resistive surface layer, underlain by a thin conductive layer, and then a thick, very resistive layer. At depth there is another conductive layer. The first two layers are well determined. The resistivity of the third layer is poorly determined, as would be expected for a resistive target. The fourth layer resistivity is moderately well determined. The thickness of all layers is well resolved. The misfit error is 1.84 percent.

\section{Sounding MIA127}

While repeatable with low measurement error, the data are of very poor quality with severe noise in the first eight channels. The noise is probably due to nearby anthropogenic conductors. The model becomes less resistive with depth (type Q). Only the first layer resistivity is well resolved. The thickness of the first layer is poorly resolved. The misfit error is 20.9 percent.

\section{Sounding MIA128}

The data quality is very poor through the first eight channels. The measurement error is small, but there are large variations of apparent resistivity from channel to channel suggesting the presence of anthropogenic conductors. Interpreted resistivity deceases with depth (type Q). Only the first layer resistivity is well determined. The misfit error is 13.5 percent.

\section{Sounding MIA129}

The first 15 channels exhibit very good data quality. A two-layer model with decreasing resistivity fits the data very well. All model parameters are well resolved. The misfit error is 2.59 percent.

\section{Sounding MIA130}

The data are characterized by very steep slopes on the apparent resistivity-time plots. Attempts to fit the data lead to models with very wild variations in resistivity between layers. The very thin $(0.1 \mathrm{~m})$ and unrealistically conductive second layer $(0.032 \mathrm{ohm}-\mathrm{m})$ is a sure sign of a metallic object in the subsurface or near the receiver coil. The inversion model has no resolution. The results are essentially unusable even though the misfit error is 8.21 percent.

\section{Sounding MIA131}

The first 10 channels are usable, while the subsequent channels are very noise. This is due to noise from electrical lines. Using just the first 10 channels, the data are fit pretty well with a two- 
layer model. The layer resistivities are very well resolved, and the first layer thickness is well resolved. The misfit error is 3.23 percent.

\section{Sounding MIA132}

Data quality is very good except for channels 5, 6, and 7, which appear to be influenced by a nearby metallic conductor. Masking these channels results in a very good fit. The interpreted resistivity decreases with successive layers until the fourth layer, where the resistivity increases (type QH). Resolution of all layers except the last is very good to good. The misfit error is 2.36 percent.

\section{Sounding MIA133}

These data, while very repeatable in the earlier channels, are strongly influenced by an anthropogenic conductor-probably a pipe under the football field. An attempt to fit a two-layer model to the first 13 data points resulted in a two-layer model with an abnormally conductive second layer. All of the model parameters have very large uncertainties. These data are not usable.

\section{Sounding MIA134}

These data were collected at another location in the same football field as sounding MIA133. The effect of the anthropogenic conductor appears to be absent. A two-layer model resolved the model parameters very well; however, the misfit is large ( 9.72 percent).

\section{Sounding MIA135}

After masking data in channel 14 and late-time channels, a very good fit was obtained with a three-layer model. Interpreted resistivity decreases with depth (type Q). The very resistive first layer is not well resolved, while the second layer is very well resolved. The basement resistivity is moderately well resolved. The misfit error is 2.06 percent.

\section{Sounding MIA136}

The data quality of the first 10 channels is very good. Starting with channel 11, the apparent resistivity curve starts undulating indicative of the presence of metallic conductors. A three-layer model with resistivity decreasing with depth can be found that goes through the middle of the data points. The misfit error is 16.5 percent. With the exception of the second layer resistivity, all model parameters are not well resolved. The second layer resistivity is moderately well resolved. Masking the data after channel 10, a two-layer model (not shown), that is essentially the same as the first two layers of the three-layer model, fits the data with a misfit error of 2.84 percent.

\section{Sounding MIA137}

Data quality is very good. A three-layer model with resistivity decreasing (type Q) with depth provides a very good fit with a misfit error of 2.84 percent. The first layer resistivity is poorly resolved. The second layer resistivity is well resolved, and the third layer resistivity is moderately well resolved. The thicknesses of the first and second layers are well resolved. 
MIA101

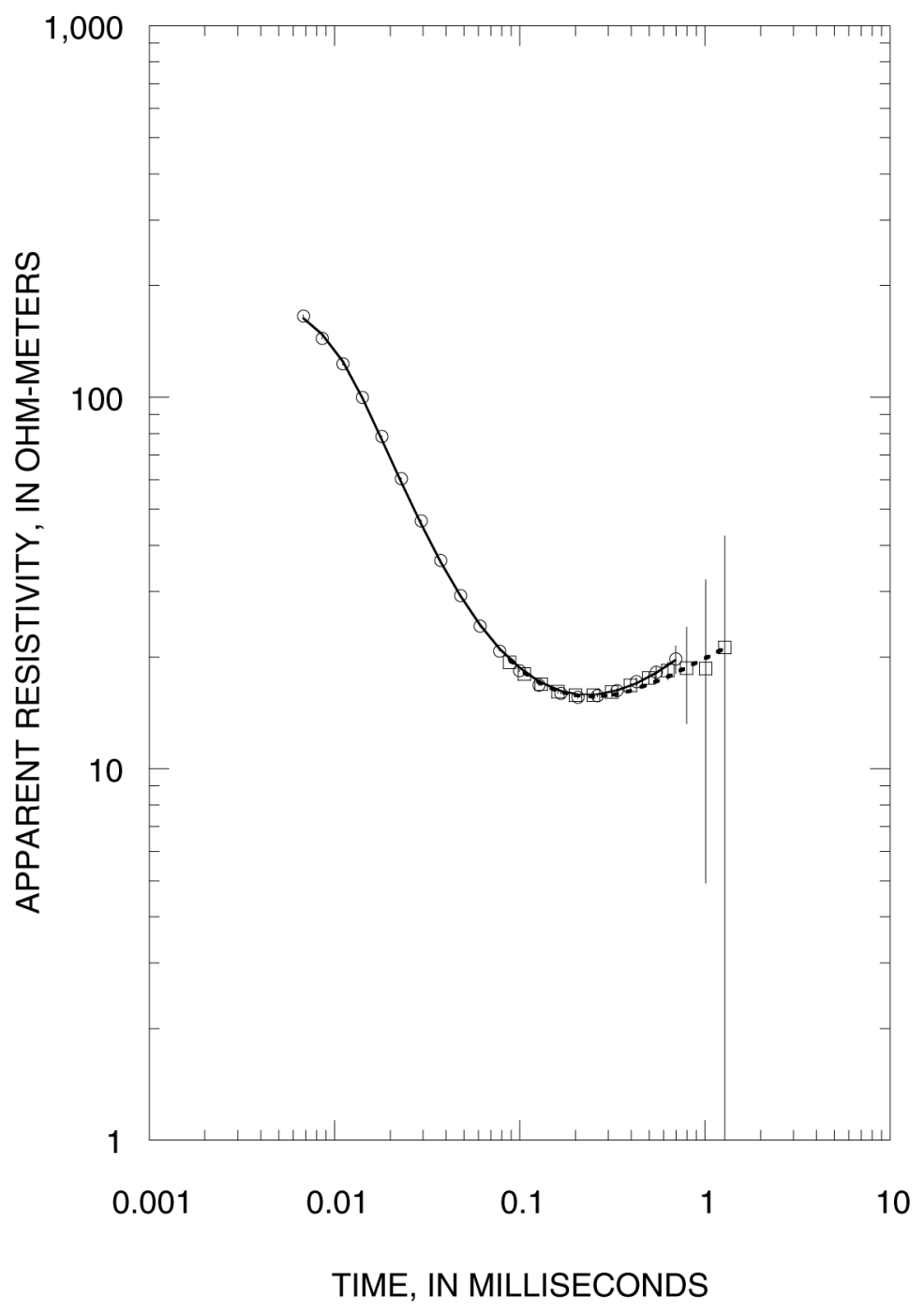

MIA101

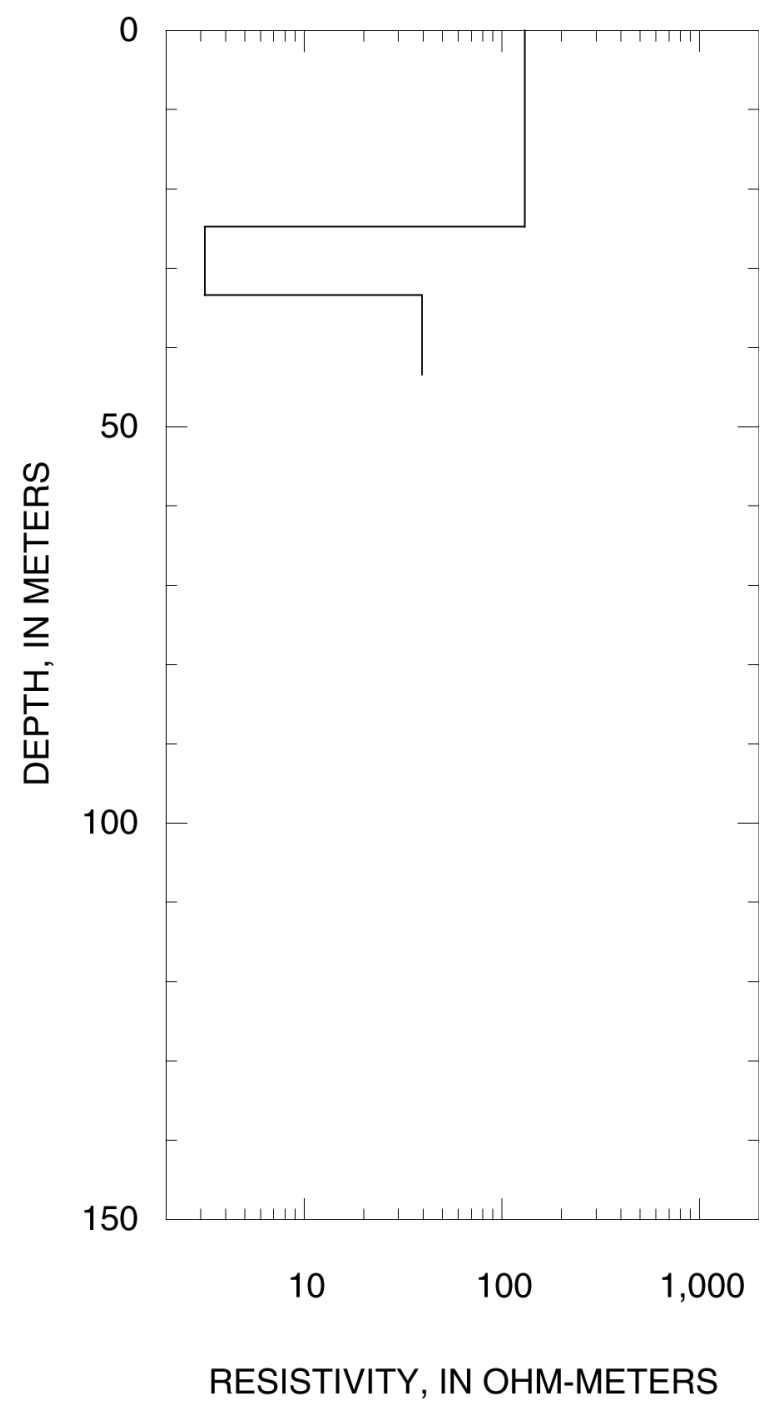

Figure 5-1. Sounding MIA101 
MIA102

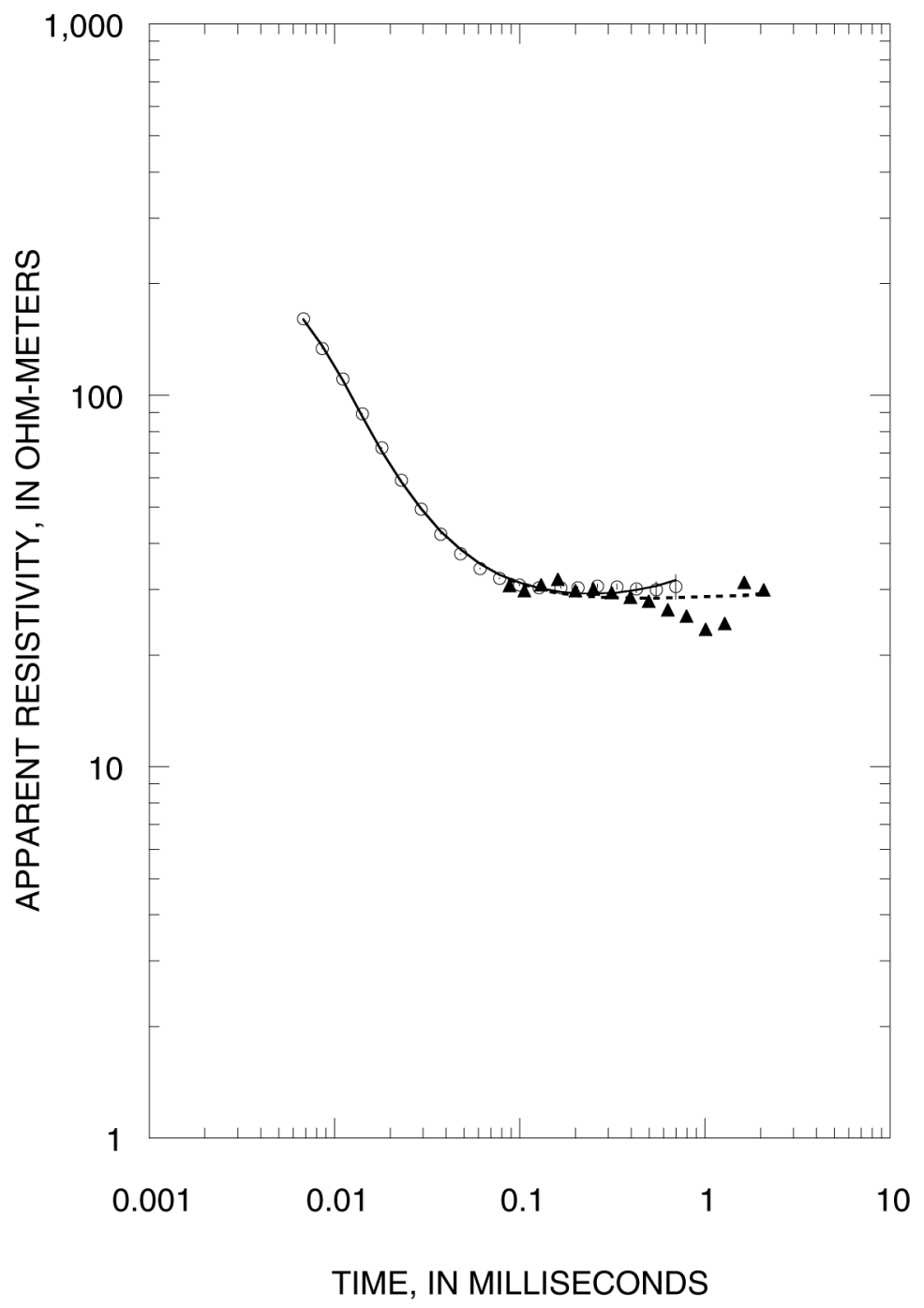

MIA102

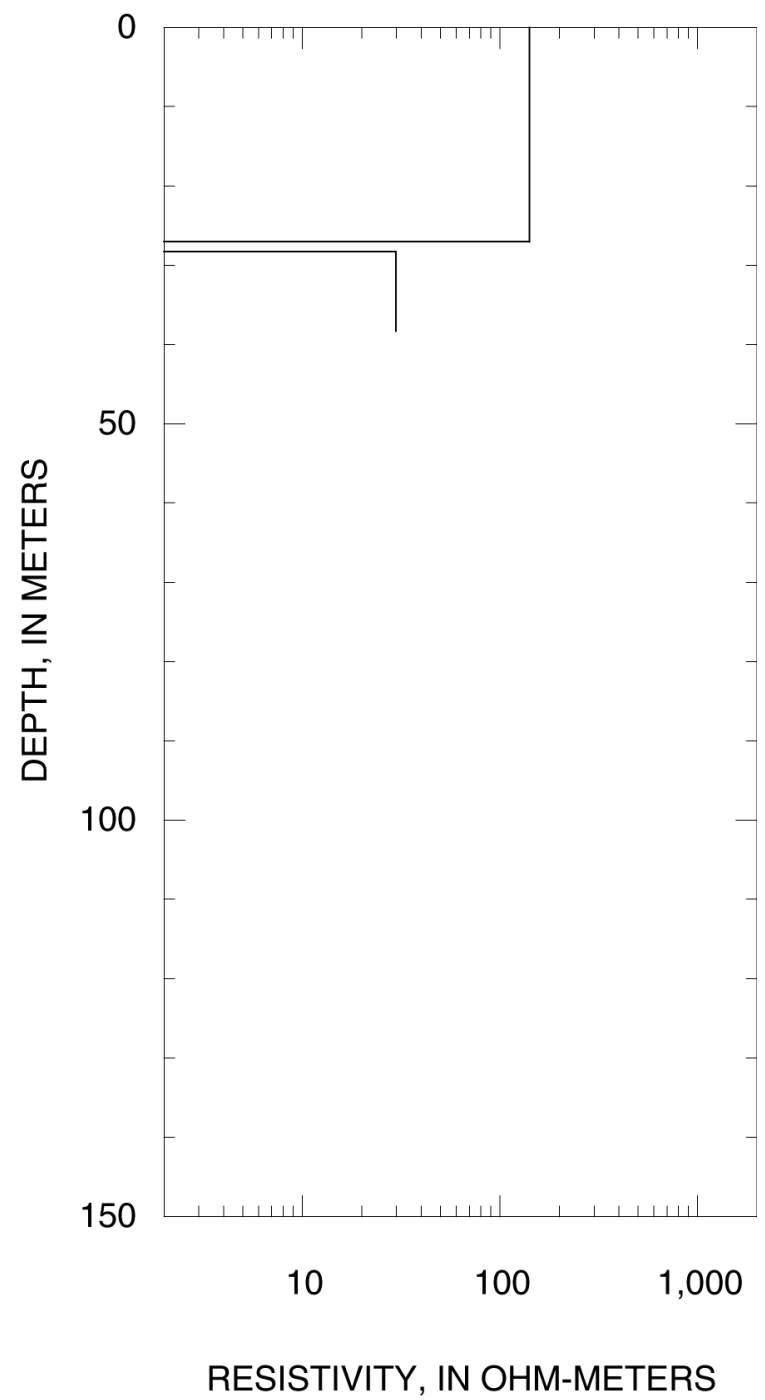

Figure 5-2. Sounding MIA102 

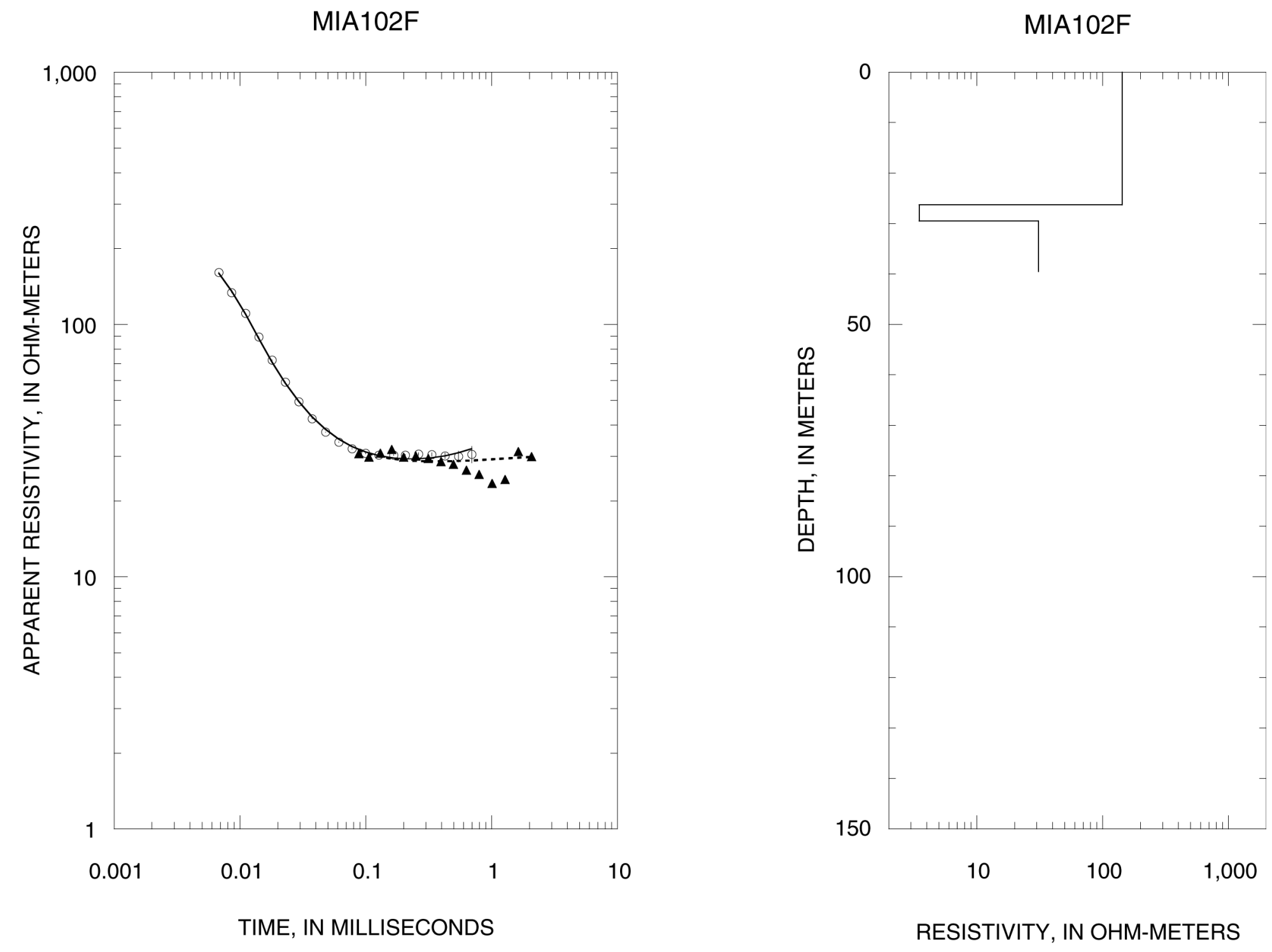

Figure 5-3. Sounding MIA102F 
MIA103

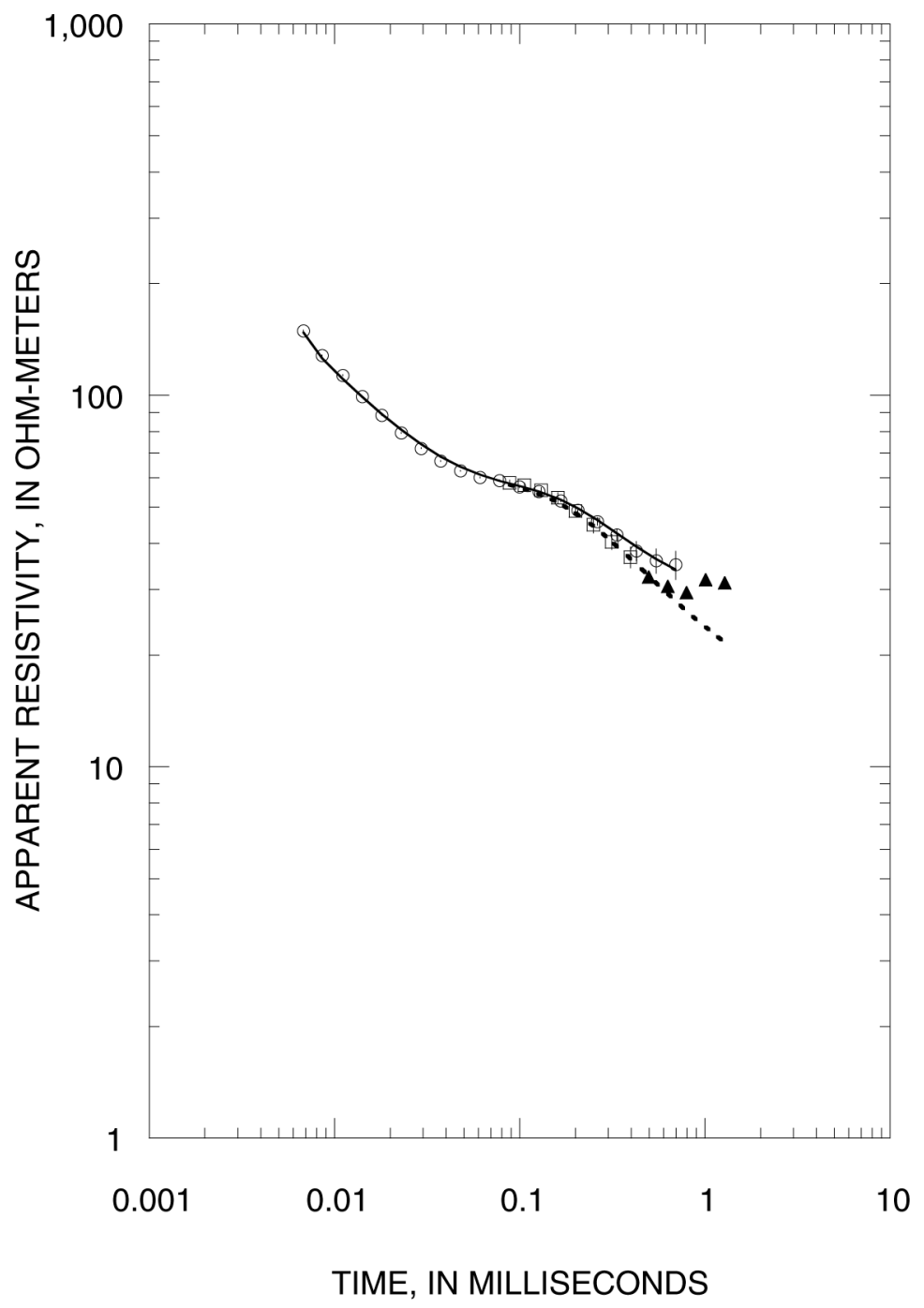

MIA103

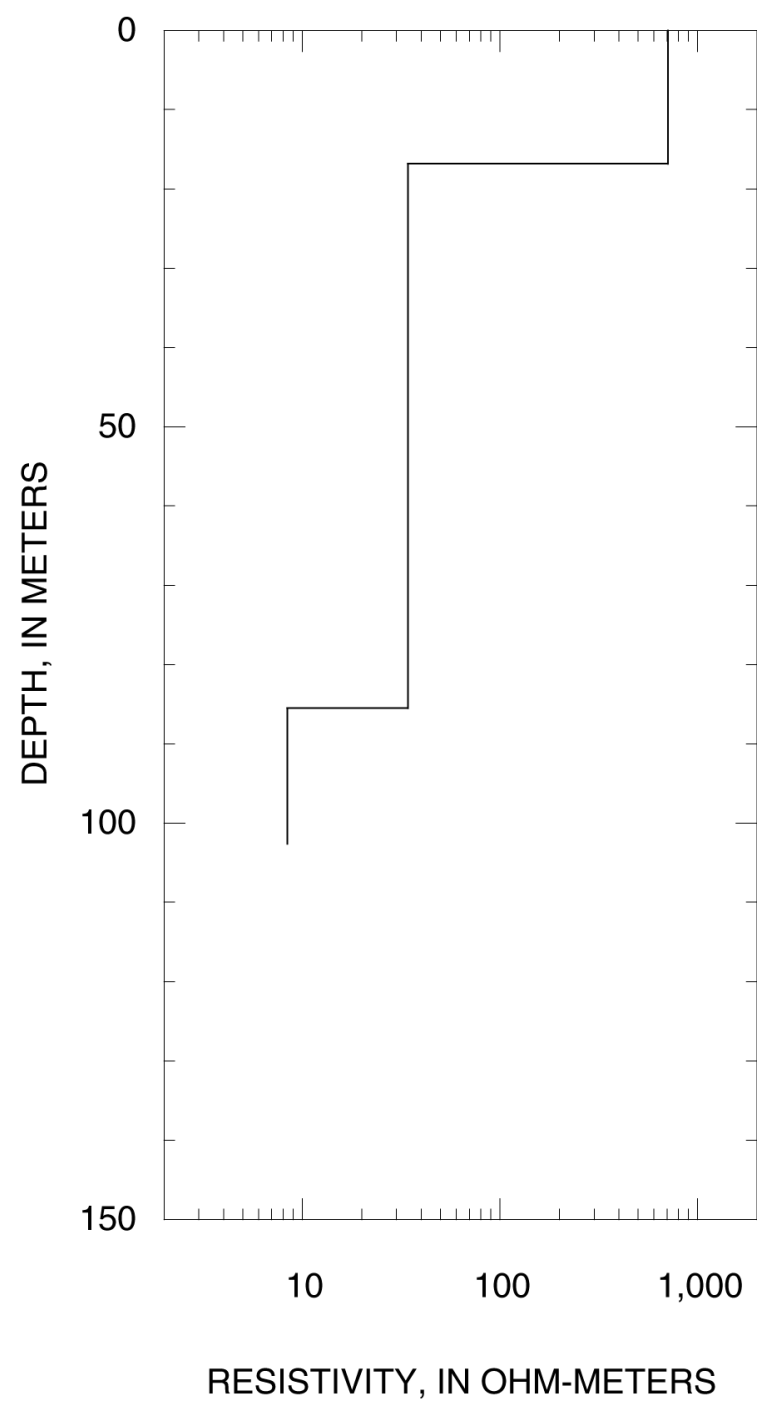

Figure 5-4. Sounding MIA103 
MIA104

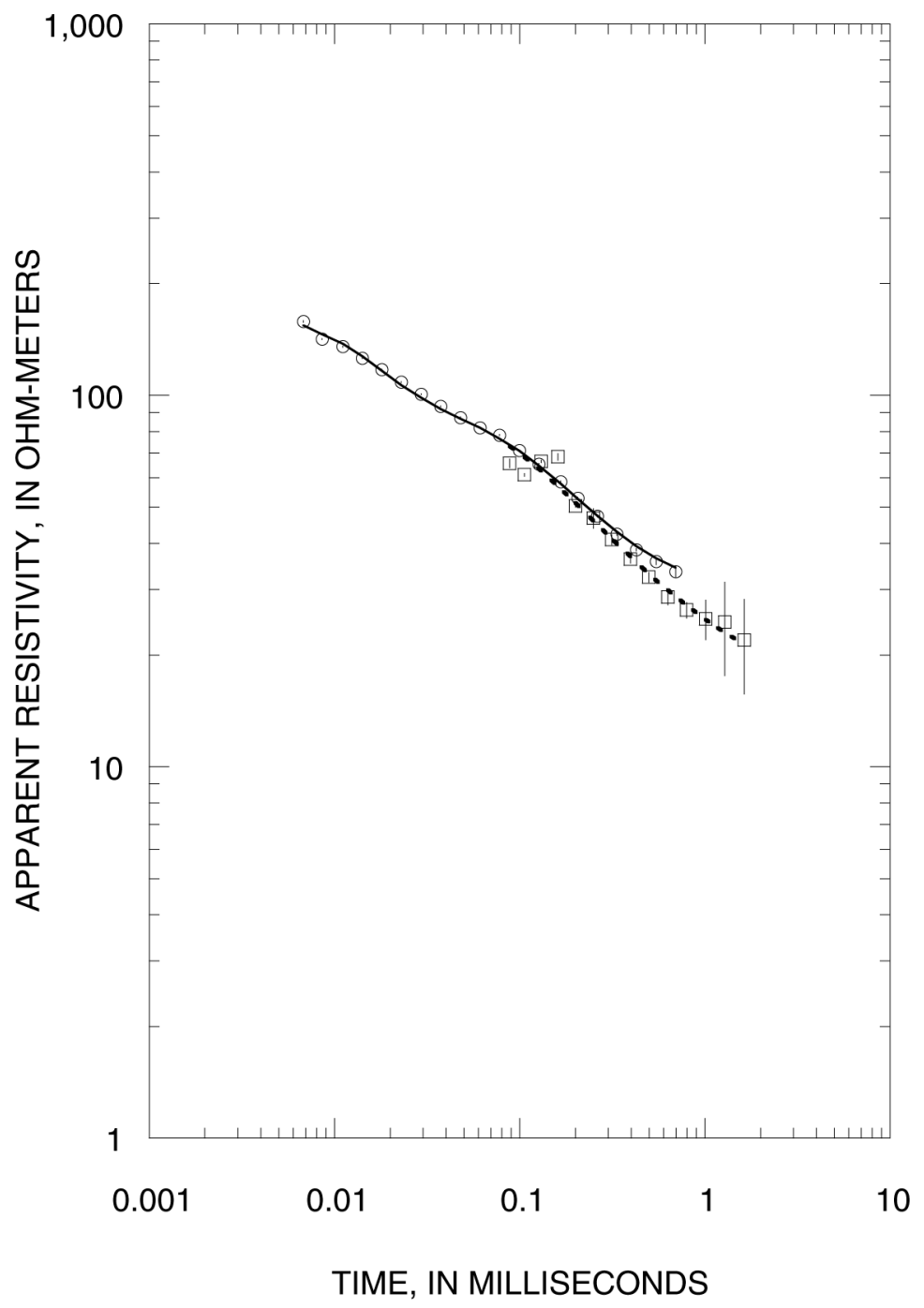

MIA104

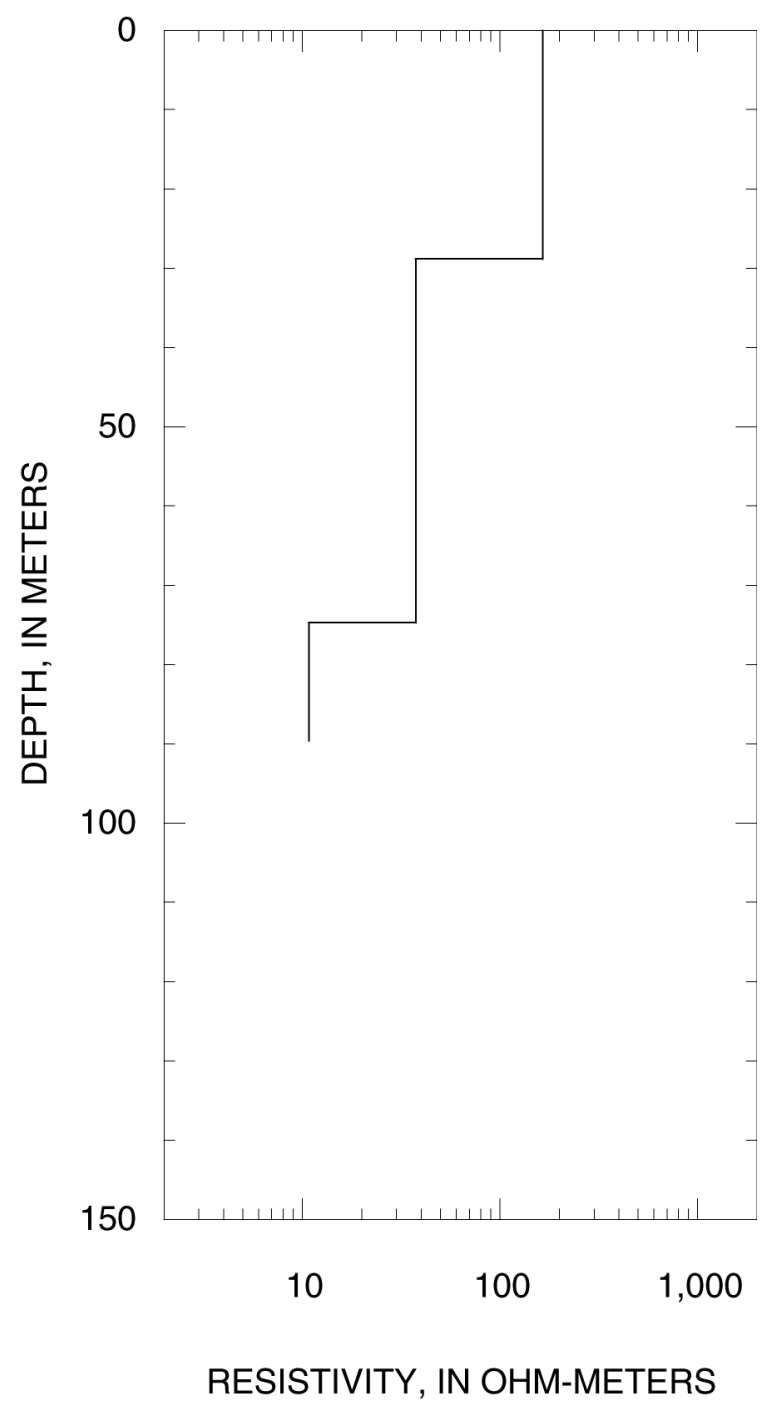

Figure 5-5. Sounding MIA104 
MIA104L4

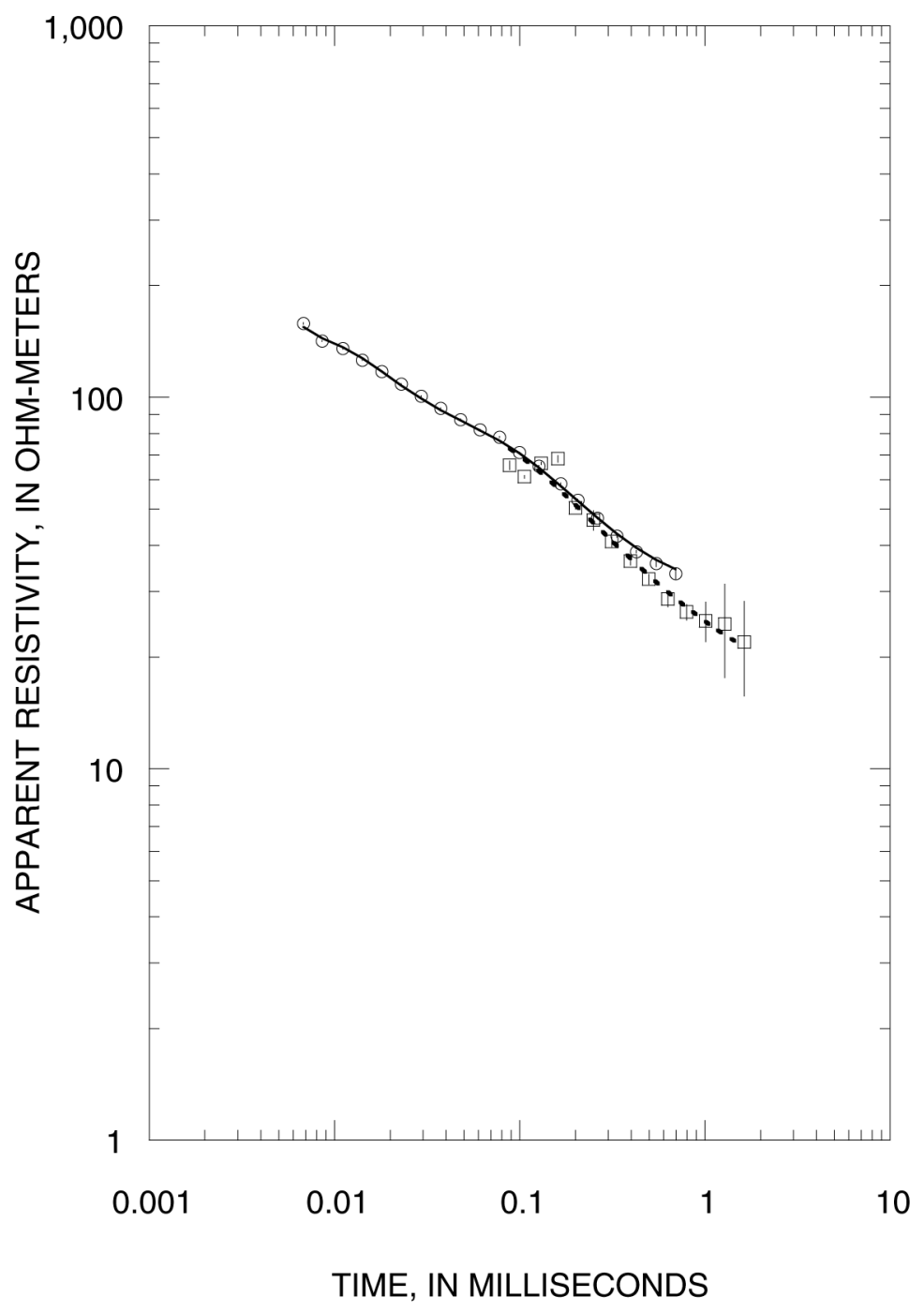

MIA104L4

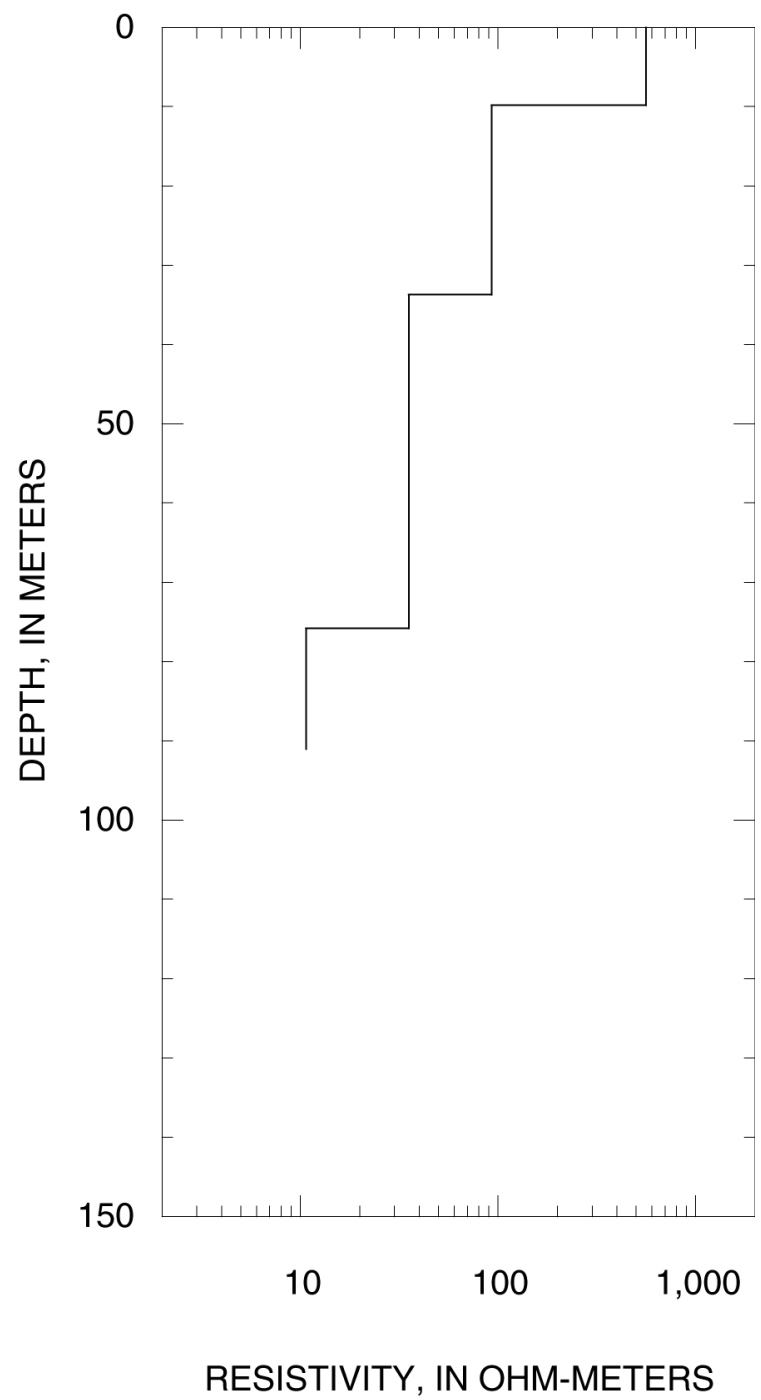

Figure 5-6. Sounding MIA104L4 
MIA105

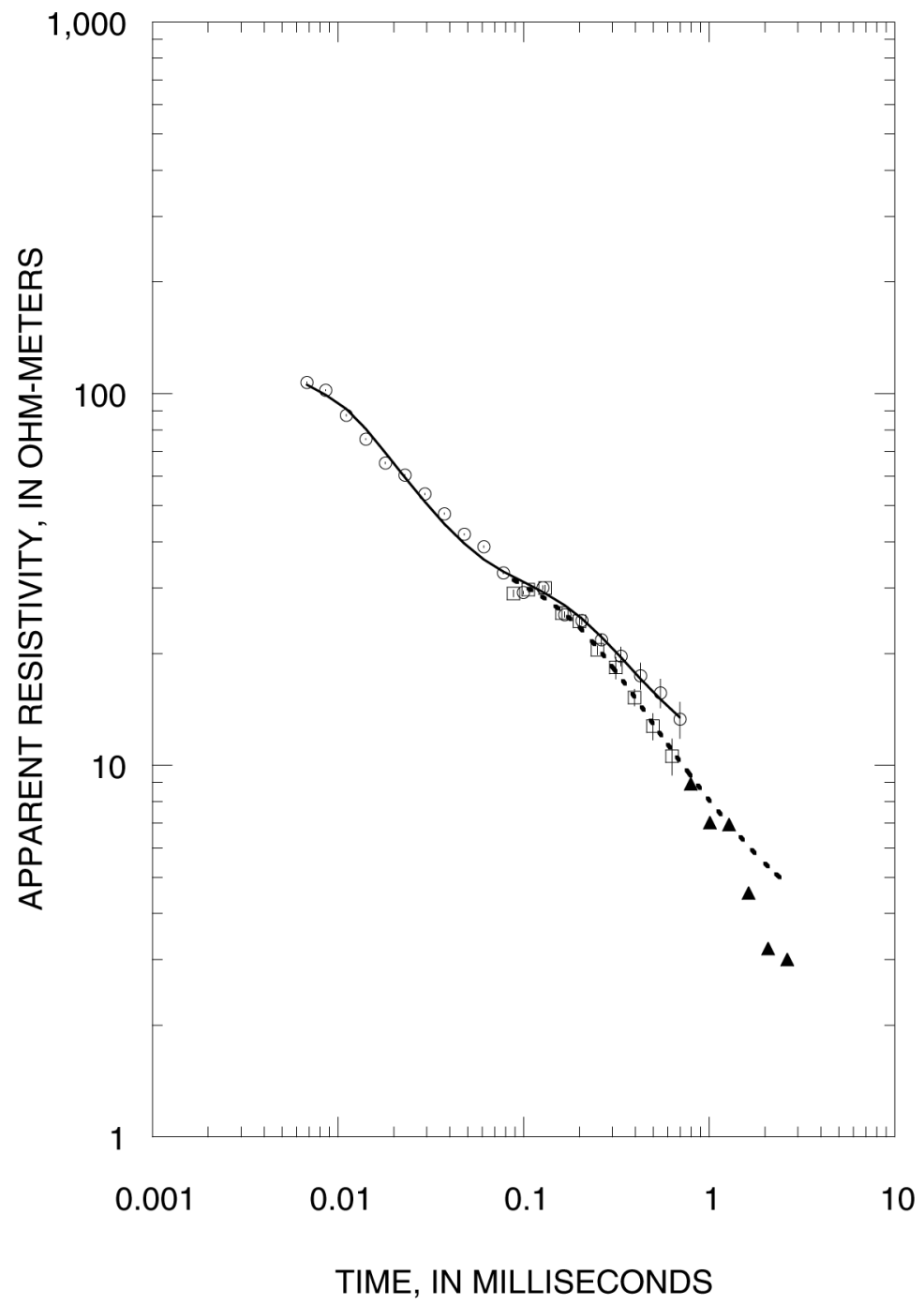

MIA105

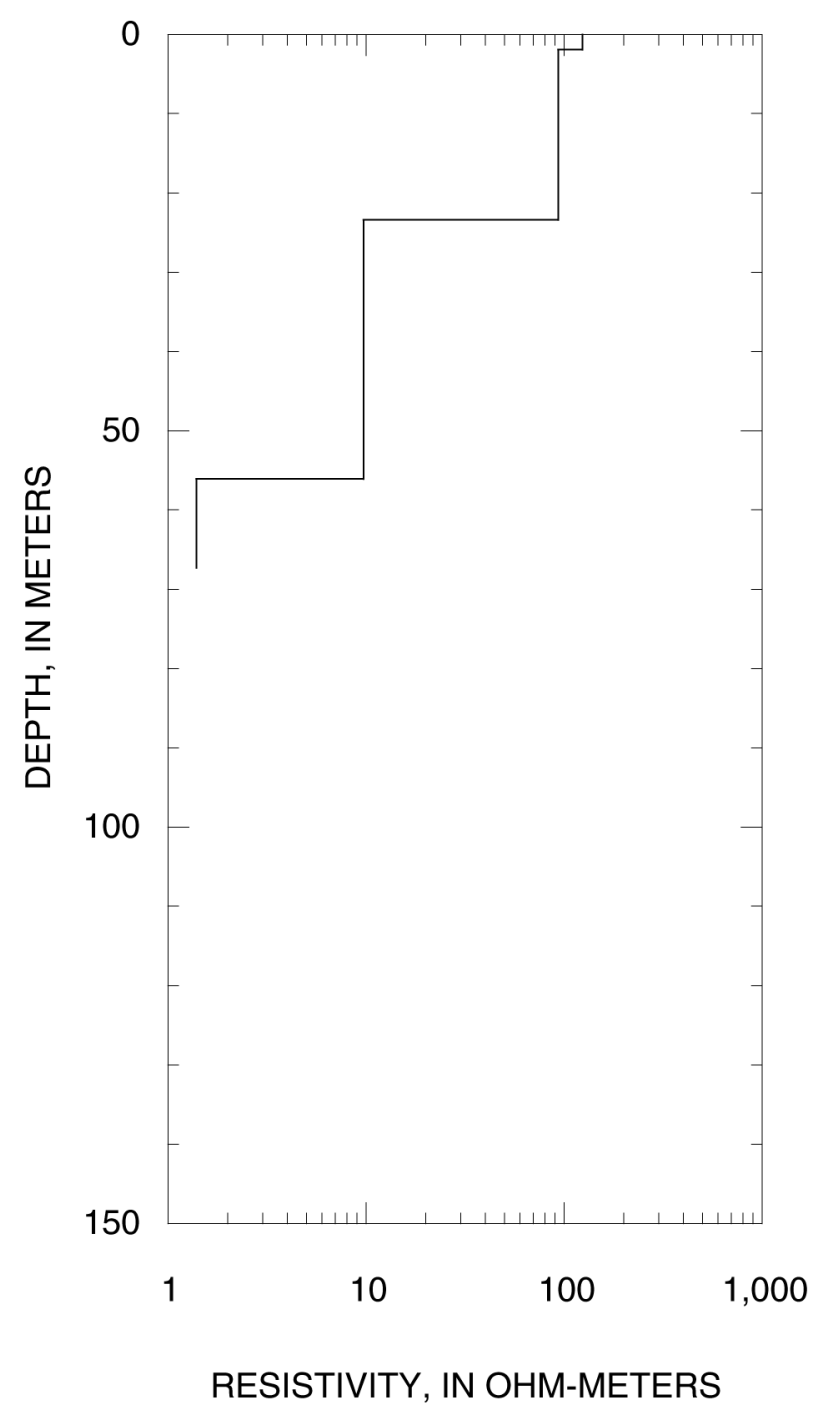

Figure 5-7. Sounding MIA105 
MIA105L5

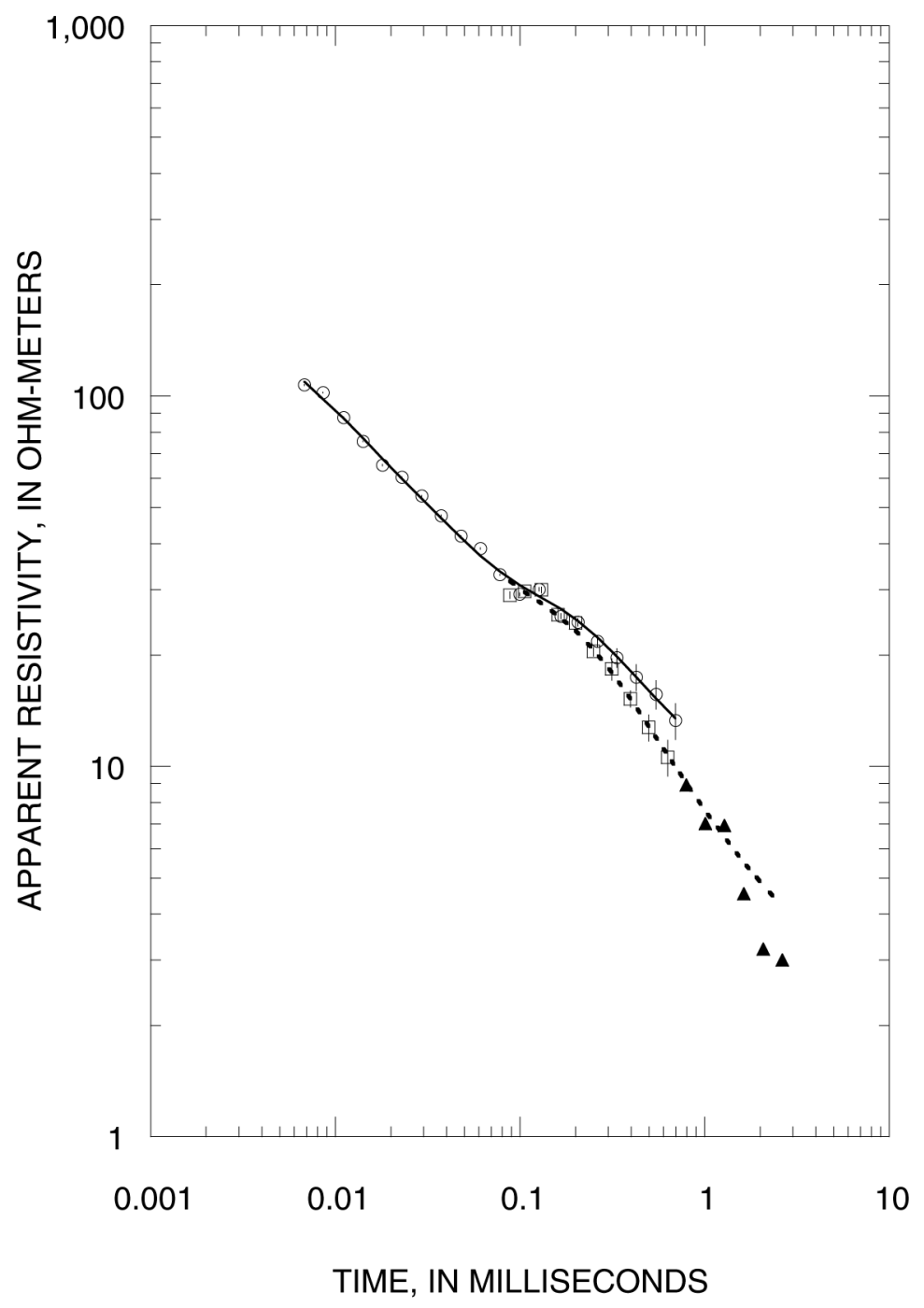

MIA105L5

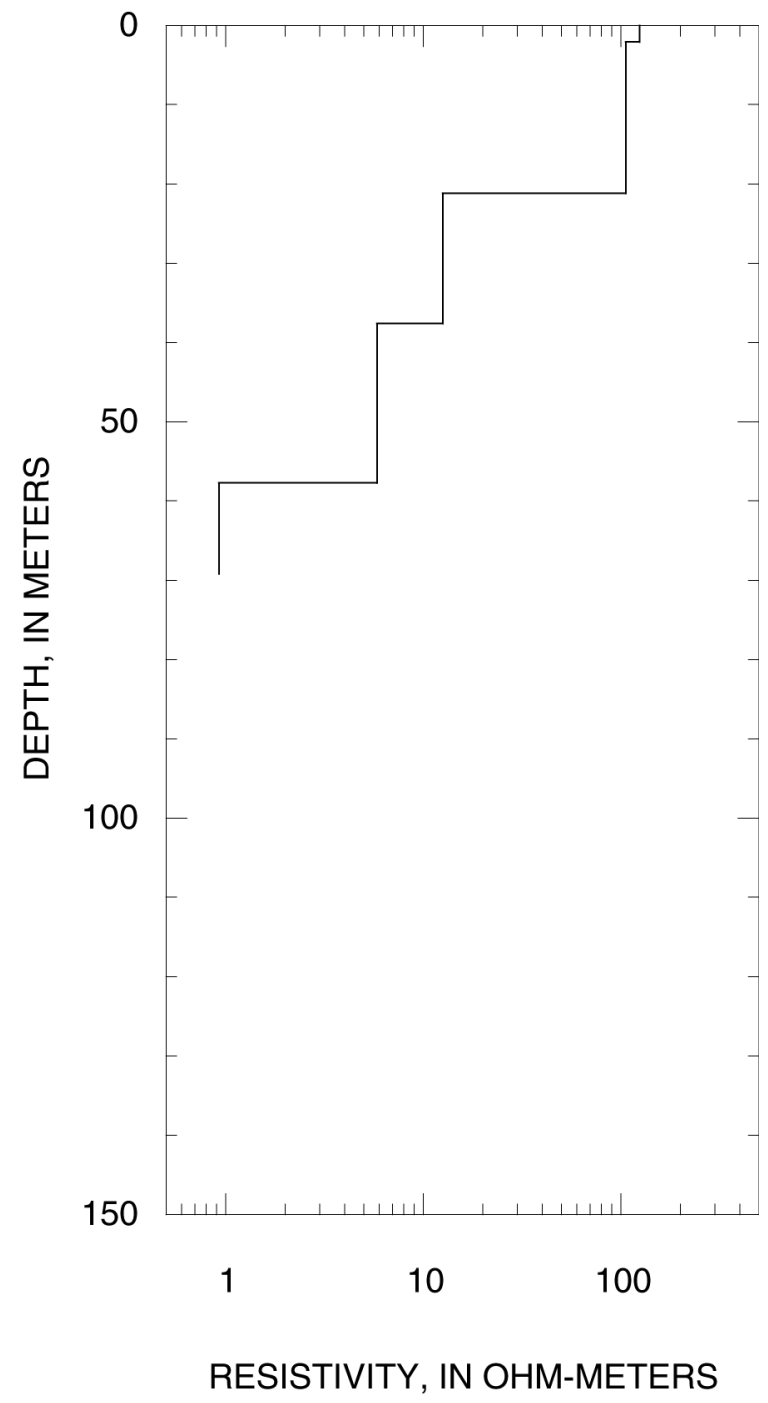

Figure 5-8. Sounding MIA105L5 
MIA106

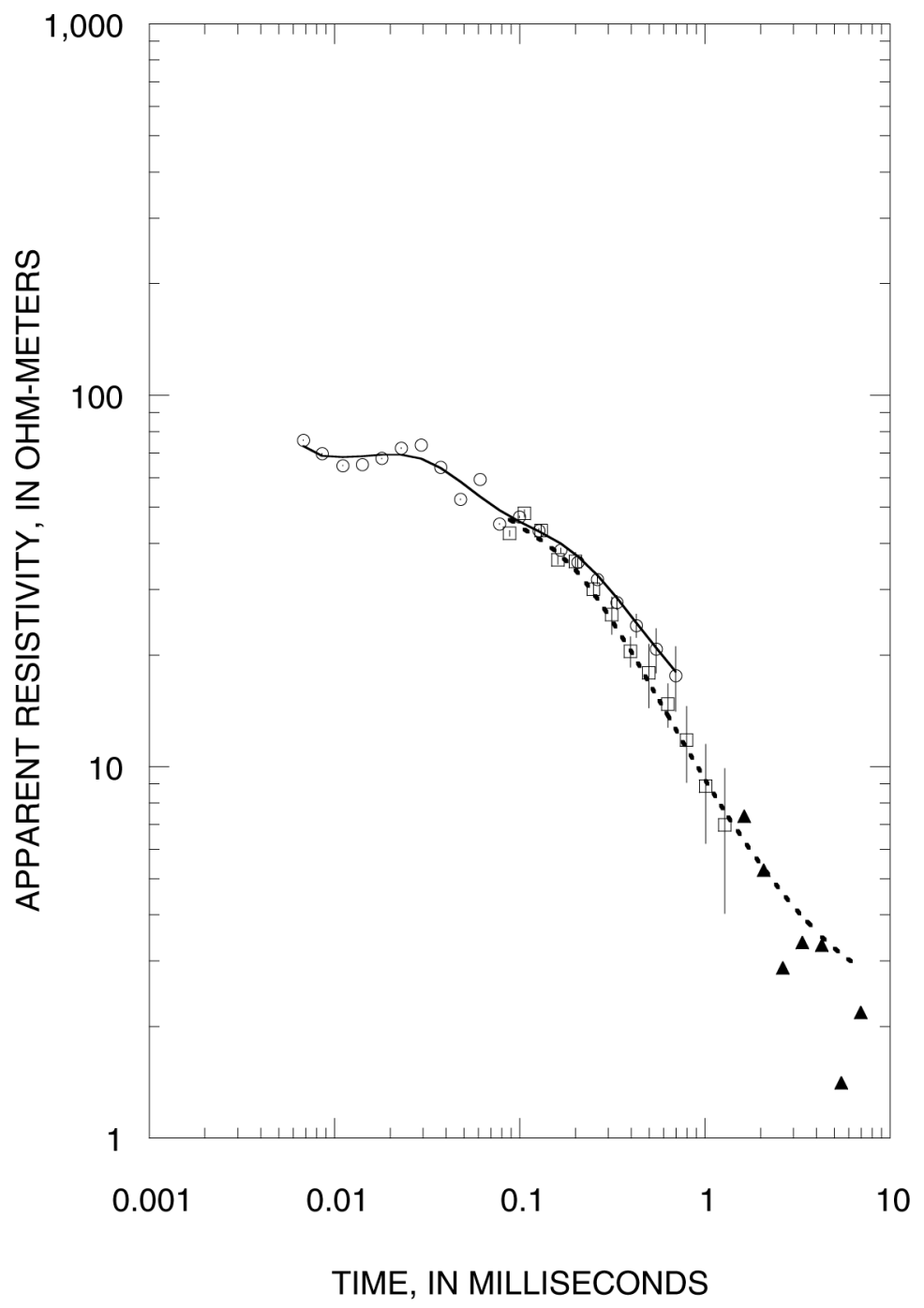

MIA106

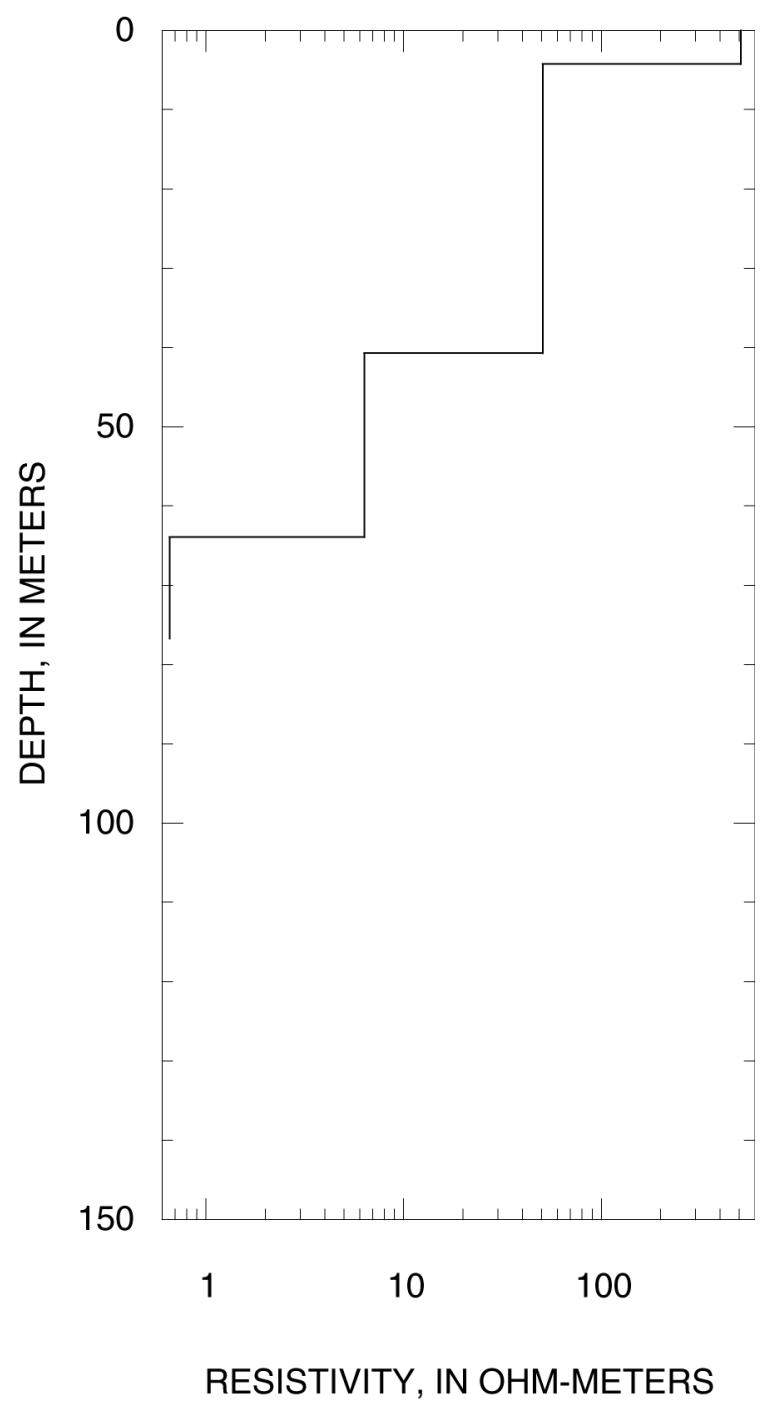

Figure 5-9. Sounding MIA106 
MIA107

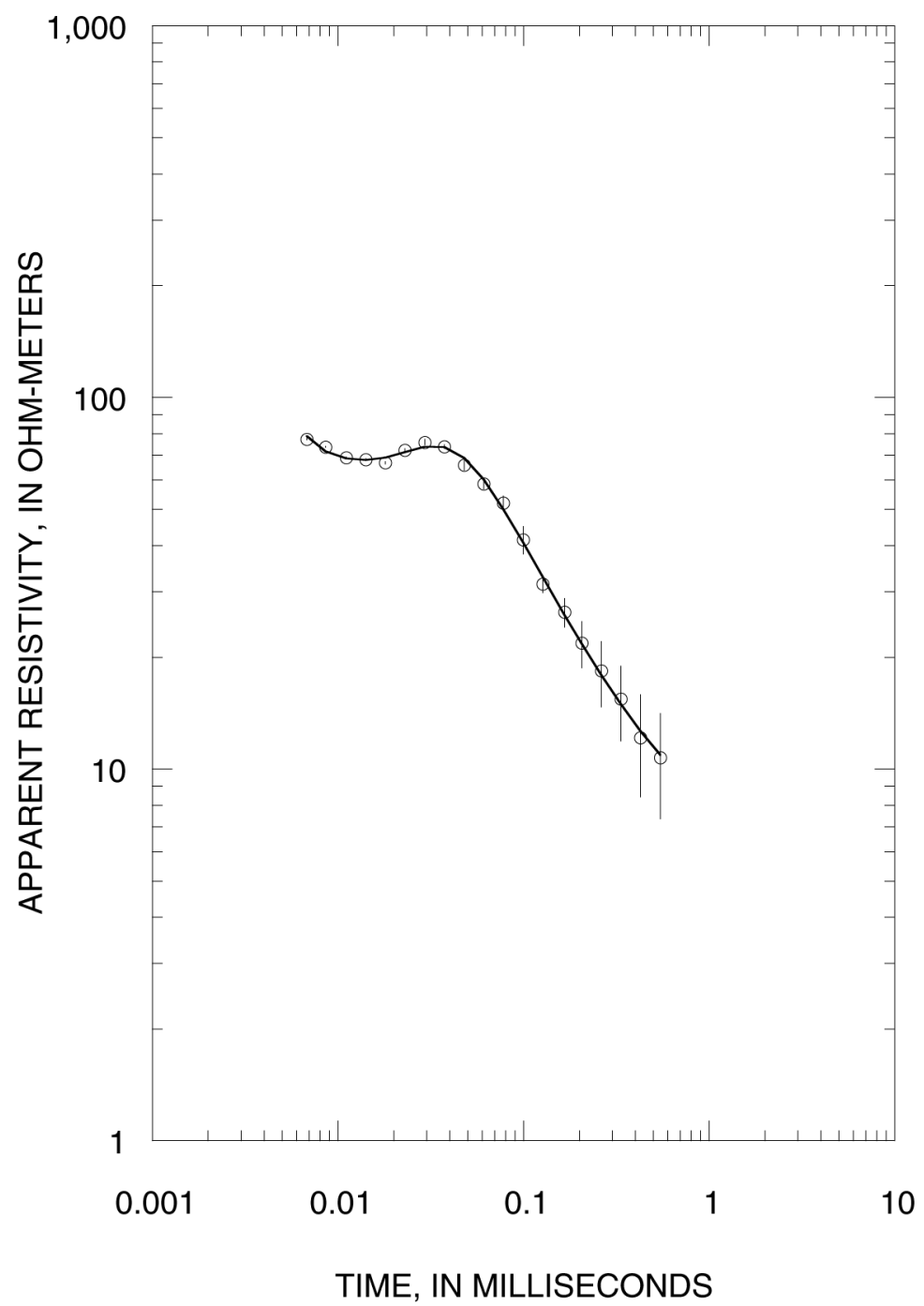

MIA107

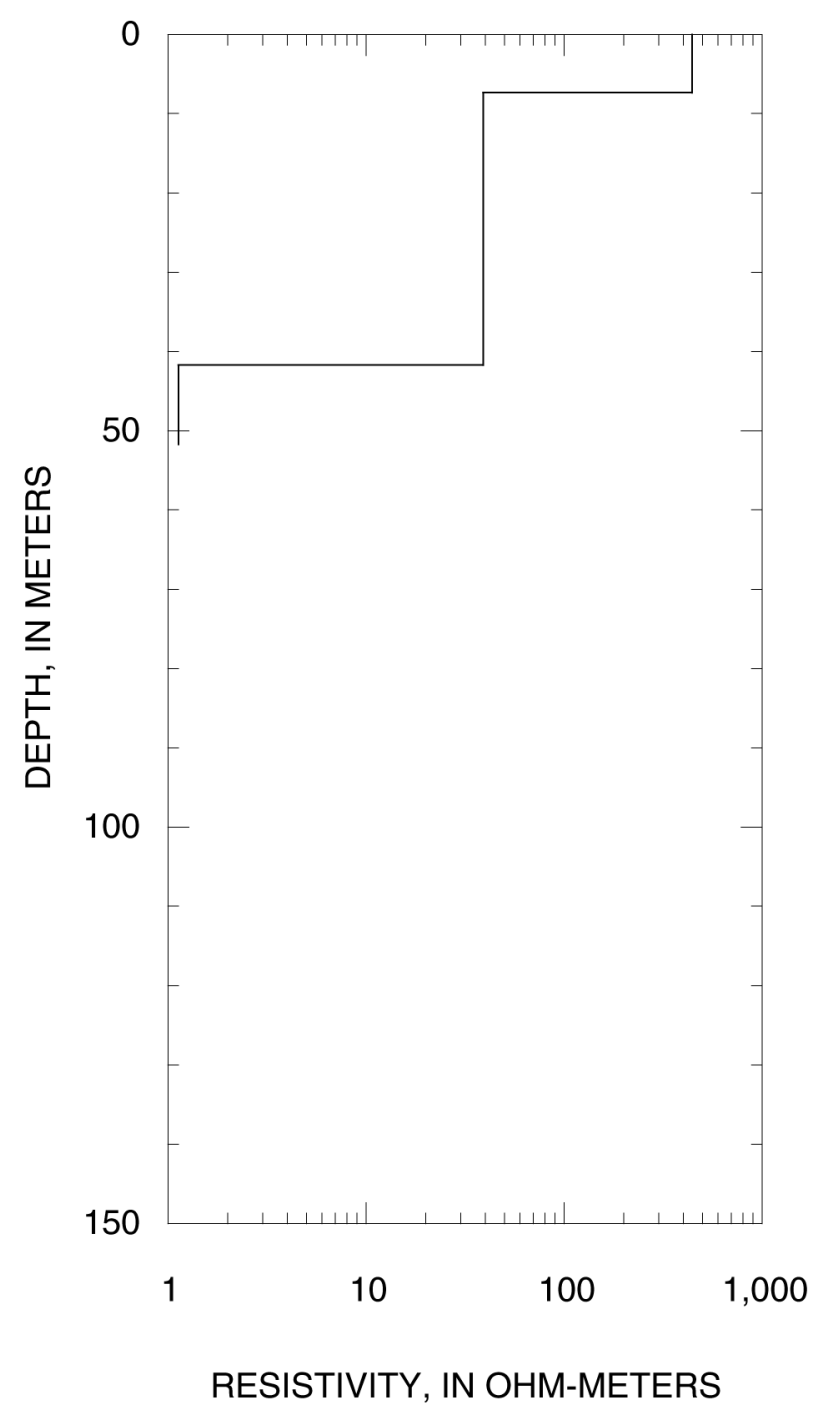

Figure 5-10. Sounding MIA107 
MIA108

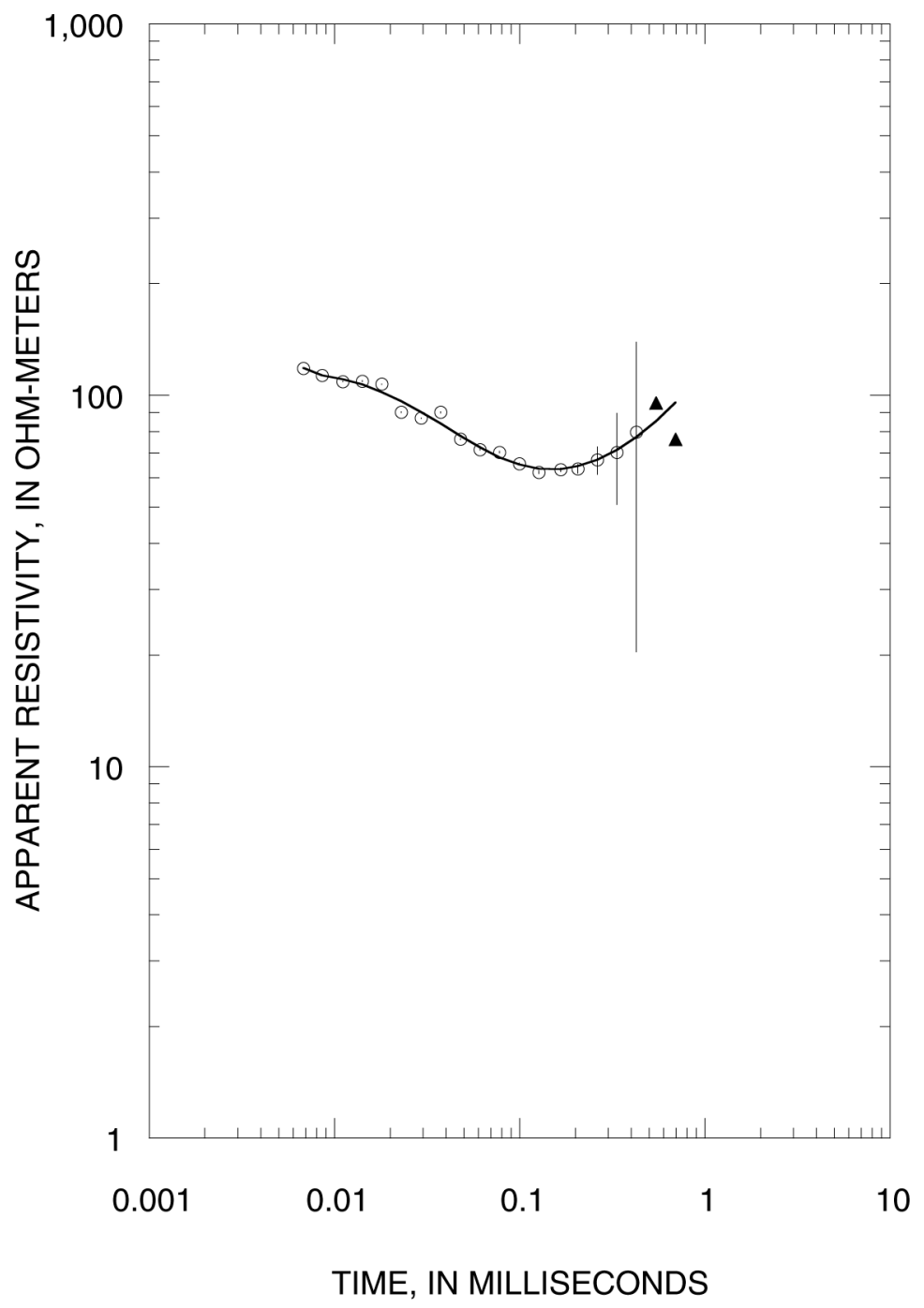

MIA108

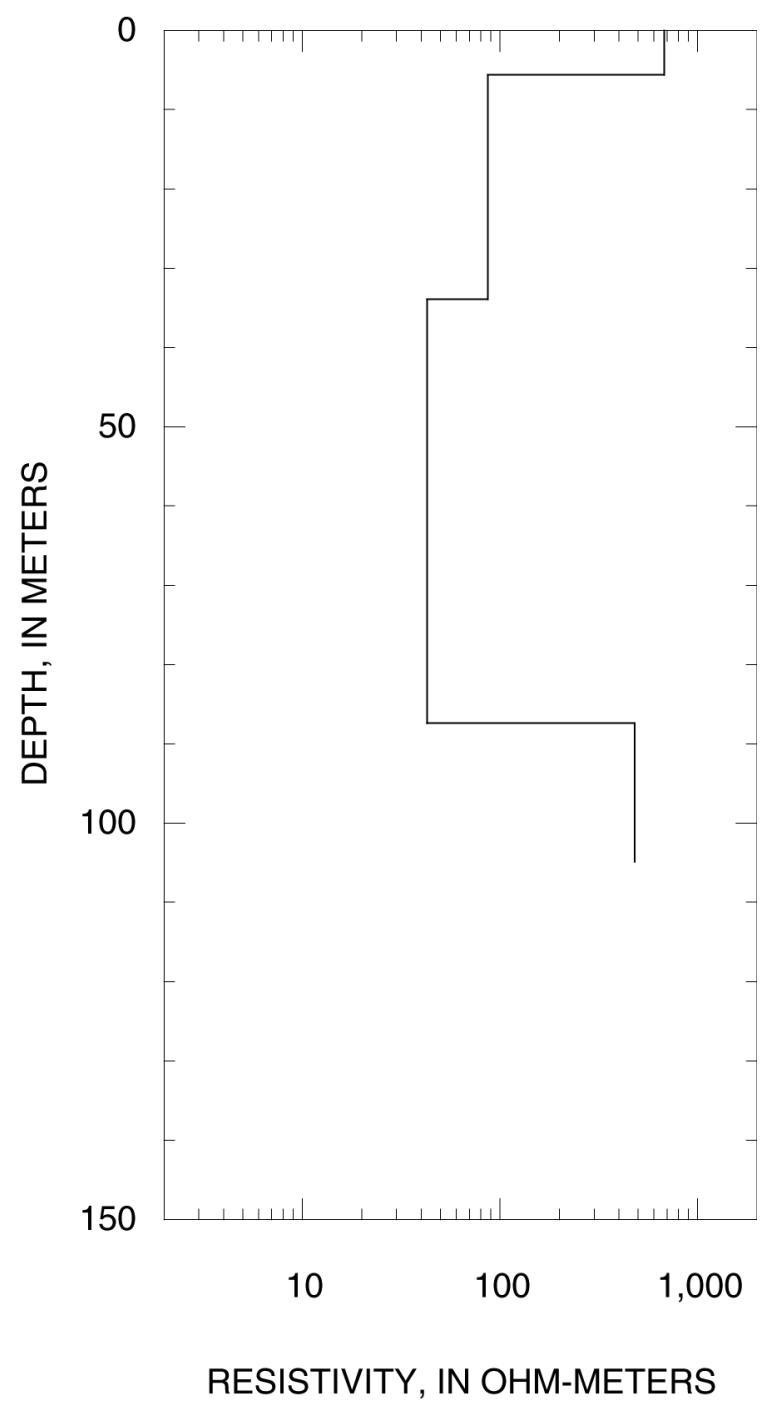

Figure 5-11. Sounding MIA108 
MIA108L3

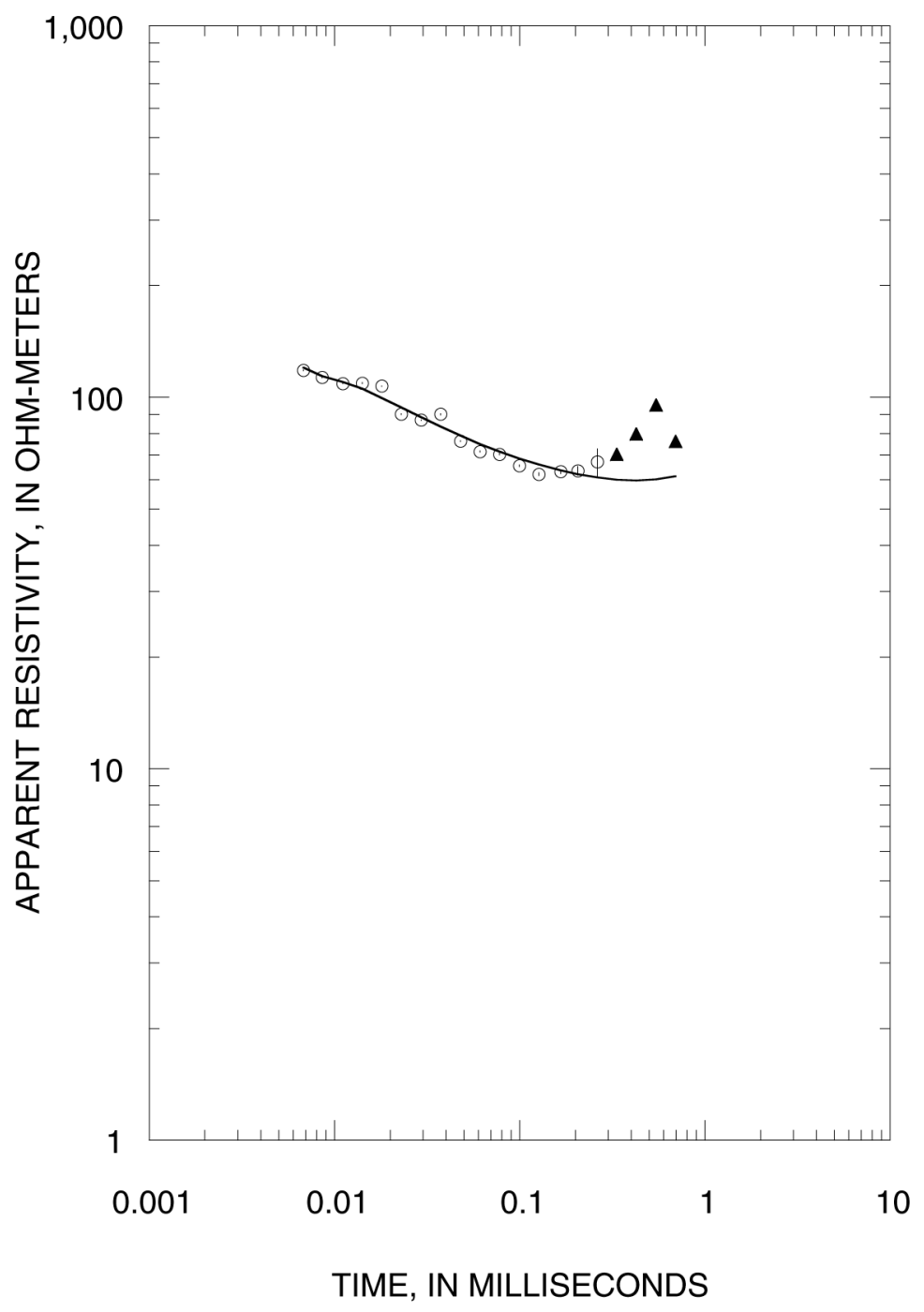

MIA108L3

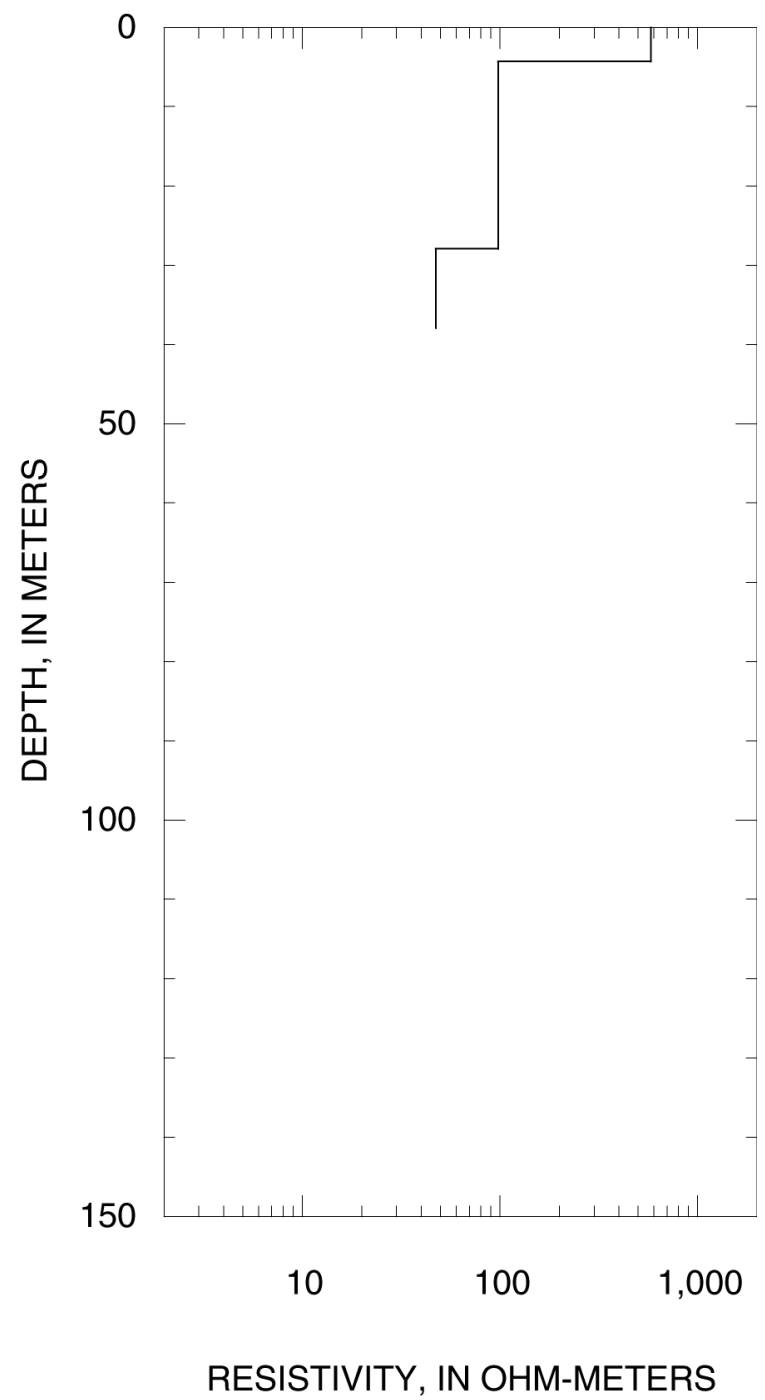

Figure 5-12. Sounding MIA108L3 
MIA109

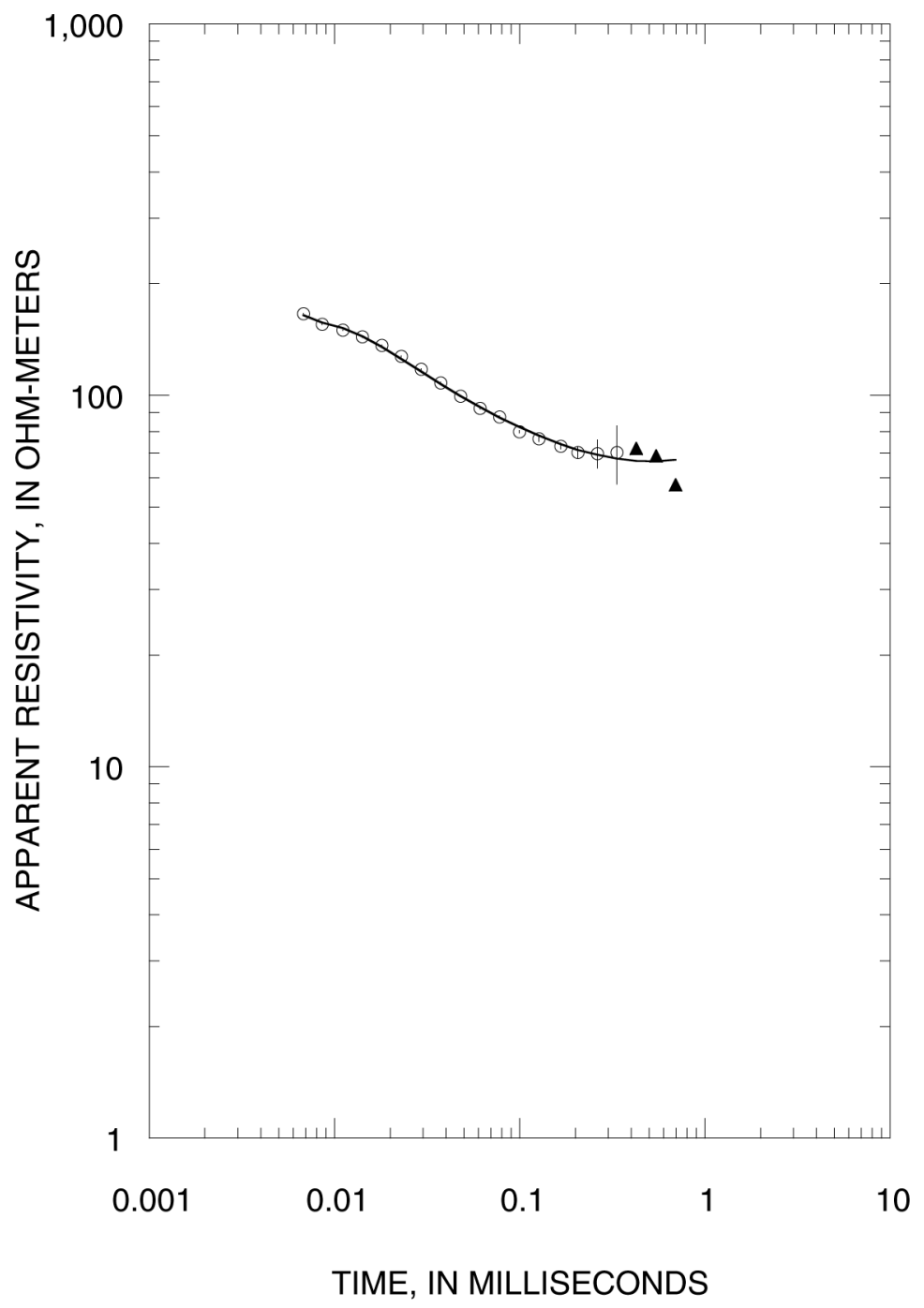

MIA109

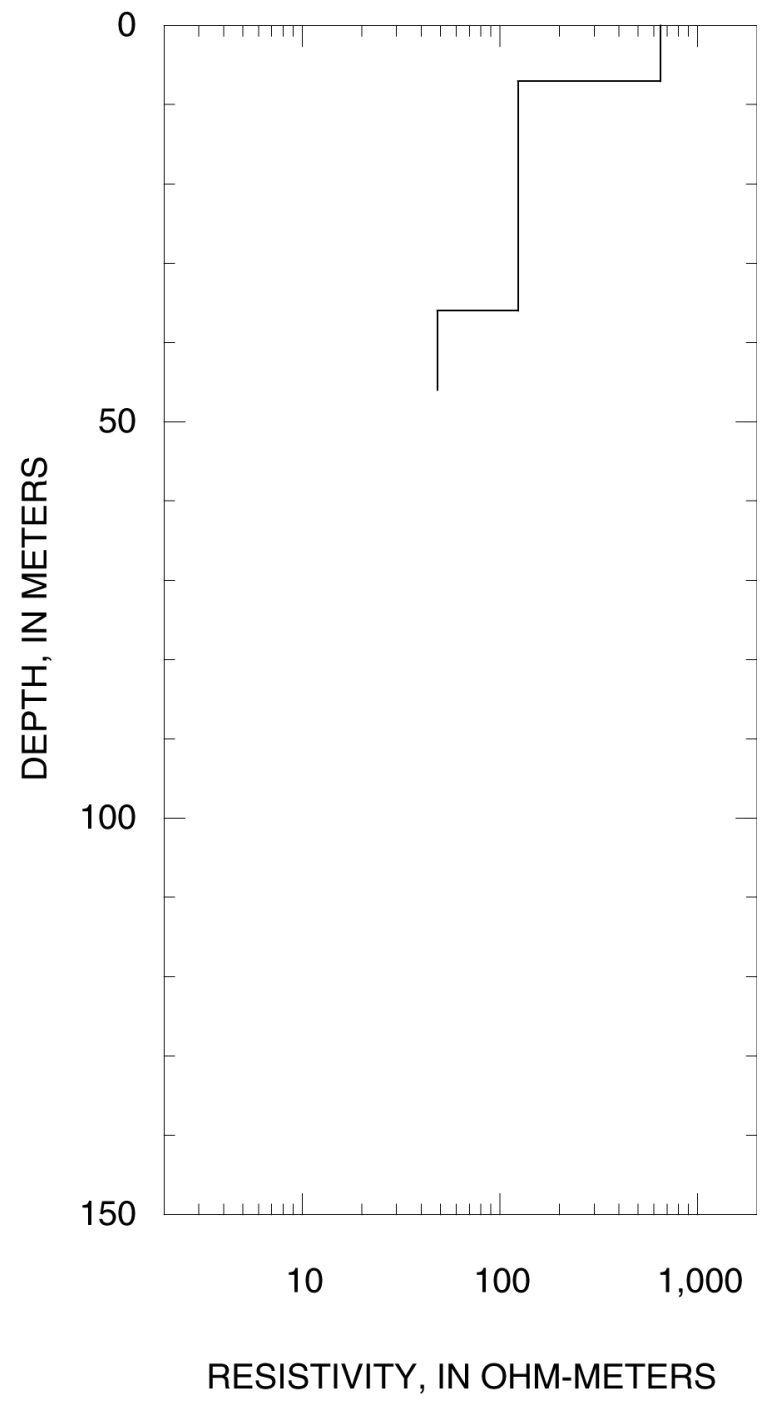

Figure 5-13. Sounding MIA109 
MIA110

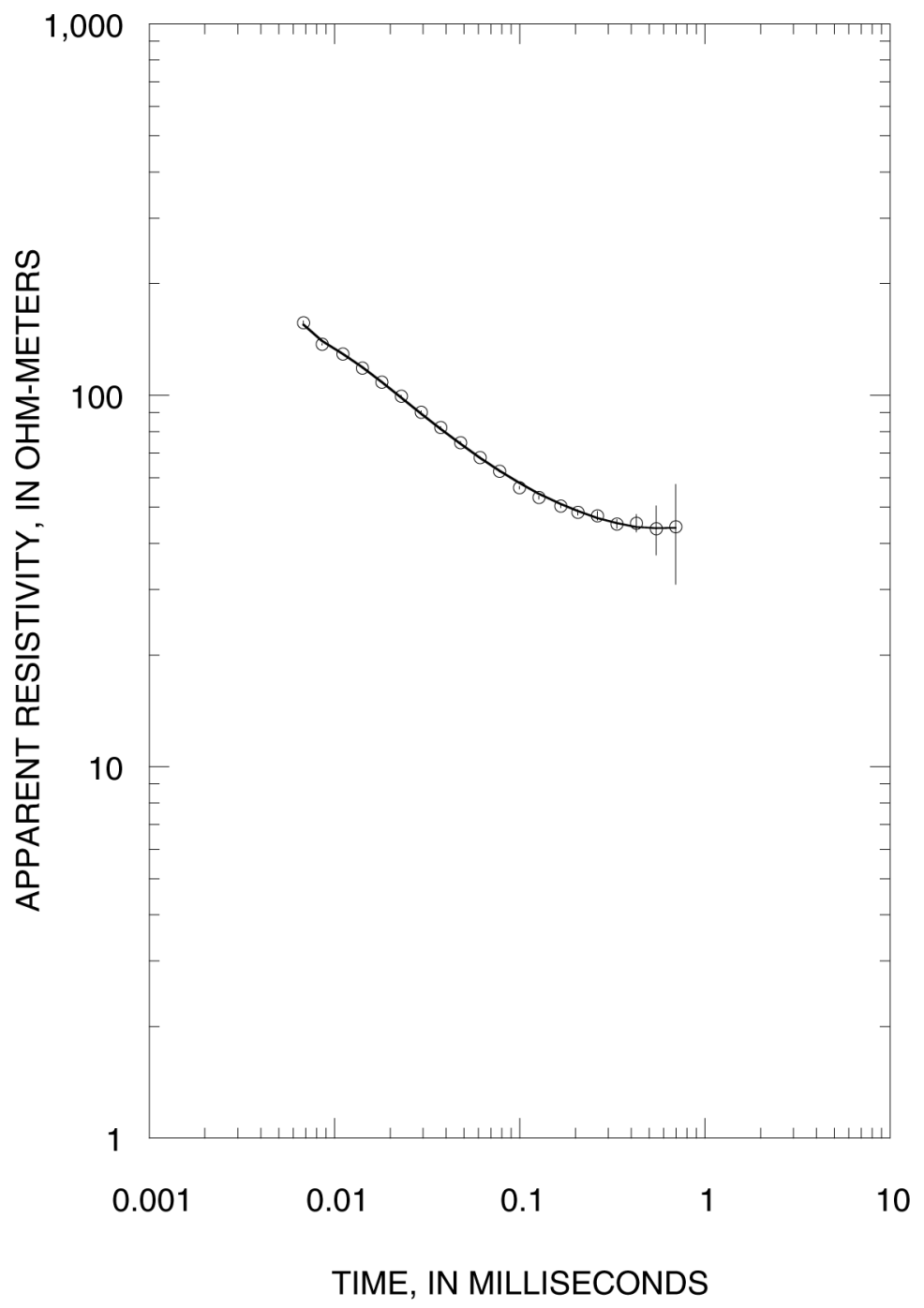

MIA110

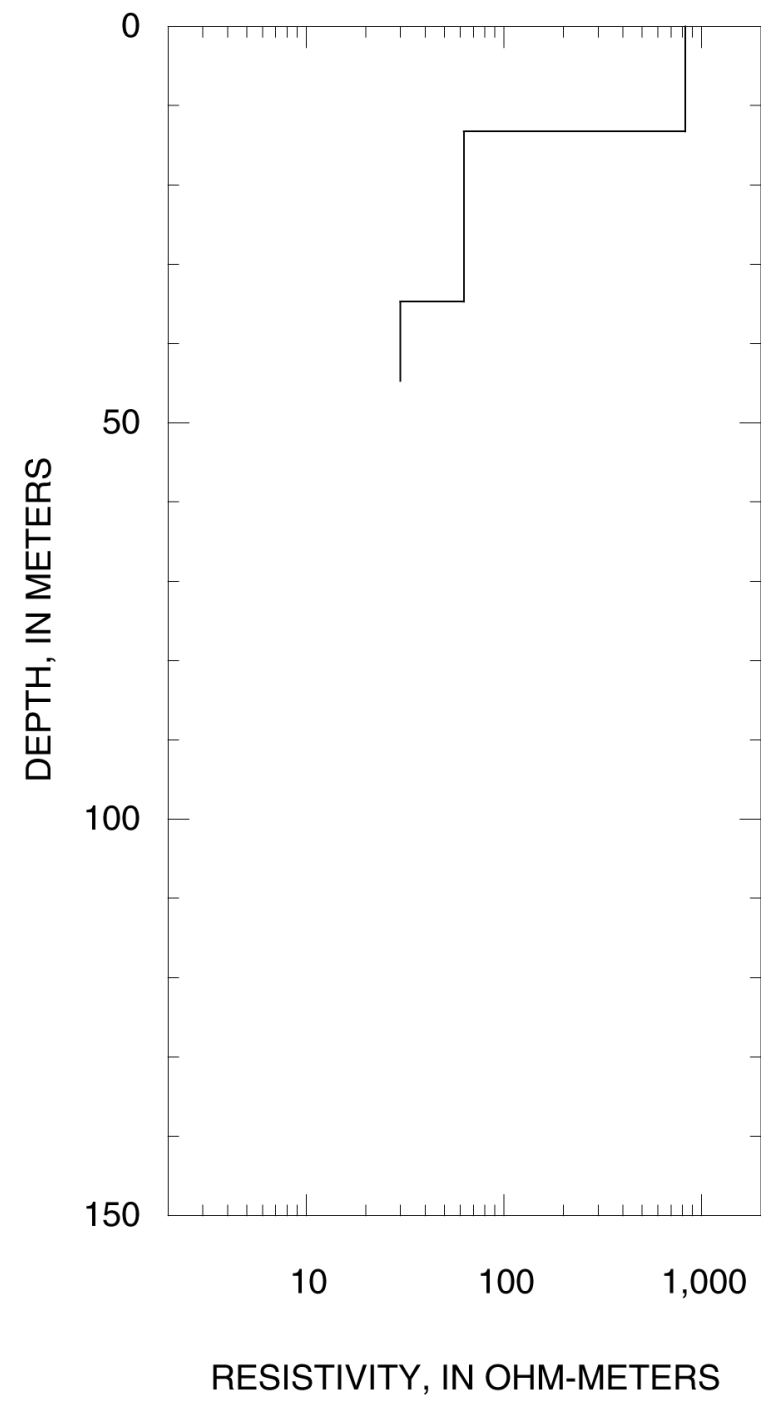

Figure 5-14. Sounding MIA110 
MIA111

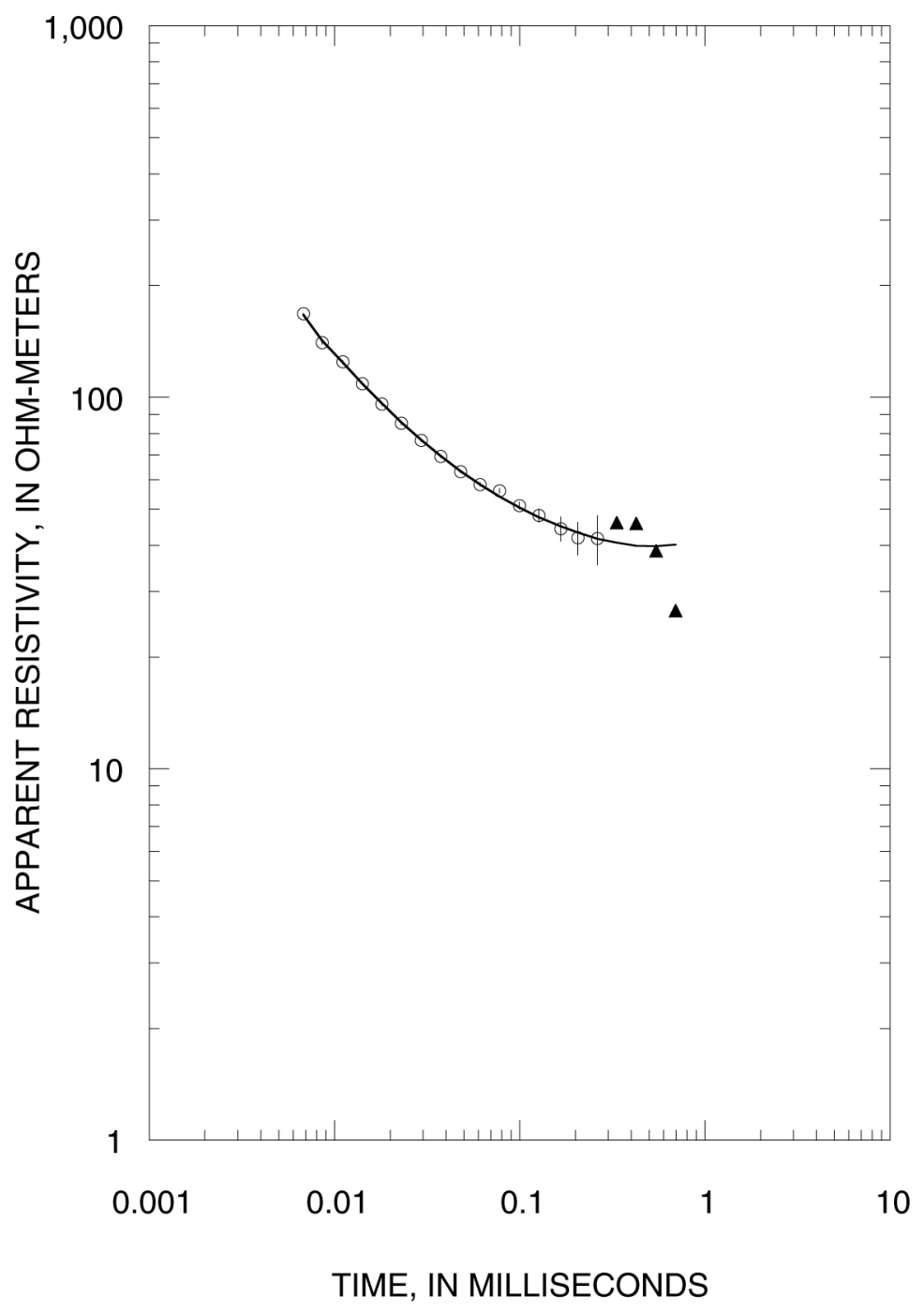

MIA111

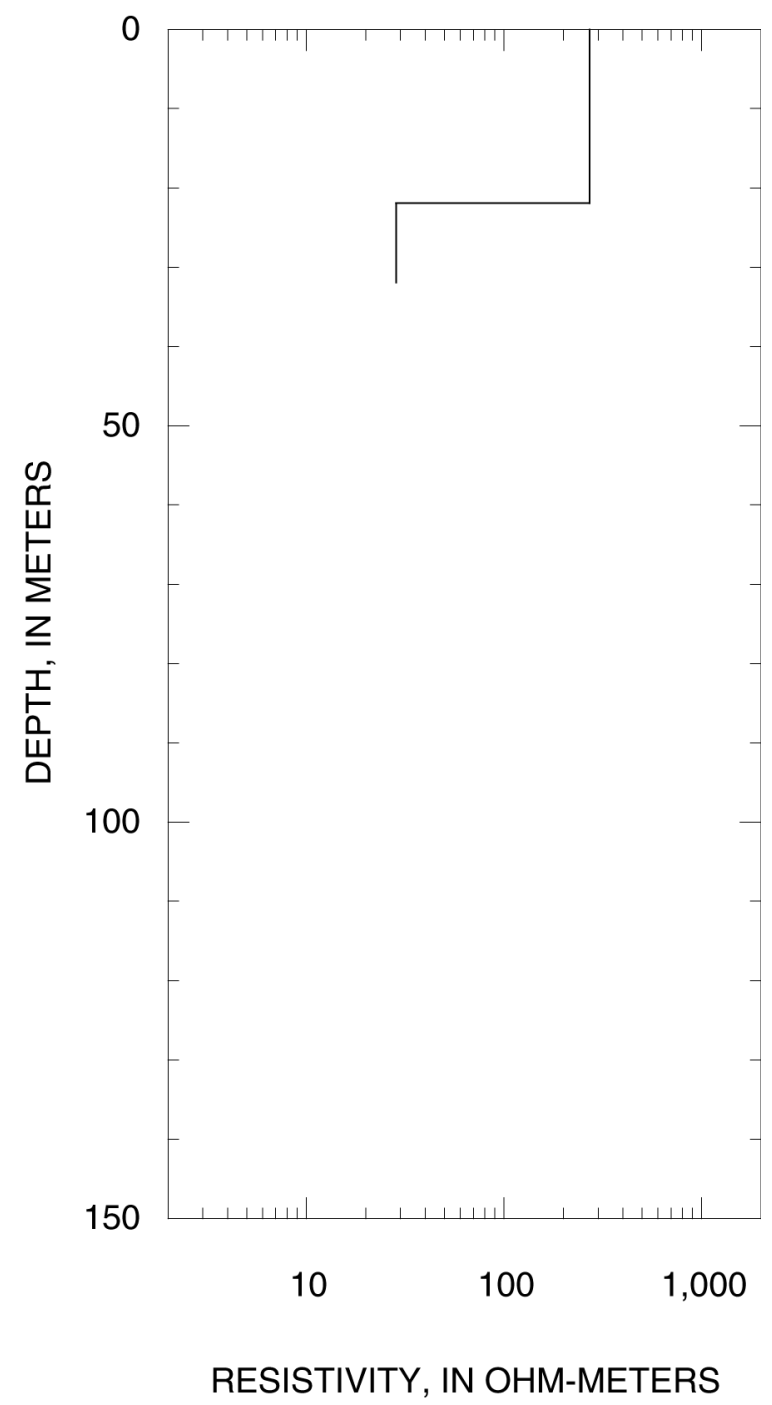

Figure 5-15. Sounding MIA111 
MIA112

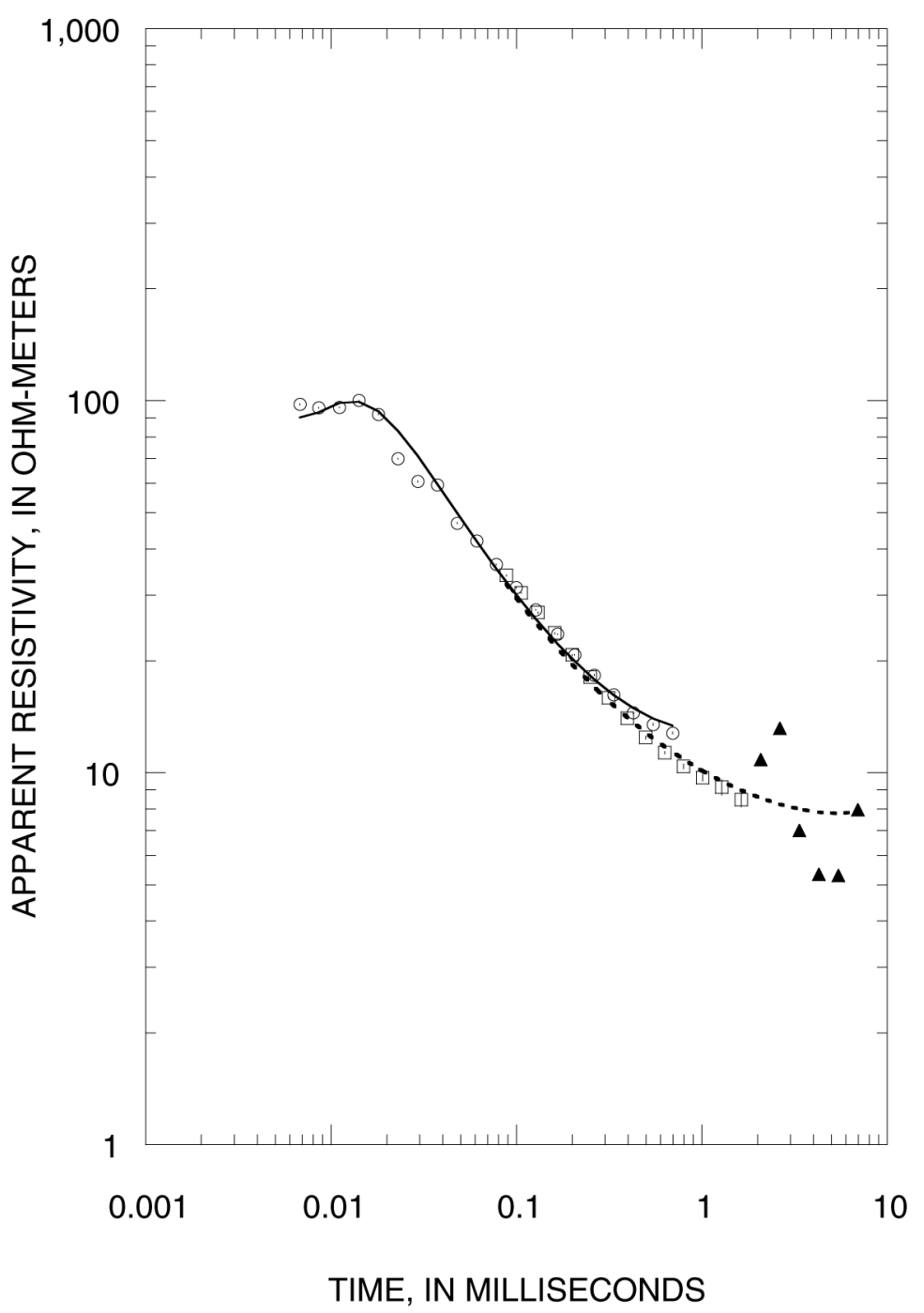

MIA112

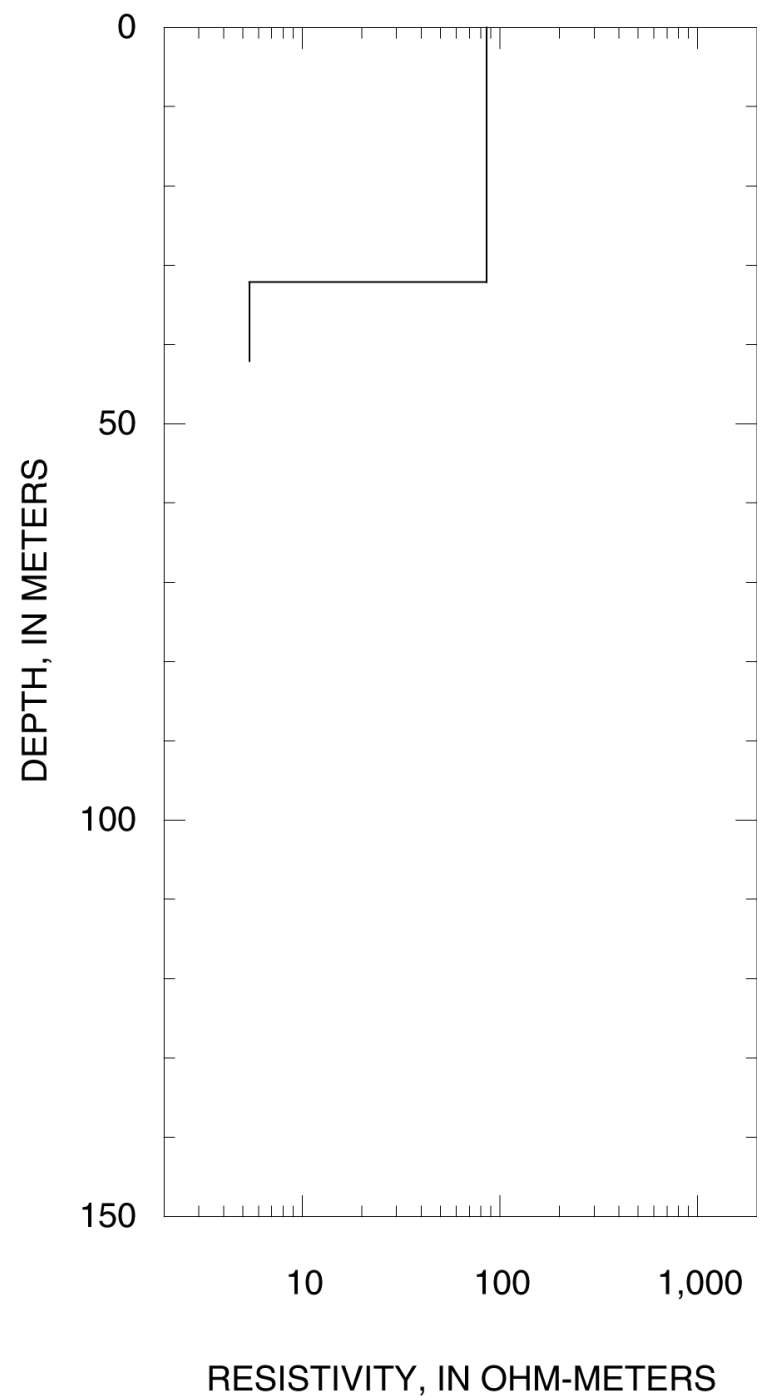

Figure 5-16. Sounding MIA112 
MIA112L3

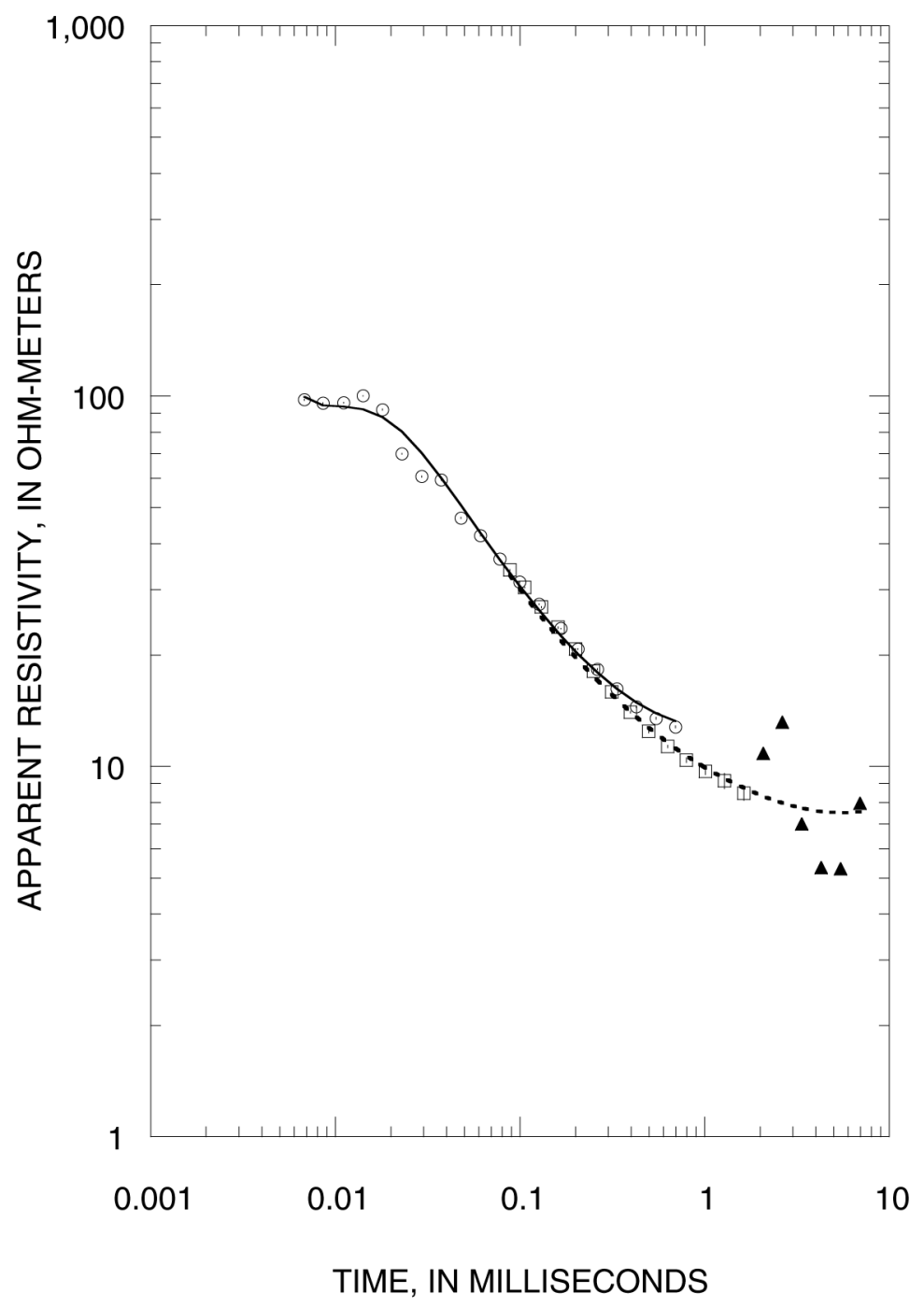

MIA112L3

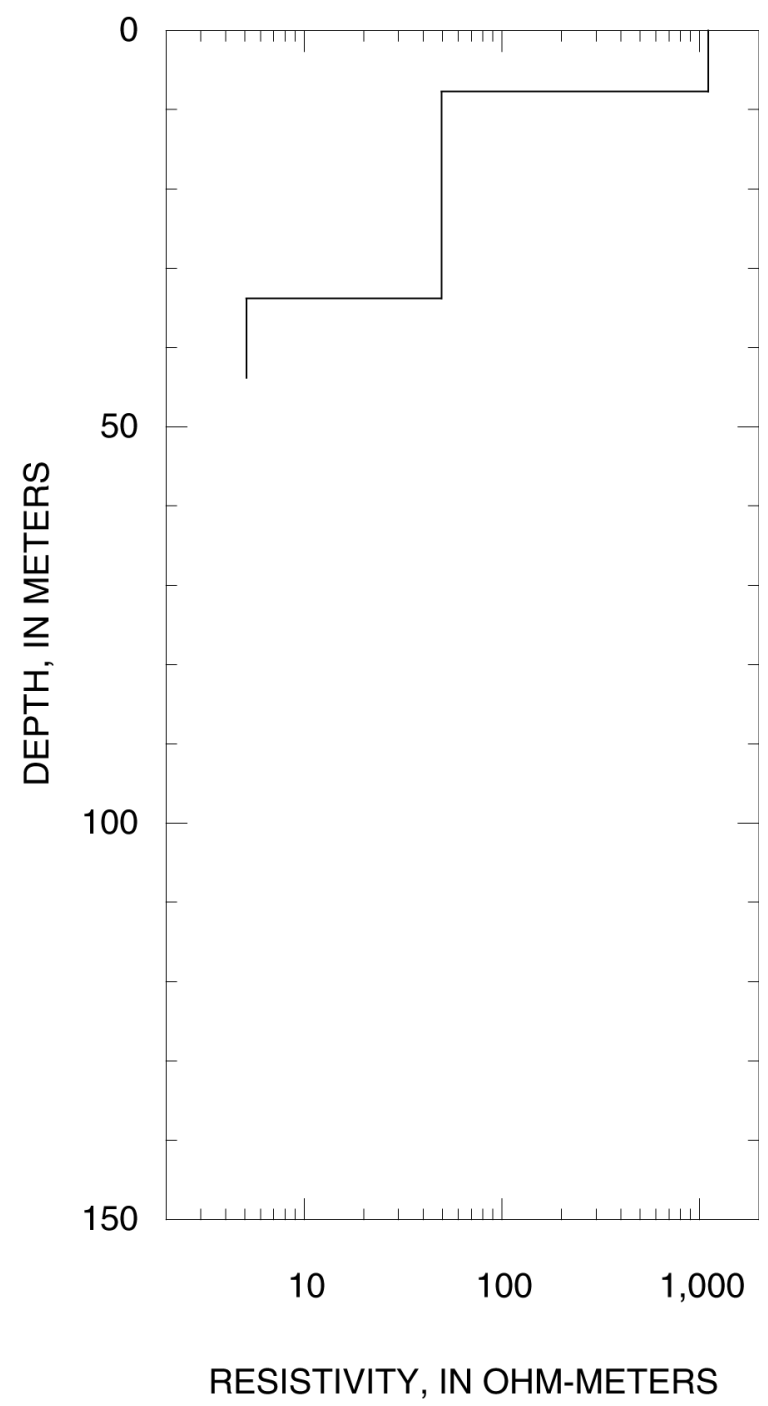

Figure 5-17. Sounding MIA112L3 
MIA113

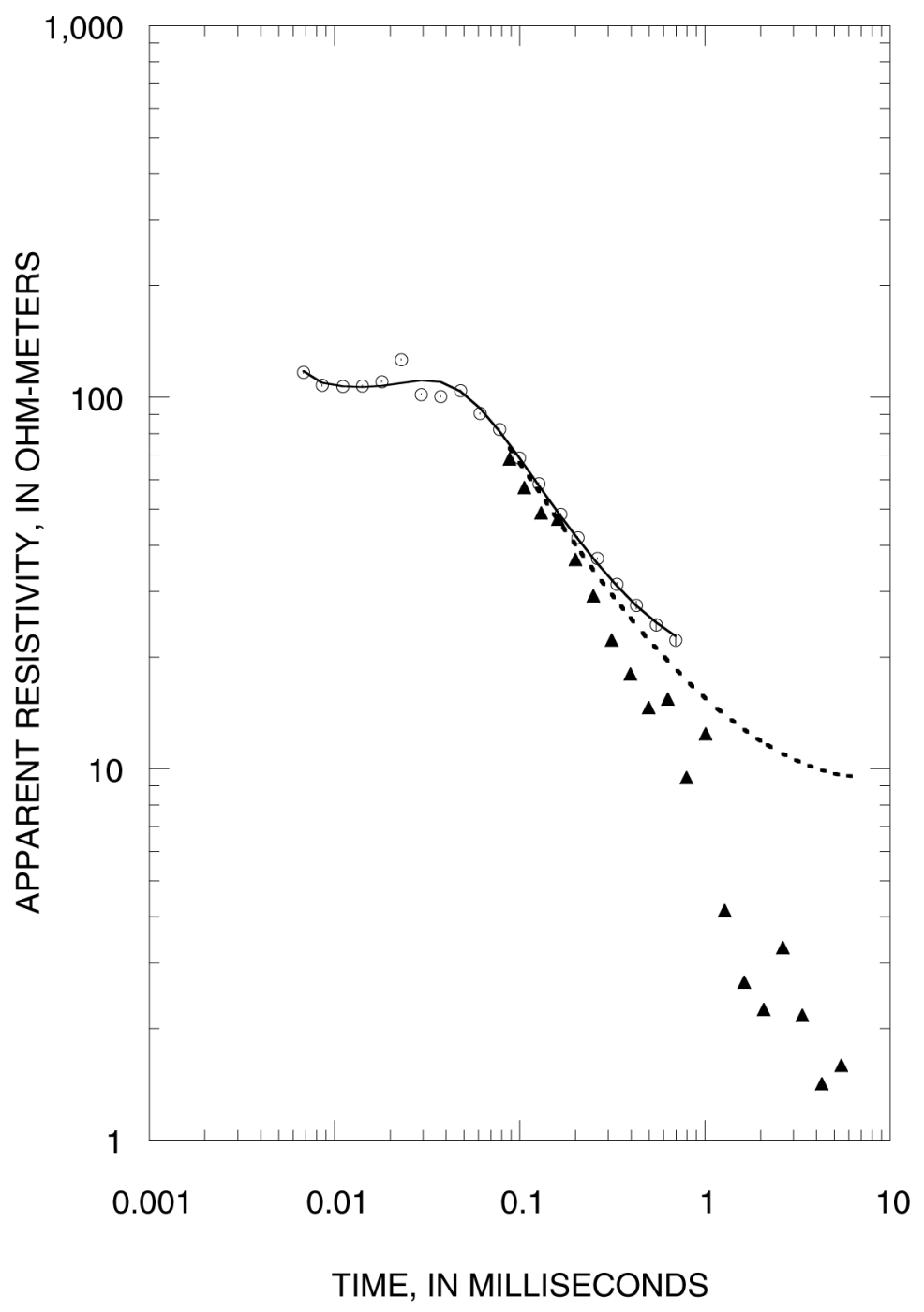

MIA113

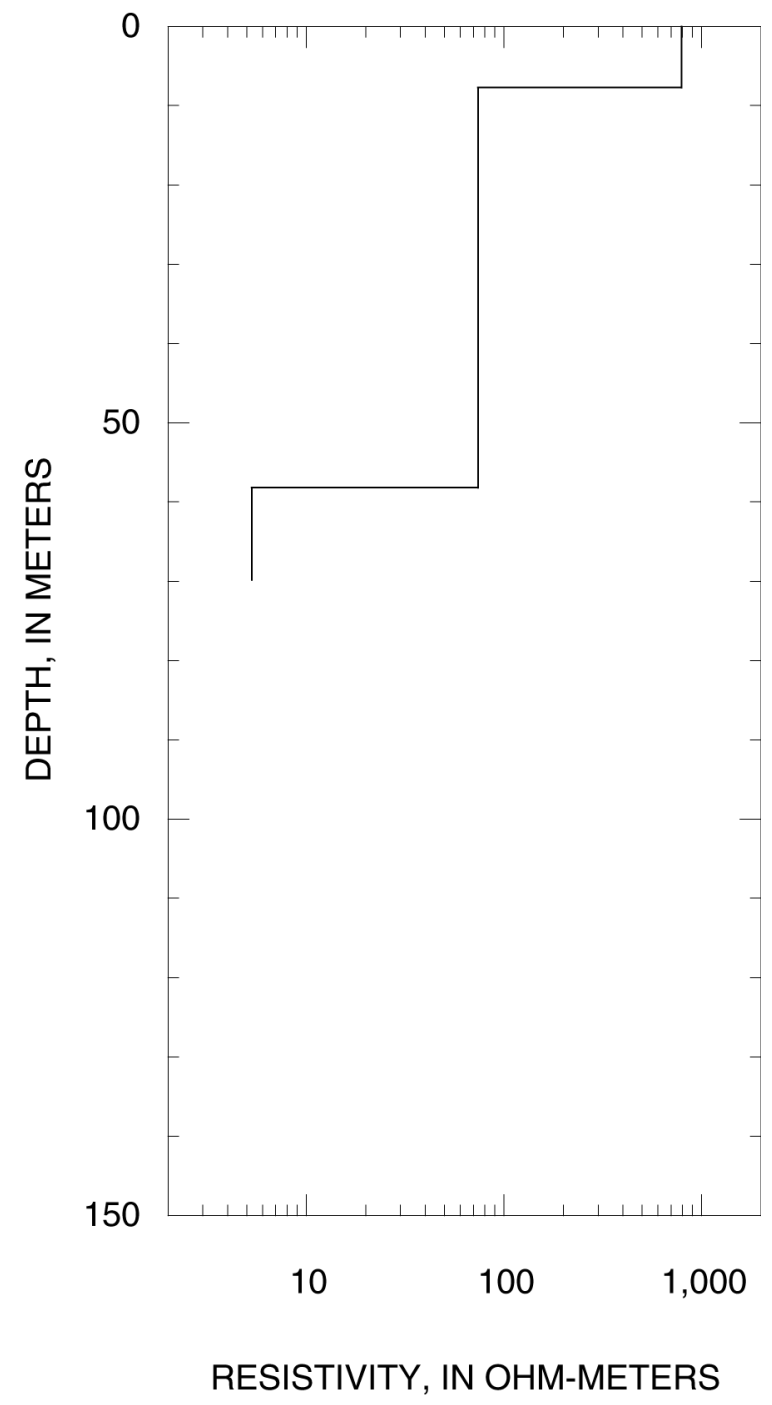

Figure 5-18. Sounding MIA113 
MIA114

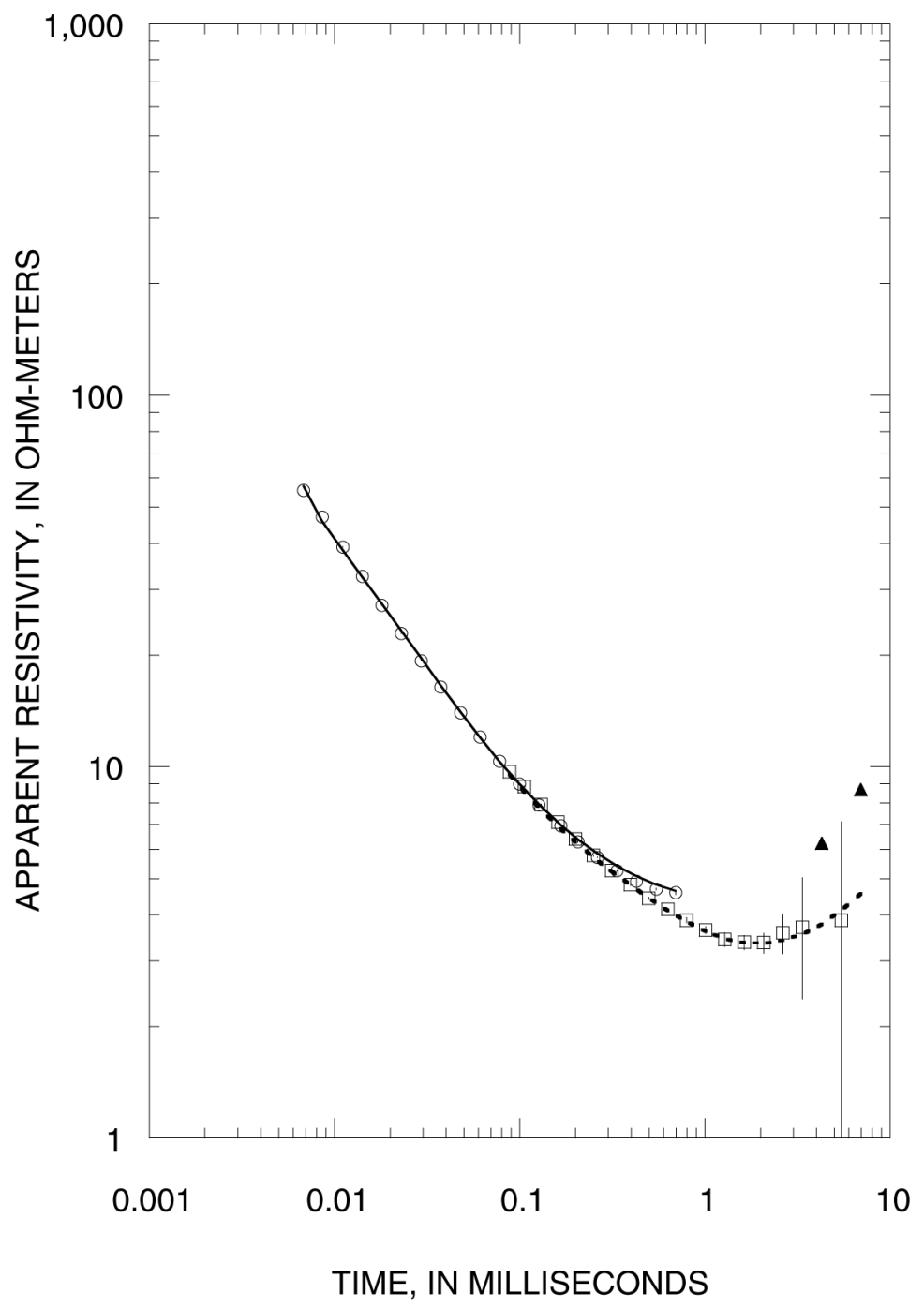

MIA114

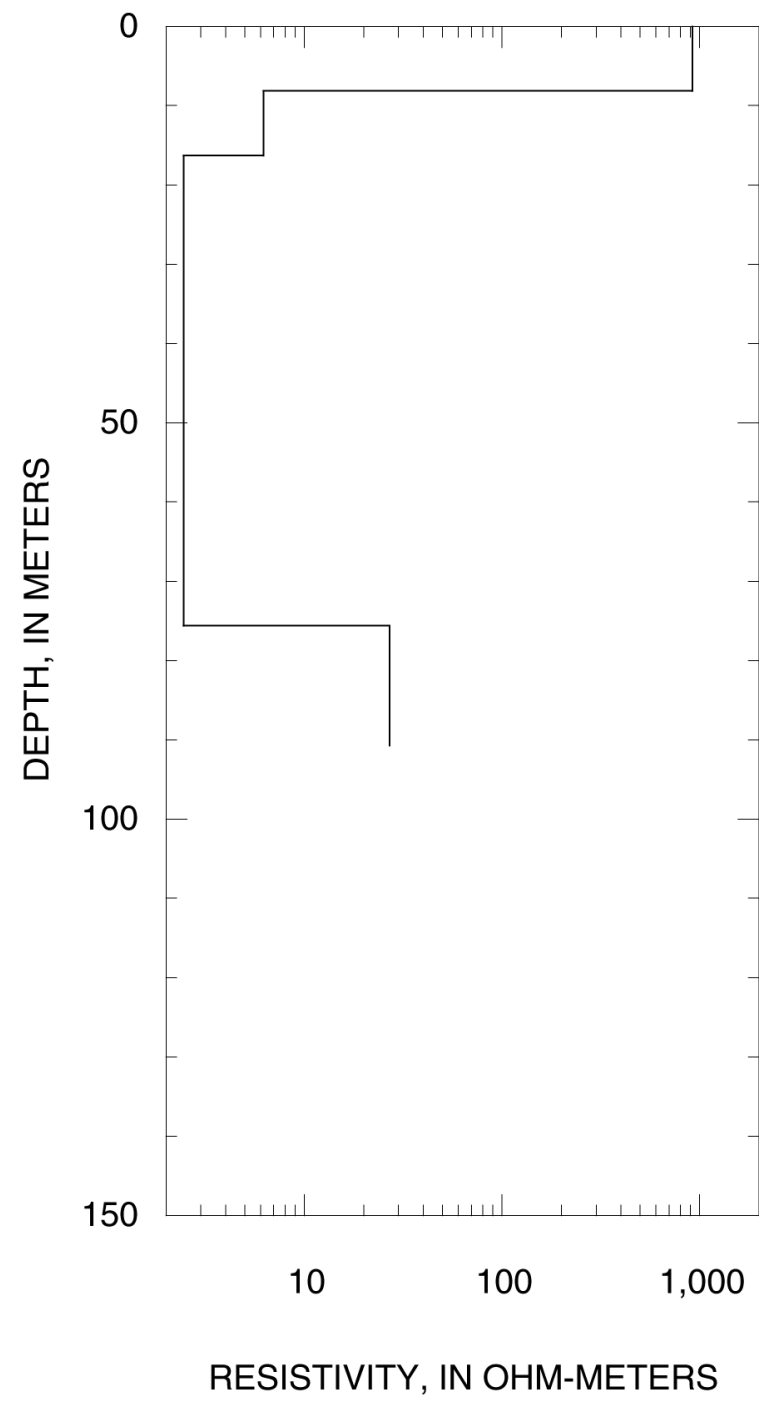

Figure 5-19. Sounding MIA114 
MIA114L3

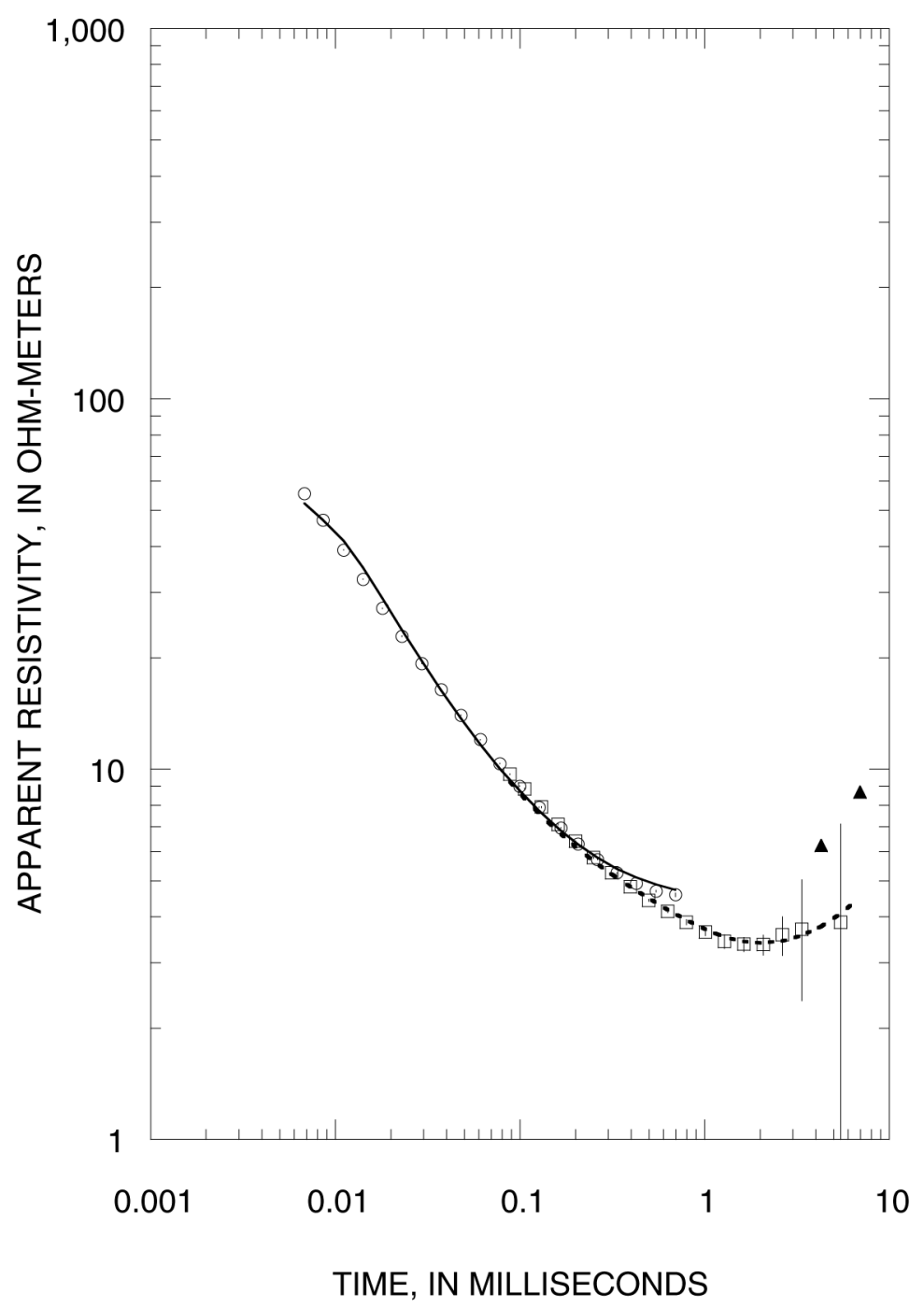

MIA114L3

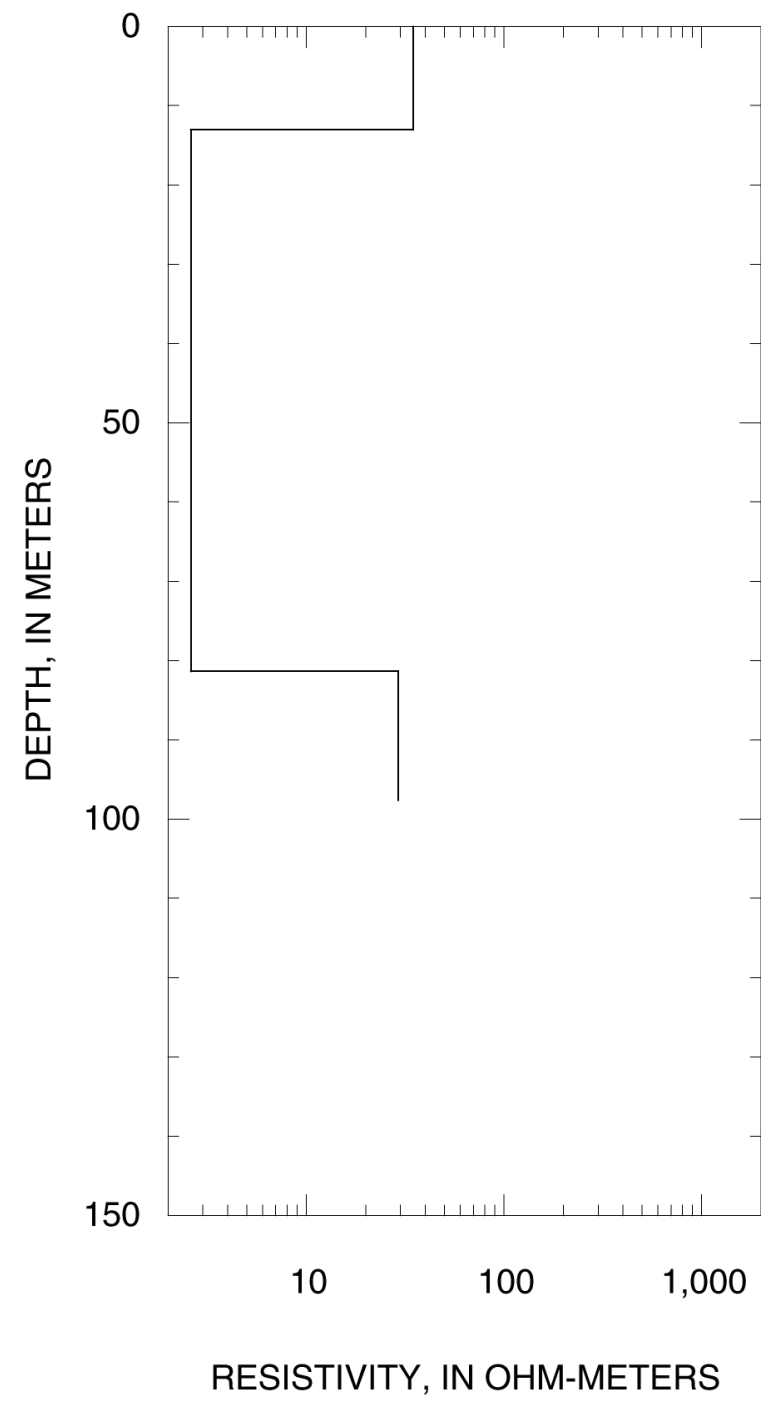

Figure 5-20. Sounding MIA114L3 
MIA115

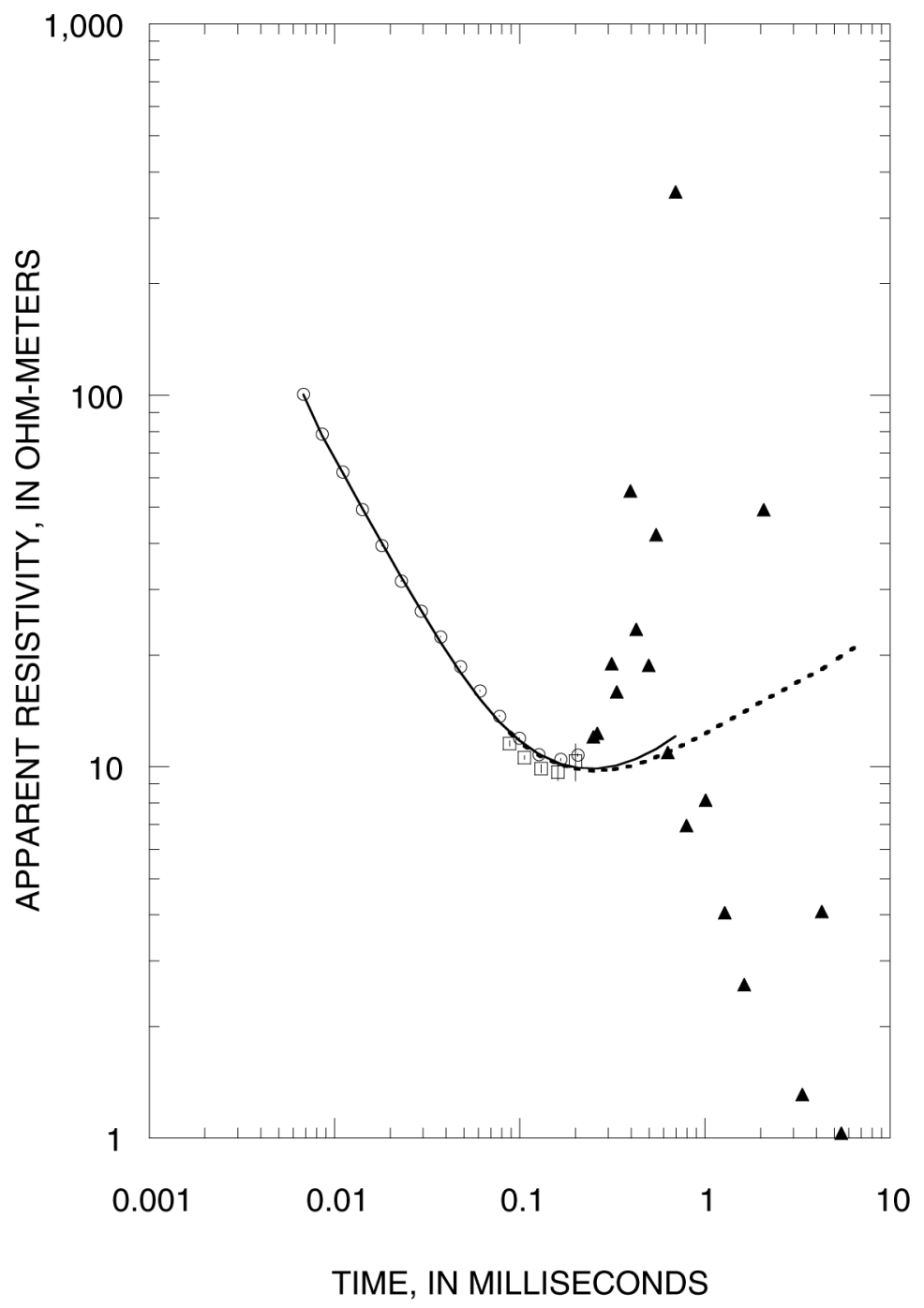

MIA115

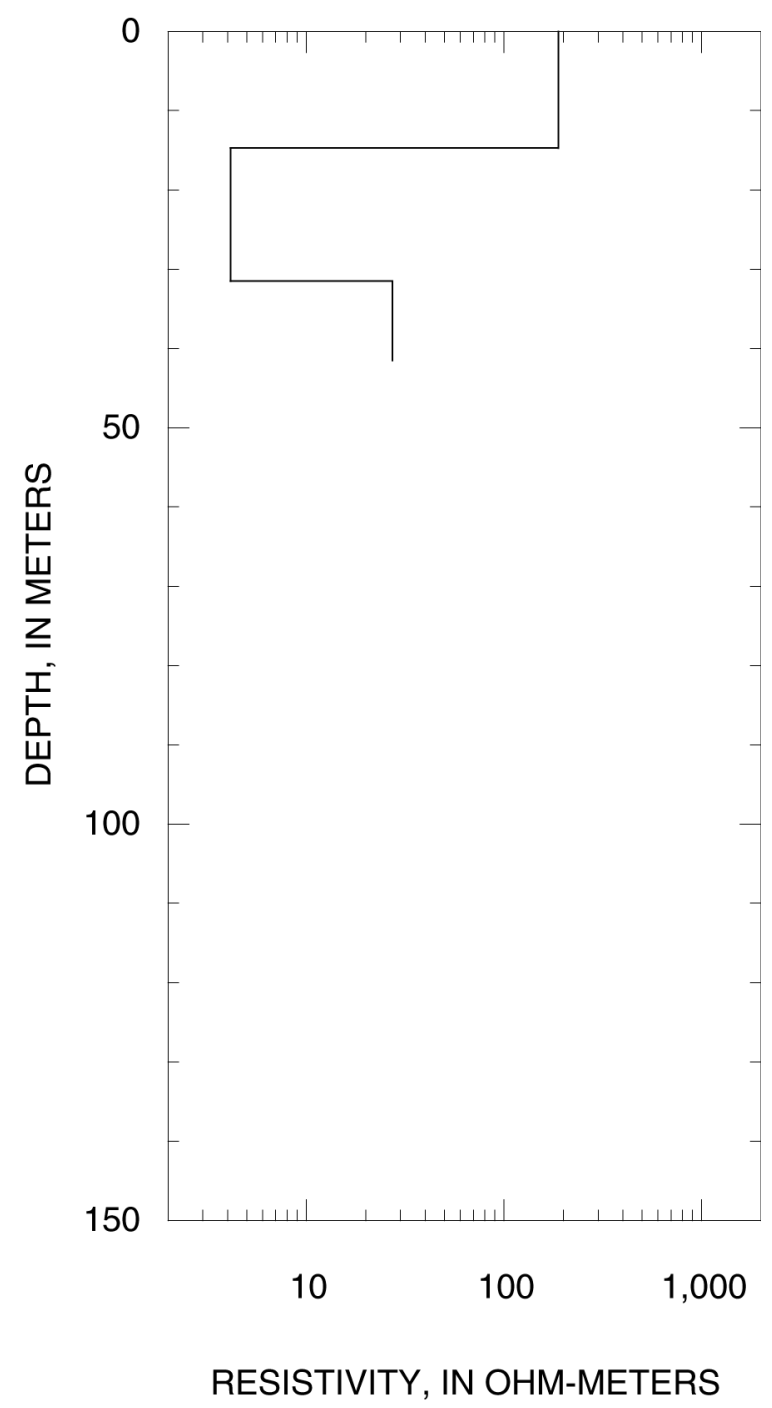

Figure 5-21. Sounding MIA115 

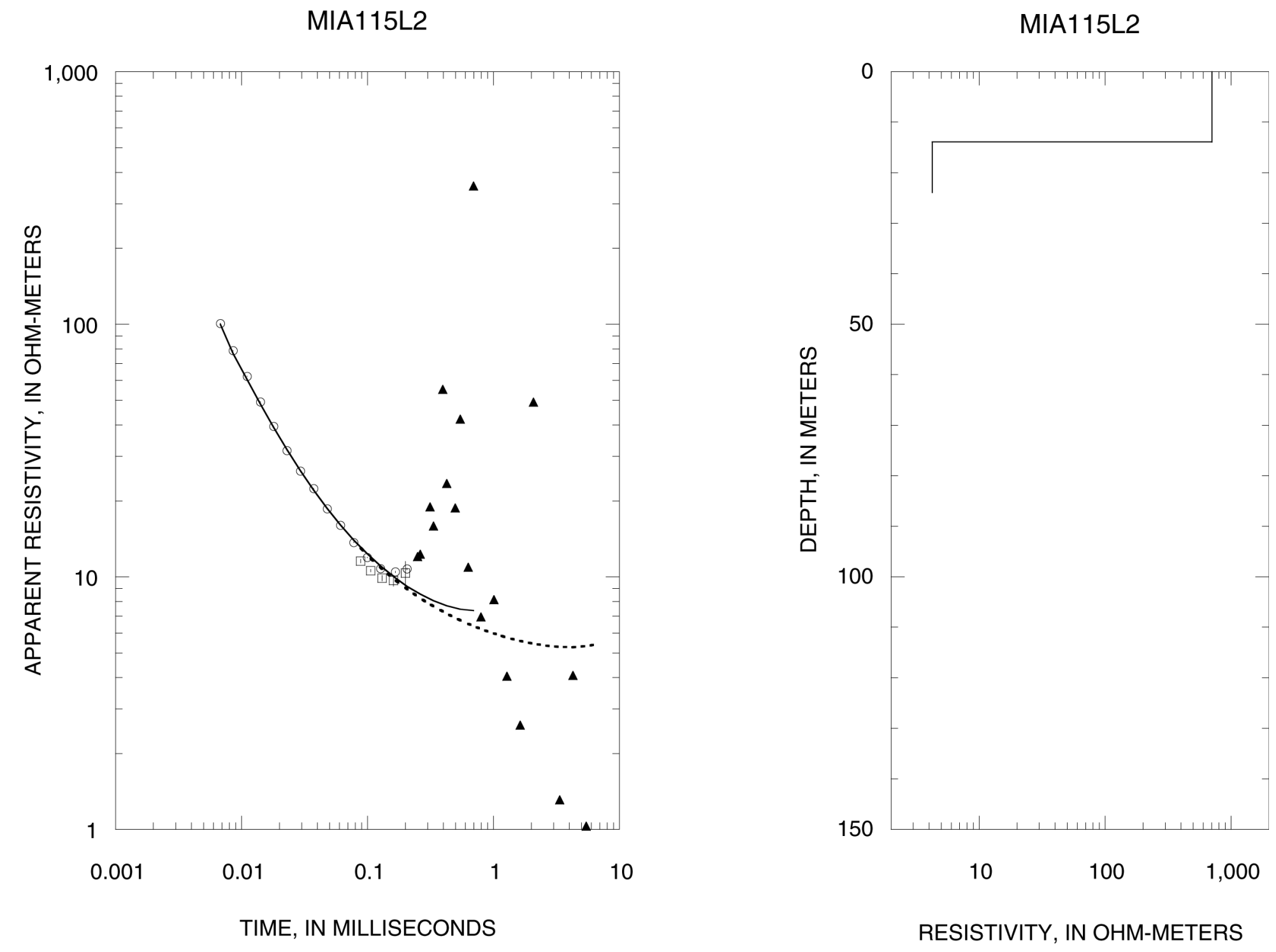

Figure 5-22. Sounding MIA115L2 
MIA116

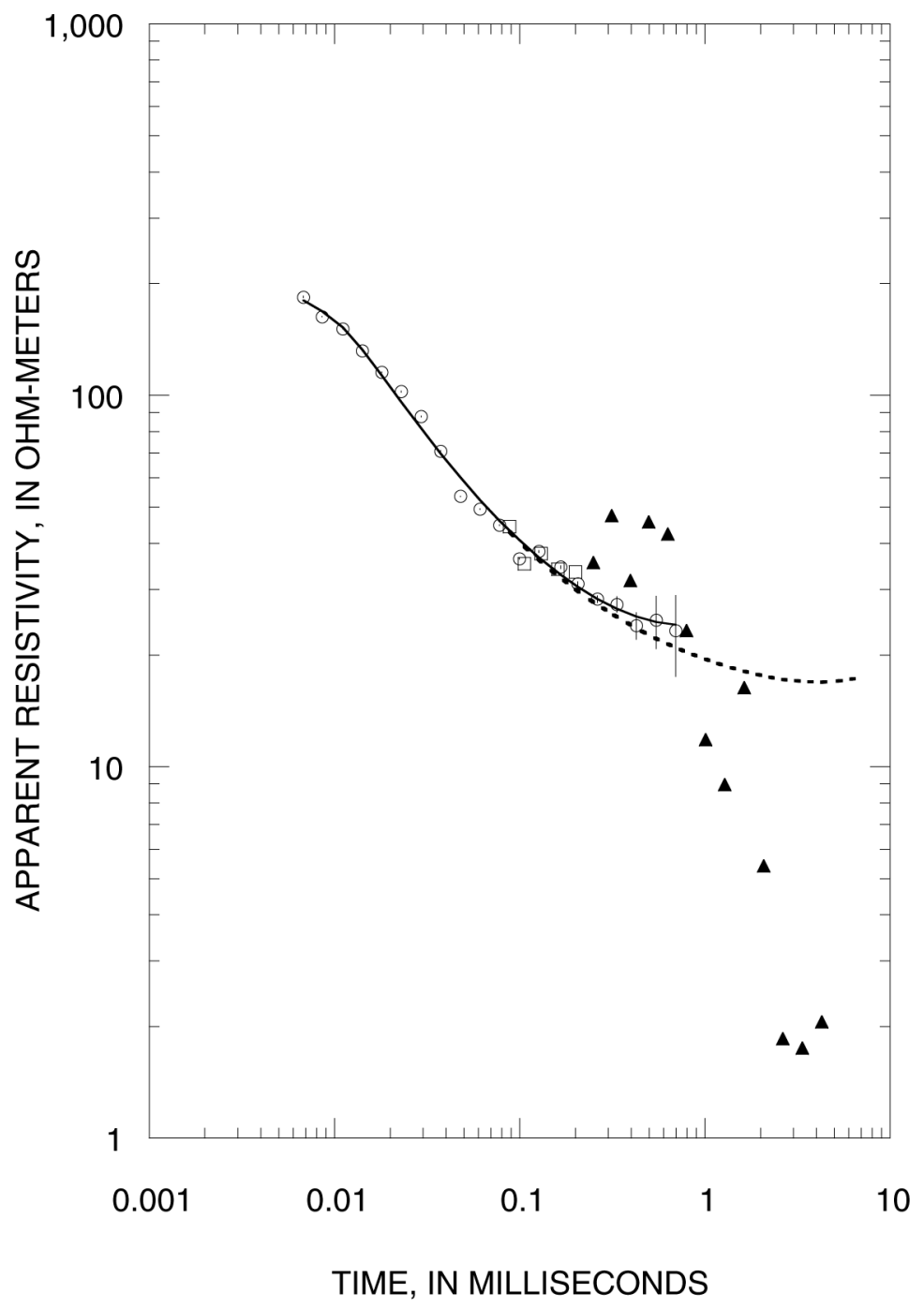

MIA116

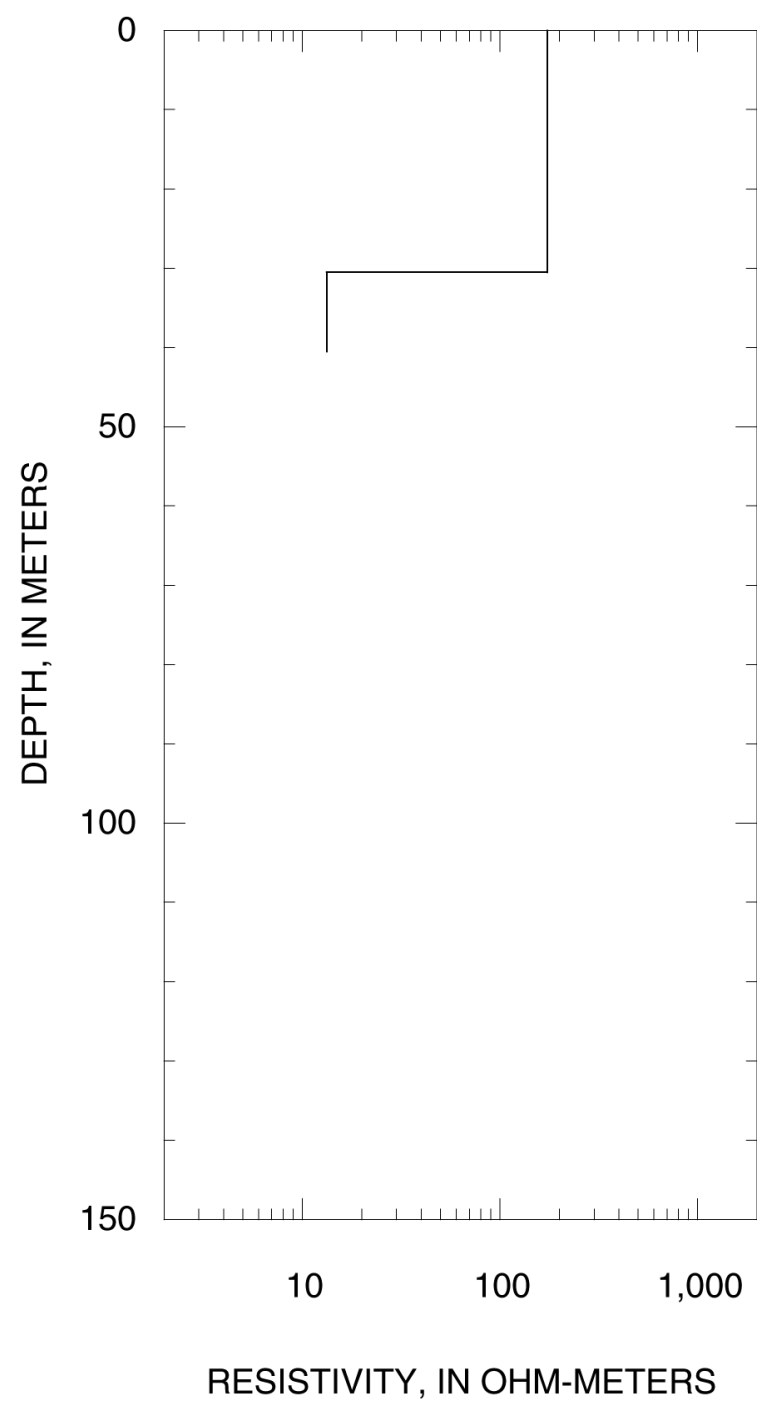

Figure 5-23. Sounding MIA116 

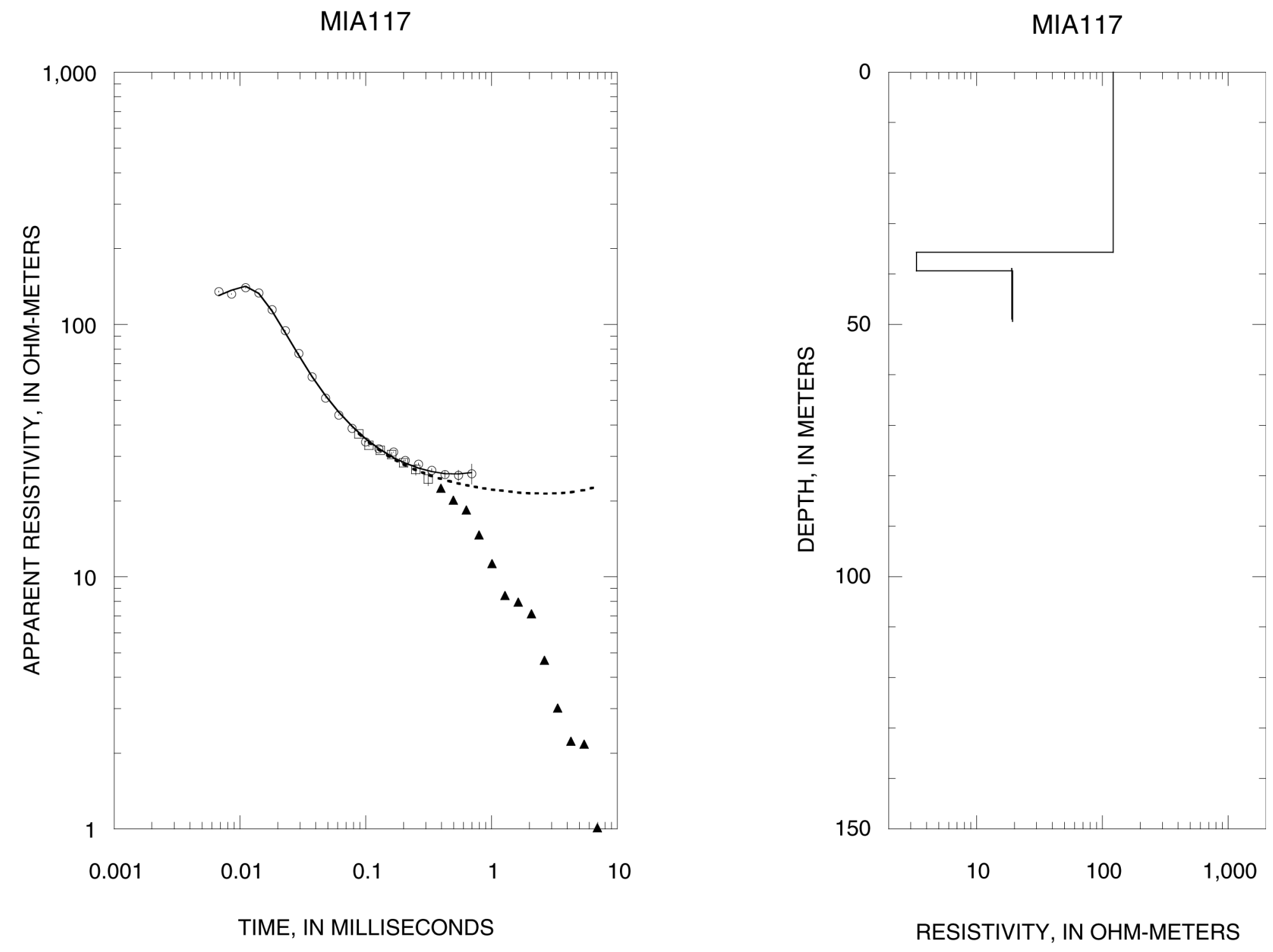

Figure 5-24. Sounding MIA117 
MIA117F3

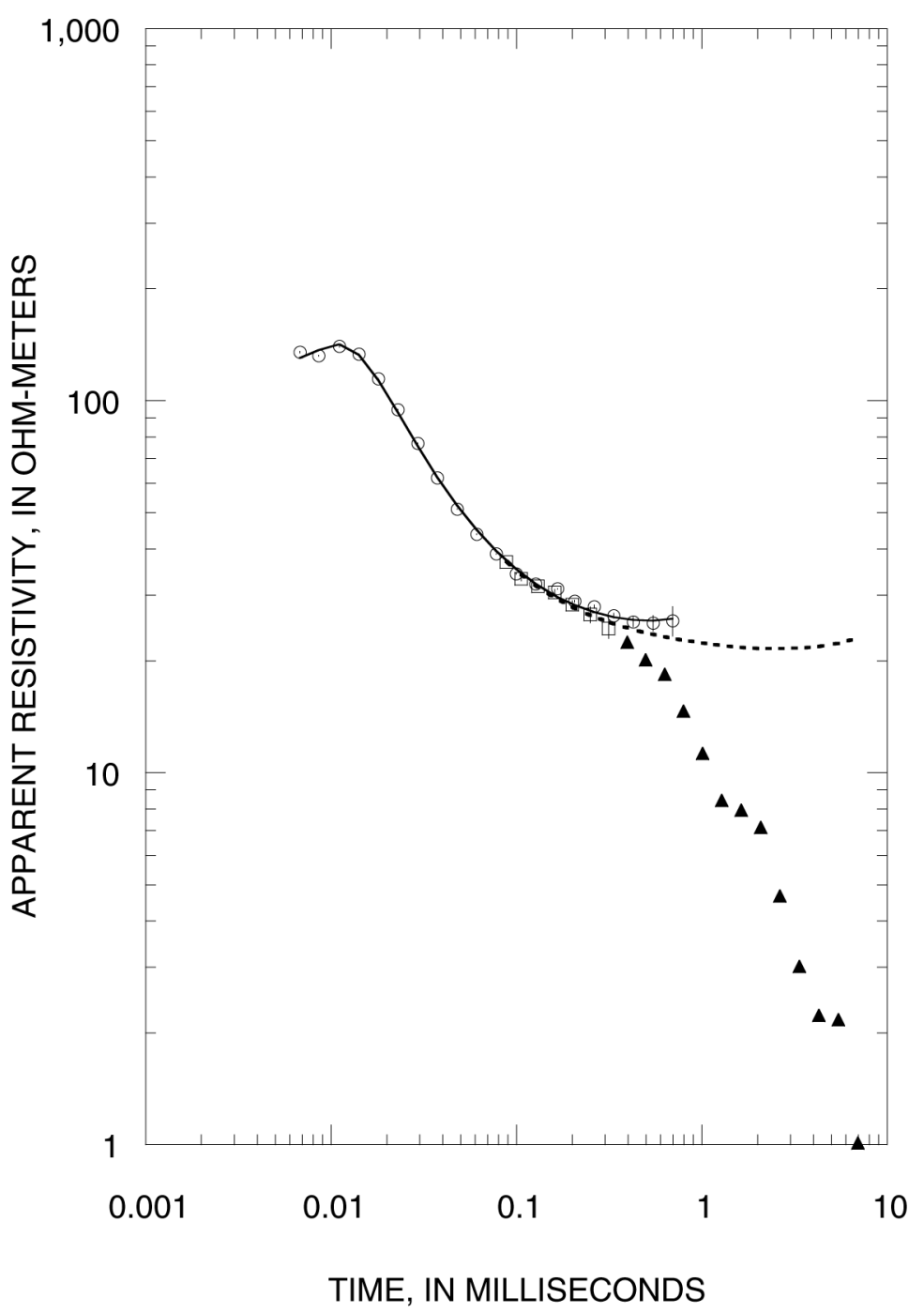

MIA117F3

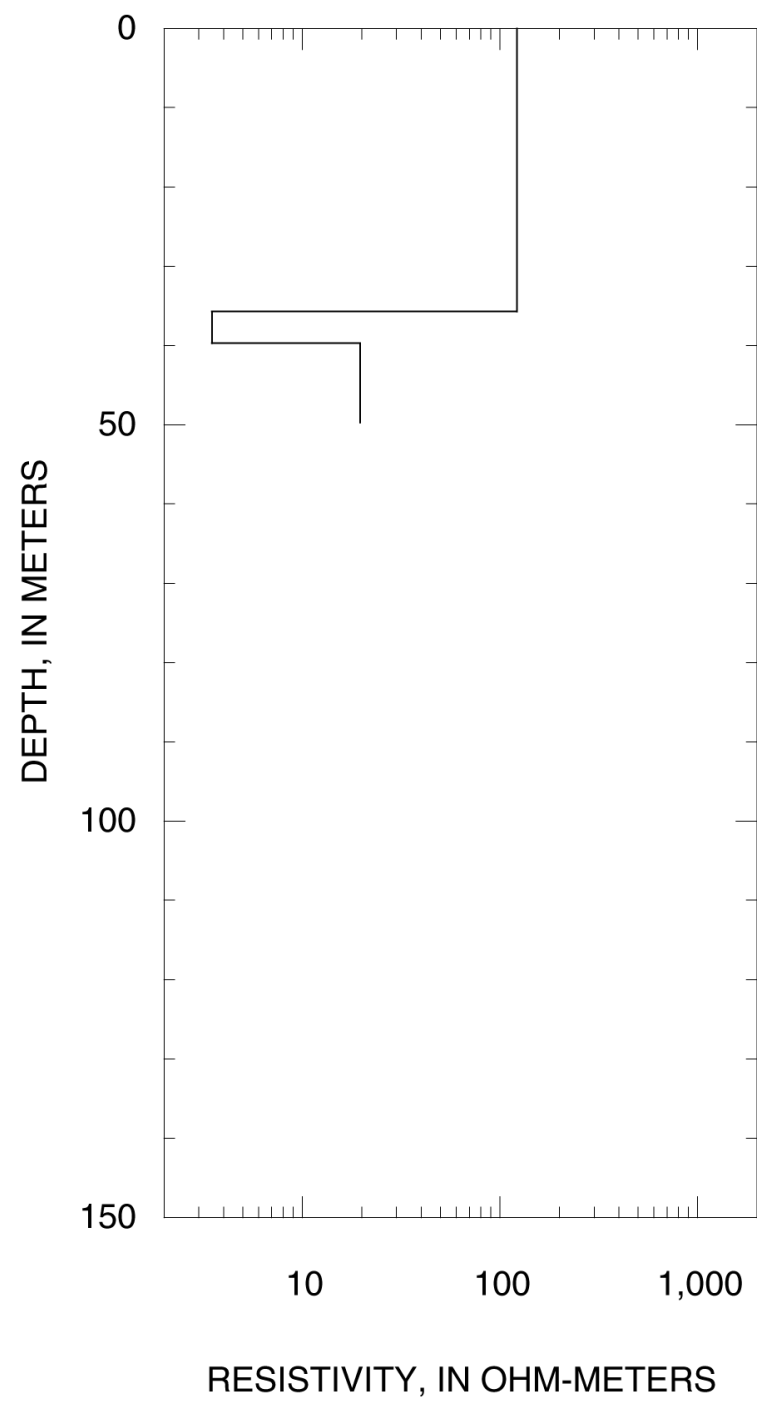

Figure 5-25. Sounding MIA117F3 
MIA117L4

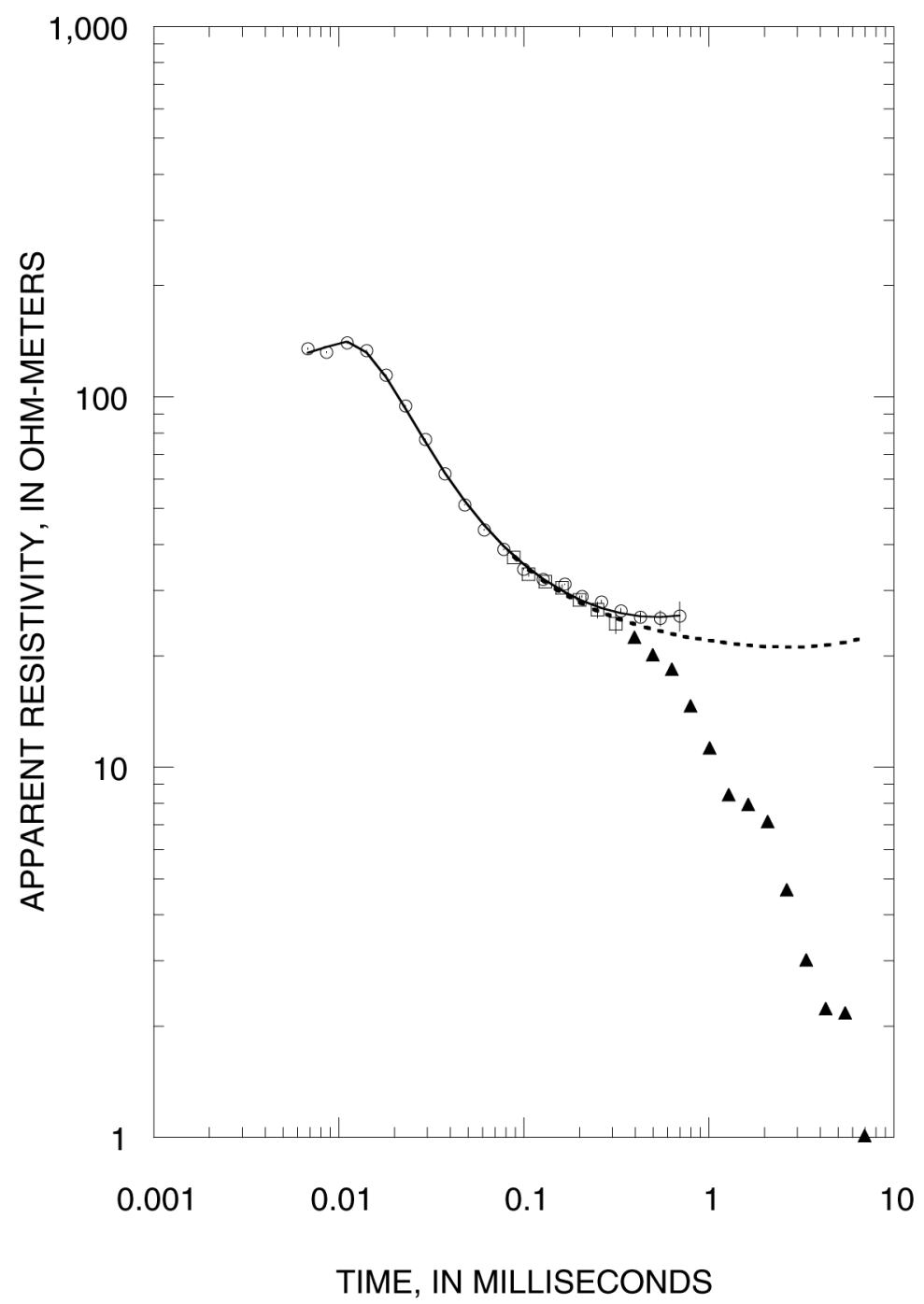

MIA117L4

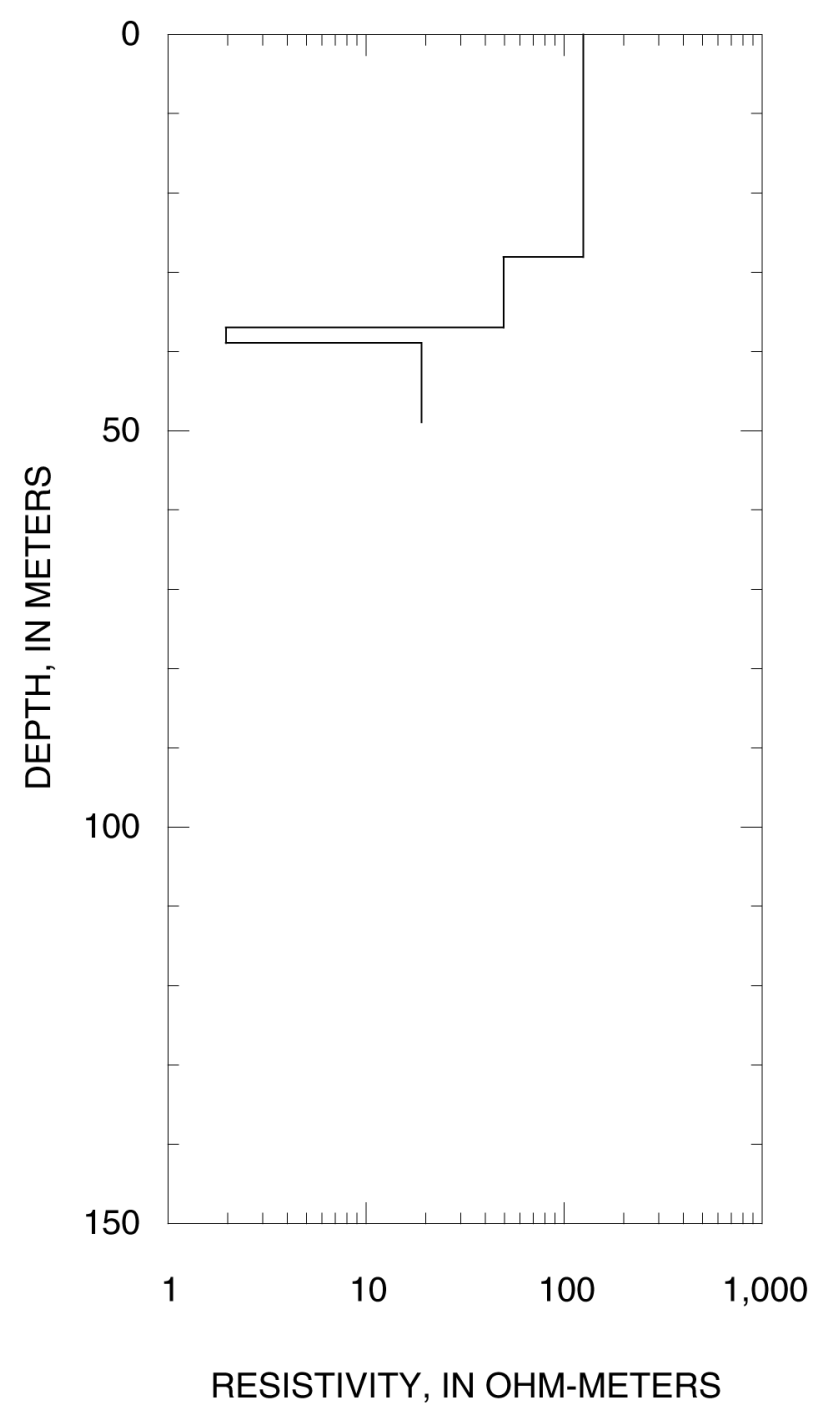

Figure 5-26. Sounding MIA117L4 
MIA118

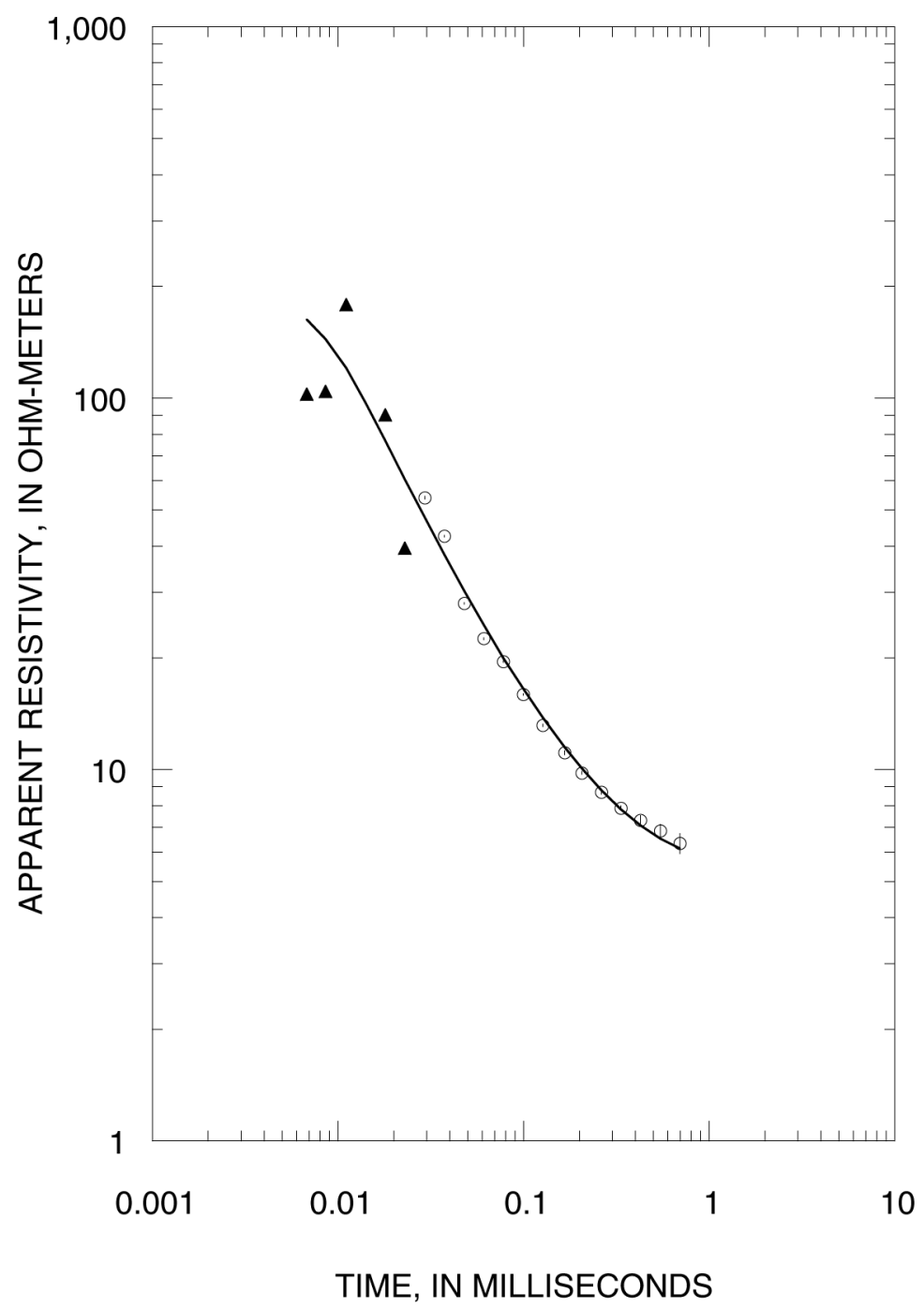

Figure 5-27. Sounding MIA118
MIA118

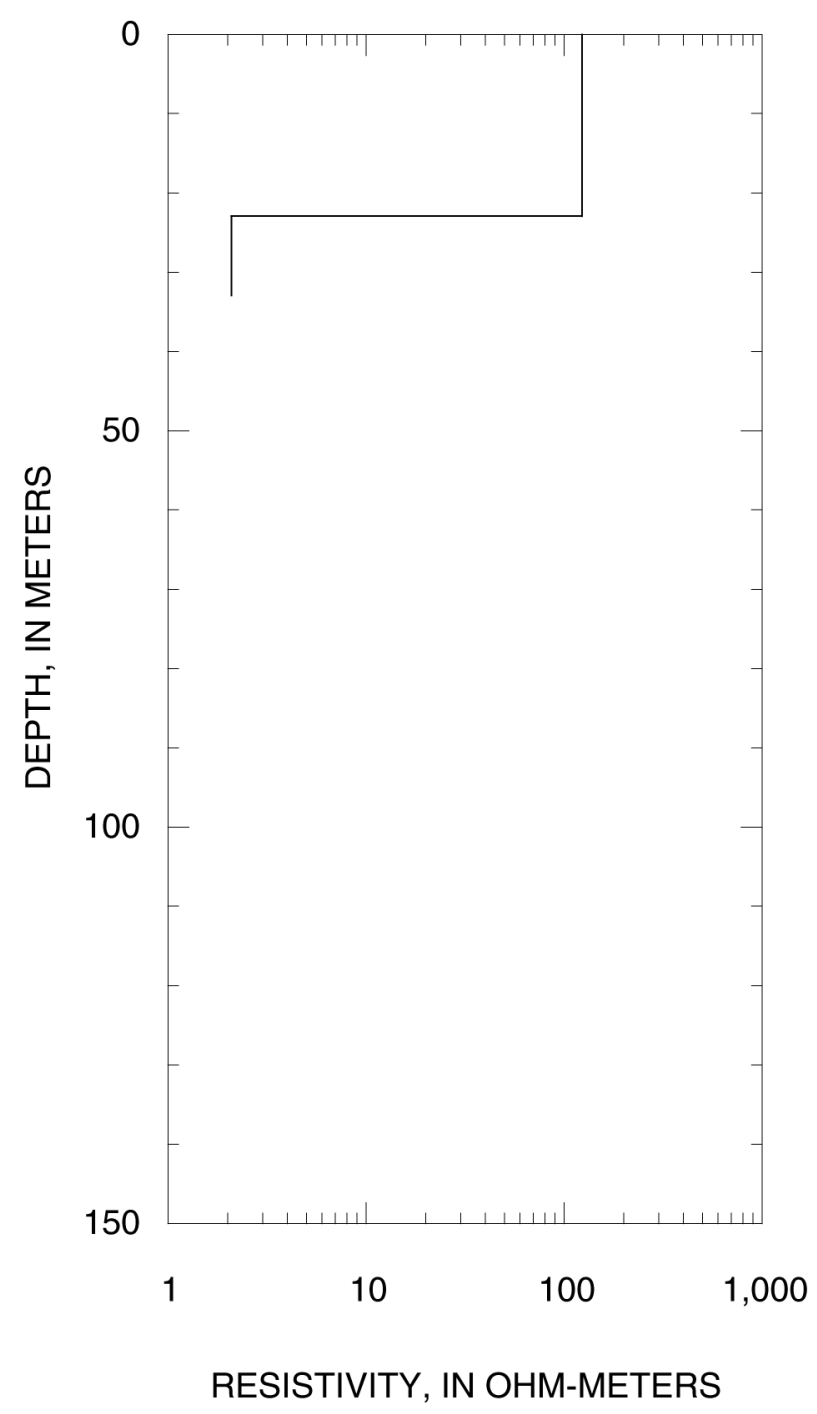


MIA119

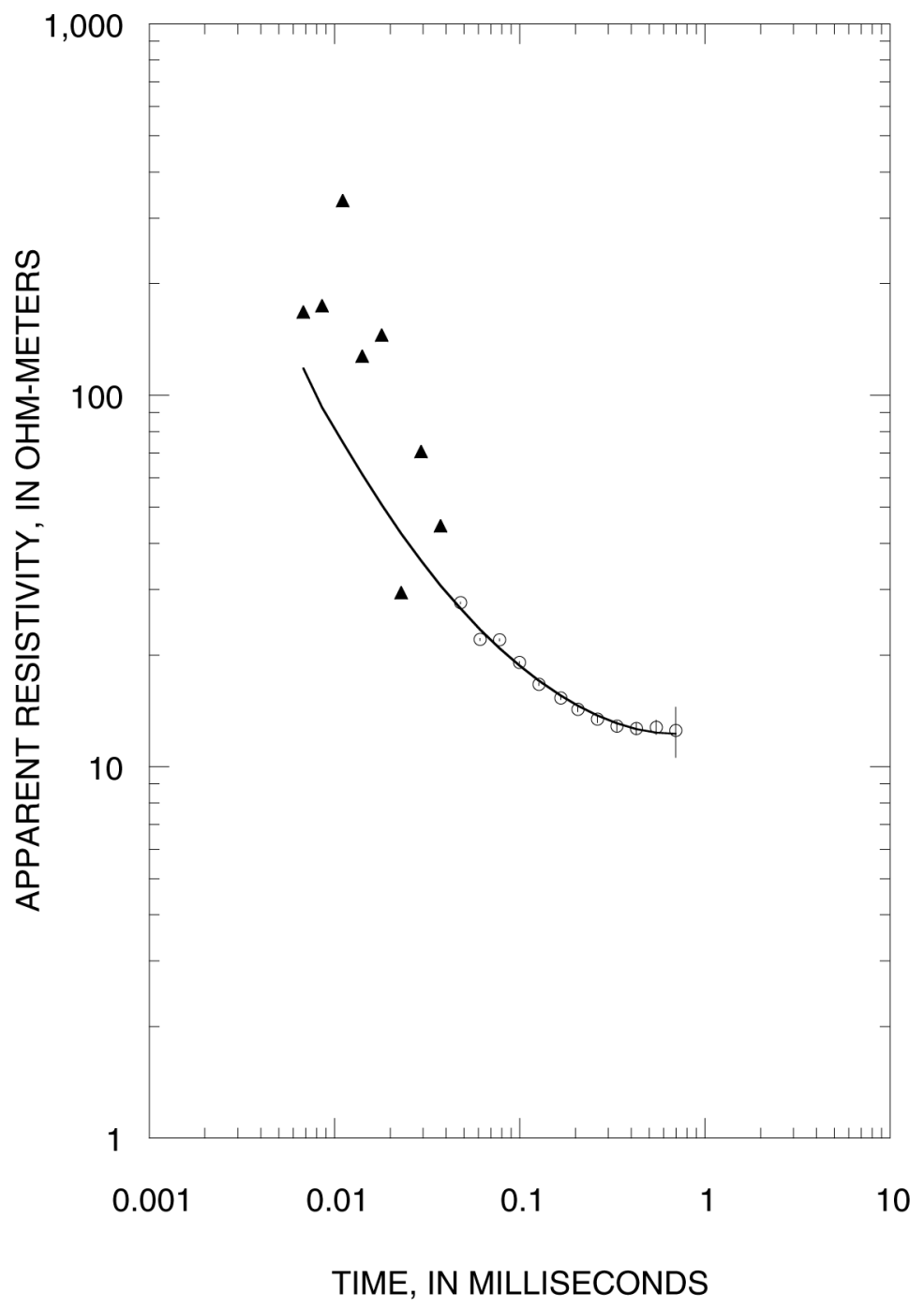

MIA119

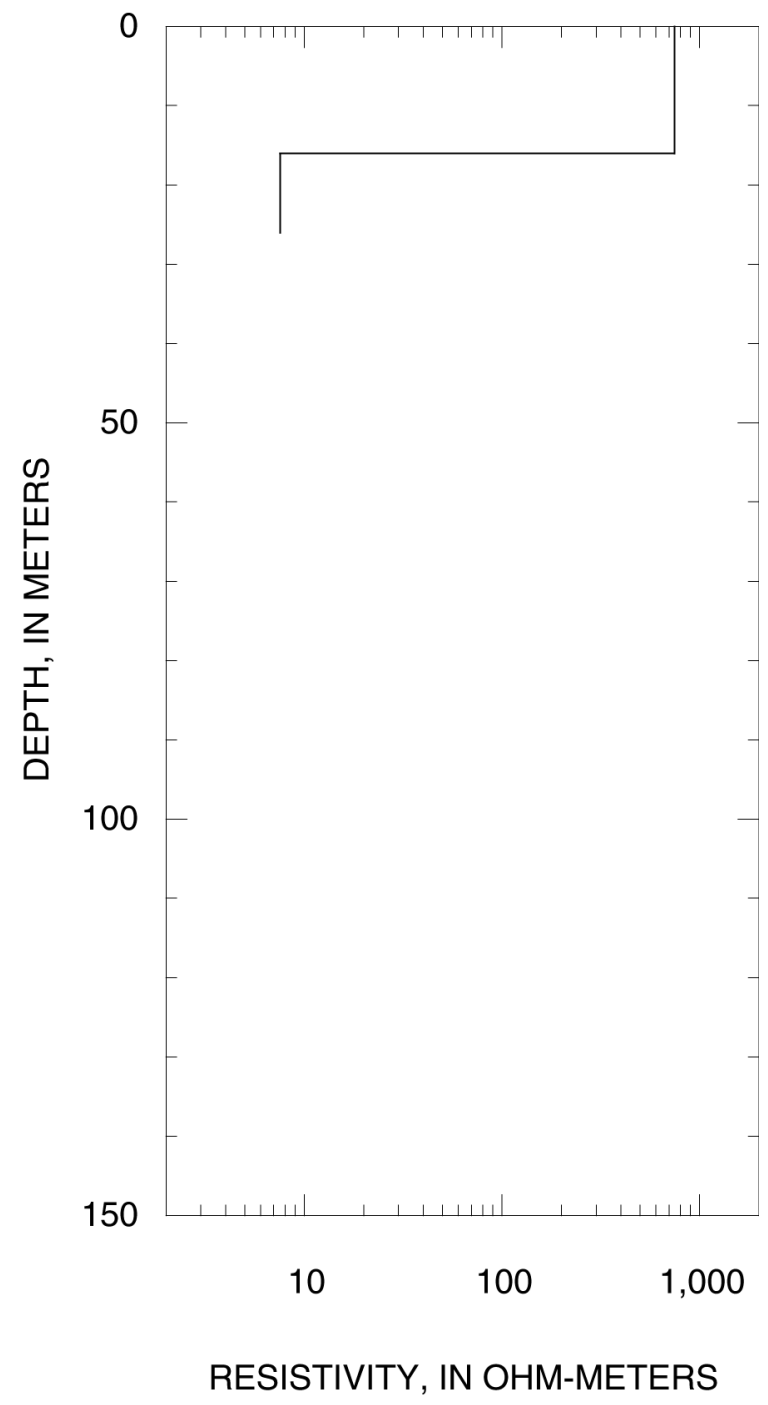

Figure 5-28. Sounding MIA119 
MIA120

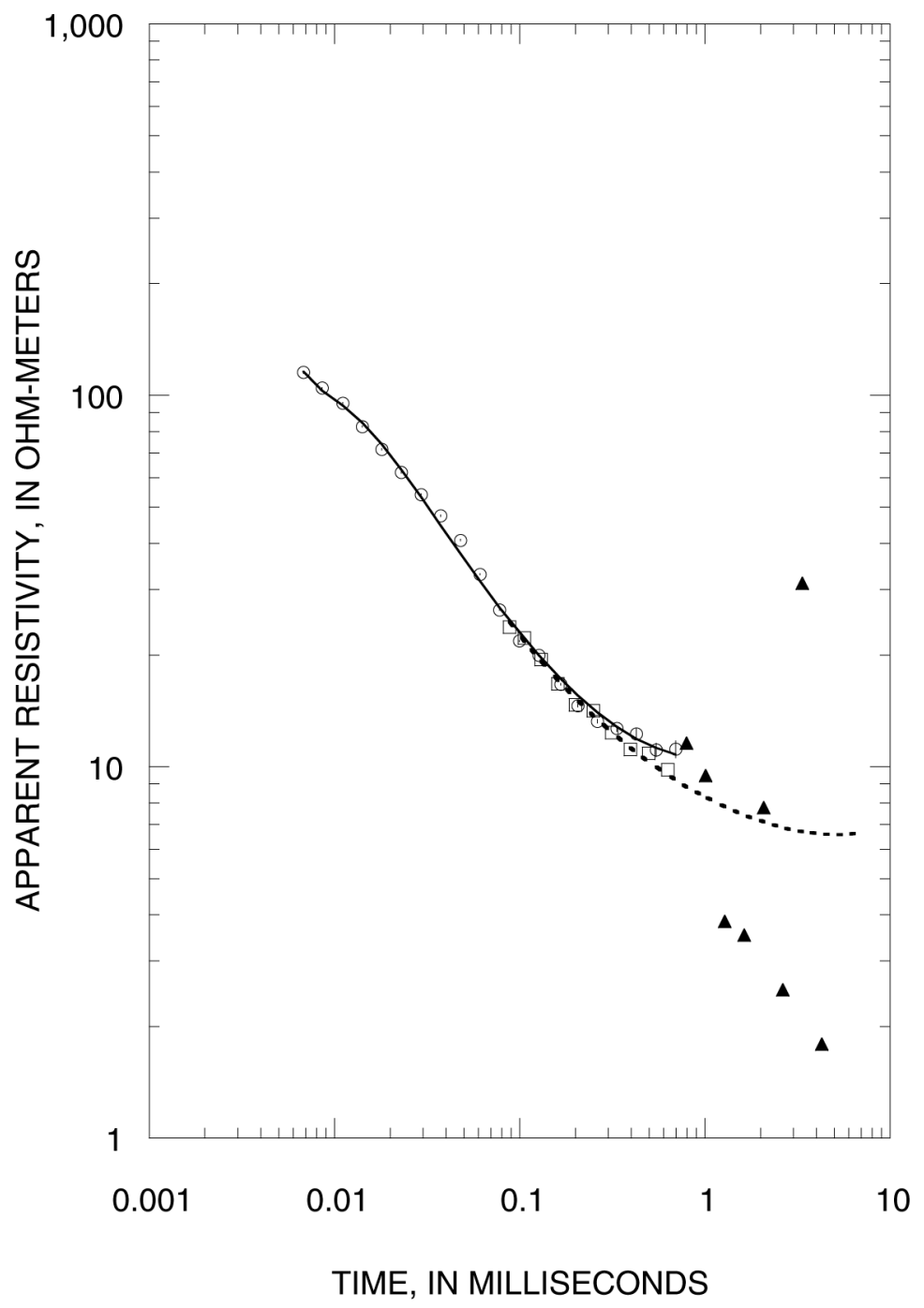

MIA120

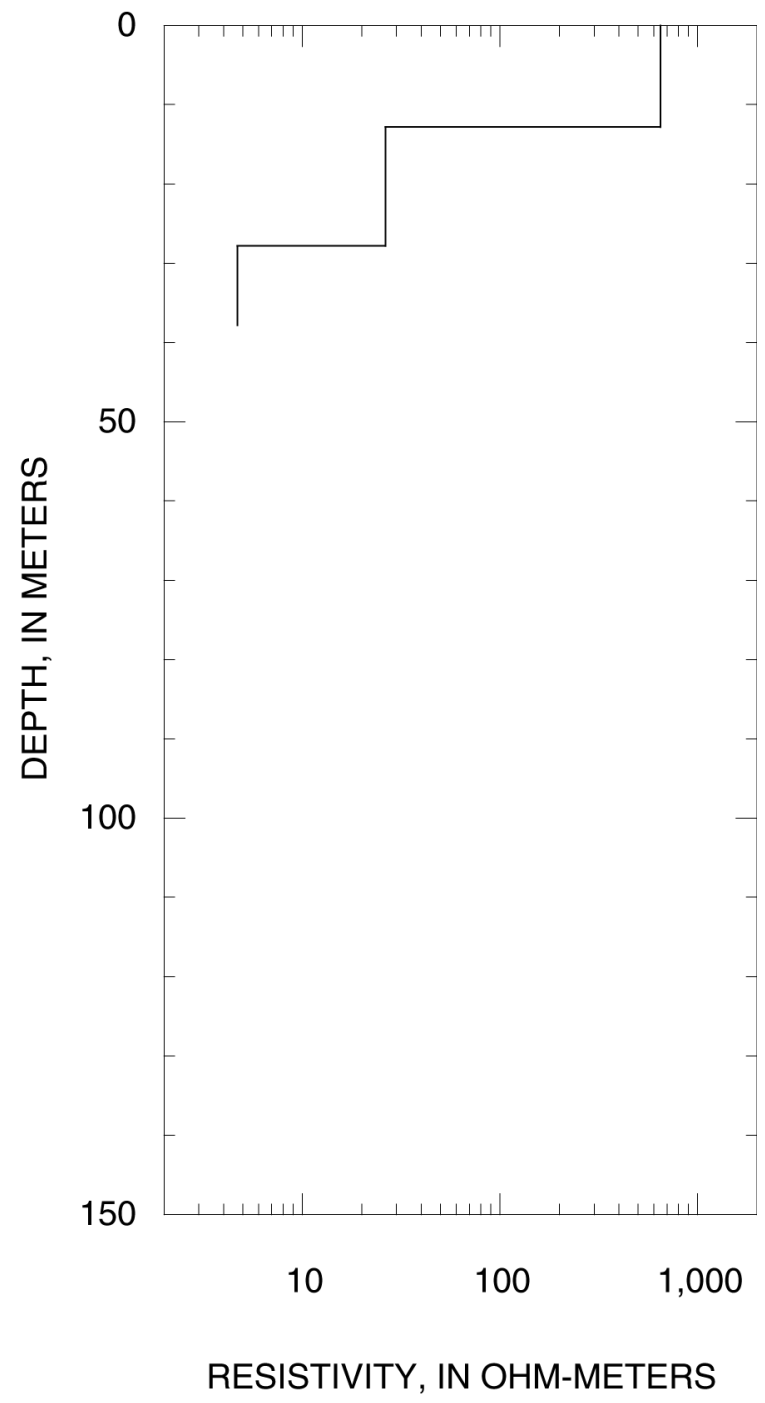

Figure 5-29. Sounding MIA120 
MIA121

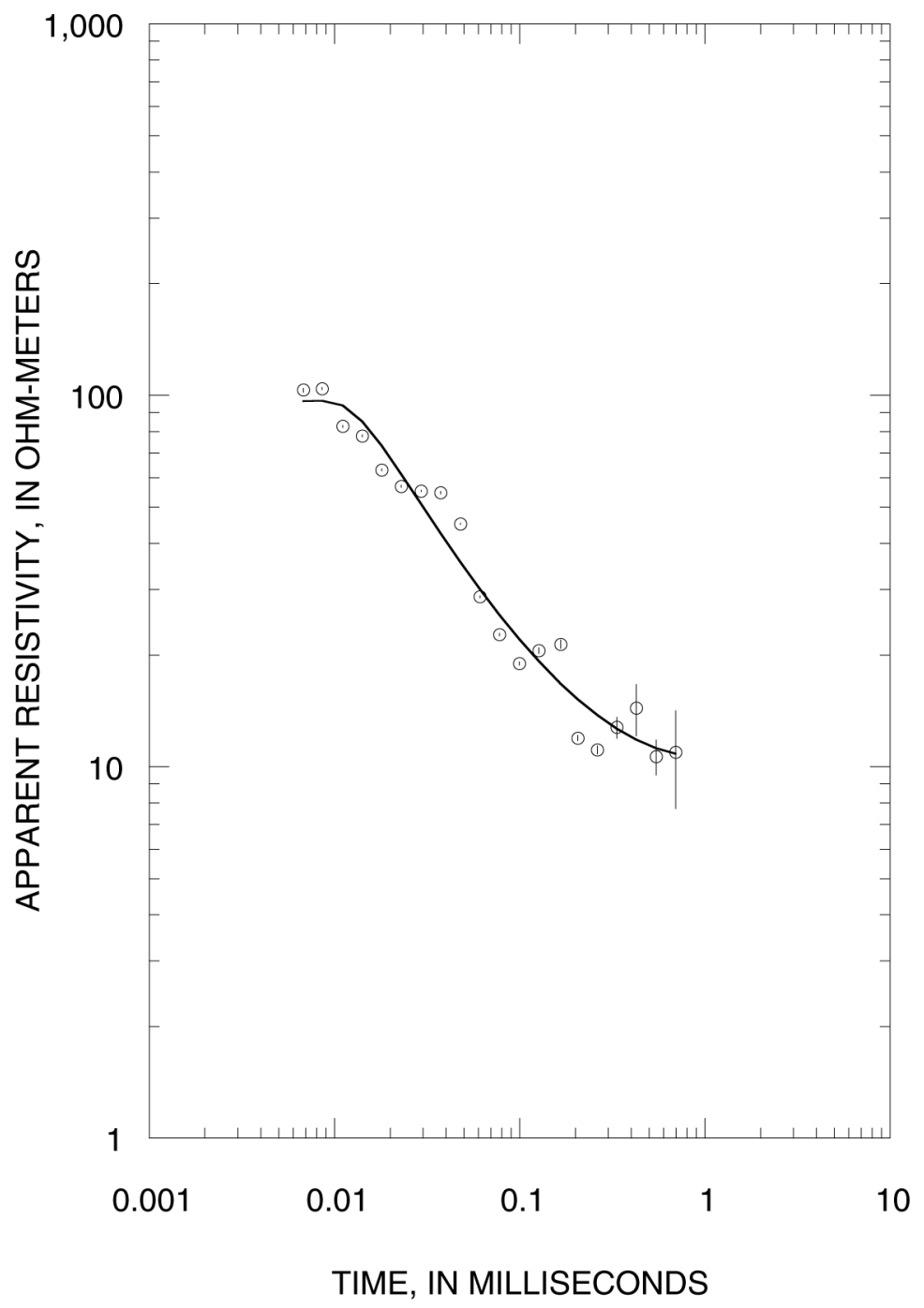

MIA121

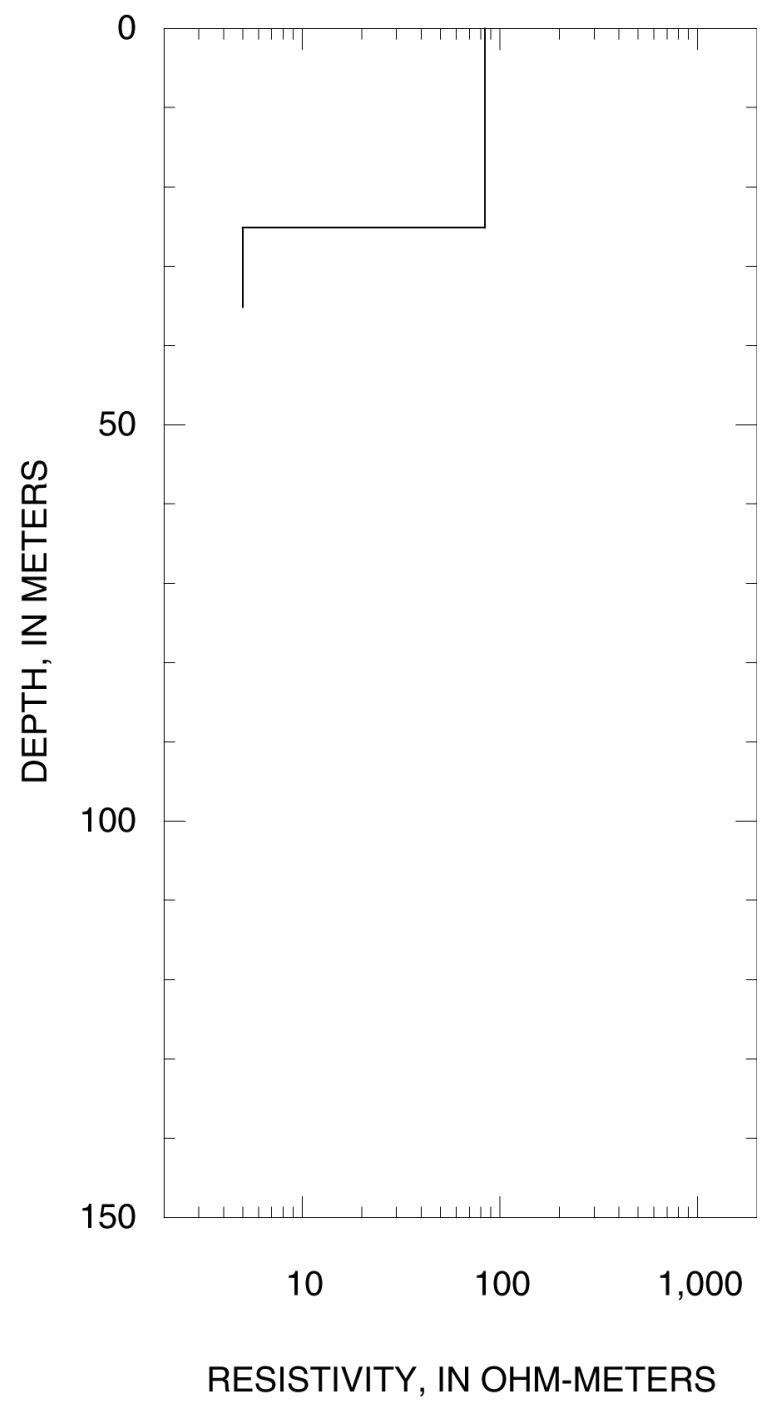

Figure 5-30. Sounding MIA121 
MIA122

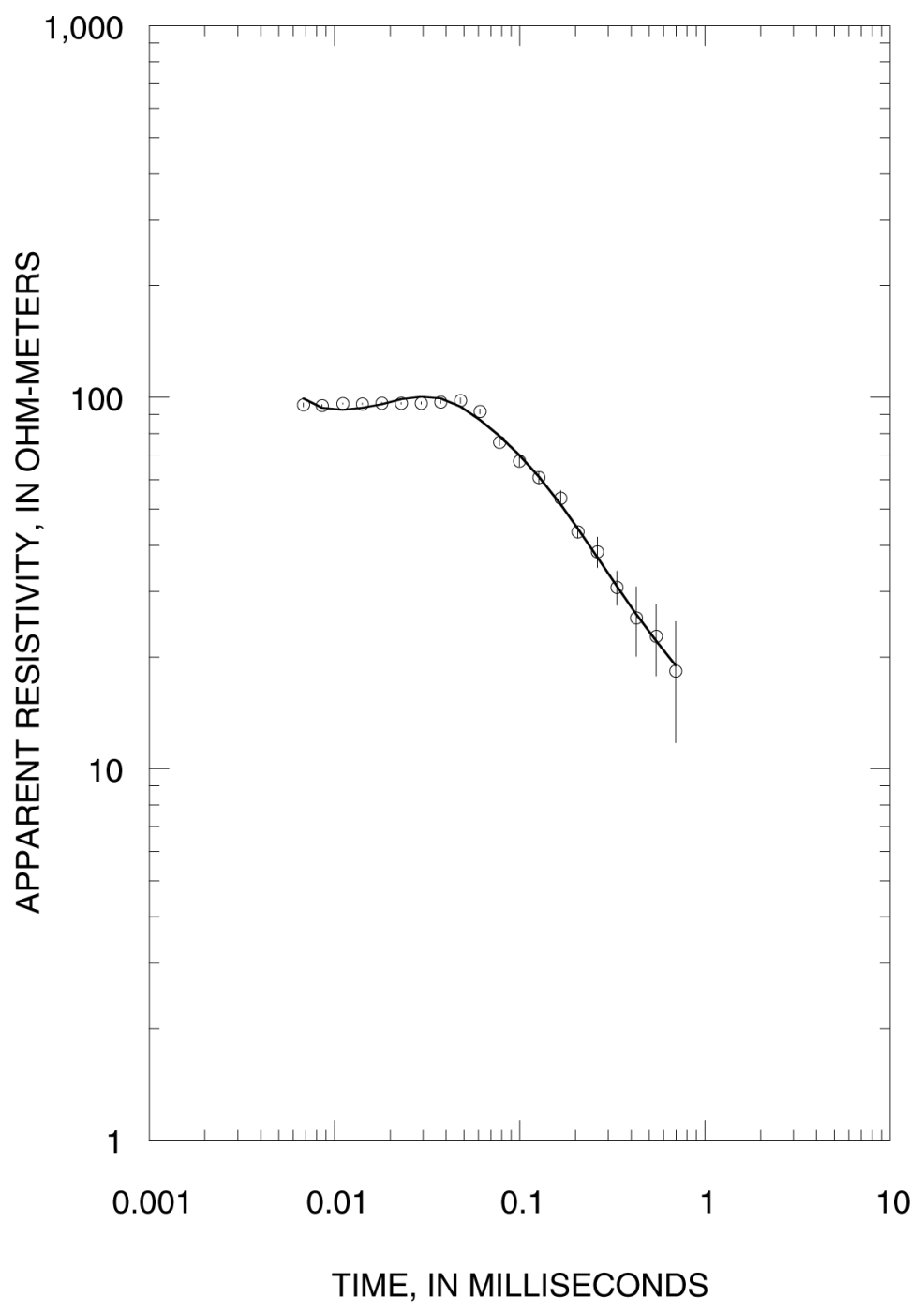

MIA122

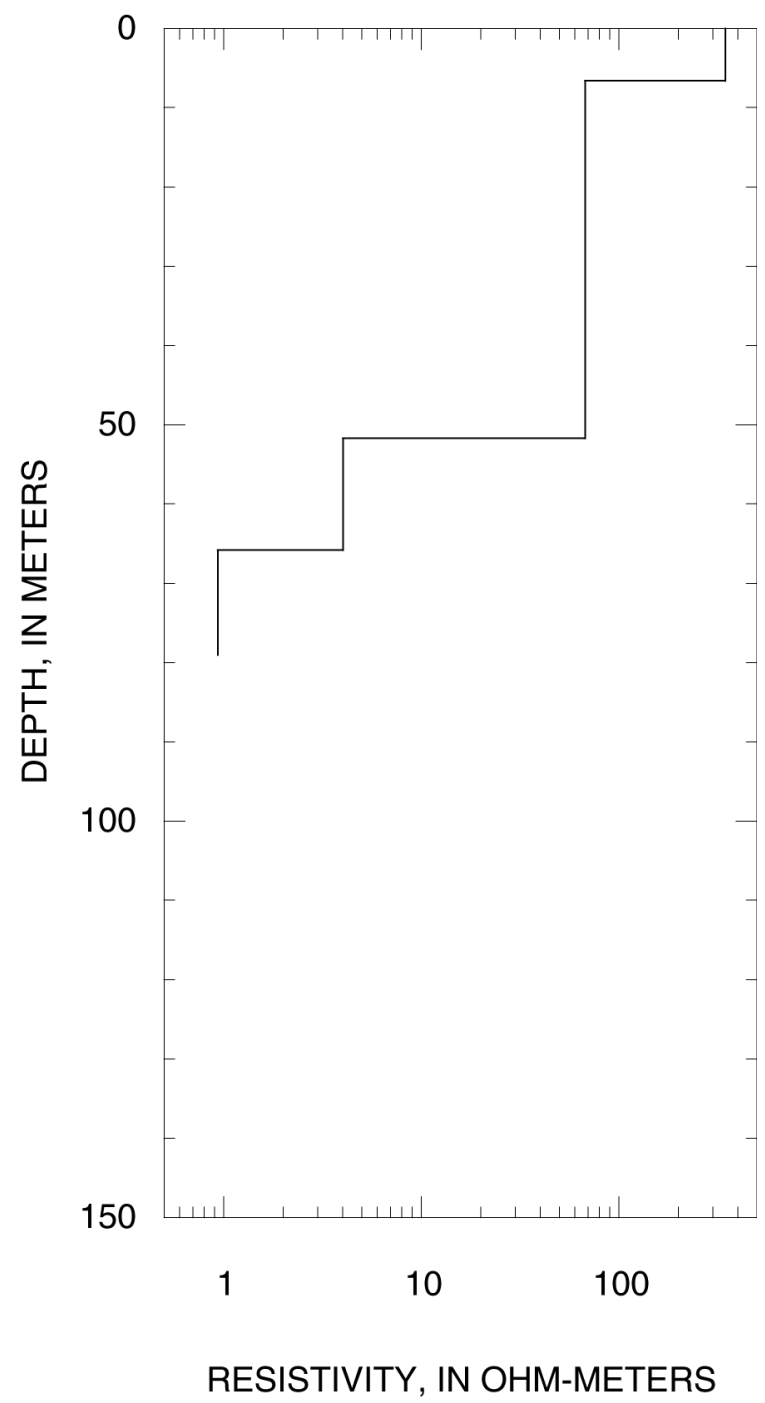

Figure 5-31. Sounding MIA122 
MIA123

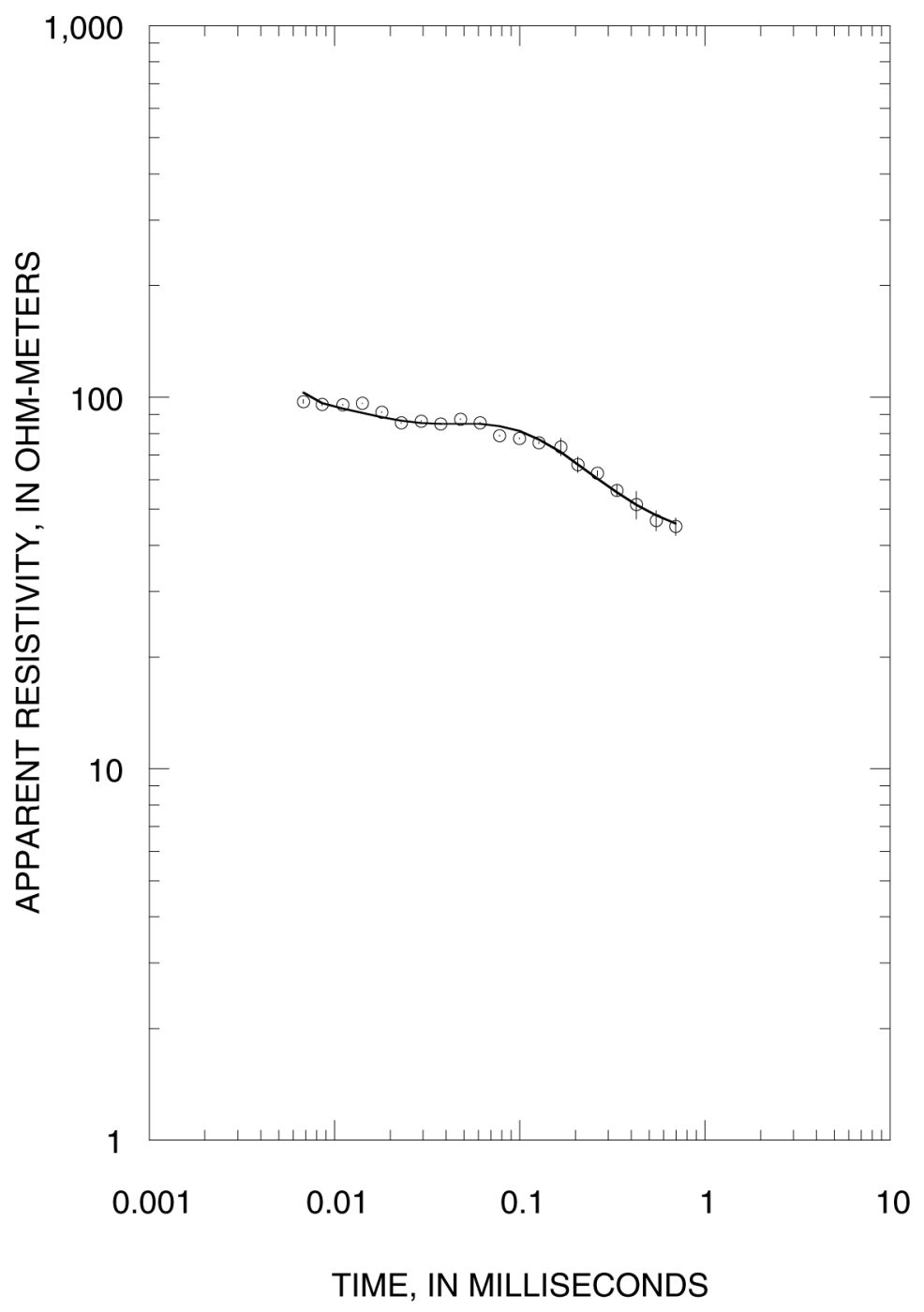

MIA123

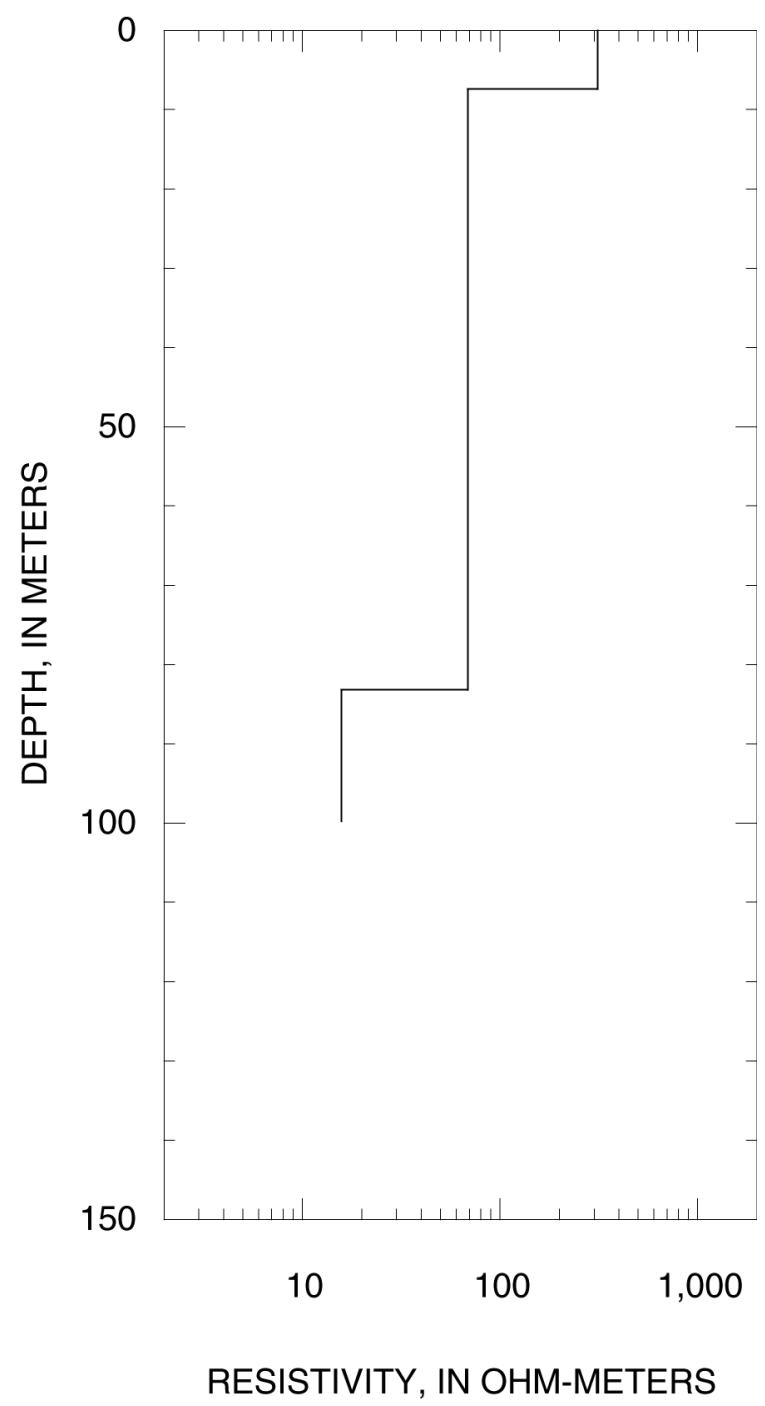

Figure 5-32. Sounding MIA123 
MIA124

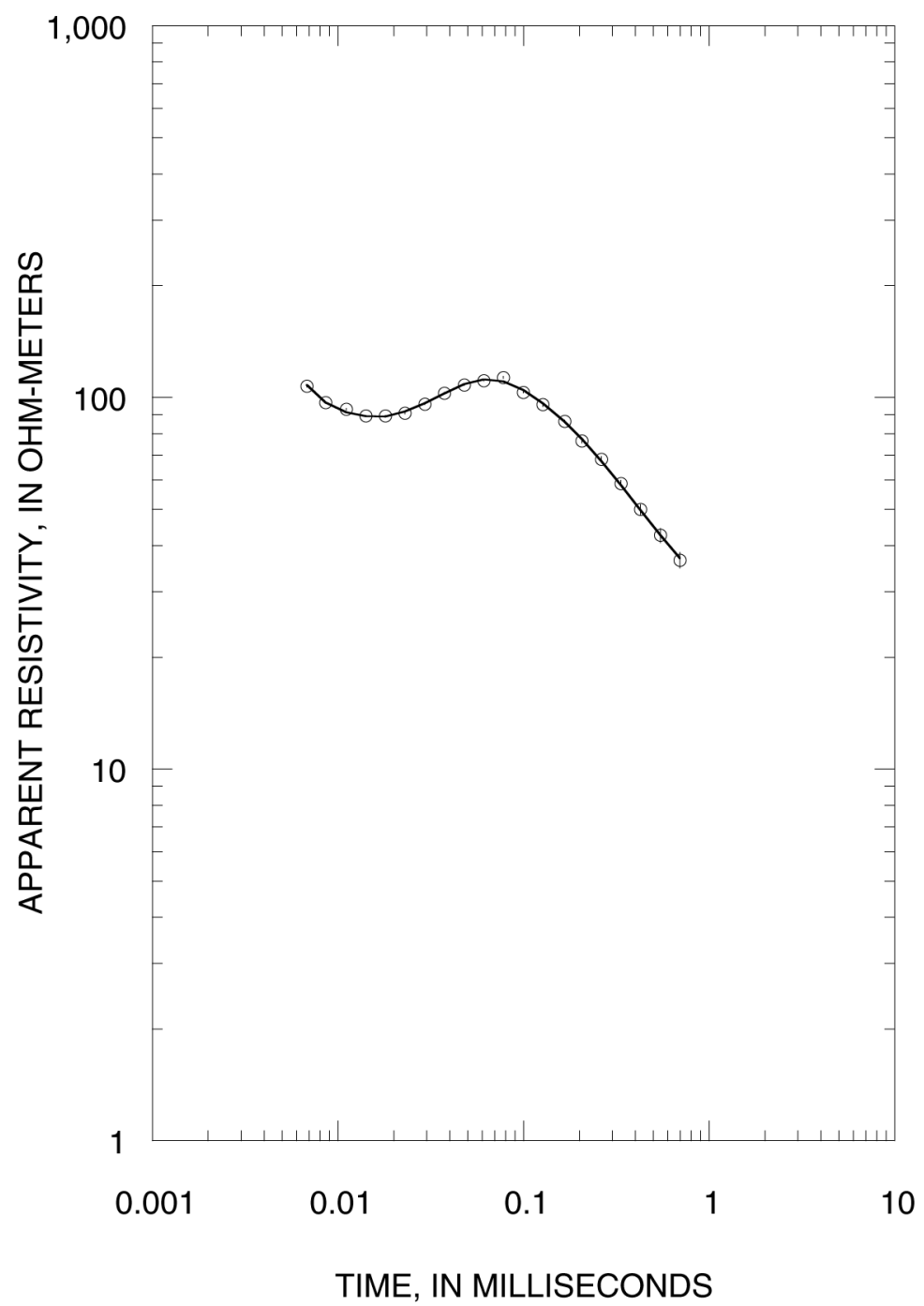

MIA124

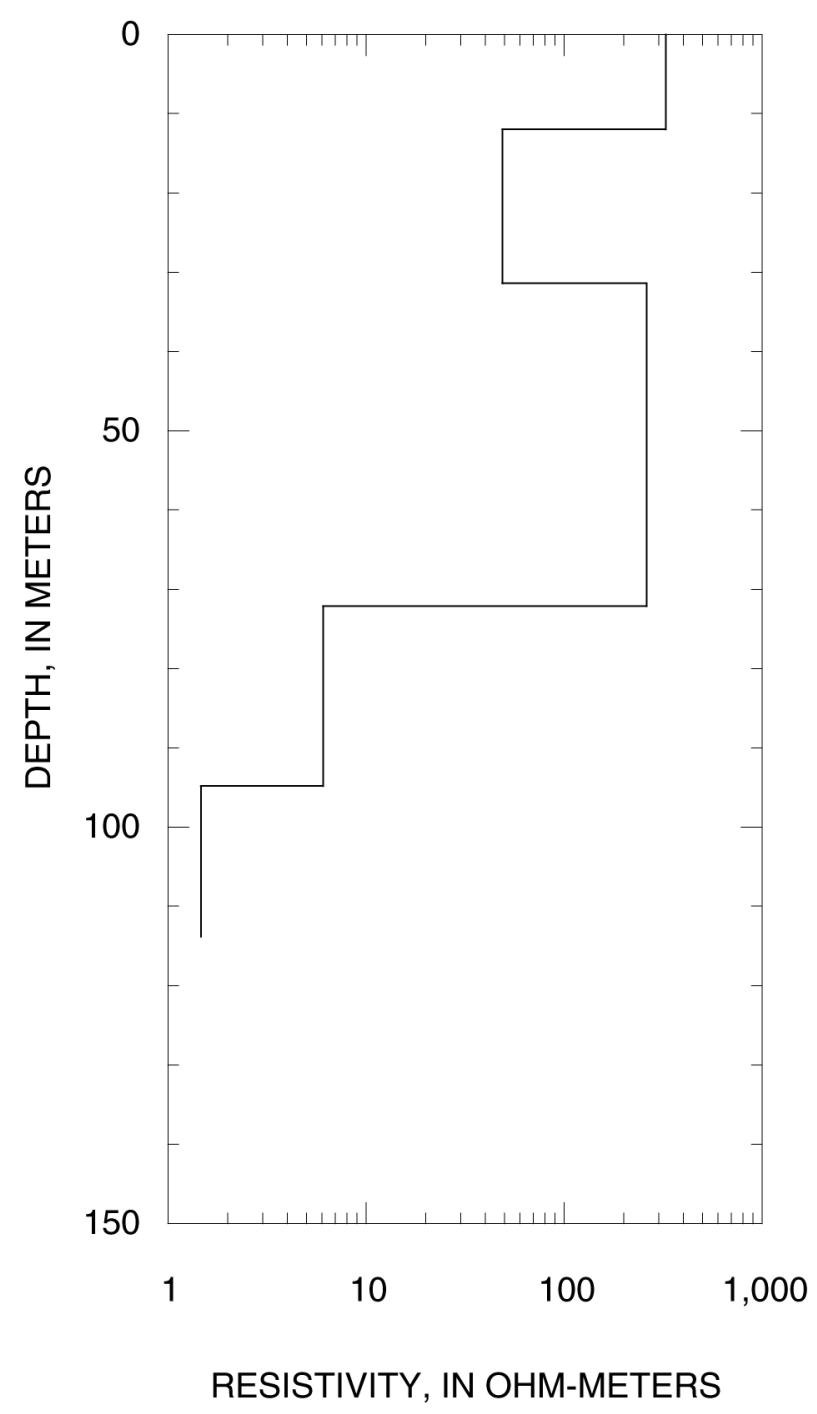

Figure 5-33. Sounding MIA124 
MIA125

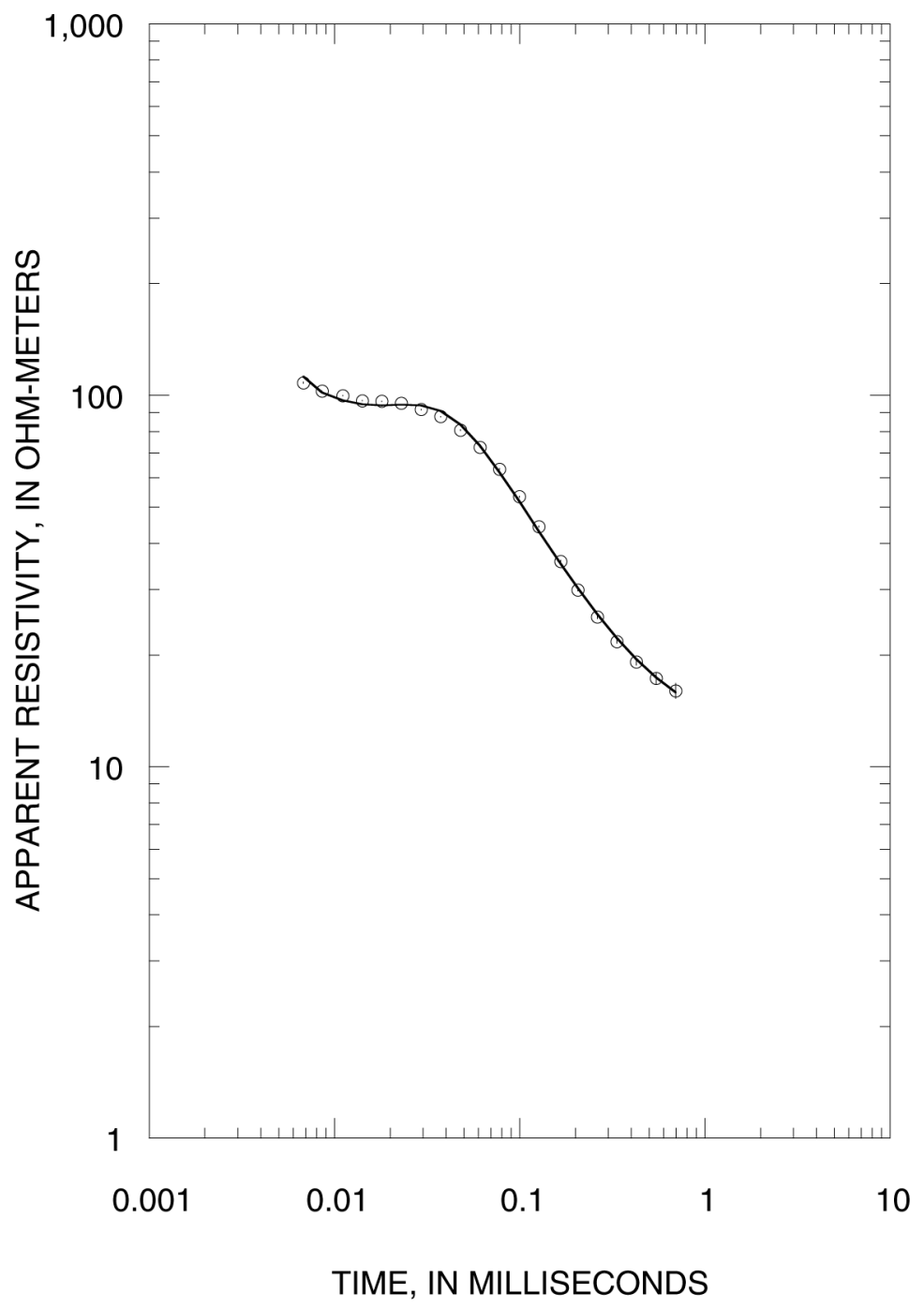

MIA125

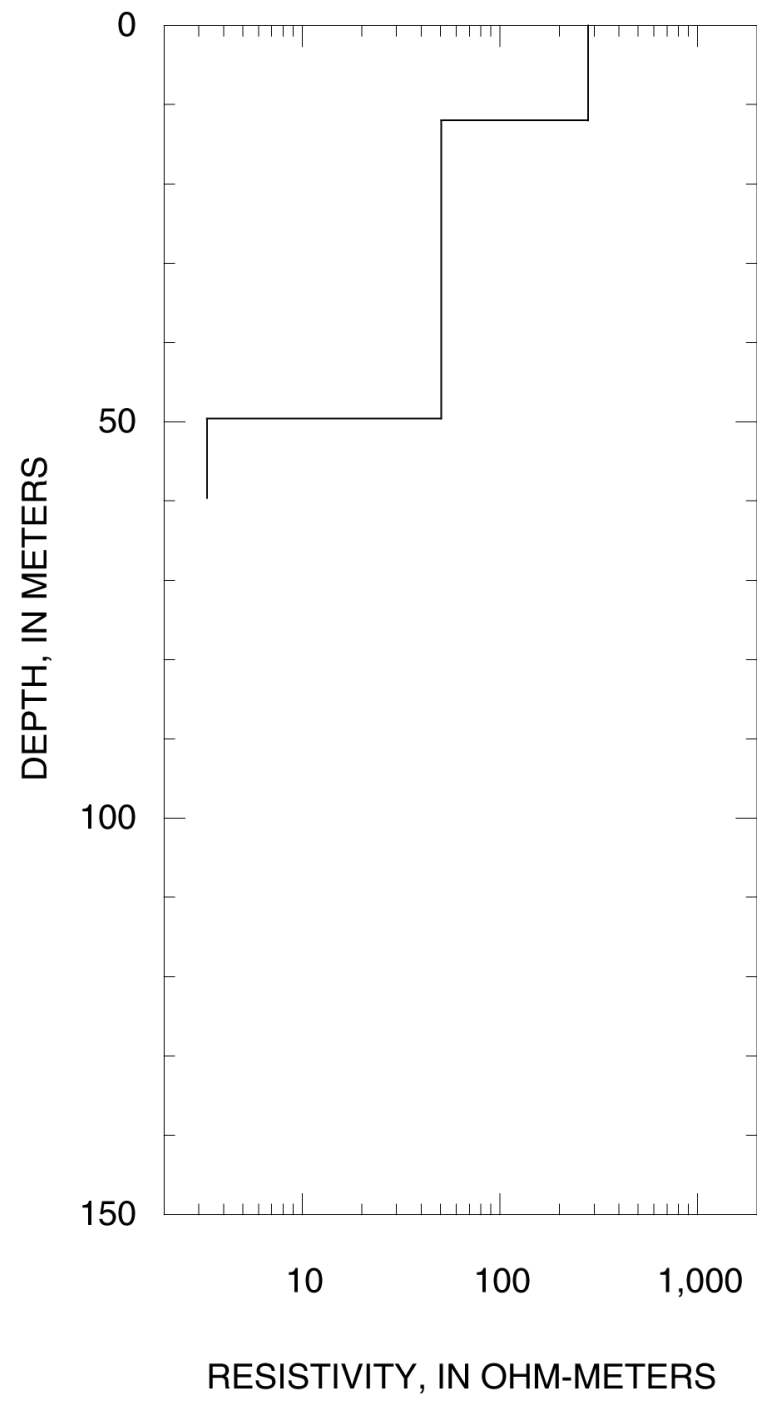

Figure 5-34. Sounding MIA125 
MIA126

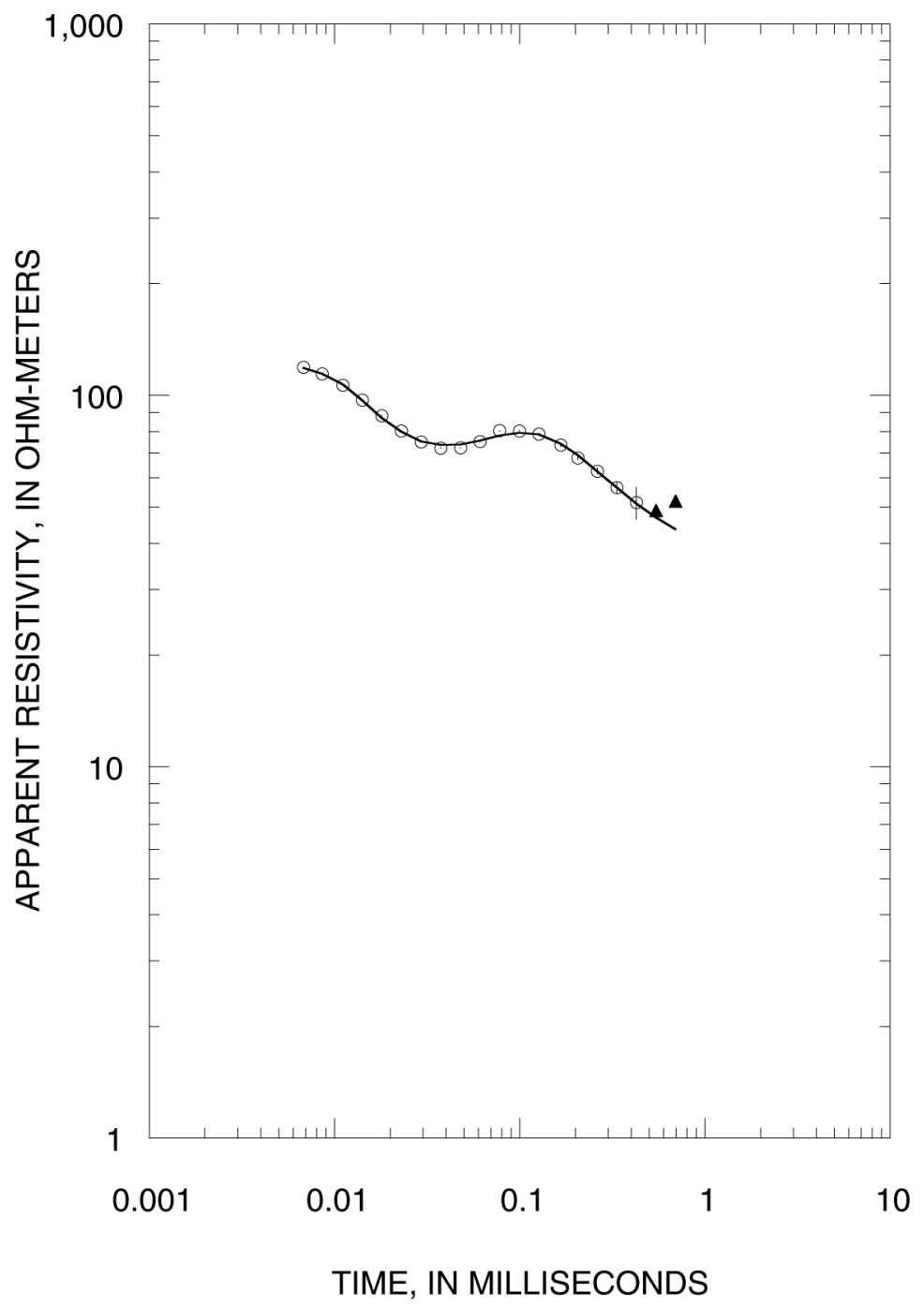

MIA126

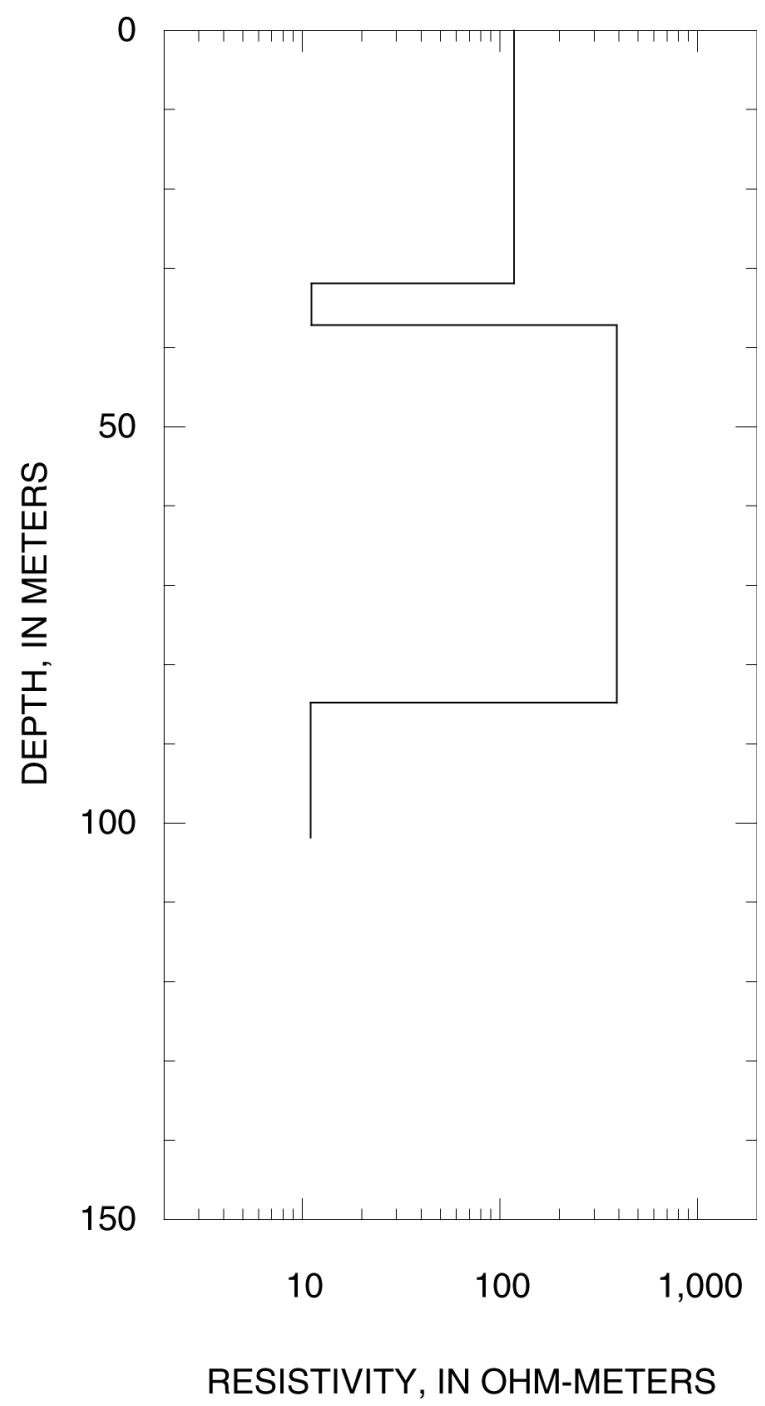

Figure 5-35. Sounding MIA126 
MIA127

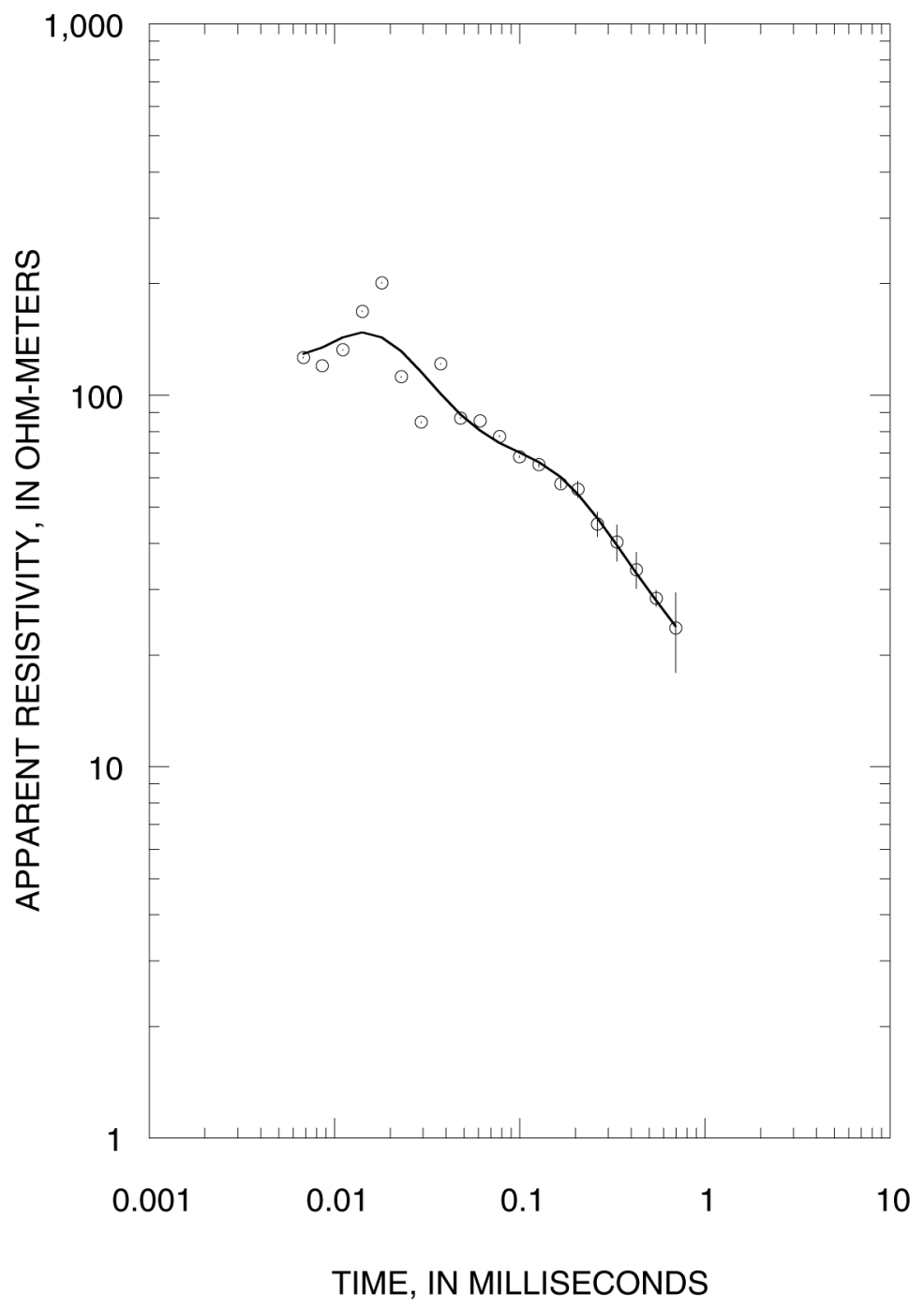

MIA127

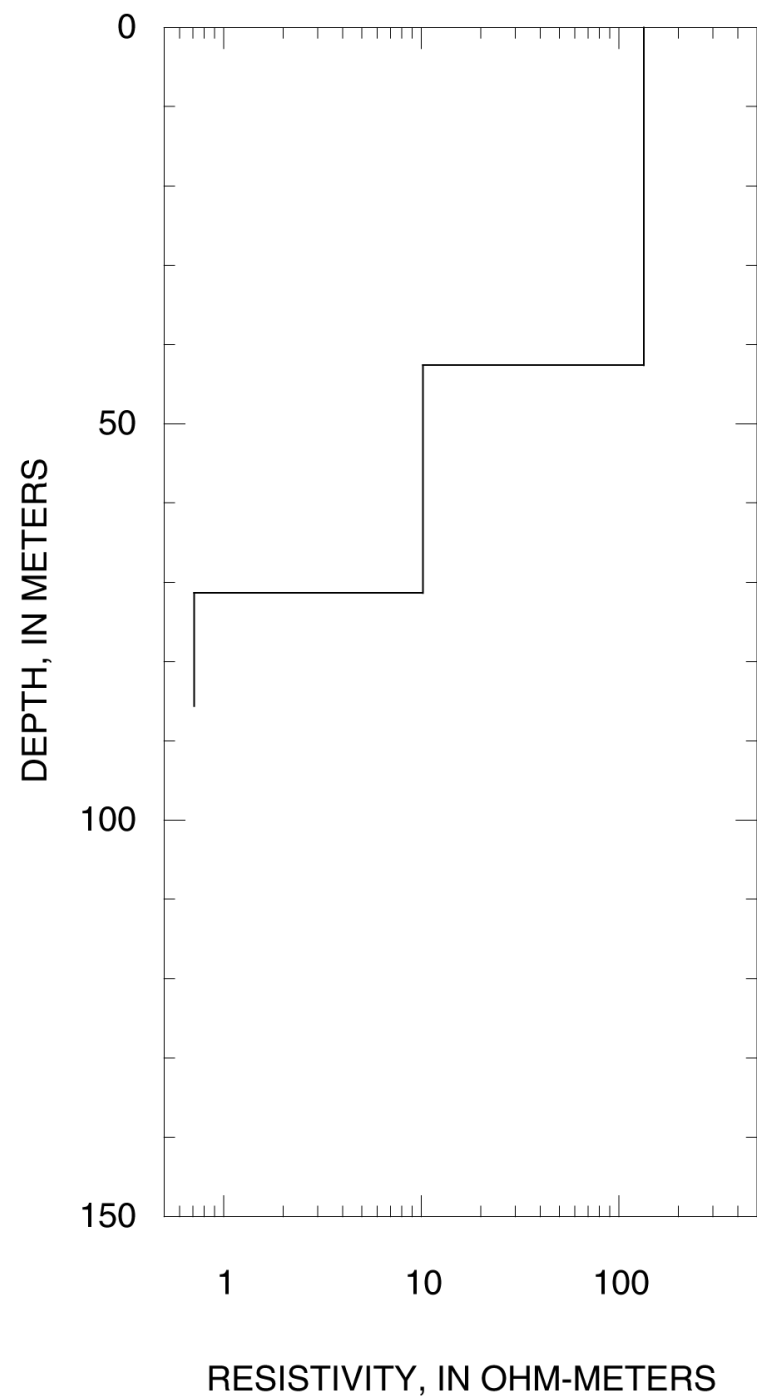

Figure 5-36. Sounding MIA127 
MIA128

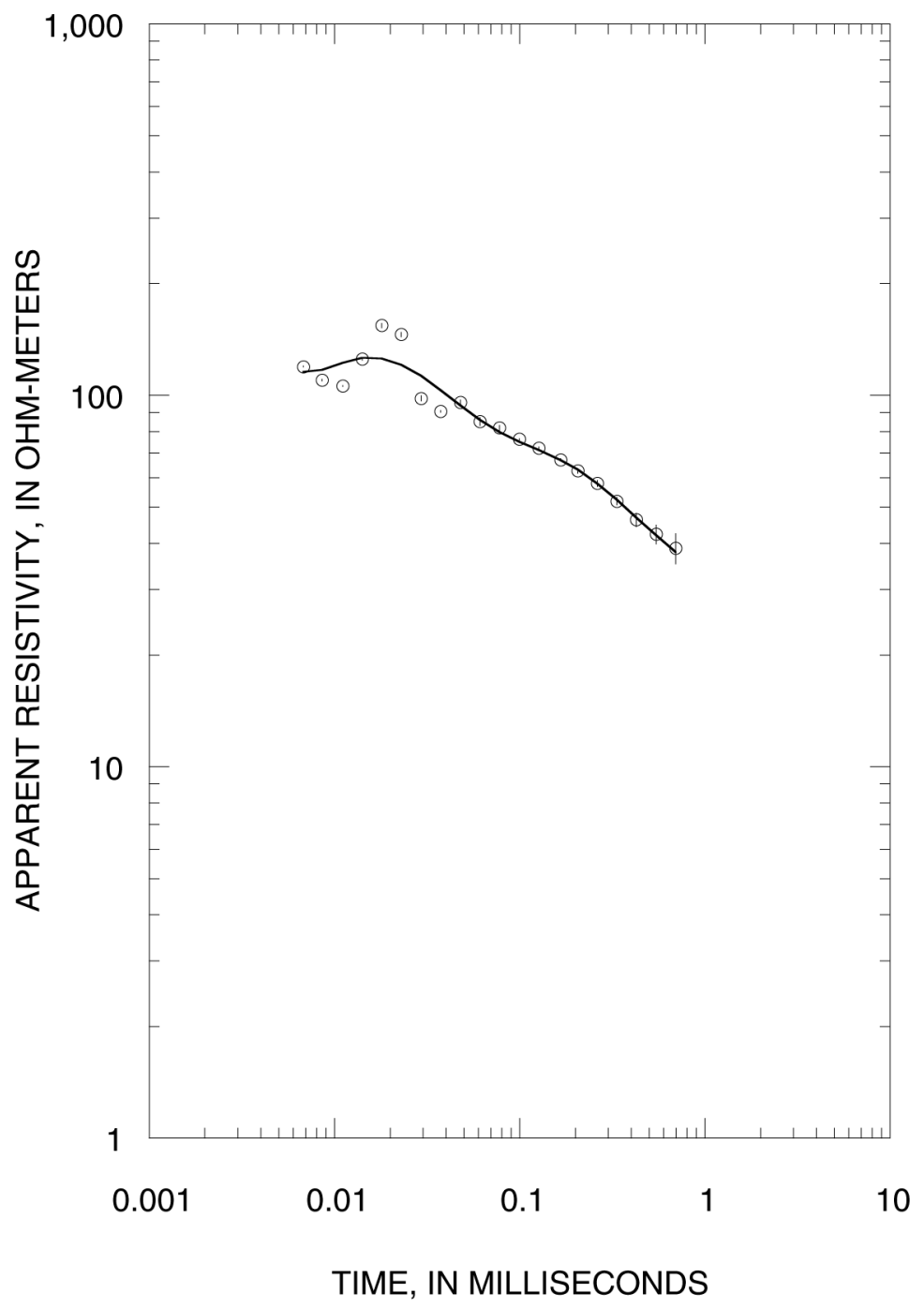

MIA128

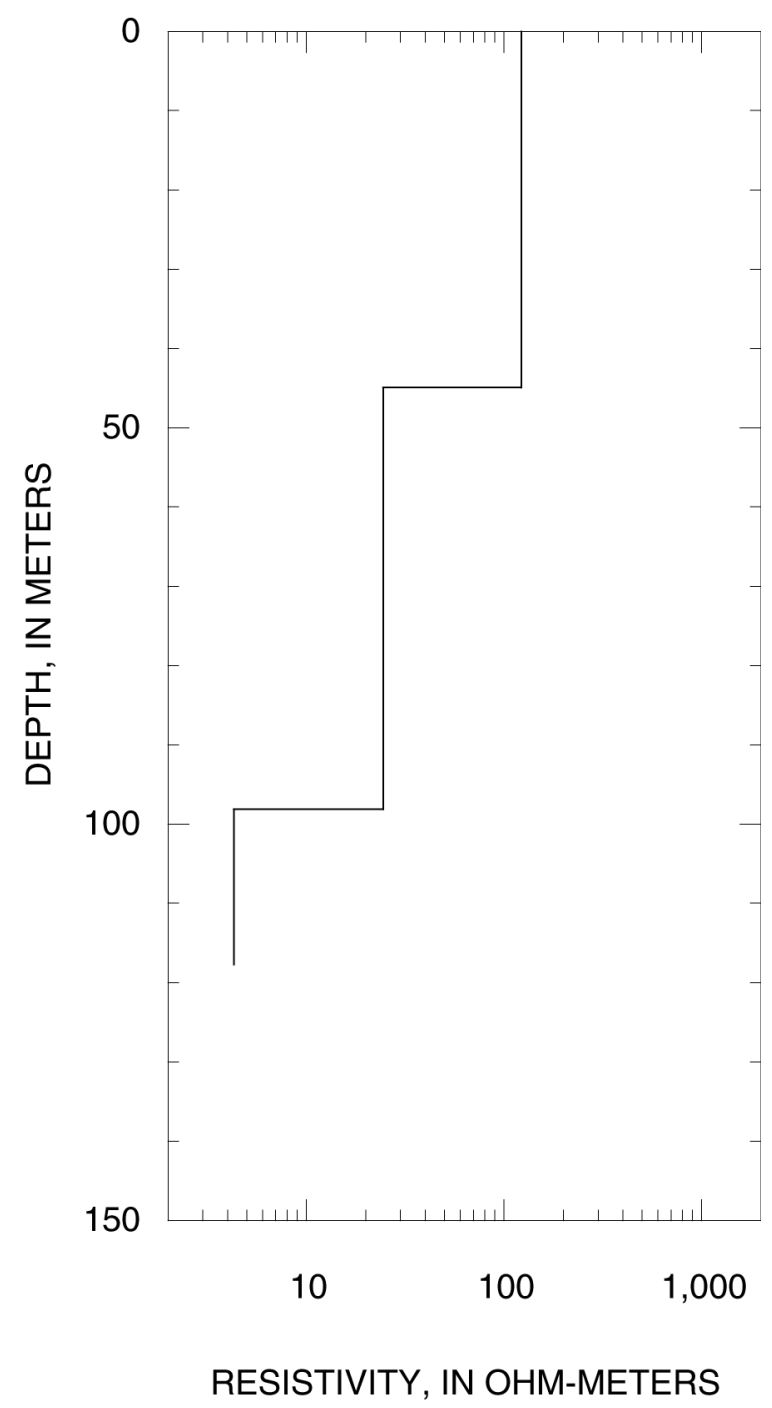

Figure 5-37. Sounding MIA128 
MIA129

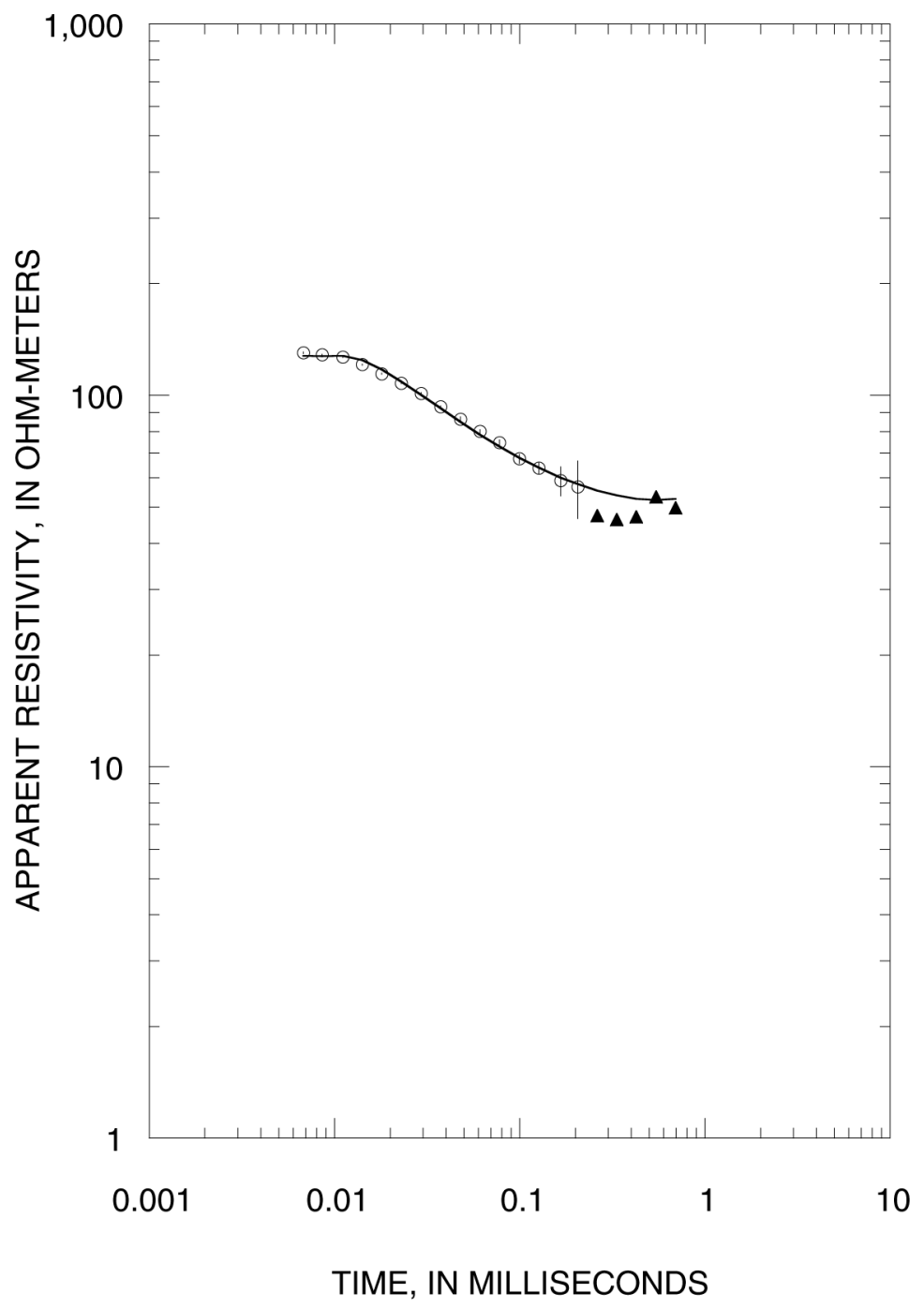

MIA129

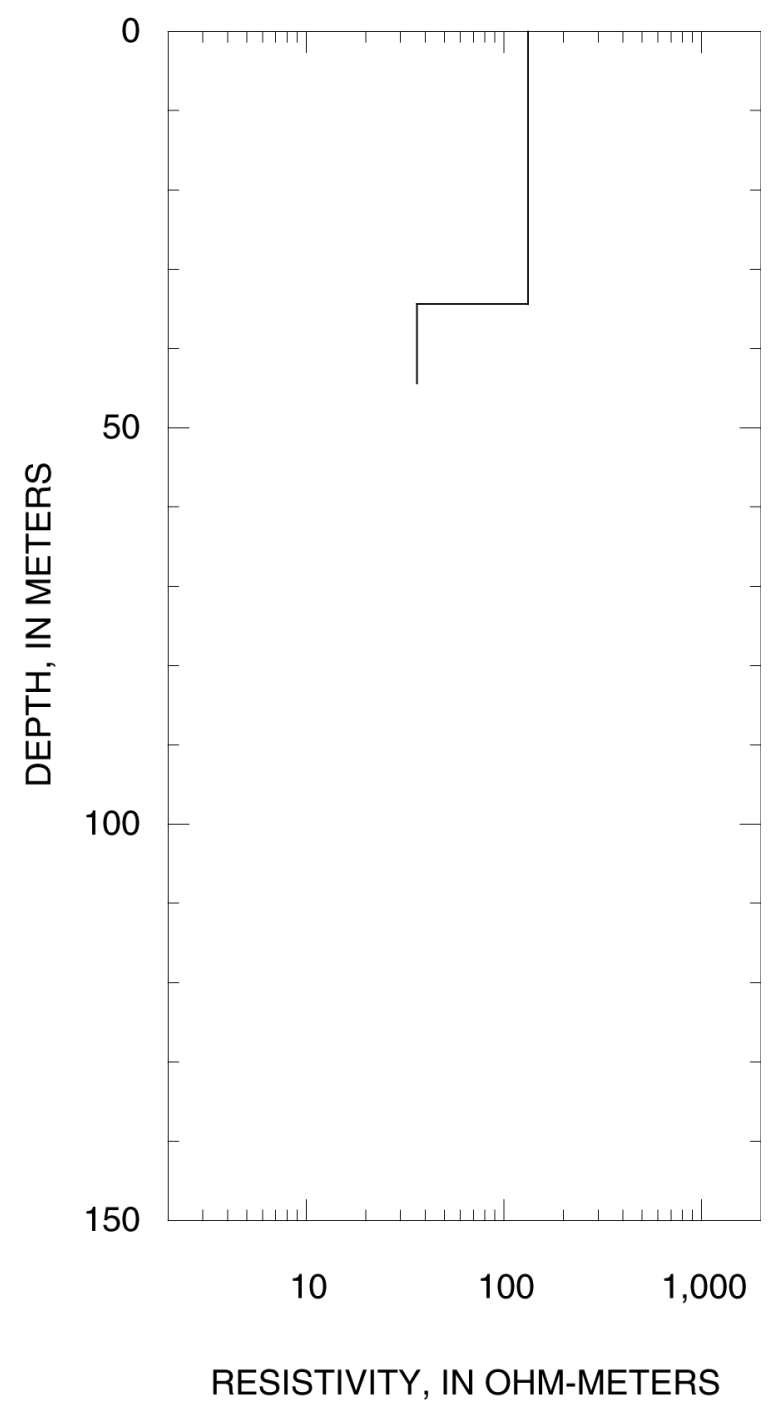

Figure 5-38. Sounding MIA129 
MIA130

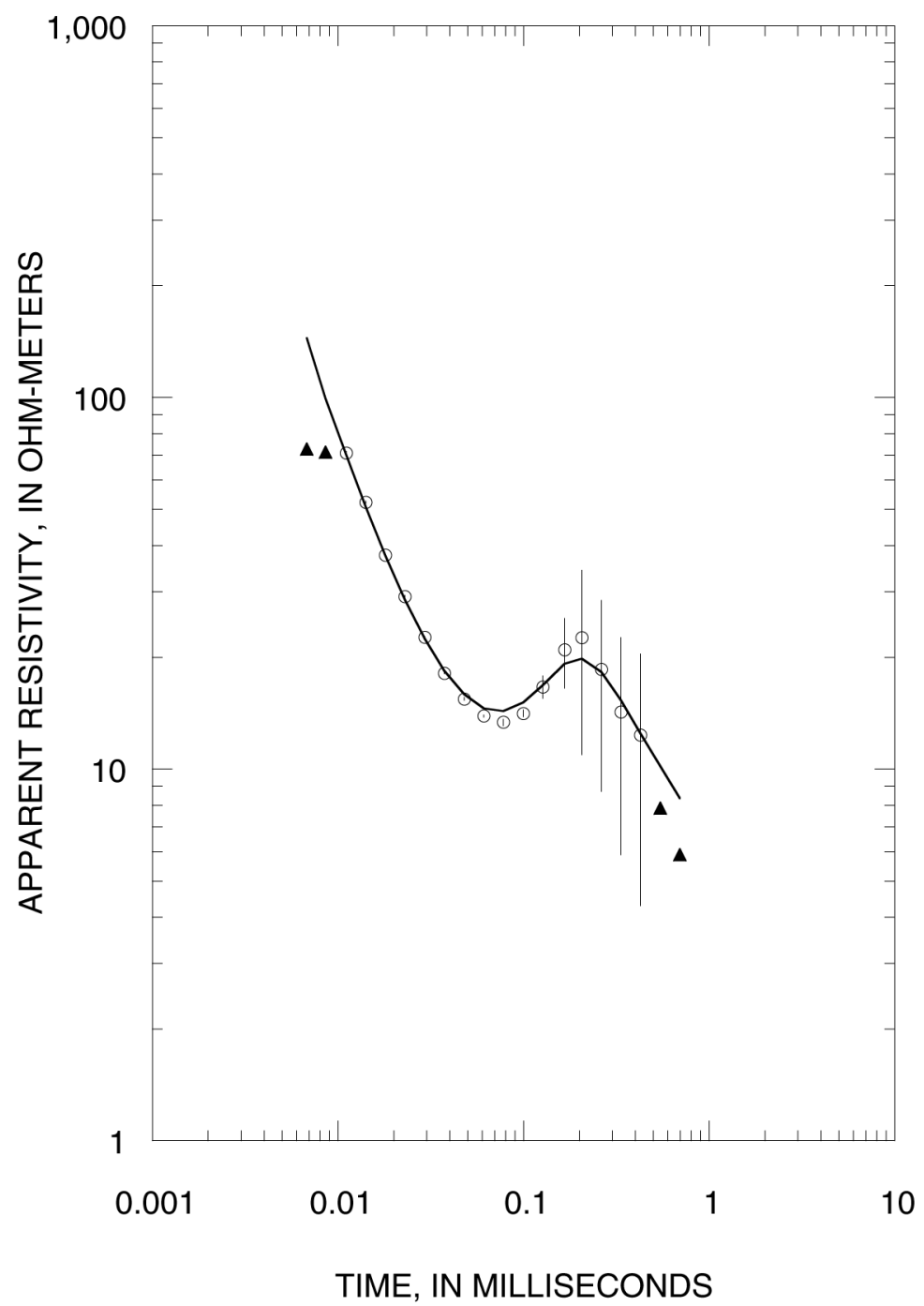

Figure 5-39. Sounding MIA130

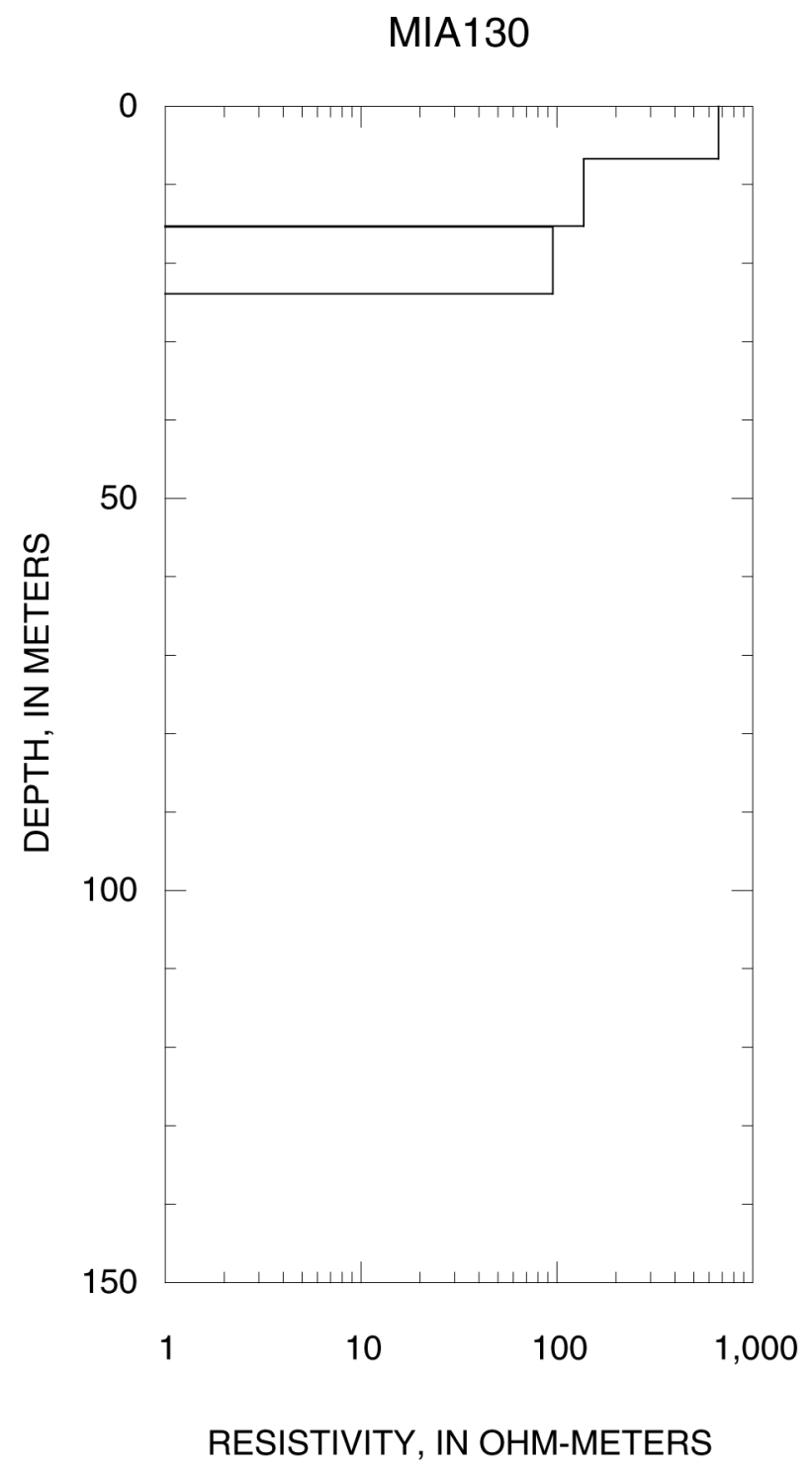


MIA131

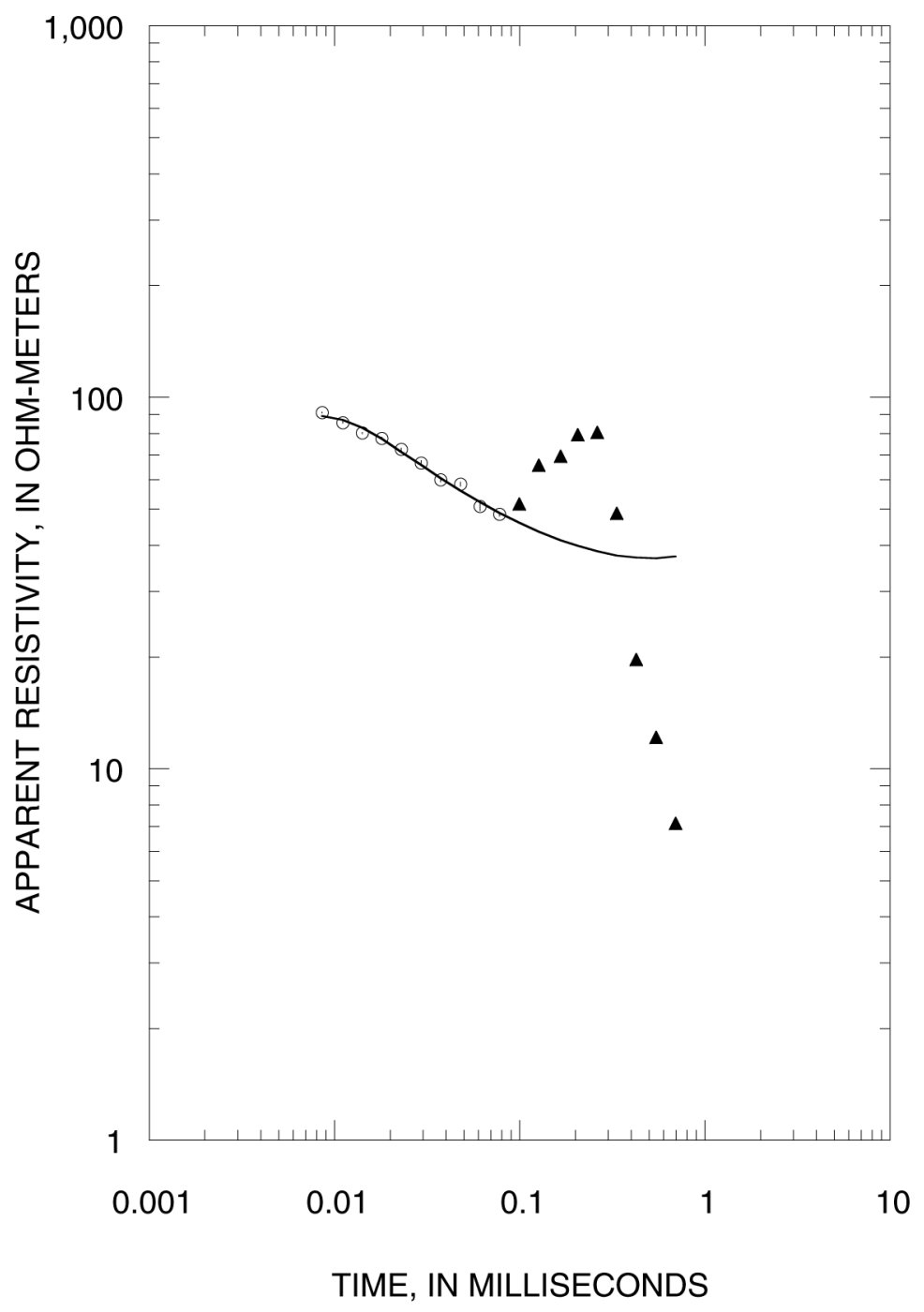

MIA131

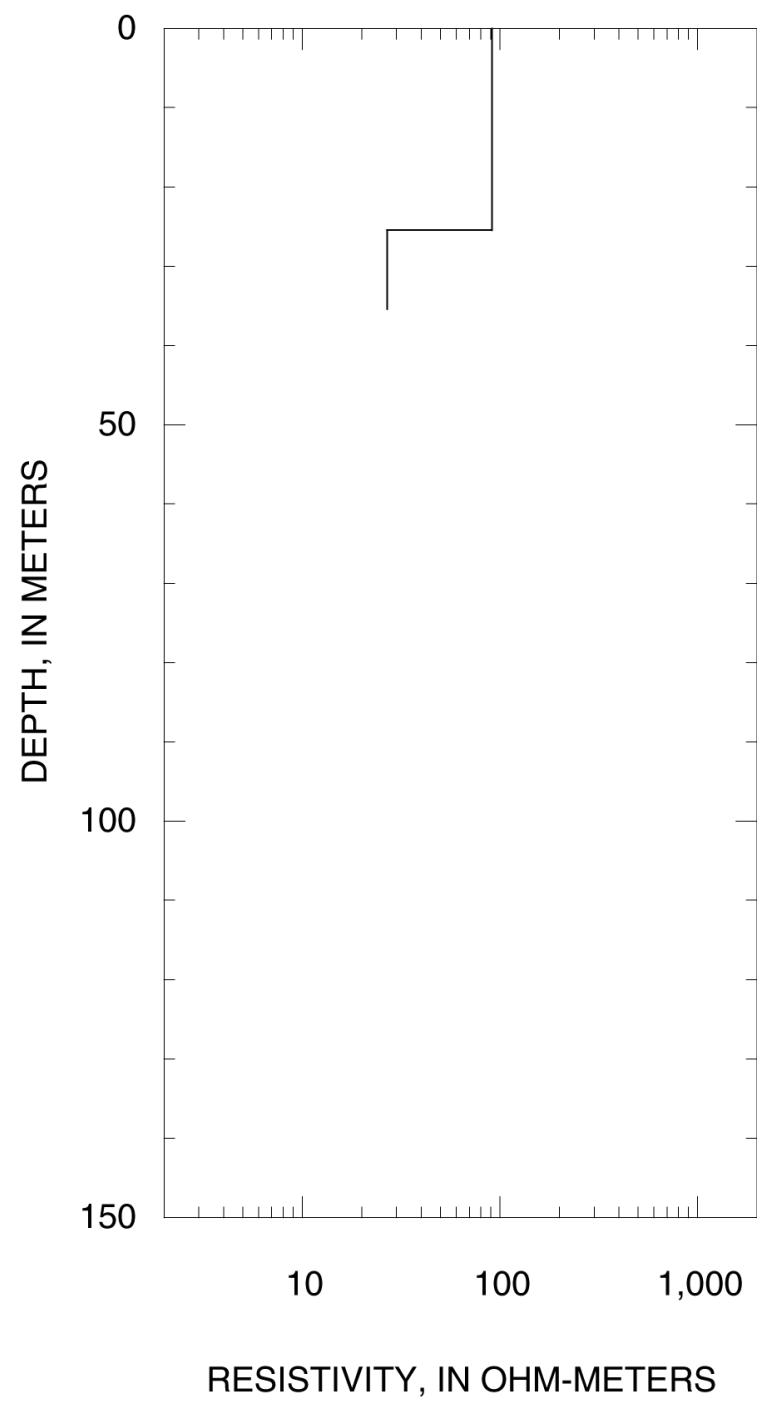

Figure 5-40. Sounding MIA131 
MIA132

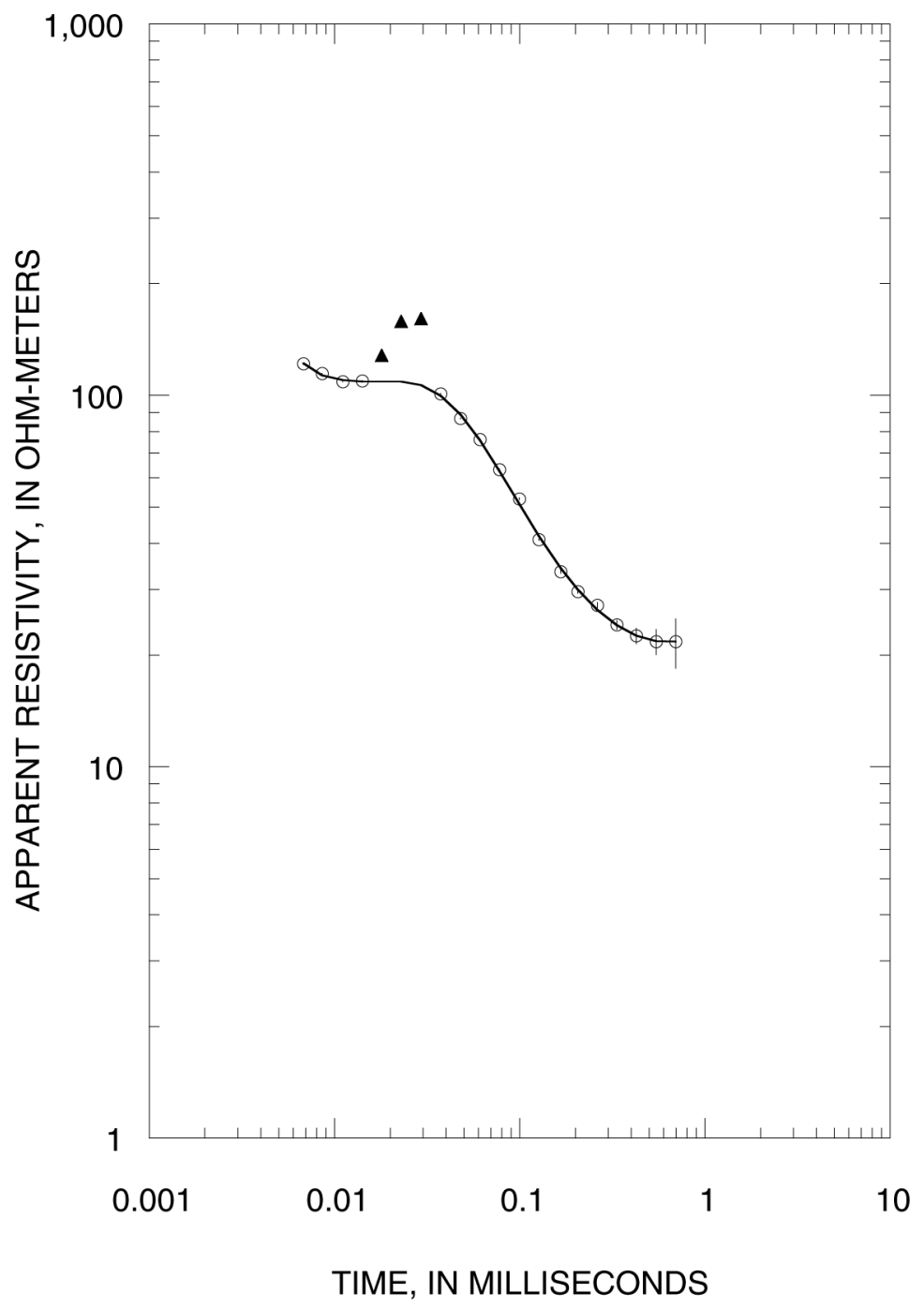

MIA132

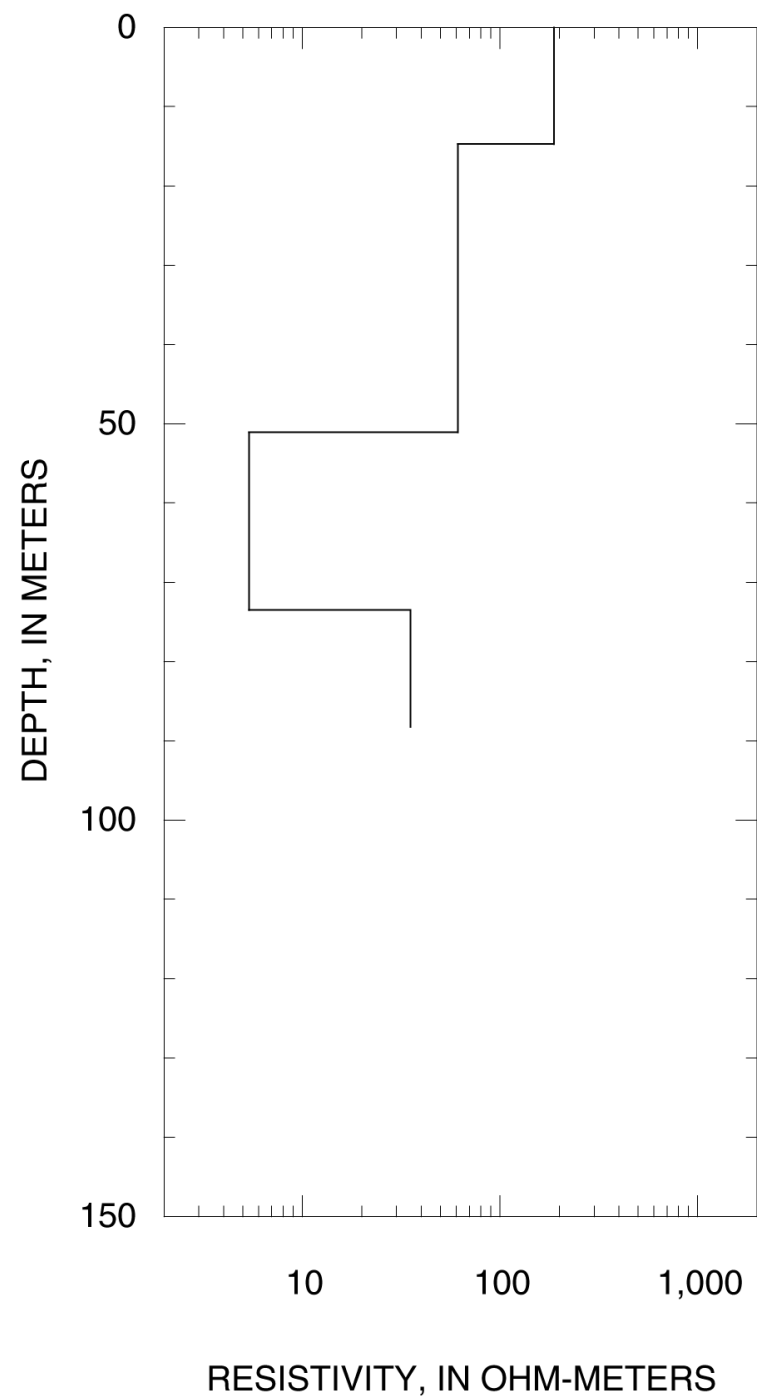

Figure 5-41. Sounding MIA132 
MIA133

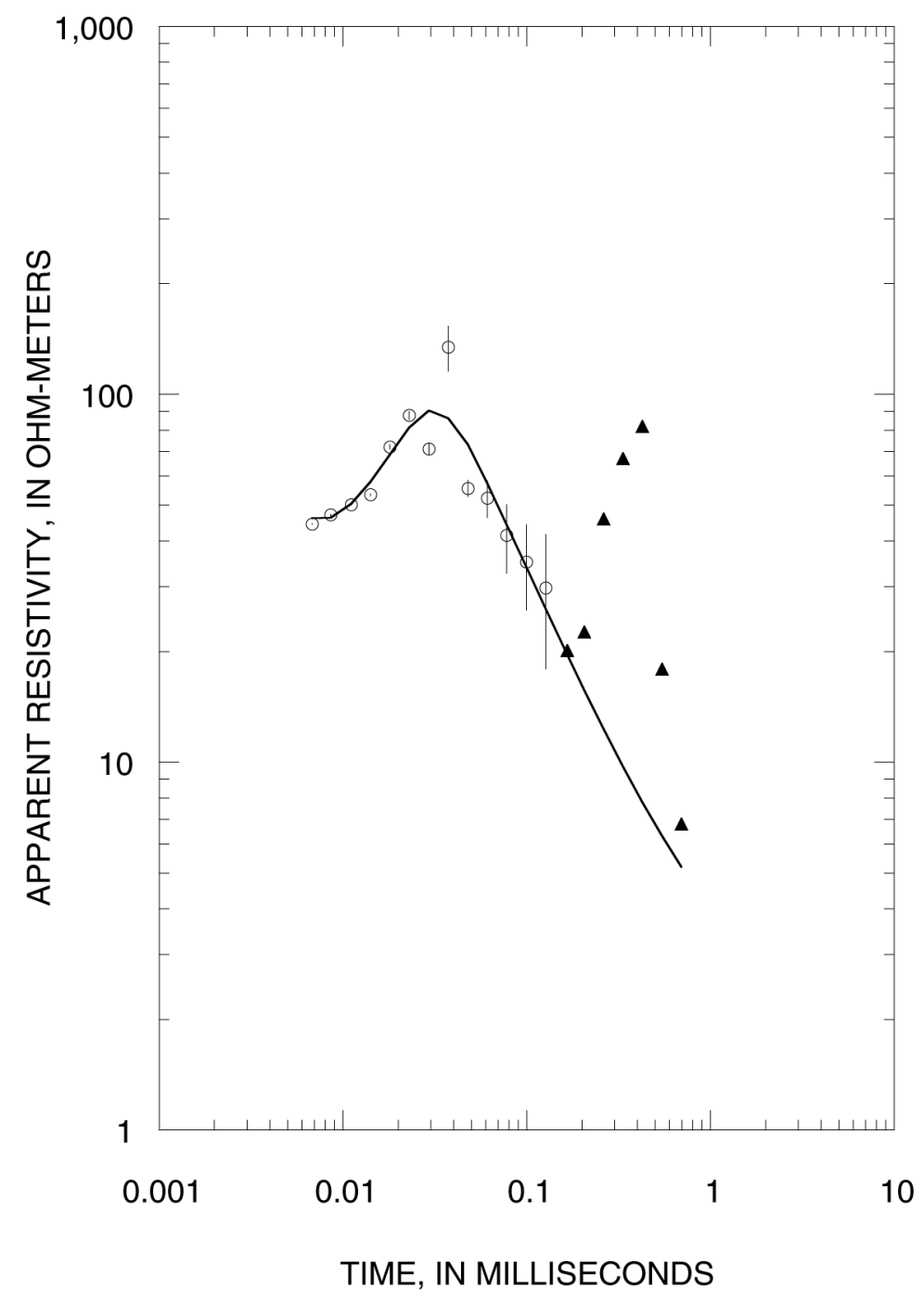

Figure 5-42. Sounding MIA133
MIA133

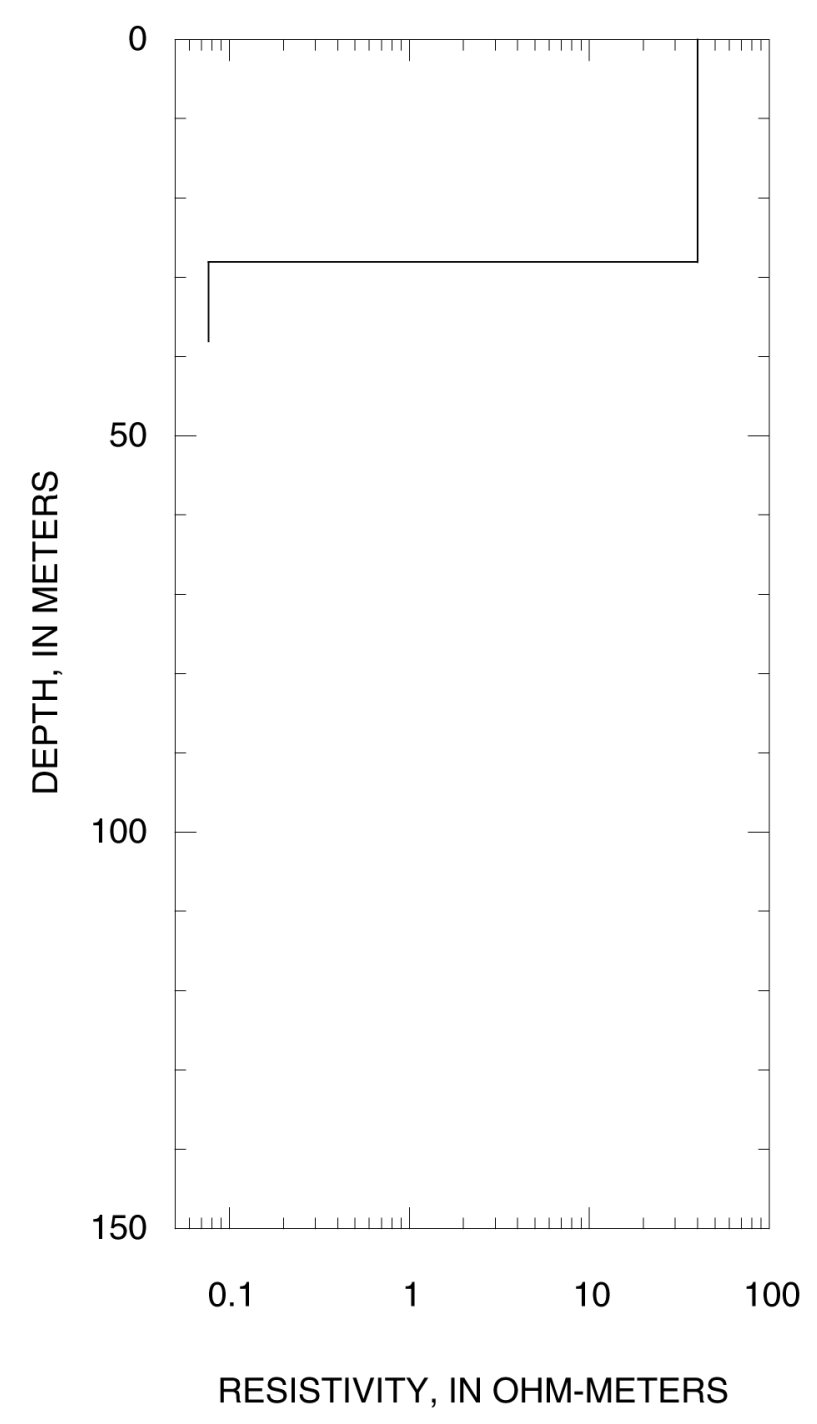


MIA134

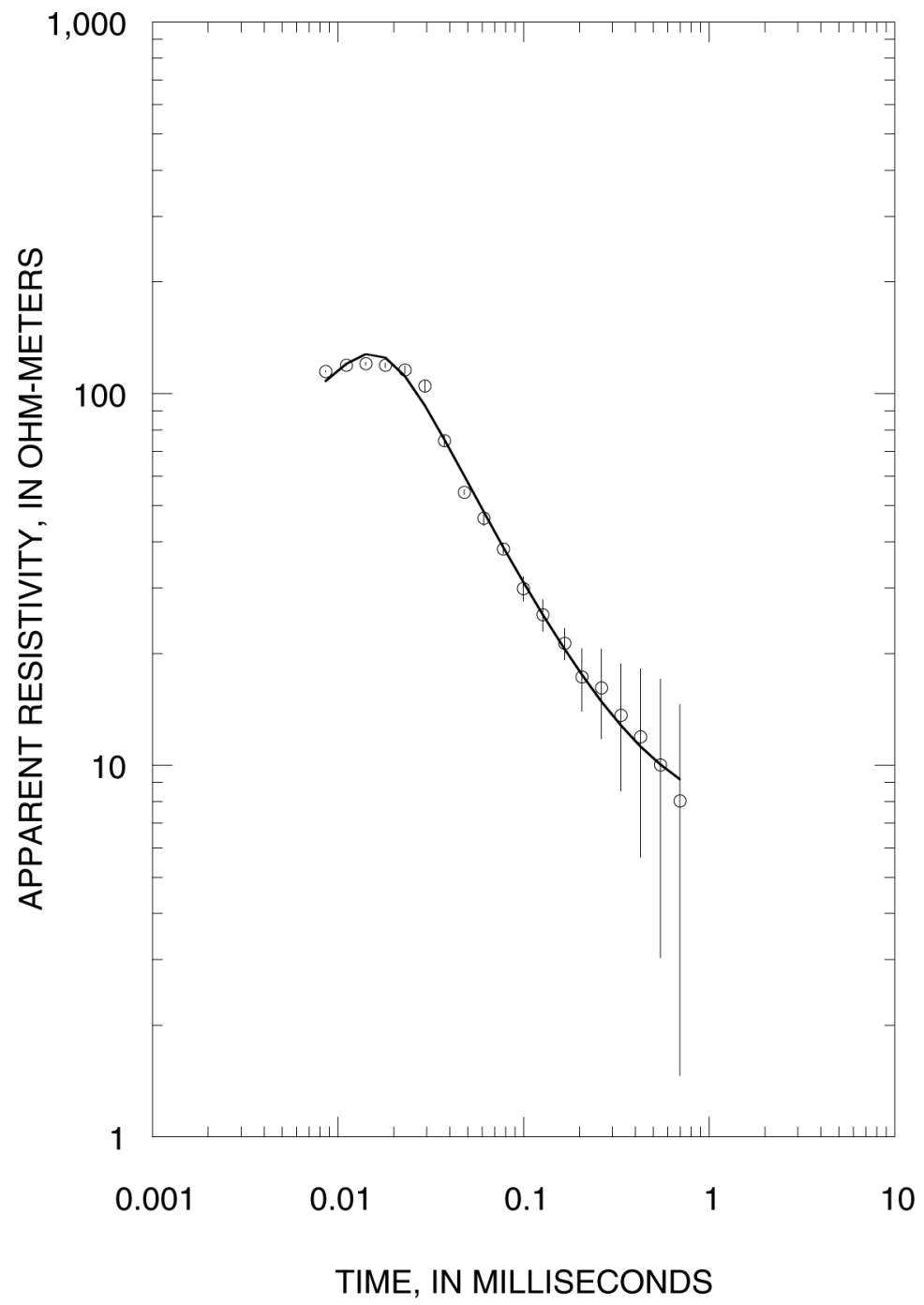

Figure 5-43. Sounding MIA134
MIA134

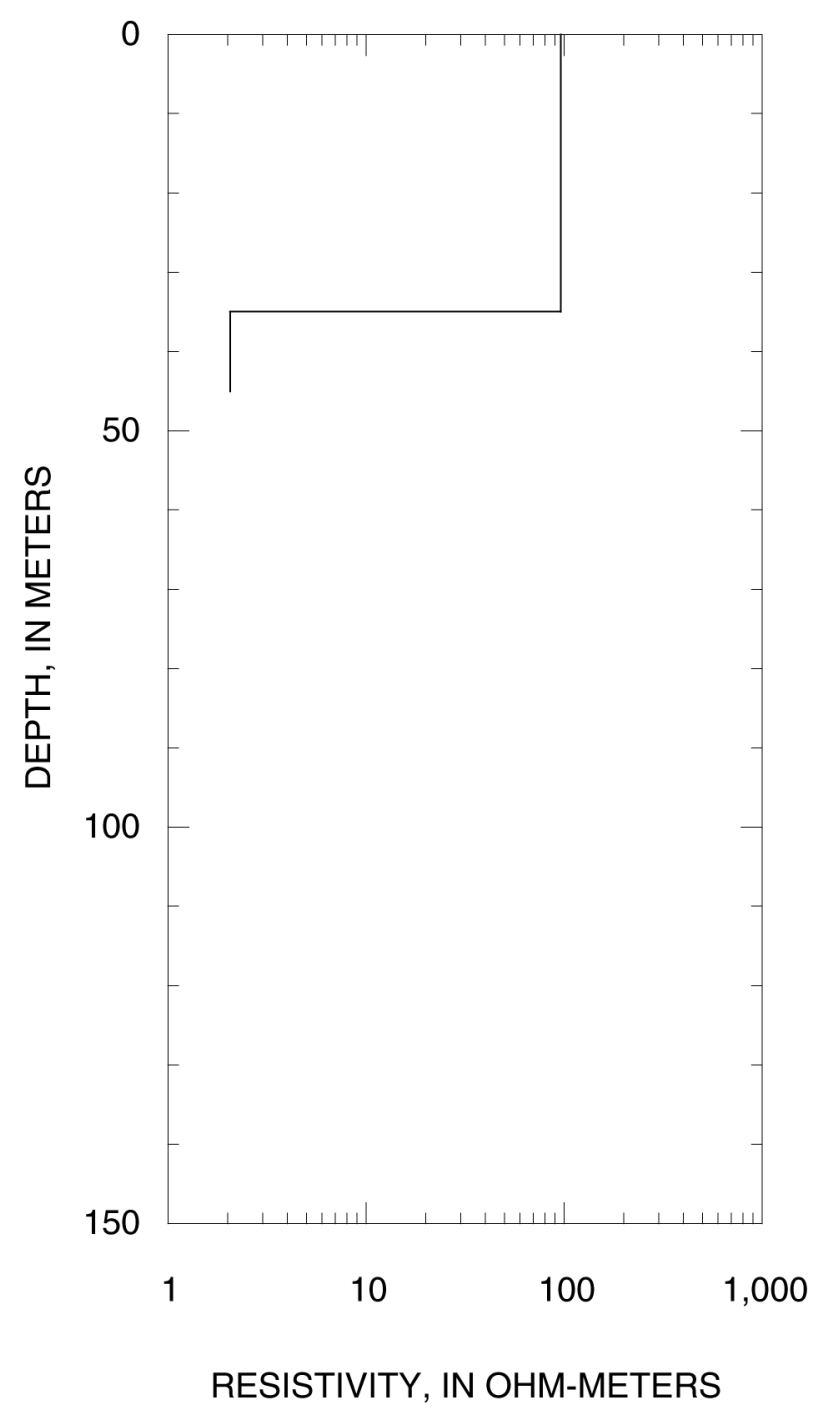


MIA135

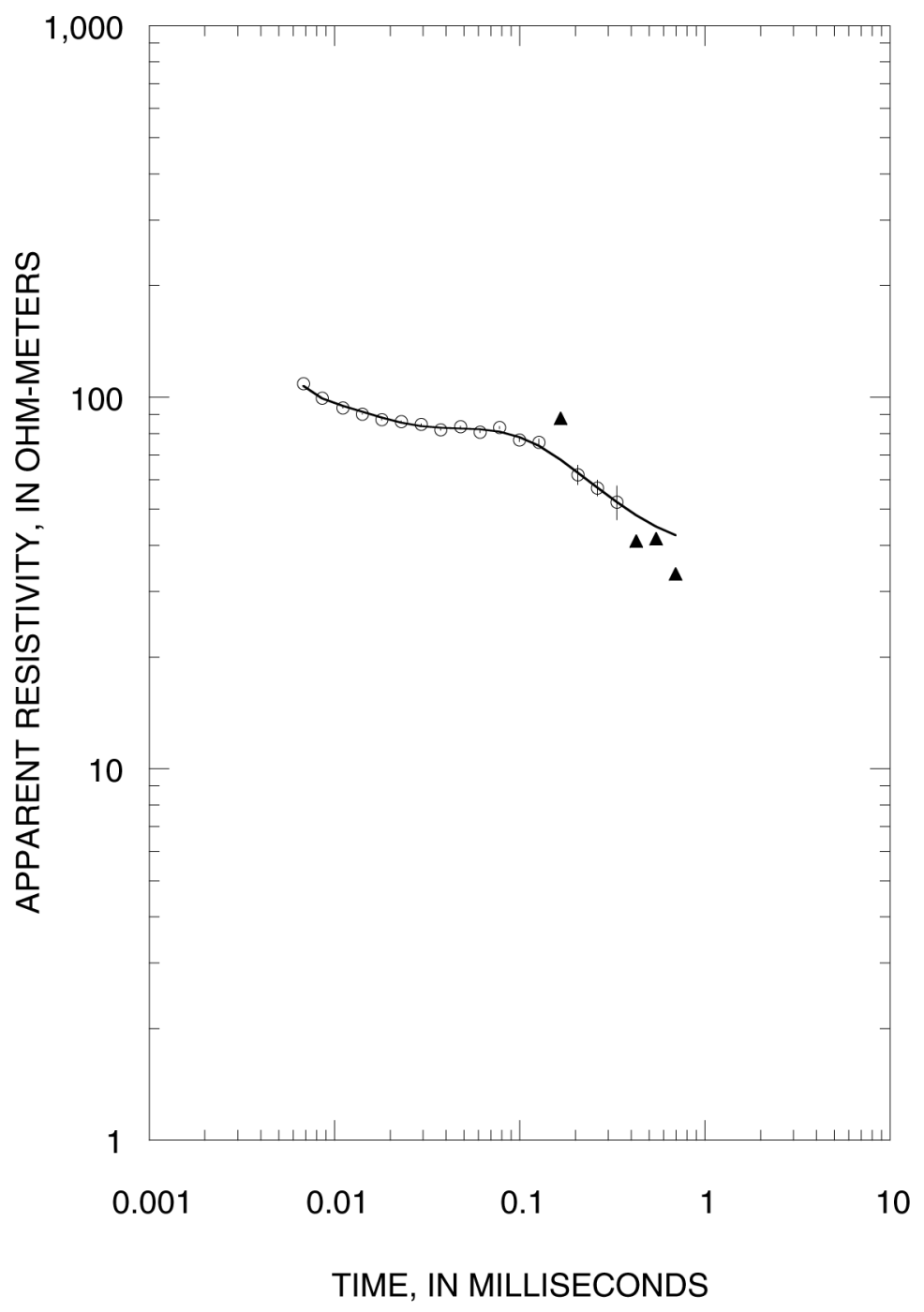

MIA135

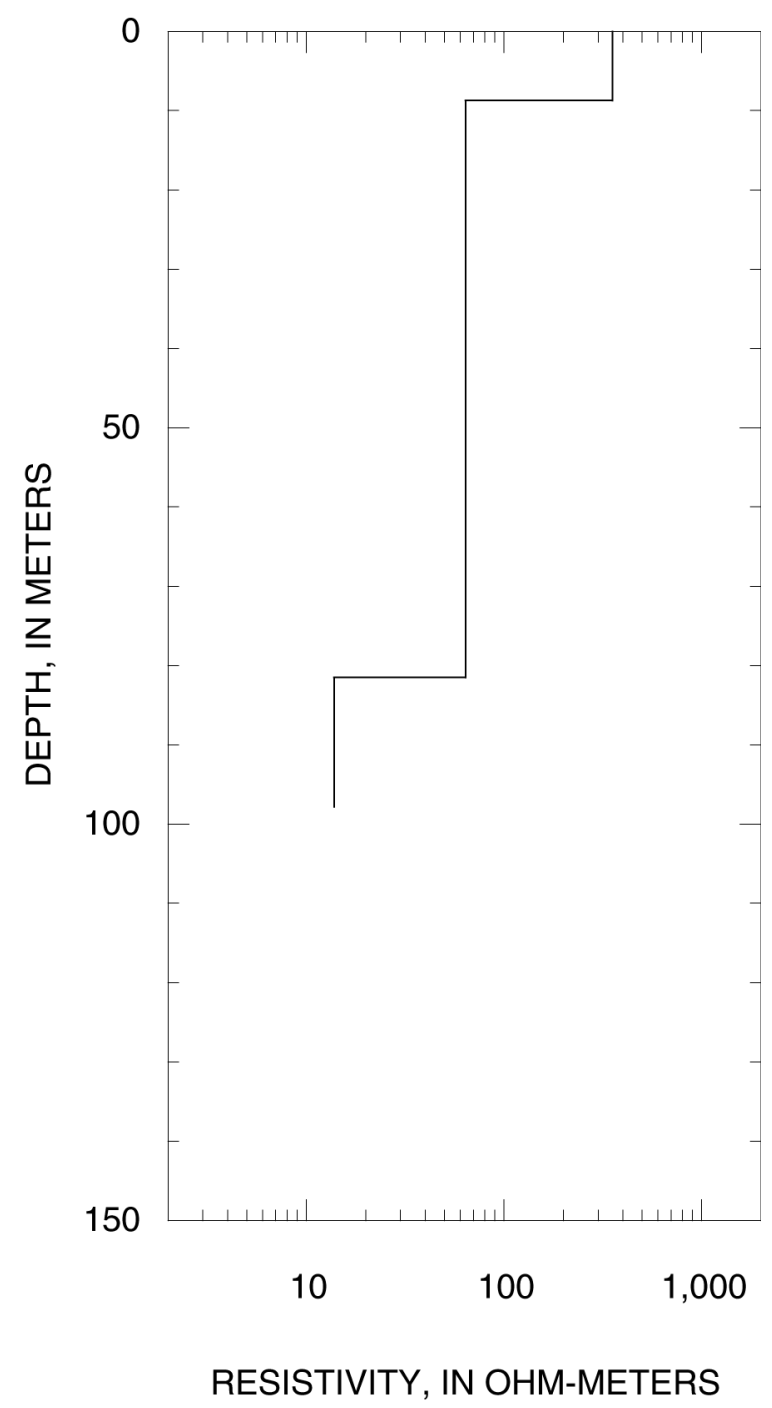

Figure 5-44. Sounding MIA135 
MIA136

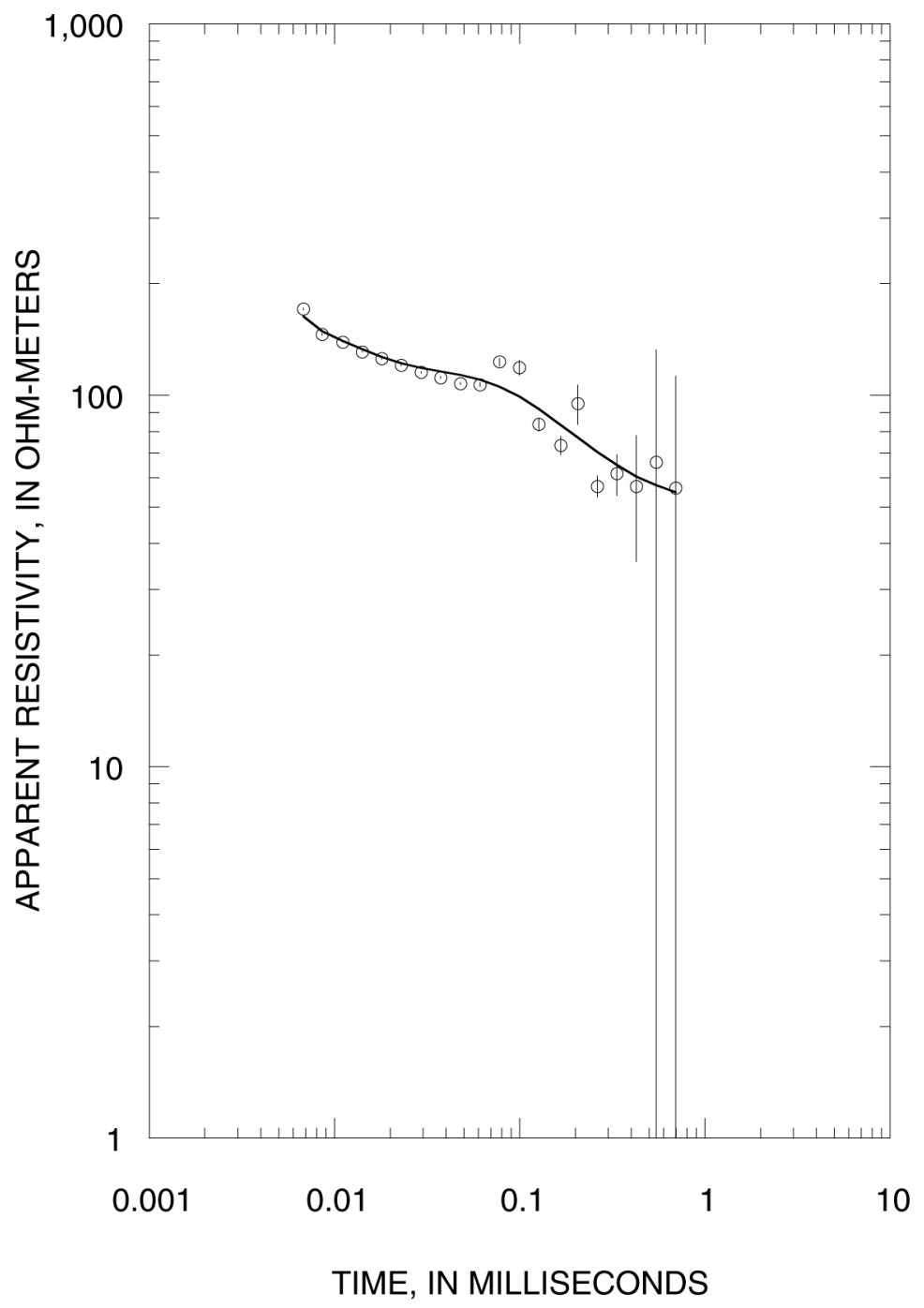

MIA136

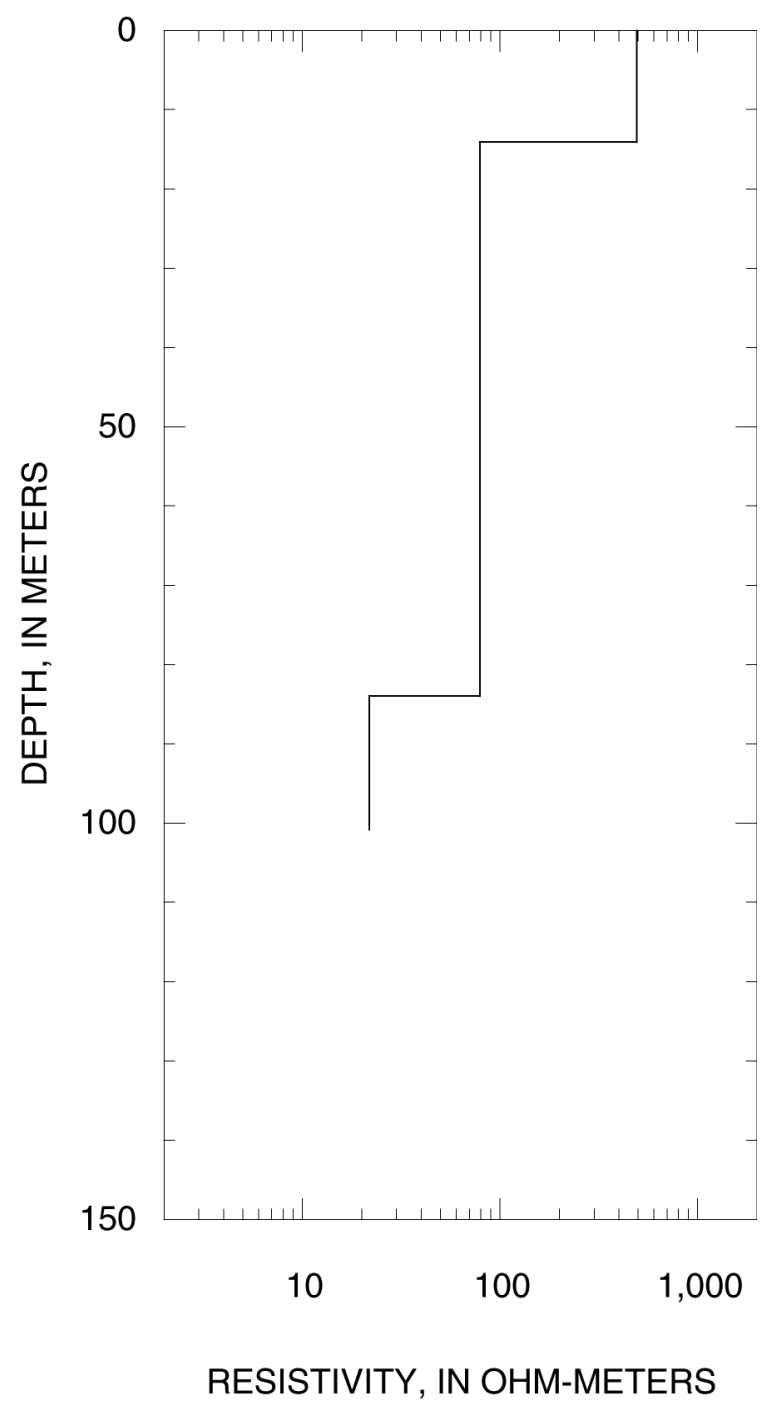

Figure 5-45. Sounding MIA136 
MIA137

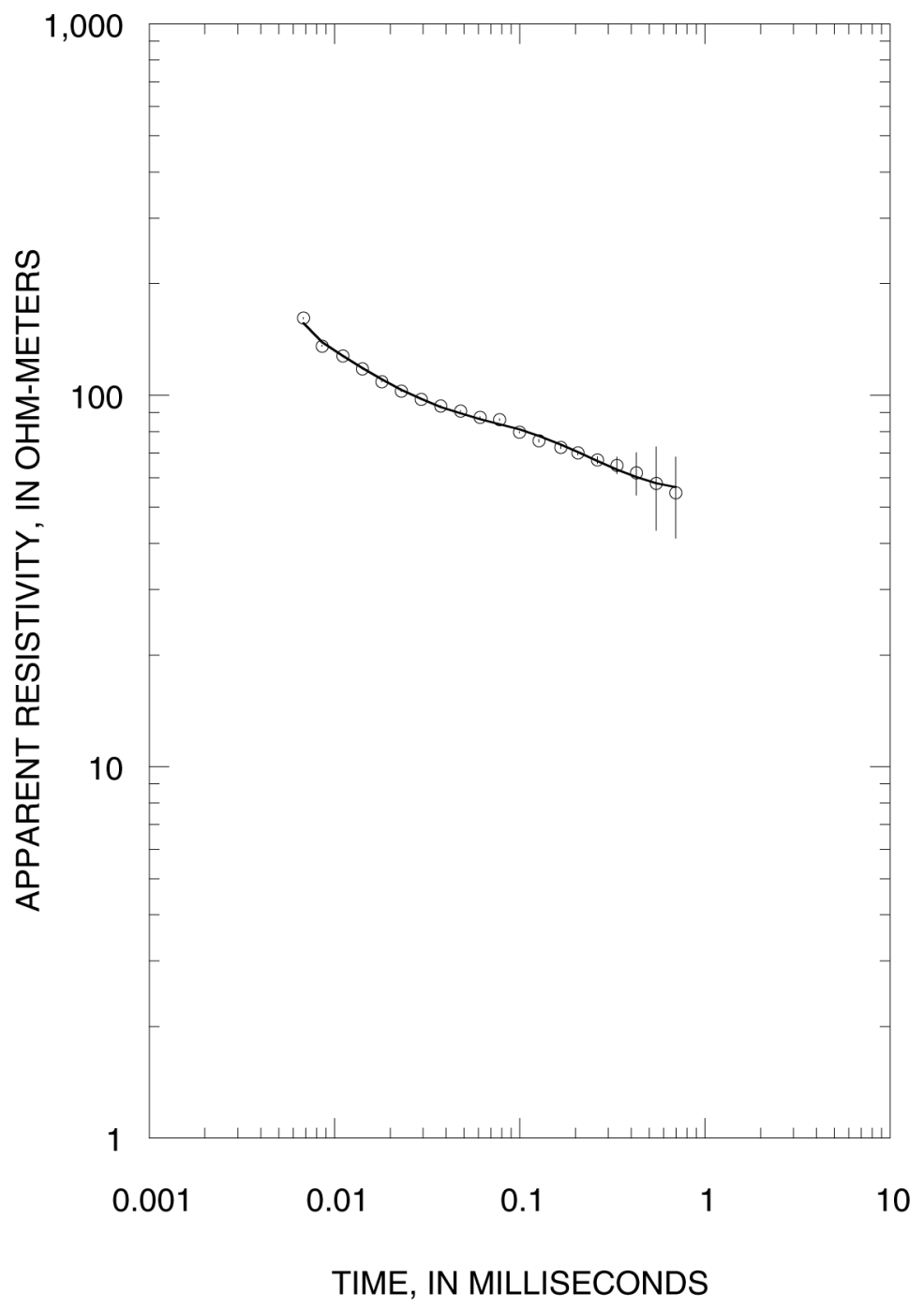

MIA137

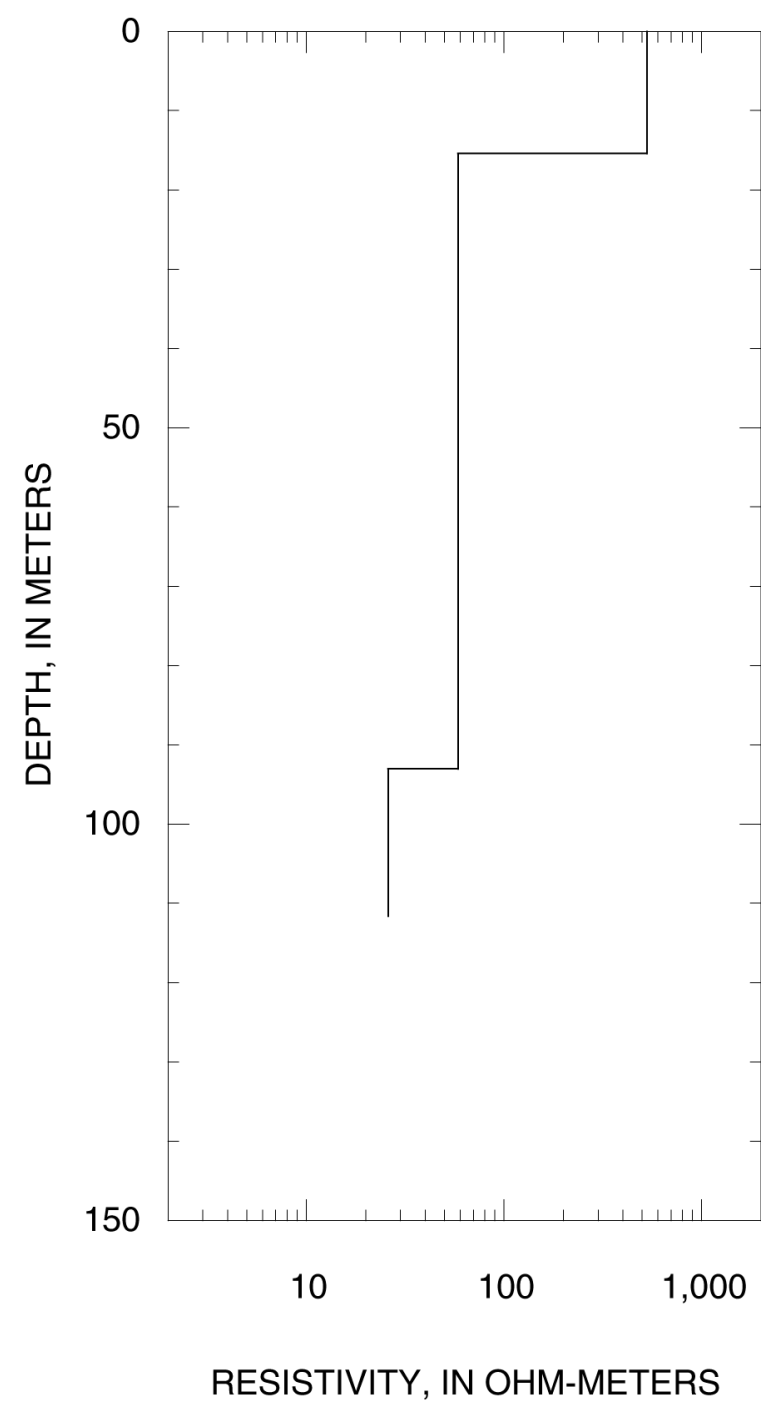

Figure 5-46. Sounding MIA137 
Appendix 6 TEM Sounding Report Files: 2009 
Sounding: MIA201

Client: US Geological Survey

(west side)

Project: Saltwater Intrusion

County: Miami-Dade County

TX loop size: X[m] $37.9 \quad \mathrm{Y}[\mathrm{m}] \quad 37.9$

$\begin{array}{cccc}\text { Model } & \text { Resistivity } & \text { Thickness } & \text { Elevation } \\ \text { Layer } & {[\text { ohm-m] }} & {[\mathrm{m}]} & {[\mathrm{m}]} \\ 1 & 132.200 & 30.6 & 0.0 \\ 2 & 28.680 & 53.4 & -30.6 \\ 3 & 6.030 & -- & -84.0\end{array}$

System: EM-58 Freq[Hz]: 285 Data Set Code: uh TX Cur[A]: 2.55 Turn Off[usec]: 2.5

RX Moment[turns-m^2]: 31.4 Gain Setting: 2

\begin{tabular}{|c|c|c|c|c|}
\hline $\begin{array}{l}\text { Time } \\
{[\mathrm{ms}]}\end{array}$ & $\begin{array}{l}\text { rhoa_obs } \\
\text { [ohm-m] }\end{array}$ & $\begin{array}{c}\text { obs err } \\
{\left[\frac{\overline{0}}{0}\right]}\end{array}$ & mask & $\begin{array}{l}\text { rhoa_ca } \\
{[\text { ohm-m ] }}\end{array}$ \\
\hline 0.00680 & 133.9 & 0.4 & $u$ & 129.7 \\
\hline 0.00860 & 124.5 & 0.2 & $u$ & 126.8 \\
\hline 0.01110 & 120.6 & 0.1 & $\mathrm{u}$ & 123.5 \\
\hline 0.01410 & 115.1 & 0.2 & $\mathrm{u}$ & 116.3 \\
\hline 0.01800 & 105.8 & 0.1 & u & 106.9 \\
\hline 0.02300 & 97.9 & 0.2 & u & 97.1 \\
\hline 0.02940 & 89.0 & 0.2 & $\mathrm{u}$ & 88.0 \\
\hline 0.03750 & 81.4 & 0.2 & u & 80.3 \\
\hline 0.04790 & 75.0 & 0.2 & u & 74. \\
\hline 0.06110 & 69.9 & 0.2 & u & 69. \\
\hline 0.07790 & 66.0 & 0.2 & u & 65.9 \\
\hline 0.09930 & 61.9 & 0.3 & u & 62. \\
\hline 0.12670 & 58.7 & 0.4 & $u$ & 59. \\
\hline 0.16640 & 54.9 & 0.6 & $u$ & 55 \\
\hline 0.20600 & 50.8 & 0.9 & $u$ & 51 \\
\hline 0.26280 & 47.0 & 1.4 & $u$ & 46 \\
\hline 0.33520 & 42.3 & 2.0 & $u$ & 42 \\
\hline 0.42770 & 38.3 & 3.1 & $u$ & 37 \\
\hline 560 & 34.6 & 5.0 & u & 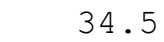 \\
\hline 0. & 32.3 & 7.8 & u & \\
\hline
\end{tabular}

Date: 23-FEB-09

UTM Coord: $E[\mathrm{~m}]$

Elevation $[\mathrm{m}]$ :

$557815.0 \quad \mathrm{~N}[\mathrm{~m}] \quad 2812968.0$ 0.0

RX location: $\mathrm{X}[\mathrm{m}]$

$0.0 \quad \mathrm{Y}[\mathrm{m}] \quad 0.0$

Fit Error [응 1.829

System: EM-58 Freq[Hz]: 30 Data Set Code: hi TX Cur $[A]: 2.40$ Turn Off [usec] : 2.5

RX Moment [turns-m^2]: 31.4 Gain Setting: 5

$\begin{array}{rrrrrr}\text { Time } & \begin{array}{c}\text { rhoa_obs } \\ \text { [ohm-m] }\end{array} & \begin{array}{c}\text { obs_err } \\ {\left[\frac{\text { o }}{0}\right]}\end{array} & \text { mask } & \begin{array}{c}\text { rhoa_cal } \\ {[\text { ohm-m] }}\end{array} \\ 1 & 0.08800 & 64.5 & 0.6 & \mathrm{u} & 63.7 \\ 2 & 0.10600 & 61.5 & 0.6 & \mathrm{u} & 61.1 \\ 3 & 0.13100 & 58.1 & 0.6 & \mathrm{u} & 57.9 \\ 4 & 0.16100 & 54.3 & 0.6 & \mathrm{u} & 54.2 \\ 5 & 0.20000 & 49.6 & 0.7 & \mathrm{u} & 49.7 \\ 6 & 0.25000 & 44.6 & 0.5 & \mathrm{u} & 44.7 \\ 7 & 0.31400 & 39.6 & 0.6 & \mathrm{u} & 39.7 \\ 8 & 0.39500 & 34.7 & 0.8 & \mathrm{u} & 34.9 \\ 9 & 0.49900 & 30.3 & 1.5 & \mathrm{u} & 30.6 \\ 10 & 0.63100 & 26.9 & 1.5 & \mathrm{u} & 26.9 \\ 11 & 0.79900 & 23.8 & 2.2 & \mathrm{u} & 23.8 \\ 12 & 1.01000 & 21.2 & 3.3 & \mathrm{u} & 21.1 \\ 13 & 1.28000 & 18.9 & 3.6 & \mathrm{u} & 18.9 \\ 14 & 1.63000 & 16.8 & 7.0 & \mathrm{u} & 17.0 \\ 15 & 2.08000 & 14.5 & 11.5 & \mathrm{~m} & 15.6 \\ 16 & 2.64000 & 12.0 & 16.8 & \mathrm{~d} & - \\ 17 & 3.37000 & 9.5 & 17.4 & \mathrm{~d} & - \\ 18 & 4.29000 & 7.3 & 16.3 & \mathrm{~d} & - \\ 19 & 5.47000 & 5.3 & 25.2 & \mathrm{~d} & - \\ 20 & 6.97000 & 3.7 & 20.1 & \mathrm{~d} & -\end{array}$


Sounding: MIA202

Location: SW 360th Street

Project: Saltwater Intrusion

County: Miami-Dade County

TX loop size: X $[\mathrm{m}] \quad 37.9 \quad \mathrm{Y}[\mathrm{m}] \quad 37.9$

$\begin{array}{cccc}\text { Model } & \text { Resistivity } & \text { Thickness } & \text { Elevation } \\ \text { Layer } & {[\text { ohm-m] }} & {[\mathrm{m}]} & {[\mathrm{m}]} \\ 1 & 161.500 & 21.5 & 0.0 \\ 2 & 11.380 & 14.2 & -21.5 \\ 3 & 114.800 & 39.8 & -35.8 \\ 4 & 7.930 & -- & -75.7\end{array}$

System: EM-58 Freq[Hz]: 285 Data Set Code: uh TX Cur[A]: 2.55 Turn Off[usec]: 2.5

RX Moment[turns-m^2]: 31.4 Gain Setting: 2

$\begin{array}{rrrrrr}\text { Time } & \begin{array}{c}\text { rhoa_obs } \\ \text { [ohm-m] }\end{array} & \begin{array}{c}\text { obs_err } \\ {\left[\frac{\mathrm{o}}{0}\right]}\end{array} & \text { mask } & \begin{array}{c}\text { rhoa_cal } \\ \text { [ohm-m] }\end{array} \\ 1 & 0.00680 & 140.2 & 0.3 & \mathrm{u} & 139.4 \\ 2 & 0.00860 & 114.1 & 0.2 & \mathrm{u} & 115.8 \\ 3 & 0.01110 & 96.6 & 0.3 & \mathrm{u} & 95.7 \\ 4 & 0.01410 & 79.0 & 0.3 & \mathrm{u} & 78.7 \\ 5 & 0.01800 & 64.7 & 0.3 & \mathrm{u} & 65.0 \\ 6 & 0.02300 & 54.5 & 0.3 & \mathrm{u} & 54.7 \\ 7 & 0.02940 & 47.3 & 0.3 & \mathrm{u} & 47.2 \\ 8 & 0.03750 & 42.4 & 0.3 & \mathrm{u} & 42.2 \\ 9 & 0.04790 & 39.1 & 0.3 & \mathrm{u} & 39.0 \\ 10 & 0.06110 & 37.1 & 0.3 & \mathrm{u} & 37.1 \\ 11 & 0.07790 & 36.2 & 0.3 & \mathrm{u} & 36.4 \\ 12 & 0.09930 & 36.0 & 0.3 & \mathrm{u} & 36.4 \\ 13 & 0.12670 & 36.3 & 0.3 & \mathrm{u} & 36.9 \\ 14 & 0.16640 & 37.1 & 0.4 & \mathrm{u} & 37.3 \\ 15 & 0.20600 & 36.8 & 0.4 & \mathrm{u} & 37.1 \\ 16 & 0.26280 & 36.4 & 0.8 & \mathrm{u} & 36.2 \\ 17 & 0.33520 & 34.9 & 1.2 & \mathrm{u} & 34.6 \\ 18 & 0.42770 & 33.0 & 2.1 & \mathrm{u} & 32.7 \\ 19 & 0.54560 & 30.9 & 3.7 & \mathrm{u} & 30.8 \\ 20 & 0.69590 & 29.4 & 3.8 & \mathrm{u} & 29.2\end{array}$

Date: $23-\mathrm{FEB}-09$

UTM Coord: $\mathrm{E}[\mathrm{m}]$

Elevation $[\mathrm{m}]$ :

$558369.0 \quad \mathrm{~N}[\mathrm{~m}] \quad 2813097.0$

0.0

RX location: X $[\mathrm{m}]$

$0.0 \quad Y[m]$

0.0
Fit Error [\%]: 1.473

System: EM-58 Freq[Hz]: 30 Data Set Code: hi TX Cur $[A]: 2.50$ Turn Off[usec]: 2.5

RX Moment [turns-m^2]: $31.4 \quad$ Gain Setting: 5

$\begin{array}{ccccc}\begin{array}{c}\text { Time } \\ \text { [ms] }\end{array} & \begin{array}{c}\text { rhoa_obs } \\ \text { [ohm-m] }\end{array} & \begin{array}{c}\text { obs_err } \\ {\left[\begin{array}{c}\bar{o} \\ 0\end{array}\right.}\end{array} & \text { mask } & \begin{array}{c}\text { rhoa_cal } \\ \text { [ohm-m] }\end{array} \\ 0.08800 & 36.6 & 0.4 & \mathrm{u} & 36.2 \\ 0.10600 & 36.6 & 0.4 & \mathrm{u} & 36.3 \\ 0.13100 & 36.8 & 0.3 & \mathrm{u} & 36.5 \\ 0.16100 & 36.8 & 0.3 & \mathrm{u} & 36.6 \\ 0.20000 & 36.3 & 0.4 & \mathrm{u} & 36.2 \\ 0.25000 & 35.0 & 0.3 & \mathrm{u} & 34.9 \\ 0.31400 & 33.0 & 0.5 & \mathrm{u} & 32.9 \\ 0.39500 & 30.3 & 1.3 & \mathrm{u} & 30.4 \\ 0.49900 & 27.6 & 1.2 & \mathrm{u} & 27.8 \\ 0.63100 & 24.8 & 1.7 & \mathrm{u} & 25.2 \\ 0.79900 & 22.3 & 2.3 & \mathrm{u} & 22.9 \\ 1.01000 & 21.0 & 5.8 & \mathrm{u} & 20.9 \\ 1.28000 & 19.6 & 11.1 & \mathrm{u} & 19.2 \\ 1.63000 & 19.0 & 17.5 & \mathrm{~m} & 17.7 \\ 2.08000 & 17.2 & 15.1 & \mathrm{~m} & 16.5 \\ 2.64000 & 21.1 & 34.0 & \mathrm{~d} & - \\ 3.37000 & 28.6 & 35.0 & \mathrm{~d} & - \\ 4.29000 & 11.1 & 51.7 & \mathrm{~d} & - \\ 5.47000 & 10.0 & 66.2 & \mathrm{~d} & - \\ 6.97000 & 17.8 & 100.0 & \mathrm{~d} & -\end{array}$


Sounding: MIA203

Client: US Geological Survey

Location: SW 360th street [east]

Project: Saltwater Intrusion

County: Miami-Dade County

TX loop size: X[m] $37.9 \quad \mathrm{Y}[\mathrm{m}] \quad 37.9$

$\begin{array}{cccc}\text { Model } & \text { Resistivity } & \text { Thickness } & \text { Elevation } \\ \text { Layer } & {[\text { ohm-m] }} & {[\mathrm{m}]} & {[\mathrm{m}]} \\ 1 & 1782.500 & 9.6 & 0.0 \\ 2 & 78.770 & 9.3 & -9.6 \\ 3 & 5.770 & 9.9 & -18.9 \\ 4 & 112.800 & 41.6 & -28.8 \\ 5 & 10.590 & -- & -70.4\end{array}$

System: EM-58 Freq[Hz]: 285 Data Set Code: uh TX Cur $[\mathrm{A}]: 2.60$ Turn Off [usec]: 2.5

RX Moment[turns-m^2]: 31.4 Gain Setting: 2

$\begin{array}{lrrrr}\begin{array}{c}\text { Time } \\ \text { [ms }]\end{array} & \begin{array}{r}\text { rhoa_obs } \\ \text { [ohm-m] }\end{array} & \begin{array}{c}\text { obs err } \\ {\left[\frac{\mathrm{o}}{0}\right]}\end{array} & \text { mask } & \begin{array}{r}\text { rhoa_cal } \\ \text { [ohm-m] }\end{array} \\ 0.00680 & 137.0 & 0.4 & u & 135.9 \\ 0.00860 & 102.9 & 0.4 & u & 104.4 \\ 0.01110 & 81.7 & 0.5 & u & 81.0 \\ 0.01410 & 63.7 & 0.4 & u & 63.3 \\ 0.01800 & 50.2 & 0.5 & u & 50.1 \\ 0.02300 & 40.7 & 0.4 & u & 40.6 \\ 0.02940 & 33.9 & 0.4 & u & 33.9 \\ 0.03750 & 29.2 & 0.4 & u & 29.3 \\ 0.04790 & 26.0 & 0.4 & u & 26.2 \\ 0.06110 & 24.0 & 0.4 & u & 24.2 \\ 0.07790 & 22.9 & 0.4 & u & 23.1 \\ 0.09930 & 22.5 & 0.4 & u & 22.8 \\ 0.12670 & 22.7 & 0.5 & u & 23.0 \\ 0.16640 & 23.7 & 0.4 & u & 23.6 \\ 0.20600 & 24.2 & 0.5 & u & 24.3 \\ 0.26280 & 25.2 & 0.7 & u & 25.0 \\ 0.33520 & 25.6 & 1.0 & u & 25.4 \\ 0.42770 & 25.9 & 1.6 & u & 25.5 \\ 0.54560 & 25.7 & 2.7 & u & 25.5 \\ 0.69590 & 25.5 & 4.6 & u & 25.3\end{array}$

Date: $23-\mathrm{FEB}-09$

UTM Coord: $\mathrm{E}[\mathrm{m}]$

Elevation $[\mathrm{m}]$ :

$559064.0 \quad \mathrm{~N}[\mathrm{~m}] \quad 2812970.0$

0.0

RX location: X $[\mathrm{m}]$

$0.0 \quad Y[m]$

0.0

Fit Error $\left[\frac{\circ}{0}\right]: \quad 1.667$

System: EM-58 Freq[Hz]: 30 Data Set Code: hi TX Cur $[\mathrm{A}]: 2.50$ Turn Off [usec]: 2.5

RX Moment [turns-m^2]: 31.4 Gain Setting: 5

\begin{tabular}{|c|c|c|c|c|}
\hline $\begin{array}{l}\text { Time } \\
{[\mathrm{ms}]}\end{array}$ & $\begin{array}{l}\text { rhoa_obs } \\
\text { [ohm-m] }\end{array}$ & $\begin{array}{c}\text { obs err } \\
{\left[\frac{0}{0}\right]}\end{array}$ & mask & $\begin{array}{c}\text { rhoa_cal } \\
\text { [ohm-m] }\end{array}$ \\
\hline 0.08800 & 22.9 & 0.3 & u & 22.8 \\
\hline 0.10600 & 22.8 & 0.3 & $\mathrm{u}$ & 22.7 \\
\hline 0.13100 & 23.0 & 0.3 & $u$ & 22.9 \\
\hline 0.16100 & 23.5 & 0.3 & $\mathrm{u}$ & 23.3 \\
\hline 0.20000 & 24.0 & 0.3 & $\mathrm{u}$ & 23.9 \\
\hline 0.25000 & 24.4 & 0.4 & $u$ & 24.2 \\
\hline 0.31400 & 24.4 & 0.4 & $\mathrm{u}$ & 24.3 \\
\hline 0.39500 & 24.0 & 0.6 & $\mathrm{u}$ & 24.0 \\
\hline 0.49900 & 23.0 & 0.5 & $\mathrm{u}$ & 23.2 \\
\hline 0.63100 & 21.8 & 0.8 & $\mathrm{u}$ & 22.3 \\
\hline 0.79900 & 20.6 & 0.9 & u & 21.1 \\
\hline 1.01000 & 19.7 & 1.4 & u & 20.0 \\
\hline 1.28000 & 19.0 & 3.0 & u & 19.0 \\
\hline 1.63000 & 18.2 & 4.2 & u & 18.1 \\
\hline 2.0 & 17.8 & 7.7 & u & 17.3 \\
\hline 2.6 & 16.5 & 7.7 & u & 16.6 \\
\hline & 15.8 & 24.1 & $\mathrm{~m}$ & 16.2 \\
\hline & 16.2 & 58.5 & d & - \\
\hline & .4 & 98.6 & d & - \\
\hline & 9.5 & 43.8 & d & - \\
\hline
\end{tabular}


Project: Saltwater Intrusion

TX loop size: X $[\mathrm{m}] \quad 37.9 \quad \mathrm{Y}[\mathrm{m}] \quad 37.9$

RX location: X $[\mathrm{m}] \quad 0.0 \quad \mathrm{Y}[\mathrm{m}] \quad 0.0$

$\begin{array}{cccc}\text { Model } & \text { Resistivity } & \text { Thickness } & \text { Elevation } \\ \text { Layer } & {[\text { ohm-m] }} & {[\mathrm{m}]} & {[\mathrm{m}]} \\ 1 & 1809.700 & 17.6 & 0.0 \\ 2 & 6.270 & 11.4 & -17.6 \\ 3 & 109.700 & 41.5 & -29.1 \\ 4 & 10.570 & -- & -70.6\end{array}$

System: EM-58 Freq[Hz]: 285 Data Set Code: uh TX Cur[A]: 2.60 Turn Off[usec]: 2.5

RX Moment[turns-m^2]: 31.4 Gain Setting: 2

\begin{tabular}{|c|c|c|c|c|}
\hline $\begin{array}{l}\text { Time } \\
\text { [ms ] }\end{array}$ & $\begin{array}{l}\text { rhoa_obs } \\
\text { [ohm-m] }\end{array}$ & $\begin{array}{c}\text { obs err } \\
{\left[\frac{\overline{0}}{0}\right]}\end{array}$ & mask & $\begin{array}{l}\text { rhoa_ca } \\
\text { [ohm-m] }\end{array}$ \\
\hline 0.00680 & 137.0 & 0.4 & $u$ & 137.9 \\
\hline 0.00860 & 102.9 & 0.4 & $\mathrm{u}$ & 104.5 \\
\hline 0.01110 & 81.7 & 0.5 & $u$ & 80. \\
\hline 0.01410 & 63.7 & 0.4 & $u$ & 63. \\
\hline 0.01800 & 50.2 & 0.5 & u & 50. \\
\hline 0.02300 & 40.7 & 0.4 & u & 40. \\
\hline 0.02940 & 33.9 & 0.4 & u & 33. \\
\hline 0.03750 & 29.2 & 0.4 & $\mathrm{u}$ & 29. \\
\hline 0.04790 & 26.0 & 0.4 & $\mathrm{u}$ & 26 . \\
\hline 0.06110 & 24.0 & 0.4 & $\mathrm{u}$ & 24.2 \\
\hline 0.07790 & 22.9 & 0.4 & $\mathrm{u}$ & 23. \\
\hline 0.09930 & 22.5 & 0.4 & $\mathrm{u}$ & 22.8 \\
\hline 0.12670 & 22.7 & 0.5 & $\mathrm{u}$ & 23. \\
\hline 0.16640 & 23.7 & 0.4 & $\mathrm{u}$ & 23.6 \\
\hline 0.20600 & 24.2 & 0.5 & $\mathrm{u}$ & 24. \\
\hline 0.26280 & 25.2 & 0.7 & $u$ & 25 . \\
\hline 0.33520 & 25.6 & 1.0 & u & 25 . \\
\hline 0.42770 & 25.9 & 1.6 & u & 3 . \\
\hline 0.5 & 25.7 & 2.7 & u & $\bullet$ \\
\hline 2 & 25.5 & 4.6 & $\mathrm{u}$ & \\
\hline
\end{tabular}

\section{Fit Error $\left[\frac{\circ}{0}\right]: 1.716$}

System: EM-58 Freq[Hz]: 30 Data Set Code: hi TX Cur $[\mathrm{A}]: \quad 2.50$ Turn off[usec] : 2.5

RX Moment [turns-m^2] : 31.4 Gain Setting: 5

$\begin{array}{crrcr}\begin{array}{c}\text { Time } \\ \text { [ms] }\end{array} & \begin{array}{c}\text { rhoa_obs } \\ \text { [ohm-m] }\end{array} & \begin{array}{c}\text { obs_err } \\ {\left[\begin{array}{c}\bar{o} \\ \mathrm{o}\end{array}\right.}\end{array} & \text { mask } & \begin{array}{c}\text { rhoa_cal } \\ \text { [ohm-m] }\end{array} \\ 0.08800 & 22.9 & 0.3 & \mathrm{u} & 22.8 \\ 0.10600 & 22.8 & 0.3 & \mathrm{u} & 22.7 \\ 0.13100 & 23.0 & 0.3 & \mathrm{u} & 22.9 \\ 0.16100 & 23.5 & 0.3 & \mathrm{u} & 23.3 \\ 0.20000 & 24.0 & 0.3 & \mathrm{u} & 23.9 \\ 0.25000 & 24.4 & 0.4 & \mathrm{u} & 24.3 \\ 0.31400 & 24.4 & 0.4 & \mathrm{u} & 24.3 \\ 0.39500 & 24.0 & 0.6 & \mathrm{u} & 24.0 \\ 0.49900 & 23.0 & 0.5 & \mathrm{u} & 23.3 \\ 0.63100 & 21.8 & 0.8 & \mathrm{u} & 22.3 \\ 0.79900 & 20.6 & 0.9 & \mathrm{u} & 21.2 \\ 1.01000 & 19.7 & 1.4 & \mathrm{u} & 20.0 \\ 1.28000 & 19.0 & 3.0 & \mathrm{u} & 19.0 \\ 1.63000 & 18.2 & 4.2 & \mathrm{u} & 18.1 \\ 2.08000 & 17.8 & 7.7 & \mathrm{u} & 17.3 \\ 2.64000 & 16.5 & 7.7 & \mathrm{u} & 16.6 \\ 3.37000 & 15.8 & 24.1 & \mathrm{~m} & 16.2 \\ 4.29000 & 16.2 & 58.5 & \mathrm{~d} & - \\ 5.47000 & 15.4 & 98.6 & \mathrm{~d} & - \\ 6.97000 & 9.5 & 43.8 & \mathrm{~d} & -\end{array}$


Sounding: MIA204

Client: US Geological Survey Location: SW 360th Street [north side]

Project: Saltwater Intrusion

County: Miami-Dade County

TX loop size: X[m] $37.9 \quad \mathrm{Y}[\mathrm{m}] \quad 37.9$

$\begin{array}{cccc}\text { Model } & \text { Resistivity } & \text { Thickness } & \text { Elevation } \\ \text { Layer } & {[\text { ohm-m] }} & {[\mathrm{m}]} & {[\mathrm{m}]} \\ 1 & 117.500 & 10.7 & 0.0 \\ 2 & 21.760 & 3.7 & -10.7 \\ 3 & 3.720 & 11.2 & -14.4 \\ 4 & 21.240 & 56.2 & -25.7 \\ 5 & 7.860 & -- & -81.9\end{array}$

System: EM-58 Freq[Hz]: 285 Data Set Code: uh TX Cur $[\mathrm{A}]: 2.60$ Turn Off [usec]: 2.5

RX Moment[turns-m^2]: 31.4 Gain Setting: 2

$\begin{array}{ccccr}\begin{array}{c}\text { Time } \\ \text { [ms }]\end{array} & \begin{array}{c}\text { rhoa_obs } \\ \text { [ohm-m] }\end{array} & \begin{array}{c}\text { obserr } \\ {\left[\begin{array}{c}\text { o } \\ 0\end{array}\right.}\end{array} & \text { mask } & \begin{array}{r}\text { rhoa_cal } \\ \text { [ohm-m] }\end{array} \\ 0.00680 & 84.4 & 0.3 & \mathrm{u} & 84.4 \\ 0.00860 & 67.8 & 0.3 & \mathrm{u} & 67.2 \\ 0.01110 & 53.8 & 0.3 & \mathrm{u} & 54.0 \\ 0.01410 & 43.2 & 0.3 & \mathrm{u} & 43.5 \\ 0.01800 & 34.7 & 0.3 & \mathrm{u} & 34.9 \\ 0.02300 & 28.0 & 0.3 & \mathrm{u} & 27.9 \\ 0.02940 & 22.6 & 0.3 & \mathrm{u} & 22.6 \\ 0.03750 & 18.6 & 0.3 & \mathrm{u} & 18.6 \\ 0.04790 & 15.7 & 0.3 & \mathrm{u} & 15.7 \\ 0.06110 & 13.6 & 0.3 & \mathrm{u} & 13.6 \\ 0.07790 & 12.1 & 0.3 & \mathrm{u} & 12.2 \\ 0.09930 & 11.1 & 0.3 & \mathrm{u} & 11.2 \\ 0.12670 & 10.5 & 0.3 & \mathrm{u} & 10.6 \\ 0.16640 & 10.3 & 0.3 & \mathrm{u} & 10.3 \\ 0.20600 & 10.2 & 0.3 & \mathrm{u} & 10.3 \\ 0.26280 & 10.4 & 0.2 & \mathrm{u} & 10.5 \\ 0.33520 & 10.9 & 0.3 & \mathrm{u} & 10.9 \\ 0.42770 & 11.5 & 0.3 & \mathrm{u} & 11.5 \\ 0.54560 & 12.2 & 0.4 & \mathrm{u} & 12.2 \\ 0.69590 & 13.1 & 0.5 & \mathrm{u} & 13.0\end{array}$

Date: $24-\mathrm{FEB}-09$

UTM Coord: $\mathrm{E}[\mathrm{m}]$

Elevation $[\mathrm{m}]$ :

$558907.0 \quad \mathrm{~N}[\mathrm{~m}] \quad 2811696.0$

0.0

RX location: X $[\mathrm{m}]$

$0.0 \quad Y[m]$

0.0
Fit Error [응 1.077

System: EM-58 Freq[Hz]: 30 Data Set Code: hi TX Cur $[\mathrm{A}]: 2.50$ Turn Off[usec]: 2.5

RX Moment [turns-m^2]: 31.4 Gain Setting: 5

$\begin{array}{ccccc}\begin{array}{c}\text { Time } \\ \text { [ms] }\end{array} & \begin{array}{c}\text { rhoa_obs } \\ \text { [ohm-m] }\end{array} & \begin{array}{c}\text { obs_err } \\ {\left[\overline{\frac{o}{0}}\right]}\end{array} & \text { mask } & \begin{array}{c}\text { rhoa_cal } \\ \text { [ohm-m] }\end{array} \\ 0.08800 & 11.8 & 0.4 & \mathrm{u} & 11.6 \\ 0.10600 & 11.1 & 0.4 & \mathrm{u} & 11.0 \\ 0.13100 & 10.6 & 0.4 & \mathrm{u} & 10.5 \\ 0.16100 & 10.3 & 0.4 & \mathrm{u} & 10.3 \\ 0.20000 & 10.3 & 0.4 & \mathrm{u} & 10.2 \\ 0.25000 & 10.4 & 0.4 & \mathrm{u} & 10.3 \\ 0.31400 & 10.6 & 0.4 & \mathrm{u} & 10.5 \\ 0.39500 & 10.9 & 0.4 & \mathrm{u} & 10.8 \\ 0.49900 & 11.2 & 0.4 & \mathrm{u} & 11.2 \\ 0.63100 & 11.5 & 0.5 & \mathrm{u} & 11.5 \\ 0.79900 & 11.7 & 0.6 & \mathrm{u} & 11.7 \\ 1.01000 & 11.7 & 1.3 & \mathrm{u} & 11.8 \\ 1.28000 & 11.8 & 1.8 & \mathrm{u} & 11.8 \\ 1.63000 & 11.7 & 2.7 & \mathrm{u} & 11.7 \\ 2.08000 & 11.5 & 3.6 & \mathrm{u} & 11.5 \\ 2.64000 & 10.6 & 5.3 & \mathrm{~d} & - \\ 3.37000 & 10.0 & 9.0 & \mathrm{~d} & - \\ 4.29000 & 8.0 & 13.6 & \mathrm{~d} & - \\ 5.47000 & 6.4 & 18.6 & \mathrm{~d} & - \\ 6.97000 & 5.0 & 24.1 & \mathrm{~d} & -\end{array}$


Sounding: MIA204L4 Location: SW 360th street [north side]

Project: Saltwater Intrusion

County: Miami-Dade County

TX loop size: X $[\mathrm{m}] \quad 37.9 \quad \mathrm{Y}[\mathrm{m}] \quad 37.9$

$\begin{array}{cccc}\text { Model } & \text { Resistivity } & \text { Thickness } & \text { Elevation } \\ \text { Layer } & {[\text { ohm-m] }} & {[\mathrm{m}]} & {[\mathrm{m}]} \\ 1 & 91.190 & 13.9 & 0.0 \\ 2 & 3.780 & 11.8 & -13.9 \\ 3 & 21.130 & 56.8 & -25.8 \\ 4 & 7.730 & -- & -82.7\end{array}$

System: EM-58 Freq[Hz]: 285 Data Set Code: uh TX Cur $[A]: 2.60$ Turn Off[usec]: 2.5

RX Moment[turns-m^2]: 31.4 Gain Setting: 2

$\begin{array}{rrrrrr}\text { Time } & \begin{array}{c}\text { rhoa_obs } \\ \text { [ohm-m] }\end{array} & \begin{array}{c}\text { obs_err } \\ {\left[\frac{\mathrm{o}}{0}\right]}\end{array} & \text { mask } & \begin{array}{c}\text { rhoa_cal } \\ \text { [ohm-m] }\end{array} \\ 1 & 0.00680 & 84.4 & 0.3 & \mathrm{u} & 84.5 \\ 2 & 0.00860 & 67.8 & 0.3 & \mathrm{u} & 67.2 \\ 3 & 0.01110 & 53.8 & 0.3 & \mathrm{u} & 54.0 \\ 4 & 0.01410 & 43.2 & 0.3 & \mathrm{u} & 43.4 \\ 5 & 0.01800 & 34.7 & 0.3 & \mathrm{u} & 34.8 \\ 6 & 0.02300 & 28.0 & 0.3 & \mathrm{u} & 27.9 \\ 7 & 0.02940 & 22.6 & 0.3 & \mathrm{u} & 22.6 \\ 8 & 0.03750 & 18.6 & 0.3 & \mathrm{u} & 18.6 \\ 9 & 0.04790 & 15.7 & 0.3 & \mathrm{u} & 15.7 \\ 10 & 0.06110 & 13.6 & 0.3 & \mathrm{u} & 13.6 \\ 11 & 0.07790 & 12.1 & 0.3 & \mathrm{u} & 12.2 \\ 12 & 0.09930 & 11.1 & 0.3 & \mathrm{u} & 11.2 \\ 13 & 0.12670 & 10.5 & 0.3 & \mathrm{u} & 10.6 \\ 14 & 0.16640 & 10.3 & 0.3 & \mathrm{u} & 10.3 \\ 15 & 0.20600 & 10.2 & 0.3 & \mathrm{u} & 10.3 \\ 16 & 0.26280 & 10.4 & 0.2 & \mathrm{u} & 10.5 \\ 17 & 0.33520 & 10.9 & 0.3 & \mathrm{u} & 10.9 \\ 18 & 0.42770 & 11.5 & 0.3 & \mathrm{u} & 11.5 \\ 19 & 0.54560 & 12.2 & 0.4 & \mathrm{u} & 12.2 \\ 20 & 0.69590 & 13.1 & 0.5 & \mathrm{u} & 13.0\end{array}$

Date: $24-\mathrm{FEB}-09$

UTM Coord: $\mathrm{E}[\mathrm{m}]$

Elevation $[\mathrm{m}]$ :

$558907.0 \quad \mathrm{~N}[\mathrm{~m}] \quad 2811696.0$

0.0

RX location: X $[\mathrm{m}]$

$0.0 \quad Y[m]$

0.0
Fit Error $[\%]: 1.069$

System: EM-58 Freq[Hz]: 30 Data Set Code: hi TX Cur $[A]: 2.50$ Turn Off[usec]: 2.5

RX Moment [turns-m^2]: 31.4 Gain Setting: 5

$\begin{array}{ccccc}\begin{array}{c}\text { Time } \\ \text { [ms] }\end{array} & \begin{array}{c}\text { rhoa_obs } \\ \text { [ohm-m] }\end{array} & \begin{array}{c}\text { obs_err } \\ {\left[\begin{array}{c}\overline{\%} \\ 0\end{array}\right.}\end{array} & \text { mask } & \begin{array}{c}\text { rhoa_cal } \\ \text { [ohm-m] }\end{array} \\ 0.08800 & 11.8 & 0.4 & \mathrm{u} & 11.6 \\ 0.10600 & 11.1 & 0.4 & \mathrm{u} & 11.0 \\ 0.13100 & 10.6 & 0.4 & \mathrm{u} & 10.5 \\ 0.16100 & 10.3 & 0.4 & \mathrm{u} & 10.3 \\ 0.20000 & 10.3 & 0.4 & \mathrm{u} & 10.2 \\ 0.25000 & 10.4 & 0.4 & \mathrm{u} & 10.3 \\ 0.31400 & 10.6 & 0.4 & \mathrm{u} & 10.5 \\ 0.39500 & 10.9 & 0.4 & \mathrm{u} & 10.8 \\ 0.49900 & 11.2 & 0.4 & \mathrm{u} & 11.2 \\ 0.63100 & 11.5 & 0.5 & \mathrm{u} & 11.5 \\ 0.79900 & 11.7 & 0.6 & \mathrm{u} & 11.7 \\ 1.01000 & 11.7 & 1.3 & \mathrm{u} & 11.8 \\ 1.28000 & 11.8 & 1.8 & \mathrm{u} & 11.8 \\ 1.63000 & 11.7 & 2.7 & \mathrm{u} & 11.6 \\ 2.08000 & 11.5 & 3.6 & \mathrm{u} & 11.4 \\ 2.64000 & 10.6 & 5.3 & \mathrm{~d} & - \\ 3.37000 & 10.0 & 9.0 & \mathrm{~d} & - \\ 4.29000 & 8.0 & 13.6 & \mathrm{~d} & - \\ 5.47000 & 6.4 & 18.6 & \mathrm{~d} & - \\ 6.97000 & 5.0 & 24.1 & \mathrm{~d} & -\end{array}$


Sounding: MIA205

Client: US Geological Survey

Location: SW 360th Street (south side)

Project: Saltwater Intrusion

County: Miami-Dade County

TX loop size: X[m] $37.9 \quad \mathrm{Y}[\mathrm{m}] \quad 37.9$

$\begin{array}{cccc}\text { Model } & \text { Resistivity } & \text { Thickness } & \text { Elevation } \\ \text { Layer } & {[\text { ohm-m] }} & {[\mathrm{m}]} & {[\mathrm{m}]} \\ 1 & 125.000 & 15.7 & 0.0 \\ 2 & 6.000 & 10.3 & -15.7 \\ 3 & 50.930 & 28.1 & -26.0 \\ 4 & 12.860 & -- & -54.1\end{array}$

System: EM-58 Freq[Hz]: 285 Data Set Code: uh TX $\operatorname{Cur}[A]: 2.60$ Turn Off [usec]: 2.5

RX Moment[turns-m^2]: 31.4 Gain Setting: 2

$\begin{array}{rrrrrr}\text { Time } & \begin{array}{c}\text { rhoa_obs } \\ \text { [ohm-m] }\end{array} & \begin{array}{c}\text { obs_err } \\ {\left[\frac{\mathrm{o}}{0}\right]}\end{array} & \text { mask } & \begin{array}{c}\text { rhoa_cal } \\ \text { [ohm-m] }\end{array} \\ 1 & 0.00680 & 97.3 & 0.5 & \mathrm{u} & 96.8 \\ 2 & 0.00860 & 76.1 & 0.3 & \mathrm{u} & 76.6 \\ 3 & 0.01110 & 60.7 & 0.4 & \mathrm{u} & 61.0 \\ 4 & 0.01410 & 48.6 & 0.4 & \mathrm{u} & 48.8 \\ 5 & 0.01800 & 39.4 & 0.4 & \mathrm{u} & 39.4 \\ 6 & 0.02300 & 32.7 & 0.4 & \mathrm{u} & 32.5 \\ 7 & 0.02940 & 27.8 & 0.4 & \mathrm{u} & 27.6 \\ 8 & 0.03750 & 24.4 & 0.4 & \mathrm{u} & 24.3 \\ 9 & 0.04790 & 22.0 & 0.4 & \mathrm{u} & 22.0 \\ 10 & 0.06110 & 20.5 & 0.4 & \mathrm{u} & 20.5 \\ 11 & 0.07790 & 19.6 & 0.4 & \mathrm{u} & 19.7 \\ 12 & 0.09930 & 19.1 & 0.4 & \mathrm{u} & 19.4 \\ 13 & 0.12670 & 19.1 & 0.4 & \mathrm{u} & 19.3 \\ 14 & 0.16640 & 19.5 & 0.4 & \mathrm{u} & 19.5 \\ 15 & 0.20600 & 19.6 & 0.4 & \mathrm{u} & 19.6 \\ 16 & 0.26280 & 19.9 & 0.5 & \mathrm{u} & 19.8 \\ 17 & 0.33520 & 20.1 & 0.6 & \mathrm{u} & 19.9 \\ 18 & 0.42770 & 20.1 & 0.7 & \mathrm{u} & 20.0 \\ 19 & 0.54560 & 20.3 & 0.9 & \mathrm{u} & 20.2 \\ 20 & 0.69590 & 20.5 & 1.3 & \mathrm{u} & 20.5\end{array}$

Date: 24-FEB-09

UTM Coord: $\mathrm{E}[\mathrm{m}]$

Elevation $[\mathrm{m}]$ :

$558086.0 \quad \mathrm{~N}[\mathrm{~m}] \quad 2811251.0$

0.0

RX location: X $[\mathrm{m}]$

$0.0 \quad Y[m]$

0.0
Fit Error $[\%]: 1.047$

System: EM-58 Freq[Hz]: 30 Data Set Code: hi TX Cur $[A]: 2.50$ Turn Off[usec]: 2.5

RX Moment [turns-m^2]: 31.4 Gain Setting: 5

$\begin{array}{ccccc}\begin{array}{c}\text { Time } \\ \text { [ms] }\end{array} & \begin{array}{c}\text { rhoa_obs } \\ \text { [ohm-m] }\end{array} & \begin{array}{c}\text { obserr } \\ {\left[\begin{array}{c}\text { o }] \\ 0.08800\end{array}\right.}\end{array} & \text { mask } & \begin{array}{c}\text { rhoa_cal } \\ \text { [ohm-m] }\end{array} \\ 0.10600 & 19.6 & 0.1 & \mathrm{u} & 19.4 \\ 0.13100 & 19.3 & 0.1 & \mathrm{u} & 19.3 \\ 0.16100 & 19.4 & 0.1 & \mathrm{u} & 19.2 \\ 0.20000 & 19.5 & 0.1 & \mathrm{u} & 19.3 \\ 0.25000 & 19.5 & 0.2 & \mathrm{u} & 19.4 \\ 0.31400 & 19.4 & 0.2 & \mathrm{u} & 19.4 \\ 0.39500 & 19.0 & 0.1 & \mathrm{u} & 19.2 \\ 0.49900 & 18.5 & 0.5 & \mathrm{u} & 18.0 \\ 0.63100 & 17.9 & 0.8 & \mathrm{u} & 18.2 \\ 0.79900 & 17.4 & 1.3 & \mathrm{u} & 17.7 \\ 1.01000 & 17.3 & 1.9 & \mathrm{u} & 17.3 \\ 1.28000 & 16.9 & 2.5 & \mathrm{u} & 16.9 \\ 1.63000 & 16.7 & 4.5 & \mathrm{u} & 16.5 \\ 2.08000 & 15.6 & 6.8 & \mathrm{~m} & 16.2 \\ 2.64000 & 15.3 & 10.2 & \mathrm{~m} & 15.9 \\ 3.37000 & 15.0 & 24.3 & \mathrm{~d} & - \\ 4.29000 & 13.9 & 36.8 & \mathrm{~d} & - \\ 5.47000 & 12.4 & 40.8 & \mathrm{~d} & - \\ 6.97000 & 12.4 & 75.4 & \mathrm{~d} & -\end{array}$


Sounding: MIA206

Client: US Geological Survey

Location: SW 360th Street (north side)

Project: Saltwater Intrusion

County: Miami-Dade County

TX loop size: X[m] $37.9 \quad \mathrm{Y}[\mathrm{m}] \quad 37.9$

$\begin{array}{cccc}\text { Model } & \text { Resistivity } & \text { Thickness } & \text { Elevation } \\ \text { Layer } & {[\text { ohm-m] }} & {[\mathrm{m}]} & {[\mathrm{m}]} \\ 1 & 132.400 & 26.8 & 0.0 \\ 2 & 46.850 & 43.5 & -26.8 \\ 3 & 10.240 & 54.0 & -70.4 \\ 4 & 28.930 & -- & -124.4\end{array}$

System: EM-58 Freq[Hz]: 285 Data Set Code: uh TX Cur[A]: 2.65 Turn Off[usec]: 2.5

RX Moment[turns-m^2]: 31.4 Gain Setting: 2

$\begin{array}{rrrrrr}\text { Time } & \begin{array}{c}\text { rhoa_obs } \\ \text { [ohm-m] }\end{array} & \begin{array}{c}\text { obs_err } \\ {\left[\frac{\mathrm{o}}{0}\right]}\end{array} & \text { mask } & \begin{array}{c}\text { rhoa_cal } \\ \text { [ohm-m] }\end{array} \\ 1 & 0.00680 & 126.3 & 0.5 & \mathrm{u} & 124.5 \\ 2 & 0.00860 & 117.7 & 0.2 & \mathrm{u} & 119.8 \\ 3 & 0.01110 & 115.6 & 0.2 & \mathrm{u} & 116.1 \\ 4 & 0.01410 & 111.4 & 0.2 & \mathrm{u} & 111.1 \\ 5 & 0.01800 & 104.9 & 0.2 & \mathrm{u} & 105.2 \\ 6 & 0.02300 & 100.9 & 0.1 & \mathrm{u} & 99.9 \\ 7 & 0.02940 & 96.5 & 0.1 & \mathrm{u} & 95.7 \\ 8 & 0.03750 & 92.7 & 0.1 & \mathrm{u} & 92.3 \\ 9 & 0.04790 & 88.5 & 0.2 & \mathrm{u} & 89.1 \\ 10 & 0.06110 & 84.0 & 0.2 & \mathrm{u} & 85.1 \\ 11 & 0.07790 & 78.9 & 0.2 & \mathrm{u} & 79.7 \\ 12 & 0.09930 & 71.8 & 0.3 & \mathrm{u} & 73.0 \\ 13 & 0.12670 & 64.8 & 0.5 & \mathrm{u} & 65.6 \\ 14 & 0.16640 & 56.5 & 0.5 & \mathrm{u} & 57.1 \\ 15 & 0.20600 & 50.3 & 0.5 & \mathrm{u} & 50.9 \\ 16 & 0.26280 & 44.7 & 0.9 & \mathrm{u} & 44.6 \\ 17 & 0.33520 & 39.6 & 1.2 & \mathrm{u} & 39.5 \\ 18 & 0.42770 & 35.9 & 2.2 & \mathrm{u} & 35.5 \\ 19 & 0.54560 & 33.0 & 3.2 & \mathrm{u} & 32.8 \\ 20 & 0.69590 & 31.6 & 4.0 & \mathrm{u} & 31.0\end{array}$

Date: $24-\mathrm{FEB}-09$

UTM Coord: $\mathrm{E}[\mathrm{m}]$

Elevation $[\mathrm{m}]$ :

$557261.0 \quad \mathrm{~N}[\mathrm{~m}]$

2811712.0

0.0

RX location: $\mathrm{X}[\mathrm{m}]$

$0.0 \quad \mathrm{Y}[\mathrm{m}]$

0.0
Fit Error [\%]: 1.703

System: EM-58 Freq[Hz]: 30 Data Set Code: hi TX Cur $[A]: 2.50$ Turn Off[usec]: 2.5

RX Moment [turns-m^2] : 31.4 Gain Setting: 5

$\begin{array}{ccccc}\begin{array}{c}\text { Time } \\ \text { [ms] }\end{array} & \begin{array}{c}\text { rhoa_obs } \\ \text { [ohm-m] }\end{array} & \begin{array}{c}\text { obserr } \\ {\left[\begin{array}{c}\text { o }] \\ 0\end{array}\right.}\end{array} & \text { mask } & \begin{array}{c}\text { rhoa_cal } \\ \text { [ohm-m] }\end{array} \\ 0.08800 & 76.9 & 0.7 & \mathrm{u} & 75.6 \\ 0.10600 & 71.1 & 0.9 & \mathrm{u} & 69.8 \\ 0.16100 & 64.1 & 0.9 & \mathrm{u} & 63.1 \\ 0.20000 & 57.1 & 1.0 & \mathrm{u} & 56.4 \\ 0.25000 & 50.1 & 0.9 & \mathrm{u} & 49.8 \\ 0.31400 & 43.6 & 0.9 & \mathrm{u} & 43.7 \\ 0.39500 & 38.1 & 1.0 & \mathrm{u} & 38.3 \\ 0.49900 & 33.5 & 0.8 & \mathrm{u} & 33.9 \\ 0.63100 & 30.0 & 0.7 & \mathrm{u} & 30.4 \\ 0.79900 & 27.4 & 0.9 & \mathrm{u} & 27.7 \\ 1.01000 & 25.4 & 1.9 & \mathrm{u} & 25.7 \\ 1.28000 & 24.5 & 2.1 & \mathrm{u} & 24.3 \\ 1.63000 & 23.7 & 5.0 & \mathrm{u} & 23.4 \\ 2.08000 & 22.5 & 10.1 & \mathrm{u} & 22.8 \\ 2.64000 & 20.8 & 11.4 & \mathrm{~m} & 22.6 \\ 3.37000 & 19.6 & 12.7 & \mathrm{~d} & - \\ 4.29000 & 17.6 & 28.2 & \mathrm{~d} & - \\ 5.47000 & 14.4 & 26.6 & \mathrm{~d} & - \\ 6.97000 & 11.5 & 46.3 & \mathrm{~d} & -\end{array}$


Sounding: MIA207

Client: US Geological Survey

Location: SW 360th Street (north side)

Project: Saltwater Intrusion

County: Miami-Dade County

TX loop size: X[m] $37.9 \quad \mathrm{Y}[\mathrm{m}] \quad 37.9$

$\begin{array}{cccc}\text { Model } & \text { Resistivity } & \text { Thickness } & \text { Elevation } \\ \text { Layer } & {[\text { ohm-m] }} & {[\mathrm{m}]} & {[\mathrm{m}]} \\ 1 & 213.200 & 18.9 & 0.0 \\ 2 & 54.160 & 54.0 & -18.9 \\ 3 & 12.270 & 46.0 & -73.0 \\ 4 & 36.670 & -- & -119.0\end{array}$

System: EM-58 Freq[Hz]: 285 Data Set Code: uh TX Cur[A]: 2.65 Turn Off[usec]: 2.5

RX Moment[turns-m^2]: 31.4 Gain Setting: 2

$\begin{array}{rrrrrr}\text { Time } & \begin{array}{c}\text { rhoa_obs } \\ \text { [ohm-m] }\end{array} & \begin{array}{c}\text { obs_err } \\ {\left[\frac{\mathrm{o}}{0}\right]}\end{array} & \text { mask } & \begin{array}{c}\text { rhoa_cal } \\ \text { [ohm-m] }\end{array} \\ 1 & 0.00680 & 146.6 & 0.2 & \mathrm{u} & 144.5 \\ 2 & 0.00860 & 126.0 & 0.2 & \mathrm{u} & 130.4 \\ 3 & 0.01110 & 122.4 & 0.3 & \mathrm{u} & 120.9 \\ 4 & 0.01410 & 114.3 & 0.3 & \mathrm{u} & 113.0 \\ 5 & 0.01800 & 106.1 & 0.3 & \mathrm{u} & 105.9 \\ 6 & 0.02300 & 100.4 & 0.3 & \mathrm{u} & 100.3 \\ 7 & 0.02940 & 96.1 & 0.3 & \mathrm{u} & 96.1 \\ 8 & 0.03750 & 93.0 & 0.3 & \mathrm{u} & 93.1 \\ 9 & 0.04790 & 89.8 & 0.2 & \mathrm{u} & 90.3 \\ 10 & 0.06110 & 86.5 & 0.3 & \mathrm{u} & 86.9 \\ 11 & 0.07790 & 82.2 & 0.3 & \mathrm{u} & 82.1 \\ 12 & 0.09930 & 74.7 & 0.4 & \mathrm{u} & 75.8 \\ 13 & 0.12670 & 67.4 & 0.2 & \mathrm{u} & 68.4 \\ 14 & 0.16640 & 59.0 & 0.4 & \mathrm{u} & 59.6 \\ 15 & 0.20600 & 53.2 & 0.6 & \mathrm{u} & 53.3 \\ 16 & 0.26280 & 47.7 & 0.6 & \mathrm{u} & 47.2 \\ 17 & 0.33520 & 43.0 & 1.8 & \mathrm{u} & 42.5 \\ 18 & 0.42770 & 39.3 & 1.4 & \mathrm{u} & 39.0 \\ 19 & 0.54560 & 37.0 & 1.4 & \mathrm{u} & 36.8 \\ 20 & 0.69590 & 36.8 & 1.9 & \mathrm{u} & 35.7\end{array}$

Date: $24-\mathrm{FEB}-09$

UTM Coord: $\mathrm{E}[\mathrm{m}]$

Elevation $[\mathrm{m}]$ :

$556572.0 \quad \mathrm{~N}[\mathrm{~m}] \quad 2811778.0$

0.0

RX location: X $[\mathrm{m}]$

$0.0 \quad Y[m]$

0.0
Fit Error $[\%]: 1.789$

System: EM-58 Freq[Hz]: 30 Data Set Code: hi TX Cur $[A]: 2.50$ Turn Off[usec]: 2.5

RX Moment[turns-m^2]: 31.4 Gain Setting: 5

$\begin{array}{crrcc}\begin{array}{c}\text { Time } \\ \text { [ms] }\end{array} & \begin{array}{c}\text { rhoa_obs } \\ \text { [ohm-m] }\end{array} & \begin{array}{c}\text { obs_err } \\ {\left[\begin{array}{c}\overline{\%} \\ 0\end{array}\right.}\end{array} & \text { mask } & \begin{array}{c}\text { rhoa_cal } \\ \text { [ohm-m] }\end{array} \\ 0.08800 & 79.9 & 0.4 & \mathrm{u} & 78.4 \\ 0.10600 & 73.6 & 0.4 & \mathrm{u} & 72.8 \\ 0.13100 & 66.6 & 0.4 & \mathrm{u} & 66.1 \\ 0.16100 & 59.4 & 0.5 & \mathrm{u} & 59.2 \\ 0.20000 & 52.5 & 0.6 & \mathrm{u} & 52.5 \\ 0.25000 & 46.4 & 0.5 & \mathrm{u} & 46.5 \\ 0.31400 & 41.2 & 0.7 & \mathrm{u} & 41.5 \\ 0.39500 & 37.1 & 0.8 & \mathrm{u} & 37.4 \\ 0.49900 & 33.9 & 0.8 & \mathrm{u} & 34.4 \\ 0.63100 & 31.5 & 1.1 & \mathrm{u} & 32.1 \\ 0.79900 & 30.1 & 1.9 & \mathrm{u} & 30.4 \\ 1.01000 & 29.4 & 2.5 & \mathrm{u} & 29.4 \\ 1.28000 & 28.8 & 3.7 & \mathrm{u} & 28.7 \\ 1.63000 & 28.3 & 6.0 & \mathrm{u} & 28.5 \\ 2.08000 & 28.8 & 9.9 & \mathrm{u} & 28.5 \\ 2.64000 & 28.5 & 22.4 & \mathrm{~m} & 28.9 \\ 3.37000 & 23.8 & 42.3 & \mathrm{~d} & - \\ 4.29000 & 27.8 & 59.3 & \mathrm{~d} & - \\ 5.47000 & 17.5 & 33.6 & \mathrm{~d} & - \\ 6.97000 & 19.2 & 100.0 & \mathrm{~d} & -\end{array}$


Sounding: MIA208

Client: US Geological Survey

Location: SW 360th Street (south side)

Project: Saltwater Intrusion

County: Miami-Dade County

TX loop size: X $[\mathrm{m}] \quad 37.9 \quad \mathrm{Y}[\mathrm{m}] \quad 37.9$

$\begin{array}{cccc}\text { Model } & \text { Resistivity } & \text { Thickness } & \text { Elevation } \\ \text { Layer } & {[\text { ohm-m] }} & {[\mathrm{m}]} & {[\mathrm{m}]} \\ 1 & 561.300 & 16.6 & 0.0 \\ 2 & 55.060 & 59.4 & -16.6 \\ 3 & 20.880 & 38.0 & -76.1 \\ 4 & 41.440 & -- & -114.1\end{array}$

System: EM-58 Freq[Hz]: 285 Data Set Code: uh TX Cur[A]: 2.65 Turn Off[usec] : 2.5

RX Moment[turns-m^2]: 31.4 Gain Setting: 2

$\begin{array}{rrrrrr}\text { Time } & \begin{array}{c}\text { rhoa_obs } \\ \text { [ohm-m] }\end{array} & \begin{array}{c}\text { obs_err } \\ {\left[\frac{\mathrm{o}}{0}\right]}\end{array} & \text { mask } & \begin{array}{c}\text { rhoa_cal } \\ \text { [ohm-m] }\end{array} \\ 1 & 0.00680 & 171.0 & 0.4 & \mathrm{u} & 164.3 \\ 2 & 0.00860 & 140.2 & 0.1 & \mathrm{u} & 144.6 \\ 3 & 0.01110 & 132.7 & 0.1 & \mathrm{u} & 131.6 \\ 4 & 0.01410 & 120.4 & 0.1 & \mathrm{u} & 121.3 \\ 5 & 0.01800 & 110.3 & 0.1 & \mathrm{u} & 112.3 \\ 6 & 0.02300 & 103.5 & 0.2 & \mathrm{u} & 105.0 \\ 7 & 0.02940 & 99.0 & 0.2 & \mathrm{u} & 99.3 \\ 8 & 0.03750 & 95.6 & 0.2 & \mathrm{u} & 94.6 \\ 9 & 0.04790 & 91.6 & 0.2 & \mathrm{u} & 90.6 \\ 10 & 0.06110 & 86.9 & 0.2 & \mathrm{u} & 86.5 \\ 11 & 0.07790 & 81.6 & 0.2 & \mathrm{u} & 81.7 \\ 12 & 0.09930 & 74.7 & 0.3 & \mathrm{u} & 76.1 \\ 13 & 0.12670 & 69.0 & 0.3 & \mathrm{u} & 70.1 \\ 14 & 0.16640 & 63.2 & 0.4 & \mathrm{u} & 63.6 \\ 15 & 0.20600 & 57.9 & 6.1 & \mathrm{u} & 59.1 \\ 16 & 0.26280 & 56.0 & 0.5 & \mathrm{u} & 55.0 \\ 17 & 0.33520 & 51.6 & 8.5 & \mathrm{u} & 51.9 \\ 18 & 0.42770 & 50.7 & 12.6 & \mathrm{u} & 49.9 \\ 19 & 0.54560 & 48.7 & 5.1 & \mathrm{u} & 48.8 \\ 20 & 0.69590 & 50.3 & 6.6 & \mathrm{u} & 48.8\end{array}$

Date: $24-\mathrm{FEB}-09$

UTM Coord: $\mathrm{E}[\mathrm{m}]$

Elevation $[\mathrm{m}]$ :

$555809.0 \quad \mathrm{~N}[\mathrm{~m}] \quad 2811154.0$

0.0

RX location: X $[\mathrm{m}]$

$0.0 \quad \mathrm{Y}[\mathrm{m}]$

0.0
Fit Error [\%]: 2.269

System: EM-58 Freq[Hz]: 30 Data Set Code: hi TX Cur $[A]: 2.50$ Turn Off[usec]: 2.5

RX Moment[turns-m^2]: 31.4 Gain Setting: 5

$\begin{array}{crrcc}\begin{array}{c}\text { Time } \\ \text { [ms] }\end{array} & \begin{array}{c}\text { rhoa_obs } \\ \text { [ohm-m] }\end{array} & \begin{array}{c}\text { obs_err } \\ {\left[\begin{array}{c}\overline{\%} \\ 0\end{array}\right.}\end{array} & \text { mask } & \begin{array}{c}\text { rhoa_cal } \\ \text { [ohm-m] }\end{array} \\ 0.08800 & 79.5 & 0.6 & \mathrm{u} & 78.5 \\ 0.10600 & 74.4 & 0.6 & \mathrm{u} & 73.8 \\ 0.13100 & 69.3 & 0.9 & \mathrm{u} & 68.5 \\ 0.16100 & 63.9 & 0.5 & \mathrm{u} & 63.3 \\ 0.20000 & 59.2 & 0.9 & \mathrm{u} & 58.5 \\ 0.25000 & 54.3 & 0.6 & \mathrm{u} & 54.2 \\ 0.31400 & 50.5 & 1.3 & \mathrm{u} & 50.6 \\ 0.39500 & 46.8 & 0.9 & \mathrm{u} & 47.7 \\ 0.49900 & 45.1 & 0.9 & \mathrm{u} & 45.5 \\ 0.63100 & 43.3 & 3.3 & \mathrm{u} & 43.8 \\ 0.79900 & 42.0 & 2.6 & \mathrm{u} & 42.5 \\ 1.01000 & 41.8 & 5.8 & \mathrm{u} & 41.7 \\ 1.28000 & 40.6 & 9.7 & \mathrm{u} & 41.0 \\ 1.63000 & 41.3 & 15.5 & \mathrm{u} & 40.8 \\ 2.08000 & 43.3 & 26.2 & \mathrm{~m} & 40.8 \\ 2.64000 & 43.1 & 67.2 & \mathrm{~d} & - \\ 3.37000 & 48.8 & 96.3 & \mathrm{~d} & - \\ 4.29000 & 45.3 & 100.0 & \mathrm{~d} & - \\ 5.47000 & 126.9 & 100.0 & \mathrm{~d} & - \\ 6.97000 & 43.2 & 100.0 & \mathrm{~d} & -\end{array}$


Sounding: MIA209

Location: E of SW162nd Ave \& S of SW380nd

County: Miami-Dade County

TX loop size: X[m] $37.9 \quad \mathrm{Y}[\mathrm{m}] \quad 37.9$

$\begin{array}{cccc}\text { Model } & \text { Resistivity } & \text { Thickness } & \text { Elevation } \\ \text { Layer } & {[\text { ohm-m] }} & {[\mathrm{m}]} & {[\mathrm{m}]} \\ 1 & 580.200 & 15.5 & 0.0 \\ 2 & 54.680 & 82.2 & -15.5 \\ 3 & 14.310 & 14.9 & -97.8 \\ 4 & 45.520 & -- & -112.7\end{array}$

System: EM-58 Freq[Hz]: 285 Data Set Code: uh TX $\operatorname{Cur}[\mathrm{A}]: 2.70$ Turn Off[usec]: 2.5

RX Moment[turns-m^2]: 31.4 Gain Setting: 2

\begin{tabular}{|c|c|c|c|c|}
\hline $\begin{array}{l}\text { Time } \\
{[\mathrm{ms}\rceil}\end{array}$ & $\begin{array}{l}\text { rhoa_obs } \\
\text { [ohm-m] }\end{array}$ & obs err & mask & rhoa_ca \\
\hline 0.00680 & 171.9 & 0.3 & $\mathrm{~m}$ & $\begin{array}{r}154.7 \\
\end{array}$ \\
\hline 0.00860 & 137.7 & 0.2 & u & 136.6 \\
\hline 0.01110 & 126.2 & 0.2 & u & 124.8 \\
\hline 0.01410 & 114.9 & 0.3 & u & 115.3 \\
\hline 0.01800 & 104.4 & 0.3 & u & 106.9 \\
\hline 0.02300 & 98.3 & 0.2 & u & 99. \\
\hline 0.02940 & 93.4 & 0.2 & u & 93. \\
\hline 0.03750 & 89.8 & 0.2 & u & 88. \\
\hline 0.04790 & 86.4 & 0.3 & u & 85. \\
\hline 0.06110 & 83.0 & 0.2 & u & 82.4 \\
\hline 0.07790 & 79.7 & 0.3 & u & 79.7 \\
\hline 0.09930 & 75.1 & 0.2 & $\mathrm{u}$ & 76.5 \\
\hline 0.12670 & 71.3 & 0.2 & u & 72.6 \\
\hline 0.16640 & 67.2 & 0.3 & u & 67.7 \\
\hline 0.20600 & 63.6 & 0.4 & u & 63. \\
\hline 0.26280 & 61.4 & 0.5 & u & 60. \\
\hline 0.33520 & 57.4 & 0.7 & u & 57. \\
\hline 0 & 55.7 & 0.8 & u & 5. \\
\hline & 54.8 & 1.4 & u & 4 \\
\hline & $\cdot 1$ & 1.8 & u & \\
\hline
\end{tabular}

Date: 25-FEB-09

UTM Coord: $\mathrm{E}[\mathrm{m}]$

Elevation $[\mathrm{m}]$ :

$555439.0 \quad \mathrm{~N}[\mathrm{~m}] \quad 2810949.0$

0.0

RX location: $\mathrm{X}[\mathrm{m}]$

$0.0 \quad Y[m]$

0.0
Fit Error [\%]: 2.422

System: EM-58 Freq[Hz]: 30 Data Set Code: hi TX Cur $[\mathrm{A}]: 2.50$ Turn Off[usec]: 2.5

RX Moment [turns-m^2]: 31.4 Gain Setting: 5

$\begin{array}{crrcc}\begin{array}{c}\text { Time } \\ \text { [ms] }\end{array} & \begin{array}{c}\text { rhoa_obs } \\ \text { [ohm-m] }\end{array} & \begin{array}{c}\text { obs_err } \\ {\left[\begin{array}{c}\overline{\%} \\ 0\end{array}\right.}\end{array} & \text { mask } & \begin{array}{c}\text { rhoa_cal } \\ \text { [ohm-m] }\end{array} \\ 0.08800 & 78.5 & 0.6 & \mathrm{u} & 77.8 \\ 0.10600 & 75.2 & 0.7 & \mathrm{u} & 75.0 \\ 0.13100 & 71.6 & 0.7 & \mathrm{u} & 71.3 \\ 0.16100 & 67.9 & 0.8 & \mathrm{u} & 67.3 \\ 0.20000 & 63.5 & 0.8 & \mathrm{u} & 63.1 \\ 0.25000 & 59.2 & 0.8 & \mathrm{u} & 59.1 \\ 0.31400 & 55.4 & 0.9 & \mathrm{u} & 55.7 \\ 0.39500 & 52.1 & 0.9 & \mathrm{u} & 52.8 \\ 0.49900 & 48.7 & 1.7 & \mathrm{u} & 50.5 \\ 0.63100 & 47.4 & 2.4 & \mathrm{u} & 48.7 \\ 0.79900 & 46.6 & 3.5 & \mathrm{u} & 47.4 \\ 1.01000 & 45.8 & 6.5 & \mathrm{u} & 46.4 \\ 1.28000 & 46.8 & 10.3 & \mathrm{u} & 45.9 \\ 1.63000 & 45.7 & 13.5 & \mathrm{u} & 45.5 \\ 2.08000 & 43.4 & 14.8 & \mathrm{~m} & 45.4 \\ 2.64000 & 45.6 & 27.9 & \mathrm{~d} & - \\ 3.37000 & 41.2 & 64.3 & \mathrm{~d} & - \\ 4.29000 & 95.6 & 100.0 & \mathrm{~d} & - \\ 5.47000 & 32.5 & 100.0 & \mathrm{~d} & - \\ 6.97000 & 82.7 & 100.0 & \mathrm{~d} & -\end{array}$


Sounding: MIA210

Client: US Geological Survey

Location: E SW 162 Ave \& S SW 380 St

Project: Saltwater Intrusion

County: Miami-Dade County

TX loop size: X[m] $37.9 \quad \mathrm{Y}[\mathrm{m}] \quad 37.9$

$\begin{array}{cccc}\text { Model } & \text { Resistivity } & \text { Thickness } & \text { Elevation } \\ \text { Layer } & {[\text { ohm-m] }} & {[\mathrm{m}]} & {[\mathrm{m}]} \\ 1 & 442.400 & 11.8 & 0.0 \\ 2 & 60.000 & 84.6 & -11.8 \\ 3 & 16.110 & 14.0 & -96.4 \\ 4 & 44.200 & -- & -110.4\end{array}$

System: EM-58 Freq[Hz]: 285 Data Set Code: uh TX $\operatorname{Cur}[A]: 2.65$ Turn off[usec] : 2.5

RX Moment[turns-m^2]: 31.4 Gain Setting: 2

$\begin{array}{rrrrrr}\text { Time } & \begin{array}{c}\text { rhoa_obs } \\ \text { [ohm-m] }\end{array} & \begin{array}{c}\text { obs_err } \\ {\left[\frac{\mathrm{o}}{0}\right]}\end{array} & \text { mask } & \begin{array}{c}\text { rhoa_cal } \\ \text { [ohm-m] }\end{array} \\ 1 & 0.00680 & 136.7 & 0.3 & \mathrm{~m} & 126.6 \\ 2 & 0.00860 & 116.2 & 0.3 & \mathrm{u} & 114.7 \\ 3 & 0.01110 & 109.2 & 0.4 & \mathrm{u} & 107.4 \\ 4 & 0.01410 & 101.2 & 0.4 & \mathrm{u} & 101.6 \\ 5 & 0.01800 & 93.6 & 0.5 & \mathrm{u} & 96.3 \\ 6 & 0.02300 & 89.7 & 0.4 & \mathrm{u} & 91.6 \\ 7 & 0.02940 & 86.7 & 0.4 & \mathrm{u} & 87.7 \\ 8 & 0.03750 & 84.8 & 0.4 & \mathrm{u} & 84.6 \\ 9 & 0.04790 & 83.0 & 0.4 & \mathrm{u} & 82.3 \\ 10 & 0.06110 & 81.1 & 0.4 & \mathrm{u} & 80.5 \\ 11 & 0.07790 & 79.0 & 0.4 & \mathrm{u} & 78.6 \\ 12 & 0.09930 & 75.3 & 0.4 & \mathrm{u} & 76.1 \\ 13 & 0.12670 & 72.2 & 0.4 & \mathrm{u} & 72.8 \\ 14 & 0.16640 & 68.8 & 0.5 & \mathrm{u} & 68.5 \\ 15 & 0.20600 & 65.2 & 0.5 & \mathrm{u} & 65.3 \\ 16 & 0.26280 & 63.0 & 0.9 & \mathrm{u} & 61.9 \\ 17 & 0.33520 & 59.6 & 1.3 & \mathrm{u} & 59.4 \\ 18 & 0.42770 & 57.8 & 1.6 & \mathrm{u} & 57.7 \\ 19 & 0.54560 & 58.0 & 4.9 & \mathrm{u} & 56.9 \\ 20 & 0.69590 & 60.1 & 2.0 & \mathrm{u} & 57.1\end{array}$

Date: $25-\mathrm{FEB}-09$

UTM Coord: $\mathrm{E}[\mathrm{m}]$

Elevation $[\mathrm{m}]$ :

$555420.0 \quad \mathrm{~N}[\mathrm{~m}] \quad 2810648.0$

0.0

RX location: X $[\mathrm{m}]$

$0.0 \quad Y[m]$

0.0
Fit Error $[\%]: 2.367$

System: EM-58 Freq[Hz]: 30 Data Set Code: hi TX Cur $[A]: 2.50$ Turn Off[usec]: 2.5

RX Moment [turns-m^2]: 31.4 Gain Setting: 5

$\begin{array}{crrcc}\begin{array}{c}\text { Time } \\ \text { [ms] }\end{array} & \begin{array}{c}\text { rhoa_obs } \\ \text { [ohm-m] }\end{array} & \begin{array}{c}\text { obs_err } \\ {\left[\begin{array}{c}\overline{\%} \\ \text { o }]\end{array}\right.}\end{array} & \text { mask } & \begin{array}{c}\text { rhoa_cal } \\ \text { [ohm-m] }\end{array} \\ 0.08800 & 77.5 & 0.3 & \mathrm{u} & 77.1 \\ 0.10600 & 75.0 & 0.4 & \mathrm{u} & 74.8 \\ 0.13100 & 71.9 & 0.4 & \mathrm{u} & 71.7 \\ 0.16100 & 68.6 & 0.4 & \mathrm{u} & 68.1 \\ 0.20000 & 64.6 & 0.5 & \mathrm{u} & 64.5 \\ 0.25000 & 60.7 & 0.7 & \mathrm{u} & 60.9 \\ 0.31400 & 57.0 & 0.8 & \mathrm{u} & 57.8 \\ 0.39500 & 53.7 & 1.0 & \mathrm{u} & 55.0 \\ 0.49900 & 51.0 & 2.1 & \mathrm{u} & 52.9 \\ 0.63100 & 50.3 & 1.8 & \mathrm{u} & 51.1 \\ 0.79900 & 49.2 & 6.2 & \mathrm{u} & 49.7 \\ 1.01000 & 48.5 & 6.3 & \mathrm{u} & 48.6 \\ 1.28000 & 48.0 & 10.2 & \mathrm{u} & 47.8 \\ 1.63000 & 44.2 & 11.5 & \mathrm{~d} & - \\ 2.08000 & 37.6 & 27.3 & \mathrm{~d} & - \\ 2.64000 & 33.6 & 47.2 & \mathrm{~d} & - \\ 3.37000 & 28.6 & 44.8 & \mathrm{~d} & - \\ 4.29000 & 31.4 & 77.4 & \mathrm{~d} & - \\ 5.47000 & 100.9 & 100.0 & \mathrm{~d} & - \\ 6.97000 & 58.3 & 100.0 & \mathrm{~d} & -\end{array}$


Sounding: MIA211

Client: US Geological Survey

Location: E SW 162 Ave \& S SW 380 St

Project: Saltwater Intrusion

County: Miami-Dade County

TX loop size: X $[\mathrm{m}] \quad 37.9 \quad \mathrm{Y}[\mathrm{m}] \quad 37.9$

$\begin{array}{cccc}\text { Model } & \text { Resistivity } & \text { Thickness } & \text { Elevation } \\ \text { Layer } & {[\text { ohm-m] }} & {[\mathrm{m}]} & {[\mathrm{m}]} \\ 1 & 176.100 & 20.0 & 0.0 \\ 2 & 9.770 & 4.1 & -20.0 \\ 3 & 82.010 & 50.2 & -24.1 \\ 4 & 34.660 & -- & -74.3\end{array}$

System: EM-58 Freq[Hz]: 285 Data Set Code: uh TX Cur[A]: 2.70 Turn Off[usec]: 2.4

RX Moment[turns-m^2]: 31.4 Gain Setting: 2

\begin{tabular}{|c|c|c|c|c|}
\hline $\begin{array}{l}\text { Time } \\
{[\mathrm{ms}]}\end{array}$ & $\begin{array}{l}\text { rhoa_obs } \\
\text { [ohm-m] }\end{array}$ & $\begin{array}{c}\text { obs err } \\
{\left[\frac{\overline{0}}{0}\right]}\end{array}$ & mask & $\begin{array}{l}\text { rhoa_ca] } \\
\text { [ohm-m] }\end{array}$ \\
\hline 0.00680 & 110.8 & 0.2 & $u$ & 110.6 \\
\hline 0.00860 & 89.7 & 0.2 & u & 90.9 \\
\hline 0.01110 & 79.0 & 0.3 & $\mathrm{u}$ & 78.3 \\
\hline 0.01410 & 70.4 & 0.2 & u & 69.9 \\
\hline 0.01800 & 63.9 & 0.2 & u & 64.0 \\
\hline 0.02300 & 60.1 & 0.2 & $u$ & 60.1 \\
\hline 0.02940 & 57.8 & 0.2 & $\mathrm{u}$ & 58. \\
\hline 0.03750 & 56.9 & 0.2 & $\mathrm{u}$ & 56. \\
\hline 0.04790 & 56.8 & 0.2 & $\mathrm{u}$ & 56. \\
\hline 0.06110 & 57.2 & 0.2 & u & 57.4 \\
\hline 0.07790 & 58.0 & 0.2 & $\mathrm{u}$ & 58. \\
\hline 0.09930 & 58.2 & 0.2 & u & 8. \\
\hline 0.12670 & 58.5 & 0.3 & $\mathrm{u}$ & 58. \\
\hline 0.16640 & 58.7 & 0.4 & $u$ & 58. \\
\hline 0.20600 & 57.4 & 0.4 & $u$ & 57. \\
\hline 0.26280 & 57.6 & 0.4 & $u$ & 6.7 \\
\hline 0.33520 & 55.5 & 0.7 & $u$ & 5. \\
\hline 0.42770 & 55.1 & 1.4 & $u$ & 4.9 \\
\hline 0.5 & 55.2 & 1.9 & u & 4. \\
\hline 0 . & 58.1 & 3.9 & $\mathrm{~m}$ & 4.8 \\
\hline
\end{tabular}

Date: $25-\mathrm{FEB}-09$

UTM Coord: $\mathrm{E}[\mathrm{m}]$

Elevation $[\mathrm{m}]$ :

$555523.0 \quad \mathrm{~N}[\mathrm{~m}] \quad 2810163.0$

0.0

RX location: X $[\mathrm{m}]$

$0.0 \quad Y[m]$

0.0
Fit Error $[\%]: 1.314$

System: EM-58 Freq[Hz]: 30 Data Set Code: hi TX Cur $[A]: 2.50$ Turn Off[usec]: 2.5

RX Moment [turns-m^2] : 31.4 Gain Setting: 5

$\begin{array}{crrcr}\begin{array}{c}\text { Time } \\ \text { [ms] }\end{array} & \begin{array}{r}\text { rhoa_obs } \\ \text { [ohm-m] }\end{array} & \begin{array}{c}\text { obs_err } \\ {\left[\begin{array}{c}\overline{\%} \\ 0\end{array}\right.}\end{array} & \text { mask } & \begin{array}{r}\text { rhoa_cal } \\ \text { [ohm-m] }\end{array} \\ 0.08800 & 58.3 & 0.3 & \mathrm{u} & 58.2 \\ 0.10600 & 58.6 & 0.3 & \mathrm{u} & 58.6 \\ 0.13100 & 58.7 & 0.4 & \mathrm{u} & 58.3 \\ 0.16100 & 58.3 & 0.3 & \mathrm{u} & 57.8 \\ 0.20000 & 57.0 & 0.7 & \mathrm{u} & 56.7 \\ 0.25000 & 55.1 & 0.6 & \mathrm{u} & 55.4 \\ 0.31400 & 52.9 & 1.1 & \mathrm{u} & 53.7 \\ 0.39500 & 51.0 & 1.2 & \mathrm{u} & 52.1 \\ 0.49900 & 49.4 & 0.8 & \mathrm{u} & 50.3 \\ 0.63100 & 49.2 & 1.7 & \mathrm{u} & 48.7 \\ 0.79900 & 48.1 & 4.0 & \mathrm{~m} & 47.1 \\ 1.01000 & 48.5 & 4.7 & \mathrm{~m} & 45.9 \\ 1.28000 & 48.0 & 9.9 & \mathrm{~m} & 44.5 \\ 1.63000 & 43.6 & 13.6 & \mathrm{~m} & 43.7 \\ 2.08000 & 41.1 & 9.9 & \mathrm{~m} & 42.7 \\ 2.64000 & 43.4 & 29.3 & \mathrm{~d} & - \\ 3.37000 & 91.0 & 100.0 & \mathrm{~d} & - \\ 4.29000 & 55.9 & 100.0 & \mathrm{~d} & - \\ 5.47000 & 30.5 & 65.8 & \mathrm{~d} & - \\ 6.97000 & 35.0 & 100.0 & \mathrm{~d} & -\end{array}$


Sounding: MIA212

Client: US Geological Survey Location: $0.34 \mathrm{~km} \mathrm{~S}$ of SW $388 \mathrm{St} \& 0.31 \mathrm{~km} \mathrm{~W}$ of SW 157 Ave Project: Saltwater Intrusion County: Miami-Dade County

TX loop size: X $[\mathrm{m}] \quad 37.9 \quad \mathrm{Y}[\mathrm{m}] \quad 37.9$

$\begin{array}{cccc}\text { Model } & \text { Resistivity } & \text { Thickness } & \text { Elevation } \\ \text { Layer } & {[\text { ohm-m] }} & {[\mathrm{m}]} & {[\mathrm{m}]} \\ 1 & 274.700 & 19.6 & 0.0 \\ 2 & .931 & .8 & -19.6 \\ 3 & 116.600 & 31.2 & -20.5 \\ 4 & 44.930 & -- & -51.7\end{array}$

System: EM-58 Freq[Hz]: 285 Data Set Code: uh TX Cur $[\mathrm{A}]: \quad 2.70$ Turn Off[usec]: 2.5

RX Moment[turns-m^2]: 31.4 Gain Setting: 2

\begin{tabular}{|c|c|c|c|c|}
\hline $\begin{array}{l}\text { Time } \\
{[\mathrm{ms}]}\end{array}$ & $\begin{array}{l}\text { rhoa_obs } \\
\text { [ohm-m] }\end{array}$ & $\begin{array}{c}\text { obs err } \\
{\left[\frac{\overline{0}}{0}\right]}\end{array}$ & mask & $\begin{array}{l}\text { rhoa_ca] } \\
\text { [ohm-m] }\end{array}$ \\
\hline 0.00680 & 114.7 & 0.3 & $u$ & 115.9 \\
\hline 0.00860 & 84.8 & 0.3 & u & 86.0 \\
\hline 0.01110 & 67.4 & 0.3 & u & 66.8 \\
\hline 0.01410 & 54.5 & 0.3 & u & 53.9 \\
\hline 0.01800 & 45.4 & 0.3 & u & 45. \\
\hline 0.02300 & 39.3 & 0.3 & u & 39. \\
\hline 0.02940 & 35.3 & 0.3 & u & 35. \\
\hline 0.03750 & 32.8 & 0.3 & $\mathrm{u}$ & 32 . \\
\hline 0.04790 & 31.5 & 0.3 & $\mathrm{u}$ & 31. \\
\hline 0.06110 & 31.1 & 0.3 & u & 31. \\
\hline 0.07790 & 31.5 & 0.3 & $\mathrm{u}$ & 31. \\
\hline 0.09930 & 32.3 & 0.3 & u & 32 . \\
\hline 0.12670 & 33.7 & 0.3 & $\mathrm{u}$ & 34. \\
\hline 0.16640 & 35.9 & 0.4 & $u$ & 35 . \\
\hline 0.20600 & 37.1 & 0.5 & u & 37. \\
\hline 0.26280 & 39.2 & 0.6 & $u$ & 38. \\
\hline 0.33520 & 40.4 & 0.8 & $u$ & 10. \\
\hline $0.42^{7}$ & 41.9 & 1.8 & u & 11. \\
\hline 0.5 & 43.8 & 3.0 & u & 3. \\
\hline & 47.3 & 5.6 & u & \\
\hline
\end{tabular}

Date: $25-\mathrm{FEB}-09$

UTM Coord: $\mathrm{E}[\mathrm{m}]$

Elevation $[\mathrm{m}]$ :

$555558.0 \quad \mathrm{~N}[\mathrm{~m}] \quad 2809832.0$

0.0

RX location: $\mathrm{X}[\mathrm{m}]$

$0.0 \quad Y[m]$

0.0
Fit Error $[\%]: 1.767$

System: EM-58 Freq[Hz]: 30 Data Set Code: hi TX Cur $[A]: 2.50$ Turn Off[usec]: 2.5

RX Moment [turns-m^2] : 31.4 Gain Setting: 5

$\begin{array}{crrcc}\begin{array}{c}\text { Time } \\ \text { [ms] }\end{array} & \begin{array}{c}\text { rhoa_obs } \\ \text { [ohm-m] }\end{array} & \begin{array}{c}\text { obs_err } \\ {\left[\begin{array}{c}\bar{o} \\ \mathrm{o}\end{array}\right.}\end{array} & \text { mask } & \begin{array}{c}\text { rhoa_cal } \\ \text { [ohm-m] }\end{array} \\ 0.08800 & 32.0 & 0.5 & \mathrm{u} & 32.1 \\ 0.10600 & 32.9 & 0.5 & \mathrm{u} & 33.0 \\ 0.13100 & 34.0 & 0.5 & \mathrm{u} & 34.1 \\ 0.16100 & 35.5 & 0.4 & \mathrm{u} & 35.3 \\ 0.20000 & 36.7 & 0.6 & \mathrm{u} & 36.5 \\ 0.25000 & 37.9 & 0.5 & \mathrm{u} & 37.7 \\ 0.31400 & 38.5 & 0.5 & \mathrm{u} & 38.7 \\ 0.39500 & 39.0 & 0.4 & \mathrm{u} & 39.6 \\ 0.49900 & 39.5 & 0.8 & \mathrm{u} & 40.4 \\ 0.63100 & 39.9 & 1.3 & \mathrm{u} & 41.0 \\ 0.79900 & 41.4 & 2.8 & \mathrm{u} & 41.6 \\ 1.01000 & 42.5 & 3.8 & \mathrm{u} & 42.0 \\ 1.28000 & 43.1 & 8.4 & \mathrm{u} & 42.5 \\ 1.63000 & 42.1 & 8.6 & \mathrm{u} & 42.8 \\ 2.08000 & 47.0 & 22.0 & \mathrm{~m} & 43.3 \\ 2.64000 & 60.9 & 66.5 & \mathrm{~d} & - \\ 3.37000 & 96.1 & 100.0 & \mathrm{~d} & - \\ 4.29000 & 90.7 & 100.0 & \mathrm{~d} & - \\ 5.47000 & 79.4 & 100.0 & \mathrm{~d} & - \\ 6.97000 & 13.9 & 54.8 & \mathrm{~d} & -\end{array}$


Sounding: MIA212F

Client: US Geological Survey Location: $0.34 \mathrm{~km} \mathrm{~S}$ of SW $388 \mathrm{St} \& 0.31 \mathrm{~km} \mathrm{~W}$ of SW 157 Ave Project: Saltwater Intrusion County: Miami-Dade County

TX loop size: X $[\mathrm{m}] \quad 37.9 \quad \mathrm{Y}[\mathrm{m}] \quad 37.9$

$\begin{array}{cccc}\text { Model } & \text { Resistivity } & \text { Thickness } & \text { Elevation } \\ \text { Layer } & {[\text { ohm-m] }} & {[\mathrm{m}]} & {[\mathrm{m}]} \\ 1 & 257.400 & 18.7 & 0.0 \\ 2 & 3.500 & 3.4 & -18.7 \\ 3 & 126.500 & 32.7 & -22.2 \\ 4 & 44.220 & -- & -55.0\end{array}$

System: EM-58 Freq[Hz]: 285 Data Set Code: uh TX Cur[A]: 2.70 Turn Off[usec]: 2.5

RX Moment[turns-m^2]: 31.4 Gain Setting: 2

\begin{tabular}{|c|c|c|c|c|}
\hline $\begin{array}{l}\text { Time } \\
{[\mathrm{ms}]}\end{array}$ & $\begin{array}{l}\text { rhoa_obs } \\
\text { [ohm-m] }\end{array}$ & $\begin{array}{c}\text { obs err } \\
{\left[\frac{\overline{0}}{0}\right]}\end{array}$ & mask & $\begin{array}{l}\text { rhoa_ca] } \\
{[\text { ohm-m] }}\end{array}$ \\
\hline 0.00680 & 114.7 & 0.3 & $u$ & 115.1 \\
\hline 0.00860 & 84.8 & 0.3 & $u$ & 85.7 \\
\hline 0.01110 & 67.4 & 0.3 & u & 66.8 \\
\hline 0.01410 & 54.5 & 0.3 & u & 54. \\
\hline 0.01800 & 45.4 & 0.3 & u & 45.3 \\
\hline 0.02300 & 39.3 & 0.3 & u & 39.2 \\
\hline 0.02940 & 35.3 & 0.3 & u & 35. \\
\hline 0.03750 & 32.8 & 0.3 & u & 32.8 \\
\hline 0.04790 & 31.5 & 0.3 & u & 31. \\
\hline 0.06110 & 31.1 & 0.3 & u & 31. \\
\hline 0.07790 & 31.5 & 0.3 & u & 31.7 \\
\hline 0.09930 & 32.3 & 0.3 & $\mathrm{u}$ & 32.6 \\
\hline 0.12670 & 33.7 & 0.3 & u & 33. \\
\hline 0.16640 & 35.9 & 0.4 & u & 35.6 \\
\hline 0.20600 & 37.1 & 0.5 & u & 37. \\
\hline 0.26280 & 39.2 & 0.6 & u & 38 . \\
\hline 0.33520 & 40.4 & 0.8 & u & 40. \\
\hline 0.4 & 41.9 & 1.8 & u & I. \\
\hline 0 & 43.8 & 3.0 & u & 3 \\
\hline & .3 & 5.6 & u & \\
\hline
\end{tabular}

Date: 25-FEB-09

UTM Coord: $\mathrm{E}[\mathrm{m}]$

Elevation $[\mathrm{m}]$ :

$555558.0 \quad \mathrm{~N}[\mathrm{~m}] \quad 2809832.0$

0.0

RX location: $\mathrm{X}[\mathrm{m}]$

$0.0 \quad Y[m]$

0.0
Fit Error $[\%]: 1.653$

System: EM-58 Freq[Hz]: 30 Data Set Code: hi TX Cur $[A]: 2.50$ Turn Off[usec]: 2.5

RX Moment [turns-m^2]: 31.4 Gain Setting: 5

$\begin{array}{crrcc}\begin{array}{c}\text { Time } \\ \text { [ms] }\end{array} & \begin{array}{c}\text { rhoa_obs } \\ \text { [ohm-m] }\end{array} & \begin{array}{c}\text { obs_err } \\ {\left[\begin{array}{c}\bar{o} \\ \mathrm{o}\end{array}\right.}\end{array} & \text { mask } & \begin{array}{c}\text { rhoa_cal } \\ \text { [ohm-m] }\end{array} \\ 0.08800 & 32.0 & 0.5 & \mathrm{u} & 32.0 \\ 0.10600 & 32.9 & 0.5 & \mathrm{u} & 32.9 \\ 0.13100 & 34.0 & 0.5 & \mathrm{u} & 34.0 \\ 0.16100 & 35.5 & 0.4 & \mathrm{u} & 35.2 \\ 0.20000 & 36.7 & 0.6 & \mathrm{u} & 36.5 \\ 0.25000 & 37.9 & 0.5 & \mathrm{u} & 37.7 \\ 0.31400 & 38.5 & 0.5 & \mathrm{u} & 38.8 \\ 0.39500 & 39.0 & 0.4 & \mathrm{u} & 39.7 \\ 0.49900 & 39.5 & 0.8 & \mathrm{u} & 40.5 \\ 0.63100 & 39.9 & 1.3 & \mathrm{u} & 41.0 \\ 0.79900 & 41.4 & 2.8 & \mathrm{u} & 41.6 \\ 1.01000 & 42.5 & 3.8 & \mathrm{u} & 41.9 \\ 1.28000 & 43.1 & 8.4 & \mathrm{u} & 42.5 \\ 1.63000 & 42.1 & 8.6 & \mathrm{u} & 42.6 \\ 2.08000 & 47.0 & 22.0 & \mathrm{~m} & 43.1 \\ 2.64000 & 60.9 & 66.5 & \mathrm{~d} & - \\ 3.37000 & 96.1 & 100.0 & \mathrm{~d} & - \\ 4.29000 & 90.7 & 100.0 & \mathrm{~d} & - \\ 5.47000 & 79.4 & 100.0 & \mathrm{~d} & - \\ 6.97000 & 13.9 & 54.8 & \mathrm{~d} & -\end{array}$


Sounding: MIA213

Client: US Geological Survey Location: $0.06 \mathrm{~km} \mathrm{~N}$ of $\mathrm{SW} 388$ \& $0.06 \mathrm{~km} \mathrm{E}$ of Kingman Rd Project: Saltwater Intrusion County: Miami-Dade County

TX loop size: X $[\mathrm{m}] \quad 37.9 \quad \mathrm{Y}[\mathrm{m}] \quad 37.9$

$\begin{array}{cccc}\text { Model } & \text { Resistivity } & \text { Thickness } & \text { Elevation } \\ \text { Layer } & {[\text { ohm-m] }} & {[\mathrm{m}]} & {[\mathrm{m}]} \\ 1 & 102.300 & 21.4 & 0.0 \\ 2 & .541 & .7 & -21.4 \\ 3 & 44.420 & 97.8 & -22.1 \\ 4 & 13.970 & -- & -119.9\end{array}$

System: EM-58 Freq[Hz]: 285 Data Set Code: uh TX Cur[A]: 2.70 Turn Off[usec]: 2.5

RX Moment[turns-m^2]: 31.4 Gain Setting: 2

$\begin{array}{rrrrrr}\text { Time } & \begin{array}{c}\text { rhoa_obs } \\ \text { [ohm-m] }\end{array} & \begin{array}{c}\text { obs_err } \\ {[\bar{\circ} \text { o }]}\end{array} & \text { mask } & \begin{array}{c}\text { rhoa_cal } \\ \text { [ohm-m] }\end{array} \\ 1 & 0.00680 & 124.2 & 0.3 & u & 122.6 \\ 2 & 0.00860 & 94.1 & 0.2 & \mathrm{u} & 97.9 \\ 3 & 0.01110 & 74.7 & 0.2 & \mathrm{u} & 75.3 \\ 4 & 0.01410 & 58.7 & 0.2 & \mathrm{u} & 58.1 \\ 5 & 0.01800 & 46.5 & 0.2 & \mathrm{u} & 45.8 \\ 6 & 0.02300 & 37.7 & 0.2 & \mathrm{u} & 37.2 \\ 7 & 0.02940 & 31.4 & 0.2 & \mathrm{u} & 31.1 \\ 8 & 0.03750 & 27.1 & 0.2 & \mathrm{u} & 26.9 \\ 9 & 0.04790 & 24.1 & 0.2 & \mathrm{u} & 24.1 \\ 10 & 0.06110 & 22.2 & 0.2 & \mathrm{u} & 22.4 \\ 11 & 0.07790 & 21.2 & 0.2 & \mathrm{u} & 21.4 \\ 12 & 0.09930 & 20.8 & 0.2 & \mathrm{u} & 21.0 \\ 13 & 0.12670 & 21.0 & 0.2 & \mathrm{u} & 21.1 \\ 14 & 0.16640 & 22.2 & 0.1 & \mathrm{u} & 21.8 \\ 15 & 0.20600 & 23.0 & 0.1 & \mathrm{u} & 22.7 \\ 16 & 0.26280 & 24.5 & 0.2 & \mathrm{u} & 24.0 \\ 17 & 0.33520 & 25.8 & 0.2 & \mathrm{u} & 25.6 \\ 18 & 0.42770 & 27.5 & 0.4 & \mathrm{u} & 27.4 \\ 19 & 0.54560 & 29.2 & 0.4 & \mathrm{u} & 29.3 \\ 20 & 0.69590 & 32.1 & 1.1 & \mathrm{u} & 31.0\end{array}$

Date: $25-\mathrm{FEB}-09$

UTM Coord: $\mathrm{E}[\mathrm{m}]$

Elevation $[\mathrm{m}]$ :

$556774.0 \quad \mathrm{~N}[\mathrm{~m}] \quad 2810192.0$

0.0

RX location: $\mathrm{X}[\mathrm{m}]$

$0.0 \quad Y[m]$

0.0
Fit Error [응: 2.353

System: EM-58 Freq[Hz]: 30 Data Set Code: hi TX Cur $[A]: 2.50$ Turn Off[usec]: 2.5

RX Moment[turns-m^2]: 31.4 Gain Setting: 5

$\begin{array}{ccccc}\begin{array}{c}\text { Time } \\ \text { [ms] }\end{array} & \begin{array}{c}\text { rhoa_obs } \\ \text { [ohm-m] }\end{array} & \begin{array}{c}\text { obs_err } \\ {\left[\begin{array}{c}\bar{o} \\ \mathrm{o}\end{array}\right.}\end{array} & \text { mask } & \begin{array}{c}\text { rhoa_cal } \\ \text { [ohm-m] }\end{array} \\ 0.08800 & 20.8 & 0.2 & \mathrm{u} & 21.1 \\ 0.10600 & 20.7 & 0.2 & \mathrm{u} & 20.9 \\ 0.13100 & 21.0 & 0.2 & \mathrm{u} & 21.1 \\ 0.16100 & 21.7 & 0.2 & \mathrm{u} & 21.6 \\ 0.20000 & 22.5 & 0.2 & \mathrm{u} & 22.4 \\ 0.25000 & 23.5 & 0.4 & \mathrm{u} & 23.4 \\ 0.31400 & 24.4 & 0.3 & \mathrm{u} & 24.5 \\ 0.39500 & 25.2 & 0.5 & \mathrm{u} & 25.7 \\ 0.49900 & 25.9 & 1.3 & \mathrm{u} & 26.7 \\ 0.63100 & 26.5 & 1.2 & \mathrm{u} & 27.3 \\ 0.79900 & 27.3 & 4.4 & \mathrm{u} & 27.4 \\ 1.01000 & 27.6 & 4.7 & \mathrm{u} & 27.1 \\ 1.28000 & 26.7 & 6.9 & \mathrm{u} & 26.4 \\ 1.63000 & 25.2 & 11.9 & \mathrm{u} & 25.5 \\ 2.08000 & 22.1 & 17.6 & \mathrm{~d} & - \\ 2.64000 & 17.9 & 26.8 & \mathrm{~d} & - \\ 3.37000 & 13.4 & 29.1 & \mathrm{~d} & - \\ 4.29000 & 10.6 & 38.7 & \mathrm{~d} & - \\ 5.47000 & 7.1 & 33.5 & \mathrm{~d} & - \\ 6.97000 & 5.1 & 30.1 & \mathrm{~d} & -\end{array}$


Sounding: MIA213F

Client: US Geological Survey Location: $0.06 \mathrm{~km} \mathrm{~N}$ of $\mathrm{SW} 388$ \& $0.06 \mathrm{~km}$ E of Kingman Rd Project: Saltwater Intrusion County: Miami-Dade County

TX loop size: X[m] $37.9 \quad \mathrm{Y}[\mathrm{m}] \quad 37.9$

$\begin{array}{cccc}\text { Model } & \text { Resistivity } & \text { Thickness } & \text { Elevation } \\ \text { Layer } & \text { [ohm-m] } & {[\mathrm{m}]} & {[\mathrm{m}]} \\ 1 & 118.100 & 19.5 & 0.0 \\ 2 & 3.500 & 5.1 & -19.5 \\ 3 & 49.430 & 90.1 & -24.7 \\ 4 & 14.390 & -- & -114.9\end{array}$

System: EM-58 Freq[Hz]: 285 Data Set Code: uh TX $\operatorname{Cur}[\mathrm{A}]: 2.70$ Turn Off [usec]: 2.5 RX Moment[turns-m^2]: 31.4 Gain Setting: 2

\begin{tabular}{|c|c|c|c|c|}
\hline $\begin{array}{l}\text { Time } \\
{[\mathrm{ms}]}\end{array}$ & $\begin{array}{l}\text { rhoa_obs } \\
\text { [ohm-m] }\end{array}$ & $\begin{array}{c}\text { obs err } \\
{\left[\frac{\overline{0}}{0}\right]}\end{array}$ & mask & $\begin{array}{l}\text { rhoa_cal } \\
\text { [ohm-m] }\end{array}$ \\
\hline 0.00680 & 124.2 & 0.3 & u & 123.4 \\
\hline 0.00860 & 94.1 & 0.2 & $u$ & 97.0 \\
\hline 0.01110 & 74.7 & 0.2 & $u$ & 74.7 \\
\hline 0.01410 & 58.7 & 0.2 & $u$ & 58.0 \\
\hline 0.01800 & 46.5 & 0.2 & $\mathrm{u}$ & 45.9 \\
\hline 0.02300 & 37.7 & 0.2 & $\mathrm{u}$ & 37.3 \\
\hline 0.02940 & 31.4 & 0.2 & $u$ & 31.3 \\
\hline 0.03750 & 27.1 & 0.2 & u & 27.1 \\
\hline 0.04790 & 24.1 & 0.2 & u & 24.3 \\
\hline 0.06110 & 22.2 & 0.2 & $u$ & 22.4 \\
\hline 0.07790 & 21.2 & 0.2 & u & 21.4 \\
\hline 0.09930 & 20.8 & 0.2 & u & 21.0 \\
\hline 0.12670 & 21.0 & 0.2 & $u$ & 21.1 \\
\hline 0.16640 & 22.2 & 0.1 & $u$ & 21.7 \\
\hline 0.20600 & 23.0 & 0.1 & $u$ & 22.6 \\
\hline 0.26280 & 24.5 & 0.2 & u & 24.0 \\
\hline 0.33520 & 25.8 & 0.2 & u & 25.6 \\
\hline 0.42770 & 27.5 & 0.4 & u & 27.4 \\
\hline 560 & 29.2 & 0.4 & u & 29 \\
\hline 20 & 32.1 & 1.1 & $u$ & 31.0 \\
\hline
\end{tabular}

Date: 25-FEB-09

UTM Coord: $\mathrm{E}[\mathrm{m}]$

Elevation $[\mathrm{m}]$ :

$556774.0 \quad \mathrm{~N}[\mathrm{~m}] \quad 2810192.0$ 0.0

RX location: X $[\mathrm{m}]$

$0.0 \quad Y[m]$

0.0
Fit Error [응: 2.282

System: EM-58 Freq[Hz]: 30 Data Set Code: hi TX Cur $[A]: 2.50$ Turn Off[usec]: 2.5

RX Moment[turns-m^2]: 31.4 Gain Setting: 5

$\begin{array}{crrcc}\begin{array}{c}\text { Time } \\ \text { [ms] }\end{array} & \begin{array}{c}\text { rhoa_obs } \\ \text { [ohm-m] }\end{array} & \begin{array}{c}\text { obs_err } \\ {\left[\begin{array}{c}\bar{o} \\ \mathrm{o}\end{array}\right.}\end{array} & \text { mask } & \begin{array}{c}\text { rhoa_cal } \\ \text { [ohm-m] }\end{array} \\ 0.08800 & 20.8 & 0.2 & \mathrm{u} & 21.1 \\ 0.10600 & 20.7 & 0.2 & \mathrm{u} & 20.9 \\ 0.13100 & 21.0 & 0.2 & \mathrm{u} & 21.1 \\ 0.16100 & 21.7 & 0.2 & \mathrm{u} & 21.5 \\ 0.20000 & 22.5 & 0.2 & \mathrm{u} & 22.3 \\ 0.25000 & 23.5 & 0.4 & \mathrm{u} & 23.3 \\ 0.31400 & 24.4 & 0.3 & \mathrm{u} & 24.5 \\ 0.39500 & 25.2 & 0.5 & \mathrm{u} & 25.8 \\ 0.49900 & 25.9 & 1.3 & \mathrm{u} & 26.7 \\ 0.63100 & 26.5 & 1.2 & \mathrm{u} & 27.4 \\ 0.79900 & 27.3 & 4.4 & \mathrm{u} & 27.4 \\ 1.01000 & 27.6 & 4.7 & \mathrm{u} & 27.1 \\ 1.28000 & 26.7 & 6.9 & \mathrm{u} & 26.4 \\ 1.63000 & 25.2 & 11.9 & \mathrm{u} & 25.5 \\ 2.08000 & 22.1 & 17.6 & \mathrm{~d} & - \\ 2.64000 & 17.9 & 26.8 & \mathrm{~d} & - \\ 3.37000 & 13.4 & 29.1 & \mathrm{~d} & - \\ 4.29000 & 10.6 & 38.7 & \mathrm{~d} & - \\ 5.47000 & 7.1 & 33.5 & \mathrm{~d} & - \\ 6.97000 & 5.1 & 30.1 & \mathrm{~d} & -\end{array}$


Sounding: MIA214

Client: US Geological Survey Location: $0.29 \mathrm{~km} \mathrm{~N}$ of $\mathrm{SW} 388$ \& $0.03 \mathrm{~km} \mathrm{E}$ of Kingman Rd Project: Saltwater Intrusion County: Miami-Dade County

TX loop size: X $[\mathrm{m}] \quad 37.9 \quad \mathrm{Y}[\mathrm{m}] \quad 37.9$

$\begin{array}{cccc}\text { Model } & \text { Resistivity } & \text { Thickness } & \text { Elevation } \\ \text { Layer } & \text { [ohm-m] } & {[\mathrm{m}]} & {[\mathrm{m}]} \\ 1 & 147.600 & 19.9 & 0.0 \\ 2 & 7.720 & 2.6 & -19.9 \\ 3 & 43.760 & 56.2 & -22.5 \\ 4 & 10.230 & 11.0 & -78.7 \\ 5 & 32.900 & -- & -89.8\end{array}$

System: EM-58 Freq[Hz]: 285 Data Set Code: uh TX Cur $[A]: 2.60$ Turn Off [usec]: 2.5 RX Moment[turns-m^2]: 31.4 Gain Setting: 2

$\begin{array}{crrrr}\begin{array}{c}\text { Time } \\ \text { [ms }\end{array} & \begin{array}{r}\text { rhoa_obs } \\ \text { [ohm-m] }\end{array} & \begin{array}{c}\text { obs_err } \\ {\left[\frac{\overline{0}}{0}\right]}\end{array} & \text { mask } & \begin{array}{r}\text { rhoa_cal } \\ \text { [ohm-m] }\end{array} \\ 0.00680 & 107.1 & 0.2 & u & 105.9 \\ 0.00860 & 87.0 & 0.2 & u & 88.4 \\ 0.01110 & 77.1 & 0.2 & u & 76.7 \\ 0.01410 & 68.5 & 0.2 & u & 68.4 \\ 0.01800 & 61.9 & 0.2 & u & 62.1 \\ 0.02300 & 57.5 & 0.2 & u & 57.5 \\ 0.02940 & 54.2 & 0.2 & u & 54.1 \\ 0.03750 & 52.0 & 0.2 & u & 51.8 \\ 0.04790 & 50.4 & 0.2 & u & 50.4 \\ 0.06110 & 49.3 & 0.2 & u & 49.5 \\ 0.07790 & 48.4 & 0.2 & u & 48.7 \\ 0.09930 & 47.0 & 0.2 & u & 47.7 \\ 0.12670 & 45.6 & 0.3 & u & 46.2 \\ 0.16640 & 43.9 & 0.1 & u & 44.0 \\ 0.20600 & 41.9 & 0.3 & u & 42.2 \\ 0.26280 & 40.6 & 0.1 & u & 40.4 \\ 0.33520 & 38.9 & 0.4 & u & 39.0 \\ 0.42770 & 38.0 & 0.7 & u & 38.1 \\ 0.54560 & 37.8 & 1.5 & u & 37.9 \\ 0.69590 & 39.9 & 1.5 & u & 38.3\end{array}$

Date: $25-\mathrm{FEB}-09$

UTM Coord: $\mathrm{E}[\mathrm{m}]$

Elevation $[\mathrm{m}]$ :

$556751.0 \quad \mathrm{~N}[\mathrm{~m}]$

2810422.0

0.0

RX location: $\mathrm{X}[\mathrm{m}]$

$0.0 \quad Y[m]$

0.0
Fit Error [응 : 1.817

System: EM-58 Freq[Hz]: 30 Data Set Code: hi TX Cur $[\mathrm{A}]: 2.50$ Turn Off[usec]: 2.5

RX Moment [turns-m^2]: 31.4 Gain Setting: 5

$\begin{array}{ccccc}\begin{array}{c}\text { Time } \\ \text { [ms] }\end{array} & \begin{array}{c}\text { rhoa_obs } \\ \text { [ohm-m] }\end{array} & \begin{array}{c}\text { obs_err } \\ {\left[\overline{\frac{o}{0}}\right]}\end{array} & \text { mask } & \begin{array}{c}\text { rhoa_cal } \\ \text { [ohm-m] }\end{array} \\ 0.08800 & 48.5 & 0.2 & \mathrm{u} & 48.1 \\ 0.10600 & 47.4 & 0.2 & \mathrm{u} & 47.0 \\ 0.13100 & 45.9 & 0.3 & \mathrm{u} & 45.6 \\ 0.16100 & 44.2 & 0.3 & \mathrm{u} & 43.7 \\ 0.20000 & 42.1 & 0.2 & \mathrm{u} & 41.7 \\ 0.25000 & 39.8 & 0.4 & \mathrm{u} & 39.7 \\ 0.31400 & 37.7 & 0.5 & \mathrm{u} & 38.0 \\ 0.39500 & 35.8 & 0.9 & \mathrm{u} & 36.4 \\ 0.49900 & 34.7 & 1.1 & \mathrm{u} & 35.3 \\ 0.63100 & 33.9 & 1.8 & \mathrm{u} & 34.3 \\ 0.79900 & 33.4 & 3.1 & \mathrm{u} & 33.6 \\ 1.01000 & 33.4 & 2.8 & \mathrm{u} & 33.1 \\ 1.28000 & 33.5 & 6.3 & \mathrm{u} & 32.9 \\ 1.63000 & 31.9 & 8.4 & \mathrm{u} & 32.6 \\ 2.08000 & 29.7 & 12.4 & \mathrm{~d} & - \\ 2.64000 & 28.2 & 22.0 & \mathrm{~d} & - \\ 3.37000 & 28.6 & 38.0 & \mathrm{~d} & - \\ 4.29000 & 18.9 & 36.0 & \mathrm{~d} & - \\ 5.47000 & 12.7 & 38.6 & \mathrm{~d} & - \\ 6.97000 & 9.0 & 28.5 & \mathrm{~d} & -\end{array}$


Sounding: MIA215

Client: US Geological Survey Location: $0.60 \mathrm{~km} \mathrm{~N}$ of $\mathrm{SW} 380 \& 0.03 \mathrm{~km} \mathrm{~W}$ of $3 \mathrm{Mile} \mathrm{Rd}$ Project: Saltwater Intrusion County: Miami-Dade County

TX loop size: X $[\mathrm{m}] \quad 37.9 \quad \mathrm{Y}[\mathrm{m}] \quad 37.9$

$\begin{array}{cccc}\text { Model } & \text { Resistivity } & \text { Thickness } & \text { Elevation } \\ \text { Layer } & {[\text { ohm-m] }} & {[\mathrm{m}]} & {[\mathrm{m}]} \\ 1 & 166.400 & 20.2 & 0.0 \\ 2 & 7.110 & 2.8 & -20.2 \\ 3 & 37.330 & 53.9 & -23.1 \\ 4 & 7.440 & 13.7 & -77.0 \\ 5 & 21.730 & -- & -90.8\end{array}$

System: EM-58 Freq[Hz]: 285 Data Set Code: uh TX Cur $[A]: 2.60$ Turn Off [usec]: 2.5 RX Moment [turns-m^2]: 31.4 Gain Setting: 2

$\begin{array}{crrrr}\begin{array}{c}\text { Time } \\ \text { [ms }\end{array} & \begin{array}{c}\text { rhoa_obs } \\ \text { [ohm-m] }\end{array} & \begin{array}{c}\text { obs_err } \\ {\left[\frac{\overline{0}}{0}\right]}\end{array} & \text { mask } & \begin{array}{r}\text { rhoa_cal } \\ \text { [ohm-m] }\end{array} \\ 0.00680 & 115.1 & 0.5 & u & 113.9 \\ 0.00860 & 91.3 & 0.5 & u & 93.1 \\ 0.01110 & 79.7 & 0.5 & u & 79.2 \\ 0.01410 & 69.6 & 0.5 & u & 69.1 \\ 0.01800 & 61.5 & 0.5 & u & 61.6 \\ 0.02300 & 56.0 & 0.5 & u & 56.0 \\ 0.02940 & 51.9 & 0.5 & u & 51.9 \\ 0.03750 & 49.0 & 0.5 & u & 49.0 \\ 0.04790 & 47.0 & 0.5 & u & 47.1 \\ 0.06110 & 45.8 & 0.5 & u & 45.9 \\ 0.07790 & 44.9 & 0.5 & u & 45.3 \\ 0.09930 & 43.7 & 0.5 & u & 44.4 \\ 0.12670 & 42.4 & 0.4 & u & 43.1 \\ 0.16640 & 40.4 & 0.5 & u & 40.7 \\ 0.20600 & 38.1 & 0.5 & u & 38.4 \\ 0.26280 & 35.9 & 0.5 & u & 35.9 \\ 0.33520 & 33.6 & 0.4 & u & 33.7 \\ 0.42770 & 31.9 & 0.6 & u & 32.0 \\ 0.54560 & 30.9 & 0.6 & u & 30.9 \\ 0.69590 & 31.1 & 1.1 & u & 30.5\end{array}$

Date: $25-\mathrm{FEB}-09$

UTM Coord: $\mathrm{E}[\mathrm{m}]$

Elevation $[\mathrm{m}]$ :

$557500.0 \quad \mathrm{~N}[\mathrm{~m}] \quad 2811056.0$

0.0

RX location: X $[\mathrm{m}]$

$0.0 \quad Y[m]$

0.0

Fit Error [\%]: 1.444

System: EM-58 Freq[Hz]: 30 Data Set Code: hi TX Cur $[\mathrm{A}]: 2.50$ Turn Off[usec]: 2.5

RX Moment[turns-m^2]: 31.4 Gain Setting: 5

$\begin{array}{ccccc}\begin{array}{c}\text { Time } \\ \text { [ms }]\end{array} & \begin{array}{c}\text { rhoa_obs } \\ \text { [ohm-m] }\end{array} & \begin{array}{c}\text { obserr } \\ {\left[\frac{\mathrm{o}}{0}\right]}\end{array} & \text { mask } & \begin{array}{c}\text { rhoa_cal } \\ \text { [ohm-m] }\end{array} \\ 0.08800 & 45.3 & 0.2 & \mathrm{u} & 44.6 \\ 0.10600 & 44.3 & 0.2 & \mathrm{u} & 43.8 \\ 0.13100 & 42.8 & 0.2 & \mathrm{u} & 42.3 \\ 0.16100 & 40.8 & 0.2 & \mathrm{u} & 40.3 \\ 0.20000 & 38.2 & 0.2 & \mathrm{u} & 37.9 \\ 0.25000 & 35.4 & 0.3 & \mathrm{u} & 35.2 \\ 0.31400 & 32.7 & 0.4 & \mathrm{u} & 32.7 \\ 0.39500 & 30.2 & 0.4 & \mathrm{u} & 30.5 \\ 0.49900 & 28.5 & 1.0 & \mathrm{u} & 28.7 \\ 0.63100 & 27.0 & 1.2 & \mathrm{u} & 27.3 \\ 0.79900 & 26.2 & 1.8 & \mathrm{u} & 26.1 \\ 1.01000 & 25.7 & 2.6 & \mathrm{u} & 25.2 \\ 1.28000 & 24.2 & 4.4 & \mathrm{u} & 24.5 \\ 1.63000 & 23.9 & 8.8 & \mathrm{u} & 24.1 \\ 2.08000 & 23.8 & 5.9 & \mathrm{u} & 23.7 \\ 2.64000 & 20.9 & 19.4 & \mathrm{~d} & - \\ 3.37000 & 17.5 & 25.9 & \mathrm{~d} & - \\ 4.29000 & 14.3 & 22.0 & \mathrm{~d} & - \\ 5.47000 & 9.3 & 44.5 & \mathrm{~d} & - \\ 6.97000 & 7.2 & 56.7 & \mathrm{~d} & -\end{array}$


Sounding: MIA216

Location: E - US 1

Client: US Geological Survey

Project: Saltwater Intrusion

County: Miami-Dade County

TX loop size: X[m] $37.9 \quad \mathrm{Y}[\mathrm{m}] \quad 37.9$

$\begin{array}{cccc}\text { Model } & \text { Resistivity } & \text { Thickness } & \text { Elevation } \\ \text { Layer } & \text { [ohm-m] } & {[\mathrm{m}]} & {[\mathrm{m}]} \\ 1 & 224.400 & 25.6 & 0.0 \\ 2 & 16.820 & 4.0 & -25.6 \\ 3 & 92.860 & 95.5 & -29.6 \\ 4 & 35.370 & -- & -125.2\end{array}$

System: EM-58 Freq[Hz]: 285 Data Set Code: uh TX $\operatorname{Cur}[A]: 2.70$ Turn Off[usec]: 2.5

RX Moment[turns-m^2]: 31.4 Gain Setting: 2

$\begin{array}{rrrrrr}\text { Time } & \begin{array}{c}\text { rhoa_obs } \\ \text { [ohm-m] }\end{array} & \begin{array}{c}\text { obs_err } \\ {\left[\frac{\mathrm{o}}{0}\right]}\end{array} & \text { mask } & \begin{array}{c}\text { rhoa_cal } \\ \text { [ohm-m] }\end{array} \\ 1 & 0.00680 & 159.9 & 0.4 & \mathrm{u} & 158.4 \\ 2 & 0.00860 & 132.6 & 0.3 & \mathrm{u} & 136.4 \\ 3 & 0.01110 & 122.6 & 0.3 & \mathrm{u} & 122.2 \\ 4 & 0.01410 & 113.6 & 0.3 & \mathrm{u} & 112.2 \\ 5 & 0.01800 & 104.5 & 0.3 & \mathrm{u} & 104.4 \\ 6 & 0.02300 & 99.4 & 0.3 & \mathrm{u} & 99.1 \\ 7 & 0.02940 & 95.1 & 0.3 & \mathrm{u} & 95.1 \\ 8 & 0.03750 & 92.3 & 0.3 & \mathrm{u} & 92.6 \\ 9 & 0.04790 & 90.7 & 0.3 & \mathrm{u} & 91.1 \\ 10 & 0.06110 & 90.2 & 0.3 & \mathrm{u} & 90.7 \\ 11 & 0.07790 & 91.1 & 0.3 & \mathrm{u} & 90.8 \\ 12 & 0.09930 & 90.9 & 0.5 & \mathrm{u} & 91.5 \\ 13 & 0.12670 & 91.6 & 0.4 & \mathrm{u} & 92.1 \\ 14 & 0.16640 & 92.4 & 0.7 & \mathrm{u} & 92.1 \\ 15 & 0.20600 & 90.6 & 1.3 & \mathrm{u} & 91.2 \\ 16 & 0.26280 & 92.0 & 1.8 & \mathrm{u} & 89.5 \\ 17 & 0.33520 & 87.8 & 2.2 & \mathrm{u} & 87.1 \\ 18 & 0.42770 & 85.6 & 6.1 & \mathrm{u} & 84.5 \\ 19 & 0.54560 & 83.4 & 11.2 & \mathrm{u} & 82.4 \\ 20 & 0.69590 & 90.8 & 15.0 & \mathrm{~m} & 80.9\end{array}$

Date: 26-FEB-09

UTM Coord: $\mathrm{E}[\mathrm{m}]$

Elevation $[\mathrm{m}]$ :

$553598.0 \quad \mathrm{~N}[\mathrm{~m}] \quad 2807227.0$

0.0

RX location: X $[\mathrm{m}]$

$0.0 \quad Y[m]$

0.0
Fit Error [\%]: 2.167

System: EM-58 Freq[Hz]: 30 Data Set Code: hi TX Cur $[A]: 2.50$ Turn Off[usec]: 2.5

RX Moment [turns-m^2] : 31.4 Gain Setting: 5

$\begin{array}{crrcc}\begin{array}{c}\text { Time } \\ \text { [ms] }\end{array} & \begin{array}{c}\text { rhoa_obs } \\ \text { [ohm-m] }\end{array} & \begin{array}{c}\text { obs_err } \\ {\left[\begin{array}{c}\overline{\%} \\ 0\end{array}\right.}\end{array} & \text { mask } & \begin{array}{c}\text { rhoa_cal } \\ \text { [ohm-m] }\end{array} \\ 0.08800 & 91.2 & 0.4 & \mathrm{u} & 90.8 \\ 0.10600 & 91.8 & 0.5 & \mathrm{u} & 91.3 \\ 0.13100 & 92.4 & 0.7 & \mathrm{u} & 91.3 \\ 0.16100 & 91.3 & 0.5 & \mathrm{u} & 90.9 \\ 0.20000 & 89.3 & 1.1 & \mathrm{u} & 89.5 \\ 0.25000 & 86.4 & 1.9 & \mathrm{u} & 87.2 \\ 0.31400 & 81.5 & 2.0 & \mathrm{u} & 83.8 \\ 0.39500 & 78.0 & 2.7 & \mathrm{u} & 79.7 \\ 0.49900 & 74.3 & 5.6 & \mathrm{u} & 75.5 \\ 0.63100 & 73.7 & 6.4 & \mathrm{u} & 71.2 \\ 0.79900 & 68.1 & 6.3 & \mathrm{u} & 67.2 \\ 1.01000 & 61.7 & 16.6 & \mathrm{u} & 63.4 \\ 1.28000 & 58.0 & 17.5 & \mathrm{~m} & 60.3 \\ 1.63000 & 50.9 & 26.8 & \mathrm{~d} & - \\ 2.08000 & 42.9 & 38.0 & \mathrm{~d} & - \\ 2.64000 & 34.2 & 41.7 & \mathrm{~d} & - \\ 3.37000 & 25.6 & 51.2 & \mathrm{~d} & - \\ 4.29000 & 25.8 & 62.9 & \mathrm{~d} & - \\ 5.47000 & 20.1 & 100.0 & \mathrm{~d} & - \\ 6.97000 & 14.7 & 74.3 & \mathrm{~d} & -\end{array}$


Sounding: MIA217

Location: W - US $1 \mathrm{~N}-424$ St

Project: Saltwater Intrusion

County: Miami-Dade County

TX loop size: X $[\mathrm{m}] \quad 37.9 \quad \mathrm{Y}[\mathrm{m}] \quad 37.9$

$\begin{array}{cccc}\text { Model } & \text { Resistivity } & \text { Thickness } & \text { Elevation } \\ \text { Layer } & {[\text { ohm-m] }} & {[\mathrm{m}]} & {[\mathrm{m}]} \\ 1 & 192.900 & 26.0 & 0.0 \\ 2 & 13.500 & 3.5 & -26.0 \\ 3 & 100.300 & 98.0 & -29.5 \\ 4 & 47.630 & -- & -127.5\end{array}$

System: EM-58 Freq[Hz]: 285 Data Set Code: uh TX Cur[A]: 2.70 Turn Off[usec]: 2.5

RX Moment[turns-m^2]: 31.4 Gain Setting: 2

$\begin{array}{rrrrrr}\text { Time } & \begin{array}{c}\text { rhoa_obs } \\ \text { [ohm-m] }\end{array} & \begin{array}{c}\text { obs_err } \\ {\left[\frac{\bar{o}}{0}\right]}\end{array} & \text { mask } & \begin{array}{c}\text { rhoa_cal } \\ \text { [ohm-m] }\end{array} \\ 1 & 0.00680 & 149.5 & 0.3 & \mathrm{u} & 148.7 \\ 2 & 0.00860 & 124.6 & 0.2 & \mathrm{u} & 128.2 \\ 3 & 0.01110 & 115.5 & 0.3 & \mathrm{u} & 114.4 \\ 4 & 0.01410 & 106.1 & 0.3 & \mathrm{u} & 104.5 \\ 5 & 0.01800 & 97.2 & 0.3 & \mathrm{u} & 97.4 \\ 6 & 0.02300 & 92.3 & 0.3 & \mathrm{u} & 92.2 \\ 7 & 0.02940 & 88.4 & 0.3 & \mathrm{u} & 89.0 \\ 8 & 0.03750 & 86.1 & 0.3 & \mathrm{u} & 86.8 \\ 9 & 0.04790 & 85.0 & 0.3 & \mathrm{u} & 86.0 \\ 10 & 0.06110 & 85.2 & 0.3 & \mathrm{u} & 86.0 \\ 11 & 0.07790 & 86.8 & 0.3 & \mathrm{u} & 86.8 \\ 12 & 0.09930 & 87.2 & 0.2 & \mathrm{u} & 87.8 \\ 13 & 0.12670 & 88.5 & 0.3 & \mathrm{u} & 89.1 \\ 14 & 0.16640 & 90.0 & 0.5 & \mathrm{u} & 90.1 \\ 15 & 0.20600 & 89.1 & 0.6 & \mathrm{u} & 90.3 \\ 16 & 0.26280 & 91.6 & 1.0 & \mathrm{u} & 90.0 \\ 17 & 0.33520 & 87.8 & 0.9 & \mathrm{u} & 89.3 \\ 18 & 0.42770 & 88.5 & 2.4 & \mathrm{u} & 88.4 \\ 19 & 0.54560 & 89.4 & 3.5 & \mathrm{u} & 87.7 \\ 20 & 0.69590 & 99.4 & 7.0 & \mathrm{~m} & 87.7\end{array}$

Date: 26-FEB-09

UTM Coord: $\mathrm{E}[\mathrm{m}]$

Elevation $[\mathrm{m}]$ :

$553577.0 \quad \mathrm{~N}[\mathrm{~m}] \quad 2806974.0$

0.0

RX location: X $[\mathrm{m}]$

$0.0 \quad Y[m]$

0.0
Fit Error $[\%]: 2.230$

System: EM-58 Freq[Hz]: 30 Data Set Code: hi TX $\operatorname{Cur}[A]: 2.50$ Turn Off[usec]: 2.5

RX Moment[turns-m^2]: 31.4 Gain Setting: 5

$\begin{array}{ccccc}\begin{array}{c}\text { Time } \\ \text { [ms] }\end{array} & \begin{array}{c}\text { rhoa_obs } \\ \text { [ohm-m] }\end{array} & \begin{array}{c}\text { obs_err } \\ {\left[\begin{array}{c}\overline{\%} \\ \mathrm{o}\end{array}\right.}\end{array} & \text { mask } & \begin{array}{c}\text { rhoa_cal } \\ {[\text { ohm-m] }}\end{array} \\ 0.08800 & 88.0 & 0.3 & \mathrm{u} & 87.0 \\ 0.10600 & 89.1 & 0.4 & \mathrm{u} & 87.9 \\ 0.13100 & 90.3 & 0.5 & \mathrm{u} & 88.7 \\ 0.16100 & 90.4 & 0.6 & \mathrm{u} & 89.1 \\ 0.20000 & 88.8 & 0.9 & \mathrm{u} & 88.9 \\ 0.25000 & 86.6 & 0.9 & \mathrm{u} & 87.9 \\ 0.31400 & 83.9 & 2.4 & \mathrm{u} & 86.0 \\ 0.39500 & 81.3 & 1.7 & \mathrm{u} & 83.6 \\ 0.49900 & 83.3 & 5.1 & \mathrm{u} & 80.7 \\ 0.63100 & 78.8 & 8.3 & \mathrm{u} & 77.7 \\ 0.79900 & 74.7 & 12.0 & \mathrm{u} & 74.7 \\ 1.01000 & 70.7 & 15.4 & \mathrm{u} & 71.9 \\ 1.28000 & 70.4 & 23.3 & \mathrm{~d} & - \\ 1.63000 & 58.8 & 21.5 & \mathrm{~d} & - \\ 2.08000 & 66.7 & 67.2 & \mathrm{~d} & - \\ 2.64000 & 41.6 & 55.8 & \mathrm{~d} & - \\ 3.37000 & 44.0 & 66.0 & \mathrm{~d} & - \\ 4.29000 & 30.9 & 64.1 & \mathrm{~d} & - \\ 5.47000 & 36.1 & 100.0 & \mathrm{~d} & - \\ 6.97000 & 22.1 & 100.0 & \mathrm{~d} & -\end{array}$


Sounding: MIA218

Location: W - US 1

Project: Saltwater Intrusion

County: Miami-Dade County

TX loop size: X $[\mathrm{m}] \quad 37.9 \quad \mathrm{Y}[\mathrm{m}] \quad 37.9$

$\begin{array}{cccc}\text { Model } & \text { Resistivity } & \text { Thickness } & \text { Elevation } \\ \text { Layer } & {[\text { ohm-m] }} & {[\mathrm{m}]} & {[\mathrm{m}]} \\ 1 & 61.920 & 15.4 & 0.0 \\ 2 & .950 & 1.6 & -15.4 \\ 3 & 19.880 & -- & -17.1\end{array}$

System: EM-58 Freq[Hz]: 285 Data Set Code: uh TX Cur $[A]: \quad 2.60$ Turn Off[usec]: 2.5

RX Moment[turns-m^2]: 31.4 Gain Setting: 2

\begin{tabular}{|c|c|c|c|c|c|}
\hline & $\begin{array}{l}\text { Time } \\
{[\mathrm{ms}]}\end{array}$ & $\begin{array}{l}\text { rhoa_obs } \\
\text { [ohm-m] }\end{array}$ & $\begin{array}{c}\text { obs err } \\
{\left[\frac{\mathrm{o}}{0}\right]}\end{array}$ & mask & $\begin{array}{l}\text { rhoa_cal } \\
{[\mathrm{ohm-m}]}\end{array}$ \\
\hline 1 & 0.00680 & 85.1 & 0.3 & u & 84.2 \\
\hline 2 & 0.00860 & 64.4 & 0.3 & $\mathrm{u}$ & 66.1 \\
\hline 3 & 0.01110 & 49.5 & 0.3 & $\mathrm{u}$ & 50.1 \\
\hline 4 & 0.01410 & 38.3 & 0.3 & $\mathrm{u}$ & 38.1 \\
\hline 5 & 0.01800 & 30.0 & 0.3 & $u$ & 29.6 \\
\hline 6 & 0.02300 & 23.9 & 0.3 & u & 23.6 \\
\hline 7 & 0.02940 & 19.6 & 0.3 & u & 19.4 \\
\hline 8 & 0.03750 & 16.6 & 0.3 & u & 16.5 \\
\hline 9 & 0.04790 & 14.5 & 0.3 & u & 14.5 \\
\hline 10 & 0.06110 & 13.1 & 0.3 & u & 13.1 \\
\hline 11 & 0.07790 & 12.2 & 0.3 & u & 12.3 \\
\hline 12 & 0.09930 & 11.7 & 0.3 & u & 11.9 \\
\hline 13 & 0.12670 & 11.5 & 0.3 & $u$ & 11.7 \\
\hline 14 & 0.16640 & 11.7 & 0.3 & u & 11.8 \\
\hline 15 & 0.20600 & 11.8 & 0.3 & $\mathrm{u}$ & 12.0 \\
\hline 16 & 0.26280 & 12.4 & 0.3 & u & 12.4 \\
\hline 17 & 0.33520 & 13.0 & 0.4 & $u$ & 12.9 \\
\hline 18 & 0.42770 & 13.7 & 0.3 & $u$ & 13.6 \\
\hline 19 & 0.54560 & 14.6 & 0.7 & $\mathrm{u}$ & 14.4 \\
\hline 20 & 0.6 & 15.7 & 0.7 & $\mathrm{u}$ & 15. \\
\hline
\end{tabular}

Date: $26-\mathrm{FEB}-09$

UTM Coord: $\mathrm{E}[\mathrm{m}]$

Elevation $[\mathrm{m}]$ :

$554678.0 \quad \mathrm{~N}[\mathrm{~m}] \quad 2801646.0$

0.0

RX location: X $[\mathrm{m}]$

$0.0 \quad Y[m]$

0.0

Fit Error $\left[\frac{\circ}{0}\right]: 1.756$

System: EM-58 Freq[Hz]: 30 Data Set Code: hi TX Cur $[A]: 2.50$ Turn Off[usec]: 2.5

RX Moment [turns-m^2]: 31.4 Gain Setting: 5

Time
$[\mathrm{ms}]$
0.08800
0.10600
0.13100
0.16100
0.20000
0.25000
0.31400
0.39500
0.49900
0.63100
0.79900
1.01000
1.28000
1.63000
2.08000
2.64000
3.37000
4.29000
5.47000
6.97000

rhoa_obs
[ohm-m]
12.1
11.8
11.7
11.7
11.9
12.2
12.6
13.1
13.5
13.9
14.3
14.7
15.0
14.5
13.3
12.5
9.8
7.8
5.2
3.7

obs err mask

rhoa_cal

[\% ]

0.2
0.2

0.2

0.2

0.2

0.2

0.2

0.2

0.2

0.7

1.0

1.6

1. 3

2.6

5.6

9.0

16.5

12.7

10.2 
Sounding: MIA218F

Location: W - US 1

Project: Saltwater Intrusion

County: Miami-Dade County

TX loop size: X $[\mathrm{m}] \quad 37.9 \quad \mathrm{Y}[\mathrm{m}] \quad 37.9$

$\begin{array}{cccc}\text { Model } & \text { Resistivity } & \text { Thickness } & \text { Elevation } \\ \text { Layer } & \text { [ohm-m] } & {[\mathrm{m}]} & {[\mathrm{m}]} \\ 1 & 184.900 & 12.7 & 0.0 \\ 2 & 3.500 & 7.8 & -12.7 \\ 3 & 21.150 & -- & -20.5\end{array}$

System: EM-58 Freq[Hz]: 285 Data Set Code: uh TX Cur $[A]: 2.60$ Turn Off[usec]: 2.5

RX Moment[turns-m^2]: 31.4 Gain Setting: 2

\begin{tabular}{|c|c|c|c|c|c|}
\hline & $\begin{array}{l}\text { Time } \\
{[\mathrm{ms}]}\end{array}$ & $\begin{array}{l}\text { rhoa_obs } \\
\text { [ohm-m] }\end{array}$ & $\begin{array}{c}\text { obs err } \\
{\left[\frac{\mathrm{o}}{0}\right]}\end{array}$ & mask & $\begin{array}{l}\text { rhoa_cal } \\
{[\mathrm{ohm-m}]}\end{array}$ \\
\hline 1 & 0.00680 & 85.1 & 0.3 & u & 84.0 \\
\hline 2 & 0.00860 & 64.4 & 0.3 & $\mathrm{u}$ & 63.6 \\
\hline 3 & 0.01110 & 49.5 & 0.3 & $\mathrm{u}$ & 48.8 \\
\hline 4 & 0.01410 & 38.3 & 0.3 & $u$ & 37.9 \\
\hline 5 & 0.01800 & 30.0 & 0.3 & $u$ & 29.8 \\
\hline 6 & 0.02300 & 23.9 & 0.3 & u & 23.9 \\
\hline 7 & 0.02940 & 19.6 & 0.3 & u & 19.8 \\
\hline 8 & 0.03750 & 16.6 & 0.3 & u & 16.8 \\
\hline 9 & 0.04790 & 14.5 & 0.3 & u & 14.8 \\
\hline 10 & 0.06110 & 13.1 & 0.3 & u & 13.4 \\
\hline 11 & 0.07790 & 12.2 & 0.3 & u & 12.5 \\
\hline 12 & 0.09930 & 11.7 & 0.3 & u & 11.9 \\
\hline 13 & 0.12670 & 11.5 & 0.3 & u & 11.7 \\
\hline 14 & 0.16640 & 11.7 & 0.3 & u & 11.7 \\
\hline 15 & 0.20600 & 11.8 & 0.3 & u & 11.9 \\
\hline 16 & 0.26280 & 12.4 & 0.3 & u & 12.3 \\
\hline 17 & 0.33520 & 13.0 & 0.4 & $u$ & 12. \\
\hline 18 & 0.42770 & 13.7 & 0.3 & $u$ & 13. \\
\hline 19 & 0.54560 & 14.6 & 0.7 & u & 14. \\
\hline 20 & 0.69 & 15.7 & 0.7 & u & 15. \\
\hline
\end{tabular}

Date: $26-\mathrm{FEB}-09$

UTM Coord: $\mathrm{E}[\mathrm{m}]$

Elevation $[\mathrm{m}]$ :

$554678.0 \quad \mathrm{~N}[\mathrm{~m}] \quad 2801646.0$

0.0

RX location: X $[\mathrm{m}]$

$0.0 \quad Y[m]$

0.0
Error [을 2.239

System: EM-58 Freq[Hz]: 30 Data Set Code: hi TX Cur $[A]: 2.50$ Turn Off[usec]: 2.5

RX Moment [turns-m^2] : 31.4 Gain Setting: 5

Time
$[\mathrm{ms}]$
0.08800
0.10600
0.13100
0.16100
0.20000
0.25000
0.31400
0.39500
0.49900
0.63100
0.79900
1.01000
1.28000
1.63000
2.08000
2.64000
3.37000
4.29000
5.47000
6.97000

obs err mask

$[\overline{\bar{o}} \overline{0}]$

0.2

0.2

0.2

0.2

0.2

0.2

0.2

0.2

0.2

1.0

1.6

1.3

3.9

2.6

5.6

16.5

16.5

12.7

10.2 rhoa_cal

[ohm-m]

12.1

11.8

11.7

11.7

11.8

12.1

12.4

12.9

13.4

13.9

14.4

15.0

15.5

16.1

16.7

17.2

$-$ 
Sounding: MIA219

Location: E - US 1

Project: Saltwater Intrusion

County: Miami-Dade County

TX loop size: X $[\mathrm{m}] \quad 37.9 \quad \mathrm{Y}[\mathrm{m}] \quad 37.9$

$\begin{array}{cccc}\text { Model } & \text { Resistivity } & \text { Thickness } & \text { Elevation } \\ \text { Layer } & {[\text { ohm-m] }} & {[\mathrm{m}]} & {[\mathrm{m}]} \\ 1 & 89.180 & 18.7 & 0.0 \\ 2 & .887 & .8 & -18.7 \\ 3 & 84.440 & -- & -19.5\end{array}$

System: EM-58 Freq[Hz]: 285 Data Set Code: uh TX Cur $[A]: 2.70$ Turn Off[usec] : 2.5

RX Moment [turns-m^2]: 31.4 Gain Setting:

\begin{tabular}{|c|c|c|c|c|c|}
\hline & $\begin{array}{l}\text { Time } \\
{[\mathrm{ms}]}\end{array}$ & $\begin{array}{l}\text { rhoa_obs } \\
\text { [ohm-m] }\end{array}$ & $\begin{array}{c}\text { obs err } \\
{\left[\frac{\mathrm{o}}{0}\right]}\end{array}$ & mask & $\begin{array}{l}\text { rhoa_cal } \\
\text { [ohm-m] }\end{array}$ \\
\hline 1 & 0.00680 & 91.5 & 0.4 & $\mathrm{u}$ & 90.3 \\
\hline 2 & 0.00860 & 69.3 & 0.4 & $u$ & 70.7 \\
\hline 3 & 0.01110 & 55.3 & 0.5 & $\mathrm{u}$ & 55.8 \\
\hline 4 & 0.01410 & 45.2 & 0.5 & $u$ & 45.3 \\
\hline 5 & 0.01800 & 38.0 & 0.5 & $u$ & 38.0 \\
\hline 6 & 0.02300 & 33.2 & 0.5 & $\mathrm{u}$ & 32.9 \\
\hline 7 & 0.02940 & 29.9 & 0.5 & u & 29.6 \\
\hline 8 & 0.03750 & 27.9 & 0.5 & u & 27.5 \\
\hline 9 & 0.04790 & 26.8 & 0.5 & u & 26.5 \\
\hline 10 & 0.06110 & 26.5 & 0.5 & u & 26.3 \\
\hline 11 & 0.07790 & 26.8 & 0.5 & u & 26.8 \\
\hline 12 & 0.09930 & 27.6 & 0.5 & u & 27.8 \\
\hline 13 & 0.12670 & 28.9 & 0.5 & u & 29.3 \\
\hline 14 & 0.16640 & 31.3 & 0.5 & $\mathrm{u}$ & 31.6 \\
\hline 15 & 0.20600 & 33.1 & 0.5 & $\mathrm{u}$ & 33.8 \\
\hline 16 & 0.26280 & 36.7 & 0.5 & $\mathrm{u}$ & 36.5 \\
\hline 17 & 0.33520 & 40.3 & 0.5 & $u$ & 39.8 \\
\hline 18 & 0.42770 & 44.9 & 0.8 & $u$ & 43.4 \\
\hline 19 & 0.5 & 49.8 & 1.7 & $\mathrm{u}$ & 47.5 \\
\hline 20 & 0.69 & 56.6 & 3.4 & $\mathrm{u}$ & $\bullet$ \\
\hline
\end{tabular}

Date: $26-\mathrm{FEB}-09$

UTM Coord: $\mathrm{E}[\mathrm{m}]$

Elevation $[\mathrm{m}]$ :

$554443.0 \quad \mathrm{~N}[\mathrm{~m}] \quad 2805290.0$

0.0

RX location: X $[\mathrm{m}]$

$0.0 \quad Y[m]$

0.0
Fit Error $\left[\frac{0}{0}\right]: 3.406$

System: EM-58 Freq[Hz]: 30 Data Set Code: hi TX Cur $[\mathrm{A}]: 2.50$ Turn Off[usec]: 2.5

RX Moment[turns-m^2]: 31.4 Gain Setting: 5

Time
$[\mathrm{ms}]$
0.08800
0.10600
0.13100
0.16100
0.20000
0.25000
0.31400
0.39500
0.49900
0.63100
0.79900
1.01000
1.28000
1.63000
2.08000
2.64000
3.37000
4.29000
5.47000
6.97000

rhoa_obs
[ohm-m]
27.3
28.1
29.2
30.9
32.8
35.3
38.1
41.0
43.9
44.8
48.0
46.8
44.8
44.0
42.3
24.4
20.0
32.9
13.8
31.6

\begin{abstract}
Gain Setting: 5
\end{abstract}

s err
$\left[\begin{array}{l}0 \\ 0\end{array}\right]$
0.2
0.2
0.2
0.2
0.3
0.3
0.6
1.2
1.4
3.2
4.6
8.5
6.9
8.5
1.9
1.7
2.4
0.0
7.2
0.0

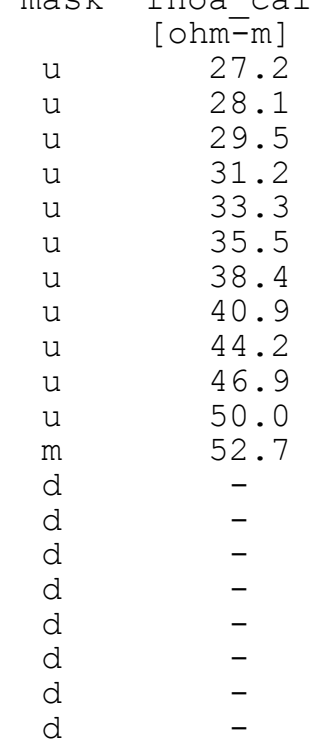


Sounding: MIA219F

Location: E - US

Project: Saltwater Intrusion

County: Miami-Dade County

TX loop size: X $[\mathrm{m}] \quad 37.9 \quad \mathrm{Y}[\mathrm{m}] \quad 37.9$

$\begin{array}{cccc}\text { Model } & \text { Resistivity } & \text { Thickness } & \text { Elevation } \\ \text { Layer } & \text { [ohm-m] } & {[\mathrm{m}]} & {[\mathrm{m}]} \\ 1 & 104.100 & 17.3 & 0.0 \\ 2 & 3.500 & 3.4 & -17.3 \\ 3 & 86.760 & -- & -20.7\end{array}$

System: EM-58 Freq[Hz]: 285 Data Set Code: uh TX $\operatorname{Cur}[A]: 2.70$ Turn Off[usec]: 2.5

RX Moment[turns-m^2]: 31.4 Gain Setting:

\begin{tabular}{|c|c|c|c|c|c|}
\hline & $\begin{array}{l}\text { Time } \\
{[\mathrm{ms}]}\end{array}$ & $\begin{array}{l}\text { rhoa_obs } \\
\text { [ohm-m] }\end{array}$ & $\begin{array}{c}\text { obs err } \\
{\left[\frac{\mathrm{o}}{0}\right]}\end{array}$ & mask & $\begin{array}{l}\text { rhoa_cal } \\
\text { [ohm-m] }\end{array}$ \\
\hline 1 & 0.00680 & 91.5 & 0.4 & $\mathrm{u}$ & 91.6 \\
\hline 2 & 0.00860 & 69.3 & 0.4 & $u$ & 70.4 \\
\hline 3 & 0.01110 & 55.3 & 0.5 & $\mathrm{u}$ & 55.4 \\
\hline 4 & 0.01410 & 45.2 & 0.5 & $u$ & 45.2 \\
\hline 5 & 0.01800 & 38.0 & 0.5 & $u$ & 37.9 \\
\hline 6 & 0.02300 & 33.2 & 0.5 & $\mathrm{u}$ & 33.0 \\
\hline 7 & 0.02940 & 29.9 & 0.5 & u & 29.7 \\
\hline 8 & 0.03750 & 27.9 & 0.5 & u & 27.6 \\
\hline 9 & 0.04790 & 26.8 & 0.5 & u & 26.6 \\
\hline 10 & 0.06110 & 26.5 & 0.5 & u & 26.3 \\
\hline 11 & 0.07790 & 26.8 & 0.5 & u & 26.8 \\
\hline 12 & 0.09930 & 27.6 & 0.5 & u & 27.8 \\
\hline 13 & 0.12670 & 28.9 & 0.5 & $u$ & 29.3 \\
\hline 14 & 0.16640 & 31.3 & 0.5 & $\mathrm{u}$ & 31.5 \\
\hline 15 & 0.20600 & 33.1 & 0.5 & $\mathrm{u}$ & 33.7 \\
\hline 16 & 0.26280 & 36.7 & 0.5 & $\mathrm{u}$ & 36.5 \\
\hline 17 & 0.33520 & 40.3 & 0.5 & $u$ & 39.8 \\
\hline 18 & 0.42770 & 44.9 & 0.8 & $u$ & 43.5 \\
\hline 19 & 0.5 & 49.8 & 1.7 & $\mathrm{u}$ & 47.7 \\
\hline 20 & 0.6 & 56.6 & 3.4 & $\mathrm{u}$ & 52 . \\
\hline
\end{tabular}

Date: 26-FEB-09

UTM Coord: $\mathrm{E}[\mathrm{m}]$

Elevation $[\mathrm{m}]$ :

$554443.0 \quad \mathrm{~N}[\mathrm{~m}] \quad 2805290.0$

0.0

RX location: X $[\mathrm{m}]$

$0.0 \quad Y[m]$

0.0

\section{Fit Error [\%]: 3.331}

System: EM-58 Freq[Hz]: 30 Data Set Code: hi TX Cur $[A]: 2.50$ Turn Off[usec]: 2.5

RX Moment [turns-m^2] : 31.4 Gain Setting: 5

Time
$[\mathrm{ms}]$
0.08800
0.10600
0.13100
0.16100
0.20000
0.25000
0.31400
0.39500
0.49900
0.63100
0.79900
1.01000
1.28000
1.63000
2.08000
2.64000
3.37000
4.29000
5.47000
6.97000

obs_err mask

rhoa_cal

[ohm-m]

$\begin{array}{lll}0.2 & u & 27.2\end{array}$

0.2

0.2
0.2

0.2

0.3

0.3

0.6

1.2

$1 \cdot 4$

3.2

4.6

8.5

16.9

38.5

41.9

31.7

22.4

100.0

37.2

100.0
28.1

29.5

31.2

33.2

35.5

38.3

41.1

44.2

47.1

50.5

53.2

$-$

$-$

- 
Sounding: MIA220

Location: Far east turn around

Project: Saltwater Intrusion

County: Miami-Dade County

TX loop size: X $[\mathrm{m}] \quad 37.9 \quad \mathrm{Y}[\mathrm{m}] \quad 37.9$

$\begin{array}{cccc}\text { Model } & \text { Resistivity } & \text { Thickness } & \text { Elevation } \\ \text { Layer } & {[\text { ohm-m] }} & {[\mathrm{m}]} & {[\mathrm{m}]} \\ 1 & 22.190 & 6.8 & 0.0 \\ 2 & 2.260 & 22.1 & -6.8 \\ 3 & 45.900 & -- & -29.0\end{array}$

System: EM-58 Freq[Hz]: 285 Data Set Code: uh TX Cur $[A]: 2.50$ Turn Off[usec]: 2.5

RX Moment[turns-m^2]: 31.4 Gain Setting:

$\begin{array}{rrrrrr}\text { Time } & \begin{array}{c}\text { rhs }] \\ \text { [ohm-m] }\end{array} & \begin{array}{c}\text { obs_err } \\ {\left[\frac{\mathrm{o}}{0}\right]}\end{array} & \text { mask } & \begin{array}{c}\text { rhoa_cal } \\ {[\mathrm{ohm-m}]}\end{array} \\ 1 & 0.00680 & 41.2 & 0.4 & \mathrm{u} & 40.8 \\ 2 & 0.00860 & 32.1 & 0.4 & \mathrm{u} & 32.1 \\ 3 & 0.01110 & 24.8 & 0.4 & \mathrm{u} & 25.4 \\ 4 & 0.01410 & 19.8 & 0.4 & \mathrm{u} & 20.3 \\ 5 & 0.01800 & 16.0 & 0.4 & \mathrm{u} & 16.3 \\ 6 & 0.02300 & 13.2 & 0.4 & \mathrm{u} & 13.2 \\ 7 & 0.02940 & 11.0 & 0.4 & \mathrm{u} & 10.8 \\ 8 & 0.03750 & 9.2 & 0.4 & \mathrm{u} & 9.0 \\ 9 & 0.04790 & 7.8 & 0.4 & \mathrm{u} & 7.7 \\ 10 & 0.06110 & 6.6 & 0.4 & \mathrm{u} & 6.6 \\ 11 & 0.07790 & 5.7 & 0.4 & \mathrm{u} & 5.7 \\ 12 & 0.09930 & 4.9 & 0.4 & \mathrm{u} & 5.0 \\ 13 & 0.12670 & 4.4 & 0.4 & \mathrm{u} & 4.4 \\ 14 & 0.16640 & 3.9 & 0.4 & \mathrm{u} & 4.0 \\ 15 & 0.20600 & 3.7 & 0.4 & \mathrm{u} & 3.8 \\ 16 & 0.26280 & 3.5 & 0.4 & \mathrm{u} & 3.6 \\ 17 & 0.33520 & 3.5 & 0.4 & \mathrm{u} & 3.5 \\ 18 & 0.42770 & 3.6 & 0.4 & \mathrm{u} & 3.6 \\ 19 & 0.54560 & 3.7 & 0.4 & \mathrm{u} & 3.8 \\ 20 & 0.69590 & 4.0 & 0.4 & \mathrm{u} & 4.0\end{array}$

Date: 26-FEB-09

UTM Coord: $\mathrm{E}[\mathrm{m}]$

Elevation $[\mathrm{m}]$ :

$560349.0 \quad \mathrm{~N}[\mathrm{~m}] \quad 2805662.0$

0.0

RX location: X $[\mathrm{m}]$

$0.0 \quad Y[m]$

0.0

\section{Fit Error $[\%]: 1.864$}

System: EM-58 Freq[Hz]: 30 Data Set Code: hi TX Cur[A]: 2.50 Turn Off[usec]: 2.5

RX Moment [turns-m^2]: 31.4 Gain Setting: 5

Time
$[\mathrm{ms}]$
0.08800
0.10600
0.13100
0.16100
0.20000
0.25000
0.31400
0.39500
0.49900
0.63100
0.79900
1.01000
1.28000
1.63000
2.08000
2.64000
3.37000
4.29000
5.47000
6.97000

rhoa_obs
[ohm-m]
5.4
4.9
4.4
4.0
3.8
3.6
3.5
3.5
3.6
3.7
3.9
4.2
4.7
5.3
6.0
6.9
7.8
8.8
9.4
9.5

obs

mask

rhoa cal

[\%]

0.2
0.2

0.2

0.2

0.2

0.2

0.2

0.2

0.2

0.2

0.3

0.5

0.8

2.2

1.5

10.1

18.5
26.4 
Sounding: MIA221

Client: US Geological Survey Location: $4.07 \mathrm{~km}$ S of SW 392 \& $0.28 \mathrm{~km}$ E Tallahassee Rd Project: Saltwater Intrusion County: Miami-Dade County

TX loop size: X[m] $37.9 \quad \mathrm{Y}[\mathrm{m}] \quad 37.9$

$\begin{array}{cccc}\text { Model } & \text { Resistivity } & \text { Thickness } & \text { Elevation } \\ \text { Layer } & {[\text { ohm-m] }} & {[\mathrm{m}]} & {[\mathrm{m}]} \\ 1 & 37.060 & 8.3 & 0.0 \\ 2 & 2.330 & 16.9 & -8.3 \\ 3 & 29.700 & -- & -25.2\end{array}$

System: EM-58 Freq[Hz]: 285 Data set Code: uh TX Cur $[A]: 2.60$ Turn Off [usec]: 2.5

RX Moment[turns-m^2]: 31.4 Gain Setting: 1

\begin{tabular}{|c|c|c|c|c|c|}
\hline & $\begin{array}{l}\text { Time } \\
{[\mathrm{ms}]}\end{array}$ & $\begin{array}{l}\text { rhoa_obs } \\
\text { [ohm-m] }\end{array}$ & $\begin{array}{c}\text { obs err } \\
{\left[\frac{\mathrm{o}}{0}\right]}\end{array}$ & mask & $\begin{array}{l}\text { rhoa_cal } \\
{[\mathrm{ohm}-\mathrm{m}]}\end{array}$ \\
\hline 1 & 0.00680 & 49.2 & 0.2 & u & 49.7 \\
\hline 2 & 0.00860 & 39.2 & 0.3 & $\mathrm{u}$ & 38.9 \\
\hline 3 & 0.01110 & 30.9 & 0.3 & $\mathrm{u}$ & 30.8 \\
\hline 4 & 0.01410 & 24.7 & 0.3 & $u$ & 24.6 \\
\hline 5 & 0.01800 & 19.9 & 0.3 & $u$ & 19.7 \\
\hline 6 & 0.02300 & 16.0 & 0.3 & $\mathrm{u}$ & 15.9 \\
\hline 7 & 0.02940 & 13.0 & 0.3 & $\mathrm{u}$ & 13.0 \\
\hline 8 & 0.03750 & 10.6 & 0.3 & $\mathrm{u}$ & 10.7 \\
\hline 9 & 0.04790 & 8.8 & 0.3 & $u$ & 8.9 \\
\hline 10 & 0.06110 & 7.4 & 0.3 & $u$ & 7.5 \\
\hline 11 & 0.07790 & 6.3 & 0.3 & $u$ & 6.4 \\
\hline 12 & 0.09930 & 5.5 & 0.3 & $u$ & 5.6 \\
\hline 13 & 0.12670 & 5.0 & 0.3 & $u$ & 5.0 \\
\hline 14 & 0.16640 & 4.6 & 0.3 & $\mathrm{u}$ & 4.6 \\
\hline 15 & 0.20600 & 4.4 & 0.3 & $\mathrm{u}$ & 4.4 \\
\hline 16 & 0.26280 & 4.4 & 0.3 & $\mathrm{u}$ & 4.4 \\
\hline 17 & 0.33520 & 4.4 & 0.3 & $u$ & 4.4 \\
\hline 18 & 0.42770 & 4.6 & 0.3 & $u$ & 4.6 \\
\hline 19 & 0.5 & 4.9 & 0.3 & $\mathrm{u}$ & 4.9 \\
\hline 20 & 0.69 & 5.4 & 0.2 & $\mathrm{u}$ & 5.4 \\
\hline
\end{tabular}

Date: $27-\mathrm{FEB}-09$

UTM Coord: $\mathrm{E}[\mathrm{m}]$

Elevation $[\mathrm{m}]$ :

$559464.0 \quad \mathrm{~N}[\mathrm{~m}] \quad 2805644.0$

0.0

RX location: X $[\mathrm{m}]$

$0.0 \quad Y[m]$

0.0

\section{Fit Error [\%]: 1.373}

System: EM-58 Freq[Hz]: 30 Data Set Code: hi TX Cur $[A]: 2.50$ Turn Off[usec]: 2.5

RX Moment[turns-m^2] : 31.4 Gain Setting: 5

rhoa_obs
[ohm-m]
6.0
5.4
5.0
4.7
4.5
4.4
4.4
4.4
4.6
4.9
5.2
5.7
6.3
7.0
7.9
9.0
10.2
12.3
15.5
39.6

obs err mask

rhoa cal

Time

[응]

$[\mathrm{ohm}-\mathrm{m}]$

\begin{abstract}
0.08800
0.10600

0.13100

0.16100

0.25000

0.31400

.39500

0.79900

1.01000

1.28000

1.63000

2.08000

2.64000

3.37000

4.29000

5.47000

6.97000
\end{abstract}

39.6

6.0

5.4

5.0

4.6

4.4

4.3

4.3

4.4

4.6

4.9

5.3

5.8

6.4

7.1

8.0

8.9

10.1

$-$ 
Sounding: MIA222

Client: US Geological Survey Location: $4.07 \mathrm{~km}$ S of SW 392 \& $0.28 \mathrm{~km}$ W Tallahassee Rd Project: Saltwater Intrusion County: Miami-Dade County

TX loop size: X $[\mathrm{m}] \quad 37.9 \quad \mathrm{Y}[\mathrm{m}] \quad 37.9$

$\begin{array}{cccc}\text { Model } & \text { Resistivity } & \text { Thickness } & \text { Elevation } \\ \text { Layer } & {[\text { ohm-m] }} & {[\mathrm{m}]} & {[\mathrm{m}]} \\ 1 & 30.500 & 9.3 & 0.0 \\ 2 & 2.050 & 15.7 & -9.3 \\ 3 & 19.820 & -- & -25.1\end{array}$

System: EM-58 Freq[Hz]: 285 Data set Code: uh TX Cur $[A]: 2.60$ Turn Off [usec]: 2.5

RX Moment[turns-m^2]: 31.4 Gain Setting: 1

\begin{tabular}{|c|c|c|c|c|c|}
\hline & $\begin{array}{l}\text { Time } \\
{[\mathrm{ms}]}\end{array}$ & $\begin{array}{l}\text { rhoa_obs } \\
\text { [ohm-m] }\end{array}$ & $\begin{array}{c}\text { obs err } \\
{\left[\frac{\mathrm{o}}{0}\right]}\end{array}$ & mask & $\begin{array}{l}\text { rhoa_cal } \\
{[\mathrm{ohm}-\mathrm{m}]}\end{array}$ \\
\hline 1 & 0.00680 & 49.9 & 0.4 & $\mathrm{u}$ & 49.7 \\
\hline 2 & 0.00860 & 40.5 & 0.4 & $\mathrm{u}$ & 40.4 \\
\hline 3 & 0.01110 & 32.4 & 0.4 & $u$ & 32.8 \\
\hline 4 & 0.01410 & 26.1 & 0.4 & $u$ & 26.4 \\
\hline 5 & 0.01800 & 21.1 & 0.4 & $u$ & 21.2 \\
\hline 6 & 0.02300 & 17.1 & 0.4 & $\mathrm{u}$ & 17.1 \\
\hline 7 & 0.02940 & 13.9 & 0.4 & $\mathrm{u}$ & 13.9 \\
\hline 8 & 0.03750 & 11.4 & 0.4 & $\mathrm{u}$ & 11.4 \\
\hline 9 & 0.04790 & 9.4 & 0.4 & $u$ & 9.4 \\
\hline 10 & 0.06110 & 7.9 & 0.4 & u & 7.8 \\
\hline 11 & 0.07790 & 6.6 & 0.4 & $u$ & 6.6 \\
\hline 12 & 0.09930 & 5.7 & 0.4 & u & 5.7 \\
\hline 13 & 0.12670 & 5.0 & 0.4 & $u$ & 5.0 \\
\hline 14 & 0.16640 & 4.5 & 0.4 & $\mathrm{u}$ & 4.5 \\
\hline 15 & 0.20600 & 4.2 & 0.4 & $\mathrm{u}$ & 4.3 \\
\hline 16 & 0.26280 & 4.1 & 0.4 & $\mathrm{u}$ & 4.2 \\
\hline 17 & 0.33520 & 4.1 & 0.4 & $u$ & 4.1 \\
\hline 18 & 0.42770 & 4.2 & 0.4 & $u$ & 4.3 \\
\hline 19 & 0.54560 & 4.5 & 0.4 & $\mathrm{u}$ & 4.5 \\
\hline 20 & 0.69 & 4.9 & 0.4 & $\mathrm{u}$ & 4.8 \\
\hline
\end{tabular}

Date: $27-\mathrm{FEB}-09$

UTM Coord: $\mathrm{E}[\mathrm{m}]$

Elevation $[\mathrm{m}]$ :

$558824.0 \quad \mathrm{~N}[\mathrm{~m}] \quad 2805659.0$

0.0

RX location: X $[\mathrm{m}]$

$0.0 \quad Y[m]$

0.0

System: EM-58 Freq[Hz]: 30 Data Set Code: hi TX Cur $[A]: 2.50$ Turn Off[usec]: 2.5

RX Moment [turns-m^2]: 31.4 Gain Setting: 5

$\begin{array}{rrrrrr} & \begin{array}{c}\text { Time } \\ {[\mathrm{ms}]}\end{array} & \begin{array}{r}\text { rhoa_obs } \\ {[\text { ohm-m] }}\end{array} & \begin{array}{c}\text { obs_err } \\ {\left[\frac{\mathrm{o}}{0}\right]}\end{array} & \mathrm{mask} & \begin{array}{c}\text { rhoa_cal } \\ {[\text { ohm-m] }}\end{array} \\ 1 & 0.08800 & 6.2 & 0.4 & \mathrm{u} & 6.1 \\ 2 & 0.10600 & 5.5 & 0.4 & \mathrm{u} & 5.4 \\ 3 & 0.13100 & 4.9 & 0.4 & \mathrm{u} & 4.9 \\ 4 & 0.16100 & 4.5 & 0.4 & \mathrm{u} & 4.5 \\ 5 & 0.20000 & 4.3 & 0.4 & \mathrm{u} & 4.3 \\ 6 & 0.25000 & 4.1 & 0.4 & \mathrm{u} & 4.1 \\ 7 & 0.31400 & 4.0 & 0.4 & \mathrm{u} & 4.1 \\ 8 & 0.39500 & 4.1 & 0.4 & \mathrm{u} & 4.1 \\ 9 & 0.49900 & 4.2 & 0.4 & \mathrm{u} & 4.2 \\ 10 & 0.63100 & 4.4 & 0.4 & \mathrm{u} & 4.4 \\ 11 & 0.79900 & 4.7 & 0.4 & \mathrm{u} & 4.7 \\ 12 & 1.01000 & 5.1 & 0.5 & \mathrm{u} & 5.0 \\ 13 & 1.28000 & 5.6 & 0.6 & \mathrm{u} & 5.5 \\ 14 & 1.63000 & 6.1 & 0.8 & \mathrm{u} & 6.0 \\ 15 & 2.08000 & 6.6 & 1.6 & \mathrm{u} & 6.7 \\ 16 & 2.64000 & 6.9 & 1.9 & \mathrm{~m} & 7.4 \\ 17 & 3.37000 & 6.7 & 5.6 & \mathrm{~m} & 8.2 \\ 18 & 4.29000 & 6.4 & 6.5 & \mathrm{~m} & 9.1 \\ 19 & 5.47000 & 5.5 & 6.0 & \mathrm{~d} & - \\ 20 & 6.97000 & 4.3 & 10.8 & \mathrm{~d} & -\end{array}$


Sounding: MIA222L4

Client: US Geological Survey Location: $4.07 \mathrm{~km}$ S of SW 392 \& $0.28 \mathrm{~km}$ W Tallahassee Rd Project: Saltwater Intrusion County: Miami-Dade County

TX loop size: X $[\mathrm{m}] \quad 37.9 \quad \mathrm{Y}[\mathrm{m}] \quad 37.9$

$\begin{array}{cccc}\text { Model } & \text { Resistivity } & \text { Thickness } & \text { Elevation } \\ \text { Layer } & {[\text { ohm-m] }} & {[\mathrm{m}]} & {[\mathrm{m}]} \\ 1 & 31.990 & 9.2 & 0.0 \\ 2 & 2.130 & 18.6 & -9.2 \\ 3 & 235.300 & 65.9 & -27.9 \\ 4 & 2.660 & -- & -93.8\end{array}$

System: EM-58 Freq[Hz]: 285 Data Set Code: uh TX $\operatorname{Cur}[A]: 2.60$ Turn Off [usec]: 2.5

RX Moment[turns-m^2]: 31.4 Gain Setting: 1

\begin{tabular}{|c|c|c|c|c|c|}
\hline & $\begin{array}{l}\text { Time } \\
{[\mathrm{ms}]}\end{array}$ & $\begin{array}{l}\text { rhoa_obs } \\
\text { [ohm-m] }\end{array}$ & $\begin{array}{c}\text { obs err } \\
{\left[\frac{\mathrm{o}}{0}\right]}\end{array}$ & mask & $\begin{array}{l}\text { rhoa_cal } \\
{[\mathrm{ohm-m}]}\end{array}$ \\
\hline 1 & 0.00680 & 49.9 & 0.4 & $u$ & 50.1 \\
\hline 2 & 0.00860 & 40.5 & 0.4 & $\mathrm{u}$ & 40.4 \\
\hline 3 & 0.01110 & 32.4 & 0.4 & $\mathrm{u}$ & 32.5 \\
\hline 4 & 0.01410 & 26.1 & 0.4 & $\mathrm{u}$ & 26.1 \\
\hline 5 & 0.01800 & 21.1 & 0.4 & $u$ & 21.0 \\
\hline 6 & 0.02300 & 17.1 & 0.4 & u & 16.9 \\
\hline 7 & 0.02940 & 13.9 & 0.4 & u & 13.8 \\
\hline 8 & 0.03750 & 11.4 & 0.4 & u & 11.4 \\
\hline 9 & 0.04790 & 9.4 & 0.4 & $u$ & 9.5 \\
\hline 10 & 0.06110 & 7.9 & 0.4 & $u$ & 7.9 \\
\hline 11 & 0.07790 & 6.6 & 0.4 & $u$ & 6.7 \\
\hline 12 & 0.09930 & 5.7 & 0.4 & $\mathrm{u}$ & 5.8 \\
\hline 13 & 0.12670 & 5.0 & 0.4 & u & 5.0 \\
\hline 14 & 0.16640 & 4.5 & 0.4 & $\mathrm{u}$ & 4.5 \\
\hline 15 & 0.20600 & 4.2 & 0.4 & $\mathrm{u}$ & 4.3 \\
\hline 16 & 0.26280 & 4.1 & 0.4 & $\mathrm{u}$ & 4.1 \\
\hline 17 & 0.33520 & 4.1 & 0.4 & $\mathrm{u}$ & 4.1 \\
\hline 18 & 0.42770 & 4.2 & 0.4 & $\mathrm{u}$ & 4.2 \\
\hline 19 & 0.54560 & 4.5 & 0.4 & $\mathrm{u}$ & 4.4 \\
\hline 20 & & 4.9 & 0.4 & $u$ & 4.9 \\
\hline
\end{tabular}

Date: 27-FEB-09

UTM Coord: $\mathrm{E}[\mathrm{m}]$

Elevation $[\mathrm{m}]$ :

$558824.0 \quad \mathrm{~N}[\mathrm{~m}] \quad 2805659.0$

0.0

RX location: X $[\mathrm{m}]$

$0.0 \quad Y[m]$

0.0
Fit Error [\%]: 1.726

System: EM-58 Freq[Hz]: 30 Data Set Code: hi TX Cur $[A]: 2.50$ Turn Off[usec]: 2.5

RX Moment [turns-m^2]: 31.4 Gain Setting: 5

Time
$[\mathrm{ms}]$
0.08800
0.10600
0.13100
0.16100
0.20000
0.25000
0.31400
0.39500
0.49900
0.63100
0.79900
1.01000
1.28000
1.63000
2.08000
2.64000
3.37000
4.29000
5.47000
6.97000

列

obs err mask

$\mathrm{hm}-\mathrm{m}]$

$\begin{array}{ll}6.2 & 0.4 \\ 5.5 & 0.4\end{array}$

$\begin{array}{ll}4.9 & 0.4\end{array}$

$\begin{array}{ll}4.5 & 0.4\end{array}$

$\begin{array}{ll}4.3 & 0.4\end{array}$

4.1 0.4

4. 0 0.4

$\begin{array}{ll}4.1 & 0.4\end{array}$

$\begin{array}{ll}4.2 & 0.4\end{array}$

4. 40.4

$4.7 \quad 0.4$

$\begin{array}{ll}5.1 & 0.5\end{array}$

$5.6 \quad 0.6$

6.10 .8

$6.6 \quad 1.6$

$6.9-1.9$

$.7 \quad 5.6$

$6.4 \quad 6.5$

$5.5 \quad 6.0$ rhoa_cal

[ohm-m]

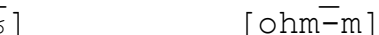

$4 \quad u \quad 6.2$

u $\quad 5.5$

u 4.9

4.5
4.2

4. 1

4.0

4.0

4.2

4.4

4.8

5.2

5.7

6.2

6.5

6.7

6.7

6.5

$-$ 
Sounding: MIA223 Client: US Geological Survey Location: $2.45 \mathrm{~km}$ S of SW $392 \mathrm{St}$ \& $0.82 \mathrm{~km}$ E Tallahasee Rd Project: Saltwater Intrusion County: Miami-Dade County

TX loop size: X $[\mathrm{m}] \quad 37.9 \quad \mathrm{Y}[\mathrm{m}] \quad 37.9$

$\begin{array}{cccc}\text { Model } & \text { Resistivity } & \text { Thickness } & \text { Elevation } \\ \text { Layer } & {[\text { ohm-m] }} & {[\mathrm{m}]} & {[\mathrm{m}]} \\ 1 & 20.350 & 7.9 & 0.0 \\ 2 & 2.180 & 17.1 & -7.9 \\ 3 & 27.610 & -- & -25.0\end{array}$

System: EM-58 Freq[Hz]: 285 Data Set Code: uh TX Cur $[A]: 2.70$ Turn Off[usec]: 2.5

RX Moment[turns-m^2]: 31.4 Gain Setting: 1

$\begin{array}{rrrrrr}\text { Time } & {[\mathrm{ms}]} & \begin{array}{c}\text { rhoa_obs } \\ {[\text { ohm-m] }}\end{array} & \begin{array}{c}\text { obserr } \\ {[\overline{\mathrm{o}}]}\end{array} & \mathrm{mask} & \begin{array}{c}\text { rhoa_cal } \\ {[\text { ohm-m] }}\end{array} \\ 1 & 0.00680 & 41.0 & 0.3 & \mathrm{u} & 40.0 \\ 2 & 0.00860 & 32.7 & 0.3 & \mathrm{u} & 32.8 \\ 3 & 0.01110 & 25.9 & 0.3 & \mathrm{u} & 26.8 \\ 4 & 0.01410 & 21.0 & 0.3 & \mathrm{u} & 21.8 \\ 5 & 0.01800 & 17.4 & 0.3 & \mathrm{u} & 17.6 \\ 6 & 0.02300 & 14.5 & 0.3 & \mathrm{u} & 14.4 \\ 7 & 0.02940 & 12.1 & 0.3 & \mathrm{u} & 11.8 \\ 8 & 0.03750 & 10.1 & 0.3 & \mathrm{u} & 9.8 \\ 9 & 0.04790 & 8.4 & 0.3 & \mathrm{u} & 8.2 \\ 10 & 0.06110 & 7.0 & 0.3 & \mathrm{u} & 6.9 \\ 11 & 0.07790 & 5.9 & 0.3 & \mathrm{u} & 5.9 \\ 12 & 0.09930 & 5.1 & 0.3 & \mathrm{u} & 5.1 \\ 13 & 0.12670 & 4.6 & 0.3 & \mathrm{u} & 4.6 \\ 14 & 0.16640 & 4.2 & 0.3 & \mathrm{u} & 4.2 \\ 15 & 0.20600 & 4.0 & 0.3 & \mathrm{u} & 4.0 \\ 16 & 0.26280 & 3.9 & 0.3 & \mathrm{u} & 3.9 \\ 17 & 0.33520 & 3.9 & 0.3 & \mathrm{u} & 3.9 \\ 18 & 0.42770 & 4.1 & 0.3 & \mathrm{u} & 4.1 \\ 19 & 0.54560 & 4.4 & 0.3 & \mathrm{u} & 4.3 \\ 20 & 0.69590 & 4.8 & 0.3 & \mathrm{u} & 4.7\end{array}$

Date: $27-\mathrm{FEB}-09$

UTM Coord: $\mathrm{E}[\mathrm{m}]$

Elevation $[\mathrm{m}]$ :

$559876.0 \quad \mathrm{~N}[\mathrm{~m}] \quad 2807328.0$

0.0

RX location: $\mathrm{X}[\mathrm{m}]$

$0.0 \quad Y[m]$

0.0

\section{Fit Error [\%]: 2.316}

System: EM-58 Freq[Hz]: 30 Data Set Code: hi TX Cur $[A]: 2.50$ Turn Off[usec]: 2.5

RX Moment[turns-m^2] : 31.4 Gain Setting: 5

Time
$[\mathrm{ms}]$
0.08800
0.10600
0.13100
0.16100
0.20000
0.25000
0.31400
0.39500
0.49900
0.63100
0.79900
1.01000
1.28000
1.63000
2.08000
2.64000
3.37000
4.29000
5.47000
6.97000

obs err mask

$[\overline{\bar{o}} \overline{0}]$

0.2
0.2

0.2

0.2

0.2

0.2

0.2

0.3

0.3

0.3

0.2

0.3

0.7

1.3

1.6

3.6

15.1

15.2

18.9

36.4 rhoa_cal [ohm-m]

5.5

4. 9

4.5

4.2

4.0

3.9

3. 9

3. 9

4.0

4.3

4.6

5.0

5.6

6.2

7.0

7.8

8.8

- 
Sounding: MIA223L4 Client: US Geological Survey Location: $2.45 \mathrm{~km} \mathrm{~S}$ of SW $392 \mathrm{St} \& 0.82 \mathrm{~km}$ E Tallahasee Rd Project: Saltwater Intrusion County: Miami-Dade County

TX loop size: X $[\mathrm{m}] \quad 37.9 \quad \mathrm{Y}[\mathrm{m}] \quad 37.9$

$\begin{array}{cccc}\text { Model } & \text { Resistivity } & \text { Thickness } & \text { Elevation } \\ \text { Layer } & \text { [ohm-m] } & {[\mathrm{m}]} & {[\mathrm{m}]} \\ 1 & 19.590 & 7.6 & 0.0 \\ 2 & 2.230 & 18.4 & -7.6 \\ 3 & 50.290 & 93.3 & -26.1 \\ 4 & 9.180 & -- & -119.5\end{array}$

System: EM-58 Freq[Hz]: 285 Data Set Code: uh TX $\operatorname{Cur}[A]: 2.60$ Turn Off [usec]: 2.5 RX Moment [turns-m^2]: 31.4 Gain Setting: 1

$\begin{array}{rrrrrr}\text { Time } & \begin{array}{r}\text { rhoa_obs } \\ \text { [ohm-m] }\end{array} & \begin{array}{c}\text { obs_err } \\ {\left[\frac{\mathrm{o}}{0}\right]}\end{array} & \text { mask } & \begin{array}{r}\text { rhoa_ca } \\ {[\text { ohm-m] }}\end{array} \\ 1 & 0.00680 & 40.0 & 0.3 & \mathrm{u} & 39.2 \\ 2 & 0.00860 & 31.9 & 0.3 & \mathrm{u} & 32.0 \\ 3 & 0.01110 & 25.2 & 0.3 & \mathrm{u} & 26.1 \\ 4 & 0.01410 & 20.5 & 0.3 & \mathrm{u} & 21.1 \\ 5 & 0.01800 & 16.9 & 0.3 & \mathrm{u} & 17.1 \\ 6 & 0.02300 & 14.1 & 0.3 & \mathrm{u} & 13.9 \\ 7 & 0.02940 & 11.8 & 0.3 & \mathrm{u} & 11.5 \\ 8 & 0.03750 & 9.8 & 0.3 & \mathrm{u} & 9.5 \\ 9 & 0.04790 & 8.2 & 0.3 & \mathrm{u} & 8.0 \\ 10 & 0.06110 & 6.8 & 0.3 & \mathrm{u} & 6.8 \\ 11 & 0.07790 & 5.8 & 0.3 & \mathrm{u} & 5.8 \\ 12 & 0.09930 & 5.0 & 0.3 & \mathrm{u} & 5.1 \\ 13 & 0.12670 & 4.5 & 0.3 & \mathrm{u} & 4.5 \\ 14 & 0.16640 & 4.1 & 0.3 & \mathrm{u} & 4.1 \\ 15 & 0.20600 & 3.9 & 0.3 & \mathrm{u} & 3.9 \\ 16 & 0.26280 & 3.8 & 0.3 & \mathrm{u} & 3.8 \\ 17 & 0.33520 & 3.8 & 0.3 & \mathrm{u} & 3.9 \\ 18 & 0.42770 & 4.0 & 0.3 & \mathrm{u} & 4.0 \\ 19 & 0.54560 & 4.3 & 0.3 & \mathrm{u} & 4.3 \\ 20 & 0.69590 & 4.7 & 0.3 & \mathrm{u} & 4.7\end{array}$

Date: $27-\mathrm{FEB}-09$

UTM Coord: $\mathrm{E}[\mathrm{m}]$

Elevation $[\mathrm{m}]$ :

$559876.0 \quad \mathrm{~N}[\mathrm{~m}] \quad 2807328.0$ 0.0

RX location: $\mathrm{X}[\mathrm{m}]$

$0.0 \quad Y[m]$

0.0
Fit Error $[\%]: 1.958$

System: EM-58 Freq[Hz]: 30 Data Set Code: hi TX Cur $[A]: \quad 2.50$ Turn Off [usec] : 2.5

RX Moment[turns-m^2]: 31.4 Gain Setting: 5

Time
$[\mathrm{ms}]$
0.08800
0.10600
0.13100
0.16100
0.20000
0.25000
0.31400
0.39500
0.49900
0.63100
0.79900
1.01000
1.28000
1.63000
2.08000
2.64000
3.37000
4.29000
5.47000
6.97000

.

mask rhoa cal

$\left[\overline{\frac{0}{0}}\right] \quad[\mathrm{ohm}-\mathrm{m}]$

0.2

0.2

0.2

0.2

0.2

0.2

0.3

0.3

0.2

0.3

1.3

1.6

3.6

6.1

15.2

18.9

36.4
4.9

4.5

4.1

3.9

3.8

3. 8

3.8

4.0

4.2

4.6

5.1

5.6

6.3

7.1

7.8

8.6

$-$

$-$ 
Sounding: MIA224

Client: US Geological Survey Location: $2.45 \mathrm{~km}$ S of SW 392 \& $1.88 \mathrm{~km}$ E Tallahassee Rd Project: Saltwater Intrusion County: Miami-Dade County

TX loop size: X $[\mathrm{m}] \quad 37.9 \quad \mathrm{Y}[\mathrm{m}] \quad 37.9$

$\begin{array}{cccc}\text { Model } & \text { Resistivity } & \text { Thickness } & \text { Elevation } \\ \text { Layer } & {[\text { ohm-m] }} & {[\mathrm{m}]} & {[\mathrm{m}]} \\ 1 & 26.100 & 6.1 & 0.0 \\ 2 & 1.960 & 22.3 & -6.1 \\ 3 & 61.540 & -- & -28.5\end{array}$

System: EM-58 Freq[Hz]: 285 Data set Code: uh TX Cur $[A]: 2.60$ Turn Off [usec]: 2.5

RX Moment[turns-m^2]: 31.4 Gain Setting: 1

\begin{tabular}{|c|c|c|c|c|c|}
\hline & $\begin{array}{l}\text { Time } \\
{[\mathrm{ms}]}\end{array}$ & $\begin{array}{l}\text { rhoa_obs } \\
\text { [ohm-m] }\end{array}$ & $\begin{array}{c}\text { obs err } \\
{\left[\frac{\mathrm{o}}{0}\right]}\end{array}$ & mask & $\begin{array}{l}\text { rhoa_cal } \\
{[\mathrm{ohm}-\mathrm{m}]}\end{array}$ \\
\hline 1 & 0.00680 & 43.2 & 0.1 & $u$ & 43.1 \\
\hline 2 & 0.00860 & 33.3 & 0.2 & $\mathrm{u}$ & 32.8 \\
\hline 3 & 0.01110 & 25.2 & 0.2 & $\mathrm{u}$ & 25.4 \\
\hline 4 & 0.01410 & 19.5 & 0.2 & $\mathrm{u}$ & 19.9 \\
\hline 5 & 0.01800 & 15.4 & 0.2 & $u$ & 15.7 \\
\hline 6 & 0.02300 & 12.4 & 0.2 & $\mathrm{u}$ & 12.5 \\
\hline 7 & 0.02940 & 10.2 & 0.2 & $\mathrm{u}$ & 10.2 \\
\hline 8 & 0.03750 & 8.5 & 0.2 & $\mathrm{u}$ & 8.4 \\
\hline 9 & 0.04790 & 7.1 & 0.2 & $u$ & 7.0 \\
\hline 10 & 0.06110 & 6.1 & 0.2 & u & 6.0 \\
\hline 11 & 0.07790 & 5.2 & 0.2 & $u$ & 5.2 \\
\hline 12 & 0.09930 & 4.5 & 0.2 & $u$ & 4.5 \\
\hline 13 & 0.12670 & 3.9 & 0.2 & $u$ & 4.0 \\
\hline 14 & 0.16640 & 3.5 & 0.2 & $\mathrm{u}$ & 3.5 \\
\hline 15 & 0.20600 & 3.3 & 0.2 & $\mathrm{u}$ & 3.3 \\
\hline 16 & 0.26280 & 3.1 & 0.2 & $\mathrm{u}$ & 3.1 \\
\hline 17 & 0.33520 & 3.0 & 0.2 & $u$ & 3.0 \\
\hline 18 & 0.42770 & 3.0 & 0.2 & $u$ & 3.0 \\
\hline 19 & 0.5 & 3.2 & 0.2 & $\mathrm{u}$ & 3.2 \\
\hline 20 & 0.69 & 3.4 & 0.2 & $\mathrm{u}$ & 3.4 \\
\hline
\end{tabular}

Date: $27-\mathrm{FEB}-09$

UTM Coord: $\mathrm{E}[\mathrm{m}]$

Elevation $[\mathrm{m}]$ :

$560925.0 \quad \mathrm{~N}[\mathrm{~m}] \quad 2807316.0$

0.0

RX location: X $[\mathrm{m}]$

$0.0 \quad Y[m]$

0.0
Fit Error $[\%]: 1.246$

System: EM-58 Freq[Hz]: 30 Data Set Code: hi TX Cur $[A]: 2.50$ Turn Off[usec]: 2.5

RX Moment [turns-m^2]: 31.4 Gain Setting: 5

Time
$[\mathrm{ms}]$
0.08800
0.10600
0.13100
0.16100
0.20000
0.25000
0.31400
0.39500
0.49900
0.63100
0.79900
1.01000
1.28000
1.63000
2.08000
2.64000
3.37000
4.29000
5.47000
6.97000

rhoa_obs
[ohm-m]
4.9
4.4
3.9
3.6
3.3
3.1
3.0
2.9
3.0
3.1
3.3
3.5
3.9
4.3
4.9
5.7
6.6
7.8
9.0
16.2

obs err mask rhoa cal

0.2
0.2

0.2

0.2

0.2

0.2

0.2

0.2

0.2

0.2

0.2

0.5

0.6

0.7

1.8

4.2

9.1

12.8

46.3 [ohm-m]

\section{u 4.8}

u 4.3

u 3.9

u 3.5

u 3.1

u 3.0

3.0

u 3.1

u $\quad 3.3$

u 3.5

u 3.9

u $\quad 4.3$

$\begin{array}{ll}u & 4.9 \\ u & 5.7\end{array}$

u 6.6

6.6

9.2 
Sounding: MIA225

Client: US Geological Survey Location: $0.05 \mathrm{~km}$ W of SW $167 \& 0.84 \mathrm{~km} \mathrm{~N}$ Card Sound Rd Project: Saltwater Intrusion County: Miami-Dade County

TX loop size: X[m] $37.9 \quad \mathrm{Y}[\mathrm{m}] \quad 37.9$

$\begin{array}{cccc}\text { Model } & \text { Resistivity } & \text { Thickness } & \text { Elevation } \\ \text { Layer } & {[\text { ohm-m] }} & {[\mathrm{m}]} & {[\mathrm{m}]} \\ 1 & 311.400 & 20.3 & 0.0 \\ 2 & 24.750 & 10.8 & -20.3 \\ 3 & 70.910 & 97.1 & -31.1 \\ 4 & 25.490 & -- & -128.2\end{array}$

System: EM-58 Freq[Hz]: 285 Data Set Code: uh TX $\operatorname{Cur}[\mathrm{A}]: 2.50$ Turn Off [usec]: 2.5 RX Moment[turns-m^2]: 31.4 Gain Setting: 2

$\begin{array}{rrrrrr}\text { Time } & \begin{array}{c}\text { rhoa_obs } \\ {[\text { ohm-m] }}\end{array} & \begin{array}{c}\text { obs_err } \\ {\left[\frac{\mathrm{o}}{0}\right]}\end{array} & \text { mask } & \begin{array}{r}\text { rhoa_cal } \\ {[\text { ohm-m] }}\end{array} \\ 1 & 0.00680 & 150.8 & 0.3 & \mathrm{u} & 146.3 \\ 2 & 0.00860 & 120.8 & 0.2 & \mathrm{u} & 121.7 \\ 3 & 0.01110 & 108.4 & 0.2 & \mathrm{u} & 105.4 \\ 4 & 0.01410 & 97.0 & 0.3 & \mathrm{u} & 94.0 \\ 5 & 0.01800 & 86.7 & 0.3 & \mathrm{u} & 85.3 \\ 6 & 0.02300 & 80.2 & 0.2 & \mathrm{u} & 78.9 \\ 7 & 0.02940 & 75.2 & 0.2 & \mathrm{u} & 74.4 \\ 8 & 0.03750 & 71.9 & 0.2 & \mathrm{u} & 71.2 \\ 9 & 0.04790 & 70.4 & 0.2 & \mathrm{u} & 69.1 \\ 10 & 0.06110 & 69.5 & 0.3 & \mathrm{u} & 67.9 \\ 11 & 0.07790 & 69.3 & 0.3 & \mathrm{u} & 67.5 \\ 12 & 0.09930 & 68.5 & 0.3 & \mathrm{u} & 67.7 \\ 13 & 0.12670 & 70.8 & 0.6 & \mathrm{u} & 68.4 \\ 14 & 0.16640 & 69.1 & 1.2 & \mathrm{u} & 69.3 \\ 15 & 0.20600 & 69.5 & 1.4 & \mathrm{u} & 69.9 \\ 16 & 0.26280 & 70.4 & 2.5 & \mathrm{u} & 70.0 \\ 17 & 0.33520 & 65.7 & 3.4 & \mathrm{u} & 69.4 \\ 18 & 0.42770 & 63.8 & 5.7 & \mathrm{u} & 68.3 \\ 19 & 0.54560 & 58.3 & 11.9 & \mathrm{u} & 67.2 \\ 20 & 0.69590 & 60.4 & 28.4 & \mathrm{~d} & -\end{array}$

Date: 02-MAR-09

UTM Coord: $\mathrm{E}[\mathrm{m}]$

Elevation $[\mathrm{m}]$ :

$554172.0 \quad \mathrm{~N}[\mathrm{~m}] \quad 2811757.0$ 0.0

RX location: $\mathrm{X}[\mathrm{m}]$

$0.0 \quad \mathrm{Y}[\mathrm{m}]$

0.0

Fit Error $[\%]: 1.735$ 
Sounding: MIA226 Client: US Geological Survey Location: $0.18 \mathrm{~km} \mathrm{~S}$ of SW $380 \mathrm{St} \& 0.32 \mathrm{~km} \mathrm{~W}$ of $\mathrm{SW} 162$ Ave Project: Saltwater Intrusion County: Miami-Dade County

TX loop size: X[m] $37.9 \quad \mathrm{Y}[\mathrm{m}] \quad 37.9$

$\begin{array}{cccc}\text { Model } & \text { Resistivity } & \text { Thickness } & \text { Elevation } \\ \text { Layer } & {[\text { ohm-m] }} & {[\mathrm{m}]} & {[\mathrm{m}]} \\ 1 & 304.200 & 18.1 & 0.0 \\ 2 & 14.490 & 8.5 & -18.1 \\ 3 & 68.290 & 77.6 & -26.6 \\ 4 & 29.720 & -- & -104.2\end{array}$

System: EM-58 Freq[Hz]: 285 Data Set Code: uh TX $\operatorname{Cur}[A]: 2.65$ Turn off[usec] : 2.5

RX Moment[turns-m^2]: 31.4 Gain Setting: 2

\begin{tabular}{|c|c|c|c|c|}
\hline $\begin{array}{l}\text { Time } \\
{[\mathrm{ms}]}\end{array}$ & $\begin{array}{l}\text { rhoa_obs } \\
\text { [ohm-m] }\end{array}$ & $\begin{array}{c}\text { obs err } \\
{\left[\overline{\frac{0}{0}}\right]}\end{array}$ & mask & $\begin{array}{l}\text { rhoa_cal } \\
\text { [ohm-m] }\end{array}$ \\
\hline 0.00680 & 119.7 & 0.6 & u & 118.9 \\
\hline 0.00860 & 94.3 & 0.4 & u & 95.6 \\
\hline 0.01110 & 80.6 & 0.5 & u & 80.4 \\
\hline 0.01410 & 70.2 & 0.5 & u & 70.0 \\
\hline 0.01800 & 62.4 & 0.5 & u & 62.4 \\
\hline 0.02300 & 57.3 & 0.5 & u & 57.1 \\
\hline 0.02940 & 53.7 & 0.5 & $\mathrm{u}$ & 53.5 \\
\hline 0.03750 & 51.5 & 0.5 & u & 51.3 \\
\hline 0.04790 & 50.2 & 0.5 & u & 50.1 \\
\hline 0.06110 & 49.7 & 0.5 & $\mathrm{u}$ & 49.8 \\
\hline 0.07790 & 50.0 & 0.5 & $\mathrm{u}$ & 50.2 \\
\hline 0.09930 & 50.5 & 0.5 & $\mathrm{u}$ & 51.1 \\
\hline 0.12670 & 51.7 & 0.5 & $\mathrm{u}$ & 52.2 \\
\hline 0.16640 & 54.0 & 0.7 & $\mathrm{u}$ & 53.6 \\
\hline 0.20600 & 54.5 & 0.7 & $u$ & 54.3 \\
\hline 0.26280 & 56.4 & 1.0 & u & 54.9 \\
\hline 0.33520 & 55.2 & 1.5 & u & 55.0 \\
\hline 0.42770 & 55.0 & 2.1 & u & 55.0 \\
\hline 0.5 & 55.0 & 3.9 & u & 54.8 \\
\hline 0.6 & 57.7 & 7.7 & $\mathrm{u}$ & 55.1 \\
\hline
\end{tabular}

Date: 02-MAR-09

UTM Coord: $\mathrm{E}[\mathrm{m}]$

Elevation $[\mathrm{m}]$ :

$554719.0 \quad \mathrm{~N}[\mathrm{~m}] \quad 2810805.0$

0.0

RX location: X $[\mathrm{m}]$

$0.0 \quad Y[m]$

0.0
Fit Error [\%]: 2.441

System: EM-58 Freq[Hz]: 30 Data Set Code: hi TX Cur $[A]: 2.50$ Turn Off[usec]: 2.5

RX Moment [turns-m^2] : 31.4 Gain Setting: 5

$\begin{array}{ccccc}\begin{array}{c}\text { Time } \\ \text { [ms] }\end{array} & \begin{array}{c}\text { rhoa_obs } \\ \text { [ohm-m] }\end{array} & \begin{array}{c}\text { obs_err } \\ {\left[\begin{array}{c}\overline{\%} \\ \text { o }]\end{array}\right.}\end{array} & \text { mask } & \begin{array}{c}\text { rhoa_cal } \\ \text { [ohm-m] }\end{array} \\ 0.08800 & 50.3 & 0.5 & \mathrm{u} & 50.5 \\ 0.10600 & 51.1 & 0.4 & \mathrm{u} & 51.3 \\ 0.13100 & 52.2 & 0.5 & \mathrm{u} & 52.1 \\ 0.16100 & 53.2 & 0.5 & \mathrm{u} & 52.9 \\ 0.20000 & 54.0 & 0.4 & \mathrm{u} & 53.4 \\ 0.25000 & 53.7 & 0.8 & \mathrm{u} & 53.5 \\ 0.31400 & 52.6 & 0.7 & \mathrm{u} & 53.0 \\ 0.39500 & 50.5 & 1.1 & \mathrm{u} & 52.0 \\ 0.49900 & 48.4 & 1.3 & \mathrm{u} & 50.5 \\ 0.63100 & 47.1 & 2.1 & \mathrm{u} & 48.8 \\ 0.79900 & 47.8 & 5.0 & \mathrm{u} & 47.0 \\ 1.01000 & 46.2 & 6.0 & \mathrm{u} & 45.4 \\ 1.28000 & 47.6 & 12.1 & \mathrm{~m} & 43.6 \\ 1.63000 & 49.7 & 27.3 & \mathrm{~d} & - \\ 2.08000 & 65.7 & 82.9 & \mathrm{~d} & - \\ 2.64000 & 48.6 & 100.0 & \mathrm{~d} & - \\ 3.37000 & 32.8 & 70.2 & \mathrm{~d} & - \\ 4.29000 & 23.3 & 100.0 & \mathrm{~d} & - \\ 5.47000 & 63.3 & 100.0 & \mathrm{~d} & - \\ 6.97000 & 19.1 & 100.0 & \mathrm{~d} & -\end{array}$


Sounding: MIA227 Client: US Geological Survey Location: $0.35 \mathrm{~km}$ S of $\mathrm{SW} 380 \mathrm{St} \& 0.32 \mathrm{~km}$ W of $\mathrm{SW} 162$ Ave Project: Saltwater Intrusion County: Miami-Dade County

TX loop size: X[m] $37.9 \quad \mathrm{Y}[\mathrm{m}] \quad 37.9$

$\begin{array}{cccc}\text { Model } & \text { Resistivity } & \text { Thickness } & \text { Elevation } \\ \text { Layer } & {[\text { ohm-m] }} & {[\mathrm{m}]} & {[\mathrm{m}]} \\ 1 & 184.200 & 15.4 & 0.0 \\ 2 & 21.080 & 17.2 & -15.4 \\ 3 & 84.420 & 58.9 & -32.6 \\ 4 & 31.600 & -- & -91.6\end{array}$

System: EM-58 Freq[Hz]: 285 Data Set Code: uh TX Cur[A]: 2.70 Turn Off[usec]: 2.5

RX Moment[turns-m^2]: 31.4 Gain Setting: 2

$\begin{array}{rrrrrr}\text { Time } & \begin{array}{c}\text { rhoa_obs } \\ \text { [ohm-m] }\end{array} & \begin{array}{c}\text { obs_err } \\ {\left[\overline{\frac{o}{0}}\right]}\end{array} & \text { mask } & \begin{array}{c}\text { rhoa_cal } \\ \text { [ohm-m] }\end{array} \\ 1 & 0.00680 & 100.3 & 2.8 & \mathrm{u} & 101.8 \\ 2 & 0.00860 & 87.7 & 0.2 & \mathrm{u} & 85.4 \\ 3 & 0.01110 & 73.3 & 0.1 & \mathrm{u} & 73.4 \\ 4 & 0.01410 & 63.6 & 0.1 & \mathrm{u} & 64.2 \\ 5 & 0.01800 & 56.4 & 0.1 & \mathrm{u} & 57.0 \\ 6 & 0.02300 & 51.5 & 0.1 & \mathrm{u} & 51.7 \\ 7 & 0.02940 & 48.2 & 0.2 & \mathrm{u} & 48.1 \\ 8 & 0.03750 & 46.2 & 0.2 & \mathrm{u} & 45.8 \\ 9 & 0.04790 & 45.1 & 0.2 & \mathrm{u} & 44.7 \\ 10 & 0.06110 & 44.7 & 0.3 & \mathrm{u} & 44.4 \\ 11 & 0.07790 & 45.0 & 0.3 & \mathrm{u} & 44.9 \\ 12 & 0.09930 & 45.6 & 0.3 & \mathrm{u} & 46.0 \\ 13 & 0.12670 & 47.0 & 0.3 & \mathrm{u} & 47.3 \\ 14 & 0.16640 & 49.1 & 0.4 & \mathrm{u} & 48.9 \\ 15 & 0.20600 & 49.8 & 0.4 & \mathrm{u} & 49.9 \\ 16 & 0.26280 & 51.7 & 0.6 & \mathrm{u} & 50.7 \\ 17 & 0.33520 & 51.0 & 0.9 & \mathrm{u} & 51.2 \\ 18 & 0.42770 & 51.0 & 1.3 & \mathrm{u} & 51.4 \\ 19 & 0.54560 & 51.2 & 1.5 & \mathrm{u} & 51.8 \\ 20 & 0.69590 & 52.5 & 6.7 & \mathrm{u} & 52.4\end{array}$

Date: $02-M A R-09$

UTM Coord: $\mathrm{E}[\mathrm{m}]$

Elevation $[\mathrm{m}]$ :

$554717.0 \quad \mathrm{~N}[\mathrm{~m}] \quad 2810623.0$

0.0

RX location: X $[\mathrm{m}]$

$0.0 \quad Y[m]$

0.0
Fit Error $[\%]: 1.336$

System: EM-58 Freq[Hz]: 30 Data Set Code: hi TX Cur $[A]: \quad 2.50$ Turn Off[usec]: 2.5

RX Moment [turns-m^2] : 31.4 Gain Setting: 5

Time
$[\mathrm{ms}]$
0.08800
0.10600
0.13100
0.16100
0.20000
0.25000
0.31400
0.39500
0.49900
0.63100
0.79900
1.01000
1.28000
1.63000
2.08000
2.64000
3.37000
4.29000
5.47000
6.97000

.

rhoa cal

[ohm-m]

$\begin{array}{rrr}{\left[\frac{\bar{o}}{0}\right]} & \text { u } \\ 0.6 & \text { u } & 45.3\end{array}$

$\begin{array}{lll}0.6 & u & 45.3 \\ 0.7 & u & 46.2\end{array}$

0.8 u 47.3

$1.4 \quad \mathrm{u} \quad 48.3$

49.1

49.4

49.4

48.7

47.8

$-$

$-$

$-$ 

County: Miami-Dade County

\begin{tabular}{|c|c|c|c|}
\hline TX loop & size: $X[m]$ & $Y[m]$ & 7.9 \\
\hline odel & Resistivity & Thickness & levation \\
\hline ayer & [ohm-m] & {$[\mathrm{m}]$} & {$[\mathrm{m}]$} \\
\hline $\begin{array}{l}1 \\
2\end{array}$ & $\begin{array}{r}7.230 \\
7.230\end{array}$ & $\begin{array}{r}10.0 \\
6.1\end{array}$ & -18.0 \\
\hline 3 & 100.700 & 23.0 & -24.1 \\
\hline 4 & 35.180 & -- & -47.2 \\
\hline
\end{tabular}

System: EM-58 Freq[Hz]: 285 Data Set Code: uh TX Cur[A]: 2.70 Turn Off[usec]: 2.5 RX Moment[turns-m^2]: 31.4 Gain Setting: 2

$\begin{array}{rrrrrr}\text { Time } & \begin{array}{c}\text { rhoa_obs } \\ \text { [ohm-m] }\end{array} & \begin{array}{c}\text { obs_err } \\ {\left[\frac{\mathrm{o}}{0}\right]}\end{array} & \text { mask } & \begin{array}{c}\text { rhoa_cal } \\ \text { [ohm-m] }\end{array} \\ 1 & 0.00680 & 111.8 & 0.3 & \mathrm{u} & 111.7 \\ 2 & 0.00860 & 85.0 & 0.3 & \mathrm{u} & 85.8 \\ 3 & 0.01110 & 69.3 & 0.3 & \mathrm{u} & 68.9 \\ 4 & 0.01410 & 58.0 & 0.3 & \mathrm{u} & 57.6 \\ 5 & 0.01800 & 49.7 & 0.3 & \mathrm{u} & 49.6 \\ 6 & 0.02300 & 44.3 & 0.3 & \mathrm{u} & 44.2 \\ 7 & 0.02940 & 40.5 & 0.3 & \mathrm{u} & 40.6 \\ 8 & 0.03750 & 38.2 & 0.3 & \mathrm{u} & 38.5 \\ 9 & 0.04790 & 37.0 & 0.3 & \mathrm{u} & 37.3 \\ 10 & 0.06110 & 36.6 & 0.3 & \mathrm{u} & 36.8 \\ 11 & 0.07790 & 36.8 & 0.3 & \mathrm{u} & 36.8 \\ 12 & 0.09930 & 37.1 & 0.3 & \mathrm{u} & 37.1 \\ 13 & 0.12670 & 37.7 & 0.3 & \mathrm{u} & 37.5 \\ 14 & 0.16640 & 38.6 & 0.4 & \mathrm{u} & 37.8 \\ 15 & 0.20600 & 38.5 & 0.2 & \mathrm{u} & 38.2 \\ 16 & 0.26280 & 39.1 & 0.4 & \mathrm{u} & 38.5 \\ 17 & 0.33520 & 39.0 & 0.5 & \mathrm{u} & 38.9 \\ 18 & 0.42770 & 39.4 & 0.7 & \mathrm{u} & 39.5 \\ 19 & 0.54560 & 40.2 & 0.7 & \mathrm{u} & 40.4 \\ 20 & 0.69590 & 42.8 & 1.0 & \mathrm{u} & 41.6\end{array}$

RX location: X $[\mathrm{m}] \quad 0.0 \quad \mathrm{Y}[\mathrm{m}] \quad 0.0$

\section{Fit Error [응: 2.080}

System: EM-58 Freq[Hz]: 30 Data Set Code: hi TX Cur $[A]: 2.50$ Turn off[usec]: 2.5

RX Moment [turns-m^2]: $31.4 \quad$ Gain Setting: 5

$\begin{array}{ccccc}\begin{array}{c}\text { Time } \\ \text { [ms] }\end{array} & \begin{array}{c}\text { rhoa_obs } \\ \text { [ohm-m] }\end{array} & \begin{array}{c}\text { obs_err } \\ {\left[\begin{array}{c}\overline{\%} \\ 0\end{array}\right.}\end{array} & \text { mask } & \begin{array}{c}\text { rhoa_cal } \\ \text { [ohm-m] }\end{array} \\ 0.08800 & 36.8 & 0.4 & \mathrm{u} & 36.9 \\ 0.10600 & 37.1 & 0.4 & \mathrm{u} & 37.1 \\ 0.13100 & 37.6 & 0.4 & \mathrm{u} & 37.3 \\ 0.16100 & 37.8 & 0.3 & \mathrm{u} & 37.5 \\ 0.20000 & 37.9 & 0.5 & \mathrm{u} & 37.7 \\ 0.25000 & 37.5 & 0.4 & \mathrm{u} & 37.7 \\ 0.31400 & 36.9 & 0.5 & \mathrm{u} & 37.7 \\ 0.39500 & 36.4 & 0.9 & \mathrm{u} & 37.5 \\ 0.49900 & 36.3 & 1.3 & \mathrm{u} & 37.5 \\ 0.63100 & 36.2 & 2.6 & \mathrm{u} & 37.2 \\ 0.79900 & 36.9 & 2.2 & \mathrm{u} & 37.1 \\ 1.01000 & 37.8 & 7.2 & \mathrm{u} & 36.9 \\ 1.28000 & 37.6 & 10.0 & \mathrm{u} & 36.9 \\ 1.63000 & 37.0 & 13.4 & \mathrm{~d} & - \\ 2.08000 & 37.9 & 23.4 & \mathrm{~d} & - \\ 2.64000 & 39.2 & 34.5 & \mathrm{~d} & - \\ 3.37000 & 43.4 & 78.8 & \mathrm{~d} & - \\ 4.29000 & 34.7 & 74.0 & \mathrm{~d} & - \\ 5.47000 & 41.6 & 100.0 & \mathrm{~d} & - \\ 6.97000 & 33.0 & 100.0 & \mathrm{~d} & -\end{array}$


Sounding: MIA229

Client: US Geological Survey Location: $0.05 \mathrm{~km} \mathrm{~N}$ of $\mathrm{SW} 384 \mathrm{St} \& 0.08 \mathrm{E}$ of $3 \mathrm{Mile} \mathrm{Rd}$ Project: Saltwater Intrusion County: Miami-Dade County

TX loop size: X[m] $37.9 \quad \mathrm{Y}[\mathrm{m}] \quad 37.9$

$\begin{array}{cccc}\text { Model } & \text { Resistivity } & \text { Thickness } & \text { Elevation } \\ \text { Layer } & \text { [ohm-m] } & {[\mathrm{m}]} & {[\mathrm{m}]} \\ 1 & 264.100 & 16.8 & 0.0 \\ 2 & 7.210 & 9.1 & -16.8 \\ 3 & 43.980 & 35.6 & -25.9 \\ 4 & 16.400 & -- & -61.6\end{array}$

System: EM-58 Freq[Hz]: 285 Data Set Code: uh TX $\operatorname{Cur}[\mathrm{A}]: 2.70$ Turn Off [usec]: 2.5

RX Moment[turns-m^2]: 31.4 Gain Setting: 2

$\begin{array}{rrrrrr}\text { Time } & \begin{array}{c}\text { rhoa_obs } \\ \text { [ohm-m] }\end{array} & \begin{array}{c}\text { obs_err } \\ {\left[\frac{\mathrm{o}}{0}\right]}\end{array} & \text { mask } & \begin{array}{c}\text { rhoa_cal } \\ \text { [ohm-m] }\end{array} \\ 1 & 0.00680 & 114.9 & 0.1 & \mathrm{u} & 114.0 \\ 2 & 0.00860 & 86.6 & 0.1 & \mathrm{u} & 87.5 \\ 3 & 0.01110 & 68.8 & 0.2 & \mathrm{u} & 68.8 \\ 4 & 0.01410 & 55.3 & 0.2 & \mathrm{u} & 55.3 \\ 5 & 0.01800 & 45.4 & 0.2 & \mathrm{u} & 45.5 \\ 6 & 0.02300 & 38.5 & 0.2 & \mathrm{u} & 38.5 \\ 7 & 0.02940 & 33.6 & 0.2 & \mathrm{u} & 33.6 \\ 8 & 0.03750 & 30.3 & 0.2 & \mathrm{u} & 30.2 \\ 9 & 0.04790 & 28.0 & 0.2 & \mathrm{u} & 27.9 \\ 10 & 0.06110 & 26.7 & 0.2 & \mathrm{u} & 26.6 \\ 11 & 0.07790 & 25.9 & 0.2 & \mathrm{u} & 25.9 \\ 12 & 0.09930 & 25.6 & 0.2 & \mathrm{u} & 25.7 \\ 13 & 0.12670 & 25.7 & 0.2 & \mathrm{u} & 25.7 \\ 14 & 0.16640 & 26.1 & 0.2 & \mathrm{u} & 25.9 \\ 15 & 0.20600 & 26.1 & 0.2 & \mathrm{u} & 26.0 \\ 16 & 0.26280 & 26.3 & 0.1 & \mathrm{u} & 26.1 \\ 17 & 0.33520 & 26.2 & 0.2 & \mathrm{u} & 26.1 \\ 18 & 0.42770 & 26.3 & 0.3 & \mathrm{u} & 26.0 \\ 19 & 0.54560 & 26.6 & 0.3 & \mathrm{u} & 26.1 \\ 20 & 0.69590 & 27.9 & 0.6 & \mathrm{~m} & 26.4\end{array}$

Date: 02-MAR-09

UTM Coord: $\mathrm{E}[\mathrm{m}]$

Elevation $[\mathrm{m}]$ :

$557588.0 \quad \mathrm{~N}[\mathrm{~m}] \quad 2810641.0$

0.0

RX location: X $[\mathrm{m}]$

$0.0 \quad Y[m]$

0.0
Fit Error $[\%]: 1.072$

System: EM-58 Freq[Hz]: 30 Data Set Code: hi TX Cur $[A]: 2.50$ Turn Off[usec]: 2.5

RX Moment[turns-m^2]: 31.4 Gain Setting: 5

Time
$[\mathrm{ms}]$
0.08800
0.10600
0.13100
0.16100
0.20000
0.25000
0.31400
0.39500
0.49900
0.63100
0.79900
1.01000
1.28000
1.63000
2.08000
2.64000
3.37000
4.29000
5.47000
6.97000

obs err mask

$\left[\overline{\frac{0}{0}}\right]$
0.4
0.4
0.4
0.4
0.4
0.4
0.6
0.4
1.2
1.3
1.2
1.5
4.3
5.1
7.6
10.8
7.9
22.3
13.6
9.2

rhoa cal

[ohm-m ]

$\begin{array}{rrrr}25.6 & 0.4 & u & 25.7 \\ 25.5 & 0.4 & u & 25.6\end{array}$

$0.4 u-25.6$

$\begin{array}{llll}25.6 & 0.4 & u & 25.6\end{array}$

$\begin{array}{llll}25.4 & 0.4 & u & 25.5\end{array}$

$\begin{array}{llll}24.9 & 0.6 & \mathrm{u} & 25.1 \\ 24.3 & 0.4 & \mathrm{u} & 24.7\end{array}$

24.1

23.5

22.8

22.2

21.6

$21 \cdot 1$

20.7

$-$

$-$ 
Project: Saltwater Intrusion

$558393.0 \quad \mathrm{~N}[\mathrm{~m}] \quad 2810648.0$ 0.0

RX location: X $[\mathrm{m}]$

0.0
TX loop size: X[m] $37.9 \quad \mathrm{Y}[\mathrm{m}] \quad 37.9$

$\begin{array}{cccc}\text { Model } & \text { Resistivity } & \text { Thickness } & \text { Elevation } \\ \text { Layer } & {[\text { ohm-m] }} & {[\mathrm{m}]} & {[\mathrm{m}]} \\ 1 & 81.830 & 8.1 & 0.0 \\ 2 & 44.710 & 6.4 & -8.1 \\ 3 & 2.660 & 8.1 & -14.5 \\ 4 & 22.870 & -- & -22.7\end{array}$

System: EM-58 Freq[Hz]: 285 Data Set Code: uh TX Cur[A]: 2.60 Turn Off[usec]: 2.5

RX Moment [turns-m^2]: 31.4 Gain Setting: 2

$\begin{array}{rrrrrr}\text { Time } & \begin{array}{r}\text { rhoa_obs } \\ \text { [ohm-m] }\end{array} & \begin{array}{c}\text { obs_err } \\ {[\bar{\circ} \text { o }]}\end{array} & \text { mask } & \begin{array}{r}\text { rhoa_cal } \\ \text { [ohm-m] }\end{array} \\ 1 & 0.00680 & 84.6 & 0.3 & u & 83.9 \\ 2 & 0.00860 & 67.2 & 0.2 & \mathrm{u} & 68.2 \\ 3 & 0.01110 & 53.6 & 0.2 & \mathrm{u} & 54.7 \\ 4 & 0.01410 & 42.8 & 0.2 & \mathrm{u} & 43.1 \\ 5 & 0.01800 & 34.0 & 0.2 & \mathrm{u} & 33.7 \\ 6 & 0.02300 & 27.0 & 0.2 & \mathrm{u} & 26.4 \\ 7 & 0.02940 & 21.5 & 0.2 & \mathrm{u} & 20.9 \\ 8 & 0.03750 & 17.4 & 0.2 & \mathrm{u} & 17.0 \\ 9 & 0.04790 & 14.4 & 0.2 & \mathrm{u} & 14.2 \\ 10 & 0.06110 & 12.3 & 0.2 & \mathrm{u} & 12.3 \\ 11 & 0.07790 & 10.8 & 0.2 & \mathrm{u} & 11.0 \\ 12 & 0.09930 & 9.9 & 0.2 & \mathrm{u} & 10.1 \\ 13 & 0.12670 & 9.4 & 0.2 & \mathrm{u} & 9.6 \\ 14 & 0.16640 & 9.2 & 0.2 & \mathrm{u} & 9.4 \\ 15 & 0.20600 & 9.2 & 0.2 & \mathrm{u} & 9.4 \\ 16 & 0.26280 & 9.6 & 0.2 & \mathrm{u} & 9.6 \\ 17 & 0.33520 & 10.1 & 0.2 & \mathrm{u} & 10.0 \\ 18 & 0.42770 & 10.8 & 0.3 & \mathrm{u} & 10.6 \\ 19 & 0.54560 & 11.7 & 0.2 & \mathrm{u} & 11.4 \\ 20 & 0.69590 & 13.0 & 0.3 & \mathrm{u} & 12.4\end{array}$

\section{Fit Error [으: 2.601}

System: EM-58 Freq[Hz]: 30 Data Set Code: hi TX Cur $[A]: \quad 2.50$ Turn off[usec] : 2.5

RX Moment [turns-m^2] : 31.4 Gain Setting: 5

\begin{tabular}{|c|c|c|c|c|}
\hline $\begin{array}{l}\text { Time } \\
{[\mathrm{ms}]}\end{array}$ & $\begin{array}{l}\text { rhoa_obs } \\
\text { [ohm-m] }\end{array}$ & $\begin{array}{c}\text { obs err } \\
{\left[\frac{\mathrm{o}}{0}\right]}\end{array}$ & mask & $\begin{array}{l}\text { rhoa_cal } \\
\text { [ohm-m] }\end{array}$ \\
\hline 0.08800 & 10.5 & 0.3 & $u$ & 10.5 \\
\hline 0.10600 & 9.8 & 0.3 & $u$ & 9.9 \\
\hline 0.13100 & 9.4 & 0.3 & $u$ & 9. \\
\hline 0.16100 & 9.3 & 0.3 & $\mathrm{u}$ & 9. \\
\hline 0.20000 & 9.3 & 0.3 & u & 9. \\
\hline 0.25000 & 9.5 & 0.3 & $\mathrm{u}$ & 9. \\
\hline 0.31400 & 9.8 & 0.3 & $\mathrm{u}$ & 9.7 \\
\hline 0.39500 & 10.3 & 0.3 & $u$ & 10.1 \\
\hline 0.49900 & 10.8 & 0.4 & u & 10. \\
\hline 0.63100 & 11.4 & 0.6 & u & 11.2 \\
\hline 0.79900 & 11.9 & 0.9 & u & 11.9 \\
\hline 1.01000 & 12.5 & 1.0 & u & 12.6 \\
\hline 1.28000 & 13.3 & 1.9 & u & 13.3 \\
\hline 1.63000 & 14.0 & 2.4 & u & 14.1 \\
\hline 2.08000 & 15.0 & 4.8 & u & 14.9 \\
\hline & 15.2 & 9.9 & u & 15.6 \\
\hline & 17.9 & 16.8 & $\mathrm{~m}$ & 16.6 \\
\hline & 24.7 & 48.0 & d & - \\
\hline & 26. & 58.1 & d & - \\
\hline & 02 & 100.0 & d & - \\
\hline
\end{tabular}


Sounding: MIA230L3

Client: US Geological Survey Location: $0.05 \mathrm{~km} \mathrm{~N}$ of $\mathrm{SW} 384 \mathrm{St} \& 087 \mathrm{~km}$ E of $3 \mathrm{Mile} \mathrm{Rd}$ Project: Saltwater Intrusion County: Miami-Dade County

TX loop size: X $[\mathrm{m}] \quad 37.9 \quad \mathrm{Y}[\mathrm{m}] \quad 37.9$

$\begin{array}{cccc}\text { Model } & \text { Resistivity } & \text { Thickness } & \text { Elevation } \\ \text { Layer } & {[\text { ohm-m] }} & {[\mathrm{m}]} & {[\mathrm{m}]} \\ 1 & 202.700 & 13.0 & 0.0 \\ 2 & 3.280 & 11.0 & -13.0 \\ 3 & 23.490 & -- & -24.0\end{array}$

System: EM-58 Freq[Hz]: 285 Data set Code: uh TX Cur $[A]: 2.60$ Turn Off [usec]: 2.5

RX Moment[turns-m^2]: 31.4 Gain Setting: 2

\begin{tabular}{|c|c|c|c|c|c|}
\hline & $\begin{array}{l}\text { Time } \\
{[\mathrm{ms}]}\end{array}$ & $\begin{array}{l}\text { rhoa_obs } \\
\text { [ohm-m] }\end{array}$ & $\begin{array}{c}\text { obs err } \\
{\left[\frac{\overline{0}}{0}\right]}\end{array}$ & mask & $\begin{array}{l}\text { rhoa_cal } \\
\text { [ohm-m] }\end{array}$ \\
\hline 1 & 0.00680 & 84.6 & 0.3 & u & 89.1 \\
\hline 2 & 0.00860 & 67.2 & 0.2 & $\mathrm{u}$ & 68.2 \\
\hline 3 & 0.01110 & 53.6 & 0.2 & $\mathrm{u}$ & 53.3 \\
\hline 4 & 0.01410 & 42.8 & 0.2 & $u$ & 41.9 \\
\hline 5 & 0.01800 & 34.0 & 0.2 & $u$ & 33.0 \\
\hline 6 & 0.02300 & 27.0 & 0.2 & $\mathrm{u}$ & 26.1 \\
\hline 7 & 0.02940 & 21.5 & 0.2 & $\mathrm{u}$ & 20.8 \\
\hline 8 & 0.03750 & 17.4 & 0.2 & u & 17.0 \\
\hline 9 & 0.04790 & 14.4 & 0.2 & u & 14.3 \\
\hline 10 & 0.06110 & 12.3 & 0.2 & u & 12.4 \\
\hline 11 & 0.07790 & 10.8 & 0.2 & u & 11.1 \\
\hline 12 & 0.09930 & 9.9 & 0.2 & u & 10.2 \\
\hline 13 & 0.12670 & 9.4 & 0.2 & $u$ & 9.7 \\
\hline 14 & 0.16640 & 9.2 & 0.2 & $\mathrm{u}$ & 9.4 \\
\hline 15 & 0.20600 & 9.2 & 0.2 & $\mathrm{u}$ & 9.4 \\
\hline 16 & 0.26280 & 9.6 & 0.2 & u & 9.6 \\
\hline 17 & 0.33520 & 10.1 & 0.2 & $u$ & 10.0 \\
\hline 18 & 0.42770 & 10.8 & 0.3 & $u$ & 10.6 \\
\hline 19 & 0.5 & 11.7 & 0.2 & $\mathrm{u}$ & 11.4 \\
\hline 20 & 0.6 & 13.0 & 0.3 & $\mathrm{u}$ & 12.4 \\
\hline
\end{tabular}

Date: 02-MAR-09

UTM Coord: $\mathrm{E}[\mathrm{m}]$

Elevation $[\mathrm{m}]$ :

$558393.0 \quad \mathrm{~N}[\mathrm{~m}] \quad 2810648.0$

0.0

RX location: X $[\mathrm{m}]$

$0.0 \quad Y[m]$

0.0

\section{Fit Error $[\%]: 3.365$}

System: EM-58 Freq[Hz]: 30 Data Set Code: hi TX Cur $[A]: 2.50$ Turn Off[usec]: 2.5

RX Moment[turns-m^2] : 31.4 Gain Setting: 5

rhoa_obs
[ohm-m]
10.5
9.8
9.4
9.3
9.3
9.5
9.8
10.3
10.8
11.4
11.9
12.5
13.3
14.0
15.0
15.2
17.9
24.7
26.3
83.0

obs err mask

[흥]

0.3
0.3

0.3
0.3

0.3

0.3

0.3

0.3

0.3

0.4

0.6
0.9

1.0

1.9

2.4

4.8

9.9

16.8

$48 \cdot 0$

58.1

100.0

$\begin{array}{cr}\text { mask } & \begin{array}{r}\text { rhoa_cal } \\ \text { [ohm-m] }\end{array} \\ \mathrm{u} & 10.6 \\ \mathrm{u} & 10.0 \\ \mathrm{u} & 9.6 \\ \mathrm{u} & 9.4 \\ \mathrm{u} & 9.3 \\ \mathrm{u} & 9.4 \\ \mathrm{u} & 9.7 \\ \mathrm{u} & 10.1 \\ \mathrm{u} & 10.6 \\ \mathrm{u} & 11.2 \\ \mathrm{u} & 11.8 \\ \mathrm{u} & 12.6 \\ \mathrm{u} & 13.3 \\ \mathrm{u} & 14.2 \\ \mathrm{u} & 15.0 \\ \mathrm{u} & 15.8 \\ \mathrm{~m} & 16.8 \\ \mathrm{~d} & - \\ \mathrm{d} & - \\ \mathrm{d} & -\end{array}$


Sounding: MIA231

Location: East of SW 137th S

Project: Saltwater Intrusion

County: Miami-Dade County

TX loop size: X $[\mathrm{m}] \quad 37.9 \quad \mathrm{Y}[\mathrm{m}] \quad 37.9$

$\begin{array}{cccc}\text { Model } & \text { Resistivity } & \text { Thickness } & \text { Elevation } \\ \text { Layer } & {[\text { ohm-m] }} & {[\mathrm{m}]} & {[\mathrm{m}]} \\ 1 & 99.070 & 10.2 & 0.0 \\ 2 & 1.900 & 16.6 & -10.2 \\ 3 & 383.600 & -- & -26.8\end{array}$

System: EM-58 Freq[Hz]: 285 Data set Code: uh TX Cur $[\mathrm{A}]: \quad 2.65$ Turn Off[usec]: 2.5

RX Moment[turns-m^2]: 31.4 Gain Setting: 1

\begin{tabular}{|c|c|c|c|c|c|}
\hline & $\begin{array}{l}\text { Time } \\
{[\mathrm{ms}]}\end{array}$ & $\begin{array}{l}\text { rhoa_obs } \\
\text { [ohm-m] }\end{array}$ & $\begin{array}{c}\text { obs err } \\
{\left[\frac{\mathrm{o}}{0}\right]}\end{array}$ & mask & $\begin{array}{l}\text { rhoa_cal } \\
{[\mathrm{ohm}-\mathrm{m}]}\end{array}$ \\
\hline 1 & 0.00680 & 69.7 & 0.5 & $u$ & 70.4 \\
\hline 2 & 0.00860 & 53.7 & 0.4 & $\mathrm{u}$ & 53.3 \\
\hline 3 & 0.01110 & 41.2 & 0.5 & $\mathrm{u}$ & 41.3 \\
\hline 4 & 0.01410 & 32.1 & 0.5 & $u$ & 32.3 \\
\hline 5 & 0.01800 & 25.2 & 0.4 & $u$ & 25.4 \\
\hline 6 & 0.02300 & 20.0 & 0.5 & $\mathrm{u}$ & 20.2 \\
\hline 7 & 0.02940 & 16.0 & 0.5 & u & 16.1 \\
\hline 8 & 0.03750 & 13.0 & 0.4 & u & 13.0 \\
\hline 9 & 0.04790 & 10.7 & 0.5 & u & 10.6 \\
\hline 10 & 0.06110 & 8.8 & 0.5 & u & 8.7 \\
\hline 11 & 0.07790 & 7.4 & 0.4 & u & 7.2 \\
\hline 12 & 0.09930 & 6.2 & 0.5 & u & 6.1 \\
\hline 13 & 0.12670 & 5.3 & 0.5 & $u$ & 5.3 \\
\hline 14 & 0.16640 & 4.7 & 0.4 & $\mathrm{u}$ & 4.7 \\
\hline 15 & 0.20600 & 4.3 & 0.5 & $\mathrm{u}$ & 4.3 \\
\hline 16 & 0.26280 & 4.1 & 0.5 & $\mathrm{u}$ & 4.2 \\
\hline 17 & 0.33520 & 4.1 & 0.4 & $u$ & 4.1 \\
\hline 18 & 0.42770 & 4.2 & 0.4 & $u$ & 4.2 \\
\hline 19 & 0.5 & 4.5 & 0.4 & $\mathrm{u}$ & 4.4 \\
\hline 20 & 0.69 & 5.0 & 0.4 & $\mathrm{u}$ & 4.8 \\
\hline
\end{tabular}

Date: 03-MAR-09

UTM Coord: $\mathrm{E}[\mathrm{m}]$

Elevation $[\mathrm{m}]$ :

$559404.0 \quad \mathrm{~N}[\mathrm{~m}] \quad 2809111.0$

0.0

RX location: X $[\mathrm{m}]$

$0.0 \quad Y[m]$

0.0
Fit Error $[\%]: 3.275$

System: EM-58 Freq[Hz]: 30 Data Set Code: hi TX Cur $[\mathrm{A}]: \quad 2.50$ Turn Off[usec]: 2.5

RX Moment [turns-m^2] : 31.4 Gain Setting: 5

Time
$[\mathrm{ms}]$
0.08800
0.10600
0.13100
0.16100
0.20000
0.25000
0.31400
0.39500
0.49900
0.63100
0.79900
1.01000
1.28000
1.63000
2.08000
2.64000
3.37000
4.29000
5.47000
6.97000

rhoa_obs
[ohm-m]
6.7
5.9
5.2
4.7
4.3
4.1
3.9
4.0
4.1
4.4
4.8
5.3
5.9
6.7
7.9
9.4
12.6
20.3
12.5
6.4

rhoa_cal

[응 ] [ohm-m]

0.4 u $\quad 6.6$

0.4 u $\quad 5.8$

0.4 u $\quad 5.1$

0.4 u

0.4

$\begin{array}{lll}0.4 & u & 4.1 \\ 0.4 & u & 4.0\end{array}$

$0.4 \quad \mathrm{u} \quad 4.1$

0.4 u 4.2

0.4 u 4.4

0.4 u $\quad 4.8$

0.5 u -5.3

0.9 u 6.0

1.0 u 6.9

2.0 u 8.1

$\begin{array}{rrr}3.1 & u & 9.6\end{array}$

24.8

$\begin{array}{lll}24.0 & d & - \\ 13.6 & d & -\end{array}$ 
Sounding: MIA232

Location: Machette Trail

Project: Saltwater Intrusion

County: Miami-Dade County

TX loop size: X[m] $37.9 \quad \mathrm{Y}[\mathrm{m}] \quad 37.9$

$\begin{array}{cccc}\text { Model } & \text { Resistivity } & \text { Thickness } & \text { Elevation } \\ \text { Layer } & {[\text { ohm-m] }} & {[\mathrm{m}]} & {[\mathrm{m}]} \\ 1 & 67.120 & 11.0 & 0.0 \\ 2 & 2.900 & 19.4 & -11.0 \\ 3 & 228.000 & 100.5 & -30.4 \\ 4 & 1.690 & -- & -130.9\end{array}$

System: EM-58 Freq[Hz]: 285 Data Set Code: uh TX Cur $[A]: 2.65$ Turn off[usec] : 2.5

RX Moment [turns-m^2]: 31.4 Gain Setting: 1

\begin{tabular}{|c|c|c|c|c|}
\hline $\begin{array}{l}\text { Time } \\
{[\mathrm{ms}]}\end{array}$ & $\begin{array}{l}\text { rhoa_obs } \\
\text { [ohm-m] }\end{array}$ & $\begin{array}{c}\text { obs err } \\
{\left[\frac{\overline{0}}{0}\right]}\end{array}$ & mask & $\begin{array}{l}\text { rhoa_ca } \\
{[\text { ohm-m ] }}\end{array}$ \\
\hline 0.00680 & 64.1 & 0.5 & $u$ & 65.7 \\
\hline 0.00860 & 52.2 & 0.3 & $u$ & 51.5 \\
\hline 0.01110 & 41.9 & 0.4 & u & 41. \\
\hline 0.01410 & 33.6 & 0.4 & u & 32. \\
\hline 0.01800 & 26.9 & 0.4 & u & 26. \\
\hline 0.02300 & 21.7 & 0.4 & $\mathrm{u}$ & 21 . \\
\hline 0.02940 & 17.5 & 0.4 & $\mathrm{u}$ & 17. \\
\hline 0.03750 & 14.3 & 0.4 & u & 14 \\
\hline 0.04790 & 11.9 & 0.4 & u & 12 \\
\hline 0.06110 & 10.1 & 0.4 & u & 10 \\
\hline 0.07790 & 8.7 & 0.4 & u & 8 \\
\hline 0.09930 & 7.6 & 0.4 & $\mathrm{u}$ & \\
\hline 0.12670 & 6.9 & 0.4 & u & 6 \\
\hline 0.16640 & 6.3 & 0.4 & $u$ & 6 \\
\hline 0.20600 & 6.0 & 0.4 & u & 5 \\
\hline 0.26280 & 5.8 & 0.4 & u & 5 \\
\hline 0.33520 & 5.9 & 0.3 & u & 5 \\
\hline 0.42770 & 6.1 & 0.3 & $u$ & \\
\hline 0.54560 & 6.7 & 0.3 & u & \\
\hline 0.6 & 7.7 & 0.4 & u & \\
\hline
\end{tabular}

Date: 03-MAR-09

UTM Coord: $\mathrm{E}[\mathrm{m}]$

Elevation $[\mathrm{m}]$ :

$558515.0 \quad \mathrm{~N}[\mathrm{~m}] \quad 2807305.0$

0.0

RX location: X $[\mathrm{m}]$

$0.0 \quad Y[m]$

0.0
Fit Error [\%]: 2.116

System: EM-58 Freq[Hz]: 30 Data Set Code: hi TX Cur $[A]: 2.50$ Turn Off[usec]: 2.5

RX Moment [turns-m^2] : 31.4 Gain Setting: 5

\begin{tabular}{|c|c|c|c|c|}
\hline $\begin{array}{l}\text { Time } \\
{[\mathrm{ms}]}\end{array}$ & $\begin{array}{l}\text { rhoa_obs } \\
\text { [ohm-m] }\end{array}$ & $\begin{array}{c}\text { obs err } \\
{\left[\overline{\frac{0}{0}}\right]}\end{array}$ & mask & $\begin{array}{l}\text { rhoa_cal } \\
\text { [ohm-m] }\end{array}$ \\
\hline 0.08800 & 8.1 & 0.3 & u & 8.1 \\
\hline 0.10600 & 7.4 & 0.3 & $\mathrm{u}$ & 7.3 \\
\hline 0.13100 & 6.8 & 0.3 & u & 6.7 \\
\hline 0.16100 & 6.3 & 0.3 & u & 6.2 \\
\hline 0.20000 & 6.0 & 0.3 & u & 5 . \\
\hline 0.25000 & 5.8 & 0.3 & u & 5. \\
\hline 0.31400 & 5.7 & 0.3 & u & 5 . \\
\hline 0.39500 & 5.8 & 0.3 & u & 5.9 \\
\hline 0.49900 & 6.2 & 0.3 & u & 6.3 \\
\hline 0.63100 & 6.7 & 0.3 & u & 6.8 \\
\hline 0.79900 & 7.5 & 0.2 & u & 7.5 \\
\hline 1.01000 & 8.4 & 0.6 & u & 8.4 \\
\hline 1.28000 & 9.4 & 0.8 & u & 9.4 \\
\hline 1.63000 & 10.4 & 2.0 & u & 10.4 \\
\hline 000 & 11.1 & 3.1 & u & 11.1 \\
\hline 2 . & 11.3 & 4.8 & u & 11 . \\
\hline & 10.3 & 14.3 & d & - \\
\hline & 9.4 & 12.6 & d & - \\
\hline & 8.0 & 14.9 & d & - \\
\hline & 6. & 17 & d & \\
\hline
\end{tabular}


Sounding: MIA232L3

Location: Machette Trail

Project: Saltwater Intrusion

County: Miami-Dade County

TX loop size: X[m] $37.9 \quad \mathrm{Y}[\mathrm{m}] \quad 37.9$

$\begin{array}{cccc}\text { Model } & \text { Resistivity } & \text { Thickness } & \text { Elevation } \\ \text { Layer } & {[\text { ohm-m] }} & {[\mathrm{m}]} & {[\mathrm{m}]} \\ 1 & 67.590 & 11.0 & 0.0 \\ 2 & 2.890 & 18.8 & -11.0 \\ 3 & 149.100 & -- & -29.8\end{array}$

System: EM-58 Freq[Hz]: 285 Data set Code: uh TX Cur $[\mathrm{A}]: \quad 2.65$ Turn Off[usec]: 2.5

RX Moment[turns-m^2]: 31.4 Gain Setting:

\begin{tabular}{|c|c|c|c|c|c|}
\hline & $\begin{array}{l}\text { Time } \\
{[\mathrm{ms}]}\end{array}$ & $\begin{array}{l}\text { rhoa_obs } \\
\text { [ohm-m] }\end{array}$ & $\begin{array}{c}\text { obs err } \\
{\left[\overline{\frac{0}{0}}\right]}\end{array}$ & mask & $\begin{array}{l}\text { rhoa_cal } \\
\text { [ohm-m] }\end{array}$ \\
\hline 1 & 0.00680 & 64.1 & 0.5 & $u$ & 65.8 \\
\hline 2 & 0.00860 & 52.2 & 0.3 & u & 51.6 \\
\hline 3 & 0.01110 & 41.9 & 0.4 & u & 41.0 \\
\hline 4 & 0.01410 & 33.6 & 0.4 & $\mathrm{u}$ & 33.0 \\
\hline 5 & 0.01800 & 26.9 & 0.4 & $u$ & 26.6 \\
\hline 6 & 0.02300 & 21.7 & 0.4 & u & 21.6 \\
\hline 7 & 0.02940 & 17.5 & 0.4 & u & 17.7 \\
\hline 8 & 0.03750 & 14.3 & 0.4 & u & 14.7 \\
\hline 9 & 0.04790 & 11.9 & 0.4 & u & 12.2 \\
\hline 10 & 0.06110 & 10.1 & 0.4 & $u$ & 10.2 \\
\hline 11 & 0.07790 & 8.7 & 0.4 & $u$ & 8.7 \\
\hline 12 & 0.09930 & 7.6 & 0.4 & $u$ & 7.6 \\
\hline 13 & 0.12670 & 6.9 & 0.4 & $\mathrm{u}$ & 6.8 \\
\hline 14 & 0.16640 & 6.3 & 0.4 & u & 6.2 \\
\hline 15 & 0.20600 & 6.0 & 0.4 & u & 6.0 \\
\hline 16 & 0.26280 & 5.8 & 0.4 & $u$ & 5.9 \\
\hline 17 & 0.33520 & 5.9 & 0.3 & $u$ & 5.9 \\
\hline 18 & 0.42770 & 6.1 & 0.3 & $\mathrm{u}$ & 6.2 \\
\hline 19 & 0.54560 & 6.7 & 0.3 & $\mathrm{u}$ & 6.7 \\
\hline 20 & 0.69590 & 7.7 & 0.4 & $u$ & 7.4 \\
\hline
\end{tabular}

Date: 03-MAR-09

UTM Coord: $\mathrm{E}[\mathrm{m}]$

Elevation $[\mathrm{m}]$ :

$558515.0 \quad \mathrm{~N}[\mathrm{~m}] \quad 2807305.0$

0.0

RX location: X $[\mathrm{m}]$

$0.0 \quad Y[m]$

0.0

\section{Fit Error $[\%]: 2.510$}

System: EM-58 Freq[Hz]: 30 Data Set Code: hi TX Cur $[A]: 2.50$ Turn Off[usec]: 2.5

RX Moment[turns-m^2]: 31.4 Gain Setting: 5

Time
$[\mathrm{ms}]$
0.08800
0.10600
0.13100
0.16100
0.20000
0.25000
0.31400
0.39500
0.49900
0.63100
0.79900
1.01000
1.28000
1.63000
2.08000
2.64000
3.37000
4.29000
5.47000
6.97000

.

\begin{tabular}{|c|c|c|c|}
\hline $\begin{array}{l}\text { rhoa_obs } \\
\text { [ohm-m] }\end{array}$ & $\begin{array}{c}\text { obs err } \\
{\left[\frac{\mathrm{o}}{0}\right]}\end{array}$ & mask & $\begin{array}{l}\text { rhoa_cal } \\
\text { [ohm-m] }\end{array}$ \\
\hline 8.1 & 0.3 & u & 8.1 \\
\hline 7.4 & 0.3 & u & 7.3 \\
\hline 6.8 & 0.3 & u & 6.7 \\
\hline 6.3 & 0.3 & u & 6.2 \\
\hline 6.0 & 0.3 & u & 5.9 \\
\hline 5.8 & 0.3 & u & 5.8 \\
\hline 5.7 & 0.3 & u & 5.8 \\
\hline 5.8 & 0.3 & $\mathrm{u}$ & 6.0 \\
\hline 6.2 & 0.3 & u & 6.3 \\
\hline 6.7 & 0.3 & $\mathrm{u}$ & 6.7 \\
\hline 7.5 & 0.2 & $\mathrm{u}$ & 7.4 \\
\hline 8.4 & 0.6 & u & 8.2 \\
\hline 9.4 & 0.8 & u & 9.4 \\
\hline 10.4 & 2.0 & u & 10.7 \\
\hline 11.1 & 3.1 & $\mathrm{~m}$ & 12.5 \\
\hline 11.3 & 4.8 & $\mathrm{~m}$ & 14.7 \\
\hline 10.3 & 14.3 & d & - \\
\hline 9.4 & 12.6 & d & - \\
\hline 8.0 & 14.9 & d & - \\
\hline 6.6 & 17.3 & d & - \\
\hline
\end{tabular}


Sounding: MIA233

Client: US Geological Survey Location: $0.11 \mathrm{~km} \mathrm{~N}$ of $\mathrm{SW} 408 \mathrm{St} \& 0.50 \mathrm{~km}$ E Card Sound Rd Project: Saltwater Intrusion County: Miami-Dade County

TX loop size: X $[\mathrm{m}] \quad 37.9 \quad \mathrm{Y}[\mathrm{m}] \quad 37.9$

$\begin{array}{cccc}\text { Model } & \text { Resistivity } & \text { Thickness } & \text { Elevation } \\ \text { Layer } & {[\text { ohm-m] }} & {[\mathrm{m}]} & {[\mathrm{m}]} \\ 1 & 194.100 & 16.1 & 0.0 \\ 2 & 1.870 & 2.1 & -16.1 \\ 3 & 59.580 & 106.4 & -18.2 \\ 4 & 25.240 & -- & -124.7\end{array}$

System: EM-58 Freq[Hz]: 285 Data Set Code: uh TX Cur $[\mathrm{A}]: \quad 2.70$ Turn Off[usec]: 2.5

RX Moment[turns-m^2]: 31.4 Gain Setting: 1

$\begin{array}{rrrrrr}\text { Time } & \begin{array}{c}\text { rhoa_obs } \\ \text { [ohm-m] }\end{array} & \begin{array}{c}\text { obs_err } \\ {\left[\frac{\mathrm{o}}{0}\right]}\end{array} & \text { mask } & \begin{array}{c}\text { rhoa_cal } \\ \text { [ohm-m] }\end{array} \\ 1 & 0.00680 & 91.7 & 0.3 & \mathrm{u} & 92.1 \\ 2 & 0.00860 & 67.6 & 0.3 & \mathrm{u} & 67.8 \\ 3 & 0.01110 & 52.4 & 0.3 & \mathrm{u} & 52.1 \\ 4 & 0.01410 & 41.8 & 0.3 & \mathrm{u} & 41.6 \\ 5 & 0.01800 & 34.3 & 0.3 & \mathrm{u} & 34.3 \\ 6 & 0.02300 & 29.3 & 0.3 & \mathrm{u} & 29.2 \\ 7 & 0.02940 & 25.9 & 0.3 & \mathrm{u} & 25.9 \\ 8 & 0.03750 & 23.7 & 0.3 & \mathrm{u} & 23.7 \\ 9 & 0.04790 & 22.4 & 0.3 & \mathrm{u} & 22.5 \\ 10 & 0.06110 & 21.9 & 0.3 & \mathrm{u} & 22.0 \\ 11 & 0.07790 & 22.0 & 0.3 & \mathrm{u} & 22.1 \\ 12 & 0.09930 & 22.6 & 0.3 & \mathrm{u} & 22.7 \\ 13 & 0.12670 & 23.7 & 0.3 & \mathrm{u} & 23.8 \\ 14 & 0.16640 & 25.6 & 0.3 & \mathrm{u} & 25.4 \\ 15 & 0.20600 & 26.9 & 0.3 & \mathrm{u} & 27.1 \\ 16 & 0.26280 & 29.4 & 0.3 & \mathrm{u} & 29.2 \\ 17 & 0.33520 & 31.5 & 0.5 & \mathrm{u} & 31.6 \\ 18 & 0.42770 & 34.1 & 0.4 & \mathrm{u} & 34.0 \\ 19 & 0.54560 & 36.5 & 1.1 & \mathrm{u} & 36.5 \\ 20 & 0.69590 & 41.3 & 2.5 & \mathrm{u} & 39.1\end{array}$

Date: 03-MAR-09

UTM Coord: $\mathrm{E}[\mathrm{m}]$

Elevation $[\mathrm{m}]$ :

$556293.0 \quad \mathrm{~N}[\mathrm{~m}] \quad 2808246.0$

0.0

RX location: $\mathrm{X}[\mathrm{m}]$

$0.0 \quad Y[m]$

0.0
Fit Error [\%]: 1.913

System: EM-58 Freq[Hz]: 30 Data Set Code: hi TX Cur $[A]: 2.50$ Turn Off[usec]: 2.5

RX Moment [turns-m^2]: 31.4 Gain Setting: 5

\begin{tabular}{|c|c|c|c|c|}
\hline $\begin{array}{l}\text { Time } \\
{[\mathrm{ms}]}\end{array}$ & $\begin{array}{l}\text { rhoa_obs } \\
\text { [ohm-m] }\end{array}$ & $\begin{array}{c}\text { obs err } \\
{\left[\overline{\frac{0}{0}}\right]}\end{array}$ & mask & $\begin{array}{l}\text { rhoa_cal } \\
{[\mathrm{ohm-m}]}\end{array}$ \\
\hline 0.08800 & 44.0 & 78.3 & d & - \\
\hline 0.10600 & 23.2 & 0.7 & u & 23.0 \\
\hline 0.13100 & 24.2 & 0.6 & $\mathrm{u}$ & 23.9 \\
\hline 0.16100 & 25.4 & 0.7 & u & 25. \\
\hline 0.20000 & 26.8 & 0.4 & u & 26. \\
\hline 0.25000 & 28.4 & 0.7 & u & 28. \\
\hline 0.31400 & 29.9 & 0.8 & u & 30.2 \\
\hline 0.39500 & 31.5 & 0.8 & u & 31.9 \\
\hline 0.49900 & 32.8 & 1.2 & u & 33.5 \\
\hline 0.63100 & 33.8 & 1.1 & u & 34.5 \\
\hline 0.79900 & 34.8 & 2.2 & u & 35.2 \\
\hline 1.01000 & 35.2 & 3.8 & u & 35.1 \\
\hline 1.28000 & 35.1 & 3.7 & u & 35.1 \\
\hline 000 & 32.1 & 10.7 & $\mathrm{~m}$ & 34.5 \\
\hline 000 & 31.7 & 16.1 & d & - \\
\hline & 30.7 & 18.2 & d & - \\
\hline & 25.1 & 19.4 & d & - \\
\hline & 27.2 & 48.8 & d & - \\
\hline & 17.8 & 35.1 & d & - \\
\hline & 7.3 & 47.5 & d & - \\
\hline
\end{tabular}


Sounding: MIA233F Client: US Geological Survey Location: $0.11 \mathrm{~km} \mathrm{~N}$ of $\mathrm{SW} 408 \mathrm{St} \& 0.50 \mathrm{~km}$ E Card Sound Rd

Project: Saltwater Intrusion

County: Miami-Dade County

TX loop size: X $[\mathrm{m}] \quad 37.9 \quad \mathrm{Y}[\mathrm{m}] \quad 37.9$

$\begin{array}{cccc}\text { Model } & \text { Resistivity } & \text { Thickness } & \text { Elevation } \\ \text { Layer } & {[\text { ohm-m] }} & {[\mathrm{m}]} & {[\mathrm{m}]} \\ 1 & 178.300 & 15.4 & 0.0 \\ 2 & 3.500 & 4.1 & -15.4 \\ 3 & 62.070 & 106.0 & -19.6 \\ 4 & 23.350 & -- & -125.6\end{array}$

System: EM-58 Freq[Hz]: 285 Data Set Code: uh TX Cur $[\mathrm{A}]: \quad 2.70$ Turn Off[usec]: 2.5

RX Moment[turns-m^2]: 31.4 Gain Setting: 1

$\begin{array}{rrrrrr}\text { Time } & \begin{array}{c}\text { rhoa_obs } \\ \text { [ohm-m] }\end{array} & \begin{array}{c}\text { obs_err } \\ {\left[\frac{\mathrm{o}}{0}\right]}\end{array} & \text { mask } & \begin{array}{r}\text { rhoa_cal } \\ \text { [ohm-m] }\end{array} \\ 1 & 0.00680 & 91.7 & 0.3 & \mathrm{u} & 91.0 \\ 2 & 0.00860 & 67.6 & 0.3 & \mathrm{u} & 67.5 \\ 3 & 0.01110 & 52.4 & 0.3 & \mathrm{u} & 52.1 \\ 4 & 0.01410 & 41.8 & 0.3 & \mathrm{u} & 41.7 \\ 5 & 0.01800 & 34.3 & 0.3 & \mathrm{u} & 34.5 \\ 6 & 0.02300 & 29.3 & 0.3 & \mathrm{u} & 29.4 \\ 7 & 0.02940 & 25.9 & 0.3 & \mathrm{u} & 26.0 \\ 8 & 0.03750 & 23.7 & 0.3 & \mathrm{u} & 23.9 \\ 9 & 0.04790 & 22.4 & 0.3 & \mathrm{u} & 22.6 \\ 10 & 0.06110 & 21.9 & 0.3 & \mathrm{u} & 22.1 \\ 11 & 0.07790 & 22.0 & 0.3 & \mathrm{u} & 22.1 \\ 12 & 0.09930 & 22.6 & 0.3 & \mathrm{u} & 22.7 \\ 13 & 0.12670 & 23.7 & 0.3 & \mathrm{u} & 23.7 \\ 14 & 0.16640 & 25.6 & 0.3 & \mathrm{u} & 25.4 \\ 15 & 0.20600 & 26.9 & 0.3 & \mathrm{u} & 27.0 \\ 16 & 0.26280 & 29.4 & 0.3 & \mathrm{u} & 29.2 \\ 17 & 0.33520 & 31.5 & 0.5 & \mathrm{u} & 31.6 \\ 18 & 0.42770 & 34.1 & 0.4 & \mathrm{u} & 34.1 \\ 19 & 0.54560 & 36.5 & 1.1 & \mathrm{u} & 36.7 \\ 20 & 0.69590 & 41.3 & 2.5 & \mathrm{u} & 39.3\end{array}$

Date: 03-MAR-09

UTM Coord: $\mathrm{E}[\mathrm{m}]$

Elevation $[\mathrm{m}]$ :

$556293.0 \quad \mathrm{~N}[\mathrm{~m}] \quad 2808246.0$

0.0

RX location: $\mathrm{X}[\mathrm{m}]$

$0.0 \quad Y[m]$

0.0
Fit Error [\%]: 1.992

System: EM-58 Freq[Hz]: 30 Data Set Code: hi TX Cur $[A]: 2.50$ Turn Off[usec]: 2.5

RX Moment [turns-m^2]: 31.4 Gain Setting: 5

$\begin{array}{rrrrrc}\text { Time } & {[\mathrm{ms}]} & \begin{array}{c}\text { rhoa_obs } \\ {[\text { ohm-m] }}\end{array} & \begin{array}{c}\text { obs_err } \\ {\left[\frac{\mathrm{o}}{0}\right]}\end{array} & \text { mask } & \begin{array}{c}\text { rhoa_cal } \\ {[\text { ohm-m] }}\end{array} \\ 1 & 0.08800 & 44.0 & 78.3 & \mathrm{~d} & - \\ 2 & 0.10600 & 23.2 & 0.7 & \mathrm{u} & 22.9 \\ 3 & 0.13100 & 24.2 & 0.6 & \mathrm{u} & 23.8 \\ 4 & 0.16100 & 25.4 & 0.7 & \mathrm{u} & 25.0 \\ 5 & 0.20000 & 26.8 & 0.4 & \mathrm{u} & 26.6 \\ 6 & 0.25000 & 28.4 & 0.7 & \mathrm{u} & 28.3 \\ 7 & 0.31400 & 29.9 & 0.8 & \mathrm{u} & 30.3 \\ 8 & 0.39500 & 31.5 & 0.8 & \mathrm{u} & 32.0 \\ 9 & 0.49900 & 32.8 & 1.2 & \mathrm{u} & 33.6 \\ 10 & 0.63100 & 33.8 & 1.1 & \mathrm{u} & 34.6 \\ 11 & 0.79900 & 34.8 & 2.2 & \mathrm{u} & 35.2 \\ 12 & 1.01000 & 35.2 & 3.8 & \mathrm{u} & 35.0 \\ 13 & 1.28000 & 35.1 & 3.7 & \mathrm{u} & 34.8 \\ 14 & 1.63000 & 32.1 & 10.7 & \mathrm{~m} & 34.0 \\ 15 & 2.08000 & 31.7 & 16.1 & \mathrm{~d} & - \\ 16 & 2.64000 & 30.7 & 18.2 & \mathrm{~d} & - \\ 17 & 3.37000 & 25.1 & 19.4 & \mathrm{~d} & - \\ 18 & 4.29000 & 27.2 & 48.8 & \mathrm{~d} & - \\ 19 & 5.47000 & 17.8 & 35.1 & \mathrm{~d} & - \\ 20 & 6.97000 & 17.3 & 47.5 & \mathrm{~d} & -\end{array}$


Sounding: MIA233L3

Client: US Geological Survey Location: $0.11 \mathrm{~km} \mathrm{~N}$ of $\mathrm{SW} 408 \mathrm{St} \& 0.50 \mathrm{~km}$ E Card Sound Rd

Project: Saltwater Intrusion

County: Miami-Dade County

TX loop size: X $[\mathrm{m}] \quad 37.9 \quad \mathrm{Y}[\mathrm{m}] \quad 37.9$

$\begin{array}{cccc}\text { Model } & \text { Resistivity } & \text { Thickness } & \text { Elevation } \\ \text { Layer } & {[\text { ohm-m] }} & {[\mathrm{m}]} & {[\mathrm{m}]} \\ 1 & 239.400 & 16.2 & 0.0 \\ 2 & 1.140 & 1.2 & -16.2 \\ 3 & 54.570 & -- & -17.5\end{array}$

System: EM-58 Freq[Hz]: 285 Data set Code: uh TX Cur $[\mathrm{A}]: \quad 2.70$ Turn Off[usec]: 2.5

RX Moment[turns-m^2]: 31.4 Gain Setting: 1

$\begin{array}{rrrrrr}\text { Time } & {[\mathrm{ms}]} & \begin{array}{c}\text { rhoa_obs } \\ \text { [ohm-m] }\end{array} & \begin{array}{c}\text { obs_err } \\ {\left[\begin{array}{c}\text { o } \\ 0\end{array}\right.}\end{array} & \text { mask } & \begin{array}{r}\text { rhoa_cal } \\ {[\text { ohm-m] }}\end{array} \\ 1 & 0.00680 & 91.7 & 0.3 & \mathrm{u} & 91.5 \\ 2 & 0.00860 & 67.6 & 0.3 & \mathrm{u} & 67.4 \\ 3 & 0.01110 & 52.4 & 0.3 & \mathrm{u} & 51.9 \\ 4 & 0.01410 & 41.8 & 0.3 & \mathrm{u} & 41.5 \\ 5 & 0.01800 & 34.3 & 0.3 & \mathrm{u} & 34.3 \\ 6 & 0.02300 & 29.3 & 0.3 & \mathrm{u} & 29.3 \\ 7 & 0.02940 & 25.9 & 0.3 & \mathrm{u} & 26.0 \\ 8 & 0.03750 & 23.7 & 0.3 & \mathrm{u} & 23.9 \\ 9 & 0.04790 & 22.4 & 0.3 & \mathrm{u} & 22.7 \\ 10 & 0.06110 & 21.9 & 0.3 & \mathrm{u} & 22.3 \\ 11 & 0.07790 & 22.0 & 0.3 & \mathrm{u} & 22.4 \\ 12 & 0.09930 & 22.6 & 0.3 & \mathrm{u} & 23.0 \\ 13 & 0.12670 & 23.7 & 0.3 & \mathrm{u} & 24.0 \\ 14 & 0.16640 & 25.6 & 0.3 & \mathrm{u} & 25.4 \\ 15 & 0.20600 & 26.9 & 0.3 & \mathrm{u} & 26.8 \\ 16 & 0.26280 & 29.4 & 0.3 & \mathrm{u} & 28.7 \\ 17 & 0.33520 & 31.5 & 0.5 & \mathrm{u} & 30.7 \\ 18 & 0.42770 & 34.1 & 0.4 & \mathrm{u} & 33.1 \\ 19 & 0.54560 & 36.5 & 1.1 & \mathrm{u} & 35.7 \\ 20 & 0.69590 & 39.3 & 2.5 & \mathrm{~d} & -\end{array}$

Date: 03-MAR-09

UTM Coord: $\mathrm{E}[\mathrm{m}]$

Elevation $[\mathrm{m}]$ :

$556293.0 \quad \mathrm{~N}[\mathrm{~m}] \quad 2808246.0$

0.0

RX location: $\mathrm{X}[\mathrm{m}]$

$0.0 \quad Y[m]$

0.0

\section{Fit Error $[\%]: 2.482$}

System: EM-58 Freq[Hz]: 30 Data Set Code: hi TX $\operatorname{Cur}[A]: 2.50$ Turn Off[usec] : 2.5

RX Moment[turns-m^2] : 31.4 Gain Setting: 5

$\begin{array}{ccccc}\begin{array}{c}\text { Time } \\ \text { [ms] }\end{array} & \begin{array}{c}\text { rhoa_obs } \\ \text { [ohm-m] }\end{array} & \begin{array}{c}\text { obserr } \\ {\left[\frac{\text { og] }}{0}\right.}\end{array} & \text { mask } & \begin{array}{c}\text { rhoa_cal } \\ \text { [ohm-m] }\end{array} \\ 0.08800 & 44.0 & 78.3 & \mathrm{~d} & - \\ 0.10600 & 23.2 & 0.7 & \mathrm{u} & 23.2 \\ 0.13100 & 24.2 & 0.6 & \mathrm{u} & 24.1 \\ 0.16100 & 25.4 & 0.7 & \mathrm{u} & 25.2 \\ 0.20000 & 26.8 & 0.4 & \mathrm{u} & 26.5 \\ 0.25000 & 28.4 & 0.7 & \mathrm{u} & 28.0 \\ 0.31400 & 29.9 & 0.8 & \mathrm{u} & 29.6 \\ 0.39500 & 31.5 & 0.8 & \mathrm{u} & 31.4 \\ 0.49900 & 32.8 & 1.2 & \mathrm{u} & 33.1 \\ 0.63100 & 33.8 & 1.1 & \mathrm{u} & 34.9 \\ 0.79900 & 34.8 & 2.2 & \mathrm{u} & 36.4 \\ 1.01000 & 35.2 & 3.8 & \mathrm{~m} & 38.4 \\ 1.28000 & 35.1 & 3.7 & \mathrm{~m} & 39.6 \\ 1.63000 & 32.1 & 10.7 & \mathrm{~d} & - \\ 2.08000 & 31.7 & 16.1 & \mathrm{~d} & - \\ 2.64000 & 30.7 & 18.2 & \mathrm{~d} & - \\ 3.37000 & 25.1 & 19.4 & \mathrm{~d} & - \\ 4.29000 & 27.2 & 48.8 & \mathrm{~d} & - \\ 5.47000 & 17.8 & 35.1 & \mathrm{~d} & - \\ 6.97000 & 17.3 & 47.5 & \mathrm{~d} & -\end{array}$


Location: $.11 \mathrm{~km} \mathrm{~N}$ of $\mathrm{SW} 408$

County: Miami-Dade County

TX loop size: X[m] $37.9 \quad \mathrm{Y}[\mathrm{m}] \quad 37.9$

$\begin{array}{cccc}\text { Model } & \text { Resistivity } & \text { Thickness } & \text { Elevation } \\ \text { Layer } & {[\text { ohm-m] }} & {[\mathrm{m}]} & {[\mathrm{m}]} \\ 1 & 218.500 & 15.2 & 0.0 \\ 2 & 3.500 & 4.0 & -15.2 \\ 3 & 57.860 & -- & -19.3\end{array}$

System: EM-58 Freq[Hz]: 285 Data set Code: uh TX Cur $[\mathrm{A}]: \quad 2.70$ Turn Off[usec]: 2.5

RX Moment[turns-m^2]: 31.4 Gain Setting: 1

\begin{tabular}{|c|c|c|c|c|c|}
\hline & $\begin{array}{l}\text { Time } \\
{[\mathrm{ms}]}\end{array}$ & $\begin{array}{l}\text { rhoa_obs } \\
\text { [ohm-m] }\end{array}$ & $\begin{array}{c}\text { obs err } \\
{\left[\frac{\mathrm{o}}{0}\right]}\end{array}$ & mask & $\begin{array}{l}\text { rhoa_cal } \\
\text { [ohm-m] }\end{array}$ \\
\hline 1 & 0.00680 & 91.7 & 0.3 & u & 90.2 \\
\hline 2 & 0.00860 & 67.6 & 0.3 & $\mathrm{u}$ & 66.9 \\
\hline 3 & 0.01110 & 52.4 & 0.3 & $\mathrm{u}$ & 51.8 \\
\hline 4 & 0.01410 & 41.8 & 0.3 & $u$ & 41.6 \\
\hline 5 & 0.01800 & 34.3 & 0.3 & $u$ & 34.5 \\
\hline 6 & 0.02300 & 29.3 & 0.3 & $\mathrm{u}$ & 29.6 \\
\hline 7 & 0.02940 & 25.9 & 0.3 & u & 26.2 \\
\hline 8 & 0.03750 & 23.7 & 0.3 & u & 24.1 \\
\hline 9 & 0.04790 & 22.4 & 0.3 & u & 22.9 \\
\hline 10 & 0.06110 & 21.9 & 0.3 & u & 22.3 \\
\hline 11 & 0.07790 & 22.0 & 0.3 & u & 22.4 \\
\hline 12 & 0.09930 & 22.6 & 0.3 & u & 22.9 \\
\hline 13 & 0.12670 & 23.7 & 0.3 & u & 23.9 \\
\hline 14 & 0.16640 & 25.6 & 0.3 & $\mathrm{u}$ & 25.4 \\
\hline 15 & 0.20600 & 26.9 & 0.3 & $\mathrm{u}$ & 26.8 \\
\hline 16 & 0.26280 & 29.4 & 0.3 & $\mathrm{u}$ & 28.7 \\
\hline 17 & 0.33520 & 31.5 & 0.5 & $u$ & 30.8 \\
\hline 18 & 0.42770 & 34.1 & 0.4 & $u$ & 33.3 \\
\hline 19 & 0.5 & 36.5 & 1.1 & $\mathrm{u}$ & 36.0 \\
\hline 20 & 0.69 & 41.3 & 2.5 & $u$ & 39. \\
\hline
\end{tabular}

RX location: X $[\mathrm{m}] \quad 0.0 \quad \mathrm{Y}[\mathrm{m}] \quad 0.0$

\section{Fit Error $\left[\frac{\circ}{0}\right]: 3.330$}

System: EM-58 Freq[Hz]: 30 Data Set Code: hi TX Cur $[A]: 2.50$ Turn Off[usec] : 2.5

RX Moment [turns-m^2] : 31.4 Gain Setting: 5

$\begin{array}{ccccc}\begin{array}{c}\text { Time } \\ \text { [ms] }\end{array} & \begin{array}{c}\text { rhoa_obs } \\ \text { [ohm-m] }\end{array} & \begin{array}{c}\text { obserr } \\ {\left[\begin{array}{c}\text { o }] \\ 0\end{array}\right.}\end{array} & \text { mask } & \begin{array}{c}\text { rhoa_cal } \\ \text { [ohm-m] }\end{array} \\ 0.08800 & 44.0 & 78.3 & \mathrm{~d} & - \\ 0.10600 & 23.2 & 0.7 & \mathrm{u} & 23.1 \\ 0.13100 & 24.2 & 0.6 & \mathrm{u} & 24.0 \\ 0.16100 & 25.4 & 0.7 & \mathrm{u} & 25.1 \\ 0.20000 & 26.8 & 0.4 & \mathrm{u} & 26.4 \\ 0.25000 & 28.4 & 0.7 & \mathrm{u} & 28.0 \\ 0.31400 & 29.9 & 0.8 & \mathrm{u} & 29.6 \\ 0.39500 & 31.5 & 0.8 & \mathrm{u} & 31.6 \\ 0.49900 & 32.8 & 1.2 & \mathrm{u} & 33.3 \\ 0.63100 & 33.8 & 1.1 & \mathrm{u} & 35.4 \\ 0.79900 & 34.8 & 2.2 & \mathrm{u} & 37.1 \\ 1.01000 & 35.2 & 3.8 & \mathrm{~m} & 39.1 \\ 1.28000 & 35.1 & 3.7 & \mathrm{~m} & 40.6 \\ 1.63000 & 32.1 & 10.7 & \mathrm{~m} & 42.7 \\ 2.08000 & 31.7 & 16.1 & \mathrm{~d} & - \\ 2.64000 & 30.7 & 18.2 & \mathrm{~d} & - \\ 3.37000 & 25.1 & 19.4 & \mathrm{~d} & - \\ 4.29000 & 27.2 & 48.8 & \mathrm{~d} & - \\ 5.47000 & 17.8 & 35.1 & \mathrm{~d} & - \\ 6.97000 & 17.3 & 47.5 & \mathrm{~d} & -\end{array}$


Sounding: MIA234

Client: US Geological Survey Location: $2.83 \mathrm{~km} \mathrm{~N}$ of $\mathrm{SW} 424 \mathrm{St} \& 0.40 \mathrm{~km}$ E of US Hwy I Project: Saltwater Intrusion County: Miami-Dade County

TX loop size: X $[\mathrm{m}] \quad 37.9 \quad \mathrm{Y}[\mathrm{m}] \quad 37.9$

$\begin{array}{cccc}\text { Model } & \text { Resistivity } & \text { Thickness } & \text { Elevation } \\ \text { Layer } & {[\text { ohm-m] }} & {[\mathrm{m}]} & {[\mathrm{m}]} \\ 1 & 161.700 & 29.3 & 0.0 \\ 2 & 5.470 & 1.3 & -29.3 \\ 3 & 101.400 & 72.6 & -30.6 \\ 4 & 31.040 & -- & -103.3\end{array}$

System: EM-58 Freq[Hz]: 285 Data Set Code: uh TX $\operatorname{Cur}[A]: 2.70$ Turn Off[usec]: 2.5

RX Moment[turns-m^2]: 31.4 Gain Setting: 2

$\begin{array}{rrrrrr}\text { Time } & \begin{array}{c}\text { rhoa_obs } \\ \text { [ohm-m] }\end{array} & \begin{array}{c}\text { obs_err } \\ {\left[\frac{\mathrm{o}}{0}\right]}\end{array} & \text { mask } & \begin{array}{c}\text { rhoa_cal } \\ \text { [ohm-m] }\end{array} \\ 1 & 0.00680 & 147.3 & 0.3 & \mathrm{u} & 144.5 \\ 2 & 0.00860 & 123.5 & 0.4 & \mathrm{u} & 128.8 \\ 3 & 0.01110 & 116.4 & 0.5 & \mathrm{u} & 116.0 \\ 4 & 0.01410 & 107.6 & 0.5 & \mathrm{u} & 106.0 \\ 5 & 0.01800 & 99.2 & 0.5 & \mathrm{u} & 98.2 \\ 6 & 0.02300 & 93.7 & 0.5 & \mathrm{u} & 92.7 \\ 7 & 0.02940 & 89.6 & 0.4 & \mathrm{u} & 89.2 \\ 8 & 0.03750 & 87.1 & 0.4 & \mathrm{u} & 87.2 \\ 9 & 0.04790 & 85.7 & 0.4 & \mathrm{u} & 86.8 \\ 10 & 0.06110 & 85.7 & 0.4 & \mathrm{u} & 87.2 \\ 11 & 0.07790 & 86.9 & 0.4 & \mathrm{u} & 88.2 \\ 12 & 0.09930 & 87.2 & 0.6 & \mathrm{u} & 88.8 \\ 13 & 0.12670 & 88.0 & 0.4 & \mathrm{u} & 88.7 \\ 14 & 0.16640 & 88.3 & 0.4 & \mathrm{u} & 87.0 \\ 15 & 0.20600 & 85.8 & 0.7 & \mathrm{u} & 84.6 \\ 16 & 0.26280 & 83.7 & 0.9 & \mathrm{u} & 81.3 \\ 17 & 0.33520 & 77.1 & 1.1 & \mathrm{u} & 77.7 \\ 18 & 0.42770 & 71.9 & 2.3 & \mathrm{u} & 74.4 \\ 19 & 0.54560 & 69.1 & 2.6 & \mathrm{u} & 71.8 \\ 20 & 0.69590 & 70.2 & 6.0 & \mathrm{u} & 70.2\end{array}$

Date: 03-MAR-09

UTM Coord: $\mathrm{E}[\mathrm{m}]$

Elevation $[\mathrm{m}]$ :

$553764.0 \quad \mathrm{~N}[\mathrm{~m}] \quad 2809315.0$

0.0

RX location: X $[\mathrm{m}]$

$0.0 \quad Y[m]$

0.0
Fit Error $[\%]: 3.215$

System: EM-58 Freq[Hz]: 30 Data Set Code: hi TX Cur $[A]: 2.50$ Turn Off[usec]: 2.5

RX Moment [turns-m^2]: 31.4 Gain Setting: 5

Time
$[\mathrm{ms}]$
0.08800
0.10600
0.13100
0.16100
0.20000
0.25000
0.31400
0.39500
0.49900
0.63100
0.79900
1.01000
1.28000
1.63000
2.08000
2.64000
3.37000
4.29000
5.47000
6.97000

rhoa_cal

$\begin{array}{rrr}{[\overline{\bar{o}} \overline{0}]} & & {[\mathrm{ohm}-\mathrm{m}]} \\ 0.3 & \text { u } & 88.1\end{array}$

$\begin{array}{lll}0.3 & u & 88.1 \\ 0.2 & u & 88.4\end{array}$

$0.2 \quad u \quad 87.6$

85.9

83.1
79.2

74.9

70.3

65.9

61.8

58.1

$-$

-

$-$ 
Sounding: MIA234F

Client: US Geological Survey Location: $2.83 \mathrm{~km} \mathrm{~N}$ of SW $424 \mathrm{St} \& 0.40 \mathrm{~km}$ E of US Hwy 1 Project: Saltwater Intrusion County: Miami-Dade County

TX loop size: X $[\mathrm{m}] \quad 37.9 \quad \mathrm{Y}[\mathrm{m}] \quad 37.9$

$\begin{array}{cccc}\text { Model } & \text { Resistivity } & \text { Thickness } & \text { Elevation } \\ \text { Layer } & {[\text { ohm-m] }} & {[\mathrm{m}]} & {[\mathrm{m}]} \\ 1 & 159.200 & 29.8 & 0.0 \\ 2 & 3.500 & .8 & -29.8 \\ 3 & 101.800 & 72.6 & -30.7 \\ 4 & 30.960 & -- & -103.3\end{array}$

System: EM-58 Freq[Hz]: 285 Data Set Code: uh TX Cur[A]: 2.70 Turn Off[usec]: 2.5

RX Moment[turns-m^2]: 31.4 Gain Setting: 2

$\begin{array}{rrrrrr}\text { Time } & \begin{array}{c}\text { rhoa_obs } \\ \text { [ohm-m] }\end{array} & \begin{array}{c}\text { obserr } \\ {\left[\frac{\mathrm{o}}{0}\right]}\end{array} & \text { mask } & \begin{array}{r}\text { rhoa_cal } \\ \text { [ohm-m] }\end{array} \\ 1 & 0.00680 & 147.3 & 0.3 & \mathrm{u} & 143.9 \\ 2 & 0.00860 & 123.5 & 0.4 & \mathrm{u} & 128.8 \\ 3 & 0.01110 & 116.4 & 0.5 & \mathrm{u} & 116.2 \\ 4 & 0.01410 & 107.6 & 0.5 & \mathrm{u} & 106.1 \\ 5 & 0.01800 & 99.2 & 0.5 & \mathrm{u} & 98.3 \\ 6 & 0.02300 & 93.7 & 0.5 & \mathrm{u} & 92.6 \\ 7 & 0.02940 & 89.6 & 0.4 & \mathrm{u} & 89.2 \\ 8 & 0.03750 & 87.1 & 0.4 & \mathrm{u} & 87.1 \\ 9 & 0.04790 & 85.7 & 0.4 & \mathrm{u} & 86.8 \\ 10 & 0.06110 & 85.7 & 0.4 & \mathrm{u} & 87.1 \\ 11 & 0.07790 & 86.9 & 0.4 & \mathrm{u} & 88.1 \\ 12 & 0.09930 & 87.2 & 0.6 & \mathrm{u} & 88.7 \\ 13 & 0.12670 & 88.0 & 0.4 & \mathrm{u} & 88.7 \\ 14 & 0.16640 & 88.3 & 0.4 & \mathrm{u} & 86.9 \\ 15 & 0.20600 & 85.8 & 0.7 & \mathrm{u} & 84.7 \\ 16 & 0.26280 & 83.7 & 0.9 & \mathrm{u} & 81.2 \\ 17 & 0.33520 & 77.1 & 1.1 & \mathrm{u} & 77.8 \\ 18 & 0.42770 & 71.9 & 2.3 & \mathrm{u} & 74.4 \\ 19 & 0.54560 & 69.1 & 2.6 & \mathrm{u} & 71.8 \\ 20 & 0.69590 & 70.2 & 6.0 & \mathrm{u} & 70.1\end{array}$

Date: 03-MAR-09

UTM Coord: $\mathrm{E}[\mathrm{m}]$

Elevation $[\mathrm{m}]$ :

$553764.0 \quad \mathrm{~N}[\mathrm{~m}] \quad 2809315.0$

0.0

RX location: X $[\mathrm{m}]$

$0.0 \quad Y[m]$

0.0
Fit Error [\%]: 3.241

System: EM-58 Freq[Hz]: 30 Data Set Code: hi TX $\operatorname{Cur}[A]: 2.50$ Turn Off[usec]: 2.5

RX Moment[turns-m^2]: 31.4 Gain Setting: 5

Time
$[\mathrm{ms}]$
0.08800
0.10600
0.13100
0.16100
0.20000
0.25000
0.31400
0.39500
0.49900
0.63100
0.79900
1.01000
1.28000
1.63000
2.08000
2.64000
3.37000
4.29000
5.47000
6.97000

rhoa_cal

[ohm-m]

$\begin{array}{rrr}{[\overline{\bar{o}}]} & & {[\mathrm{ohm}-\mathrm{m}]} \\ 0.3 & u & 88.1\end{array}$

$\begin{array}{lll}0.3 & u & 88.1 \\ 0.2 & u & 88.3\end{array}$

85.8

83.1

83.1
79.1

75.0

70.2

65.9

61.7

58.1

$-$

-

$-$

- $\begin{array}{lll}0.2 & u & 87.7\end{array}$ 
Sounding: MIA235

Location: S CEMEX quarry E of US1

County: Miami-Dade County

TX loop size: X $[\mathrm{m}] \quad 37.9 \quad \mathrm{Y}[\mathrm{m}] \quad 37.9$

$\begin{array}{cccc}\text { Model } & \text { Resistivity } & \text { Thickness } & \text { Elevation } \\ \text { Layer } & {[\text { ohm-m] }} & {[\mathrm{m}]} & {[\mathrm{m}]} \\ 1 & 276.000 & 20.8 & 0.0 \\ 2 & 3.430 & 1.7 & -20.8 \\ 3 & 106.700 & 95.7 & -22.5 \\ 4 & 27.570 & -- & -118.2\end{array}$

System: EM-58 Freq[Hz]: 285 Data Set Code: uh TX Cur $[\mathrm{A}]: \quad 2.70$ Turn Off[usec]: 2.5

RX Moment[turns-m^2]: 31.4 Gain Setting: 2

$\begin{array}{rrrrrr}\text { Time } & \begin{array}{c}\text { rhoa_obs } \\ {[\text { ohm-m] }}\end{array} & \begin{array}{c}\text { obs_err } \\ {\left[\frac{\mathrm{o}}{0}\right]}\end{array} & \text { mask } & \begin{array}{r}\text { rhoa_cal } \\ \text { [ohm-m] }\end{array} \\ 1 & 0.00680 & 118.4 & 0.5 & \mathrm{u} & 118.5 \\ 2 & 0.00860 & 93.3 & 0.5 & \mathrm{u} & 94.2 \\ 3 & 0.01110 & 79.2 & 0.5 & \mathrm{u} & 78.9 \\ 4 & 0.01410 & 69.3 & 0.5 & \mathrm{u} & 68.9 \\ 5 & 0.01800 & 61.9 & 0.5 & \mathrm{u} & 62.0 \\ 6 & 0.02300 & 57.7 & 0.5 & \mathrm{u} & 57.6 \\ 7 & 0.02940 & 55.0 & 0.5 & \mathrm{u} & 55.1 \\ 8 & 0.03750 & 54.1 & 0.5 & \mathrm{u} & 54.2 \\ 9 & 0.04790 & 54.4 & 0.5 & \mathrm{u} & 54.4 \\ 10 & 0.06110 & 55.8 & 0.5 & \mathrm{u} & 55.7 \\ 11 & 0.07790 & 58.1 & 0.5 & \mathrm{u} & 58.0 \\ 12 & 0.09930 & 60.6 & 0.4 & \mathrm{u} & 60.9 \\ 13 & 0.12670 & 63.9 & 0.5 & \mathrm{u} & 64.2 \\ 14 & 0.16640 & 68.1 & 0.6 & \mathrm{u} & 67.8 \\ 15 & 0.20600 & 69.5 & 0.6 & \mathrm{u} & 69.8 \\ 16 & 0.26280 & 73.2 & 1.6 & \mathrm{u} & 71.3 \\ 17 & 0.33520 & 72.5 & 1.1 & \mathrm{u} & 71.3 \\ 18 & 0.42770 & 72.9 & 2.2 & \mathrm{u} & 70.6 \\ 19 & 0.54560 & 75.0 & 3.4 & \mathrm{~m} & 69.2 \\ 20 & 0.69590 & 81.6 & 7.4 & \mathrm{~m} & 68.4\end{array}$

Date: $04-M A R-09$

UTM Coord: $\mathrm{E}[\mathrm{m}]$

Elevation $[\mathrm{m}]$ :

$554067.0 \quad \mathrm{~N}[\mathrm{~m}] \quad 2807176.0$

0.0

RX location: X $[\mathrm{m}]$

$0.0 \quad Y[m]$

0.0
Fit Error $[\%]: 1.903$

System: EM-58 Freq[Hz]: 30 Data Set Code: hi TX Cur $[A]: 2.50$ Turn Off[usec]: 2.5

RX Moment[turns-m^2]: 31.4 Gain Setting: 5

$\begin{array}{ccccc}\begin{array}{c}\text { Time } \\ \text { [ms] }\end{array} & \begin{array}{c}\text { rhoa_obs } \\ \text { [ohm-m] }\end{array} & \begin{array}{c}\text { obs_err } \\ {\left[\begin{array}{c}\overline{\%} \\ 0\end{array}\right.}\end{array} & \text { mask } & \begin{array}{c}\text { rhoa_cal } \\ \text { [ohm-m] }\end{array} \\ 0.08800 & 59.3 & 0.8 & \mathrm{u} & 59.2 \\ 0.10600 & 61.7 & 0.6 & \mathrm{u} & 61.6 \\ 0.13100 & 64.2 & 0.7 & \mathrm{u} & 64.2 \\ 0.16100 & 66.7 & 0.8 & \mathrm{u} & 66.7 \\ 0.20000 & 67.8 & 0.6 & \mathrm{u} & 68.4 \\ 0.25000 & 68.0 & 1.2 & \mathrm{u} & 69.1 \\ 0.31400 & 66.5 & 2.3 & \mathrm{u} & 68.2 \\ 0.39500 & 64.7 & 1.8 & \mathrm{u} & 66.3 \\ 0.49900 & 62.3 & 4.5 & \mathrm{u} & 63.2 \\ 0.63100 & 61.3 & 5.0 & \mathrm{u} & 60.0 \\ 0.79900 & 55.7 & 7.7 & \mathrm{u} & 56.4 \\ 1.01000 & 54.2 & 6.2 & \mathrm{u} & 53.5 \\ 1.28000 & 49.7 & 12.7 & \mathrm{u} & 50.2 \\ 1.63000 & 49.4 & 16.3 & \mathrm{~d} & - \\ 2.08000 & 36.2 & 16.3 & \mathrm{~d} & - \\ 2.64000 & 36.2 & 29.6 & \mathrm{~d} & - \\ 3.37000 & 34.6 & 47.6 & \mathrm{~d} & - \\ 4.29000 & 24.9 & 38.0 & \mathrm{~d} & - \\ 5.47000 & 18.9 & 43.3 & \mathrm{~d} & - \\ 6.97000 & 28.2 & 100.0 & \mathrm{~d} & -\end{array}$


Sounding: MIA235F

Location: S CEMEX quarry

Client: US Geological Survey

Project: Saltwater Intrusion

County: Miami-Dade County

TX loop size: X[m] $37.9 \quad \mathrm{Y}[\mathrm{m}] \quad 37.9$

$\begin{array}{cccc}\text { Model } & \text { Resistivity } & \text { Thickness } & \text { Elevation } \\ \text { Layer } & {[\text { ohm-m] }} & {[\mathrm{m}]} & {[\mathrm{m}]} \\ 1 & 274.500 & 20.8 & 0.0 \\ 2 & 3.500 & 1.7 & -20.8 \\ 3 & 105.900 & 96.3 & -22.5 \\ 4 & 27.460 & -- & -118.8\end{array}$

System: EM-58 Freq[Hz]: 285 Data Set Code: uh TX Cur $[A]: 2.70$ Turn off[usec] : 2.5

RX Moment[turns-m^2]: 31.4 Gain Setting: 2

$\begin{array}{rrrrrr}\text { Time } & \begin{array}{c}\text { rhoa_obs } \\ \text { [ohm-m] }\end{array} & \begin{array}{c}\text { obs_err } \\ {\left[\frac{\mathrm{o}}{0}\right]}\end{array} & \text { mask } & \begin{array}{c}\text { rhoa_cal } \\ \text { [ohm-m] }\end{array} \\ 1 & 0.00680 & 118.4 & 0.5 & \mathrm{u} & 118.2 \\ 2 & 0.00860 & 93.3 & 0.5 & \mathrm{u} & 94.0 \\ 3 & 0.01110 & 79.2 & 0.5 & \mathrm{u} & 78.8 \\ 4 & 0.01410 & 69.3 & 0.5 & \mathrm{u} & 68.8 \\ 5 & 0.01800 & 61.9 & 0.5 & \mathrm{u} & 62.0 \\ 6 & 0.02300 & 57.7 & 0.5 & \mathrm{u} & 57.7 \\ 7 & 0.02940 & 55.0 & 0.5 & \mathrm{u} & 55.2 \\ 8 & 0.03750 & 54.1 & 0.5 & \mathrm{u} & 54.2 \\ 9 & 0.04790 & 54.4 & 0.5 & \mathrm{u} & 54.5 \\ 10 & 0.06110 & 55.8 & 0.5 & \mathrm{u} & 55.8 \\ 11 & 0.07790 & 58.1 & 0.5 & \mathrm{u} & 58.0 \\ 12 & 0.09930 & 60.6 & 0.4 & \mathrm{u} & 60.8 \\ 13 & 0.12670 & 63.9 & 0.5 & \mathrm{u} & 64.1 \\ 14 & 0.16640 & 68.1 & 0.6 & \mathrm{u} & 67.8 \\ 15 & 0.20600 & 69.5 & 0.6 & \mathrm{u} & 69.8 \\ 16 & 0.26280 & 73.2 & 1.6 & \mathrm{u} & 71.3 \\ 17 & 0.33520 & 72.5 & 1.1 & \mathrm{u} & 71.3 \\ 18 & 0.42770 & 72.9 & 2.2 & \mathrm{u} & 70.7 \\ 19 & 0.54560 & 75.0 & 3.4 & \mathrm{~m} & 69.3 \\ 20 & 0.69590 & 81.6 & 7.4 & \mathrm{~m} & 68.5\end{array}$

Date: $04-M A R-09$

UTM Coord: $\mathrm{E}[\mathrm{m}]$

Elevation $[\mathrm{m}]$ :

$554067.0 \quad \mathrm{~N}[\mathrm{~m}] \quad 2807176.0$

0.0

RX location: X $[\mathrm{m}]$

$0.0 \quad \mathrm{Y}[\mathrm{m}]$

0.0
Fit Error [응 1.897

System: EM-58 Freq[Hz]: 30 Data Set Code: hi TX Cur $[A]: 2.50$ Turn Off[usec]: 2.5

RX Moment [turns-m^2]: 31.4 Gain Setting: 5

$\begin{array}{ccccc}\begin{array}{c}\text { Time } \\ \text { [ms] }\end{array} & \begin{array}{c}\text { rhoa_obs } \\ \text { [ohm-m] }\end{array} & \begin{array}{c}\text { obs_err } \\ {\left[\begin{array}{c}\overline{\%} \\ 0\end{array}\right.}\end{array} & \text { mask } & \begin{array}{c}\text { rhoa_cal } \\ \text { [ohm-m] }\end{array} \\ 0.08800 & 59.3 & 0.8 & \mathrm{u} & 59.2 \\ 0.10600 & 61.7 & 0.6 & \mathrm{u} & 61.6 \\ 0.13100 & 64.2 & 0.7 & \mathrm{u} & 64.2 \\ 0.16100 & 66.7 & 0.8 & \mathrm{u} & 66.7 \\ 0.20000 & 67.8 & 0.6 & \mathrm{u} & 68.4 \\ 0.25000 & 68.0 & 1.2 & \mathrm{u} & 69.1 \\ 0.31400 & 66.5 & 2.3 & \mathrm{u} & 68.3 \\ 0.39500 & 64.7 & 1.8 & \mathrm{u} & 66.3 \\ 0.49900 & 62.3 & 4.5 & \mathrm{u} & 63.3 \\ 0.63100 & 61.3 & 5.0 & \mathrm{u} & 60.1 \\ 0.79900 & 55.7 & 7.7 & \mathrm{u} & 56.5 \\ 1.01000 & 54.2 & 6.2 & \mathrm{u} & 53.4 \\ 1.28000 & 49.7 & 12.7 & \mathrm{u} & 50.1 \\ 1.63000 & 49.4 & 16.3 & \mathrm{~d} & - \\ 2.08000 & 36.2 & 16.3 & \mathrm{~d} & - \\ 2.64000 & 36.2 & 29.6 & \mathrm{~d} & - \\ 3.37000 & 34.6 & 47.6 & \mathrm{~d} & - \\ 4.29000 & 24.9 & 38.0 & \mathrm{~d} & - \\ 5.47000 & 18.9 & 43.3 & \mathrm{~d} & - \\ 6.97000 & 28.2 & 100.0 & \mathrm{~d} & -\end{array}$


Project: Saltwater Intrusion

County: Miami-Dade County

TX loop size: X $[\mathrm{m}] \quad 37.9 \quad \mathrm{Y}[\mathrm{m}] \quad 37.9$

Date: $04-M A R-09$

UTM Coord: $\mathrm{E}[\mathrm{m}]$

Elevation $[\mathrm{m}]$ :

$554391.0 \quad \mathrm{~N}[\mathrm{~m}] \quad 2809645.0$

0.0

RX location: X $[\mathrm{m}]$

$0.0 \quad Y[m]$

0.0

$\begin{array}{cccc}\text { Model } & \text { Resistivity } & \text { Thickness } & \text { Elevation } \\ \text { Layer } & {[\text { ohm-m] }} & {[\mathrm{m}]} & {[\mathrm{m}]} \\ 1 & 130.500 & 24.0 & 0.0 \\ 2 & 2.920 & 1.4 & -24.0 \\ 3 & 116.300 & 64.2 & -25.5 \\ 4 & 40.530 & -- & -89.7\end{array}$

System: EM-58 Freq[Hz]: 285 Data Set Code: uh TX Cur $[A]: 2.70$ Turn Off [usec]: 2.5

RX Moment[turns-m^2]: 31.4 Gain Setting: 2

\begin{tabular}{|c|c|c|c|c|}
\hline $\begin{array}{l}\text { Time } \\
{[\mathrm{ms}]}\end{array}$ & $\begin{array}{l}\text { rhoa_obs } \\
\text { [ohm-m] }\end{array}$ & $\begin{array}{c}\text { obs err } \\
{\left[\frac{\mathrm{o}}{0}\right]}\end{array}$ & mask & $\begin{array}{l}\text { rhoa_cal } \\
\text { [ohm-m] }\end{array}$ \\
\hline 0.00680 & 119.9 & 0.5 & $u$ & 118.1 \\
\hline 0.00860 & 95.1 & 0.4 & $\mathrm{u}$ & 98.0 \\
\hline 0.01110 & 82.3 & 0.5 & $\mathrm{u}$ & 82.6 \\
\hline 0.01410 & 71.9 & 0.5 & $u$ & 71.3 \\
\hline 0.01800 & 64.0 & 0.5 & u & 63.4 \\
\hline 0.02300 & 58.8 & 0.5 & u & 58.1 \\
\hline 0.02940 & 55.4 & 0.5 & u & 54.9 \\
\hline 0.03750 & 53.6 & 0.5 & u & 53.4 \\
\hline 0.04790 & 53.0 & 0.5 & u & 53.1 \\
\hline 0.06110 & 53.5 & 0.5 & u & 54.1 \\
\hline 0.07790 & 55.1 & 0.5 & $\mathrm{u}$ & 55.6 \\
\hline 0.09930 & 56.9 & 0.5 & $\mathrm{u}$ & 57.8 \\
\hline 0.12670 & 59.6 & 0.5 & u & 59.8 \\
\hline 0.16640 & 62.9 & 0.6 & u & 61.9 \\
\hline 0.20600 & 63.9 & 0.7 & u & 62.8 \\
\hline 0.26280 & 66.5 & 1.3 & u & 63.5 \\
\hline 0.33520 & 64.5 & 2.5 & u & 63.5 \\
\hline 0.4 & 63.4 & 5.2 & u & 63.6 \\
\hline & 61.9 & 5.7 & u & 63. \\
\hline 0 & & 16.5 & $\mathrm{~m}$ & 64. \\
\hline
\end{tabular}

Fit Error [응: 2.855

System: EM-58 Freq[Hz]: 30 Data Set Code: hi TX Cur $[A]: 2.50$ Turn Off[usec]: 2.5

RX Moment [turns-m^2]: 31.4 Gain Setting: 5

$\begin{array}{crrcc}\begin{array}{c}\text { Time } \\ \text { [ms }]\end{array} & \begin{array}{r}\text { rhoa_obs } \\ \text { [ohm-m] }\end{array} & \begin{array}{c}\text { obs_err } \\ {\left[\frac{\bar{o}}{0}\right]}\end{array} & \text { mask } & \begin{array}{c}\text { rhoa_cal } \\ \text { [ohm-m] }\end{array} \\ 0.08800 & 55.7 & 0.3 & \mathrm{u} & 56.6 \\ 0.10600 & 57.6 & 0.2 & \mathrm{u} & 58.2 \\ 0.13100 & 59.8 & 0.4 & \mathrm{u} & 59.8 \\ 0.16100 & 61.8 & 0.5 & \mathrm{u} & 61.1 \\ 0.20000 & 62.6 & 1.1 & \mathrm{u} & 61.8 \\ 0.25000 & 62.5 & 1.3 & \mathrm{u} & 62.0 \\ 0.31400 & 60.5 & 2.4 & \mathrm{u} & 61.3 \\ 0.39500 & 58.8 & 2.9 & \mathrm{u} & 60.3 \\ 0.49900 & 56.1 & 5.1 & \mathrm{u} & 58.8 \\ 0.63100 & 56.1 & 10.1 & \mathrm{u} & 57.3 \\ 0.79900 & 57.2 & 7.8 & \mathrm{u} & 55.8 \\ 1.01000 & 54.6 & 9.4 & \mathrm{u} & 54.2 \\ 1.28000 & 54.1 & 15.9 & \mathrm{u} & 52.9 \\ 1.63000 & 51.0 & 45.2 & \mathrm{~d} & - \\ 2.08000 & 50.4 & 92.0 & \mathrm{~d} & - \\ 2.64000 & 32.8 & 69.3 & \mathrm{~d} & - \\ 3.37000 & 29.2 & 64.3 & \mathrm{~d} & - \\ 4.29000 & 26.2 & 100.0 & \mathrm{~d} & - \\ 5.47000 & 24.3 & 82.9 & \mathrm{~d} & - \\ 6.97000 & 95.5 & 100.0 & \mathrm{~d} & -\end{array}$


Project: Saltwater Intrusion

County: Miami-Dade County

TX loop size: X[m] $37.9 \quad \mathrm{Y}[\mathrm{m}] \quad 37.9$

Date: $04-M A R-09$

UTM Coord: $\mathrm{E}[\mathrm{m}]$

Elevation $[\mathrm{m}]$ :

$554391.0 \quad \mathrm{~N}[\mathrm{~m}] \quad 2809645.0$

0.0

RX location: X $[\mathrm{m}]$

$0.0 \quad Y[m]$

0.0

$\begin{array}{cccc}\text { Model } & \text { Resistivity } & \text { Thickness } & \text { Elevation } \\ \text { Layer } & {[\text { ohm-m] }} & {[\mathrm{m}]} & {[\mathrm{m}]} \\ 1 & 132.400 & 23.8 & 0.0 \\ 2 & 3.500 & 1.6 & -23.8 \\ 3 & 112.300 & 65.7 & -25.5 \\ 4 & 40.290 & -- & -91.2\end{array}$

System: EM-58 Freq[Hz]: 285 Data Set Code: uh TX Cur $[A]: 2.70$ Turn Off [usec]: 2.5

RX Moment[turns-m^2]: 31.4 Gain Setting: 2

\begin{tabular}{|c|c|c|c|c|}
\hline $\begin{array}{l}\text { Time } \\
{[\mathrm{ms}]}\end{array}$ & $\begin{array}{l}\text { rhoa_obs } \\
\text { [ohm-m] }\end{array}$ & $\begin{array}{c}\text { obs err } \\
{\left[\overline{\frac{0}{0}}\right]}\end{array}$ & mask & $\begin{array}{l}\text { rhoa_ca] } \\
{[\text { ohm-m] }}\end{array}$ \\
\hline 0.00680 & 119.9 & 0.5 & $u$ & 118.2 \\
\hline 0.00860 & 95.1 & 0.4 & $u$ & 97.8 \\
\hline 0.01110 & 82.3 & 0.5 & u & 82.5 \\
\hline 0.01410 & 71.9 & 0.5 & u & 71 . \\
\hline 0.01800 & 64.0 & 0.5 & u & 63. \\
\hline 0.02300 & 58.8 & 0.5 & u & 58.2 \\
\hline 0.02940 & 55.4 & 0.5 & u & 55. \\
\hline 0.03750 & 53.6 & 0.5 & u & 53. \\
\hline 0.04790 & 53.0 & 0.5 & u & 53. \\
\hline 0.06110 & 53.5 & 0.5 & u & 54.1 \\
\hline 0.07790 & 55.1 & 0.5 & u & 55.7 \\
\hline 0.09930 & 56.9 & 0.5 & $\mathrm{u}$ & 57.7 \\
\hline 0.12670 & 59.6 & 0.5 & u & 59.8 \\
\hline 0.16640 & 62.9 & 0.6 & u & $61 . \varepsilon$ \\
\hline 0.20600 & 63.9 & 0.7 & u & 62 . \\
\hline 0.26280 & 66.5 & 1.3 & u & 63. \\
\hline 0.33520 & 64.5 & 2.5 & u & 53. \\
\hline 0 & 63.4 & 5.2 & u & 3. \\
\hline & 61.9 & 5.7 & u & 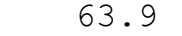 \\
\hline & & 16.5 & $\mathrm{~m}$ & \\
\hline
\end{tabular}

Fit Error [응: 2.861

System: EM-58 Freq[Hz]: 30 Data Set Code: hi TX Cur $[\mathrm{A}]: \quad 2.50$ Turn Off[usec]: 2.5

RX Moment [turns-m^2]: 31.4 Gain Setting: 5

$\begin{array}{ccrcc}\begin{array}{c}\text { Time } \\ \text { [ms] }\end{array} & \begin{array}{c}\text { rhoa_obs } \\ \text { [ohm-m] }\end{array} & \begin{array}{c}\text { obs_err } \\ {\left[\begin{array}{c}\overline{\%} \\ 0\end{array}\right.}\end{array} & \text { mask } & \begin{array}{c}\text { rhoa_cal } \\ \text { [ohm-m] }\end{array} \\ 0.08800 & 55.7 & 0.3 & \mathrm{u} & 56.6 \\ 0.10600 & 57.6 & 0.2 & \mathrm{u} & 58.2 \\ 0.13100 & 59.8 & 0.4 & \mathrm{u} & 59.8 \\ 0.16100 & 61.8 & 0.5 & \mathrm{u} & 61.0 \\ 0.20000 & 62.6 & 1.1 & \mathrm{u} & 61.9 \\ 0.25000 & 62.5 & 1.3 & \mathrm{u} & 61.9 \\ 0.31400 & 60.5 & 2.4 & \mathrm{u} & 61.4 \\ 0.39500 & 58.8 & 2.9 & \mathrm{u} & 60.3 \\ 0.49900 & 56.1 & 5.1 & \mathrm{u} & 58.9 \\ 0.63100 & 56.1 & 10.1 & \mathrm{u} & 57.3 \\ 0.79900 & 57.2 & 7.8 & \mathrm{u} & 55.8 \\ 1.01000 & 54.6 & 9.4 & \mathrm{u} & 54.1 \\ 1.28000 & 54.1 & 15.9 & \mathrm{u} & 52.9 \\ 1.63000 & 51.0 & 45.2 & \mathrm{~d} & - \\ 2.08000 & 50.4 & 92.0 & \mathrm{~d} & - \\ 2.64000 & 32.8 & 69.3 & \mathrm{~d} & - \\ 3.37000 & 29.2 & 64.3 & \mathrm{~d} & - \\ 4.29000 & 26.2 & 100.0 & \mathrm{~d} & - \\ 5.47000 & 24.3 & 82.9 & \mathrm{~d} & - \\ 6.97000 & 95.5 & 100.0 & \mathrm{~d} & -\end{array}$


Sounding: MIA237

Client: US Geological Survey Location: Hallandale High School field

Project: Saltwater Intrusion

County: Broward County

TX loop size: X $[\mathrm{m}] \quad 37.9 \quad \mathrm{Y}[\mathrm{m}] \quad 37.9$

$\begin{array}{cccc}\text { Model } & \text { Resistivity } & \text { Thickness } & \text { Elevation } \\ \text { Layer } & {[\text { ohm-m] }} & {[\mathrm{m}]} & {[\mathrm{m}]} \\ 1 & 149.800 & 11.4 & 0.0 \\ 2 & 49.210 & 37.2 & -11.4 \\ 3 & 14.060 & -- & -48.6\end{array}$

System: EM-58 Freq[Hz]: 285 Data set Code: uh TX Cur $[\mathrm{A}]: \quad 2.60$ Turn Off[usec]: 2.5

RX Moment [turns-m^2]: 31.4 Gain Setting:

\begin{tabular}{|c|c|c|c|c|c|}
\hline & $\begin{array}{l}\text { Time } \\
{[\mathrm{ms}]}\end{array}$ & $\begin{array}{l}\text { rhoa_obs } \\
\text { [ohm-m] }\end{array}$ & $\begin{array}{c}\text { obs err } \\
{\left[\frac{\mathrm{o}}{0}\right]}\end{array}$ & mask & $\begin{array}{l}\text { rhoa_cal } \\
\text { [ohm-m] }\end{array}$ \\
\hline 1 & 0.00680 & 93.5 & 0.1 & u & 93.2 \\
\hline 2 & 0.00860 & 86.8 & 0.1 & $\mathrm{u}$ & 85.7 \\
\hline 3 & 0.01110 & 80.6 & 0.2 & $u$ & 81.7 \\
\hline 4 & 0.01410 & 77.9 & 0.2 & $u$ & 79.0 \\
\hline 5 & 0.01800 & 76.3 & 0.2 & $u$ & 76.8 \\
\hline 6 & 0.02300 & 75.2 & 0.1 & $\mathrm{u}$ & 74.8 \\
\hline 7 & 0.02940 & 73.4 & 0.2 & $\mathrm{u}$ & 72.6 \\
\hline 8 & 0.03750 & 70.5 & 0.2 & $\mathrm{u}$ & 69.6 \\
\hline 9 & 0.04790 & 66.0 & 0.2 & $u$ & 65.5 \\
\hline 10 & 0.06110 & 60.4 & 0.3 & u & 60.6 \\
\hline 11 & 0.07790 & 54.7 & 0.3 & u & 55.4 \\
\hline 12 & 0.09930 & 49.0 & 0.3 & u & 50.2 \\
\hline 13 & 0.12670 & 44.7 & 0.4 & u & 45.5 \\
\hline 14 & 0.16640 & 41.3 & 0.5 & $\mathrm{u}$ & 40.9 \\
\hline 15 & 0.20600 & 38.7 & 0.6 & $\mathrm{u}$ & 37.9 \\
\hline 16 & 0.26280 & 36.5 & 0.7 & $\mathrm{u}$ & 34.9 \\
\hline 17 & 0.33520 & 34.0 & 1.1 & $u$ & 32.6 \\
\hline 18 & 0.42770 & 31.8 & 1.3 & $u$ & 30.8 \\
\hline 19 & 0.5 & 30.0 & 1.8 & $\mathrm{u}$ & 29.5 \\
\hline 20 & 0.6 & 28.6 & 3.6 & $u$ & 8.8 \\
\hline
\end{tabular}

Date: $04-M A R-09$

UTM Coord: $\mathrm{E}[\mathrm{m}]$

Elevation $[\mathrm{m}]$ :

$583733.0 \quad \mathrm{~N}[\mathrm{~m}] \quad 2875167.0$

0.0

RX location: X $[\mathrm{m}]$

$0.0 \quad Y[m]$

0.0

Fit Error [응 $\quad 3.479$

System: EM-58 Freq[Hz]: 30 Data Set Code: hi TX Cur $[A]: \quad 2.50$ Turn Off[usec]: 2.5

RX Moment [turns-m^2] : 31.4 Gain Setting: 5

Time
$[\mathrm{ms}]$
0.08800
0.10600
0.13100
0.16100
0.20000
0.25000
0.31400
0.39500
0.49900
0.63100
0.79900
1.01000
1.28000
1.63000
2.08000
2.64000
3.37000
4.29000
5.47000
6.97000

rhoa_obs
[ohm-m]
52.0
47.8
44.0
40.7
37.6
34.5
31.3
28.0
25.2
22.0
19.5
16.9
14.7
12.7
11.6
9.6
10.3
9.7
8.4
7.9

obs err mask

rhoa cal

0.6

[ohm-m]

0.4 u 44.3

0.3 u $\quad 40.6$

0.6 u $\quad 37.2$

0.9 u $\quad 34.2$

1.2 u 31.5

$\begin{array}{lll}2.1 & \text { u } & 29.2 \\ 2.3 & \text { u } & 27.2\end{array}$

2.3 u

$4.4 \mathrm{~m} \quad 25.4$

4.3 $\mathrm{m} \quad 24.0$

4. 6 m 22.7

$9.6 \quad \mathrm{~m} \quad 21.6$

20.7

$-$

$-$ 
Sounding: MIA238

Client: US Geological Survey Location: South of Hallandale Cemetery

Project: Saltwater Intrusion

County: Broward County

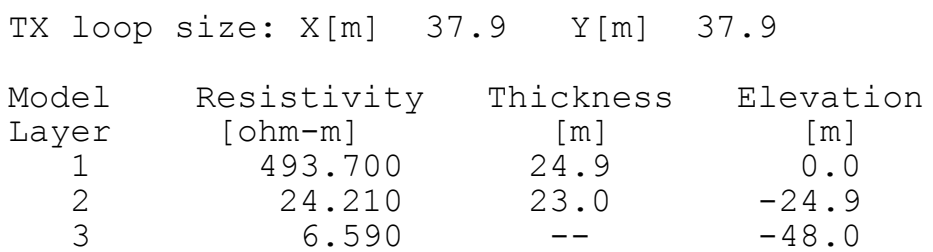

System: EM-58 Freq[Hz]: 285 Data Set Code: uh TX Cur $[\mathrm{A}]: \quad 2.50$ Turn Off[usec]: 2.5

RX Moment[turns-m^2]: 31.4 Gain Setting: 2

$\begin{array}{rrrrrr}\text { Time } & \begin{array}{c}\text { rhoa_obs } \\ \text { [ohm-m] }\end{array} & \begin{array}{c}\text { obs_err } \\ {\left[\frac{\mathrm{o}}{0}\right]}\end{array} & \text { mask } & \begin{array}{c}\text { rhoa_cal } \\ \text { [ohm-m] }\end{array} \\ 1 & 0.00680 & 232.1 & 0.3 & \mathrm{u} & 221.7 \\ 2 & 0.00860 & 169.0 & 0.2 & \mathrm{u} & 183.4 \\ 3 & 0.01110 & 164.2 & 0.4 & \mathrm{u} & 157.2 \\ 4 & 0.01410 & 134.3 & 0.2 & \mathrm{u} & 137.8 \\ 5 & 0.01800 & 122.6 & 0.2 & \mathrm{u} & 122.2 \\ 6 & 0.02300 & 112.8 & 0.2 & \mathrm{u} & 108.9 \\ 7 & 0.02940 & 98.4 & 0.2 & \mathrm{u} & 96.9 \\ 8 & 0.03750 & 82.6 & 0.2 & \mathrm{u} & 85.4 \\ 9 & 0.04790 & 74.2 & 0.2 & \mathrm{u} & 74.3 \\ 10 & 0.06110 & 62.7 & 0.2 & \mathrm{u} & 64.0 \\ 11 & 0.07790 & 54.1 & 0.4 & \mathrm{u} & 54.8 \\ 12 & 0.09930 & 48.2 & 0.3 & \mathrm{u} & 46.9 \\ 13 & 0.12670 & 38.9 & 0.3 & \mathrm{u} & 40.3 \\ 14 & 0.16640 & 33.3 & 0.6 & \mathrm{u} & 34.3 \\ 15 & 0.20600 & 31.7 & 0.7 & \mathrm{u} & 30.5 \\ 16 & 0.26280 & 28.2 & 1.1 & \mathrm{u} & 27.0 \\ 17 & 0.33520 & 25.0 & 1.1 & \mathrm{u} & 24.2 \\ 18 & 0.42770 & 22.7 & 2.0 & \mathrm{u} & 22.0 \\ 19 & 0.54560 & 20.0 & 3.5 & \mathrm{u} & 20.4 \\ 20 & 0.69590 & 18.3 & 4.6 & \mathrm{u} & 19.3\end{array}$

Date: $04-M A R-09$

UTM Coord: $\mathrm{E}[\mathrm{m}]$

Elevation $[\mathrm{m}]$ :

$584560.0 \quad \mathrm{~N}[\mathrm{~m}]$

2875121.0

RX location: $\mathrm{X}[\mathrm{m}]$

$0.0 \quad Y[m]$

0.0

Fit Error [응: 5.562 
Sounding: MIA239

Client: US Geological Survey

Location: Hallandale Cemetery NE

Project: Saltwater Int rusion

County: Broward County

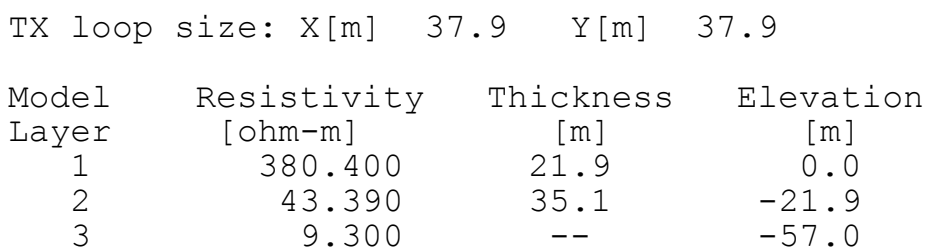

System: EM-58 Freq[Hz]:285 Data set Code: uh TX Cur $[A]: 2.50$ Turn Off[usec]: 2.5

RX Moment[turns-m^2]: 31.4 Gain Setting: 2

\begin{tabular}{|c|c|c|c|c|c|}
\hline & $\begin{array}{l}\text { Time } \\
{[\mathrm{ms}]}\end{array}$ & $\begin{array}{l}\text { rhoa_obs } \\
\text { [ohm-m] }\end{array}$ & $\begin{array}{c}\text { obs err } \\
{\left[\frac{\mathrm{o}}{0}\right]}\end{array}$ & mask & $\begin{array}{l}\text { rhoa_cal } \\
{[\mathrm{ohm}-\mathrm{m}]}\end{array}$ \\
\hline 1 & 0.00680 & 194.4 & 0.7 & u & 190.0 \\
\hline 2 & 0.00860 & 153.7 & 0.3 & $\mathrm{u}$ & 164.0 \\
\hline 3 & 0.01110 & 148.0 & 0.5 & $u$ & 147.1 \\
\hline 4 & 0.01410 & 138.1 & 0.4 & $u$ & 134.3 \\
\hline 5 & 0.01800 & 125.4 & 0.5 & $u$ & 124.3 \\
\hline 6 & 0.02300 & 118.0 & 0.4 & $\mathrm{u}$ & 116.2 \\
\hline 7 & 0.02940 & 109.4 & 0.4 & $\mathrm{u}$ & 108.9 \\
\hline 8 & 0.03750 & 100.0 & 0.4 & $\mathrm{u}$ & 100.9 \\
\hline 9 & 0.04790 & 89.3 & 0.4 & $u$ & 91.9 \\
\hline 10 & 0.06110 & 79.1 & 0.4 & u & 82.1 \\
\hline 11 & 0.07790 & 71.0 & 0.3 & $u$ & 72.1 \\
\hline 12 & 0.09930 & 63.4 & 0.3 & $u$ & 62.8 \\
\hline 13 & 0.12670 & 54.6 & 0.4 & $u$ & 54.7 \\
\hline 14 & 0.16640 & 45.5 & 0.5 & $\mathrm{u}$ & 47.0 \\
\hline 15 & 0.20600 & 42.9 & 0.4 & $\mathrm{u}$ & 42.0 \\
\hline 16 & 0.26280 & 39.6 & 0.7 & $\mathrm{u}$ & 37.3 \\
\hline 17 & 0.33520 & 35.9 & 0.8 & $u$ & 33.6 \\
\hline 18 & 0.42770 & 32.5 & 1.5 & $u$ & 30.7 \\
\hline 19 & 0.5 & 29.1 & 1.7 & $\mathrm{u}$ & 28.5 \\
\hline 20 & 0.6 & 26.1 & 2.2 & $u$ & 26.9 \\
\hline
\end{tabular}

Date: $04-\mathrm{MAR}-09$

UTM Coord: $\mathrm{E}[\mathrm{m}]$

Elevation $[\mathrm{m}]$ :

$584546.0 \quad \mathrm{~N}[\mathrm{~m}] \quad 2875285.0$

0.0

RX location: X[m]

$0.0 \quad Y[m]$

0.0

Fit Error [응 : 5.629

System: EM-58 Freq[Hz]: 30 Data Set Code: hi TX Cur $[A]: \quad 2.50$ Turn Off[usec]: 2.5

RX Moment [turns-m^2] : 31.4 Gain Setting: 5

Time
$[\mathrm{ms}]$
0.08800
0.10600
0.13100
0.16100
0.20000
0.25000
0.31400
0.39500
0.49900
0.63100
0.79900
1.01000
1.28000
1.63000
2.08000
2.64000
3.37000
4.29000
5.47000
6.97000

rhoa_obs
[ohm-m]
68.2
61.5
52.7
44.9
40.5
36.1
30.7
26.2
21.6
17.6
14.1
11.2
9.0
7.2
6.2
5.4
4.7
3.9
3.5
3.1

obs err mask

rhoa_cal

[\%]

0.5
0.8

0.8

1.1

1.6

1. 7

2.5

2. 2

3.6
5.7

6. 9

11.4

11.1

9.6

11.4

13.7

14.9

45.5 
Sounding: MIA239X

Client: US Geological Survey

Location: Hallandale Cemetery NE

Project: Saltwater Intrusion

County: Broward County

TX loop size: X $[\mathrm{m}] \quad 37.9 \quad \mathrm{Y}[\mathrm{m}] \quad 37.9$

$\begin{array}{cccc}\text { Model } & \text { Resistivity } & \text { Thickness } & \text { Elevation } \\ \text { Layer } & {[\text { ohm-m] }} & {[\mathrm{m}]} & {[\mathrm{m}]} \\ 1 & 439.200 & 22.2 & 0.0 \\ 2 & 34.400 & 31.0 & -22.2 \\ 3 & 3.130 & 20.0 & -53.2 \\ 4 & .431 & -- & -73.3\end{array}$

System: EM-58 Freq[Hz]: 285 Data Set Code: uh TX Cur[A]: 2.50 Turn Off[usec]: 2.5

RX Moment [turns-m^2]: 31.4 Gain Setting: 2

$\begin{array}{rrrrrr}\text { Time } & \begin{array}{c}\text { rhoa_obs } \\ \text { [ohm-m] }\end{array} & \begin{array}{c}\text { obs_err } \\ {\left[\frac{\mathrm{o}}{0}\right]}\end{array} & \text { mask } & \begin{array}{c}\text { rhoa_cal } \\ \text { [ohm-m] }\end{array} \\ 1 & 0.00680 & 194.4 & 0.7 & \mathrm{u} & 193.3 \\ 2 & 0.00860 & 153.7 & 0.3 & \mathrm{u} & 164.0 \\ 3 & 0.01110 & 148.0 & 0.5 & \mathrm{u} & 145.1 \\ 4 & 0.01410 & 138.1 & 0.4 & \mathrm{u} & 131.9 \\ 5 & 0.01800 & 125.4 & 0.5 & \mathrm{u} & 122.5 \\ 6 & 0.02300 & 118.0 & 0.4 & \mathrm{u} & 116.0 \\ 7 & 0.02940 & 109.4 & 0.4 & \mathrm{u} & 110.3 \\ 8 & 0.03750 & 100.0 & 0.4 & \mathrm{u} & 103.2 \\ 9 & 0.04790 & 89.3 & 0.4 & \mathrm{u} & 93.8 \\ 10 & 0.06110 & 79.1 & 0.4 & \mathrm{u} & 82.5 \\ 11 & 0.07790 & 71.0 & 0.3 & \mathrm{u} & 71.3 \\ 12 & 0.09930 & 63.4 & 0.3 & \mathrm{u} & 61.8 \\ 13 & 0.12670 & 54.6 & 0.4 & \mathrm{u} & 54.4 \\ 14 & 0.16640 & 45.5 & 0.5 & \mathrm{u} & 48.2 \\ 15 & 0.20600 & 42.9 & 0.4 & \mathrm{u} & 44.3 \\ 16 & 0.26280 & 39.6 & 0.7 & \mathrm{u} & 40.1 \\ 17 & 0.33520 & 35.9 & 0.8 & \mathrm{u} & 36.0 \\ 18 & 0.42770 & 32.5 & 1.5 & \mathrm{u} & 31.8 \\ 19 & 0.54560 & 29.1 & 1.7 & \mathrm{u} & 27.7 \\ 20 & 0.69590 & 26.1 & 2.2 & \mathrm{u} & 24.1\end{array}$

Date: $04-M A R-09$

UTM Coord: $\mathrm{E}[\mathrm{m}]$

Elevation $[\mathrm{m}]$ :

$584546.0 \quad \mathrm{~N}[\mathrm{~m}] \quad 2875285.0$

0.0

RX location: X $[\mathrm{m}]$

$0.0 \quad Y[m]$

0.0
Fit Error [\%]: 5.593

System: EM-58 Freq[Hz]: 30 Data Set Code: hi TX Cur $[A]: 2.50$ Turn Off[usec]: 2.5

RX Moment [turns-m^2] : 31.4 Gain Setting: 5

$\begin{array}{crrcr}\begin{array}{c}\text { Time } \\ \text { [ms] }\end{array} & \begin{array}{c}\text { rhoa_obs } \\ \text { [ohm-m] }\end{array} & \begin{array}{c}\text { obs_err } \\ {\left[\begin{array}{c}\bar{o} \\ \mathrm{o}\end{array}\right.}\end{array} & \text { mask } & \begin{array}{c}\text { rhoa_cal } \\ \text { [ohm-m] }\end{array} \\ 0.08800 & 68.2 & 0.5 & \mathrm{u} & 64.6 \\ 0.10600 & 61.5 & 0.8 & \mathrm{u} & 57.5 \\ 0.13100 & 52.7 & 0.8 & \mathrm{u} & 51.1 \\ 0.16100 & 44.9 & 1.1 & \mathrm{u} & 45.8 \\ 0.20000 & 40.5 & 1.3 & \mathrm{u} & 40.9 \\ 0.25000 & 36.1 & 1.6 & \mathrm{u} & 36.1 \\ 0.31400 & 30.7 & 1.7 & \mathrm{u} & 31.2 \\ 0.39500 & 26.2 & 2.5 & \mathrm{u} & 26.3 \\ 0.49900 & 21.6 & 2.9 & \mathrm{u} & 21.7 \\ 0.63100 & 17.6 & 2.2 & \mathrm{u} & 17.7 \\ 0.79900 & 14.1 & 3.6 & \mathrm{u} & 14.3 \\ 1.01000 & 11.2 & 5.7 & \mathrm{u} & 11.6 \\ 1.28000 & 9.0 & 6.9 & \mathrm{u} & 9.4 \\ 1.63000 & 7.2 & 11.4 & \mathrm{u} & 7.7 \\ 2.08000 & 6.2 & 11.1 & \mathrm{u} & 6.3 \\ 2.64000 & 5.4 & 9.6 & \mathrm{u} & 5.3 \\ 3.37000 & 4.7 & 11.4 & \mathrm{u} & 4.5 \\ 4.29000 & 3.9 & 13.7 & \mathrm{~d} & - \\ 5.47000 & 3.5 & 14.9 & \mathrm{~d} & - \\ 6.97000 & 3.1 & 45.5 & \mathrm{~d} & -\end{array}$


Sounding: MIA240A

Client: US Geological Survey Location: Hallandale Cemetery SE \#16919

Project: Saltwater Intrusion

County: Broward County

TX loop size: X $[\mathrm{m}] \quad 37.9 \quad \mathrm{Y}[\mathrm{m}] \quad 37.9$

$\begin{array}{cccc}\text { Model } & \text { Resistivity } & \text { Thickness } & \text { Elevation } \\ \text { Layer } & {[\text { ohm }-\mathrm{m}]} & {[\mathrm{m}]} & {[\mathrm{m}]} \\ 1 & 189.400 & 72.9 & 0.0 \\ 2 & 28.690 & -- & -72.9\end{array}$

System: EM-58 Freq[Hz]: 285 Data Set Code: uh TX $\operatorname{Cur}[A]: 2.50$ Turn Off[usec]: 2.5 RX Moment [turns-m^2]: 31.4 Gain Setting:

\begin{tabular}{|c|c|c|c|c|}
\hline $\begin{array}{l}\text { Time } \\
{[\mathrm{ms}]}\end{array}$ & $\begin{array}{l}\text { rhoa obs } \\
\text { [ohm-m] }\end{array}$ & $\begin{array}{c}\text { obs err } \\
{\left[\frac{\overline{0}}{0}\right]}\end{array}$ & mask & $\begin{array}{l}\text { rhoa_cal } \\
\text { [ohm-m] }\end{array}$ \\
\hline 0.00680 & 172.2 & 0.0 & $\mathrm{~m}$ & 168.8 \\
\hline 0.00860 & 153.5 & 0.0 & $u$ & 170.3 \\
\hline 0.01110 & 177.0 & 0.0 & $u$ & 178.2 \\
\hline 0.01410 & 199.0 & 0.0 & u & 187.2 \\
\hline 0.01800 & 204.1 & 0.0 & $\mathrm{u}$ & 194.7 \\
\hline 0.02300 & 205.5 & 0.0 & $\mathrm{u}$ & 199.6 \\
\hline 0.02940 & 191.9 & 0.0 & $\mathrm{u}$ & 198.5 \\
\hline 0.03750 & 184.0 & 0.0 & $u$ & 190.4 \\
\hline 0.04790 & 174.4 & 0.0 & $u$ & 176.3 \\
\hline 0.06110 & 167.2 & 0.0 & $\mathrm{u}$ & 159.0 \\
\hline 0.07790 & 161.0 & 0.0 & $\mathrm{~m}$ & 141.7 \\
\hline 0.09930 & 164.7 & 0.0 & $\mathrm{~m}$ & 125.5 \\
\hline 0.12670 & 193.5 & 0.0 & $\mathrm{~m}$ & 111.6 \\
\hline 0.16640 & 320.2 & 0.0 & $\mathrm{~m}$ & 98.3 \\
\hline 0.20600 & 334.8 & 0.0 & $\mathrm{~m}$ & 89.9 \\
\hline 0.26280 & 132.2 & 0.0 & $\mathrm{~m}$ & 81.8 \\
\hline 0.33520 & 95.1 & 0.0 & $\mathrm{~m}$ & 75.4 \\
\hline 0.42770 & 63.6 & 0.0 & $\mathrm{~m}$ & 70.5 \\
\hline 0.54560 & 51.8 & 0.0 & $\mathrm{~m}$ & 67.0 \\
\hline 0.69590 & 36.9 & 0.0 & $\mathrm{~m}$ & 64.6 \\
\hline
\end{tabular}

Date: $04-M A R-09$

UTM Coord: $\mathrm{E}[\mathrm{m}]$

Elevation $[\mathrm{m}]$ :

$584541.0 \quad \mathrm{~N}[\mathrm{~m}] \quad 2875221.0$

0.0

RX location: $\mathrm{X}[\mathrm{m}]$

$0.0 \quad Y[m]$

0.0

Fit Error $\left[\frac{\circ}{0}\right]: 7.796$ 
Sounding: MIA240B

Client: US Geological Survey Location: Hallandale Cemetery SE \#16919

Project: Saltwater Intrusion

County: Broward County

TX loop size: X $[\mathrm{m}] \quad 37.9 \quad \mathrm{Y}[\mathrm{m}] \quad 37.9$

$\begin{array}{cccc}\text { Model } & \text { Resistivity } & \text { Thickness } & \text { Elevation } \\ \text { Layer } & {[\text { ohm }-\mathrm{m}]} & {[\mathrm{m}]} & {[\mathrm{m}]} \\ 1 & 146.800 & 81.2 & 0.0 \\ 2 & 61.530 & -- & -81.2\end{array}$

System: EM-58 Freq[Hz]: 285 Data set Code: uh TX Cur $[A]: 2.50$ Turn Off[usec] : 2.5 RX Moment [turns-m^2]: $31.4 \quad$ Gain Setting:

$\begin{array}{rrrrrr}\text { Time } & \begin{array}{c}\text { rhoa_obs } \\ {[\text { ohm-m] }}\end{array} & \begin{array}{c}\text { obserr } \\ {\left[\frac{\mathrm{o}}{0}\right]}\end{array} & \text { mask } & \begin{array}{c}\text { rhoa_cal } \\ \text { [ohm-m] } \\ 132.6\end{array} \\ 1 & 0.00680 & 172.2 & 0.0 & \mathrm{~m} & 132.2 \\ 2 & 0.00860 & 153.5 & 0.0 & \mathrm{u} & 135.8 \\ 3 & 0.01110 & 177.0 & 0.0 & \mathrm{u} & 139.5 \\ 4 & 0.01410 & 199.0 & 0.0 & \mathrm{u} & 142.1 \\ 5 & 0.01800 & 204.1 & 0.0 & \mathrm{u} & 144.7 \\ 6 & 0.02300 & 205.5 & 0.0 & \mathrm{u} & 146.7 \\ 7 & 0.02940 & 191.9 & 0.0 & \mathrm{u} & 147.4 \\ 8 & 0.03750 & 184.0 & 0.0 & \mathrm{u} & 146.2 \\ 9 & 0.04790 & 174.4 & 0.0 & \mathrm{u} & 143.0 \\ 10 & 0.06110 & 167.2 & 0.0 & \mathrm{u} & 138.0 \\ 11 & 0.07790 & 161.0 & 0.0 & \mathrm{u} & 131.9 \\ 12 & 0.09930 & 164.7 & 0.0 & \mathrm{u} & 125.5 \\ 13 & 0.12670 & 193.5 & 0.0 & \mathrm{u} & 118.3 \\ 14 & 0.16640 & 320.2 & 0.0 & \mathrm{u} & 113.4 \\ 15 & 0.20600 & 334.8 & 0.0 & \mathrm{~m} & 108.3 \\ 16 & 0.26280 & 132.2 & 0.0 & \mathrm{~m} & 104.3 \\ 17 & 0.33520 & 95.1 & 0.0 & \mathrm{~m} & 101.4 \\ 18 & 0.42770 & 63.6 & 0.0 & \mathrm{~m} & 99.9 \\ 19 & 0.54560 & 51.8 & 0.0 & \mathrm{~m} & 99.5 \\ 20 & 0.69590 & 36.9 & 0.0 & \mathrm{~m} & 9\end{array}$

Date: $04-M A R-09$

UTM Coord: $\mathrm{E}[\mathrm{m}]$

Elevation $[\mathrm{m}]$ :

$584541.0 \quad \mathrm{~N}[\mathrm{~m}] \quad 2875221.0$

0.0

RX location: $\mathrm{X}[\mathrm{m}]$

$0.0 \quad Y[m]$

0.0

Fit Error $\left[\frac{\circ}{0}\right]: 7.074$ 
Sounding: MIA240C

Client: US Geological Survey

Location: Hallandale Cemetery SE combo

Project: Saltwater Intrusion

County: Broward County

TX loop size: X $[\mathrm{m}] \quad 37.9 \quad \mathrm{Y}[\mathrm{m}] \quad 37.9$

$\begin{array}{cccc}\text { Model } & \text { Resistivity } & \text { Thickness } & \text { Elevation } \\ \text { Layer } & {[\text { ohm }-\mathrm{m}]} & {[\mathrm{m}]} & {[\mathrm{m}]} \\ 1 & 168.200 & 67.3 & 0.0 \\ 2 & 96.420 & -- & -67.3\end{array}$

System: EM-58 Freq[Hz]: 285 Data Set Code: uh TX $\operatorname{Cur}[A]: 2.50$ Turn Off [usec] : 2.5 RX Moment [turns-m^2]: $31.4 \quad$ Gain Setting:

\begin{tabular}{|c|c|c|c|c|}
\hline $\begin{array}{l}\text { Time } \\
{[\mathrm{ms}]}\end{array}$ & $\begin{array}{l}\text { rhoa_obs } \\
\text { [ohm-m] }\end{array}$ & $\begin{array}{c}\text { obs err } \\
{\left[\frac{\overline{0}}{0}\right]}\end{array}$ & mask & $\begin{array}{l}\text { rhoa_cal } \\
\text { [ohm-m] }\end{array}$ \\
\hline 0.00680 & 172.2 & 0.0 & $\mathrm{~m}$ & 150.6 \\
\hline 0.00860 & 153.5 & 0.0 & $u$ & 150.9 \\
\hline 0.01110 & 177.0 & 0.0 & $u$ & 155.7 \\
\hline 0.01410 & 199.0 & 0.0 & $u$ & 160.3 \\
\hline 0.01800 & 204.1 & 0.0 & u & 163.0 \\
\hline 0.02300 & 205.5 & 0.0 & $u$ & 164.4 \\
\hline 0.02940 & 191.9 & 0.0 & $u$ & 164.2 \\
\hline 0.03750 & 184.0 & 0.0 & $u$ & 162.3 \\
\hline 0.04790 & 174.4 & 0.0 & $u$ & 158.9 \\
\hline 0.06110 & 167.2 & 0.0 & $u$ & 154.6 \\
\hline 0.07790 & 161.0 & 0.0 & $\mathrm{u}$ & 149.8 \\
\hline 0.09930 & 164.7 & 0.0 & $\mathrm{u}$ & 144.8 \\
\hline 0.12670 & 193.5 & 0.0 & $\mathrm{u}$ & 140.2 \\
\hline 0.16640 & 320.2 & 0.0 & $\mathrm{~m}$ & 135.4 \\
\hline 0.20600 & 334.8 & 0.0 & $\mathrm{~m}$ & 132.3 \\
\hline 0.26280 & 132.2 & 0.0 & $\mathrm{~m}$ & 129.3 \\
\hline 0.33520 & 95.1 & 0.0 & $\mathrm{~m}$ & 127.4 \\
\hline 0.4 & 63.6 & 0.0 & $\mathrm{~m}$ & 126.4 \\
\hline 0.54560 & 51.8 & 0.0 & $\mathrm{~m}$ & 126.9 \\
\hline 0.69590 & 36.9 & 0.0 & $\mathrm{~m}$ & 128.8 \\
\hline
\end{tabular}

Date: $04-M A R-09$

UTM Coord: $\mathrm{E}[\mathrm{m}]$

Elevation $[\mathrm{m}]$ :

$584541.0 \quad \mathrm{~N}[\mathrm{~m}] \quad 2875221.0$

0.0

RX location: $\mathrm{X}[\mathrm{m}]$

$0.0 \quad Y[m]$

0.0

Fit Error $\left[\frac{\circ}{0}\right]: 4.238$ 
Sounding: MIA241

Client: US Geological Survey Location: Hallandale water Plant west

Project: Saltwater Intrusion

County: Broward County

TX loop size: X $[\mathrm{m}] \quad 37.9 \quad \mathrm{Y}[\mathrm{m}] \quad 37.9$

$\begin{array}{cccc}\text { Model } & \text { Resistivity } & \text { Thickness } & \text { Elevation } \\ \text { Layer } & {[\text { ohm-m] }} & {[\mathrm{m}]} & {[\mathrm{m}]} \\ 1 & 740.700 & 14.4 & 0.0 \\ 2 & 71.100 & 50.6 & -14.4 \\ 3 & 5.480 & 23.0 & -65.1 \\ 4 & .789 & -- & -88.2\end{array}$

System: EM-58 Freq[Hz]: 285 Data Set Code: uh TX Cur[A]: 2.50 Turn Off[usec]: 2.5

RX Moment[turns-m^2]: 31.4 Gain Setting: 2

\begin{tabular}{|c|c|c|c|c|}
\hline $\begin{array}{l}\text { Time } \\
{[\mathrm{ms}]}\end{array}$ & $\begin{array}{l}\text { rhoa_obs } \\
\text { [ohm-m] }\end{array}$ & $\begin{array}{c}\text { obs err } \\
{\left[\frac{\overline{0}}{0}\right]}\end{array}$ & mask & $\begin{array}{l}\text { rhoa_ca] } \\
\text { [ohm-m] }\end{array}$ \\
\hline 0.00680 & 170.6 & 0.6 & u & 166.1 \\
\hline 0.00860 & 142.1 & 0.6 & u & 149.4 \\
\hline 0.01110 & 140.4 & 0.7 & $\mathrm{u}$ & 140.5 \\
\hline 0.01410 & 137.5 & 0.7 & u & 134.7 \\
\hline 0.01800 & 133.2 & 0.8 & u & 131.4 \\
\hline 0.02300 & 132.8 & 0.7 & u & 130. \\
\hline 0.02940 & 130.4 & 0.6 & u & 130. \\
\hline 0.03750 & 126.6 & 0.7 & u & 128. \\
\hline 0.04790 & 119.7 & 0.7 & $\mathrm{u}$ & 122. \\
\hline 0.06110 & 110.8 & 0.7 & u & 113. \\
\hline 0.07790 & 102.2 & 0.8 & $\mathrm{u}$ & 102 . \\
\hline 0.09930 & 92.0 & 0.8 & u & 92. \\
\hline 0.12670 & 82.9 & 0.9 & $\mathrm{u}$ & 83. \\
\hline 0.16640 & 74.3 & 0.9 & $u$ & 75 . \\
\hline 0.20600 & 67.0 & 1.3 & u & 69. \\
\hline 0.26280 & 61.6 & 1.7 & u & 61. \\
\hline 0.33520 & 54.1 & 2.3 & $u$ & 54. \\
\hline 0.42770 & 48.8 & 4.3 & $u$ & 46 \\
\hline 0.54560 & 42.3 & 6.9 & $u$ & 0 \\
\hline 0.6 & 35.9 & 9.3 & u & \\
\hline
\end{tabular}

Date: $04-M A R-09$

UTM Coord: $\mathrm{E}[\mathrm{m}]$

Elevation $[\mathrm{m}]$ :

$584295.0 \quad \mathrm{~N}[\mathrm{~m}] \quad 2874643.0$

0.0

RX location: X $[\mathrm{m}]$

$0.0 \quad Y[m]$

0.0

Fit Error [으: 3.997

System: EM-58 Freq[Hz]: 30 Data Set Code: hi TX Cur $[A]: 2.50$ Turn Off[usec]: 2.5

RX Moment [turns-m^2] : 31.4 Gain Setting: 5

$\begin{array}{crrcr}\begin{array}{c}\text { Time } \\ \text { [ms] }\end{array} & \begin{array}{c}\text { rhoa_obs } \\ \text { [ohm-m] }\end{array} & \begin{array}{c}\text { obs_err } \\ {\left[\begin{array}{c}\overline{\%} \\ 0\end{array}\right.}\end{array} & \text { mask } & \begin{array}{r}\text { rhoa_cal } \\ \text { [ohm-m] }\end{array} \\ 0.08800 & 98.5 & 0.4 & \mathrm{u} & 94.8 \\ 0.10600 & 90.0 & 0.8 & \mathrm{u} & 86.6 \\ 0.13100 & 81.4 & 1.0 & \mathrm{u} & 78.7 \\ 0.16100 & 72.3 & 1.5 & \mathrm{u} & 71.1 \\ 0.20000 & 62.9 & 2.3 & \mathrm{u} & 63.4 \\ 0.25000 & 53.9 & 3.4 & \mathrm{u} & 55.2 \\ 0.31400 & 45.5 & 3.3 & \mathrm{u} & 46.8 \\ 0.39500 & 38.1 & 3.3 & \mathrm{u} & 38.8 \\ 0.49900 & 31.2 & 3.3 & \mathrm{u} & 31.7 \\ 0.63100 & 24.8 & 7.2 & \mathrm{u} & 25.7 \\ 0.79900 & 20.2 & 8.2 & \mathrm{u} & 20.7 \\ 1.01000 & 16.2 & 12.4 & \mathrm{~m} & 16.8 \\ 1.28000 & 12.6 & 13.5 & \mathrm{~m} & 13.7 \\ 1.63000 & 9.9 & 17.1 & \mathrm{~m} & 11.2 \\ 2.08000 & 8.5 & 17.2 & \mathrm{~m} & 9.3 \\ 2.64000 & 7.1 & 24.6 & \mathrm{~m} & 7.8 \\ 3.37000 & 5.6 & 33.0 & \mathrm{~m} & 6.7 \\ 4.29000 & 4.4 & 60.4 & \mathrm{~m} & 5.8 \\ 5.47000 & 4.9 & 60.4 & \mathrm{~m} & 5.1 \\ 6.97000 & 3.9 & 71.2 & \mathrm{~d} & -\end{array}$


Sounding: MIA241X

Client: US Geological Survey Location: Hallandale water Plant west

Project: Saltwater Intrusion

County: Broward County

TX loop size: X $[\mathrm{m}] \quad 37.9 \quad \mathrm{Y}[\mathrm{m}] \quad 37.9$

$\begin{array}{cccc}\text { Model } & \text { Resistivity } & \text { Thickness } & \text { Elevation } \\ \text { Layer } & {[\text { ohm-m] }} & {[\mathrm{m}]} & {[\mathrm{m}]} \\ 1 & 775.600 & 15.1 & 0.0 \\ 2 & 65.600 & 48.4 & -15.1 \\ 3 & 3.530 & 19.2 & -63.6 \\ 4 & .374 & -- & -82.8\end{array}$

System: EM-58 Freq[Hz]: 285 Data Set Code: uh TX Cur[A]: 2.50 Turn Off[usec]: 2.5

RX Moment[turns-m^2]: 31.4 Gain Setting: 2

$\begin{array}{rrrrrr}\text { Time } & \begin{array}{c}\text { rhoa_obs } \\ \text { [ohm-m] }\end{array} & \begin{array}{c}\text { obs_err } \\ {\left[\frac{\mathrm{o}}{0}\right]}\end{array} & \text { mask } & \begin{array}{c}\text { rhoa_cal } \\ \text { [ohm-m] }\end{array} \\ 1 & 0.00680 & 170.6 & 0.6 & \mathrm{u} & 167.5 \\ 2 & 0.00860 & 142.1 & 0.6 & \mathrm{u} & 149.5 \\ 3 & 0.01110 & 140.4 & 0.7 & \mathrm{u} & 139.7 \\ 4 & 0.01410 & 137.5 & 0.7 & \mathrm{u} & 133.4 \\ 5 & 0.01800 & 133.2 & 0.8 & \mathrm{u} & 130.1 \\ 6 & 0.02300 & 132.8 & 0.7 & \mathrm{u} & 129.6 \\ 7 & 0.02940 & 130.4 & 0.6 & \mathrm{u} & 130.4 \\ 8 & 0.03750 & 126.6 & 0.7 & \mathrm{u} & 129.8 \\ 9 & 0.04790 & 119.7 & 0.7 & \mathrm{u} & 124.8 \\ 10 & 0.06110 & 110.8 & 0.7 & \mathrm{u} & 115.0 \\ 11 & 0.07790 & 102.2 & 0.8 & \mathrm{u} & 103.1 \\ 12 & 0.09930 & 92.0 & 0.8 & \mathrm{u} & 92.1 \\ 13 & 0.12670 & 82.9 & 0.9 & \mathrm{u} & 83.1 \\ 14 & 0.16640 & 74.3 & 0.9 & \mathrm{u} & 75.2 \\ 15 & 0.20600 & 67.0 & 1.3 & \mathrm{u} & 69.6 \\ 16 & 0.26280 & 61.6 & 1.7 & \mathrm{u} & 62.8 \\ 17 & 0.33520 & 54.1 & 2.3 & \mathrm{u} & 55.6 \\ 18 & 0.42770 & 48.8 & 4.3 & \mathrm{u} & 48.2 \\ 19 & 0.54560 & 42.3 & 6.9 & \mathrm{u} & 41.2 \\ 20 & 0.69590 & 35.9 & 9.3 & \mathrm{u} & 35.2\end{array}$

Date: $04-M A R-09$

UTM Coord: $\mathrm{E}[\mathrm{m}]$

Elevation $[\mathrm{m}]$ :

$584295.0 \quad \mathrm{~N}[\mathrm{~m}] \quad 2874643.0$

0.0

RX location: X $[\mathrm{m}]$

$0.0 \quad Y[m]$

0.0
Fit Error $[\%]: 4.531$

System: EM-58 Freq[Hz]: 30 Data Set Code: hi TX Cur $[A]: 2.50$ Turn Off[usec]: 2.5

RX Moment [turns-m^2] : 31.4 Gain Setting: 5

\begin{tabular}{|c|c|c|c|c|}
\hline $\begin{array}{l}\text { Time } \\
{[\mathrm{ms}]}\end{array}$ & $\begin{array}{l}\text { rhoa_obs } \\
\text { [ohm-m] }\end{array}$ & $\begin{array}{c}\text { obs err } \\
{\left[\frac{\mathrm{o}}{0}\right]}\end{array}$ & mask & $\begin{array}{l}\text { rhoa_cal } \\
\text { [ohm-m] }\end{array}$ \\
\hline 0.08800 & 98.5 & 0.4 & $u$ & 94.6 \\
\hline 0.10600 & 90.0 & 0.8 & $u$ & 85.9 \\
\hline 0.13100 & 81.4 & 1.0 & $u$ & 77.7 \\
\hline 0.16100 & 72.3 & 1.5 & $\mathrm{u}$ & 70.3 \\
\hline 0.20000 & 62.9 & 2.3 & u & 63.0 \\
\hline 0.25000 & 53.9 & 3.4 & $\mathrm{u}$ & 55.1 \\
\hline 0.31400 & 45.5 & 3.3 & $\mathrm{u}$ & 46.7 \\
\hline 0.39500 & 38.1 & 3.3 & $\mathrm{u}$ & 38.7 \\
\hline 0.49900 & 31.2 & 3.3 & u & 31.2 \\
\hline 0.63100 & 24.8 & 7.2 & u & 25.0 \\
\hline 0.79900 & 20.2 & 8.2 & u & 19.9 \\
\hline 1.01000 & 16.2 & 12.4 & u & 15.8 \\
\hline 1.28000 & 12.6 & 13.5 & u & 12.7 \\
\hline 1.63000 & 9.9 & 17.1 & u & 10.2 \\
\hline 00 & 8.5 & 17.2 & u & 8.3 \\
\hline & 7.1 & 24.6 & u & 6.8 \\
\hline & 5.6 & 33.0 & u & 5.6 \\
\hline & 4.4 & 60.4 & u & 4.8 \\
\hline & 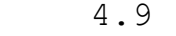 & 60.4 & $\mathrm{~m}$ & 4 . \\
\hline & & 7 & d & \\
\hline
\end{tabular}


Sounding: MIA242

Location: O.B. Johnson Park

Client: US Geological Survey

Project: Saltwater Intrus

County: Broward County

TX loop size: X $[\mathrm{m}] \quad 37.9 \quad \mathrm{Y}[\mathrm{m}] \quad 37.9$

$\begin{array}{cccc}\text { Model } & \text { Resistivity } & \text { Thickness } & \text { Elevation } \\ \text { Layer } & {[\text { ohm-m] }} & {[\mathrm{m}]} & {[\mathrm{m}]} \\ 1 & 85.040 & 76.4 & 0.0 \\ 2 & 8.920 & -- & -76.4\end{array}$

System: EM-58 Freq[Hz]: 315 Data Set Code: uh TX $\operatorname{Cur}[A]: 2.50$ Turn Off[usec]: 2.5

RX Moment [turns-m^2]: $31.4 \quad$ Gain Setting:

$\begin{array}{rrrrrr}\text { Time } & \begin{array}{r}\text { rhs }] \\ \text { [ohm-m] }\end{array} & \begin{array}{c}\text { obs_err } \\ {\left[\frac{\mathrm{o}}{0}\right]}\end{array} & \text { mask } & \begin{array}{r}\text { rhoa_cal } \\ \text { [ohm-m] }\end{array} \\ 1 & 0.00680 & 109.0 & 0.5 & \mathrm{~m} & 81.2 \\ 2 & 0.00860 & 195.9 & 0.6 & \mathrm{~m} & 79.9 \\ 3 & 0.01110 & 106.8 & 0.3 & \mathrm{~m} & 81.1 \\ 4 & 0.01410 & 190.0 & 1.2 & \mathrm{~m} & 82.5 \\ 5 & 0.01800 & 64.1 & 0.5 & \mathrm{~m} & 83.6 \\ 6 & 0.02300 & 260.3 & 0.5 & \mathrm{~m} & 85.2 \\ 7 & 0.02940 & 57.5 & 0.5 & \mathrm{~m} & 87.7 \\ 8 & 0.03750 & 176.6 & 0.7 & \mathrm{~m} & 90.9 \\ 9 & 0.04790 & 70.8 & 0.5 & \mathrm{~m} & 94.0 \\ 10 & 0.06110 & 105.9 & 0.9 & \mathrm{u} & 95.4 \\ 11 & 0.07790 & 89.0 & 0.8 & \mathrm{u} & 93.5 \\ 12 & 0.09930 & 78.1 & 0.9 & \mathrm{u} & 88.2 \\ 13 & 0.12670 & 81.0 & 1.4 & \mathrm{u} & 79.9 \\ 14 & 0.16640 & 67.5 & 1.5 & \mathrm{u} & 69.9 \\ 15 & 0.20600 & 64.6 & 2.1 & \mathrm{u} & 62.4 \\ 16 & 0.26280 & 58.3 & 2.4 & \mathrm{u} & 54.9 \\ 17 & 0.33520 & 51.7 & 3.4 & \mathrm{u} & 48.8 \\ 18 & 0.42770 & 44.5 & 6.3 & \mathrm{u} & 43.8 \\ 19 & 0.54560 & 39.2 & 10.3 & \mathrm{u} & 40.0 \\ 20 & 0.69590 & 34.8 & 12.7 & \mathrm{u} & 37.2\end{array}$

Date: $04-M A R-09$

UTM Coord: $\mathrm{E}[\mathrm{m}]$

Elevation $[\mathrm{m}]$ :

$584221.0 \quad \mathrm{~N}[\mathrm{~m}] \quad 2875414.0$

0.0

RX location: $\mathrm{X}[\mathrm{m}]$

$0.0 \quad Y[m]$

0.0

Fit Error $\left[\frac{\circ}{0}\right]: 9.798$ 
Sounding: MIA242X

Location: O.B. Johnson Park

Client: US Geological Survey

Project: Saltwater Intrus

County: Broward County

TX loop size: X $[\mathrm{m}] \quad 37.9 \quad \mathrm{Y}[\mathrm{m}] \quad 37.9$

$\begin{array}{cccc}\text { Model } & \text { Resistivity } & \text { Thickness } & \text { Elevation } \\ \text { Layer } & {[\text { ohm }-\mathrm{m}]} & {[\mathrm{m}]} & {[\mathrm{m}]} \\ 1 & 158.800 & 57.8 & 0.0 \\ 2 & 16.150 & -- & -57.8\end{array}$

System: EM-58 Freq[Hz]: 315 Data Set Code: uh TX $\operatorname{Cur}[A]: 2.50$ Turn Off[usec]: 2.5

RX Moment [turns-m^2]: 31.4 Gain Setting:

$\begin{array}{rrrrrr}\text { Time } & \begin{array}{r}\text { rhs }] \\ \text { [ohm-m] }\end{array} & \begin{array}{c}\text { obs_err } \\ {\left[\frac{\mathrm{o}}{0}\right]}\end{array} & \text { mask } & \begin{array}{c}\text { rhoa_cal } \\ \text { [ohm-m] }\end{array} \\ 1 & 0.00680 & 109.0 & 0.5 & \mathrm{~m} & 144.9 \\ 2 & 0.00860 & 195.9 & 0.6 & \mathrm{~m} & 147.7 \\ 3 & 0.01110 & 106.8 & 0.3 & \mathrm{~m} & 156.7 \\ 4 & 0.01410 & 190.0 & 1.2 & \mathrm{~m} & 166.3 \\ 5 & 0.01800 & 64.1 & 0.5 & \mathrm{~m} & 172.1 \\ 6 & 0.02300 & 260.3 & 0.5 & \mathrm{~m} & 171.4 \\ 7 & 0.02940 & 57.5 & 0.5 & \mathrm{~m} & 162.9 \\ 8 & 0.03750 & 176.6 & 0.7 & \mathrm{~m} & 147.9 \\ 9 & 0.04790 & 70.8 & 0.5 & \mathrm{~m} & 130.3 \\ 10 & 0.06110 & 105.9 & 0.9 & \mathrm{u} & 113.1 \\ 11 & 0.07790 & 89.0 & 0.8 & \mathrm{u} & 97.7 \\ 12 & 0.09930 & 78.1 & 0.9 & \mathrm{u} & 84.8 \\ 13 & 0.12670 & 81.0 & 1.4 & \mathrm{u} & 73.9 \\ 14 & 0.16640 & 67.5 & 1.5 & \mathrm{u} & 64.2 \\ 15 & 0.20600 & 64.6 & 2.1 & \mathrm{u} & 58.0 \\ 16 & 0.26280 & 58.3 & 2.4 & \mathrm{u} & 52.3 \\ 17 & 0.33520 & 51.7 & 3.4 & \mathrm{u} & 47.8 \\ 18 & 0.42770 & 44.5 & 6.3 & \mathrm{u} & 44.4 \\ 19 & 0.54560 & 39.2 & 10.3 & \mathrm{u} & 42.0 \\ 20 & 0.69590 & 34.8 & 12.7 & \mathrm{u} & 40.5\end{array}$

Date: $04-M A R-09$

UTM Coord: $\mathrm{E}[\mathrm{m}]$

Elevation $[\mathrm{m}]$ :

$584221.0 \quad \mathrm{~N}[\mathrm{~m}] \quad 2875414.0$

0.0

RX location: $\mathrm{X}[\mathrm{m}]$

$0.0 \quad Y[m]$

0.0

Fit Error $\left[\frac{\circ}{0}\right]: 14.350$ 


\section{Appendix 7 TEM Sounding Descriptions and Plots: 2009}

Descriptions of the sounding results are given below, along with plots of the measured apparent resistivity, calculated apparent resistivity for the inversion model, and the inversion estimated resistivity-depth function. The TEM data were measured at two transmitter repetition frequencies. The $285-\mathrm{Hz}$ data $(\mathrm{UH})$ are plotted as open circles $(\mathrm{O})$, and the $30-\mathrm{Hz}$ data $(\mathrm{HI})$ are plotted as open squares $(\square)$. The apparent resistivity measurement error bars are plotted as vertical lines through the data points. In most cases the error bars are smaller than the plotting symbol. The calculated $285-\mathrm{Hz}$ model response is plotted as a solid line, and the $30-\mathrm{Hz}$ model response is plotted as a dashed line. Some plots show only the $285-\mathrm{Hz}$ data because the lower repetition rate data were too noisy to be of value. Masked data points are plotted as solid diamonds $(\checkmark)$ and were not used in the inversion. All soundings were made with a square transmitter loop with a side length of $38.1 \mathrm{~m}$. Unusable soundings are noted in table 1.

Geoelectrical models can be classified by the variation of resistivity with depth (Keller and Frischknecht, 1966, p. 135). A three-layer model with a resistivity minimum $\left(\rho_{1}>\rho_{2}<\rho_{3}\right)$ is called a type-H curve. A model with a resistivity maximum $\left(\rho_{1}<\rho_{2}>\rho_{3}\right)$ is called a type-K curve. A model with a monotonic increase in resistivity $\left(\rho_{1}<\rho_{2}<\rho_{3}\right)$ is called a type-A curve. A model with a monotonic decrease in resistivity $\left(\rho_{1}>\rho_{2}>\rho_{3}\right)$ is called a type-Q curve. For curves with more than three layers, this classification is applied to the first through third layer, the second through fourth layer, and so on. For example, a curve with $\left(\rho_{1}>\rho_{2}<\rho_{3}>\rho_{4}\right)$ is classified as type HK.

The resistivity-depth models determined by inversion are usually not unique; that is, there is uncertainty in the estimated model parameters caused by errors in the observations and parameterization errors. The latter is due to naturally occurring variations in geology that are not describable by the one-dimensional (layered earth) model used for the inversion. A qualitative estimate of the parameter uncertainty is given in the descriptions below. Another issue in the inversion is the resolution of the model parameters. To understand model resolution, assume that there is an actual set of model parameters that we can only view through a set of glasses. If the glasses give a good image of the actual parameters, then the resolution is good. If, on the other hand, the glasses distort the viewing of the actual parameters, then the resolution is poor. When a descriptive resolution is given for a sequence of layer resistivities or thicknesses, such as "good, very good, poor," the order is from the first layer downward.

\section{Sounding MIA201}

A three-layer model with decreasing resistivity with depth (type Q) fits the data with a misfit error of 1.83 percent. All model parameters are well resolved. The interpreted resistivity drops to $6.0 \mathrm{ohm}-\mathrm{m}$ at a depth of $84 \mathrm{~m}$.

\section{Sounding MIA202}

The data are well modeled with a four-layer, type-HK model (conductive second and fourth layers). The second and fourth layer resistivities are very well resolved. The first layer resistivity is moderately resolved, and the third layer resistivity is poorly resolved. The first and third layer 
thicknesses are well resolved, while the second layer thickness is moderately well determined. Misfit error is 1.47 percent.

\section{Sounding MIA203}

A five-layer, type-QHK type model (MIA203) does a very good job of modeling the data with a misfit error of 1.67 percent. The conductive third layer's resistivity and thickness are very well determined. The resistive first layer is not well determined, while the second layer with a transitional value of resistivity has better parameter estimates. The resistive fourth layer is moderately well determined. Originally a four-layer, type-QK model (MIA203L4) was used. It gives a misfit error of 1.72 percent; however, comparison of the resistivity-depth plot with the induction log from a nearby well (Sec34-MW-03-FS) indicates that a more gradual transition from the surface layer to the conductive layer was appropriate. Thus, the five-layer model is preferred.

\section{Sounding MIA204}

A five-layer, type-QHK model (MIA204) fits the data with a misfit of 1.08 percent. Determination of the surface resistivity is moderate, while the resolution of the more conductive second and third layers is much better. The thickness of the fourth layer is not well determined. Initially a four-layer, type-HK model was chosen (misfit error 1.07 percent). As with sounding MIA203, comparison with the induction log from well Sec34-MW-03-FS shows that a more gradual transition to the conductive layer is required. Based on the well log the five-layer model is preferred.

\section{Sounding MIA205}

Similar to the previous four soundings, the conductive layers of this four-layer, type-HK model is well resolved, while the other layers are not as well resolved. The misfit error is 1.05 percent.

\section{Sounding MIA206}

The data are modeled with a four-layer, type-QH model with layer resistivities decreasing from the resistive surface layer until the fourth (basement) layer whose resistivity is higher. The resistivities of the first three layers and thicknesses of the first two layer are well determined, whereas the thickness of the conductive third layer and the resistivity of the fourth layer are not well constrained. The misfit error is 1.70 percent.

\section{Sounding MIA207}

The model is similar to the previous type-QH sounding. The second layer resistivity is well resolved, the third layer resistivity is moderately well resolved, and the resistivities of the first and fourth layers are poorly determined. The thickness of the second layer is well determined. The misfit error is 1.79 percent.

\section{Sounding MIA208}

The resistivity decreases with depth down to the fourth layer, where it has a slight increase (type QH). The resistivity of the second layer is well determined. All other resistivities are poorly 
to moderately well determined. The thicknesses of the first two layers are well determined. Misfit error is 2.27 percent.

\section{Sounding MIA209}

A four-layer, type-QH model gives a good fit to the data (misfit error 2.42 percent). The model is essentially a two-layer model with an additional conductive layer embedded near 100 meters depth. The second layer resistivity is very well constrained, while the resistivities of the other layers are not well determined. The thickness of the first layer is moderately well constrained.

\section{Sounding MIA210}

This sounding is similar to MIA209. A four-layer, type-QH model results in a misfit error of 2.37 percent. The second layer resistivity is well determined. The thin, conductive third layer is needed for a good fit, though the layer parameters are poorly constrained.

\section{Sounding MIA211}

A four-layer, type-HK model with a thin, conductive second layer fits the data very well (misfit error 1.31 percent). The first layer thickness is well determined. The second layer exhibits an S-equivalence meaning that only the ratio of layer thickness to layer resistivity is well determined — not the individual layer parameters.

\section{Sounding MIA212}

The four-layer, type-HK model MIA212 fits the data with a misfit error of 1.77 percent; however, there is a very severe S-equivalence for the second layer. A preferred alternative model (MIA212F) with the second layer resistivity constrained to $3.5 \mathrm{ohm}-\mathrm{m}$ based upon well $\log$ information for saltwater intruded zones gives a fit of 1.65 percent. The first and second layer thicknesses for this model are very well determined, while the first and third layer resistivities are not well determined. The resistivity of layer four is moderately well determined.

\section{Sounding MIA213}

The model is a four-layer type HK. The second layer is very thin and conductive resulting in an S-equivalence. The misfit error is 2.35 percent. The first and third layer resistivities are well determined, whereas the fourth layer resistivity is poorly determined. The second layer parameters are very poorly determined because of the $\mathrm{S}$-equivalence. Resolution of the first layer thickness is good, while that of the third layer is poor.

Model MIA213F is the preferred alternative: also a four-layer, type-HK model, but with the second layer resistivity constrained to a value of $3.5 \mathrm{ohm}-\mathrm{m}$. The misfit error is 2.28 percent. The first and third layer resistivities are moderately well determined, and the fourth layer resistivity is poorly determined. The first layer thickness is very well determined, while the third layer thickness if moderately well determined.

\section{Sounding MIA214}

A five-layer, type-HKH model fits the data with a misfit of 1.82 percent. The third and fifth layer resistivities are well determined. The second and fourth layer resistivities are moderately resolved, whereas the first layer resistivity is poorly resolved. The first and third layer thicknesses 
are moderately resolved. The second layer thickness is moderately resolved, whereas the fourth layer thickness is poorly resolved.

\section{Sounding MIA215}

A five-layer, type-HKH model fits the data with a misfit of 1.44 percent. The resolution results are similar to those of sounding MIA214.

\section{Sounding MIA216}

Sounding MIA216 fits the data using a four-layer, type-HK model with a misfit of 2.17 percent. The third and fourth layer resistivities are well resolved. The first and third layer thicknesses are well resolved. All other parameters are moderately resolved.

\section{Sounding MIA217}

A four-layer, type-HK model gives a misfit of 2.23 percent. The thin second layer shows an $\mathrm{S}$-equivalence behavior. The third layer resistivity is well resolved, the fourth layer resistivity is moderately resolved, and the first and second layer resistivities are poorly resolved. The first layer thickness is well resolved, the second layer thickness is poorly resolved, and the third layer thickness is moderately resolved.

\section{Sounding MIA218}

A three-layer, type-H model (MIA218) fits the data with an error of 1.75 percent. The resistivities of the first and third layers are well determined, as are the thickness of the first layer. The second layer parameters suffer from an S-equivalence. A preferred, alternative model (MIA218F) with the second layer resistivity fixed at $3.5 \mathrm{ohm}-\mathrm{m}$ gives a misfit error of 2.24 percent. The first layer resistivity, however, is now poorly determined.

\section{Sounding MIA219}

A three-layer, type-H model (MIA219) fits the data with an error of 3.41 percent. Because the second layer is very thin compared to its depth, there is an S-equivalence that does not allow the layer resistivity and thickness to be independently determined. The first layer resistivity is moderately well resolved, while the third layer resistivity is well resolved. Model MIA219F with the second layer resistivity constrained to $3.5 \mathrm{ohm}-\mathrm{m}$ is preferred. The misfit error for this model is 3.33 percent. All of the unconstrained model parameters are well resolved with the exception of the first layer resistivity which is poorly resolved.

\section{Sounding MIA220}

Sounding MIA220 is fit with a three-layer type-H model. The conductive second layer is thick enough that there is no S-equivalence. The misfit error is 1.86 percent. The first layer resistivity is moderately well determined, the second layer resistivity is well determined, and the third layer resistivity is poorly resolved. All layer thicknesses are well resolved. 


\section{Sounding MIA221}

A three-layer, type-H model gives a misfit of 1.37 percent. The first and third layer resistivities are moderately resolved. The second layer resistivity is very well resolved. The layer thicknesses are well resolved.

\section{Sounding MIA222}

A three-layer type-H model fits the data very well after masking the last three datum (misfit error of 1.14 percent). The layer resistivity resolutions are good, very good, and very good from surface to depth, respectively. The layer thickness resolutions are both very good.

An alternative four-layer, type-HK model (MIA222L4) that uses all of the data points gives a misfit error of 1.73 percent. This model requires a conductive basement layer. The resolutions of the layer resistivities from surface to depth are good, very good, very poor, and very poor, respectively. The resolutions of layer thicknesses are very good, very good, and good, respectively. The three-layer model is preferred because of better resolution.

\section{Sounding MIA223}

A three-layer, type-H model gives a misfit error of 2.32 percent. The first and second layer resistivities are very well determined, and the third layer resistivity is moderately well determined. The first and second layer thicknesses are very well determined. An alternative four-layer, type-HK model (MIA223L4) gives a misfit error of 1.96 percent. The resistivities and layer thicknesses of the first two layers are very well determined. The third layer thickness is moderately well determined. The third and fourth layer resistivities are very poorly determined. While the misfit for the four-layer model is smaller, the decreased resolution of the third and fourth layers makes the three-layer model the preferred one.

\section{Sounding MIA224}

The misfit error for a three-layer, type-H model is 1.25 percent. The resolution of the first layer resistivity is poor, the second layer resistivity is very good, and the third layer resistivity is very poor. The first and second layer thicknesses are very well determined.

\section{Sounding MIA225}

A four-layer, type-HK model was used. Resolution of the layer resistivities from the surface to depth are very poor, moderate, very good, and very poor, respectively. The layer thickness resolutions from the surface to depth are very good, poor, and moderate, respectively. The misfit error is 1.74 percent.

\section{Sounding MIA226}

A four-layer, type-HK model has a misfit error of 2.44 percent. The first layer resistivity is very poorly determined, however, the first layer thickness is well determined. The second layer resistivity is moderately well resolved, and the second layer thickness is poorly resolved. The third and fourth layer resistivity resolutions are moderate. The third layer thickness is moderately well resolved. 


\section{Sounding MIA227}

A four-layer, type-HK model was used. The resolutions of the layer resistivities from the surface to depth are very poor, very good, good, and good, respectively. The layer thickness resolutions from the surface to depth are very good, good, and good, respectively. The misfit error is 1.34 percent.

\section{Sounding MIA228}

A four-layer, type-HK model gives a misfit error of 2.08 percent. The resolutions of the layer resistivities from the surface to depth are very poor, good, very poor, and very good, respectively. The layer thickness resolutions from the surface to depth are very good, good, and good, respectively.

\section{Sounding MIA229}

The four-layer, type-HK model gives a misfit error of 1.07 percent. The resolutions of the layer resistivities from the surface to depth are very poor, very good, poor, and good, respectively. The layer thickness resolutions from the surface to depth are very good, good, and good, respectively.

\section{Sounding MIA230}

The four-layer, type-QH model gives a misfit error of 2.60 percent. The resolutions of the layer resistivities from the surface to depth are good, very poor, very good, and very good, respectively. The layer thickness resolutions from the surface to depth are good, poor, and very good, respectively. An alternative three-layer, type-H model (MIA230L3) give a slightly larger misfit error of 3.37 percent. The resolutions of the layer resistivities from the surface to depth are very poor, very good, and very good, respectively. Both layer thickness resolutions are very good. The four-layer model was chosen over the three-layer model primarily because it is better able to fit the curvature of the data in the first eight channels where the observational errors are very small.

\section{Sounding MIA231}

A three-layer, type-H model does a very good job of fitting the data with a misfit error of 3.28 percent. The resolutions of the layer resistivities from the surface to depth are very poor, very good, and very poor, respectively. Both layer thickness resolutions are very good.

\section{Sounding MIA232}

A four-layer, type-HK model gives a misfit error of 2.12 percent. The resolutions of the layer resistivities from the surface to depth are poor, very good, very poor, and very poor, respectively. The layer thickness resolutions are all very good. An alternative three-layer, type-H model (MIA232L3) was attempted after masking the last two data points. The misfit error was 2.51 percent. The resolutions of the layer resistivities from the surface to depth are poor, very good, and very poor, respectively. Both layer thickness resolutions are very good. The four-layer model is preferred. 


\section{Sounding MIA233}

A four-layer, type-HK model gives a misfit error of 3.02 percent. The conductive second layer suffers from S-equivalence, suggesting the need for fixing the second layer resistivity based on well information. This was done in model MIA233F with a misfit error of 1.99 percent. For model MIA233 the resolution of the layer resistivities from the surface to depth are very poor, poor, good, and poor, respectively. The resolutions of the corresponding layer thicknesses are very good, poor, and poor, respectively. For model MIA233F, the resistivity resolutions are very poor, not specified (because of fixed resistivity value), very good, and poor, respectively. The thickness resolutions are very good, very good, and good, respectively.

Alternate three-layer, type-H models were determined by masking some of the data points at the end of the transient. The alternative models are MIA233L3 and MIA233F3, and their misfit errors are 2.48 percent and 3.33 percent, respectively. Model MIA233F is preferred.

\section{Sounding MIA234}

A four-layer, type-HK model gives a misfit error of 3.22 percent. The thin, conductive second layer exhibits S-equivalence. The layer resistivity resolutions are good, poor, good, and good, respectively. The thickness resolutions are good, poor, and good, respectively. When the second layer resistivity is constrained (model MIA234F) to $3.5 \mathrm{ohm}-\mathrm{m}$, the resistivity resolutions stay about the same: good, not specified, good, and good, respectively. The thickness resolutions, however, improve slightly: very good, good, and good, respectively. The original model MIA234 is preferred.

\section{Sounding MIA235}

This four-layer, type-HK model is very similar to several of the previous soundings. The misfit error is 1.90 percent with a significant $\mathrm{S}$-equivalence for the second layer. The layer resistivity resolutions are very poor, poor, good, and good, respectively. The layer thickness resolutions are very good, poor, and good, respectively. Constraining the second layer resistivity (model MIA235F) to $3.5 \mathrm{ohm}-\mathrm{m}$ gives the same misfit error and only slightly improves the second layer, thickness resolution. The constrained model (MIA235F) is preferred.

\section{Sounding MIA236}

A four-layer, type-HK model gives a misfit error of 2.86 percent. The layer resistivity resolutions are good, poor, poor, and good, respectively. The layer thickness resolutions are very good, poor, and good, respectively. The thin, conductive second layer exhibits S-equivalence. Fixing the second layer resistivity (MIA236F) at $3.5 \mathrm{ohm}-\mathrm{m}$ improves the resolution of the second layer thickness, while all other resolutions remain essentially unchanged. The unconstrained model MIA236 is preferred.

\section{Sounding MIA237}

A three-layer, type-Q model does a reasonable job of fitting the data; misfit error is 3.48 percent. The last five data points had to been masked to obtain this fit. This suggests that there may be a buried pipe at this location. The layer resistivity resolutions are poor, very good, and very good, respectively. The thickness resolutions are good and very good, respectively. 


\section{Sounding MIA238}

A three-layer, type-Q model can only fit the data with a misfit error of 5.56 percent. The resolutions of the layer resistivities are very poor, very good, and very good, respectively. Both layer-thickness resolutions are very good.

\section{Sounding MIA239}

This sounding was made in Hallandale Cemetery and appears to be influenced by manmade objects. Even unused grave sites have concrete grave liners in place, so the likelihood of noise in the data is high. The last nine data points were masked before fitting otherwise these apparent resistivity values would have required a model with very low layer resistivities. A threelayer, type-Q model MIA239 gives a misfit error of 5.56 percent. While the first-layer resistivity resolution is very poor, all other model parameters are well resolved.

An inversion was also tried using all of the data (MIA239X). A four-layer, type-QQ model with the layer resistivities decreasing with depth resulted. The fourth layer resistivity was 0.43 ohm-m. The layer resistivity resolutions were: poor, very good, good, and good, respectively. The layer thickness resolutions were very good, very good, and very good, respectively. Because of the very low fourth layer resistivity, which may be due to metal caskets or the chain-link fence that surrounds the cemetery, this model is rejected. While the three-layer model is preferred, it must be used with caution because of the buried anthropogenic features at the site.

\section{Sounding MIA240}

This sounding was made in Hallandale Cemetery. The data show the effect of nearby metal objects. Model MIA240A masked many of the data points, retaining only those that were felt could be reasonably modeled. The influence of non-geologic objects in the subsurface should not be under estimated. The misfit error was 7.80 percent. Models MIA204B and MIA240C mask fewer data points, and the misfits errors are larger. Use of these data is very questionable other than for estimating the near surface resistivity.

\section{Sounding MIA241}

A four-layer, type-QQ model MIA241 gives a 4.00 percent misfit. Eight data points at the end of the transient were masked in the inversion because retaining them resulted in an abnormally conductive basement. The layer resistivity resolutions are very poor, very good, poor, and good, respectively. The layer thickness resolutions are good, very good, and very good, respectively. Model MIA241X unmasked all but the last time gate. The misfit error for this model was 4.53 percent. The layer resistivity resolutions are very poor, very good, poor, and poor, respectively. The layer thickness resolutions are good, very good, and very good, respectively. The fourth layer resistivity for model MIA241X is $0.37 \mathrm{ohm}-\mathrm{m}$, which is very low, suggesting the presence of a pipe. Model MIA241 is preferred.

\section{Sounding MIA242}

The early channel data were very repeatable, but produced a very discontinuous sounding curve, suggesting the presence of metallic objects near the receiver coil. Moving the receiver coil 5 meters did not lessen the noise. The usefulness of these data is questionable. Two attempts at interpretation (MIA242 and MIA242X) resulted in two-layer models whose resistivity decreases 
with depth. The misfit error for these models was 9.80 and 14.4 percent, respectively. The variability between these two models is rather large. The reliability of this sounding is questionable. 
MIA201

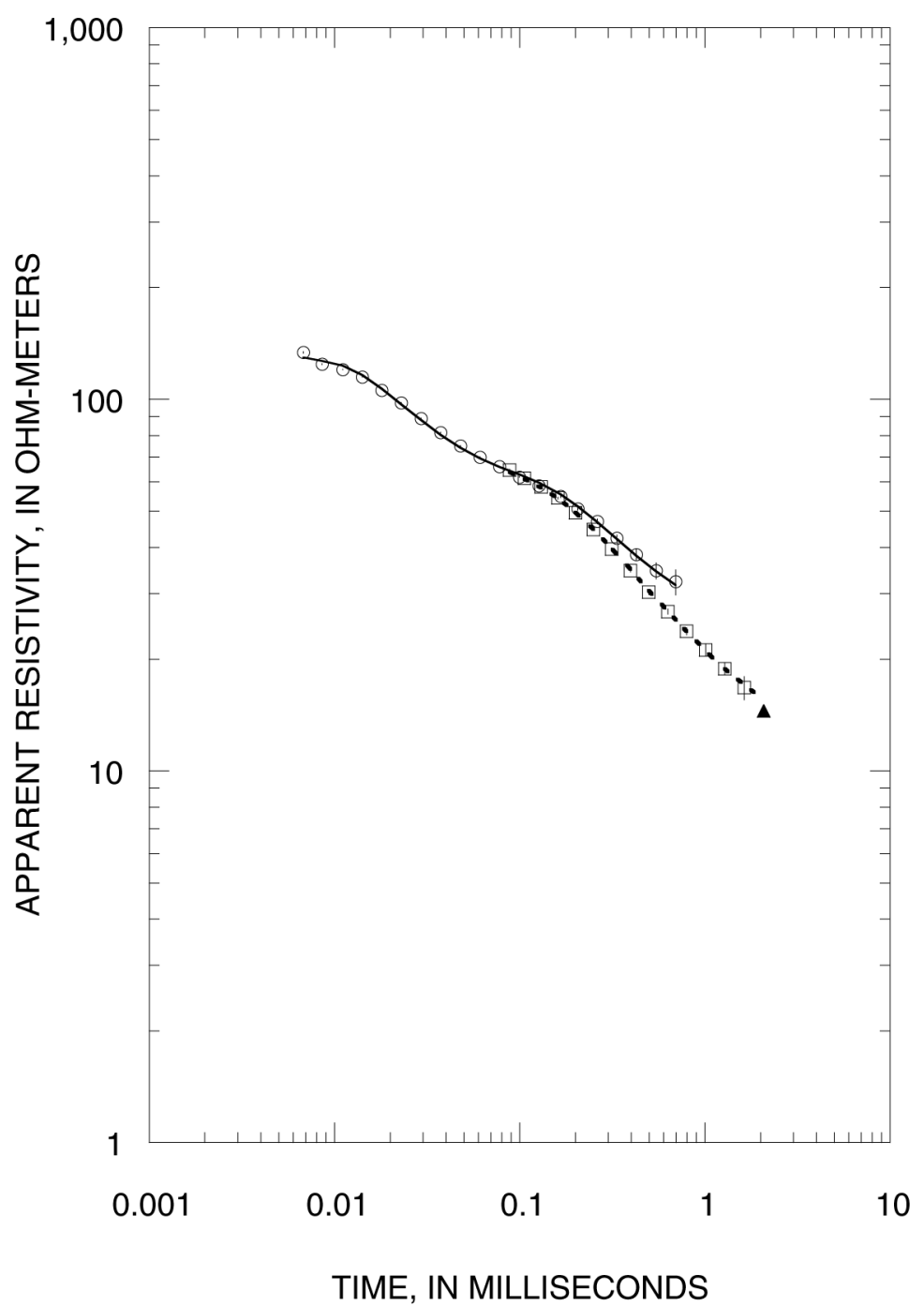

MIA201

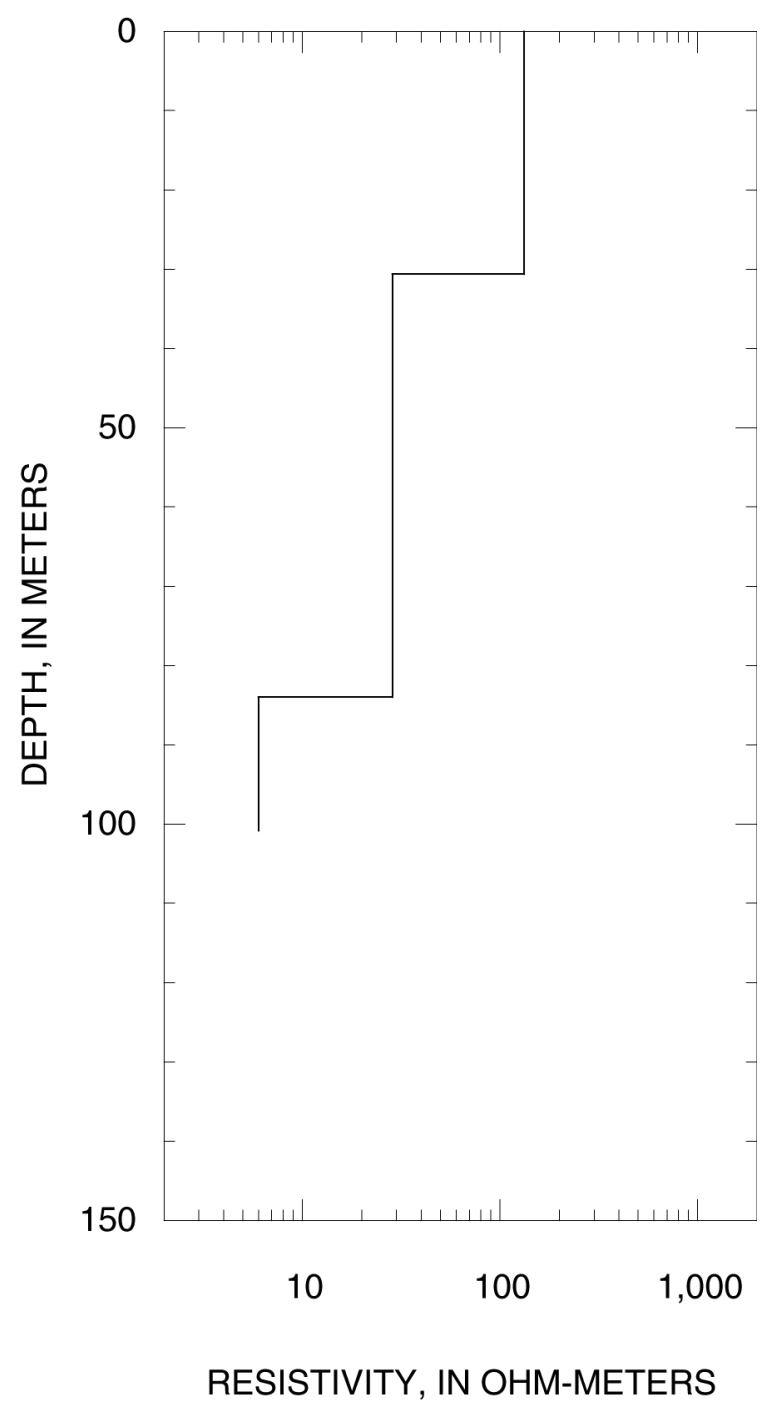

Figure 7-1. Sounding MIA201 
MIA202

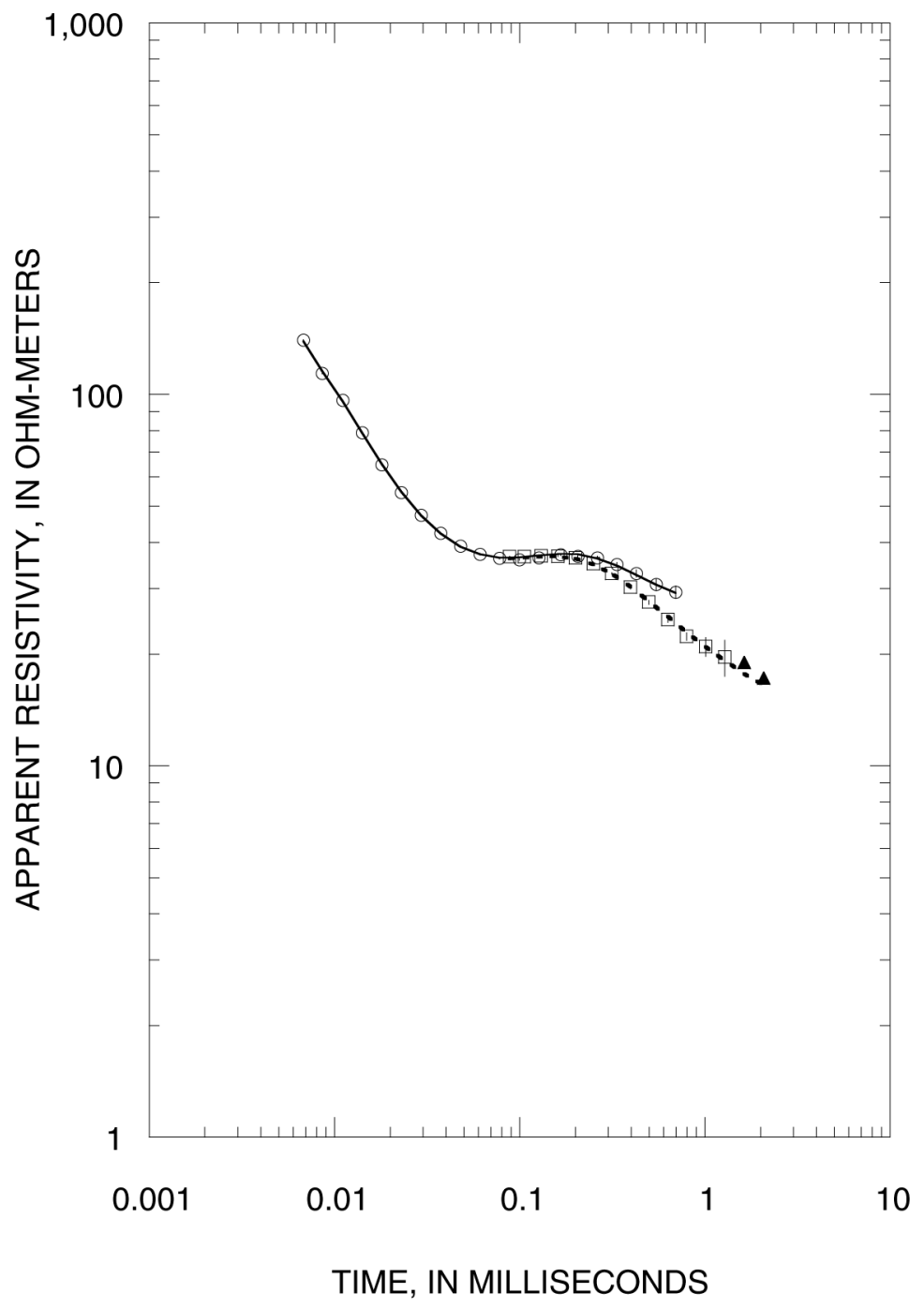

MIA202

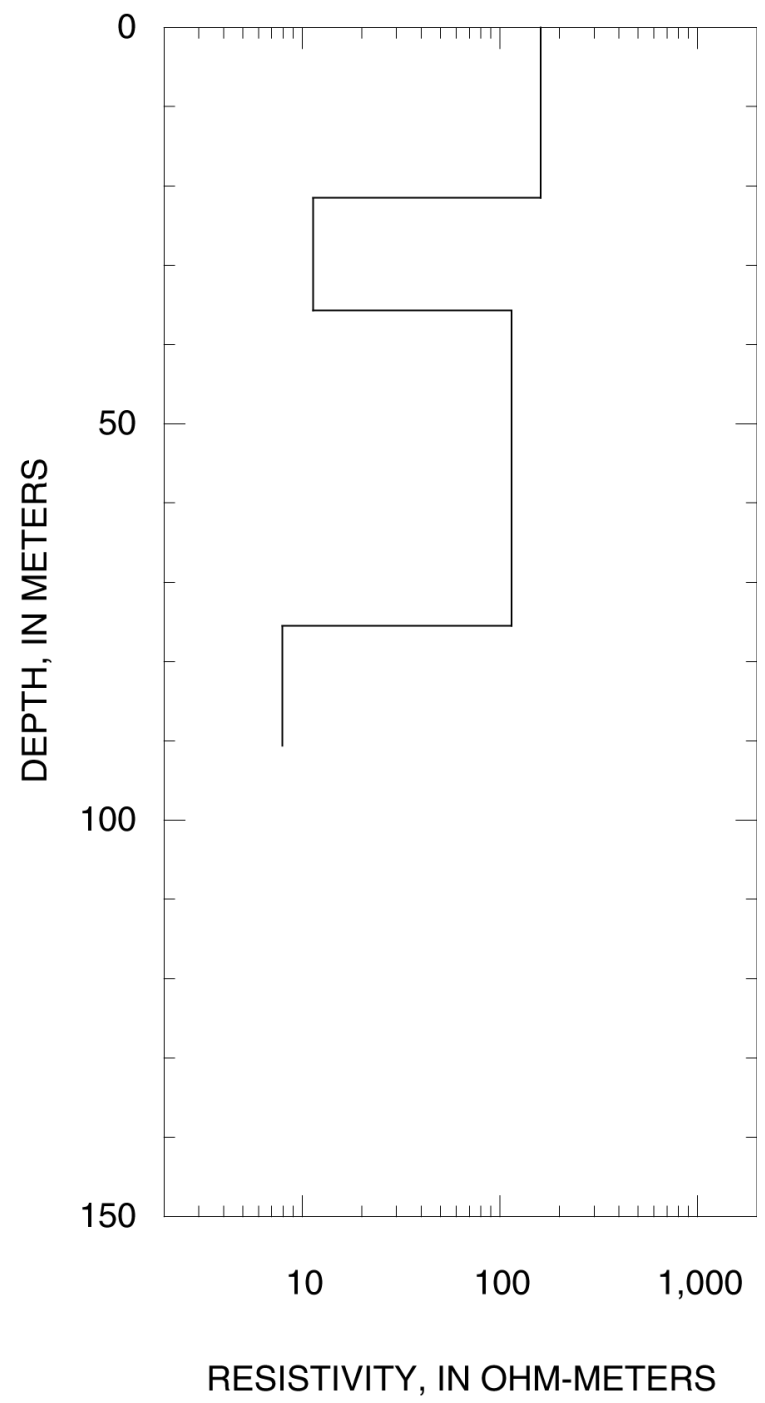

Figure 7-2. Sounding MIA202 
MIA203

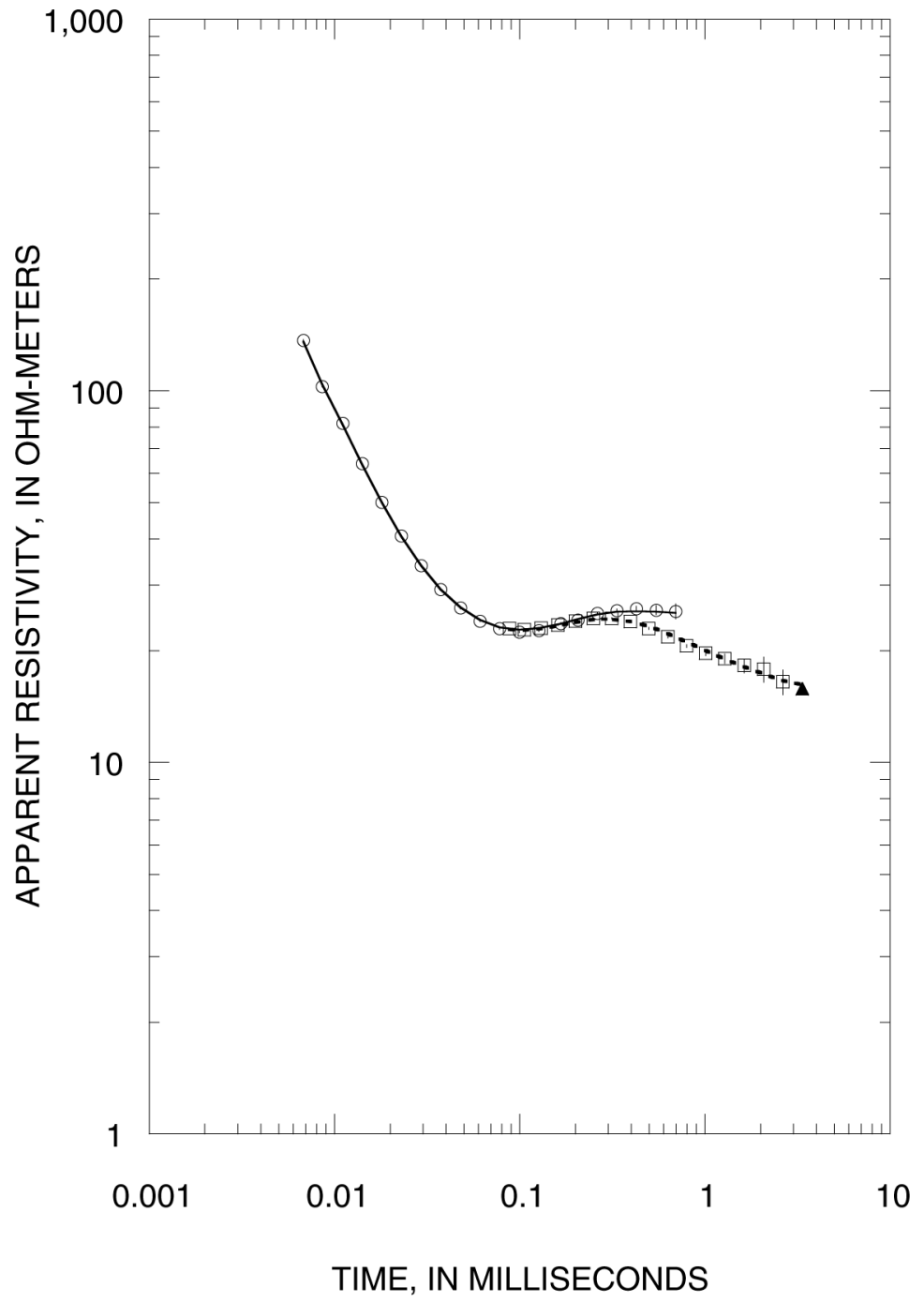

Figure 7-3. Sounding MIA203

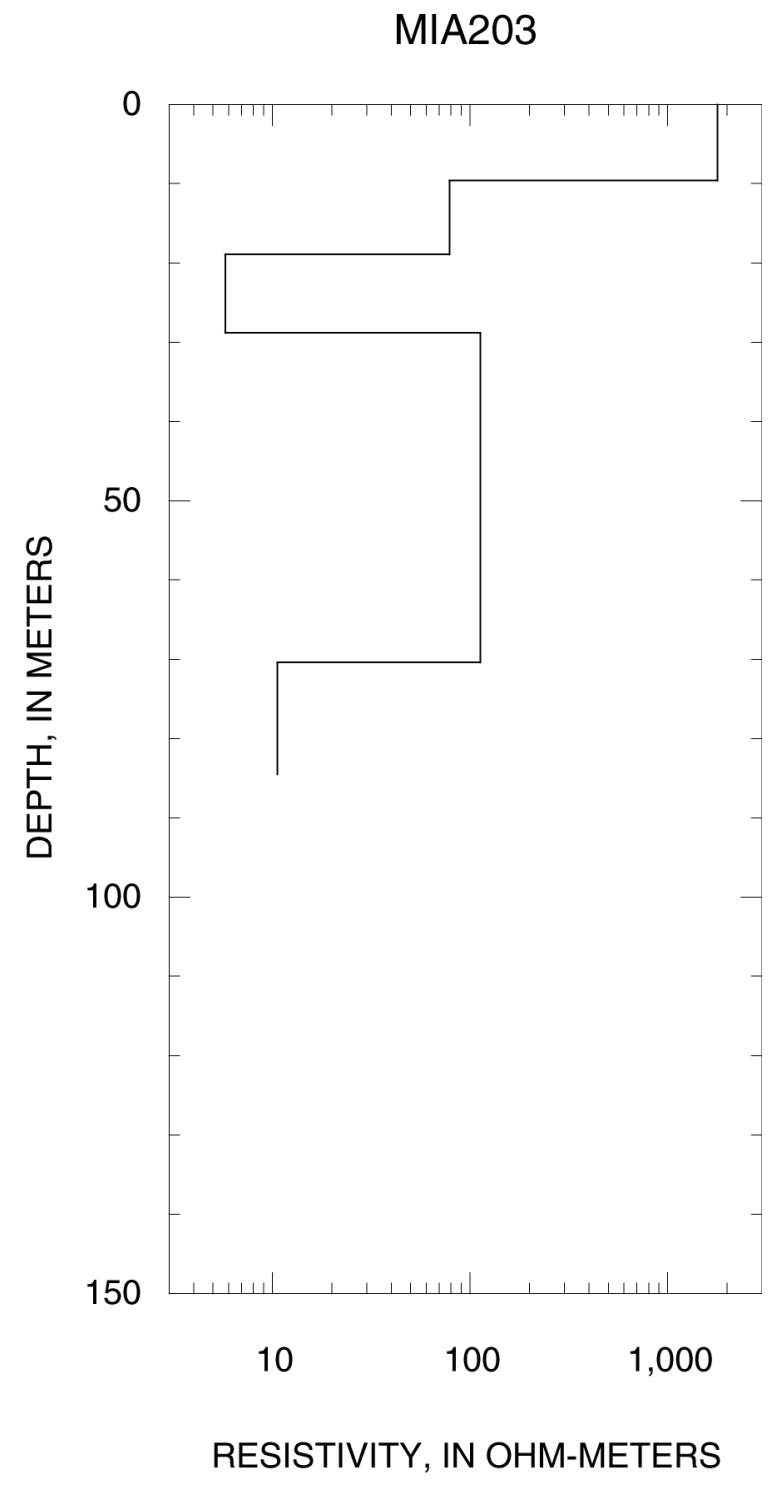


MIA203L4

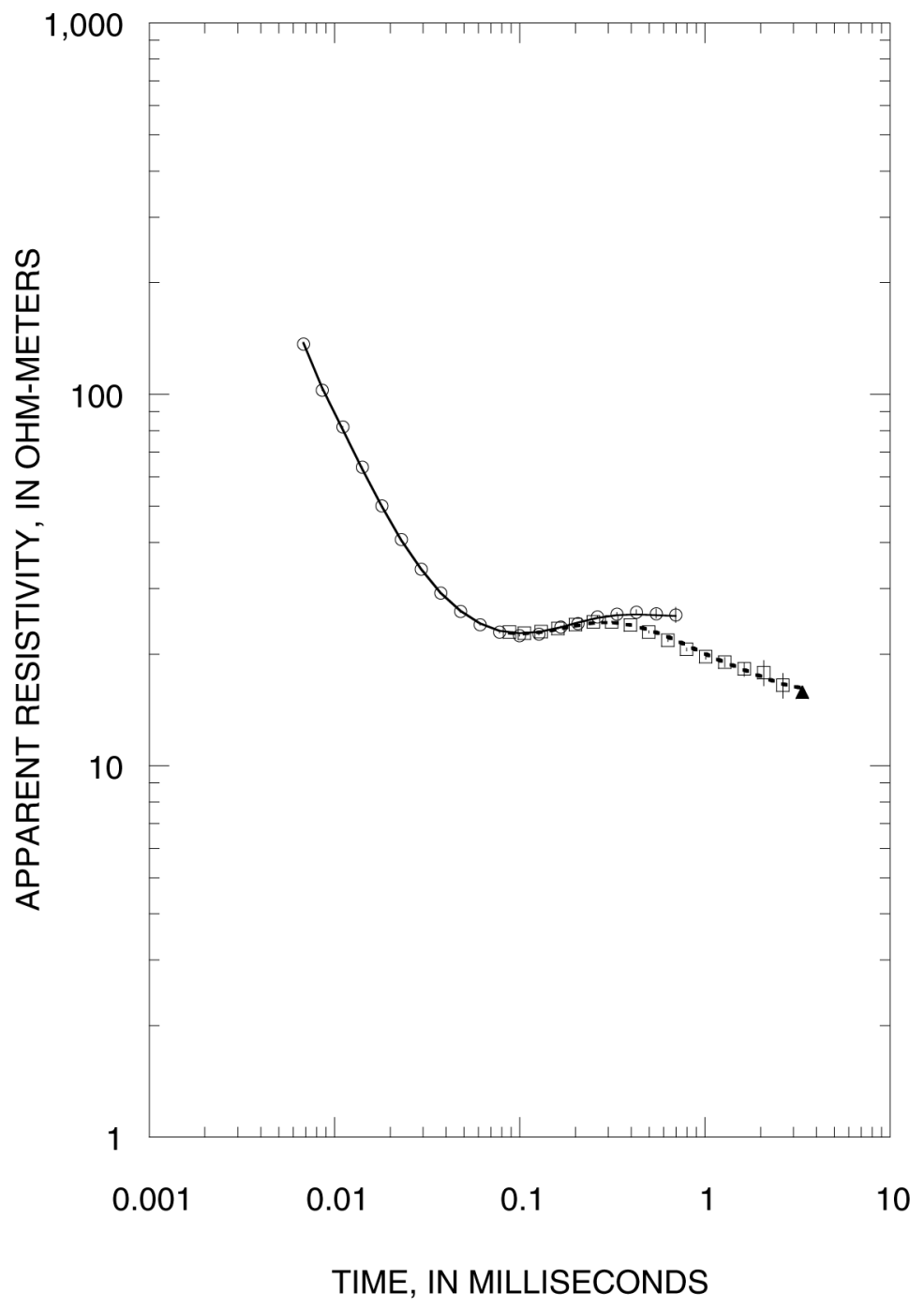

MIA203L4

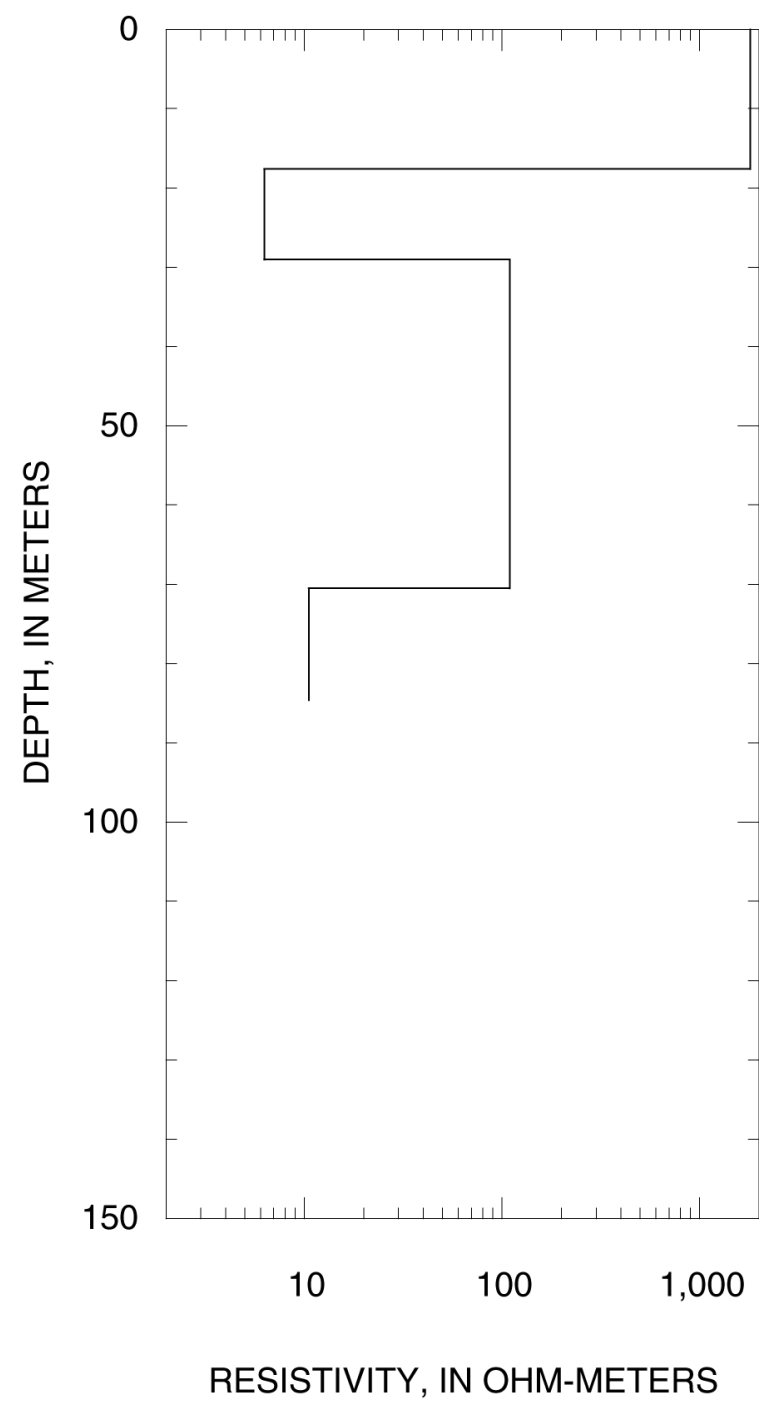

Figure 7-4. Sounding MIA203L4 
MIA204

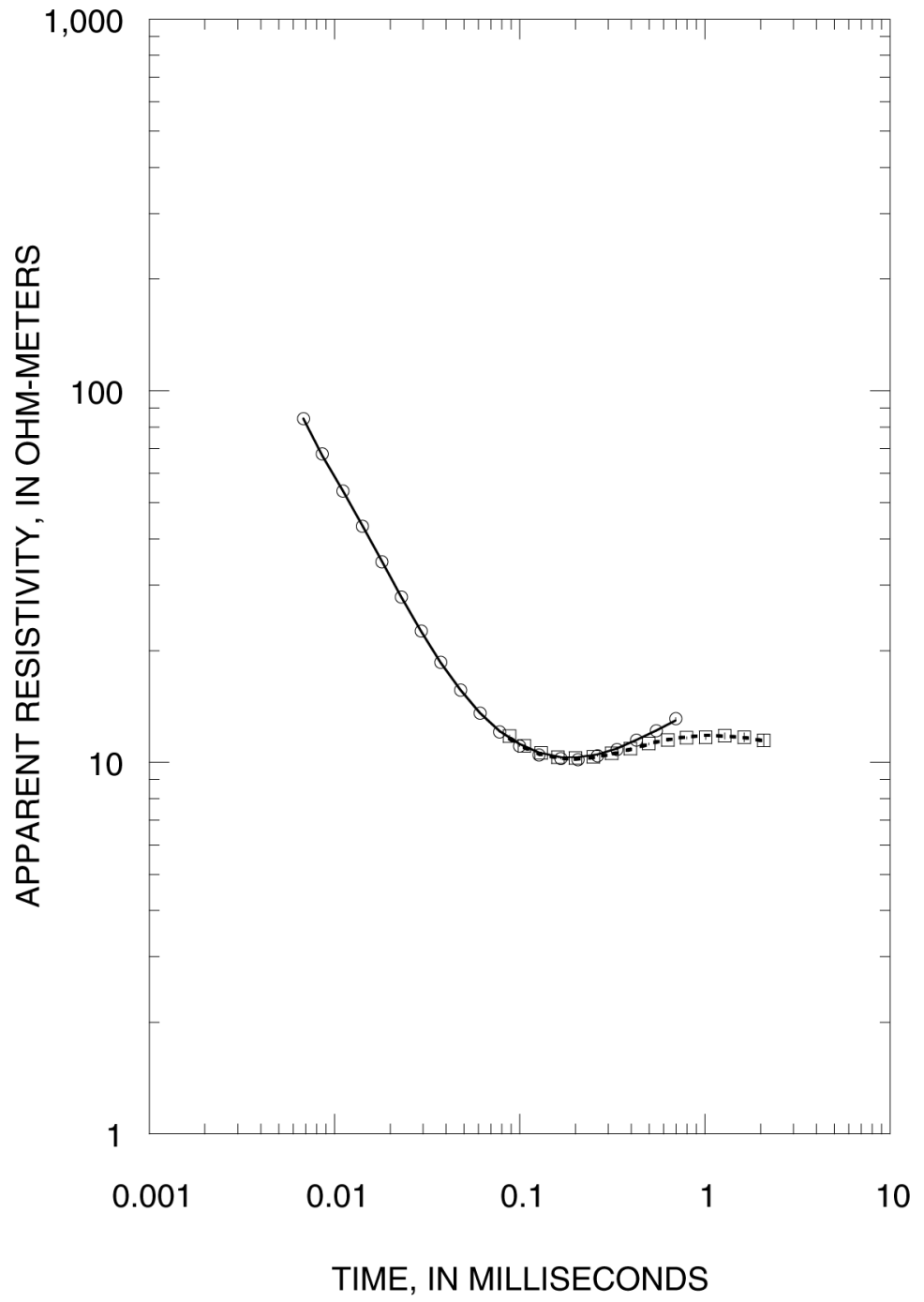

Figure 7-5. Sounding MIA204

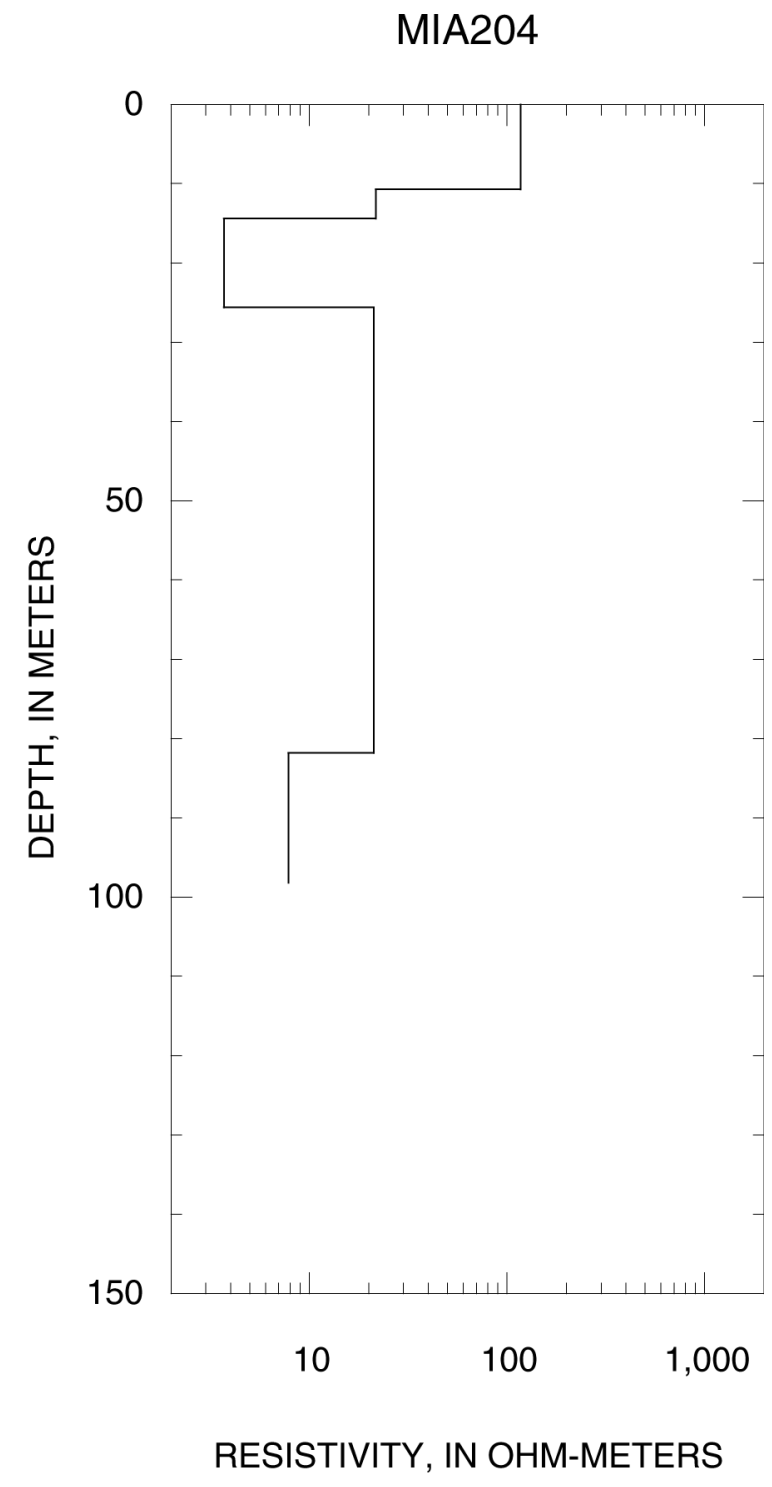


MIA204L4

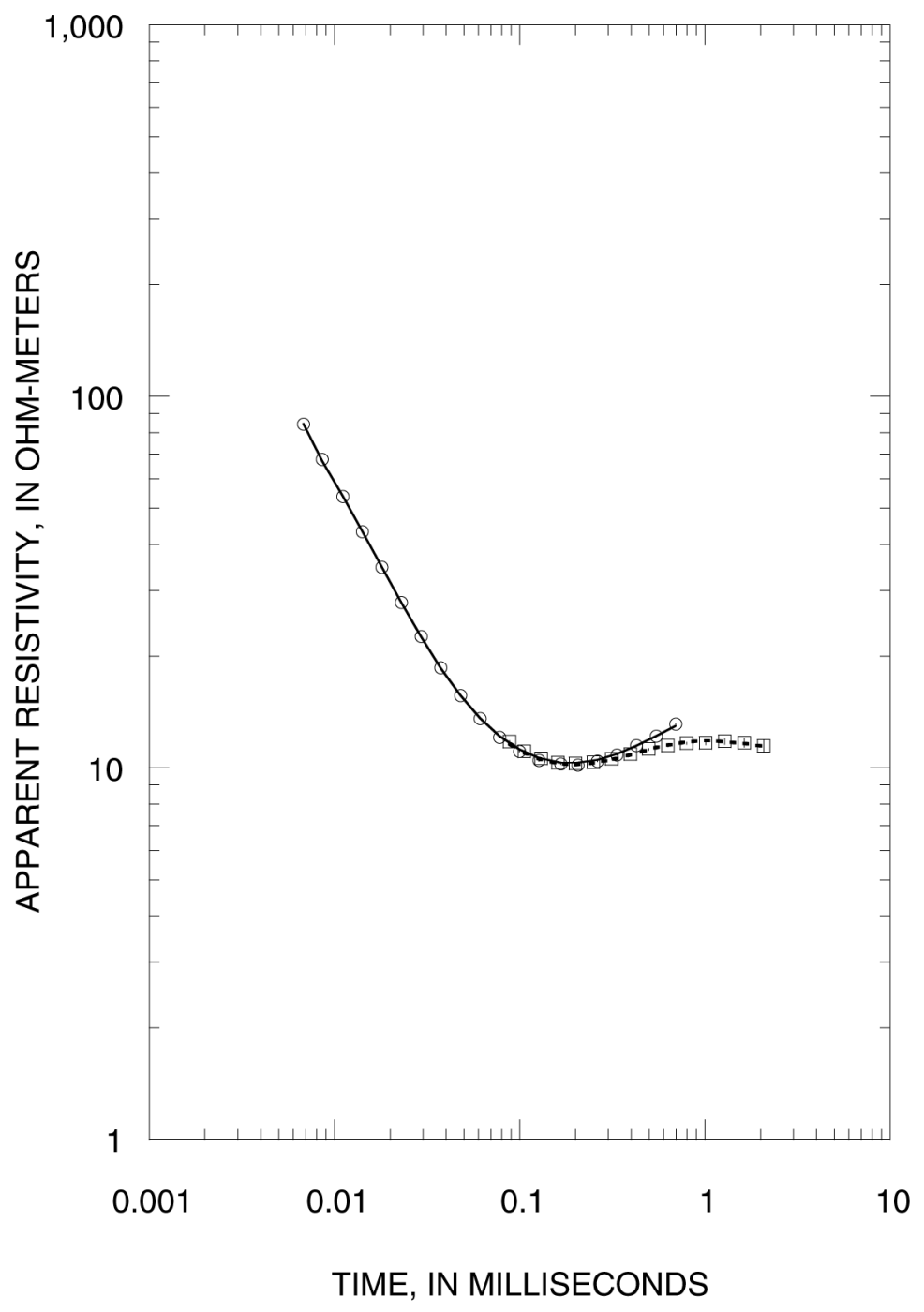

MIA204L4

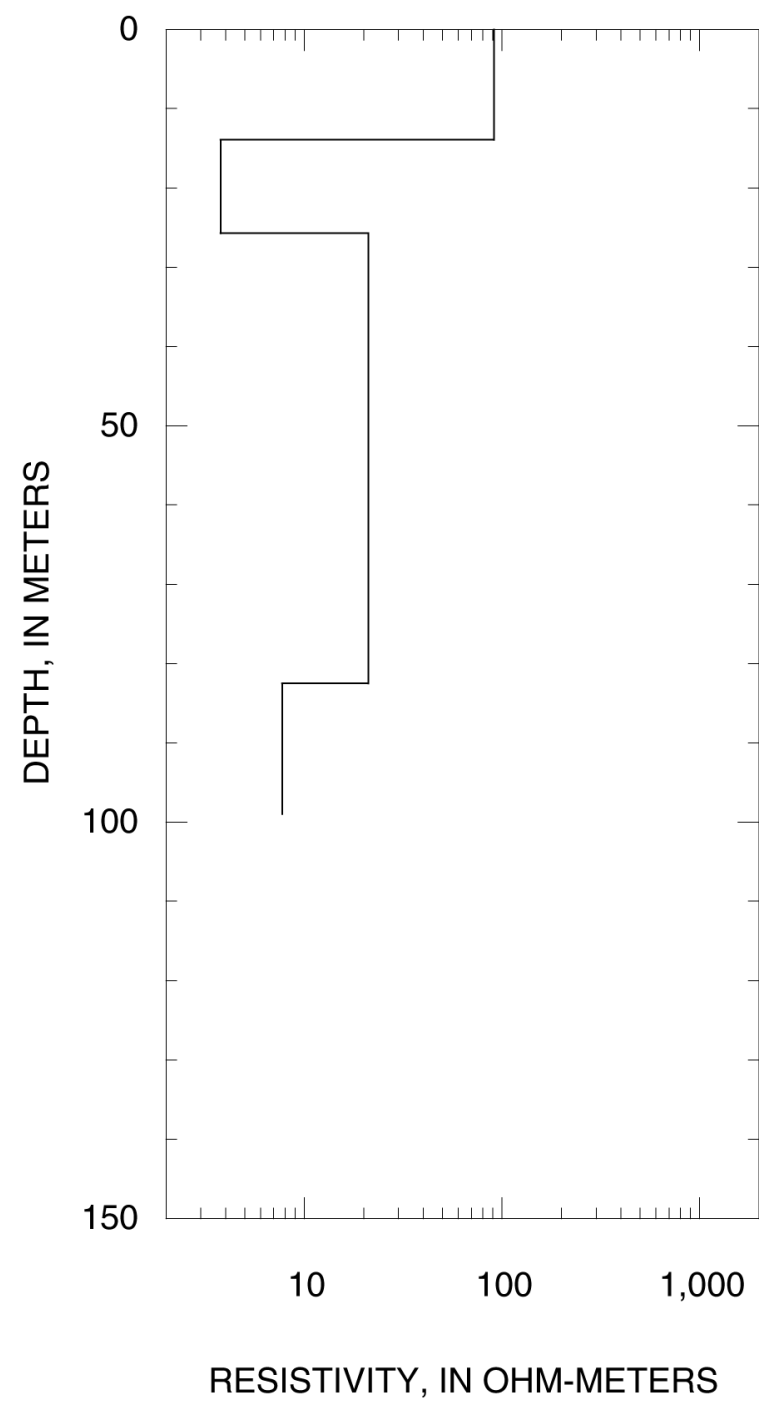

Figure 7-6. Sounding MIA204L4 
MIA205

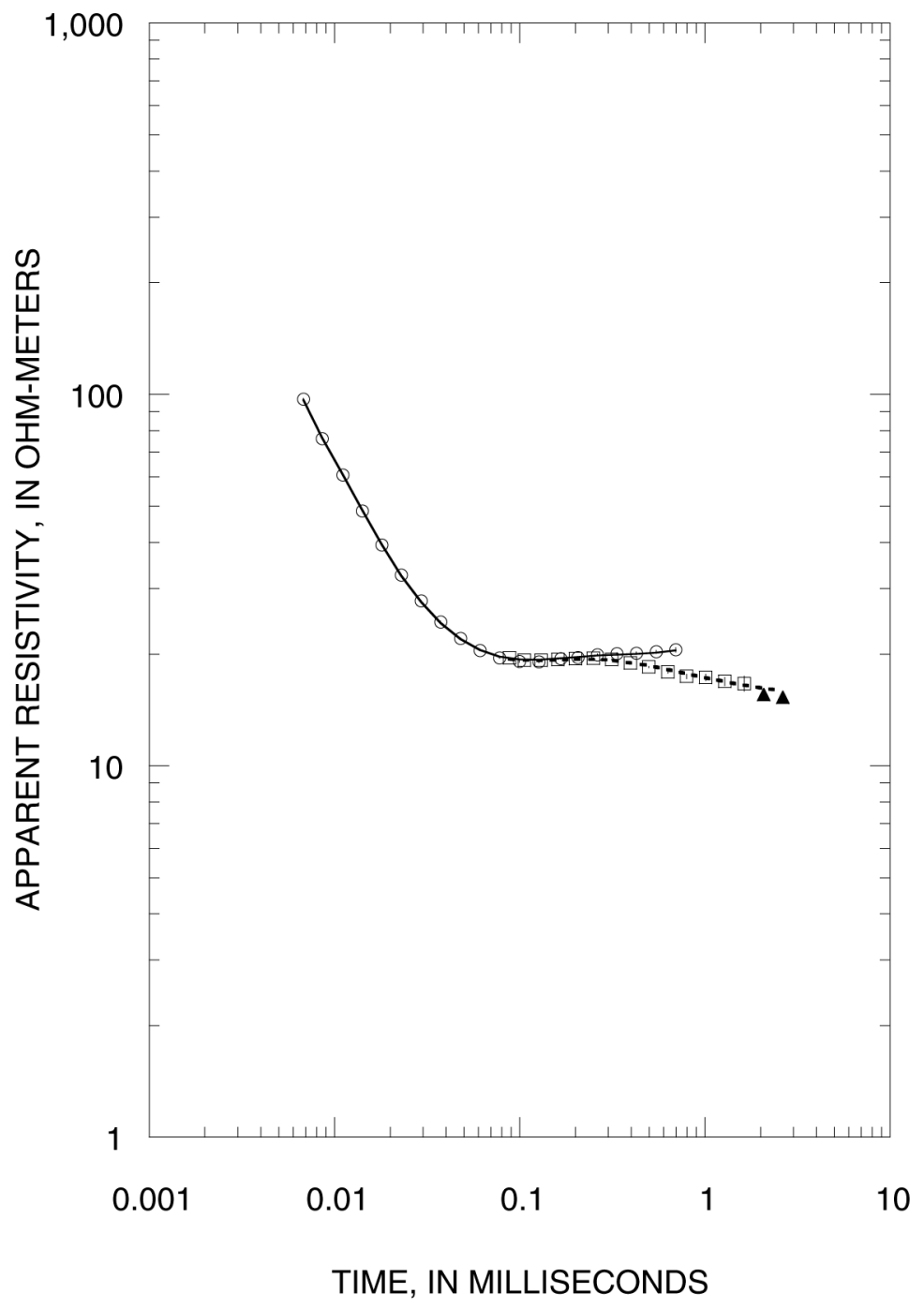

MIA205

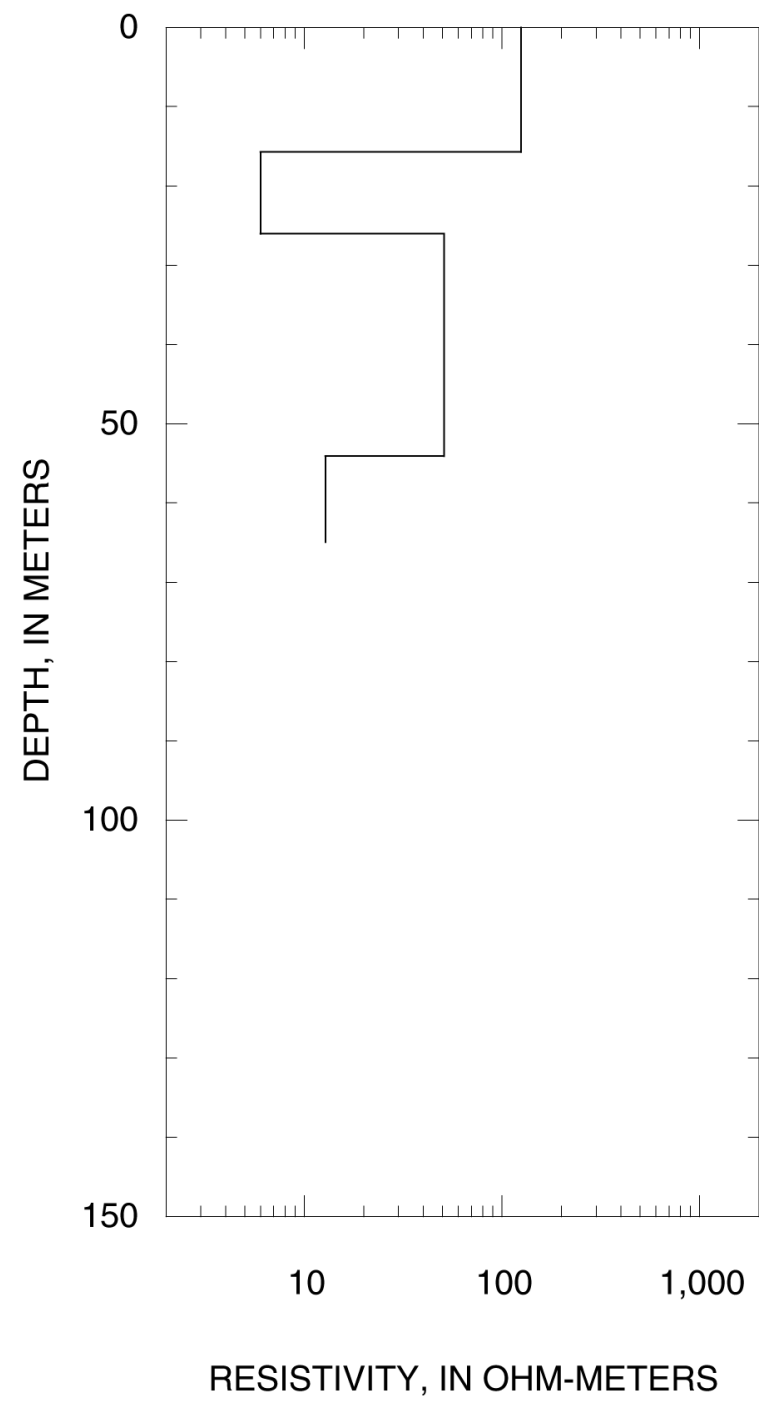

Figure 7-7. Sounding MIA205 
MIA206

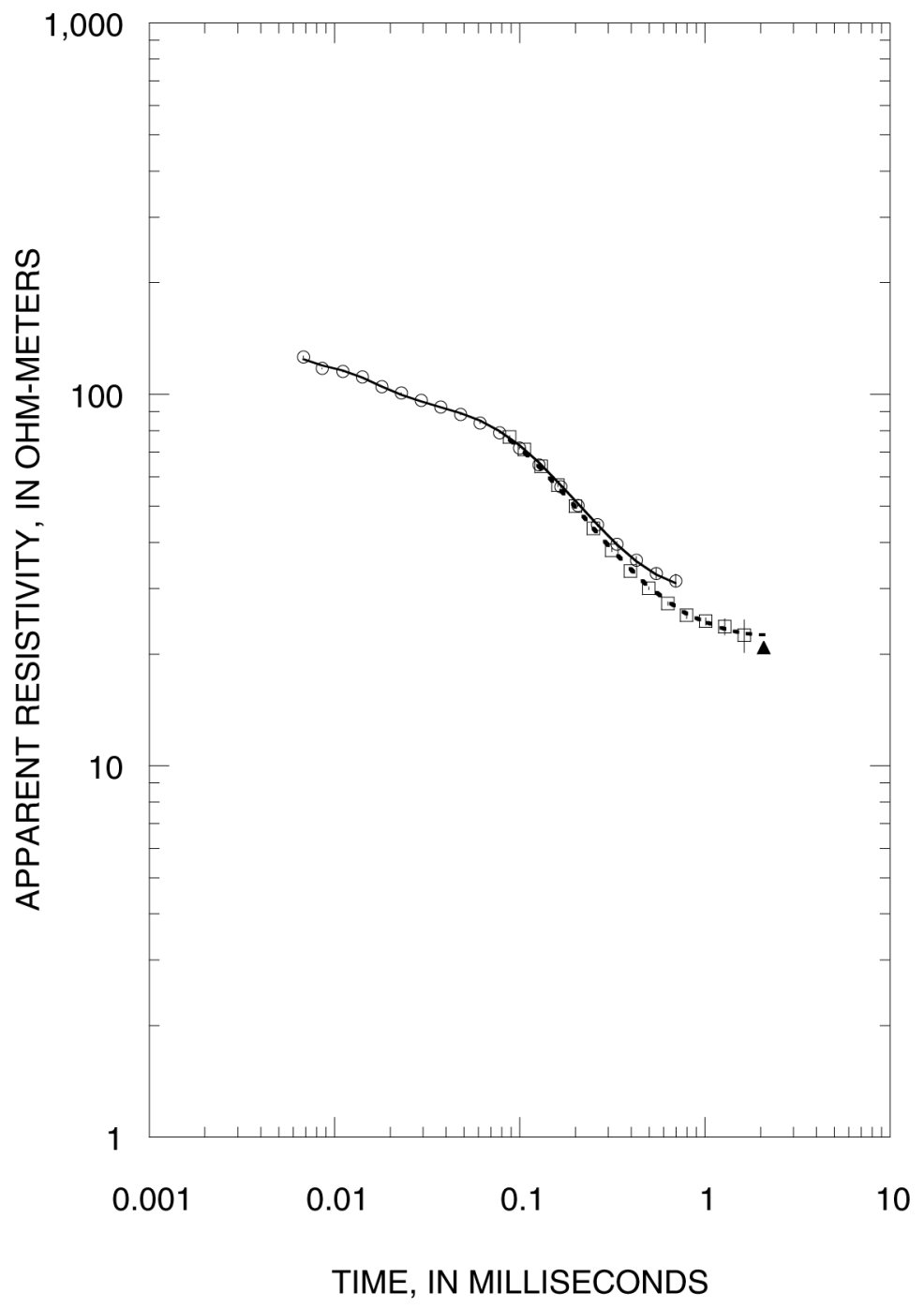

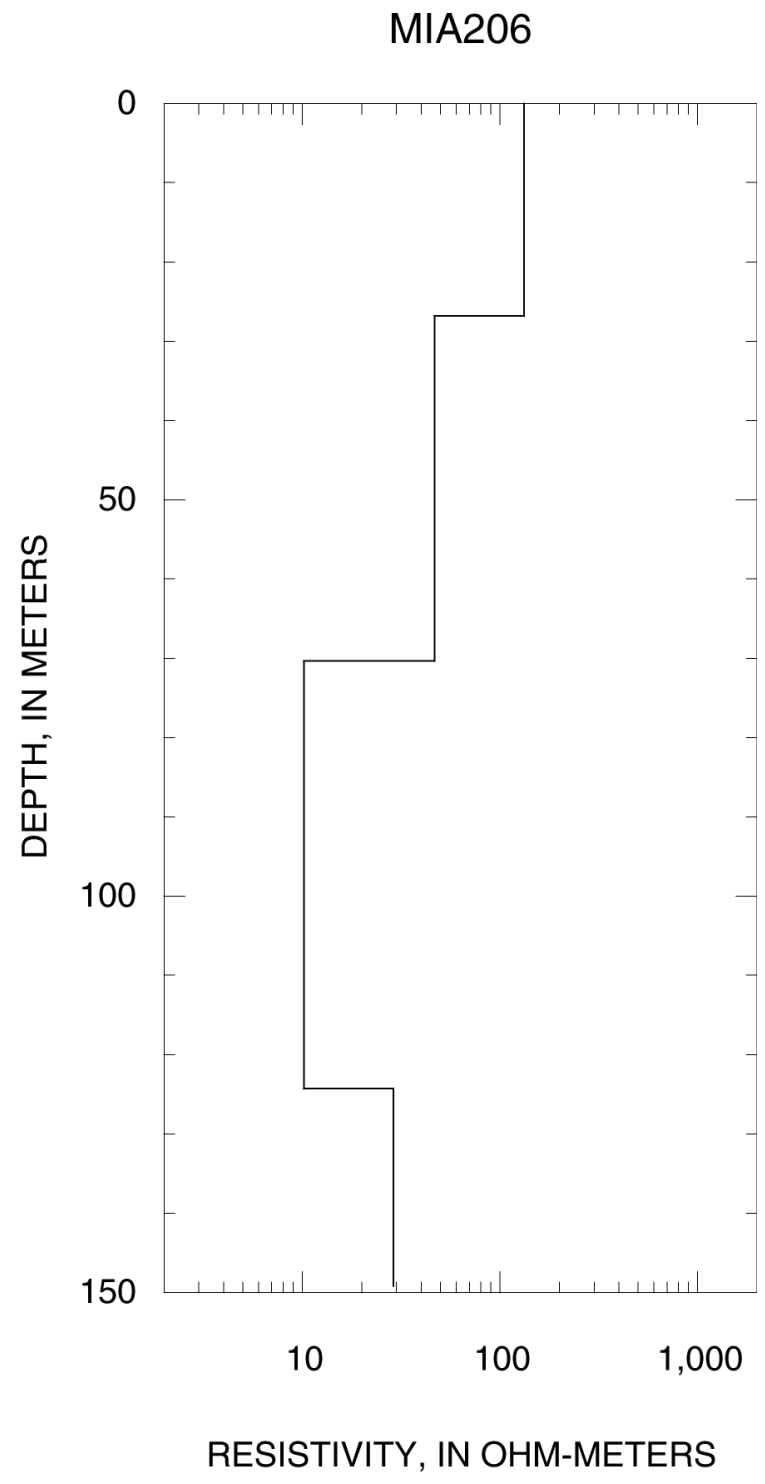

Figure 7-8. Sounding MIA206 

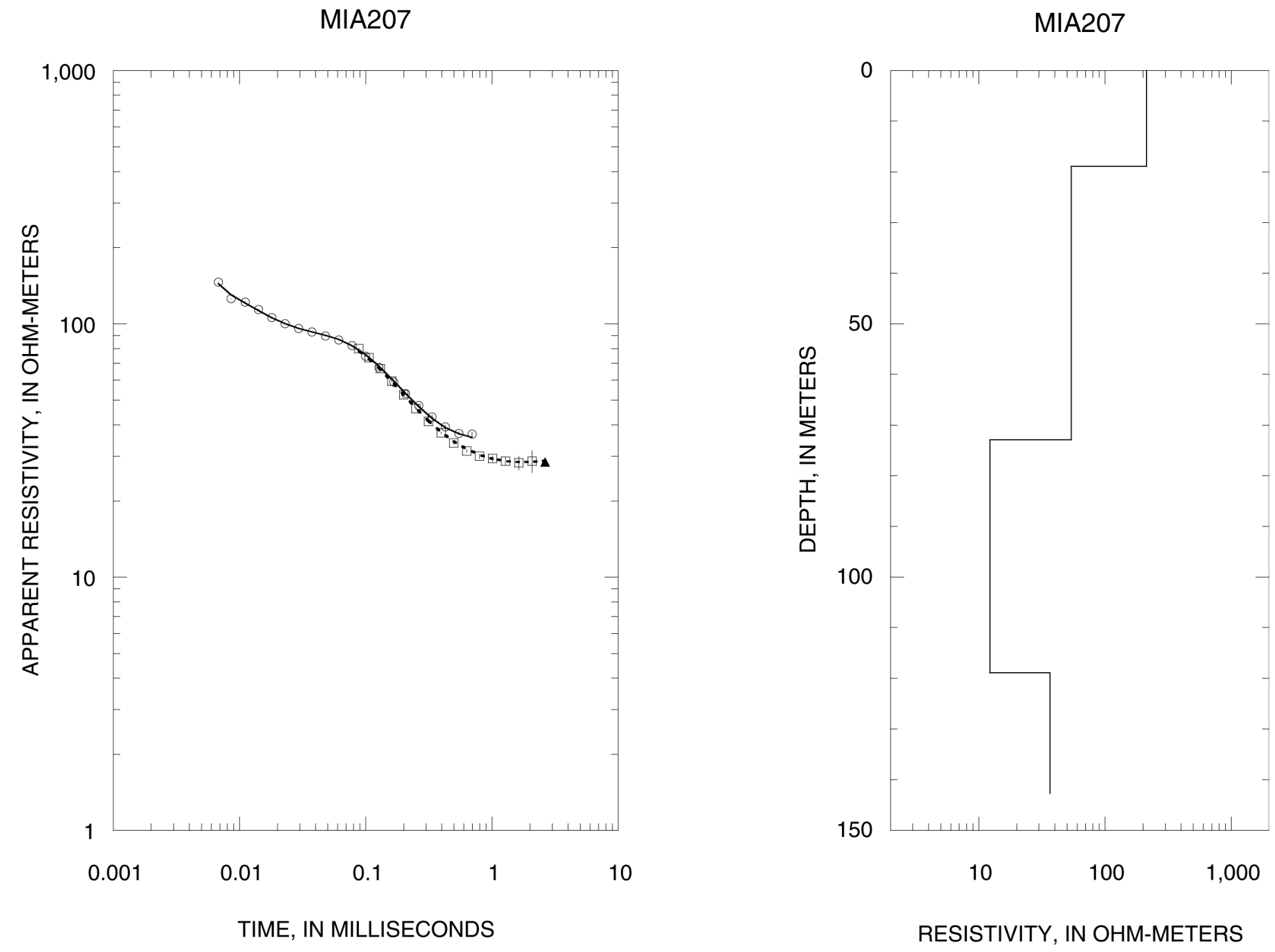

Figure 7-9. Sounding MIA207 
MIA208

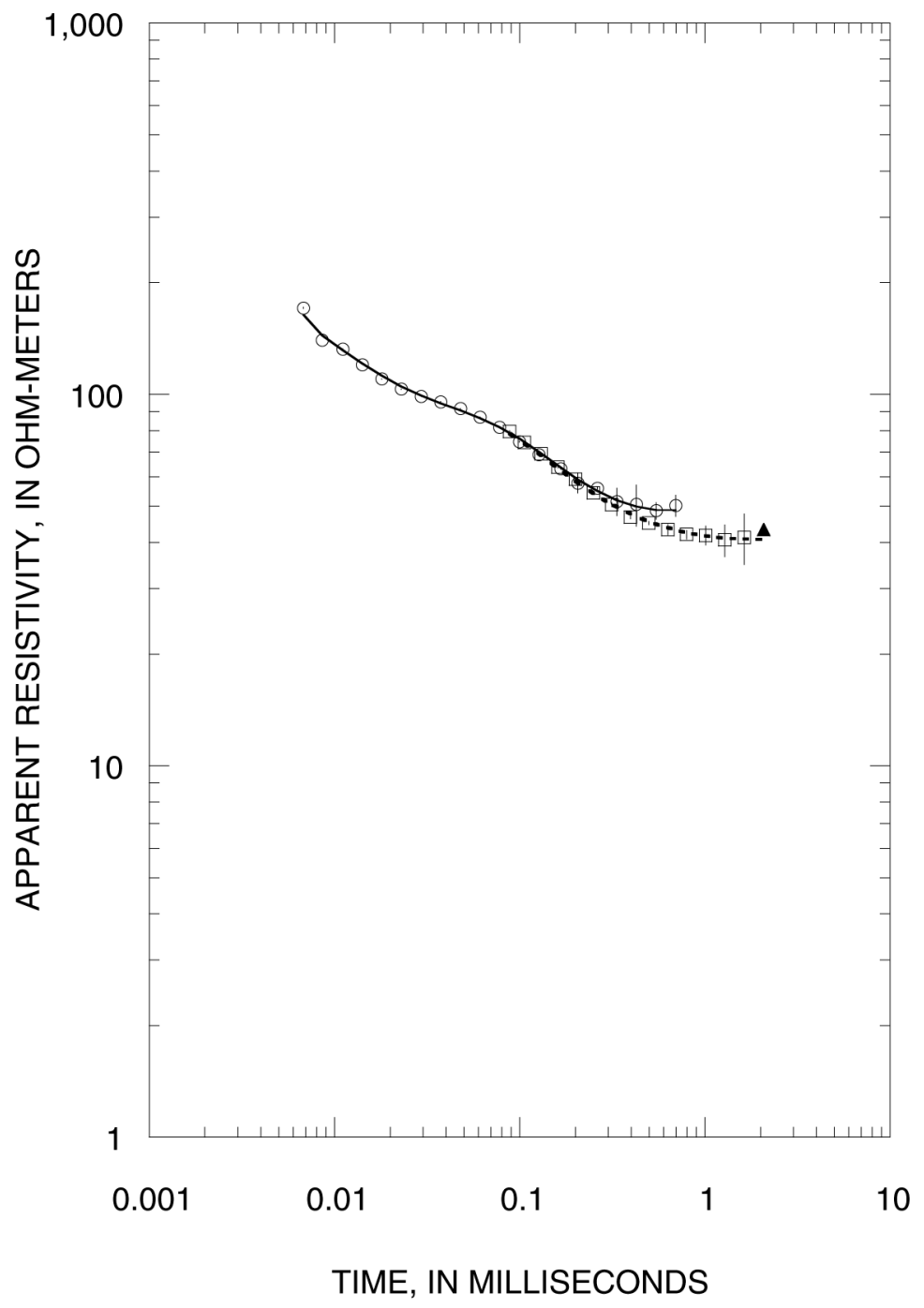

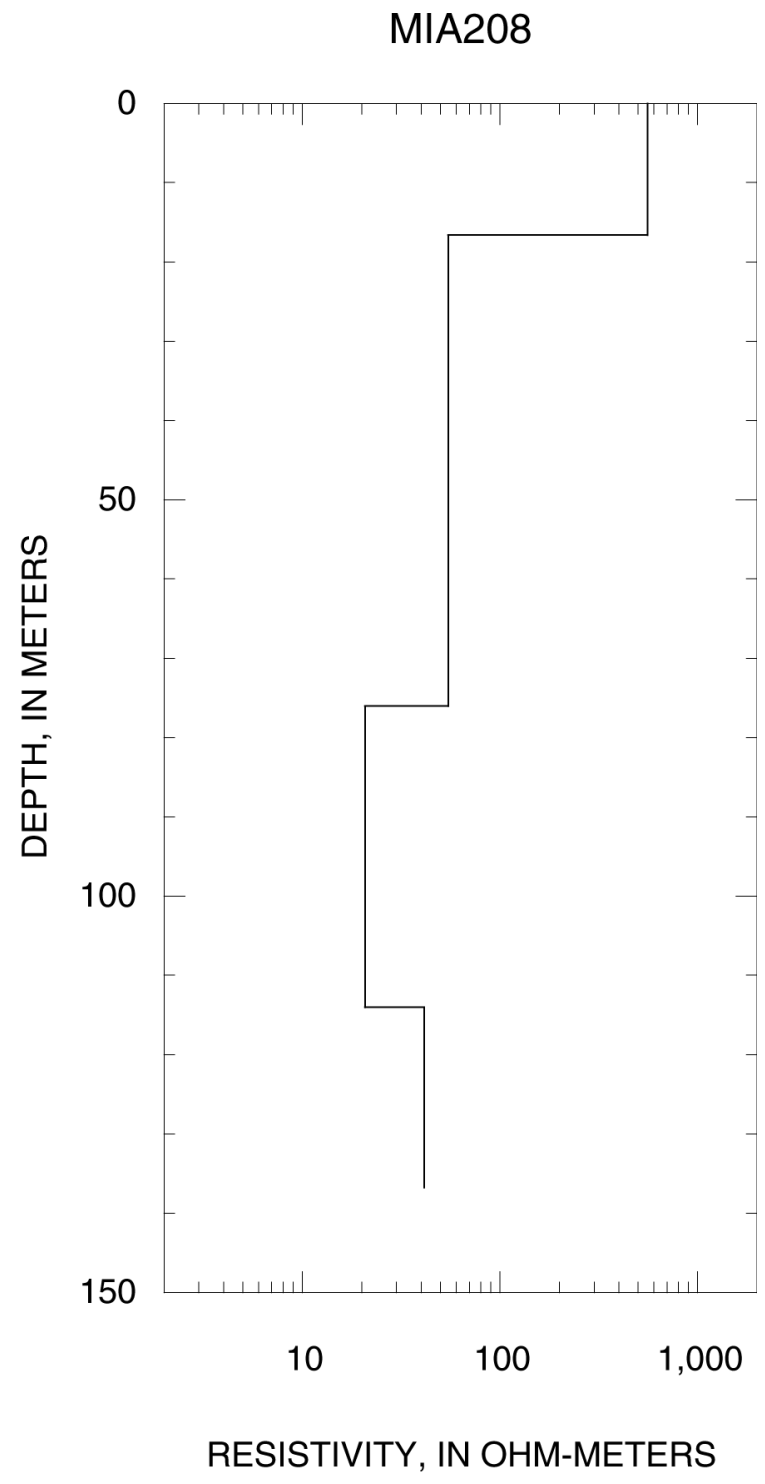

Figure 7-10. Sounding MIA208 
MIA209

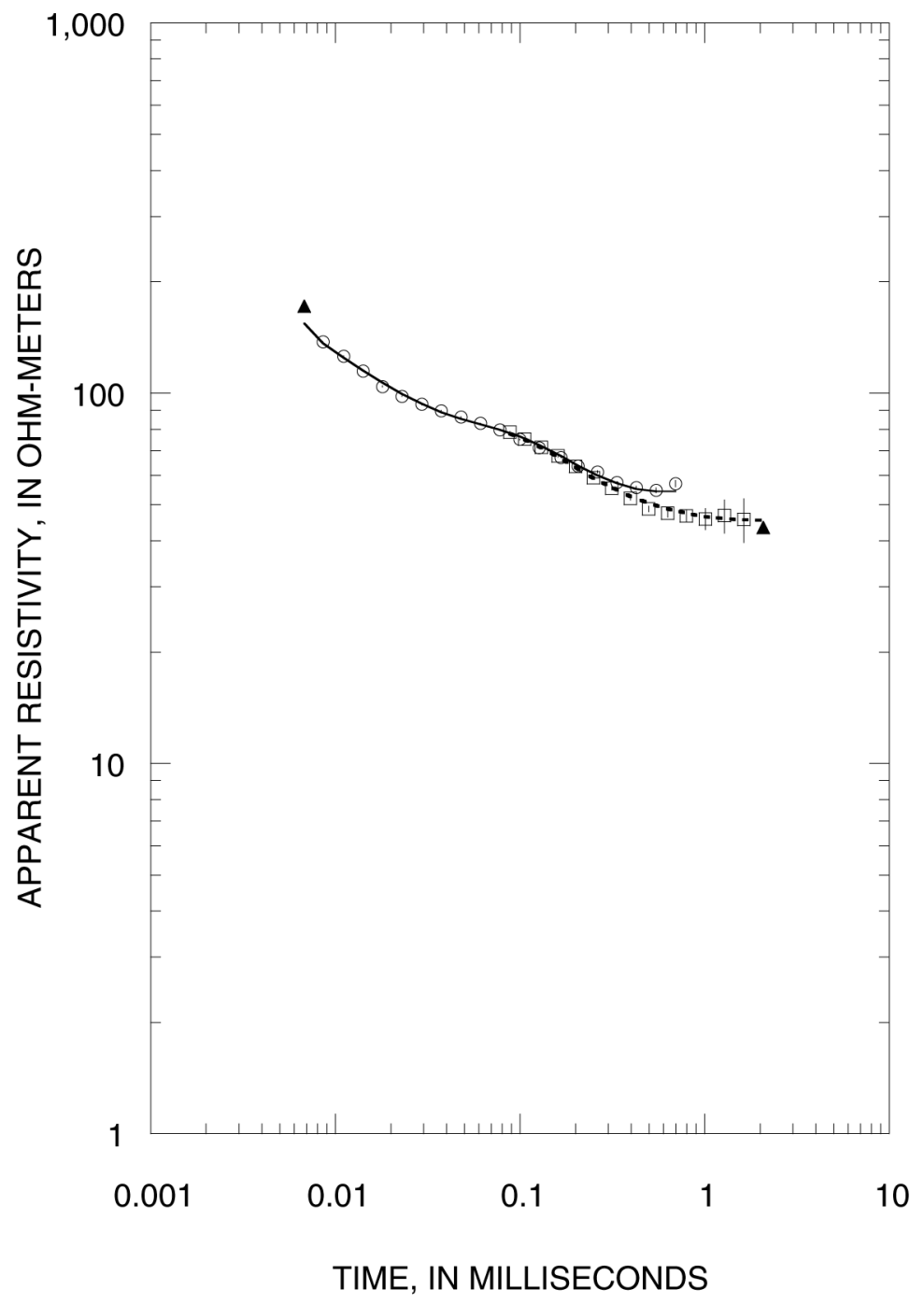

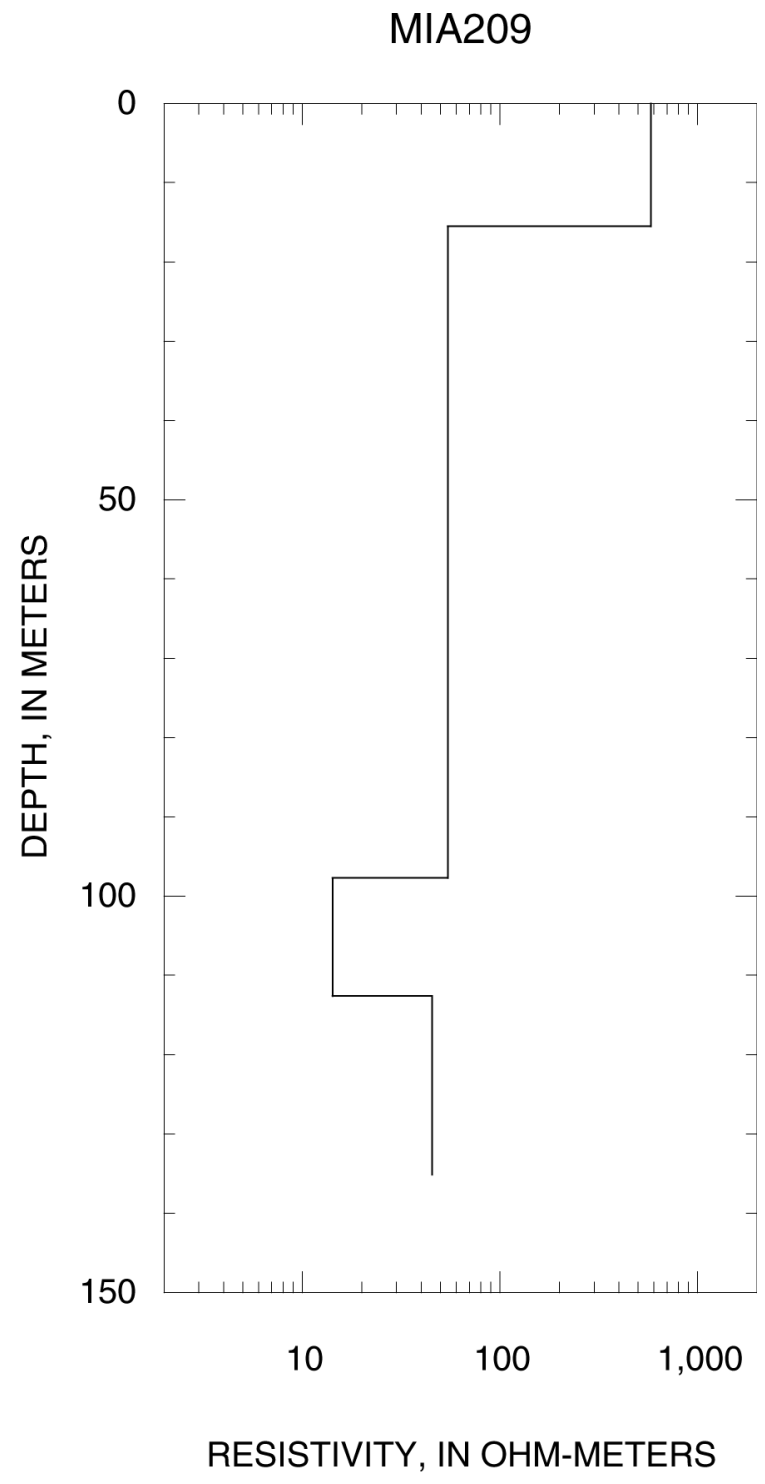

Figure 7-11. Sounding MIA209 
MIA210

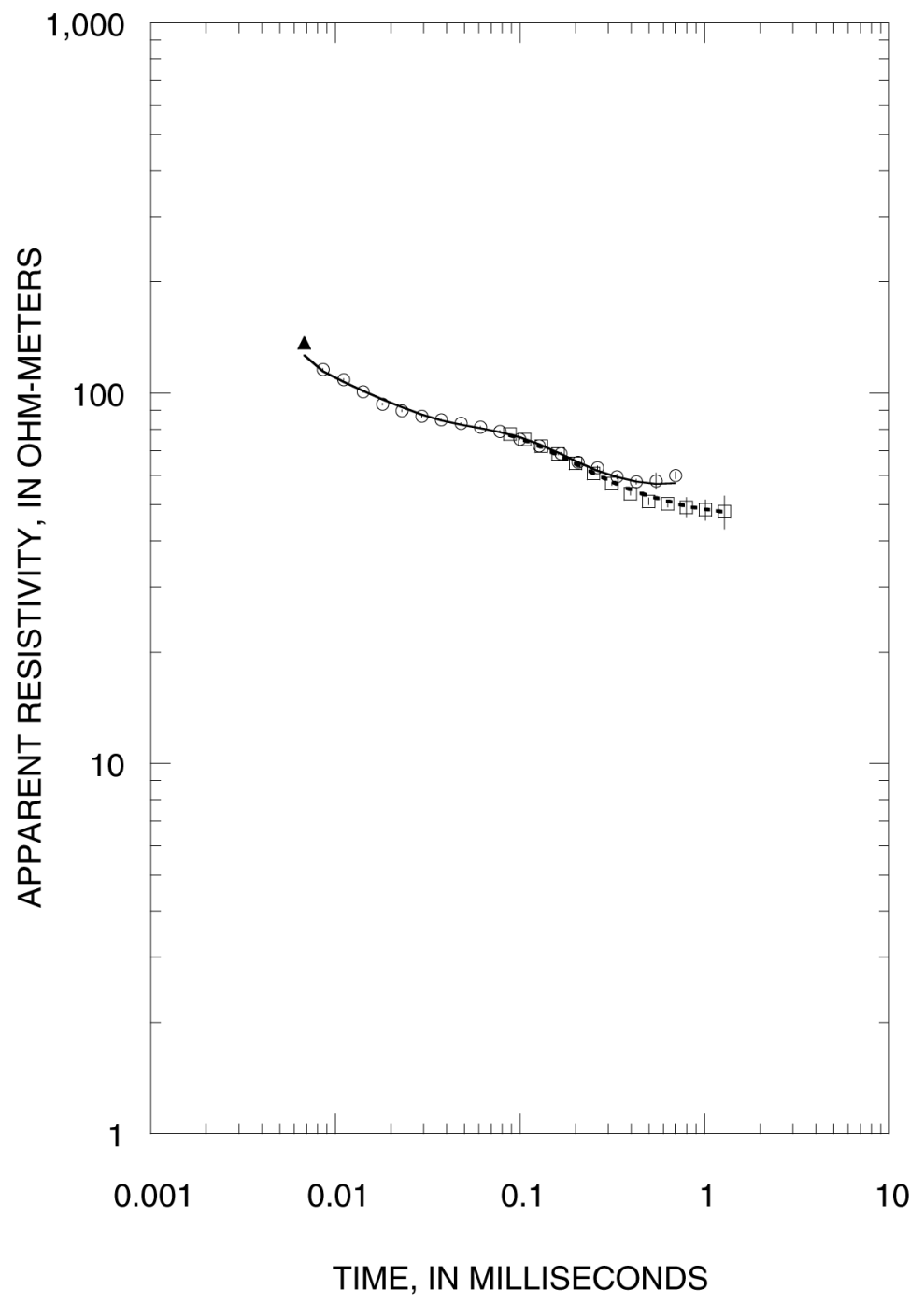

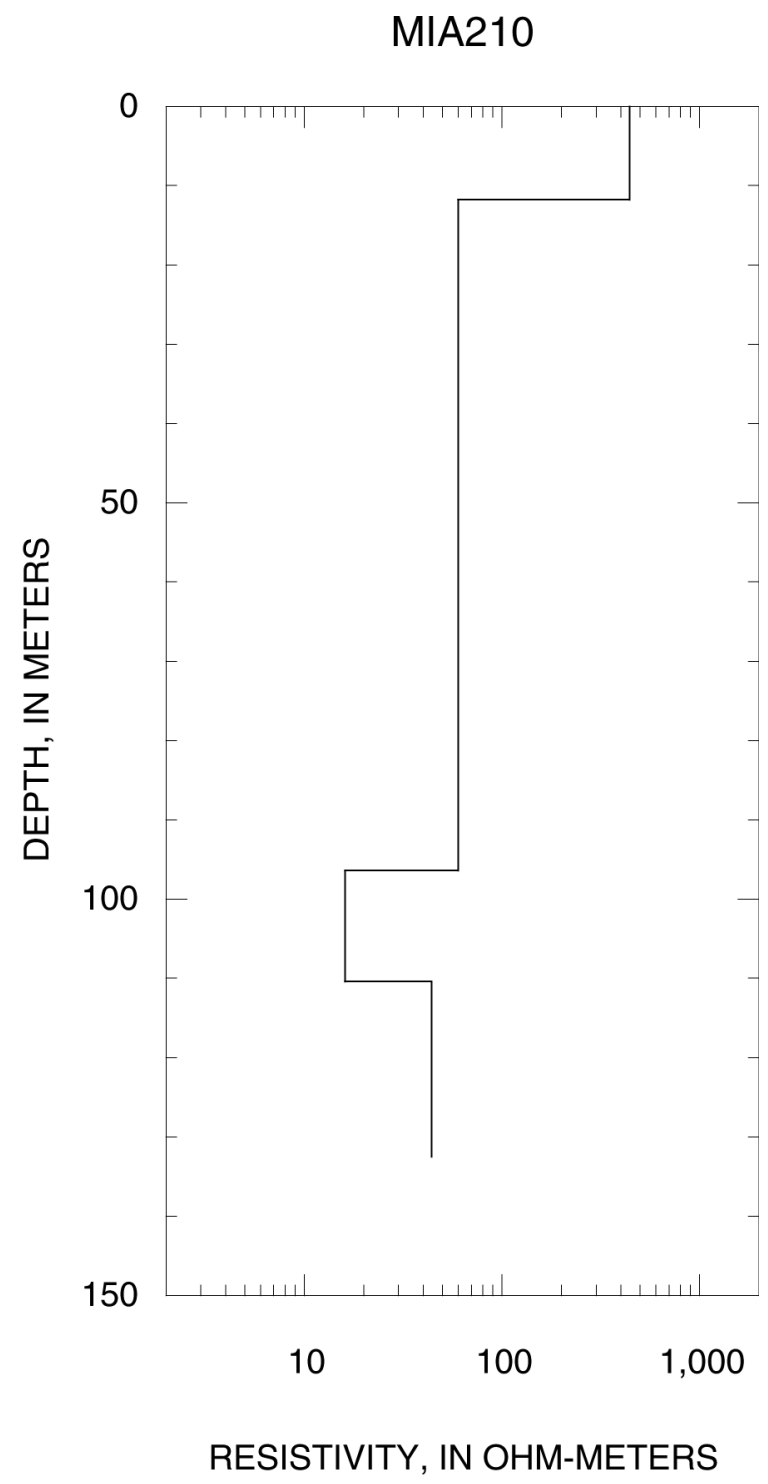

Figure 7-12. Sounding MIA210 
MIA211

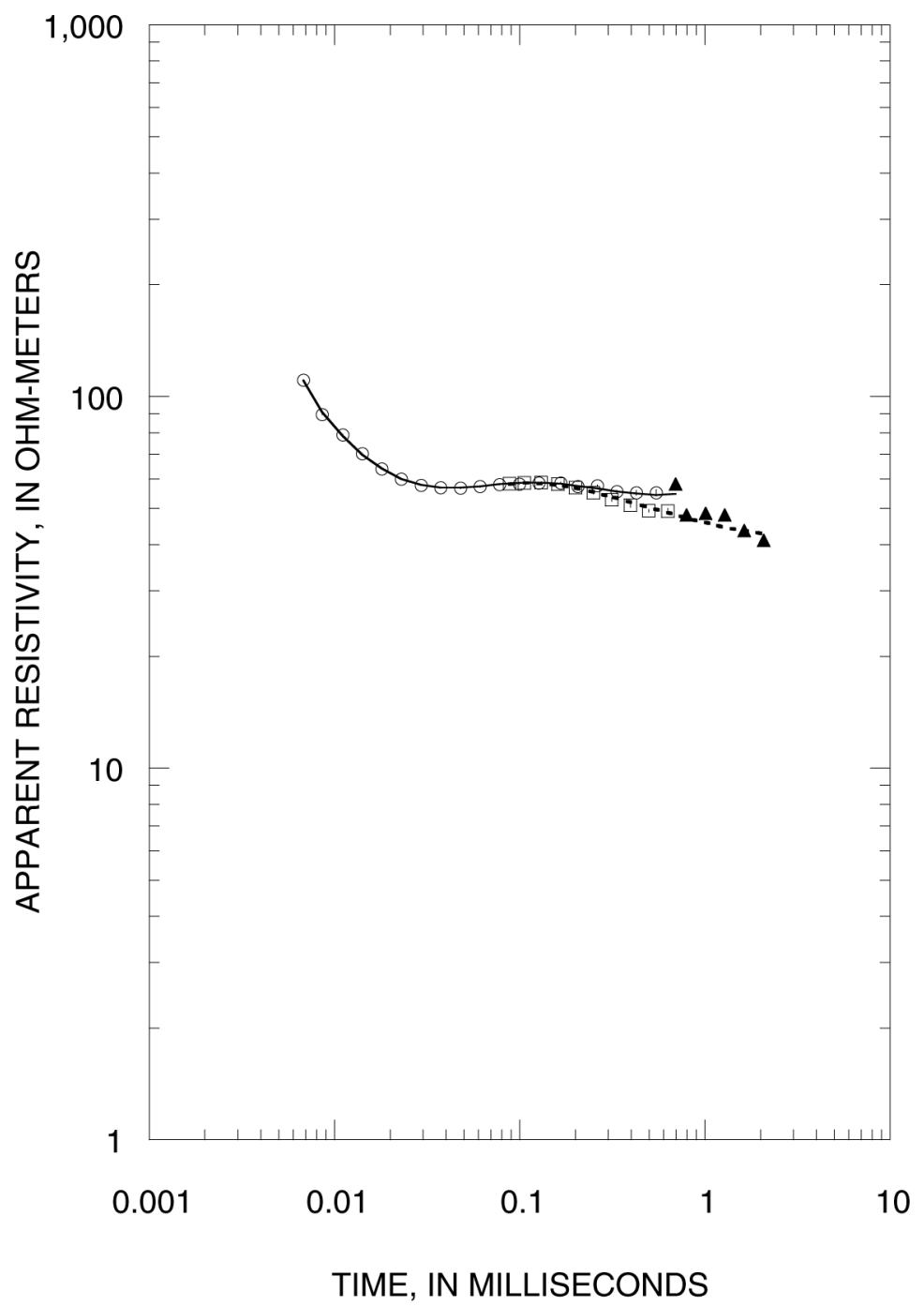

Figure 7-13. Sounding MIA211

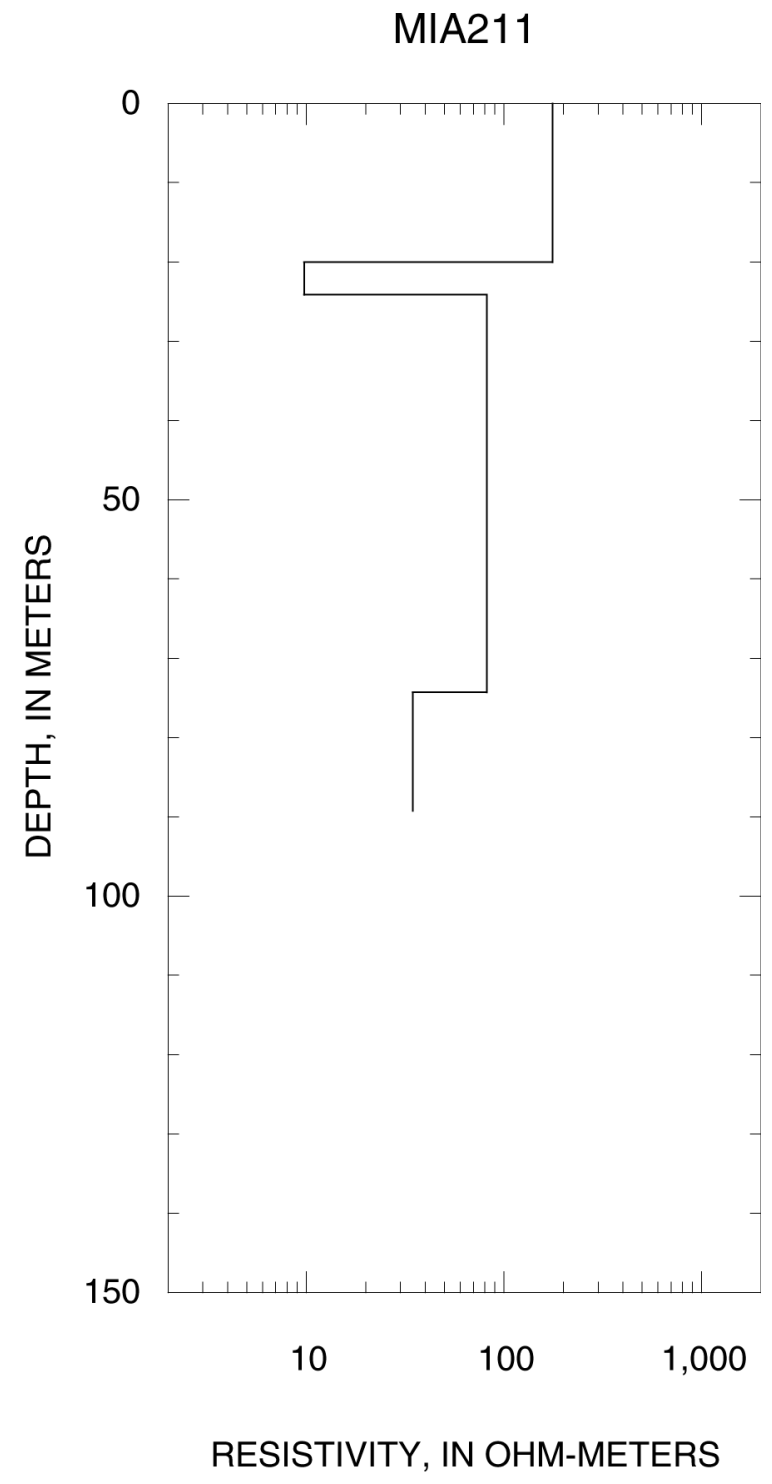


MIA212

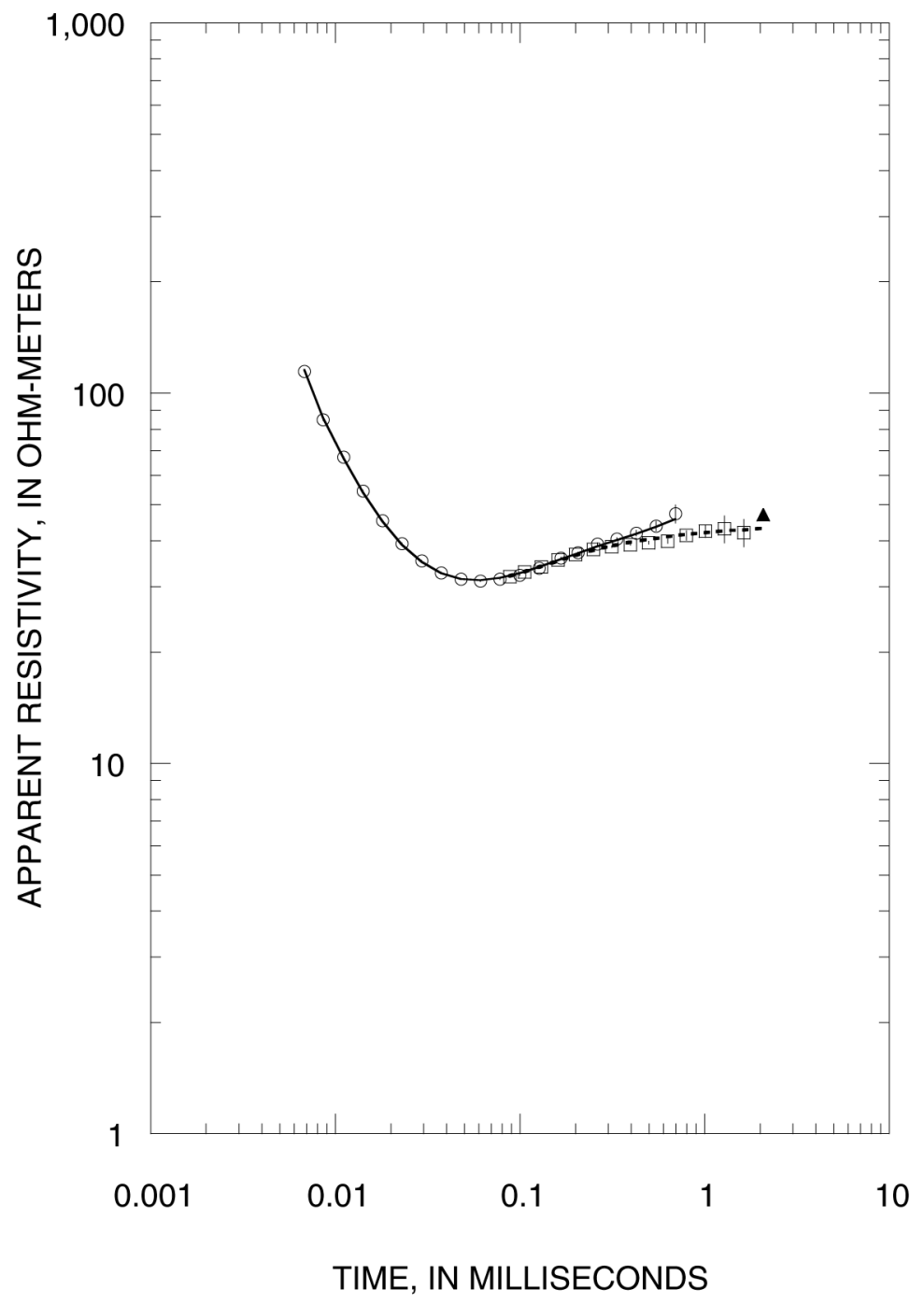

MIA212

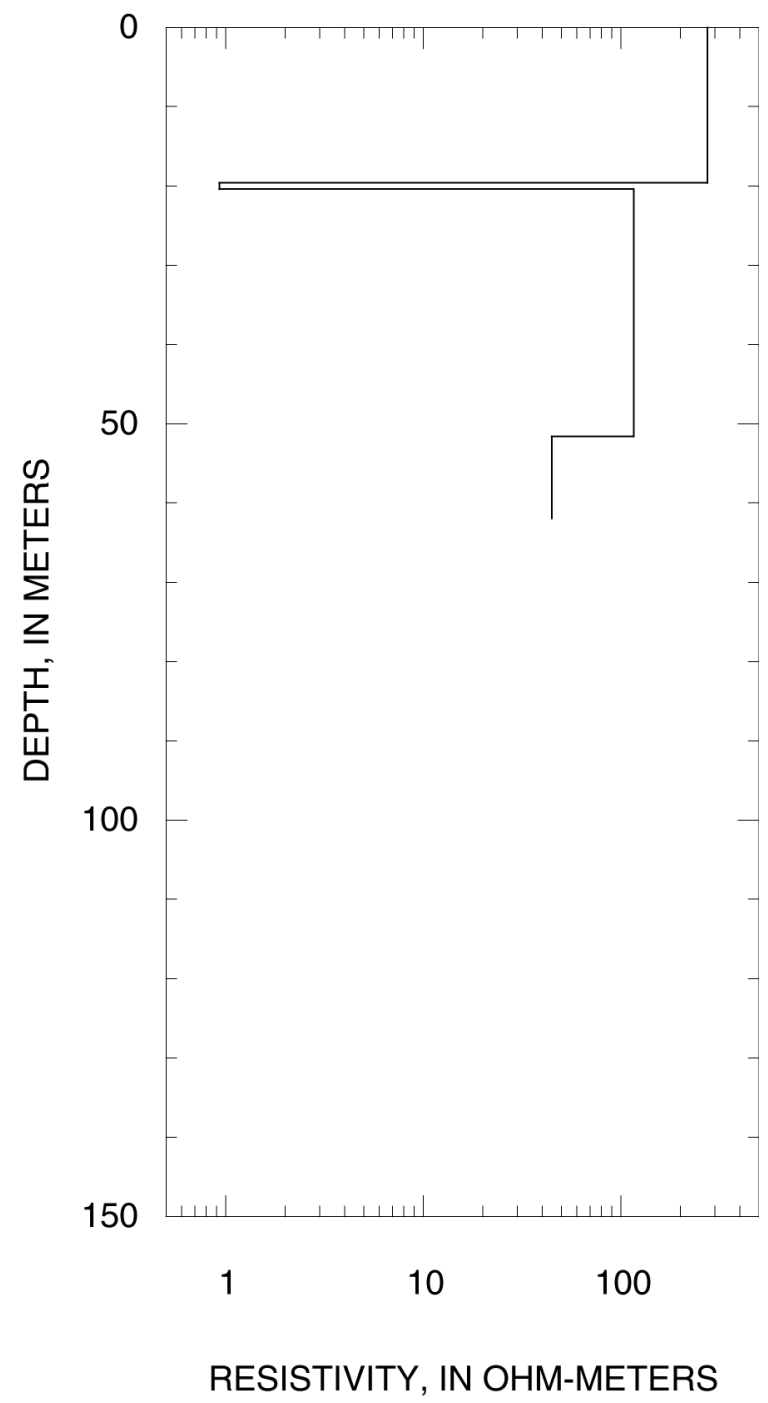

Figure 7-14. Sounding MIA212 

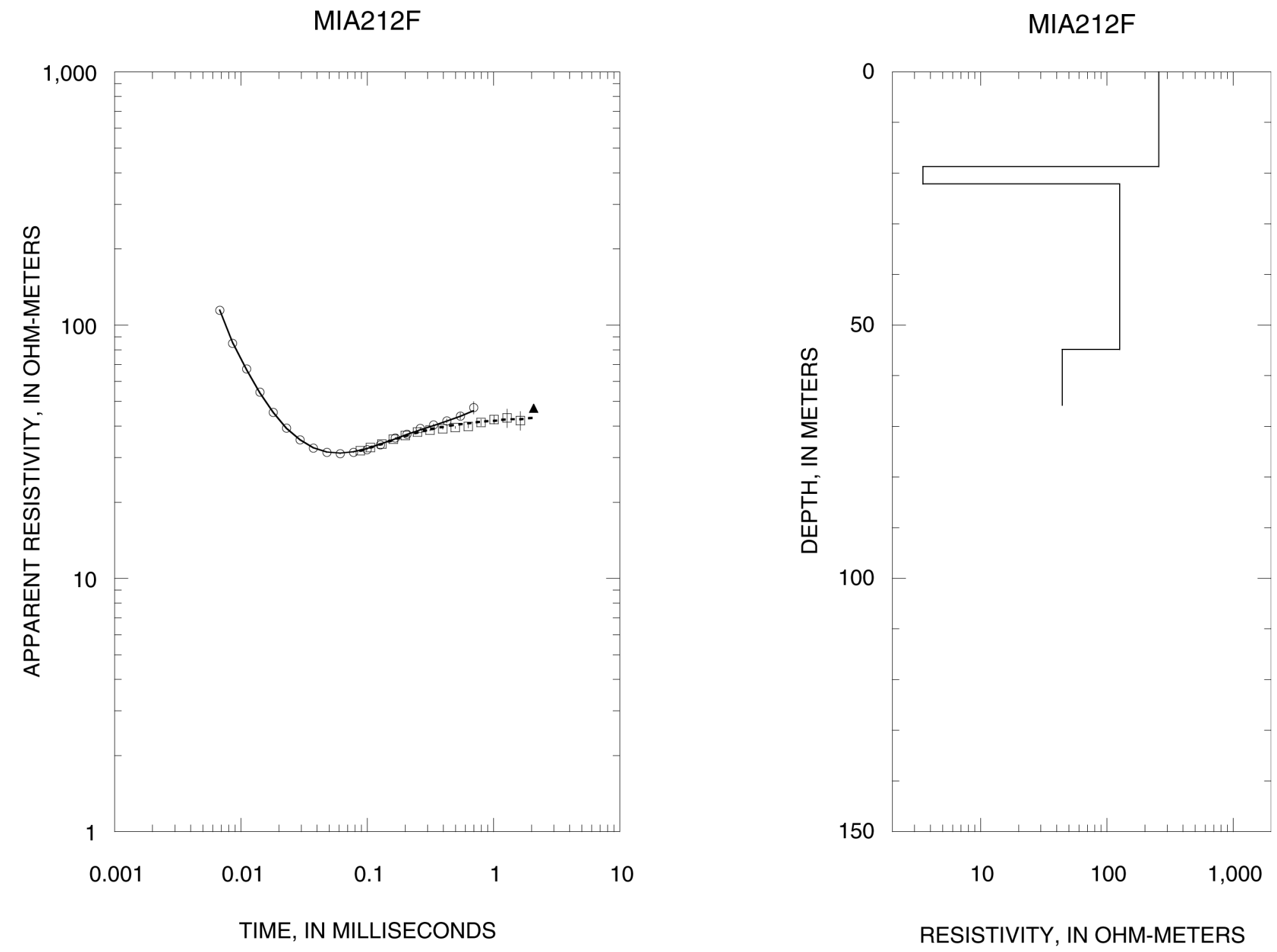

Figure 7-15. Sounding MIA212F 
MIA213

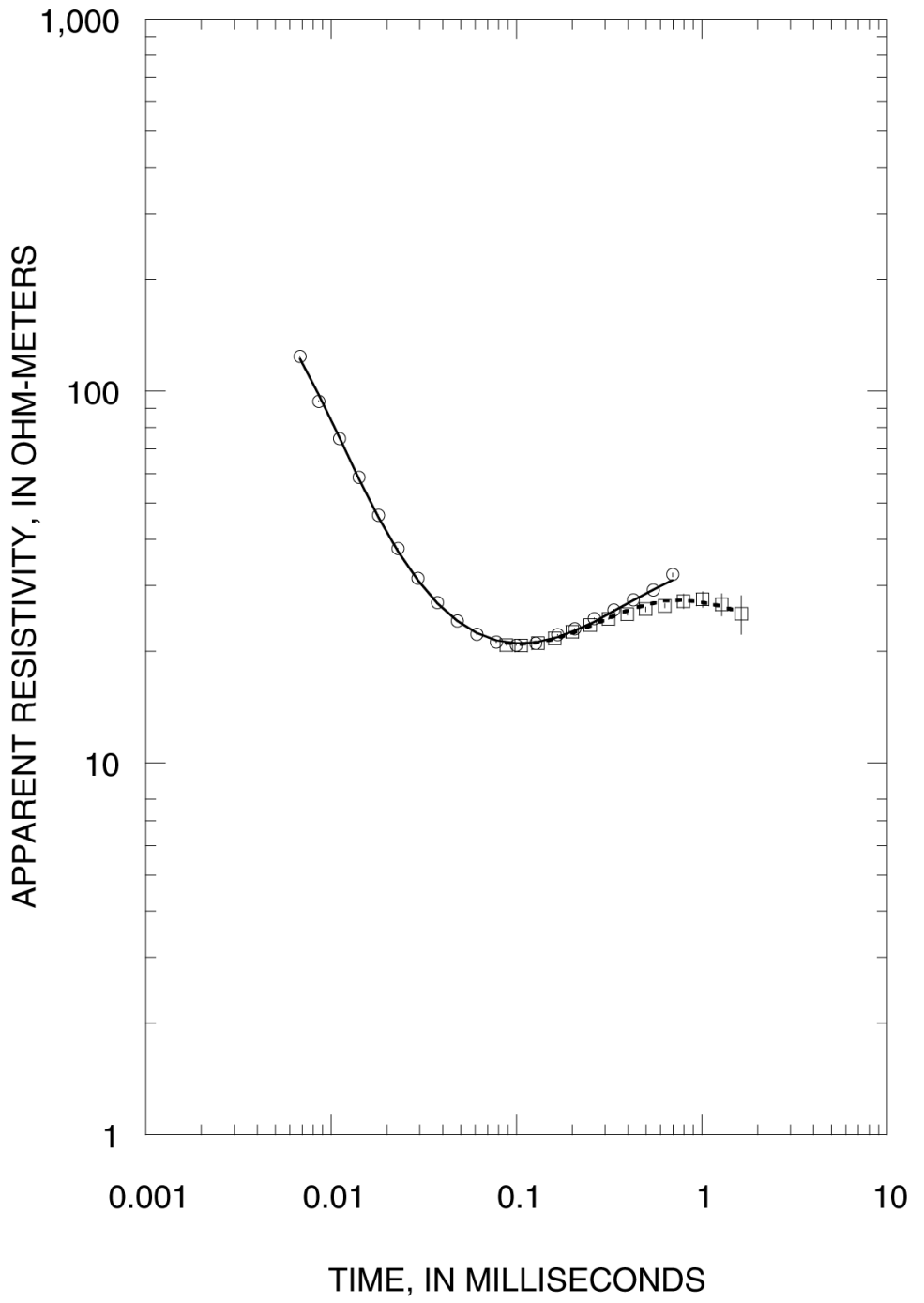

Figure 7-16. Sounding MIA213
MIA213

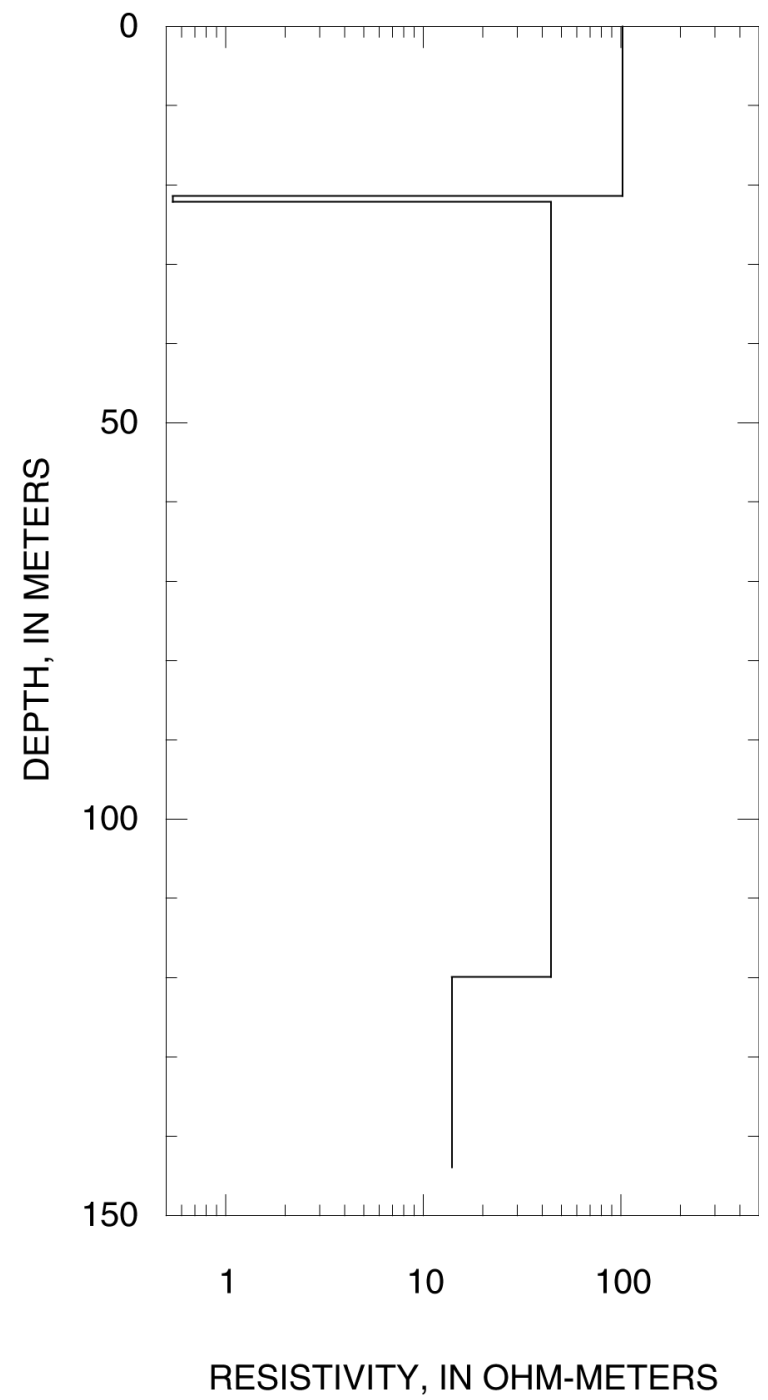



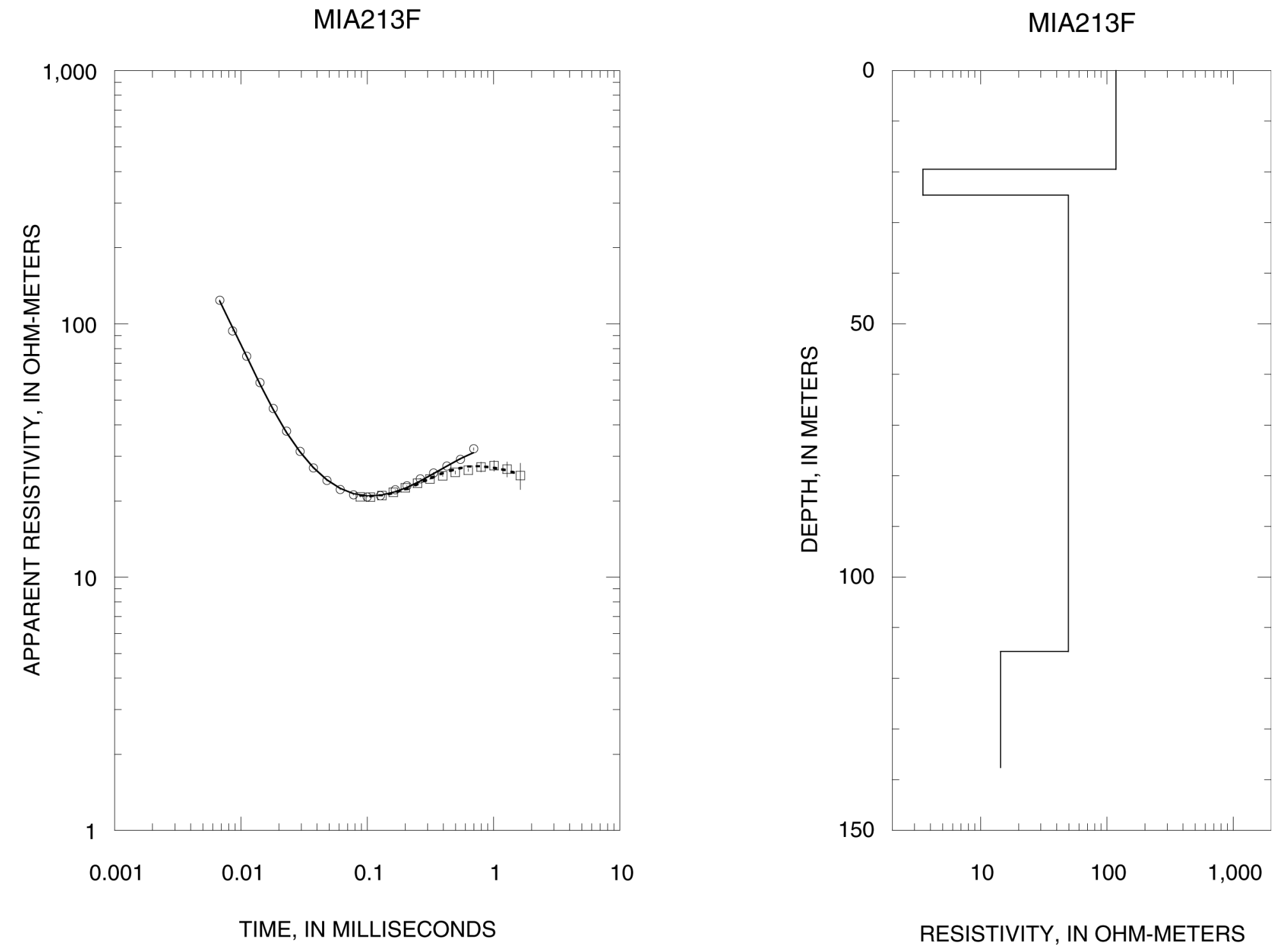

Figure 7-17. Sounding MIA213F 
MIA214

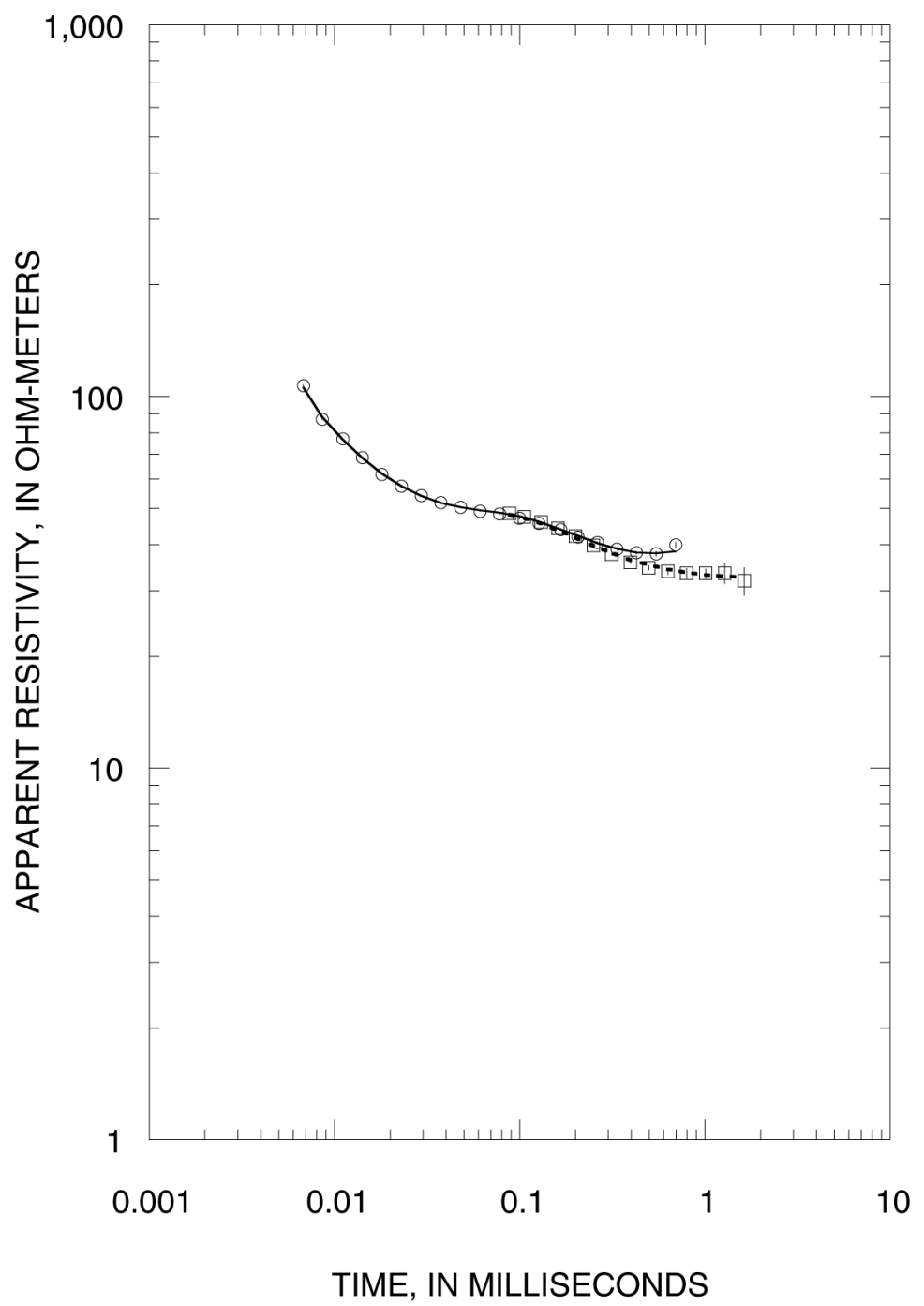

Figure 7-18. Sounding MIA214

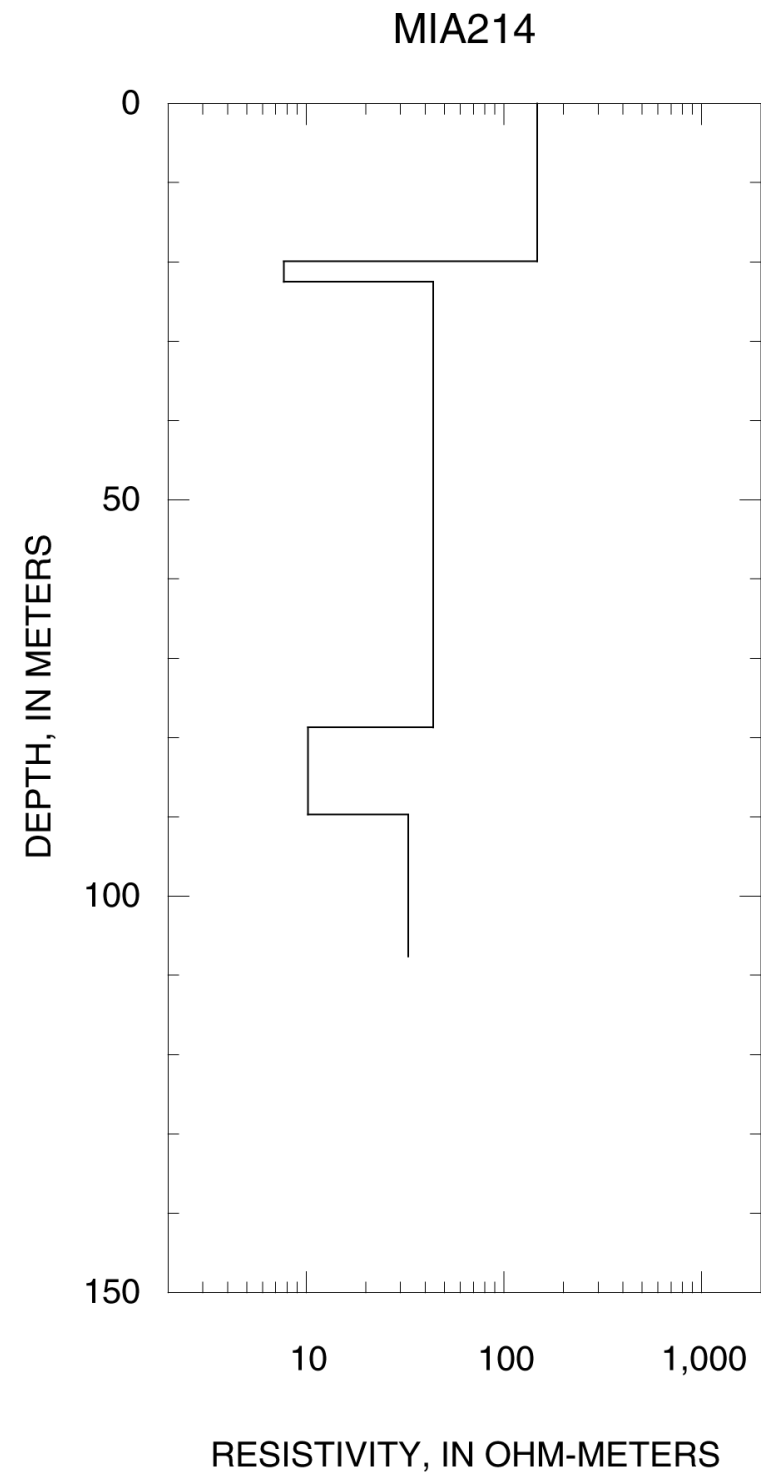


MIA215

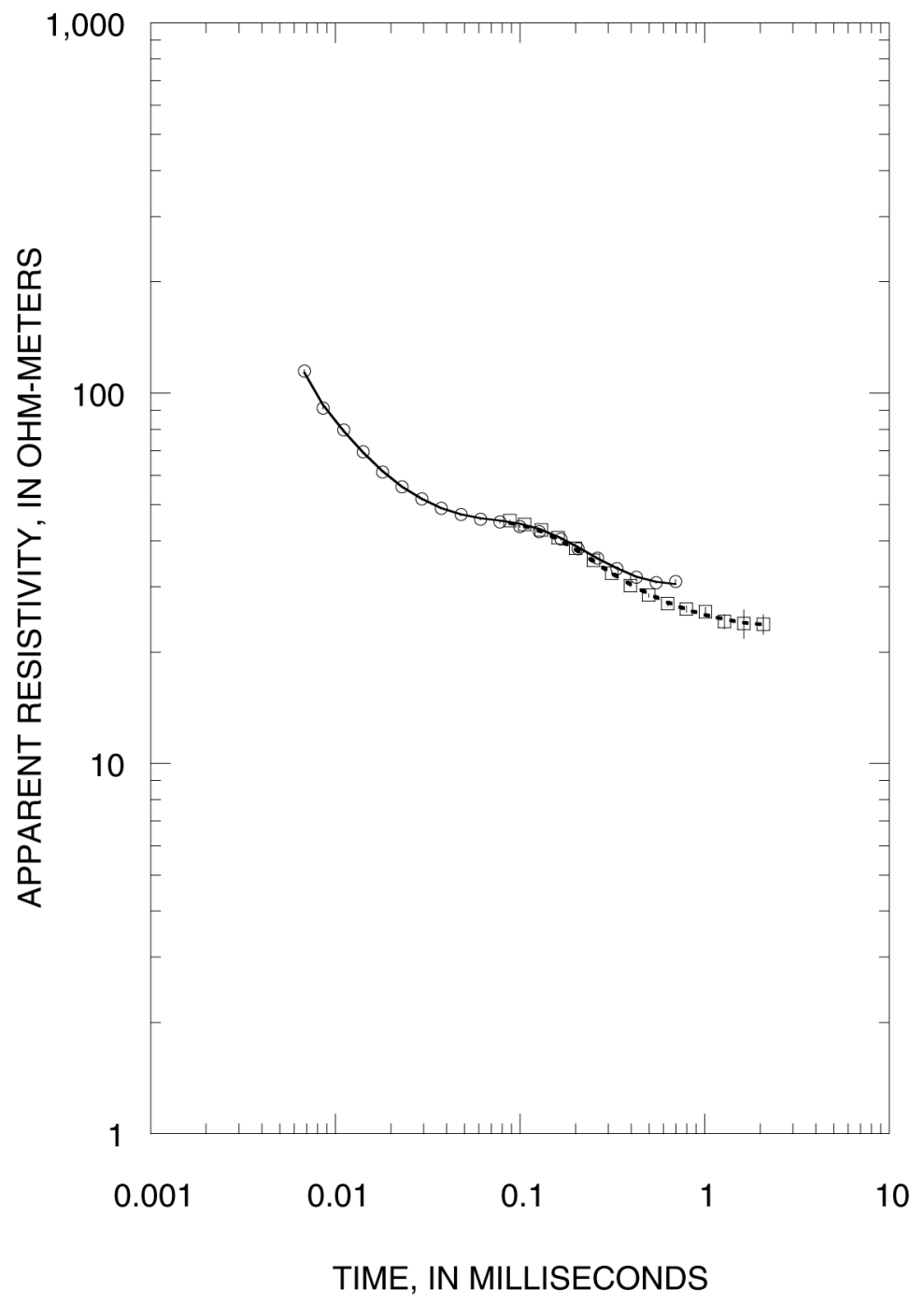

MIA215

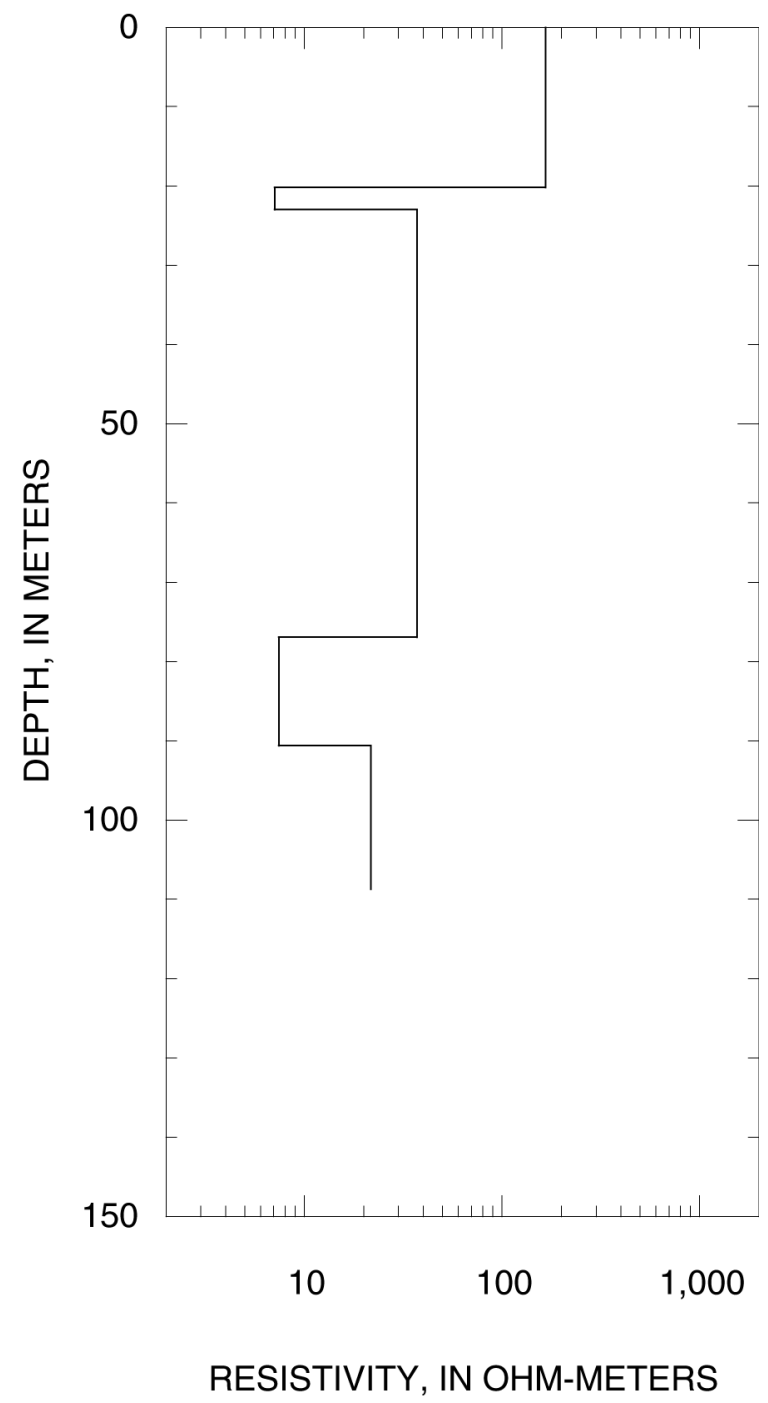

Figure 7-19. Sounding MIA215 
MIA216

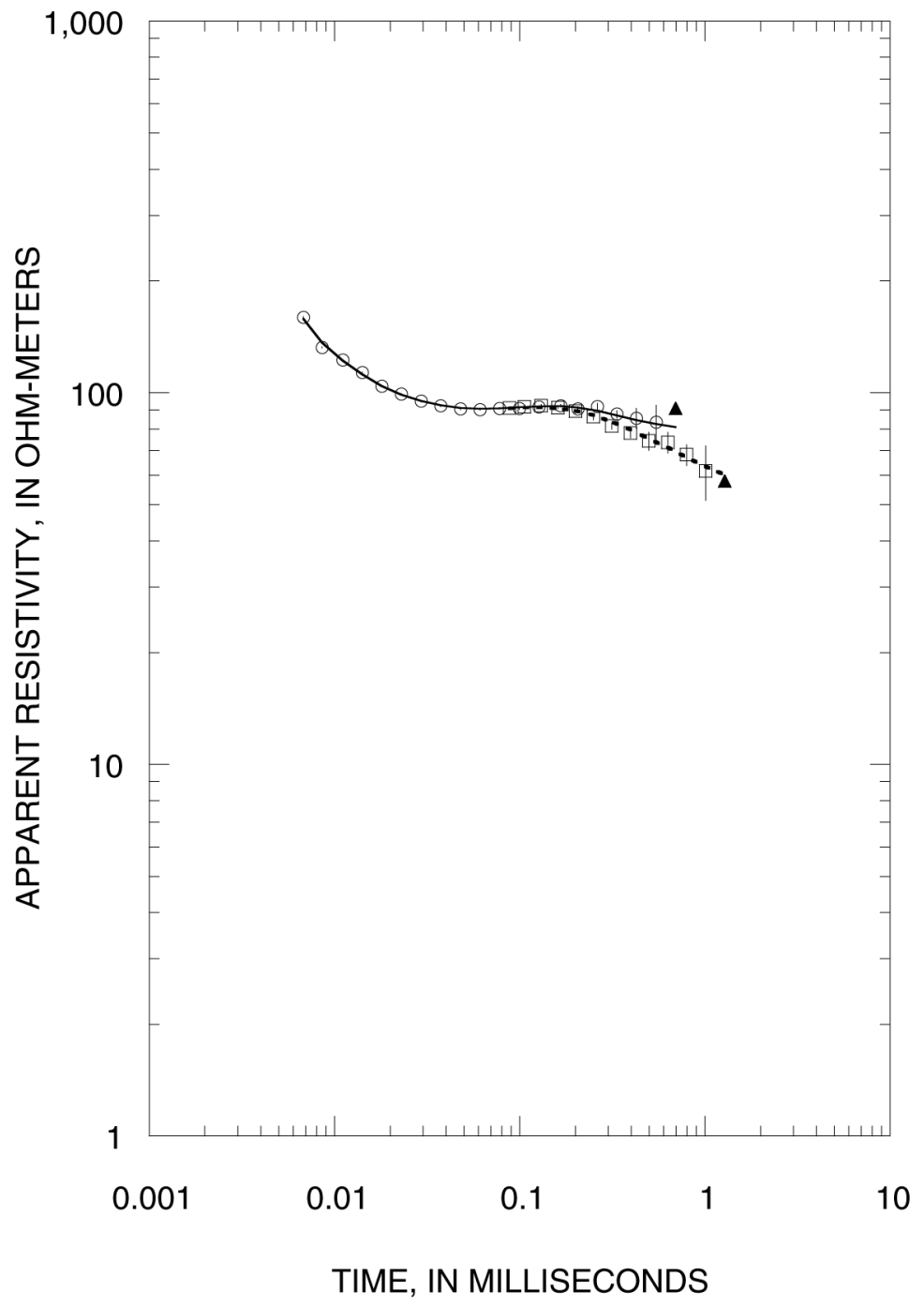

Figure 7-20. Sounding MIA216

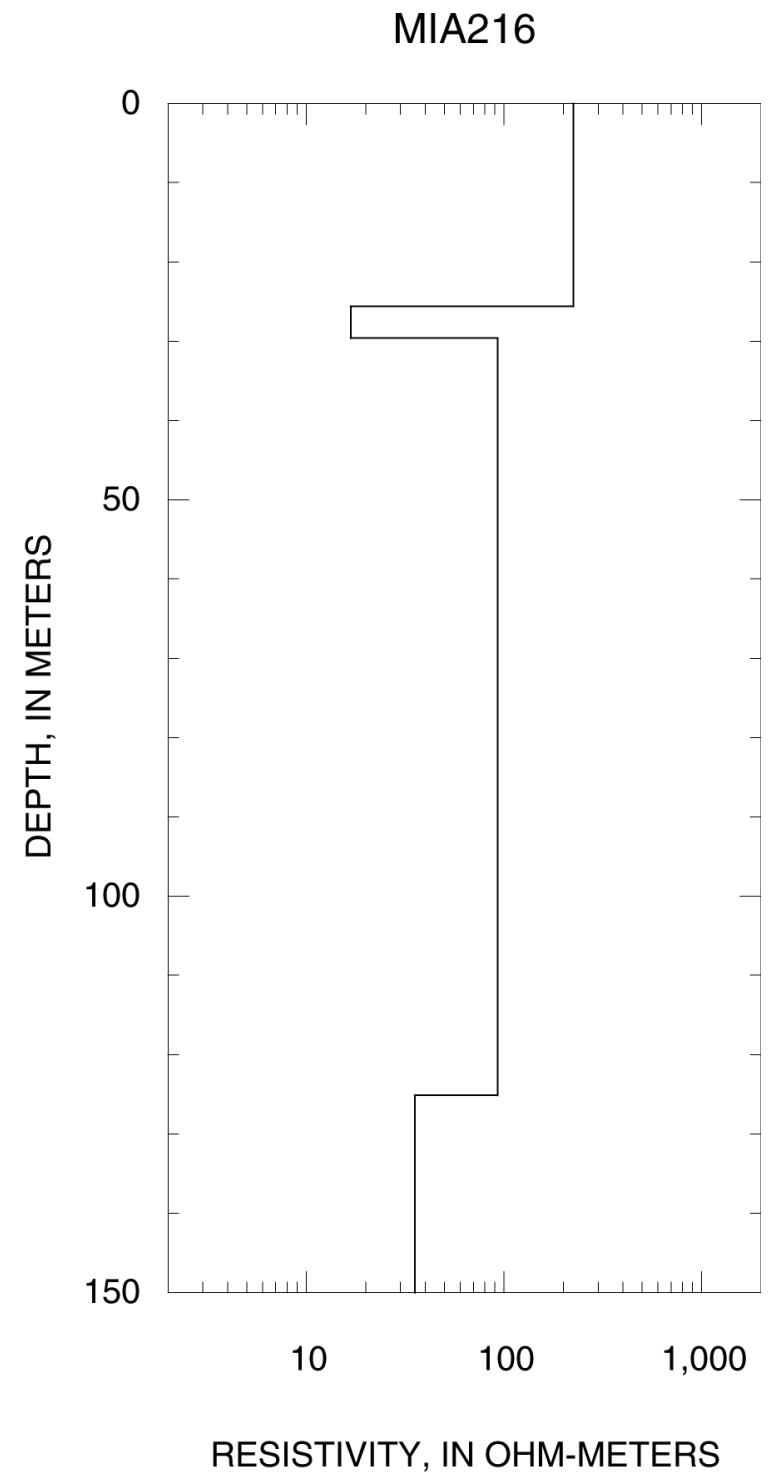



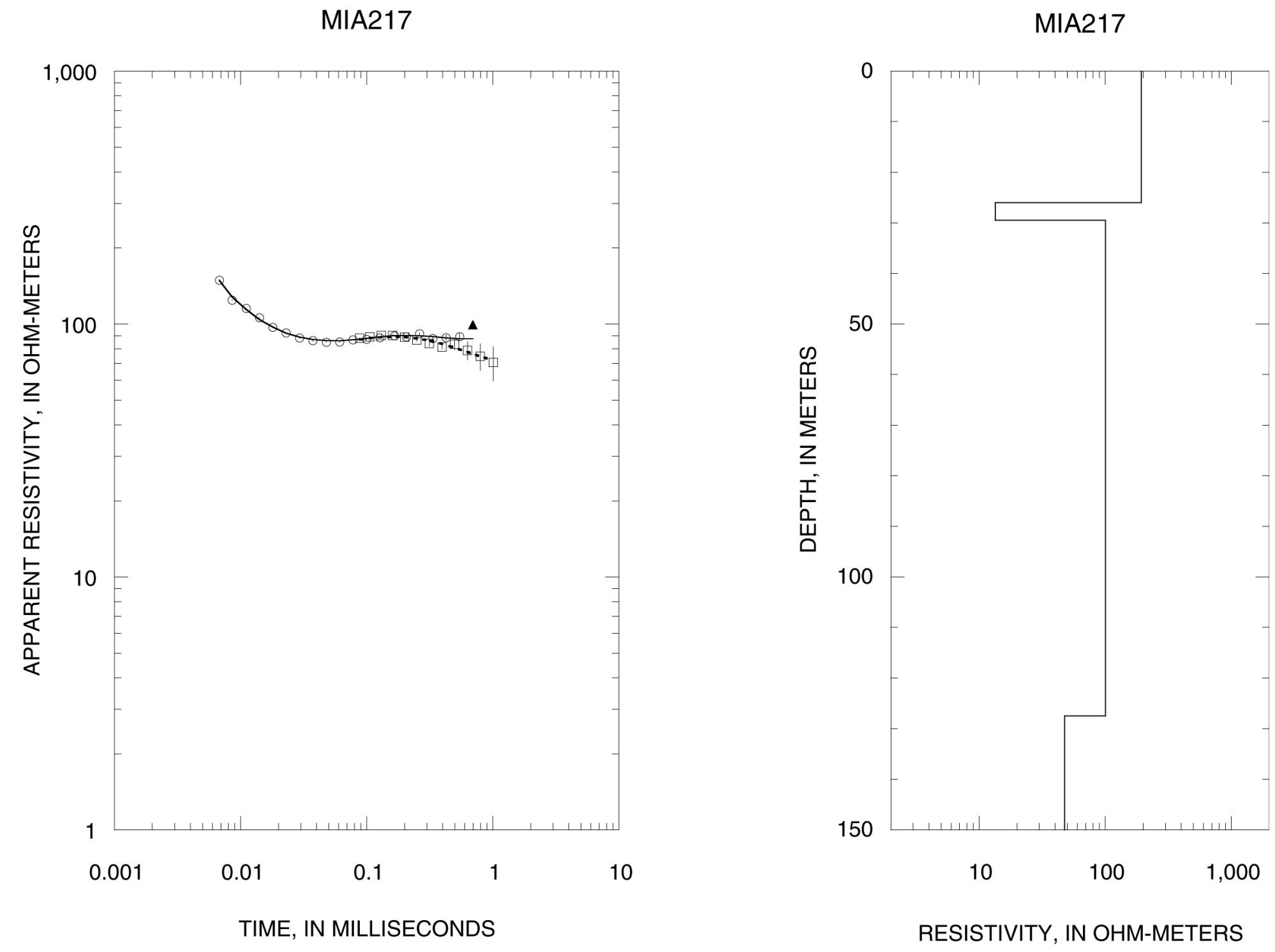

Figure 7-21. Sounding MIA217 
MIA218

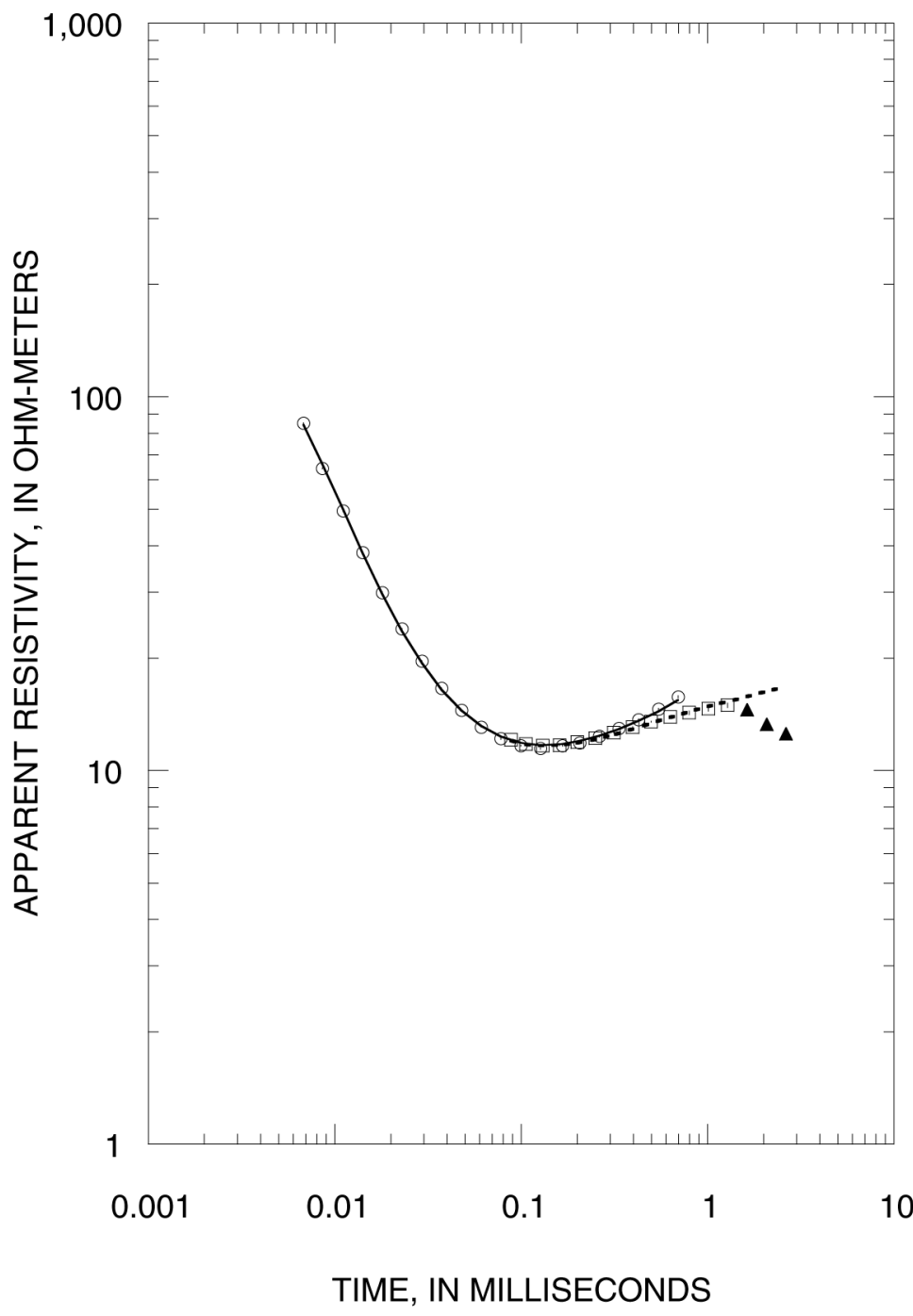

MIA218

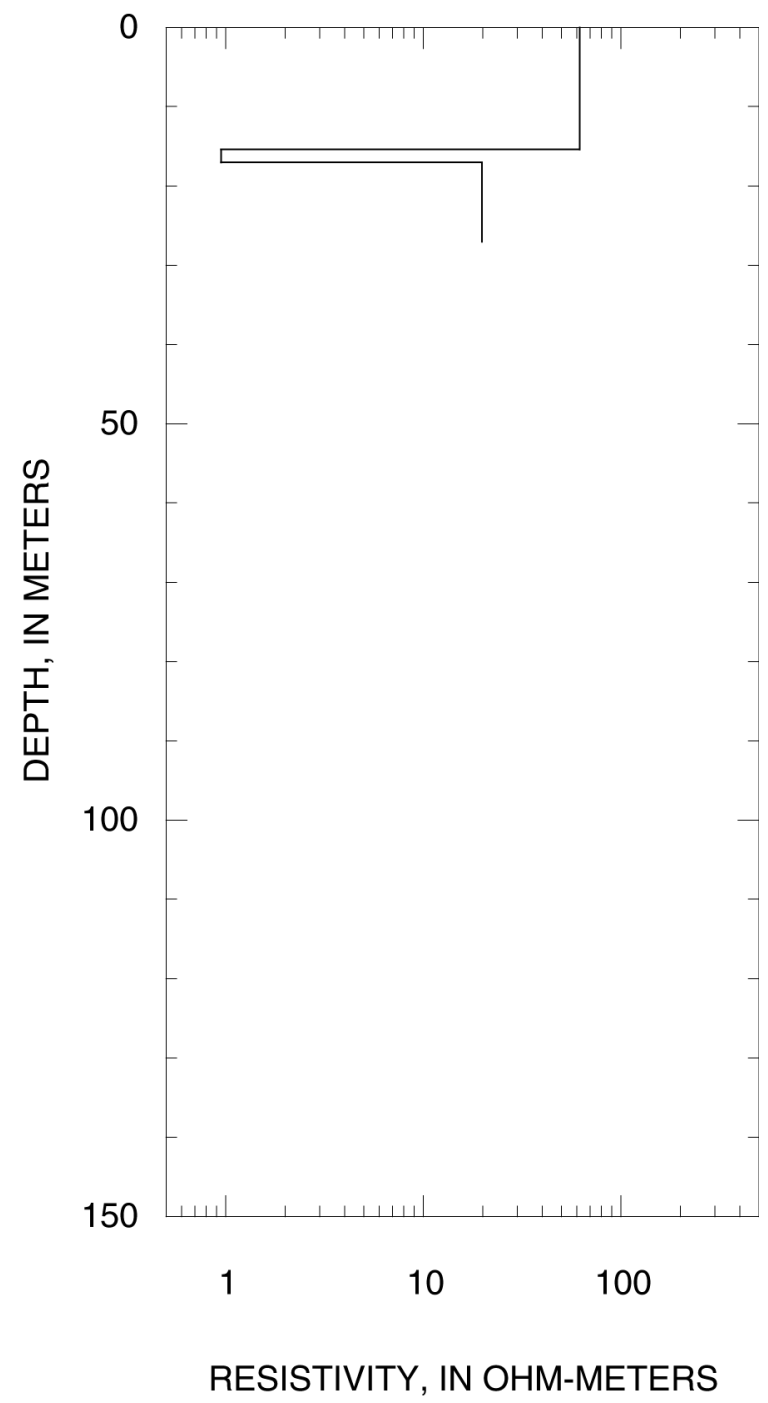

Figure 7-22. Sounding MIA218 

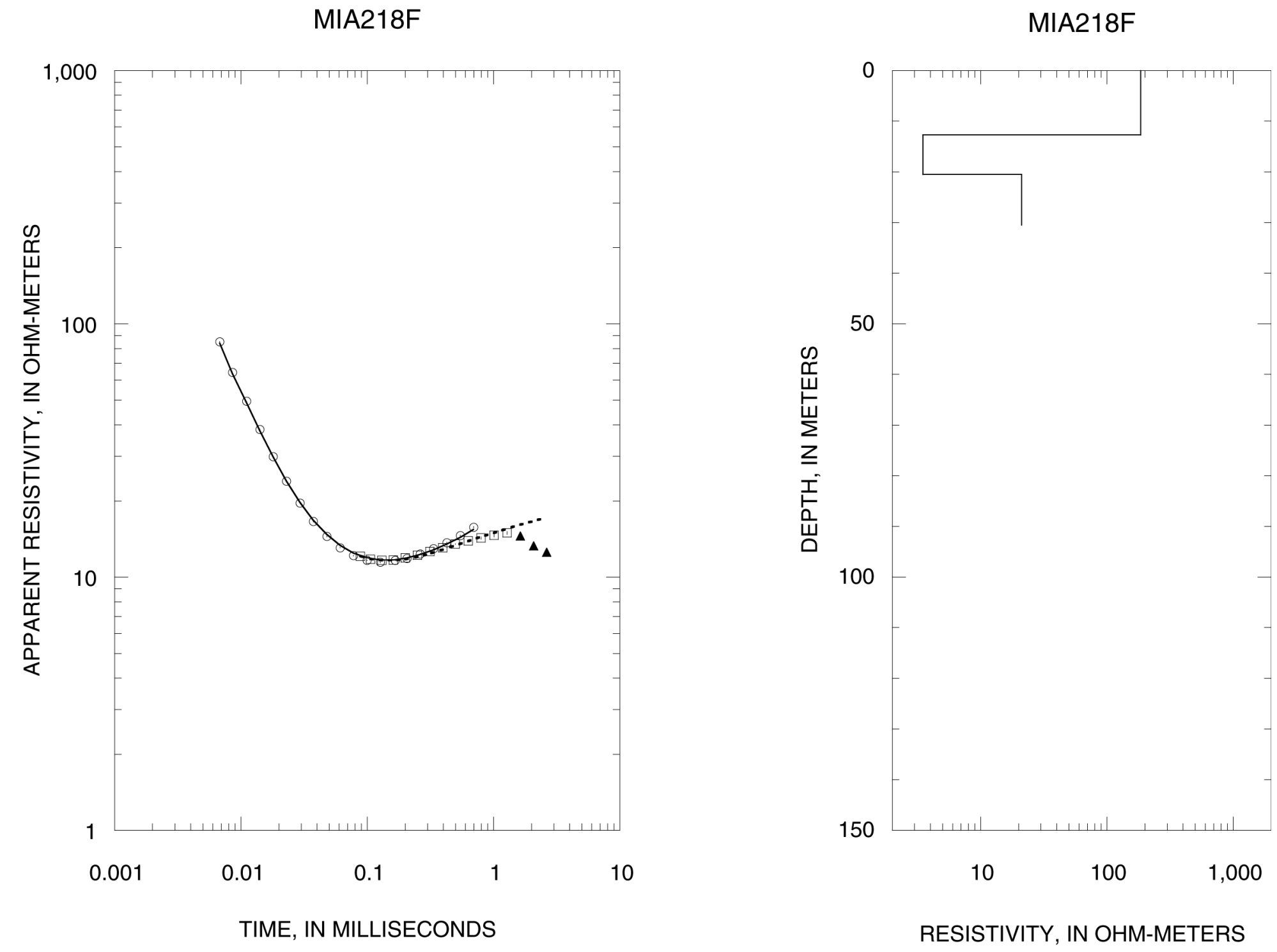

Figure 7-23. Sounding MIA218F 
MIA219

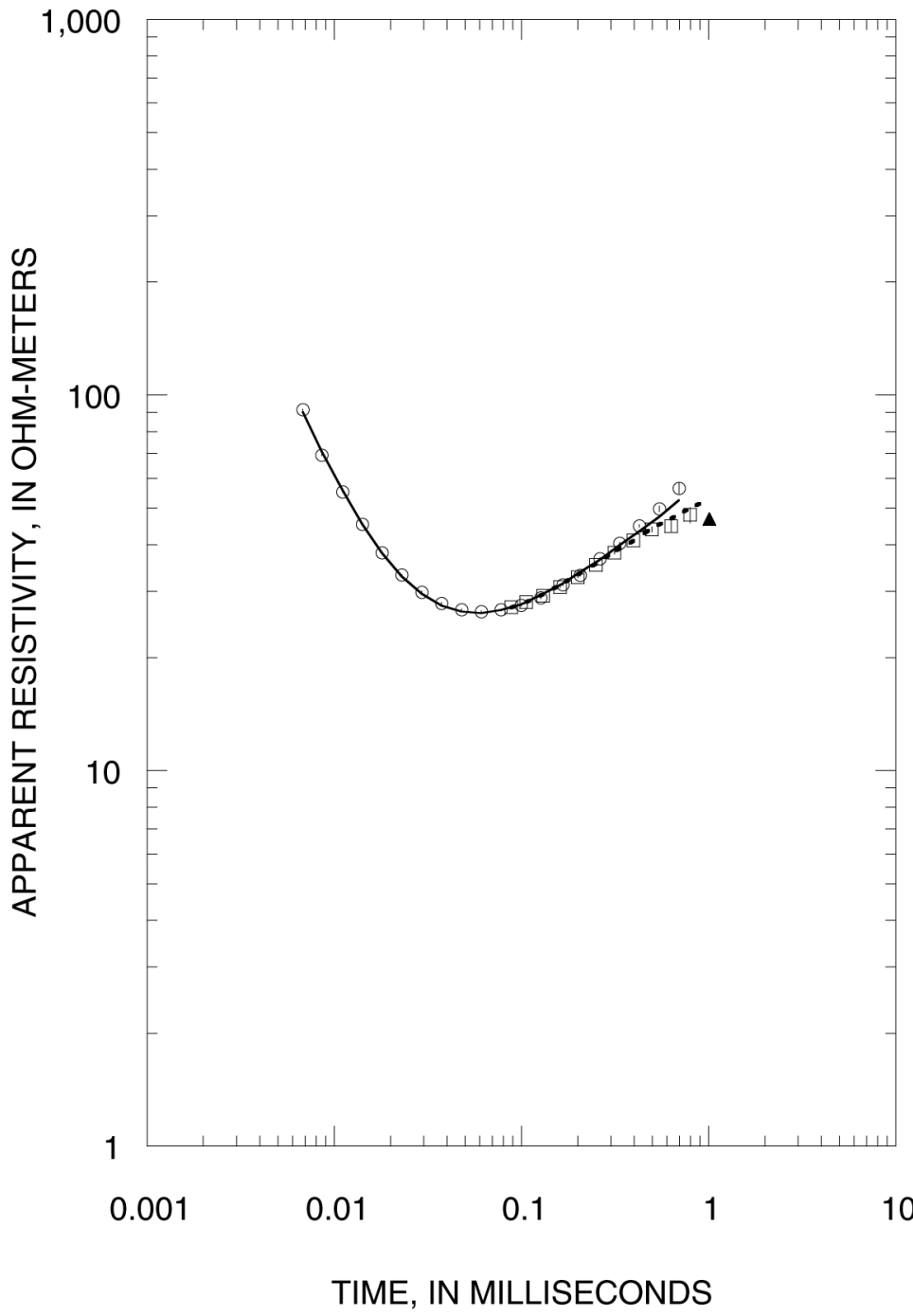

Figure 7-24. Sounding MIA219

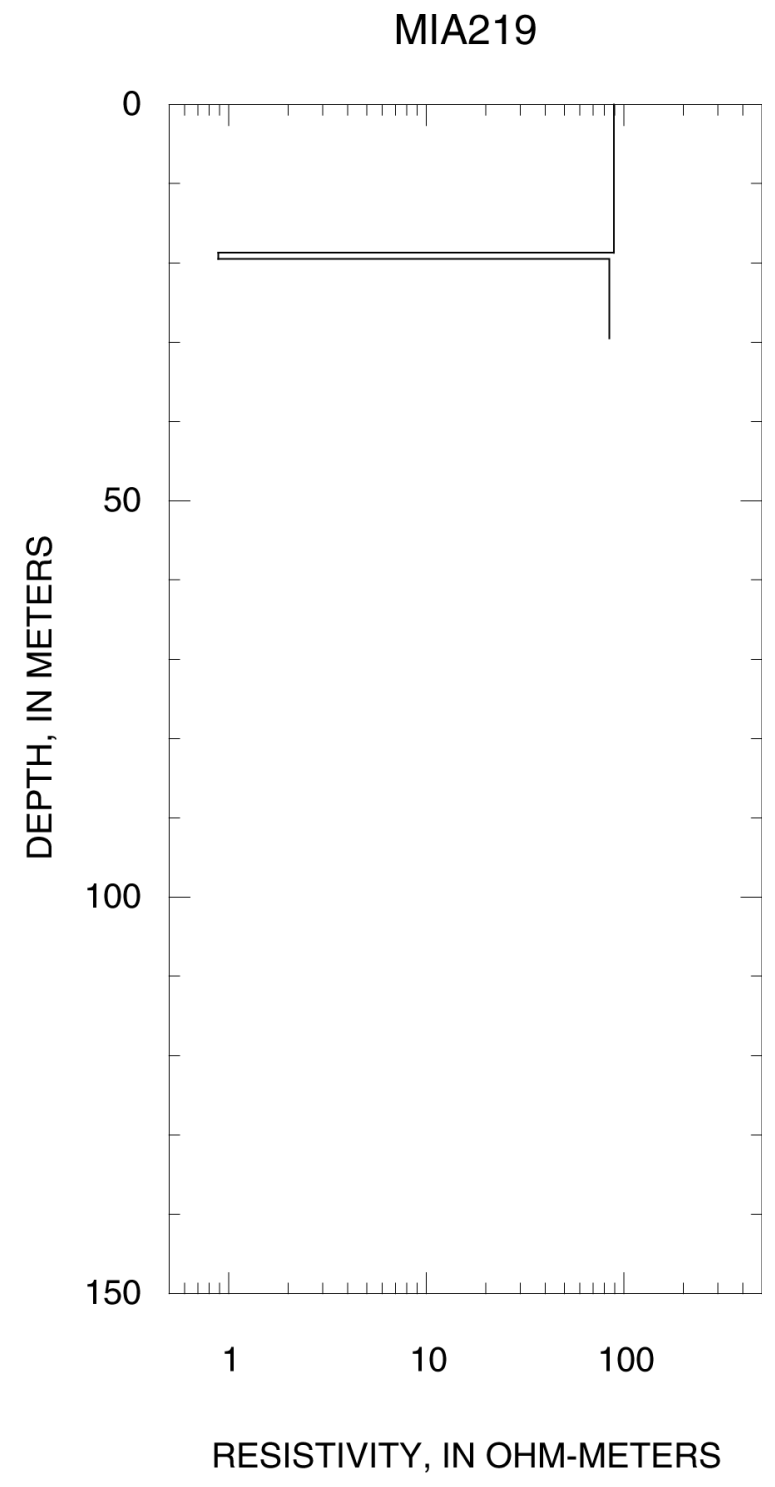



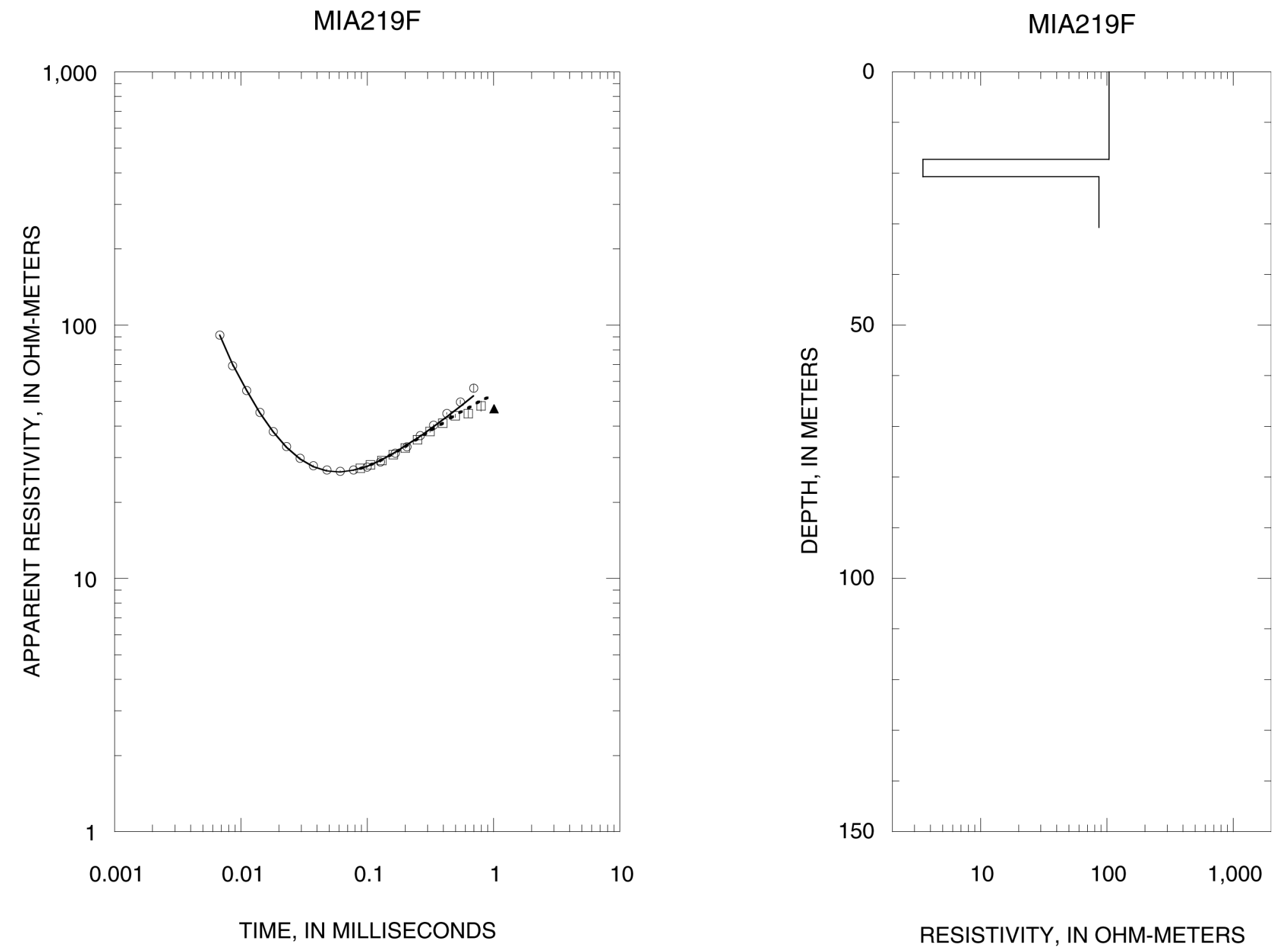

Figure 7-25. Sounding MIA219F 
MIA220

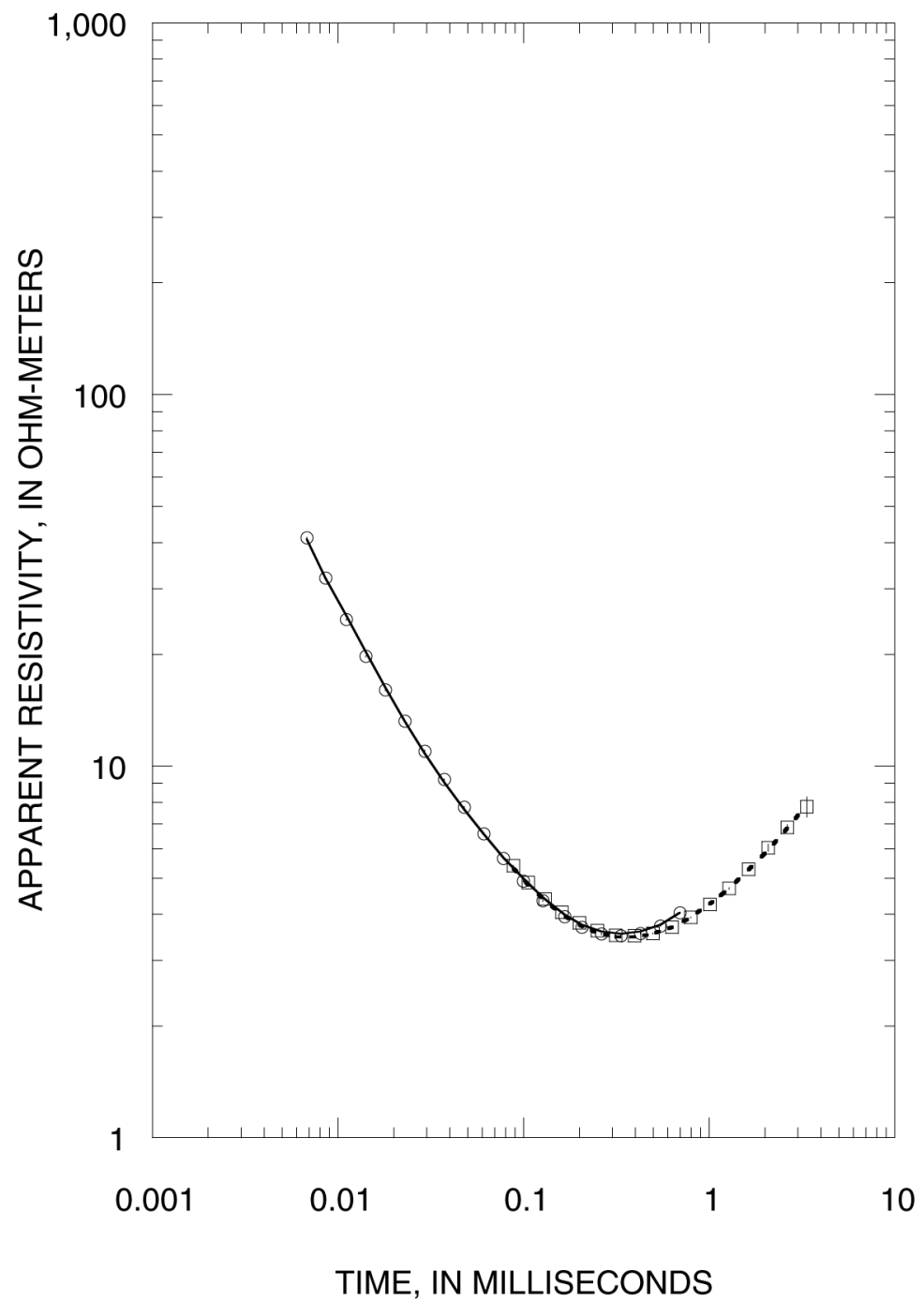

Figure 7-26. Sounding MIA220

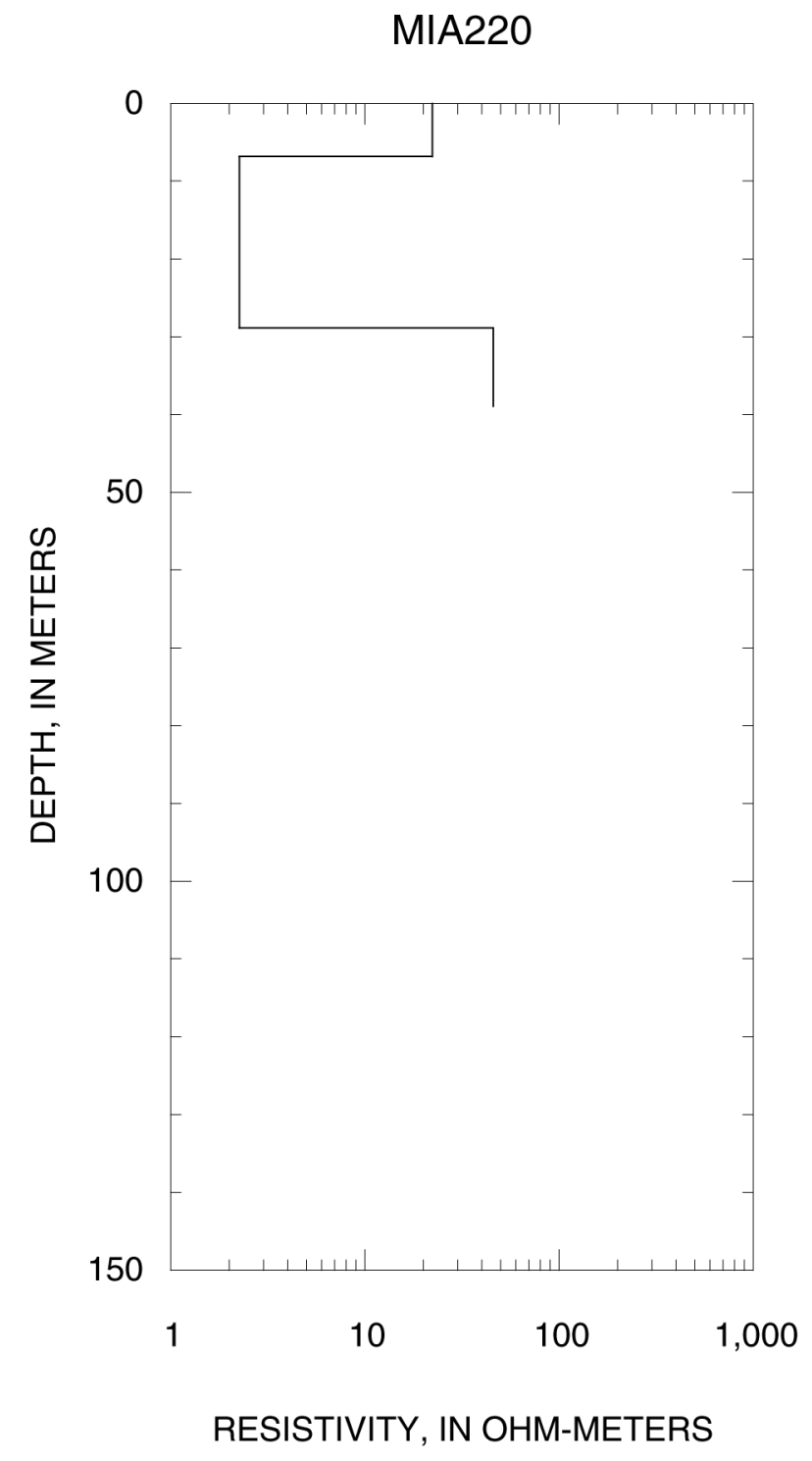


MIA221

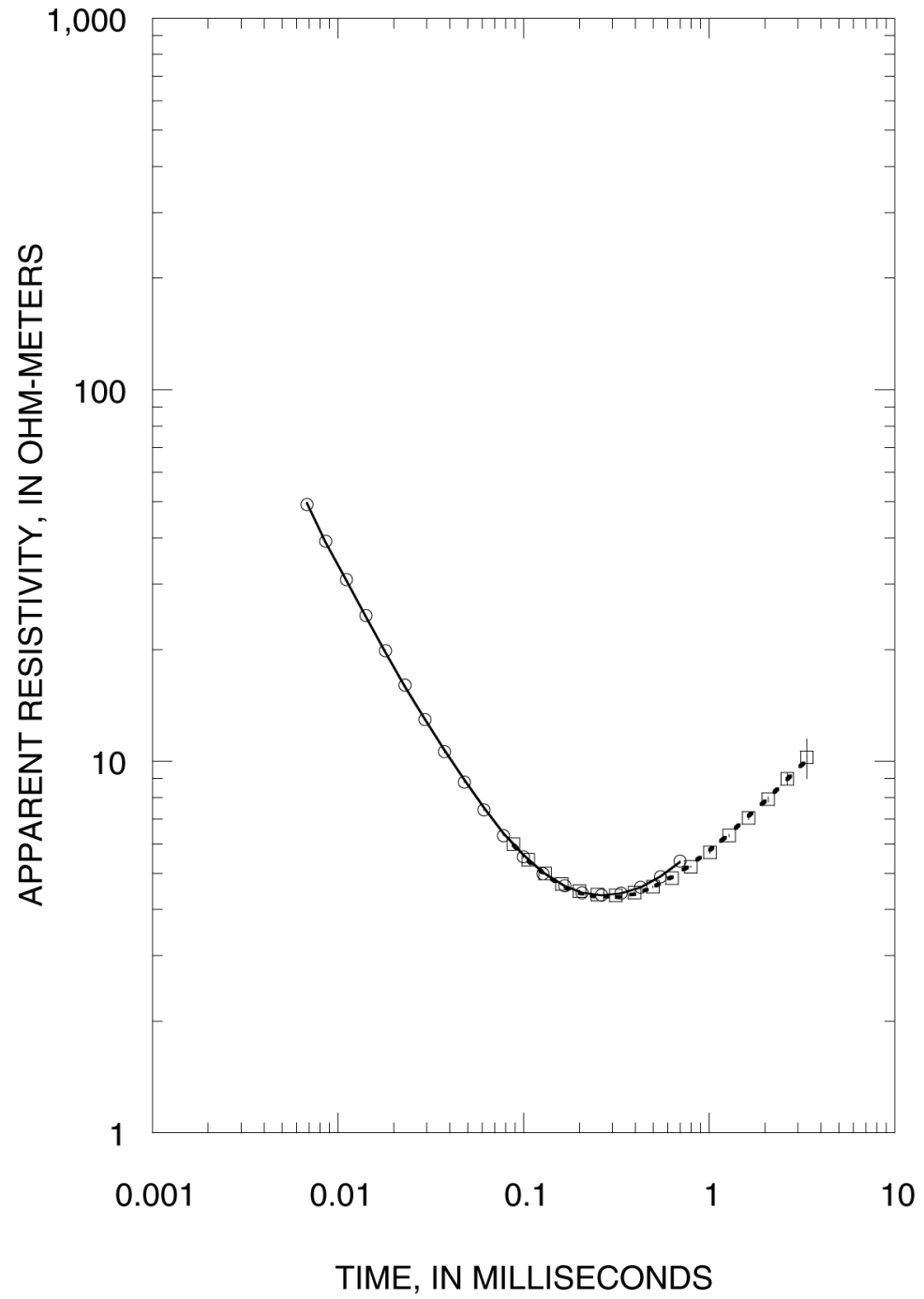

Figure 7-27. Sounding MIA221

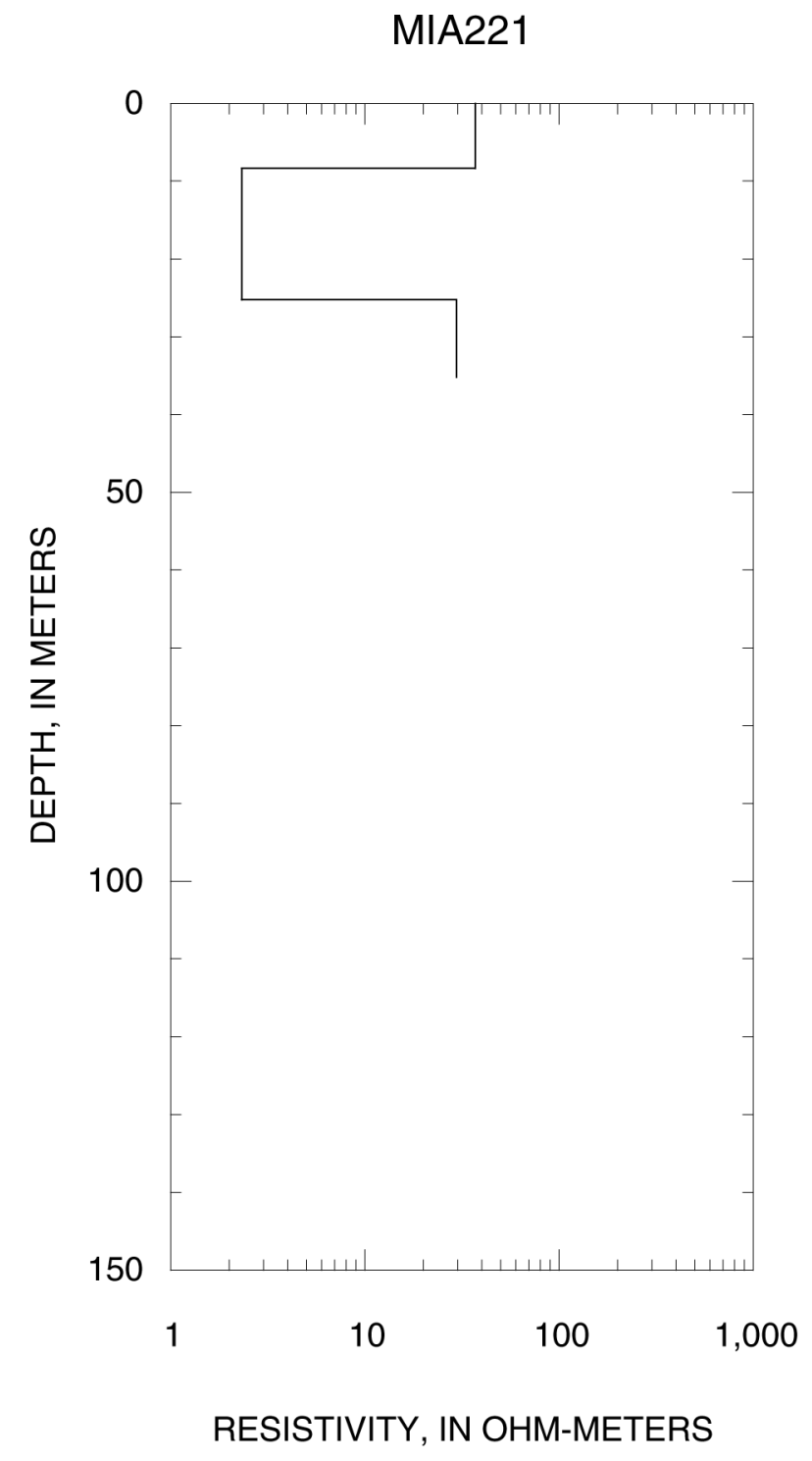


MIA222

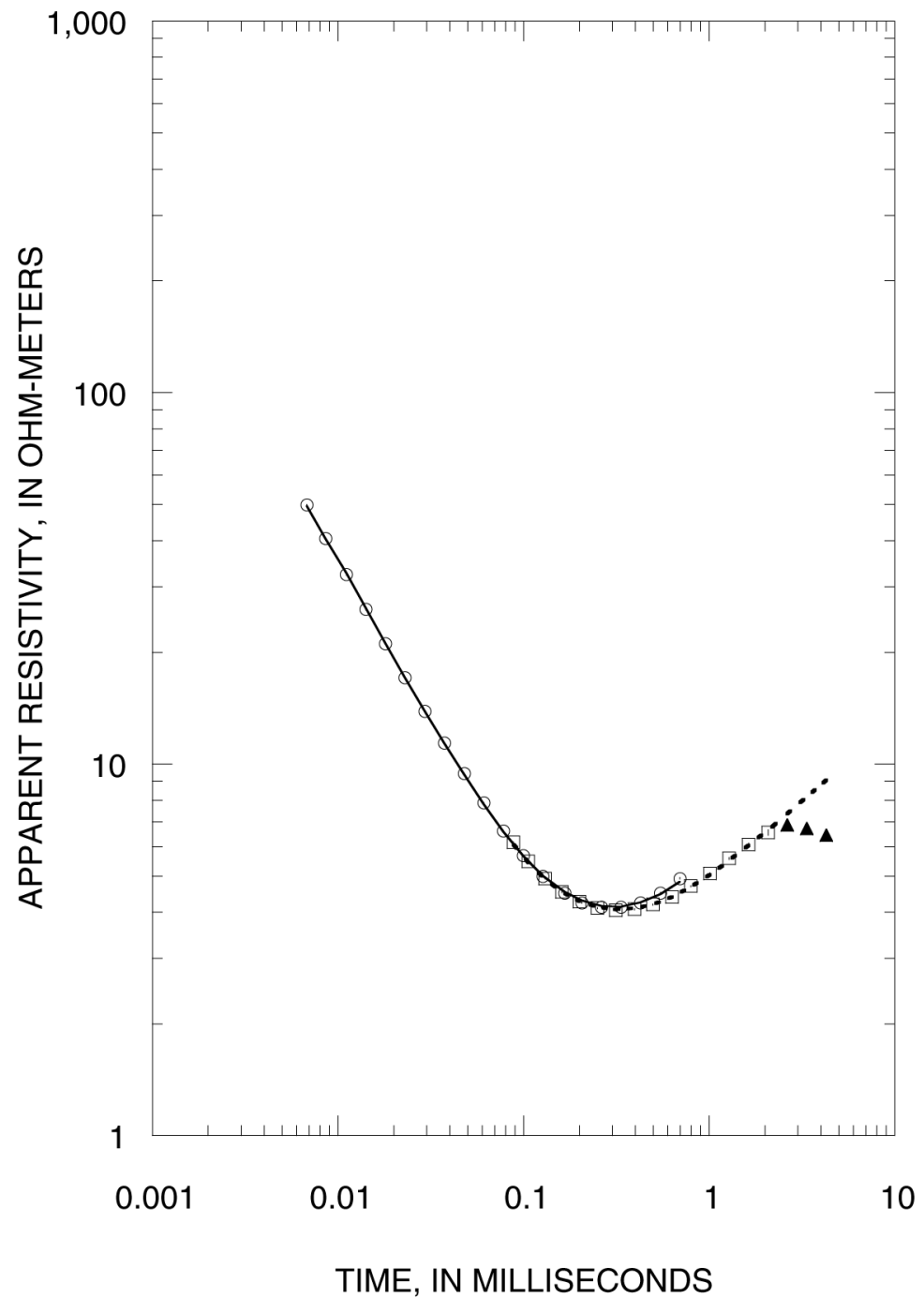

Figure 7-28. Sounding MIA222

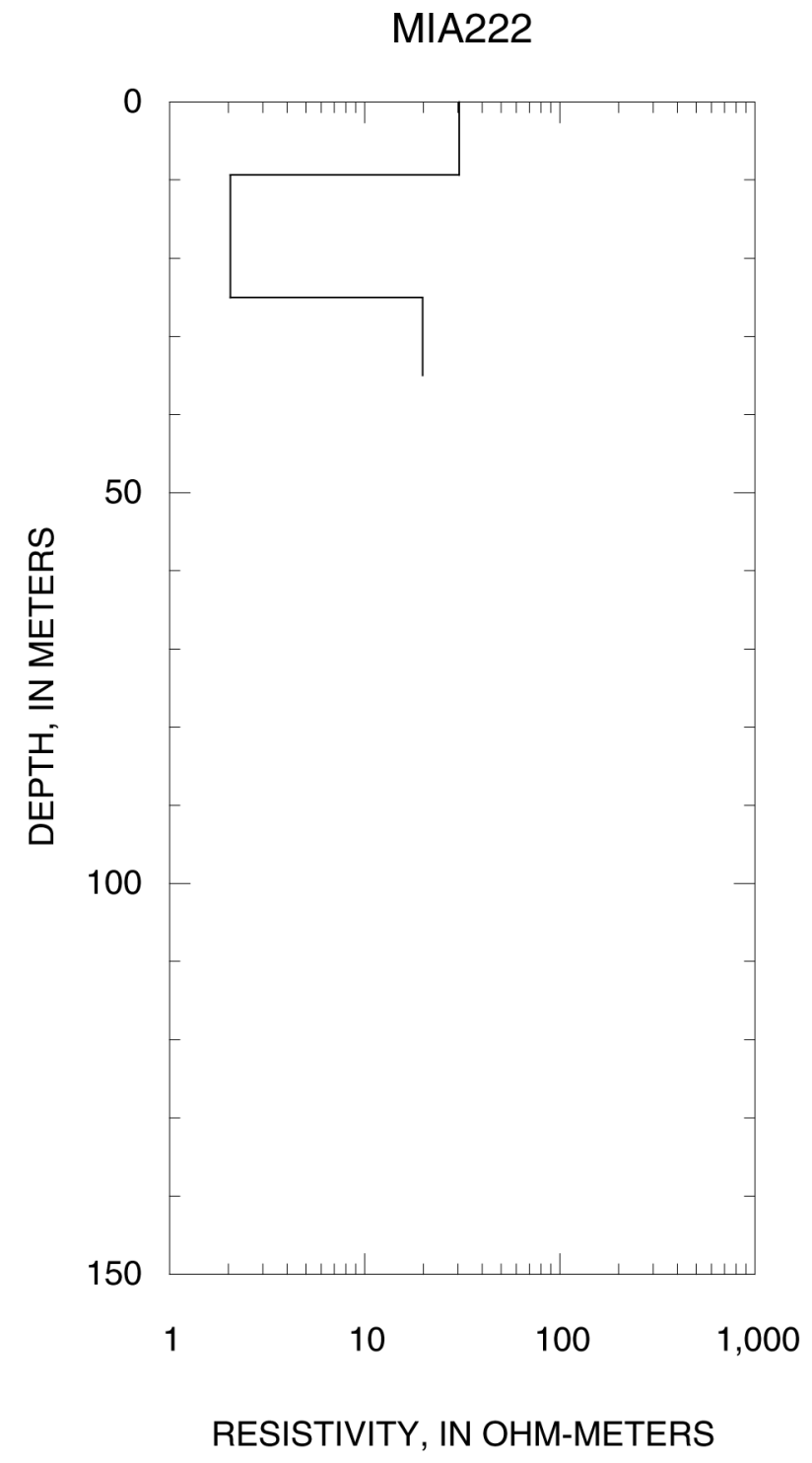


MIA222L4

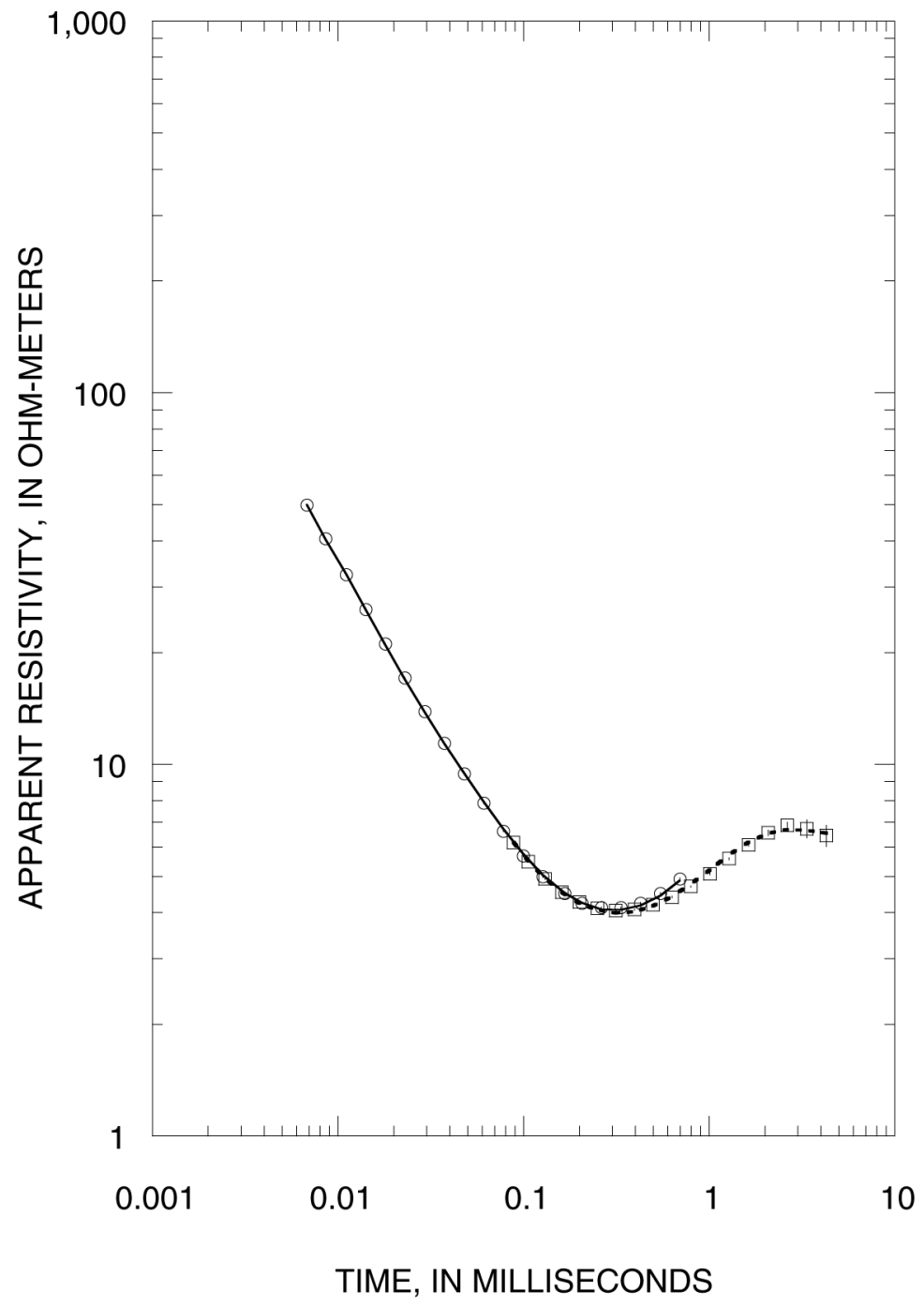

Figure 7-29. Sounding MIA222L4

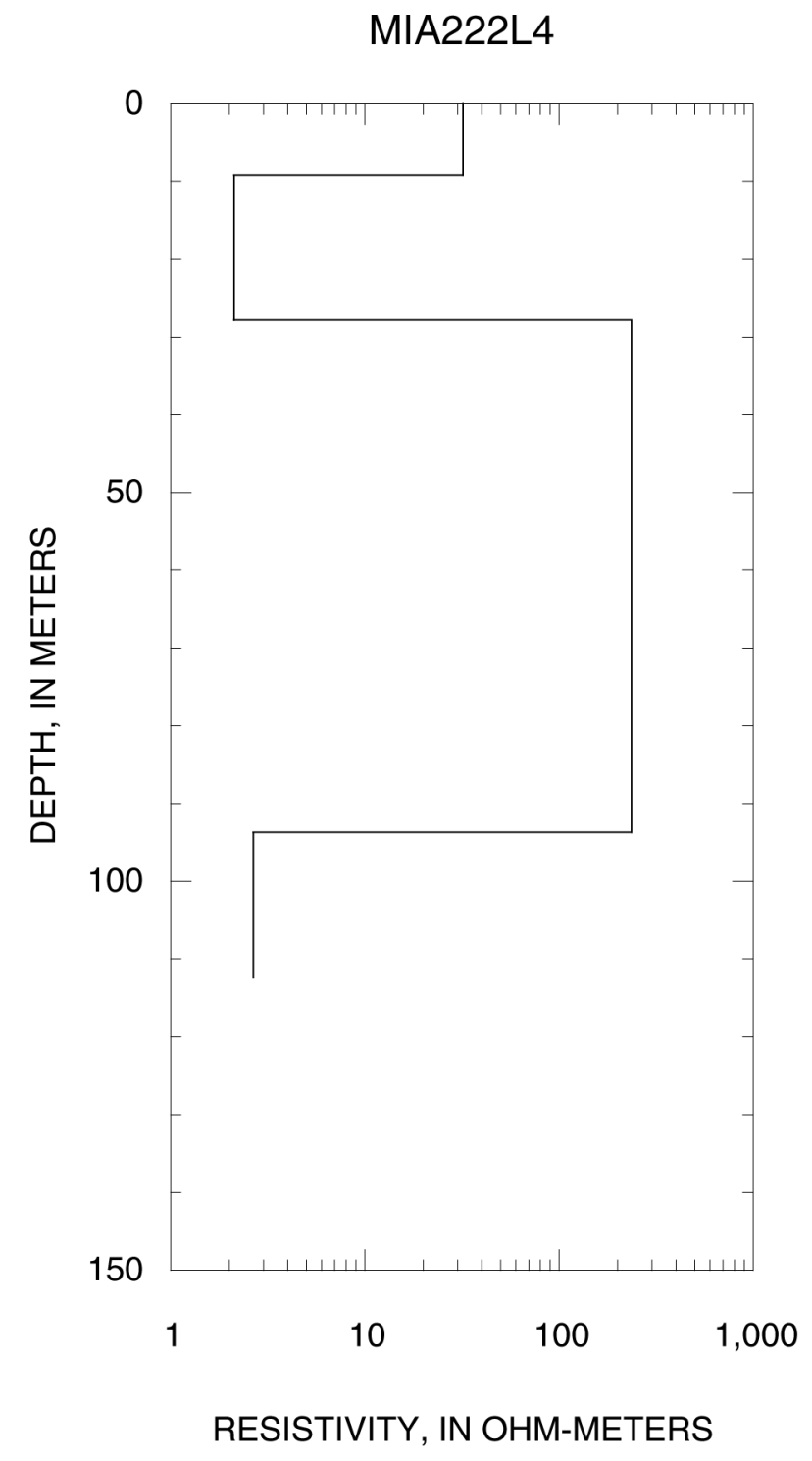


MIA223

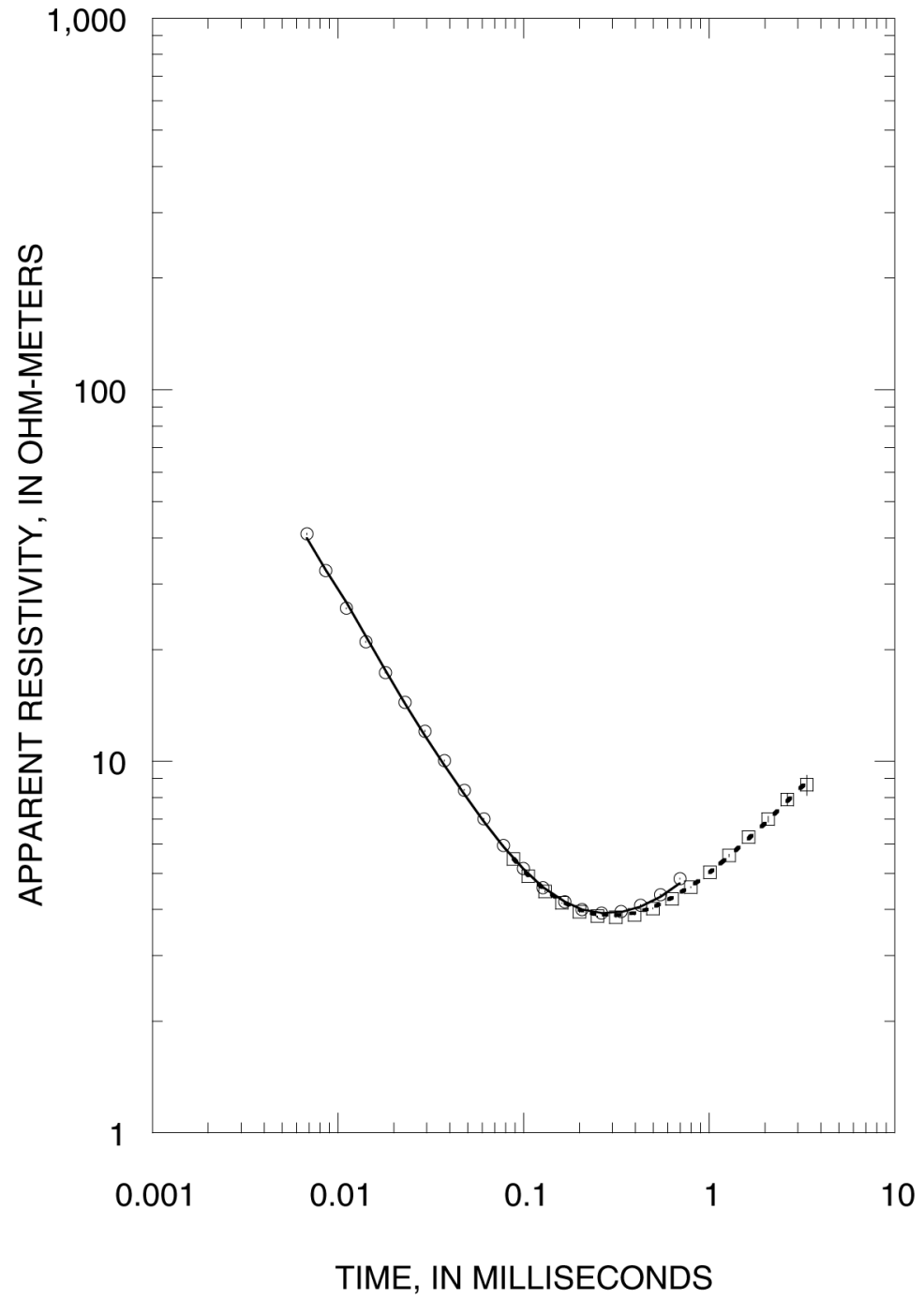

Figure 7-30. Sounding MIA223

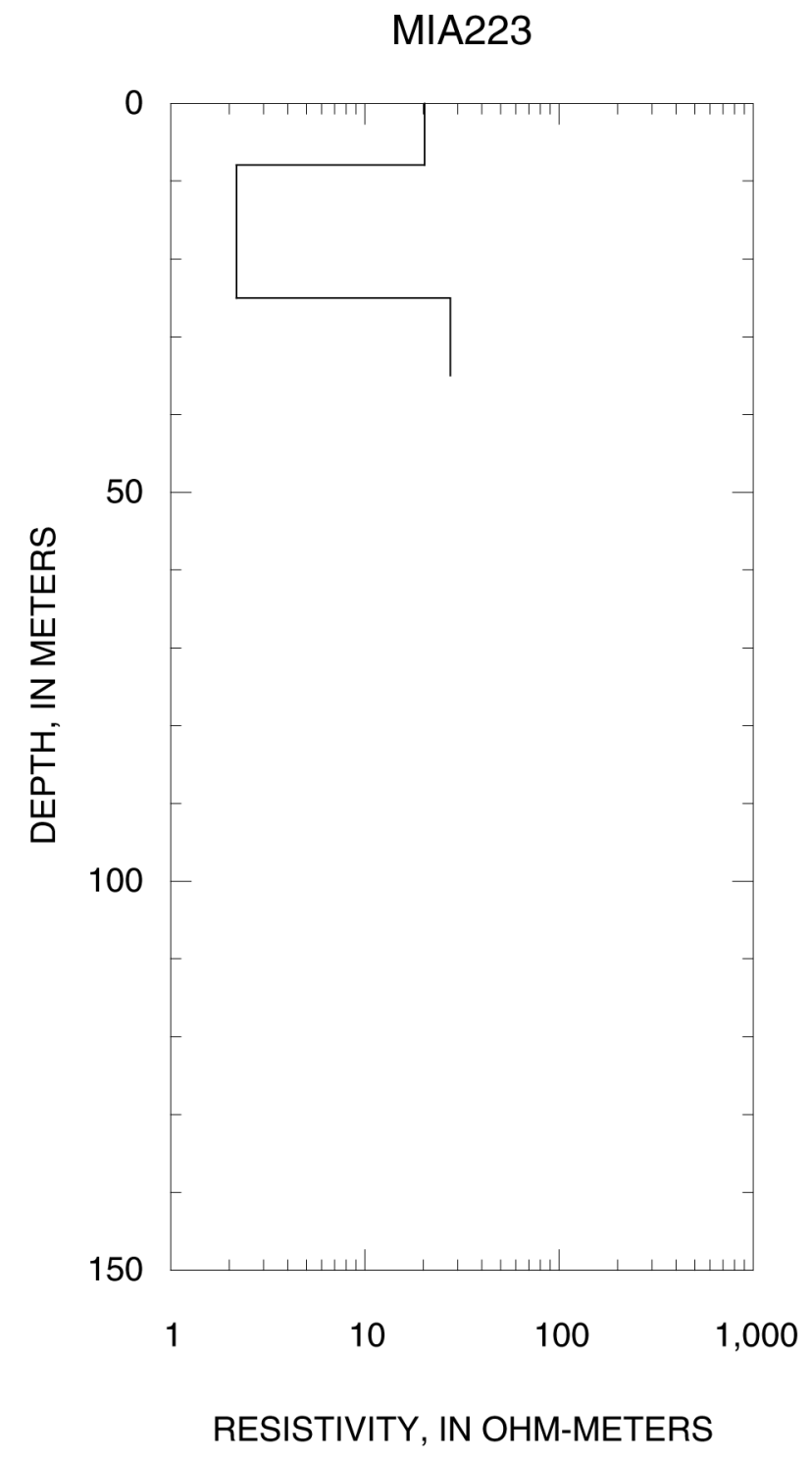


MIA223L4

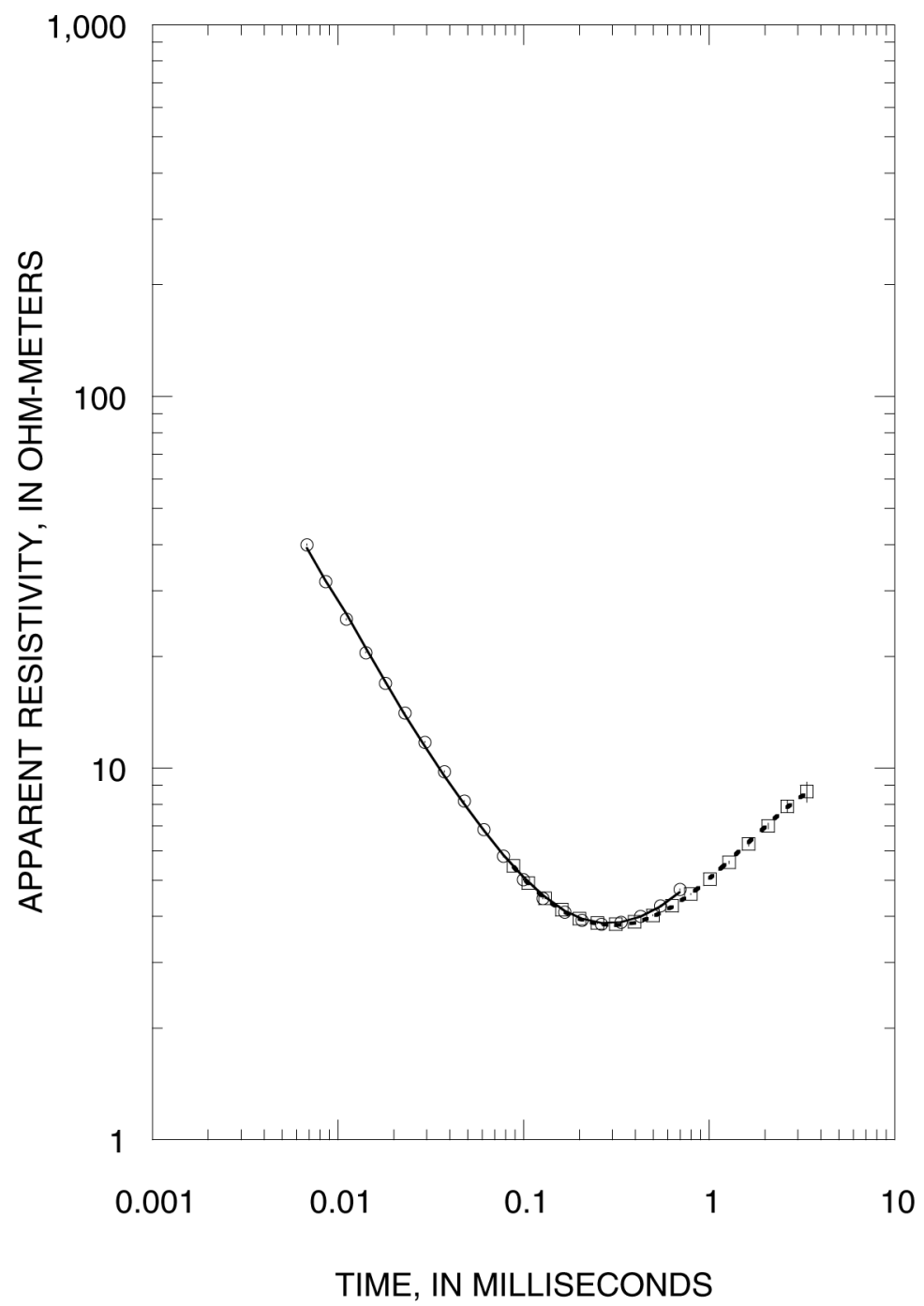

Figure 7-31. Sounding MIA223L4

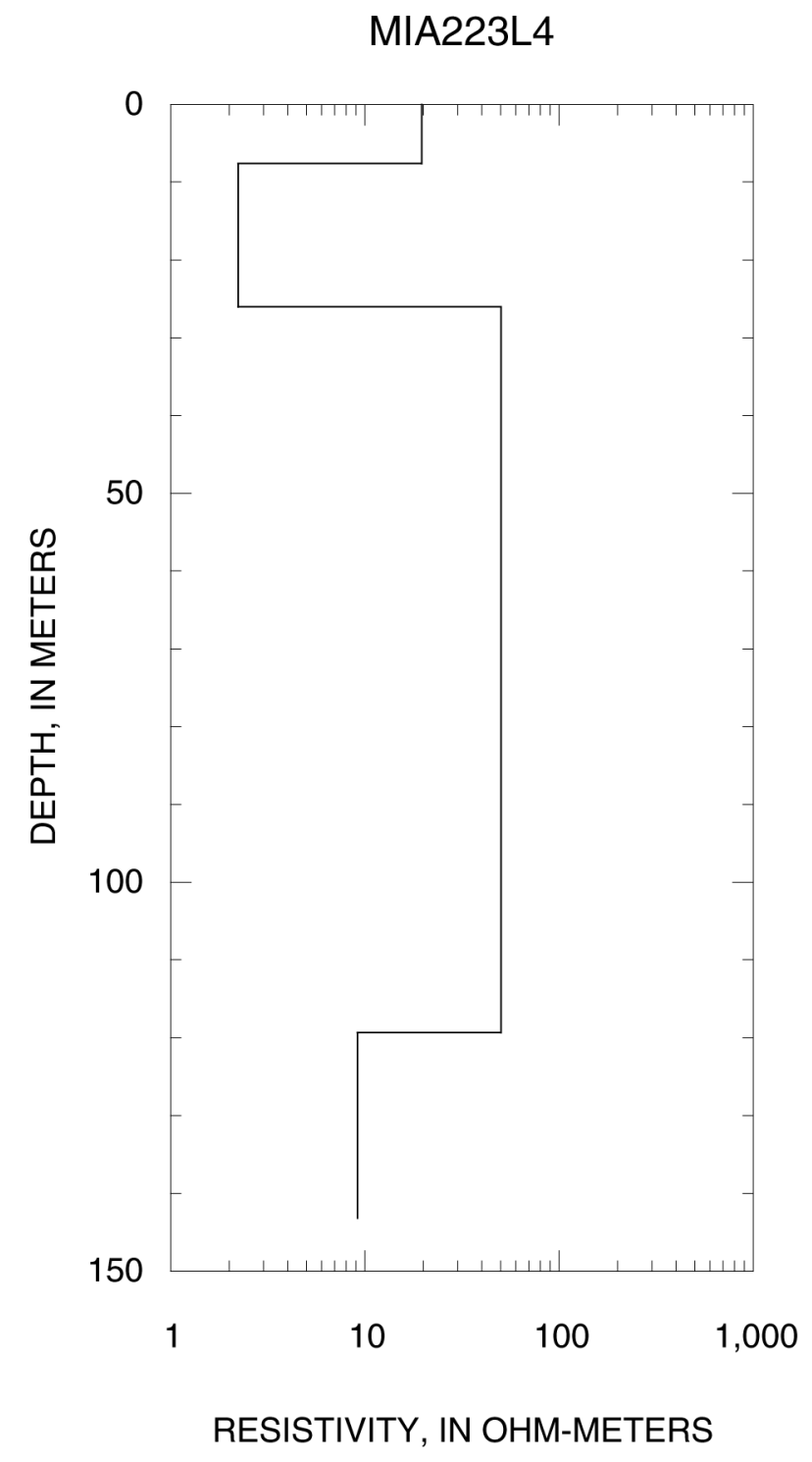


MIA224

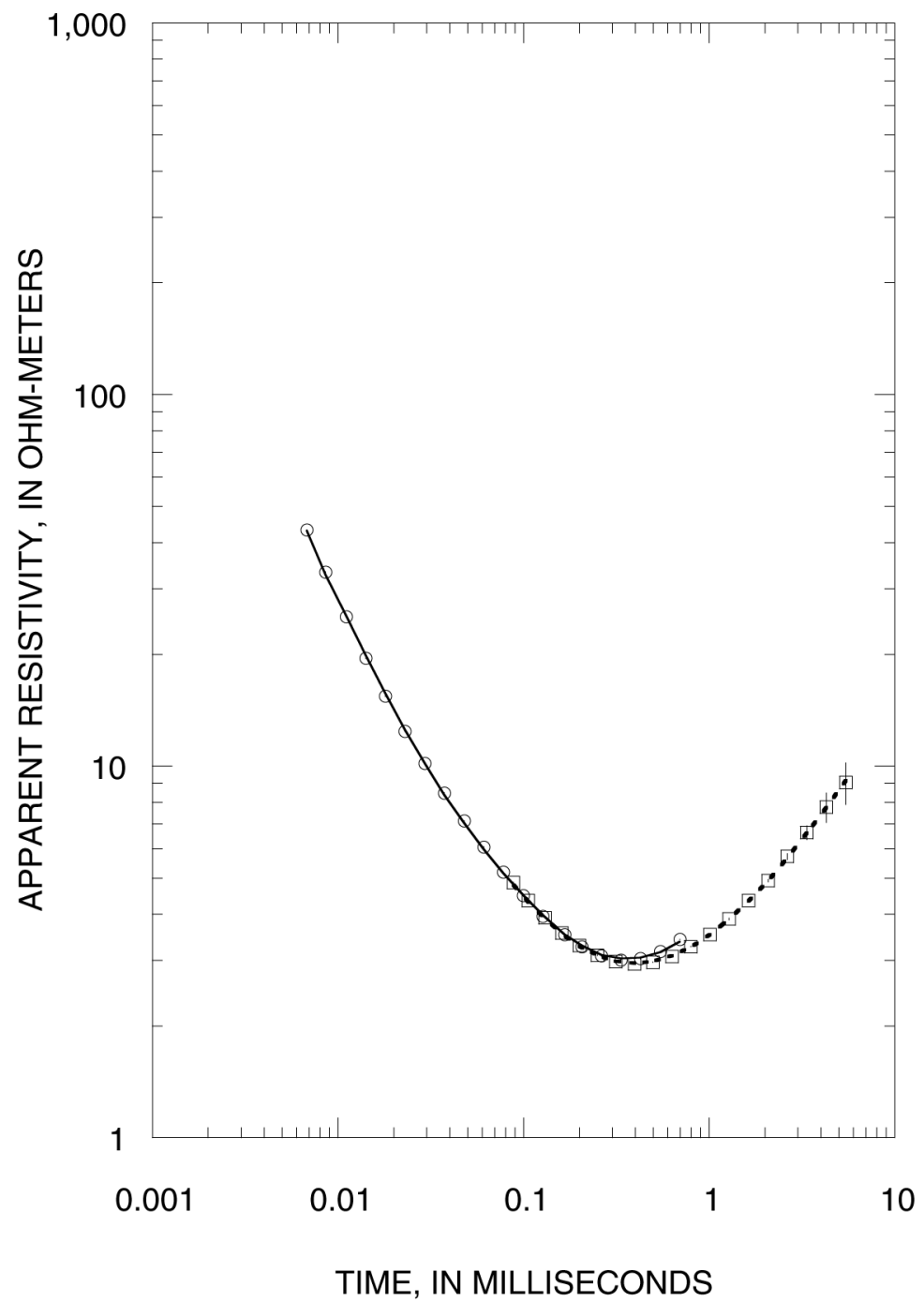

Figure 7-32. Sounding MIA224

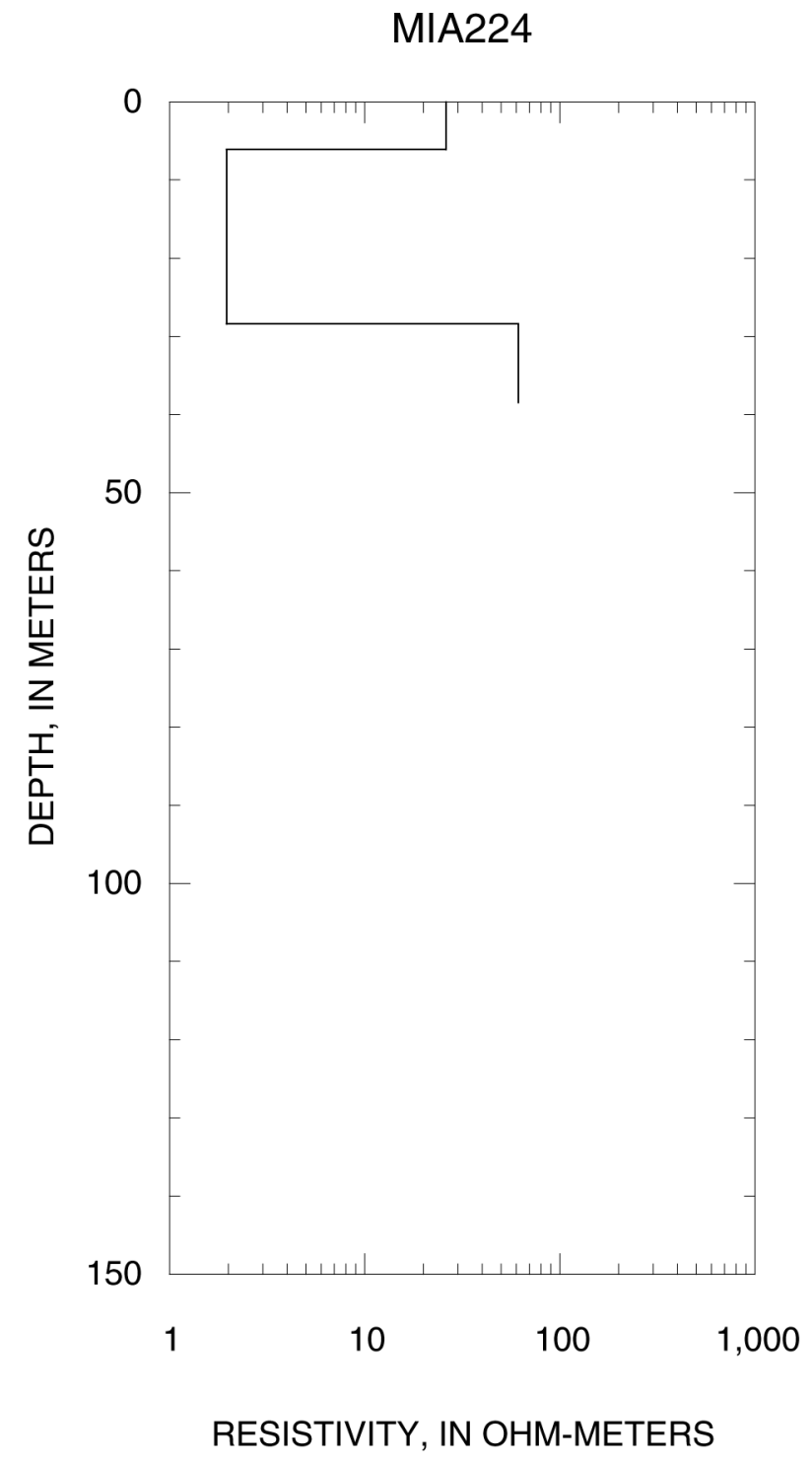


MIA225

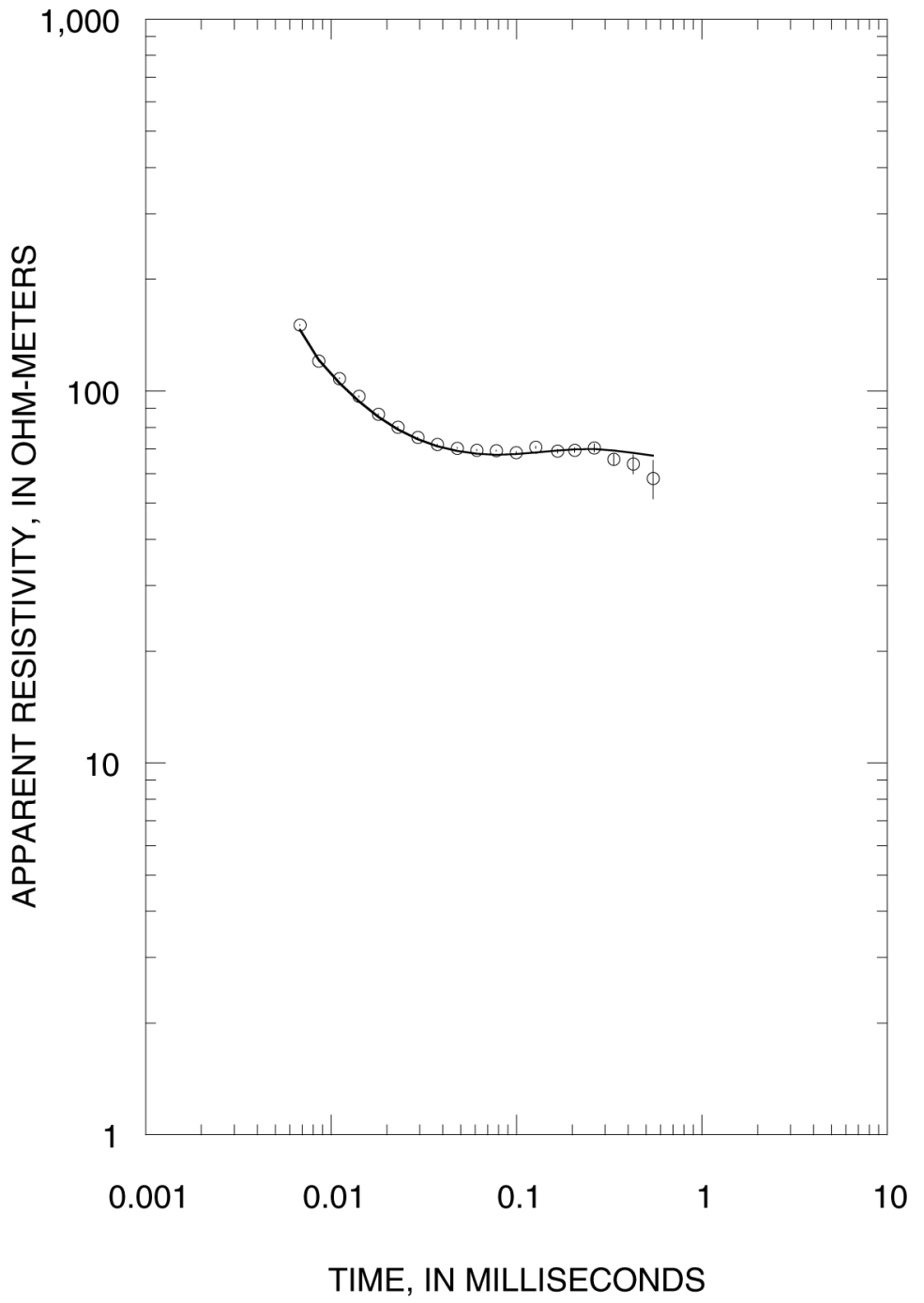

Figure 7-33. Sounding MIA225

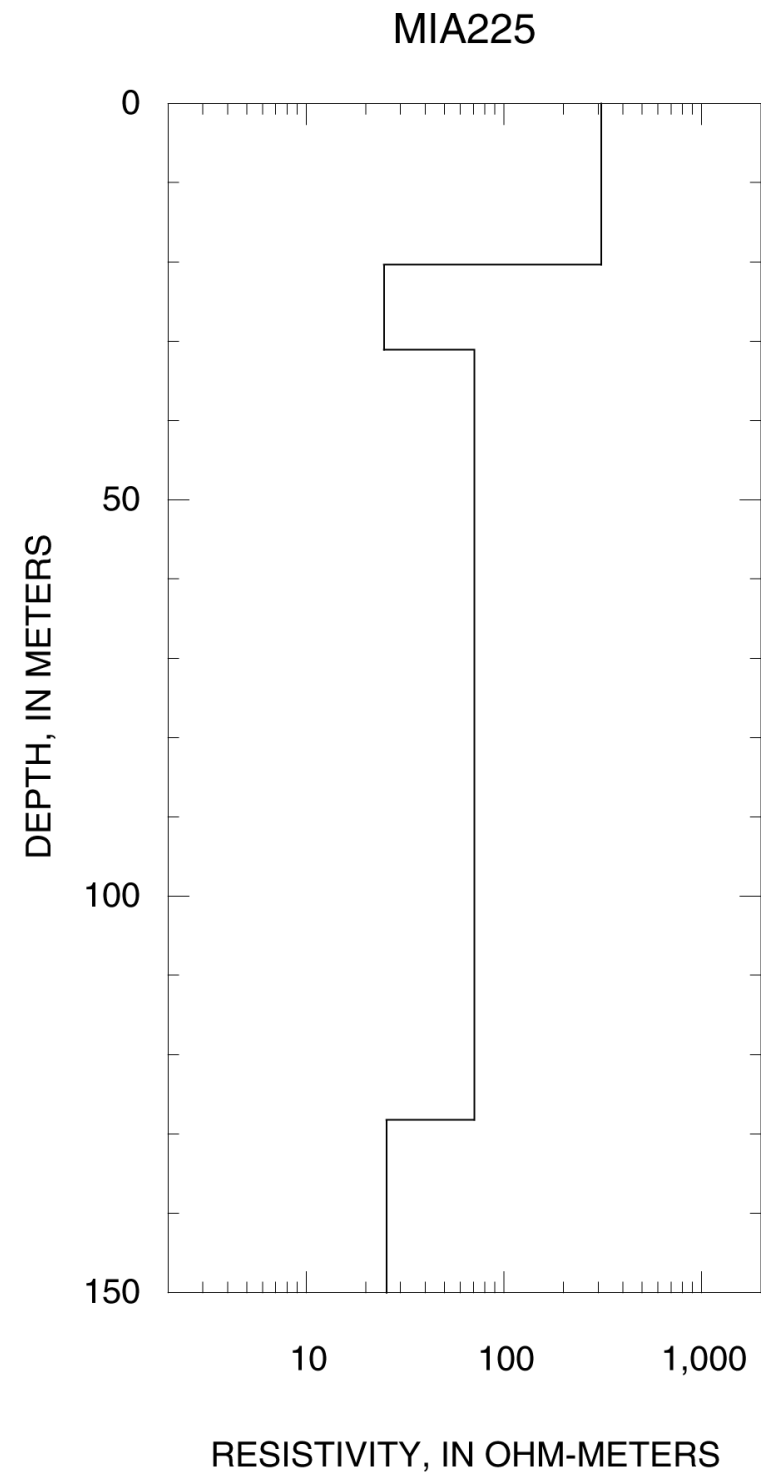


MIA226

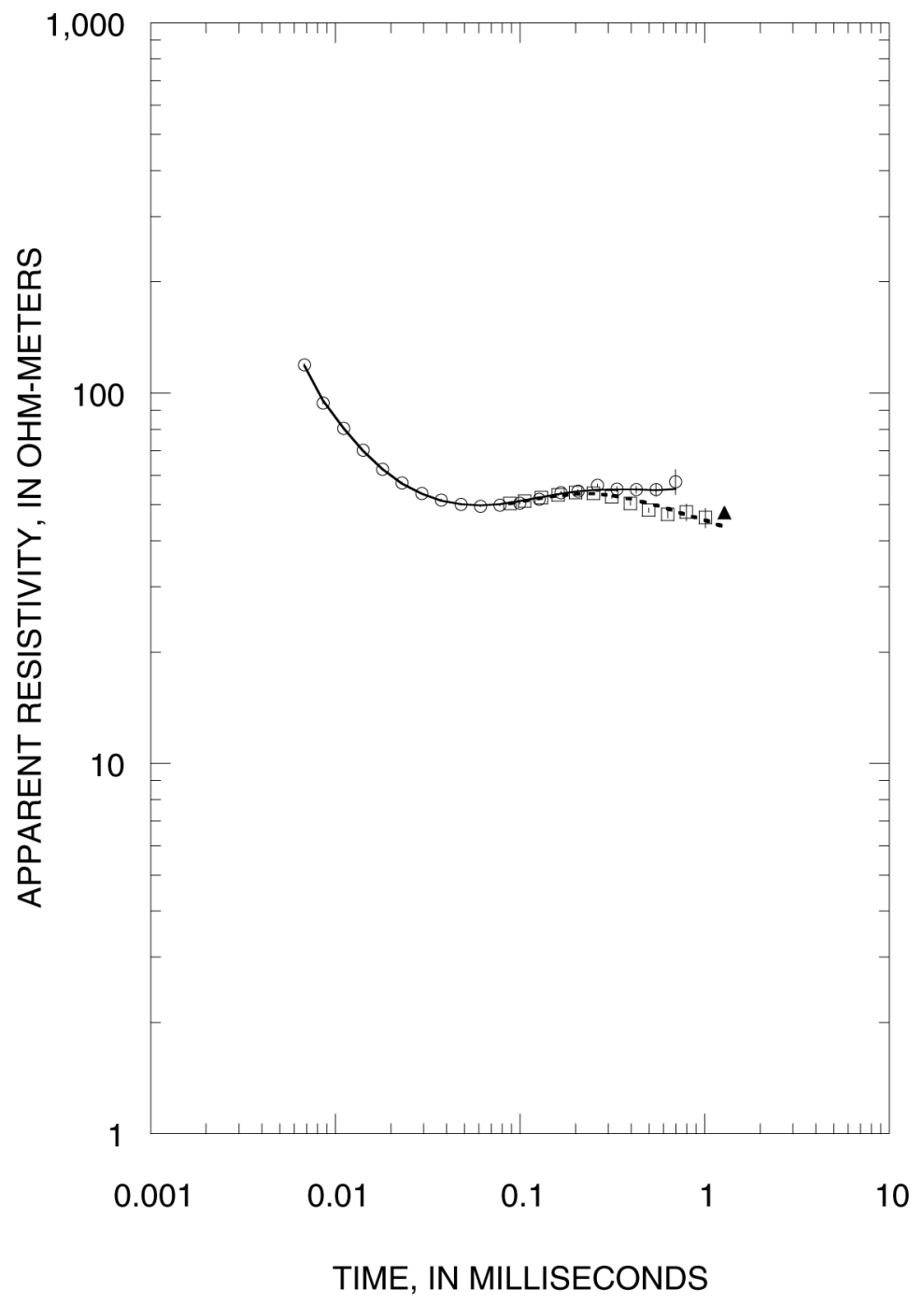

MIA226

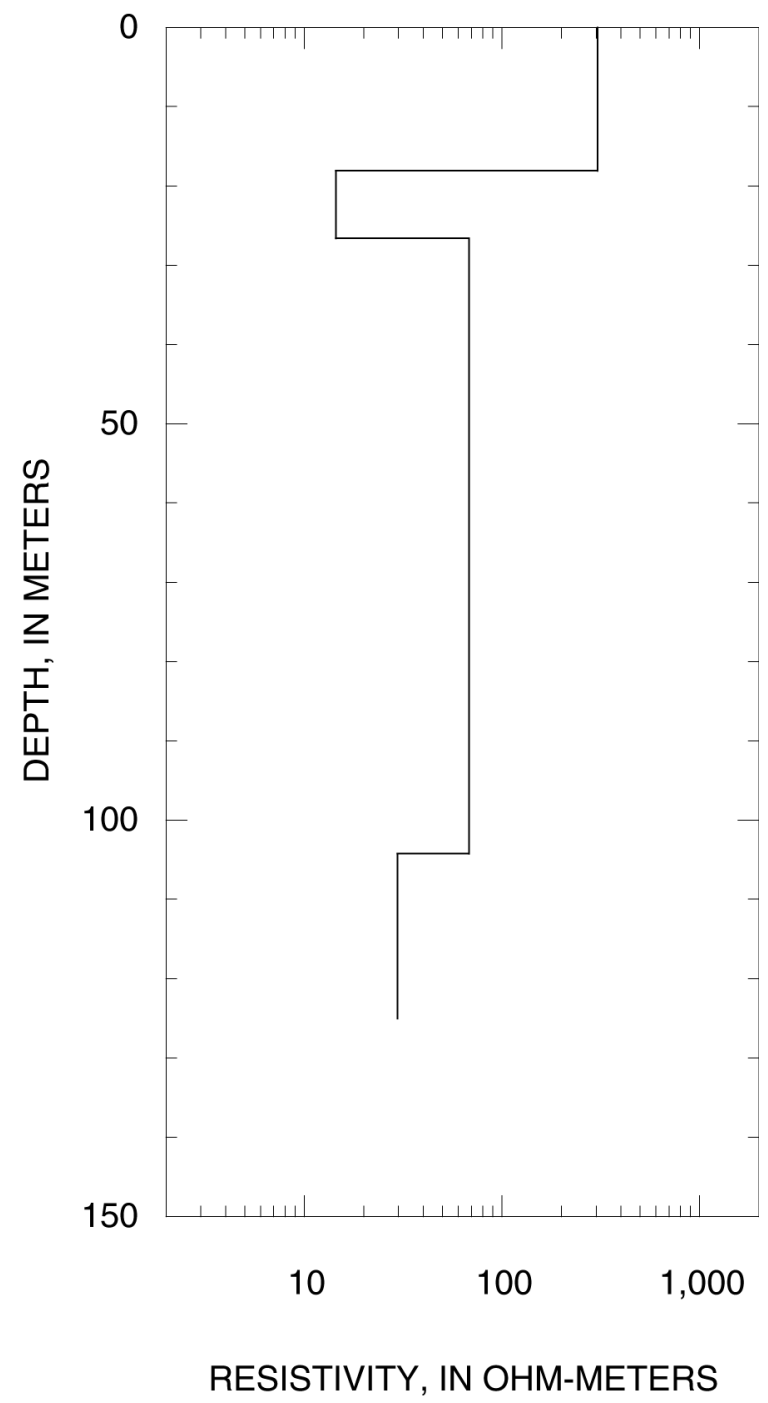

Figure 7-34. Sounding MIA226 
MIA227

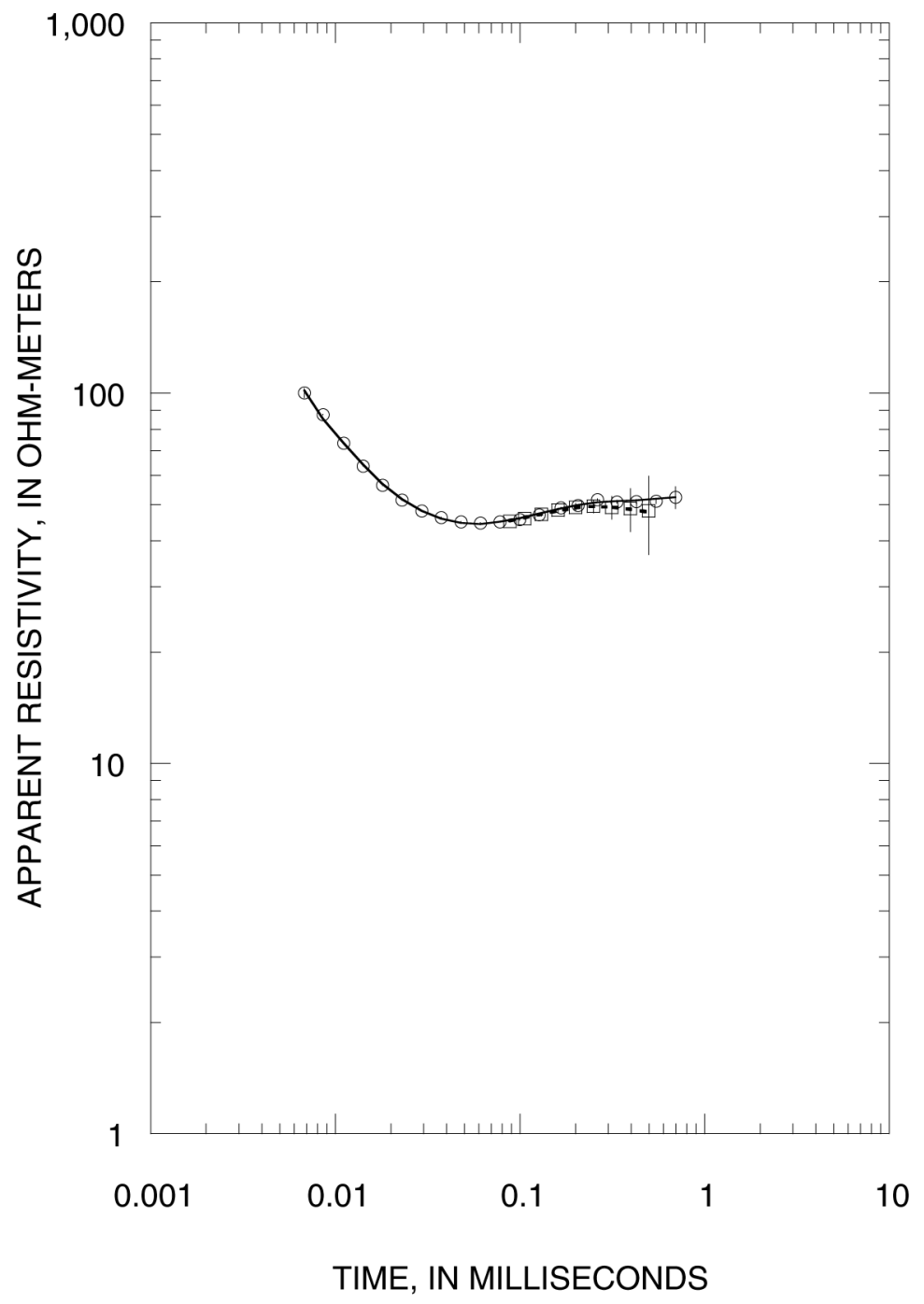

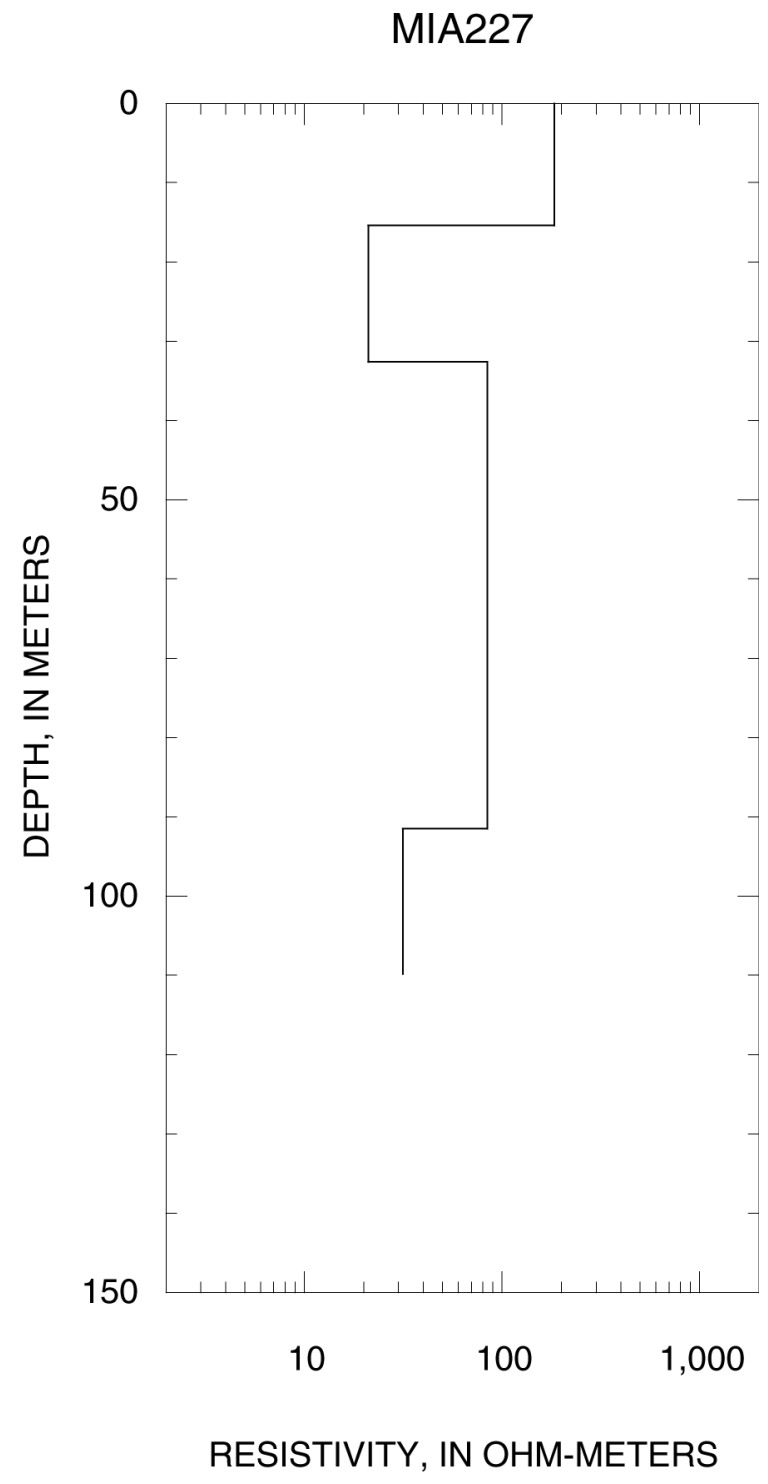

Figure 7-35. Sounding MIA227 
MIA228

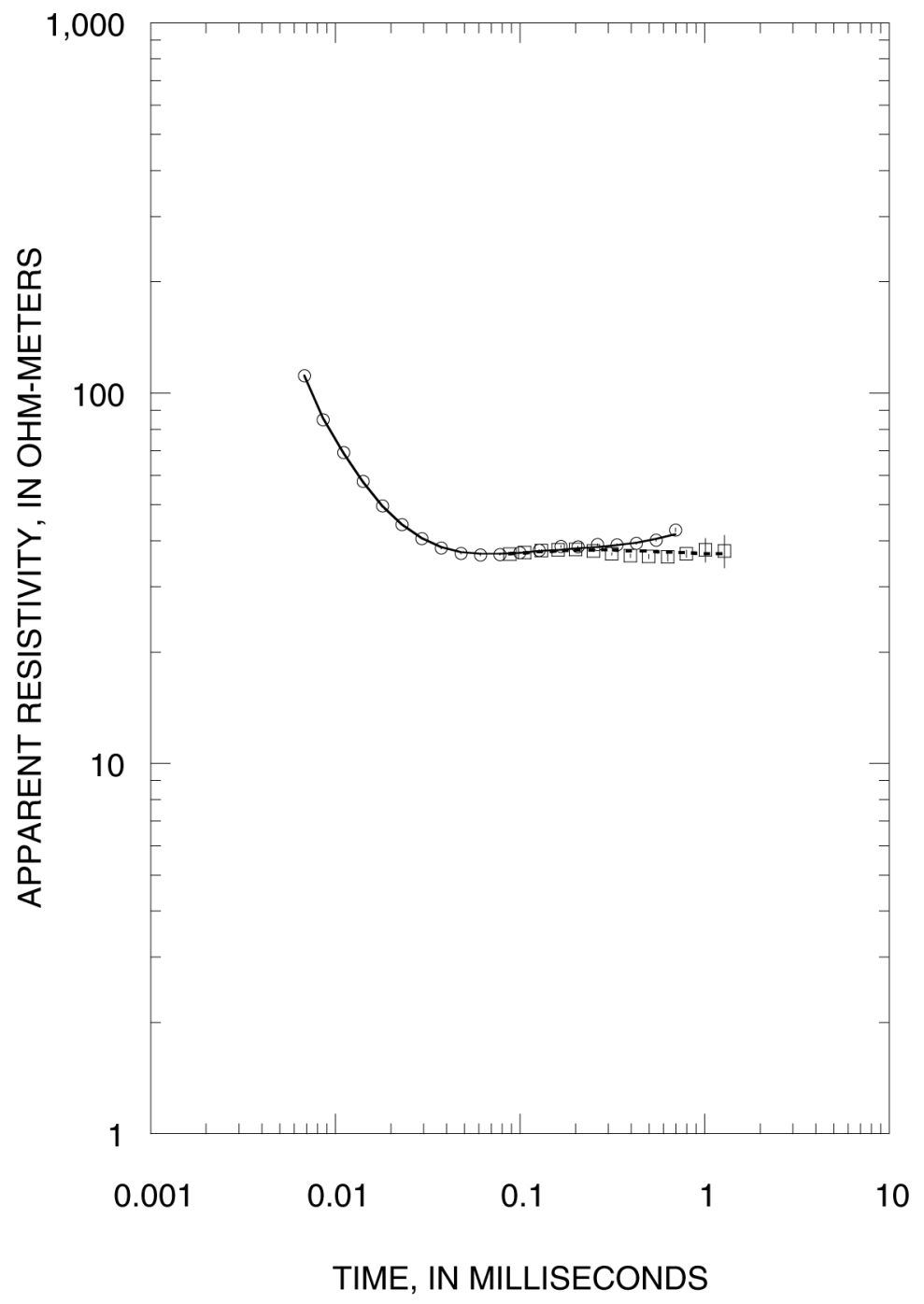

MIA228

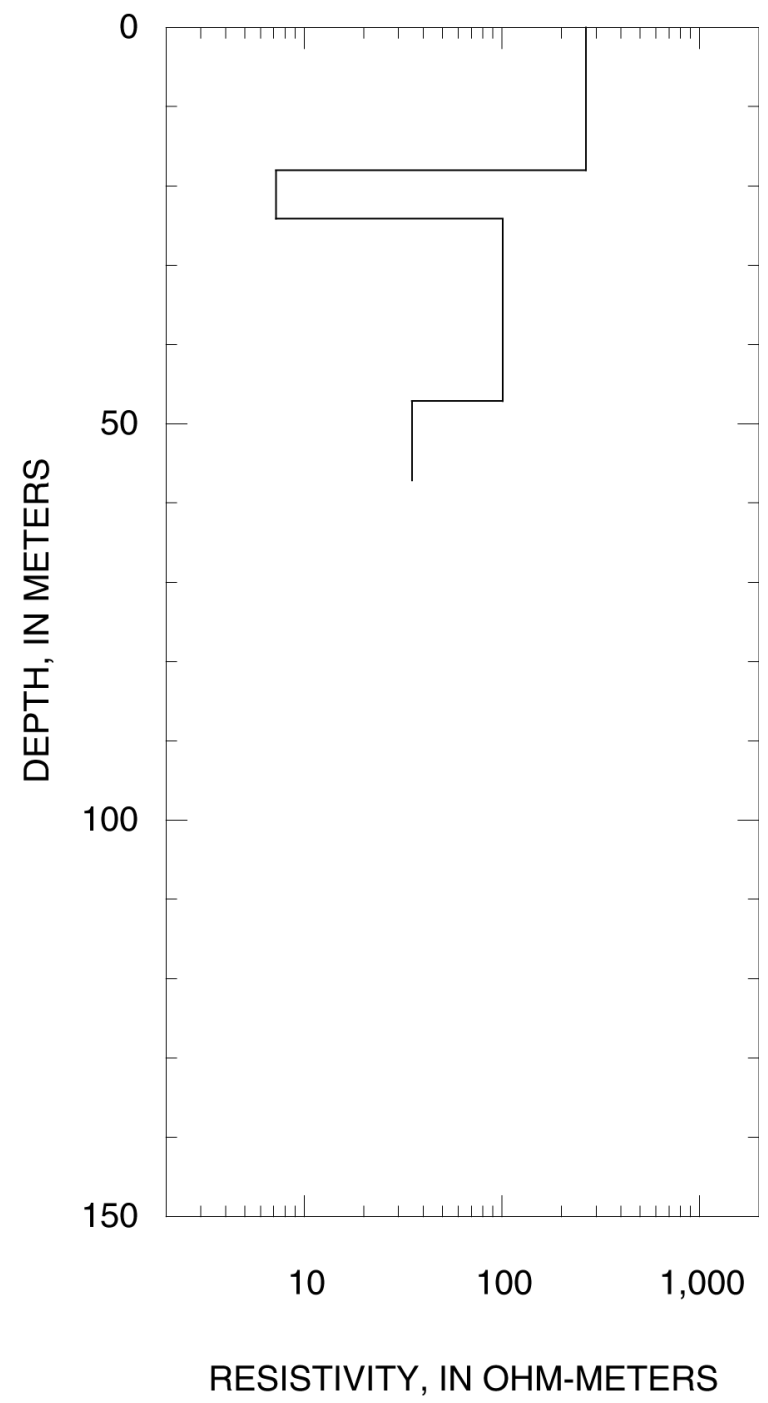

Figure 7-36. Sounding MIA228 
MIA229

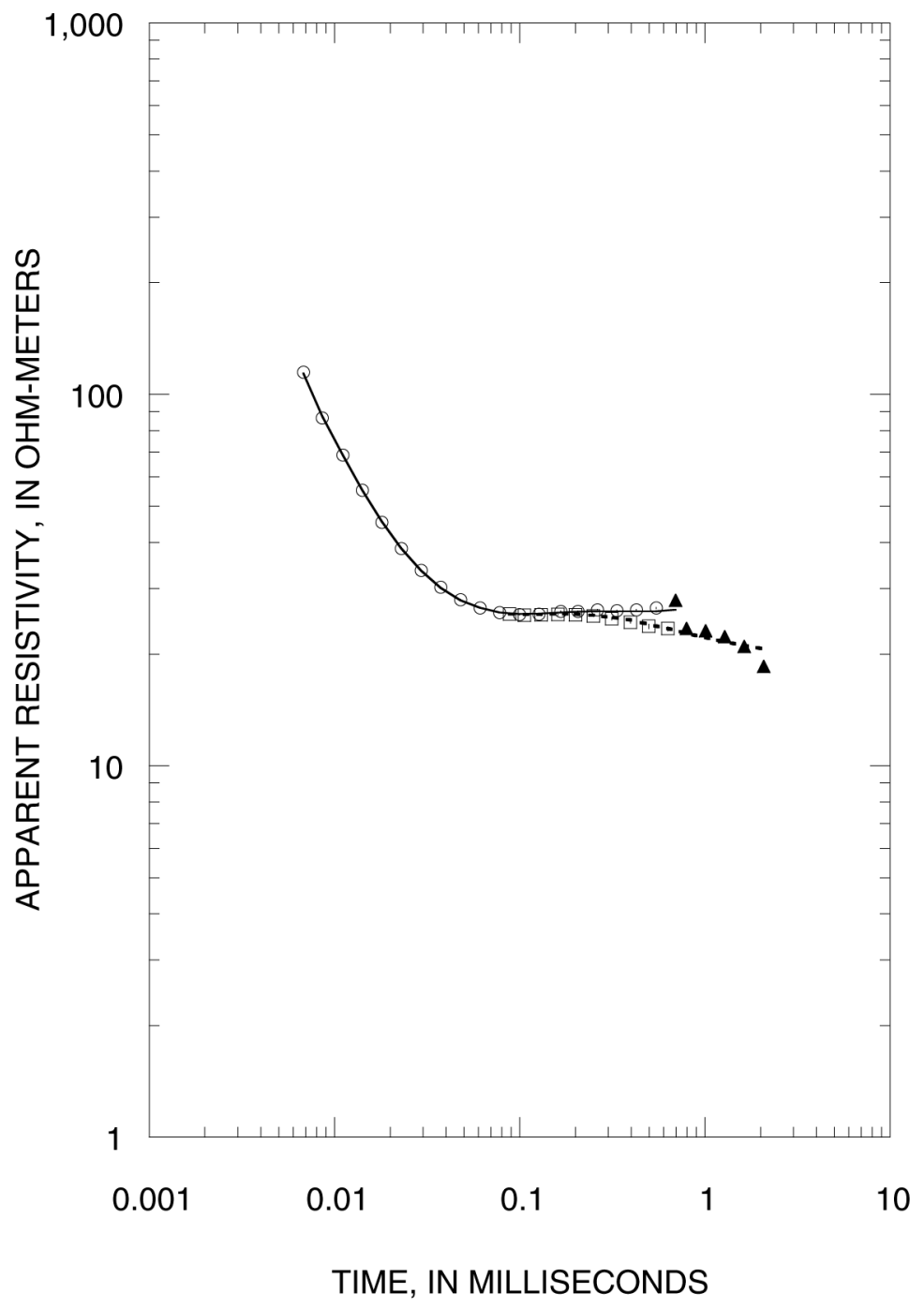

MIA229

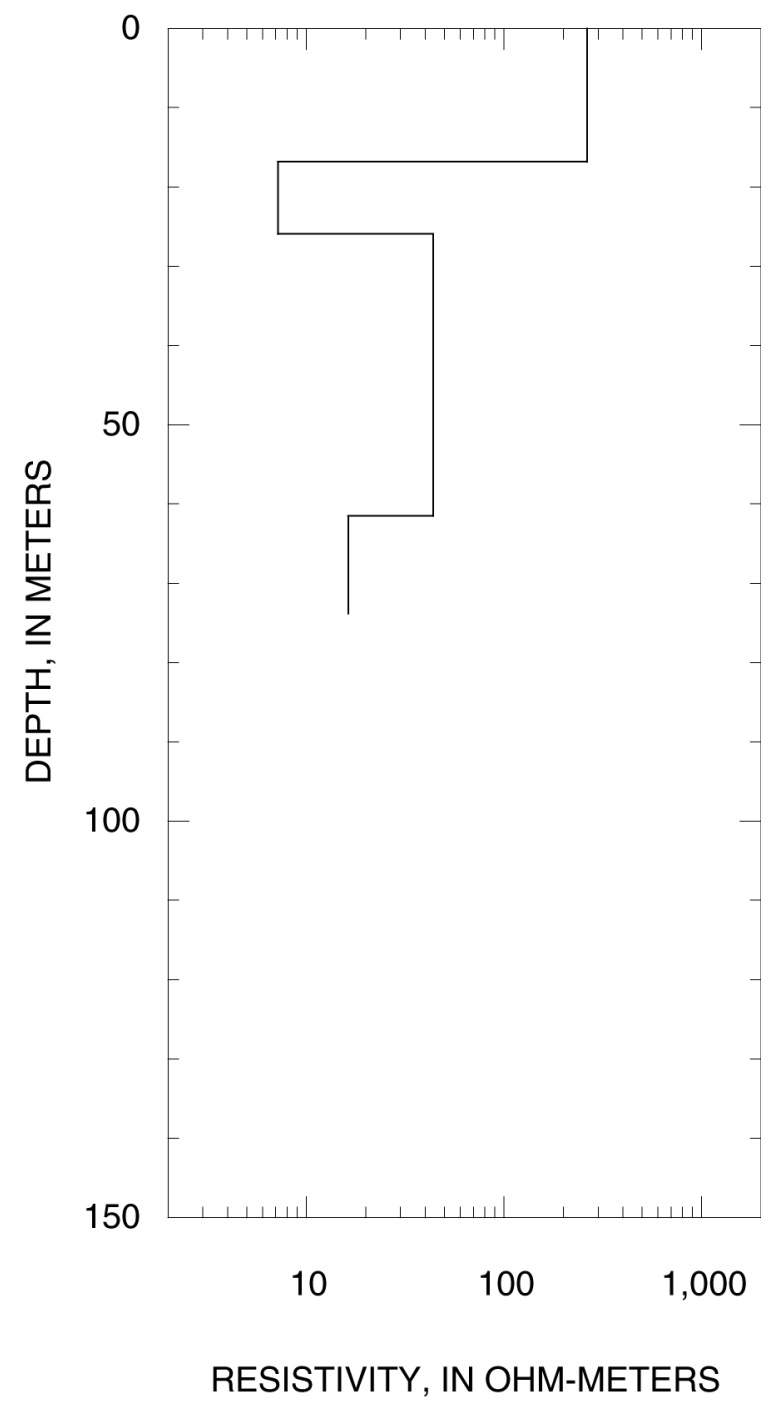

Figure 7-37. Sounding MIA229 
MIA230

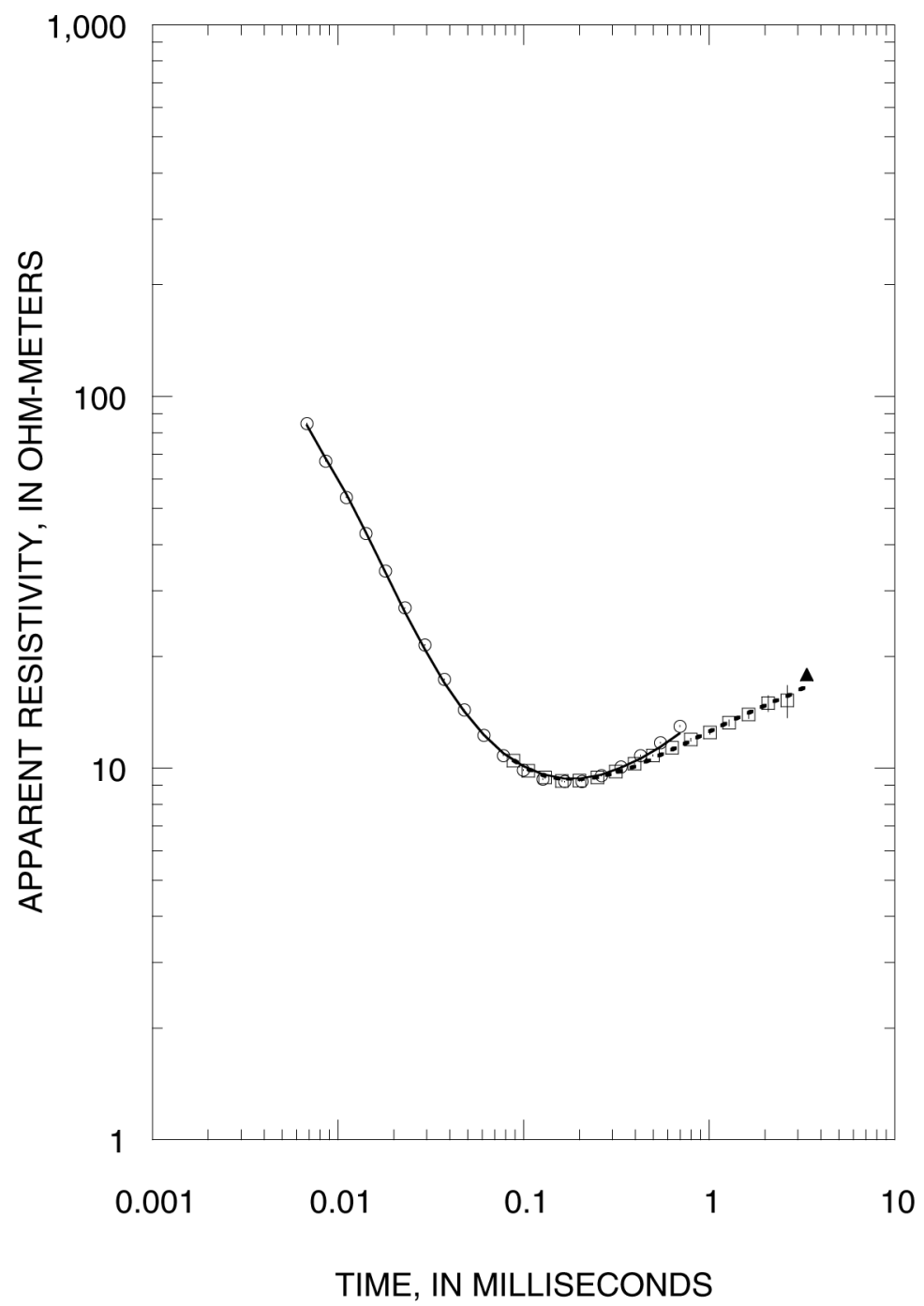

Figure 7-38. Sounding MIA230

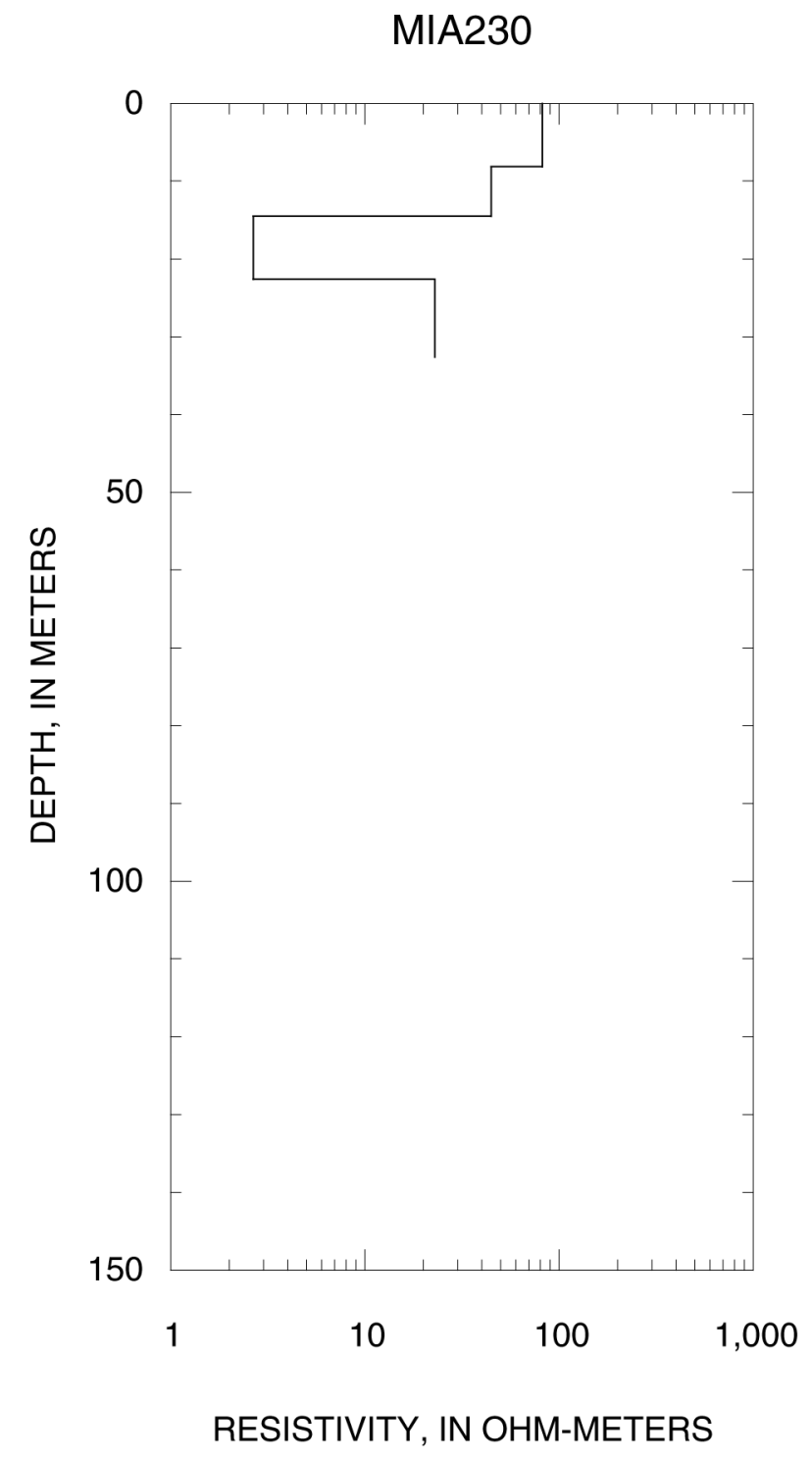


MIA230L3

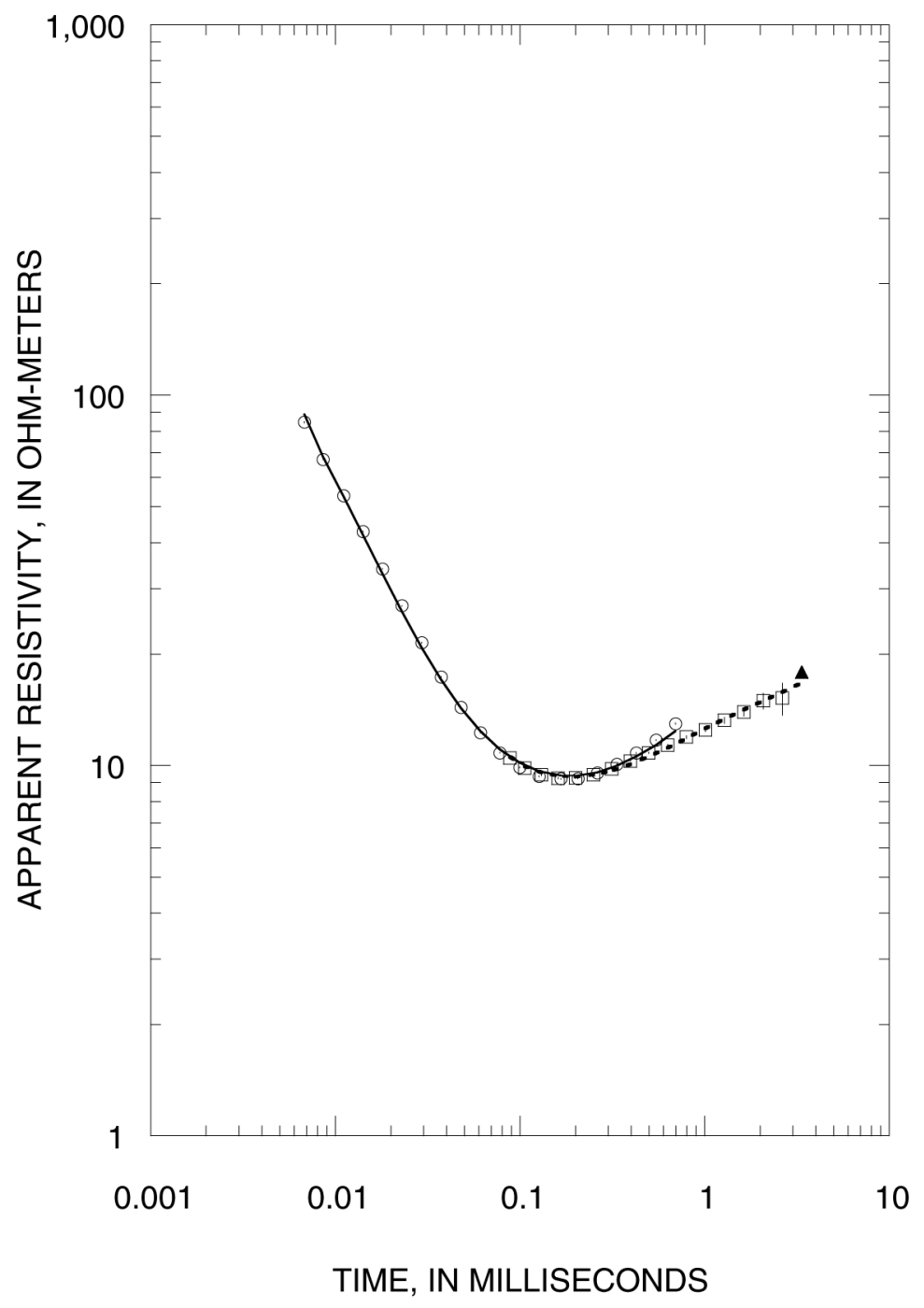

MIA230L3

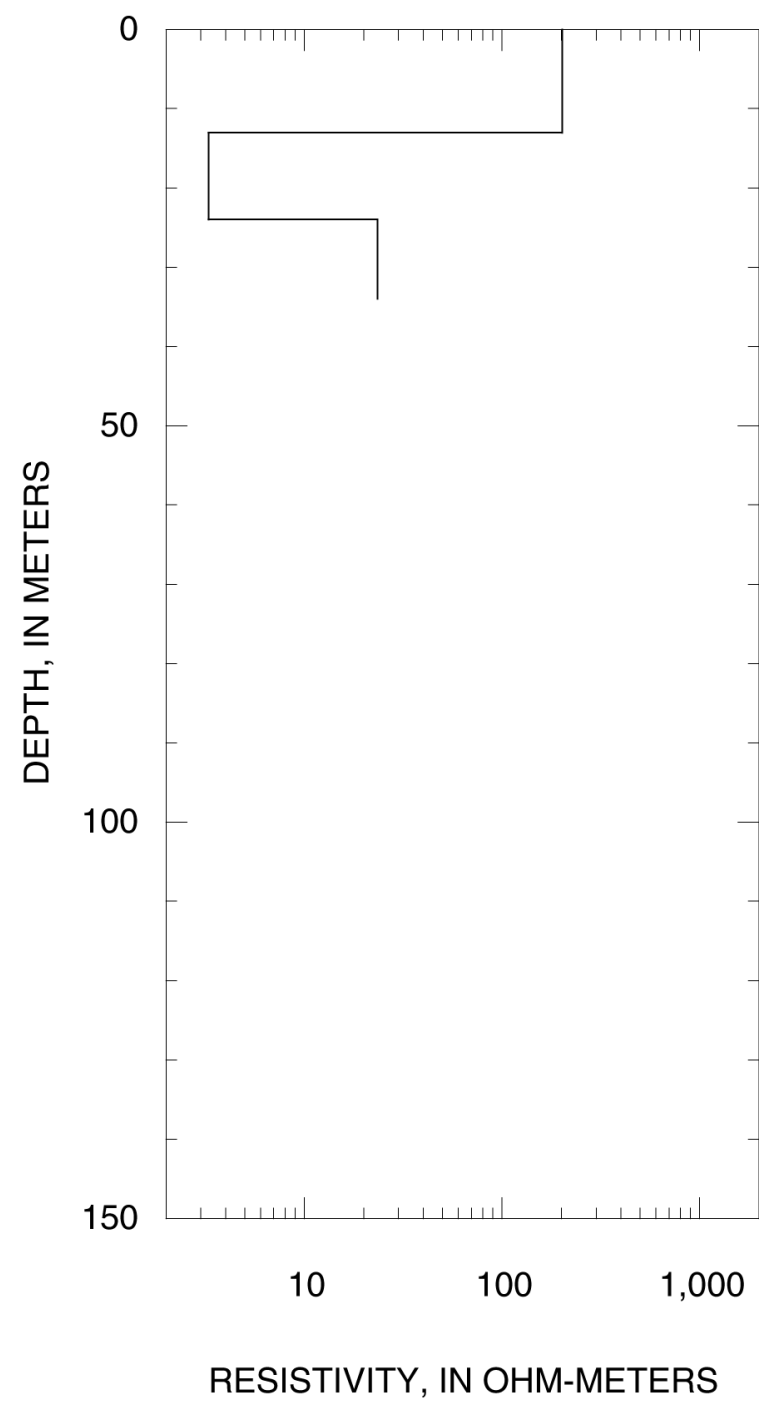

Figure 7-39. Sounding MIA230L3 
MIA231

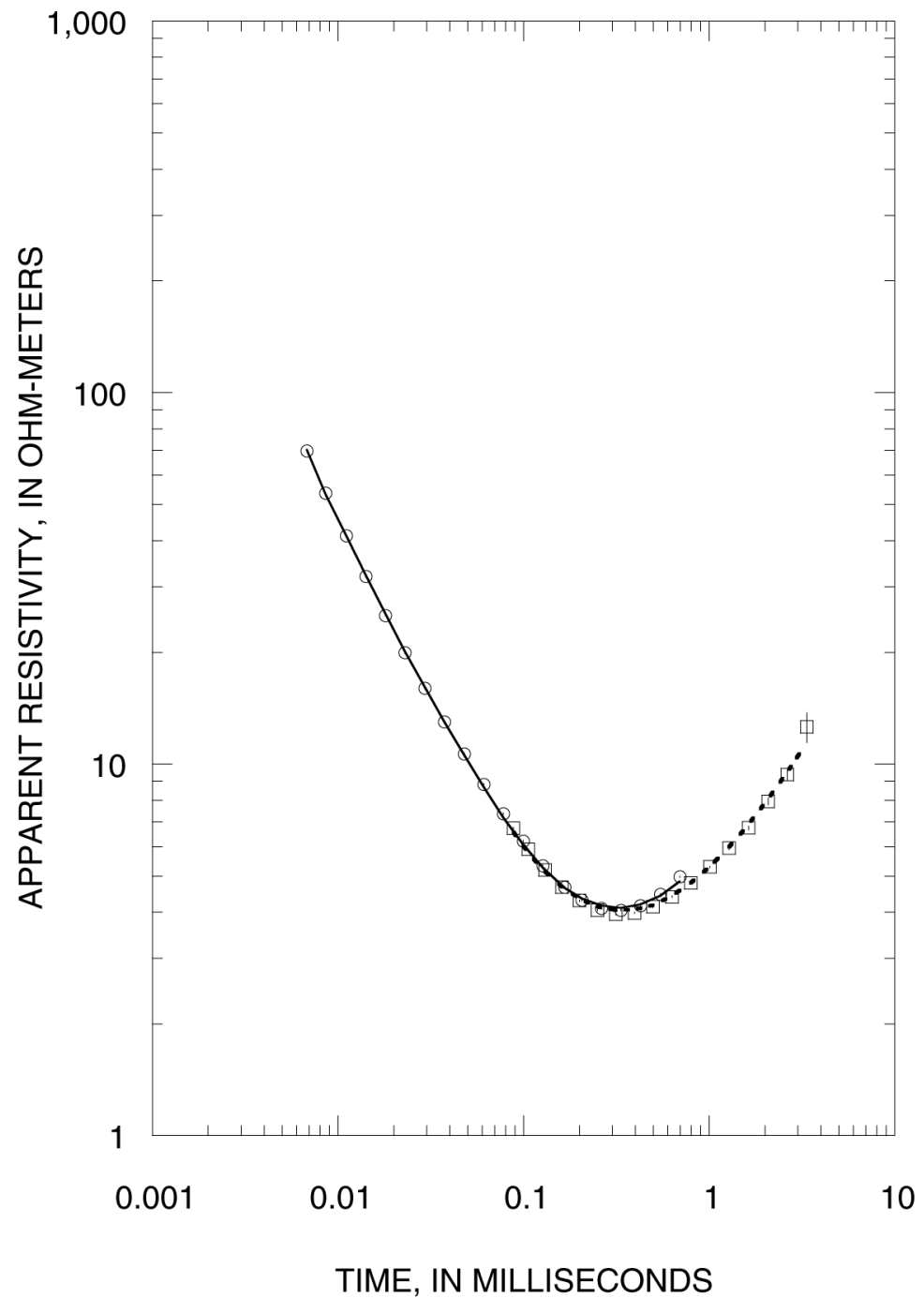

Figure 7-40. Sounding MIA231

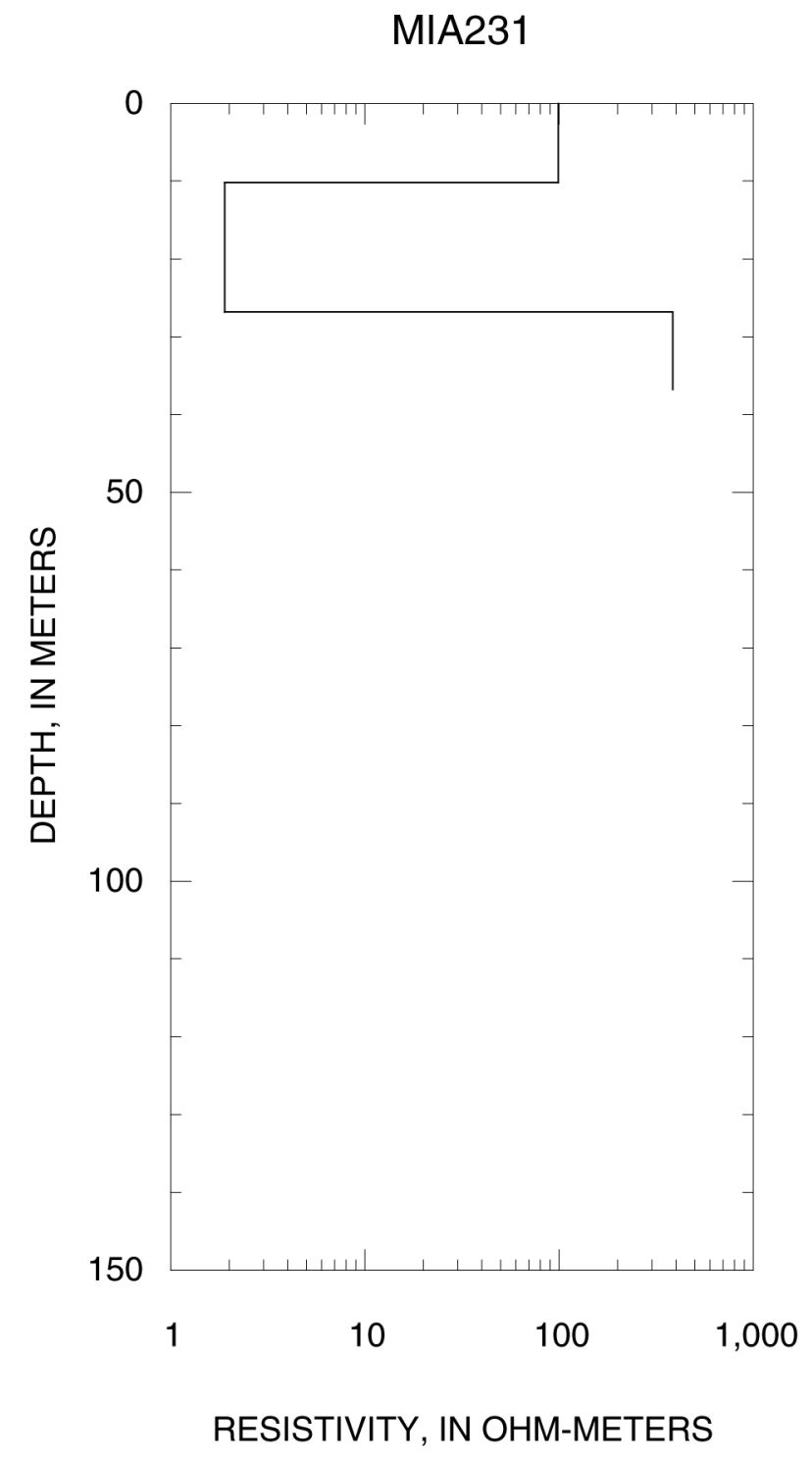


MIA232

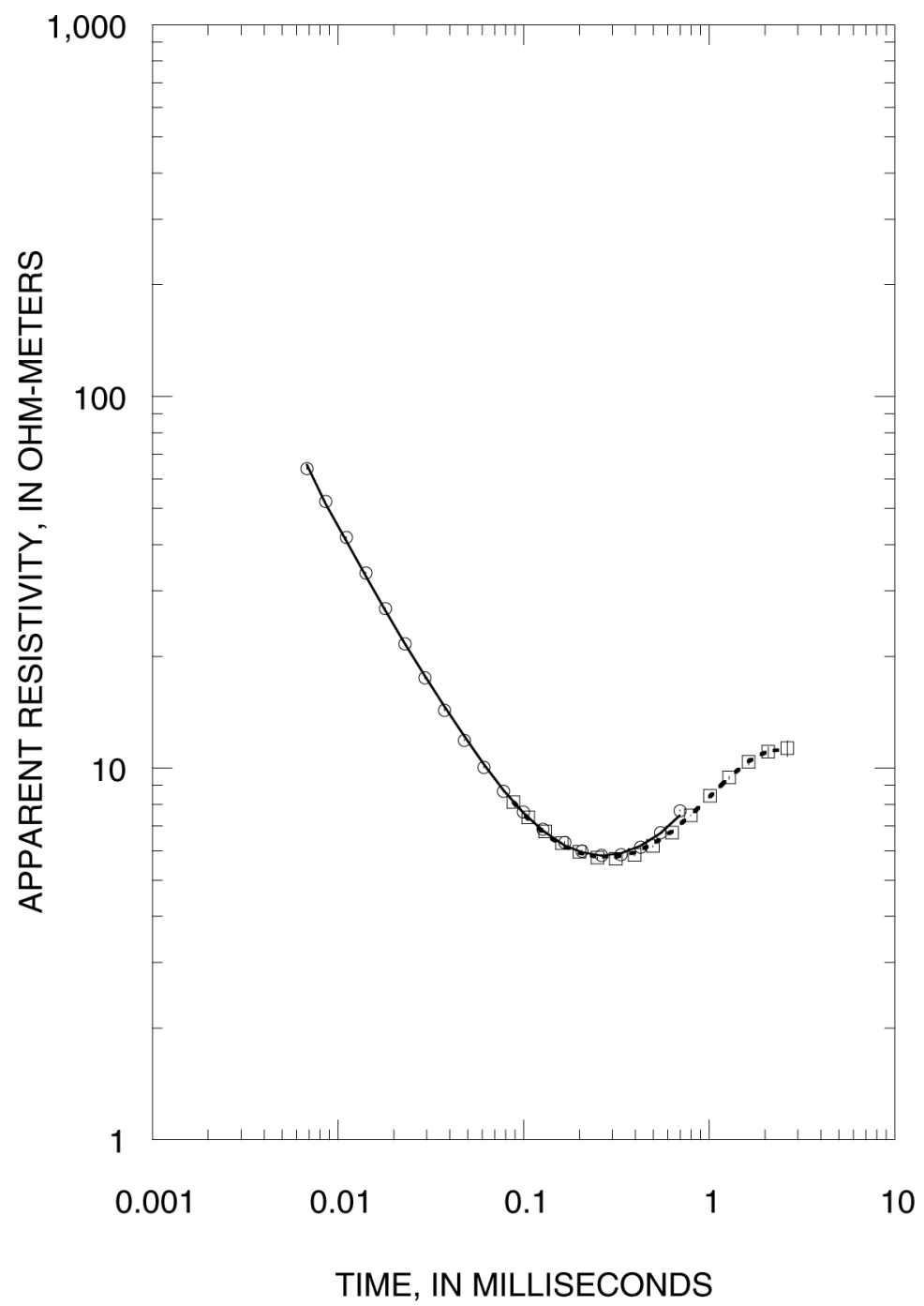

Figure 7-41. Sounding MIA232

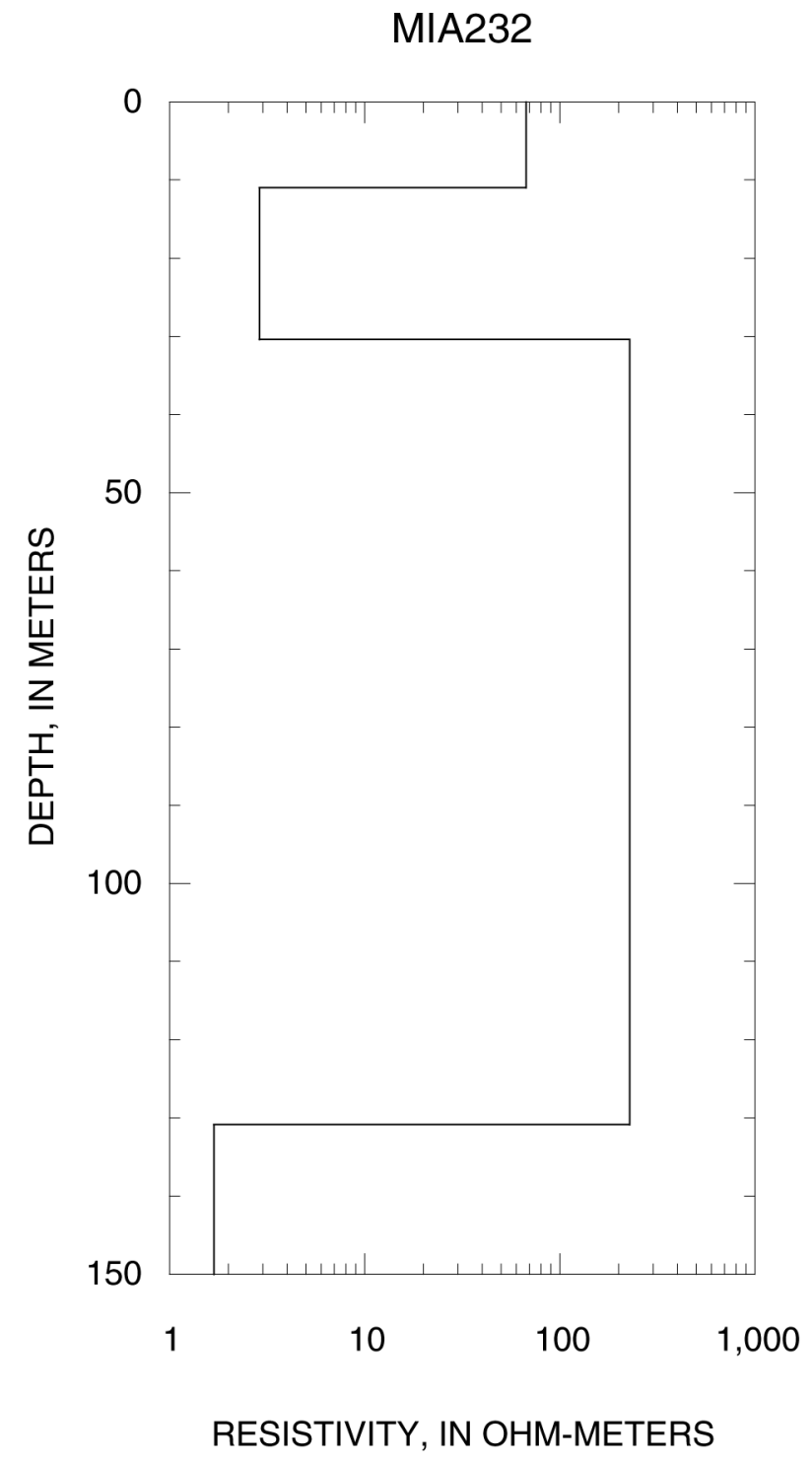


MIA233L3

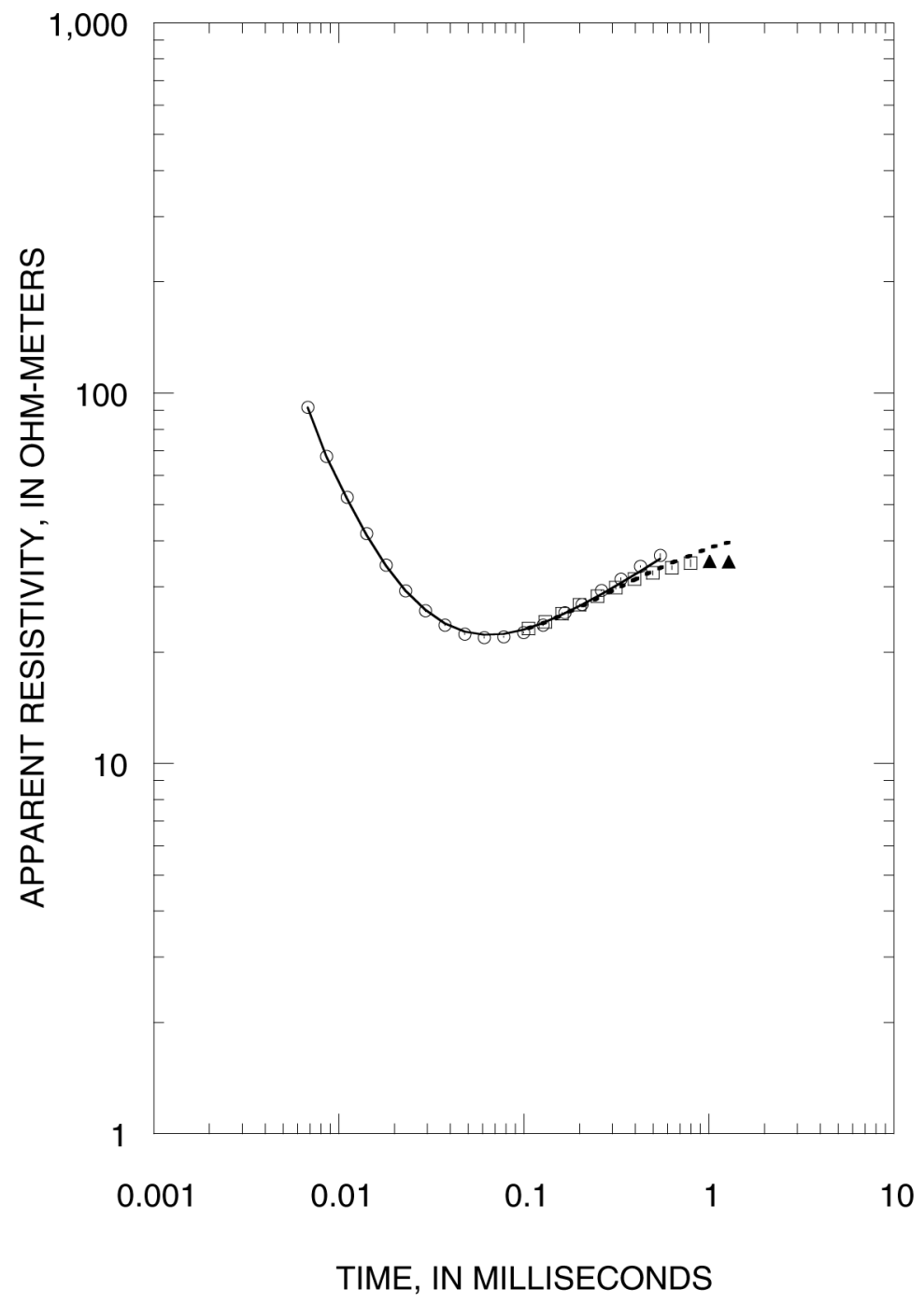

Figure 7-42. Sounding MIA232L3

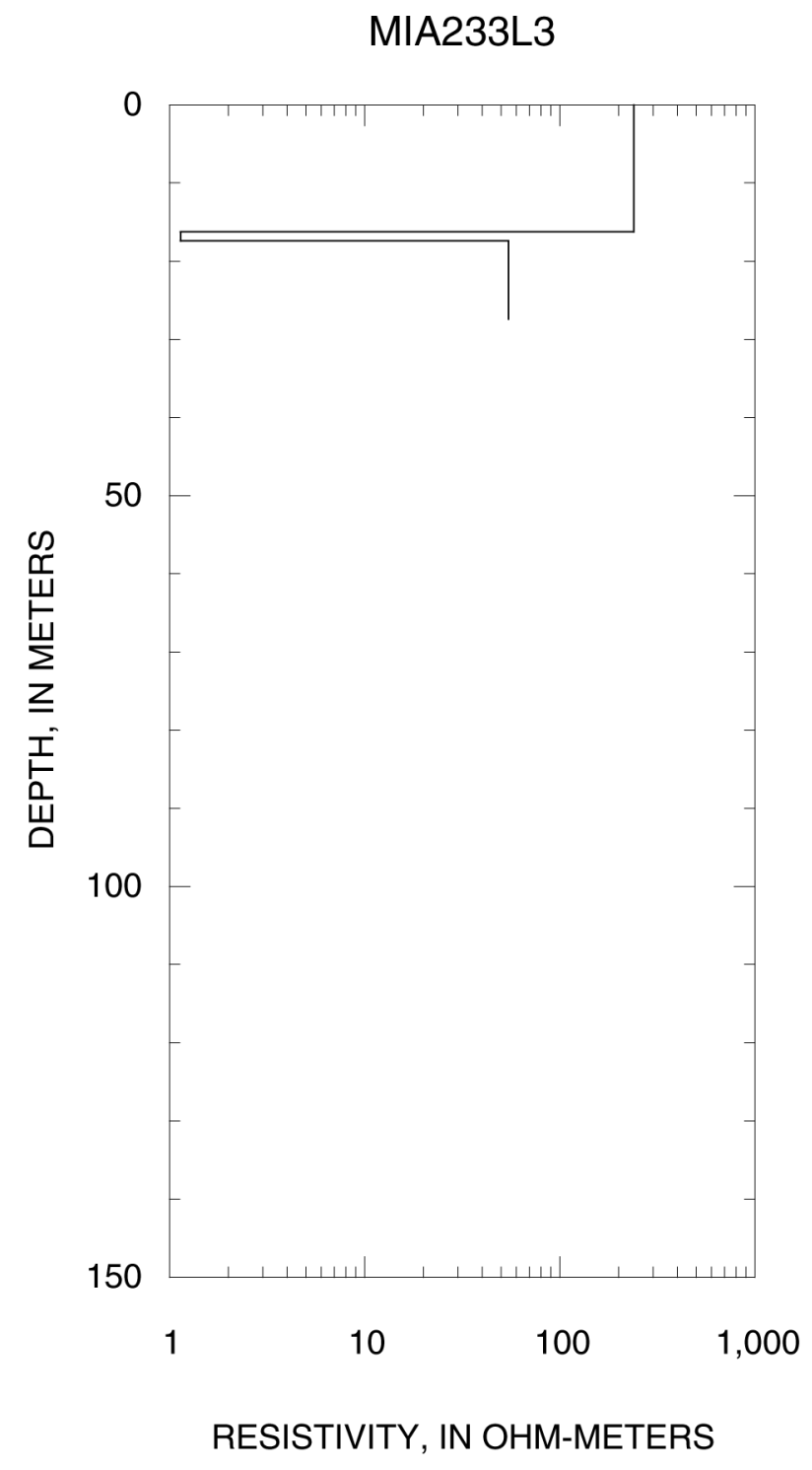


MIA233

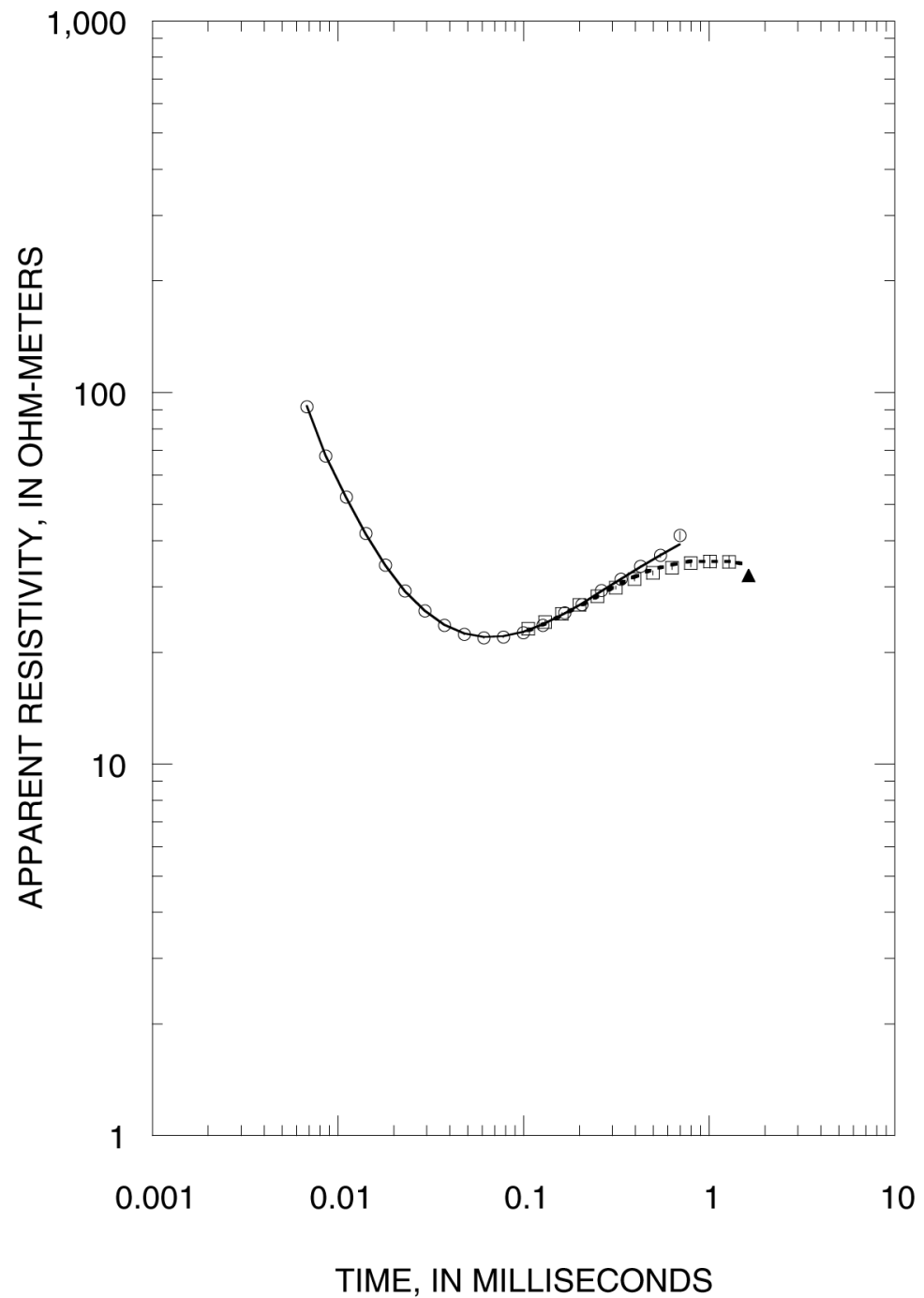

Figure 7-43. Sounding MIA233

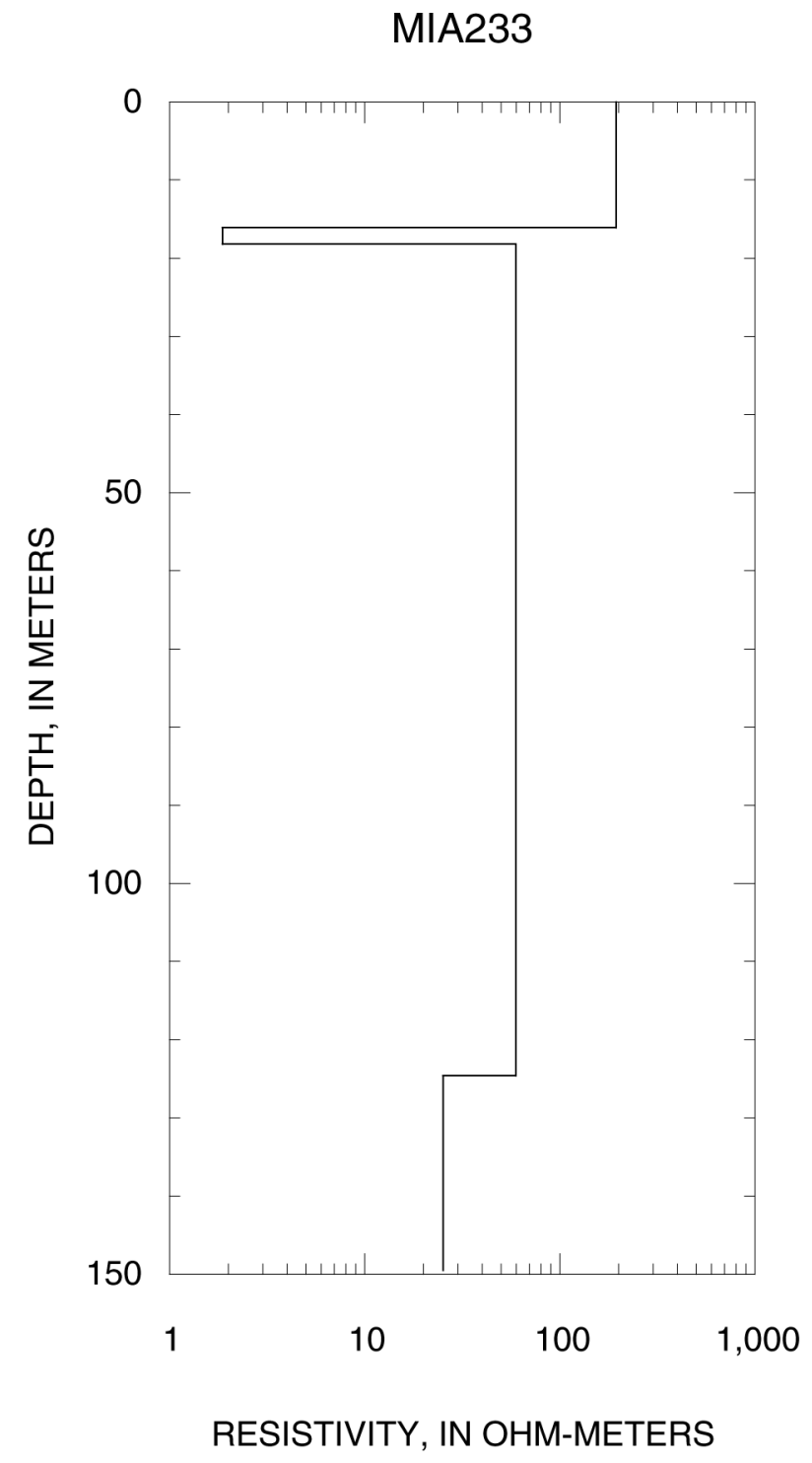



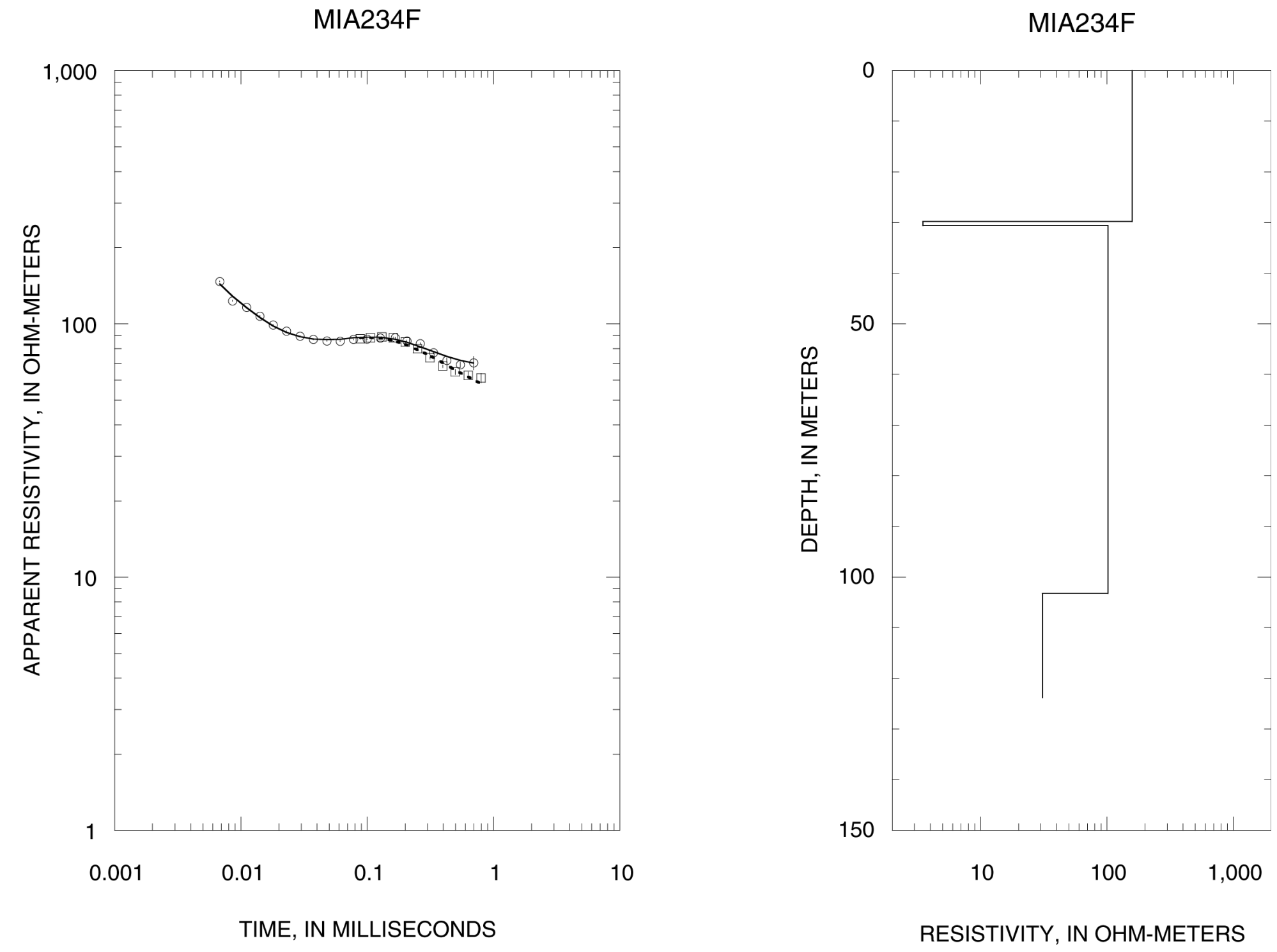

Figure 7-44. Sounding MIA233F 
MIA233L3

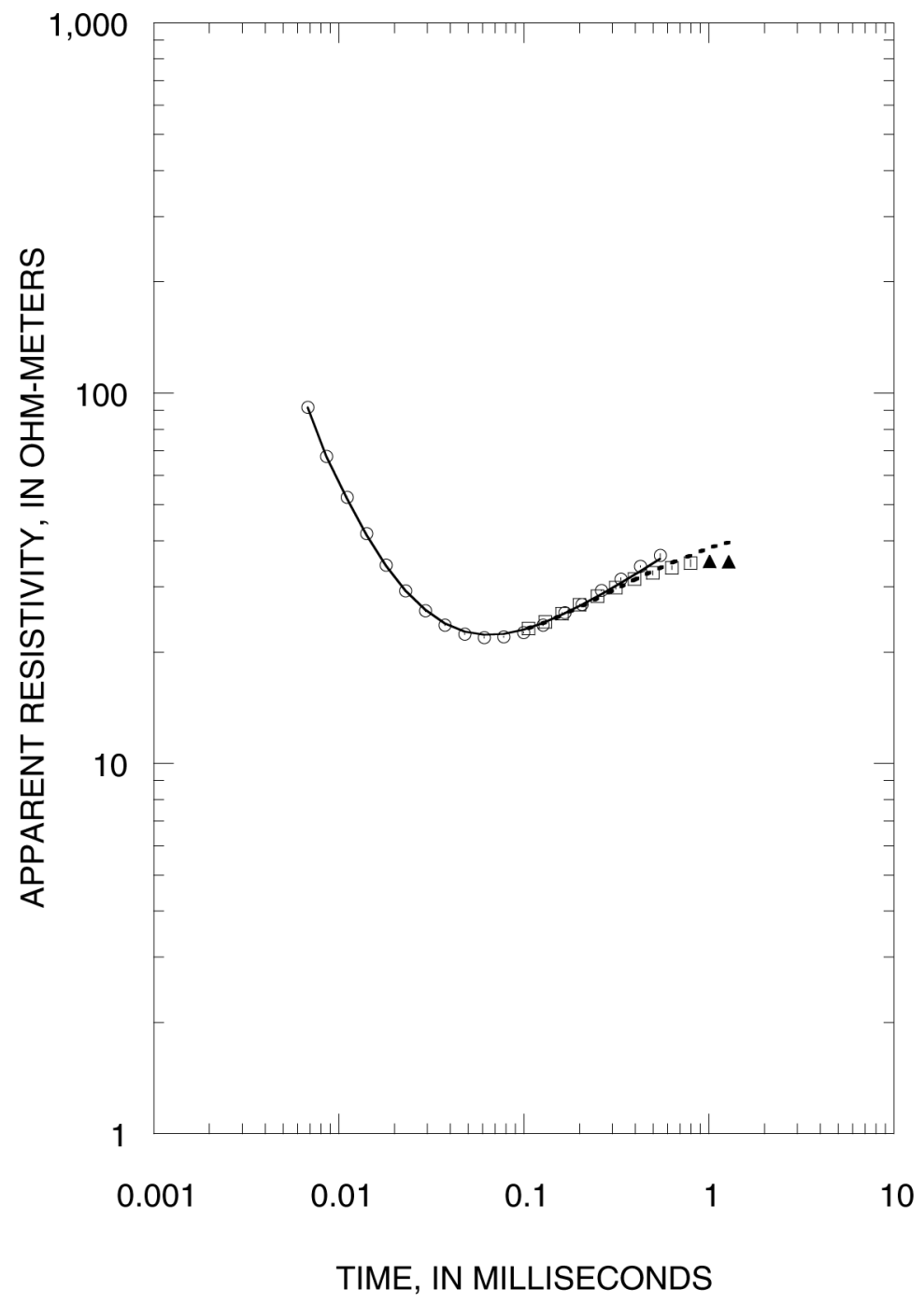

Figure 7-45. Sounding MIA233L3

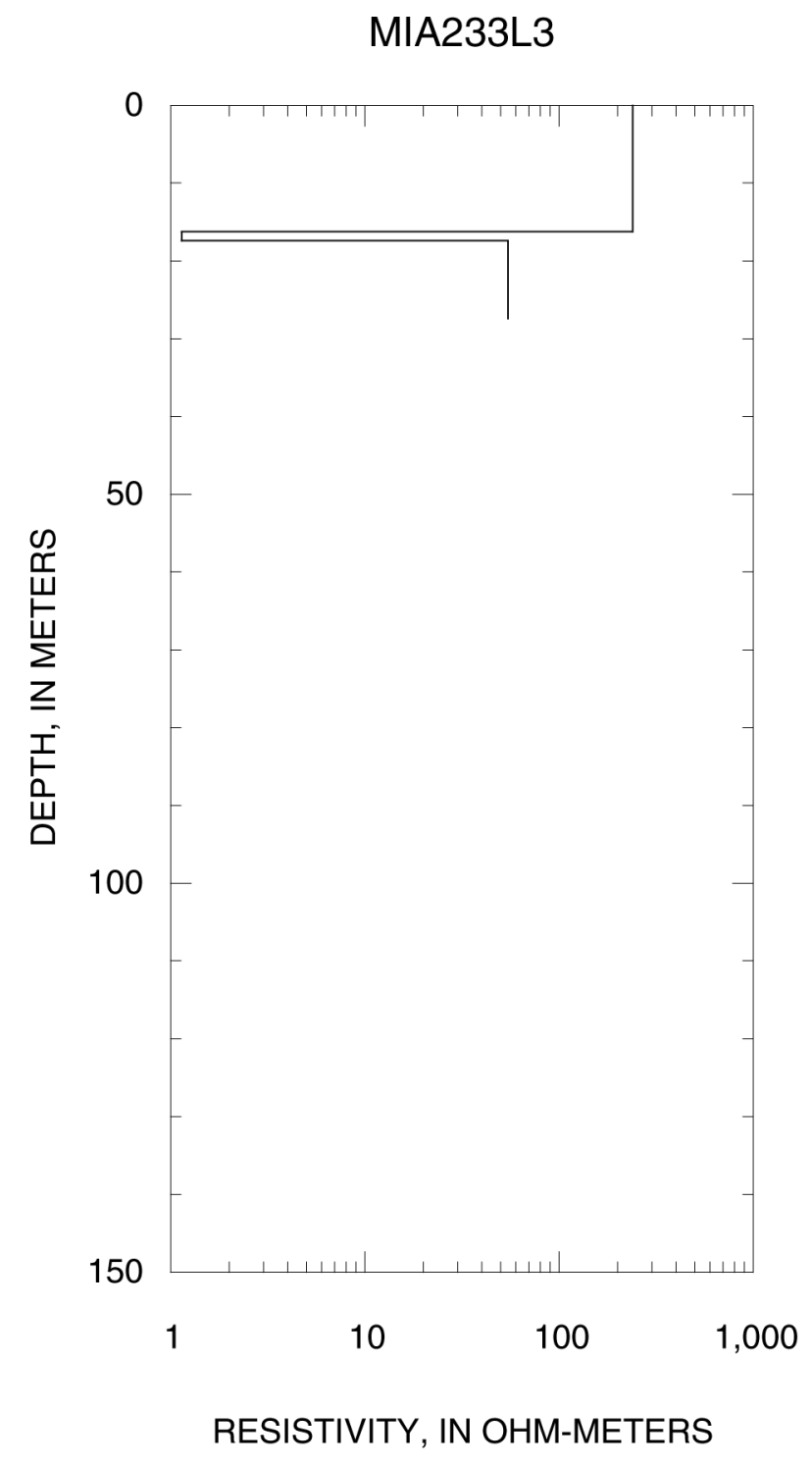


MIA233F3

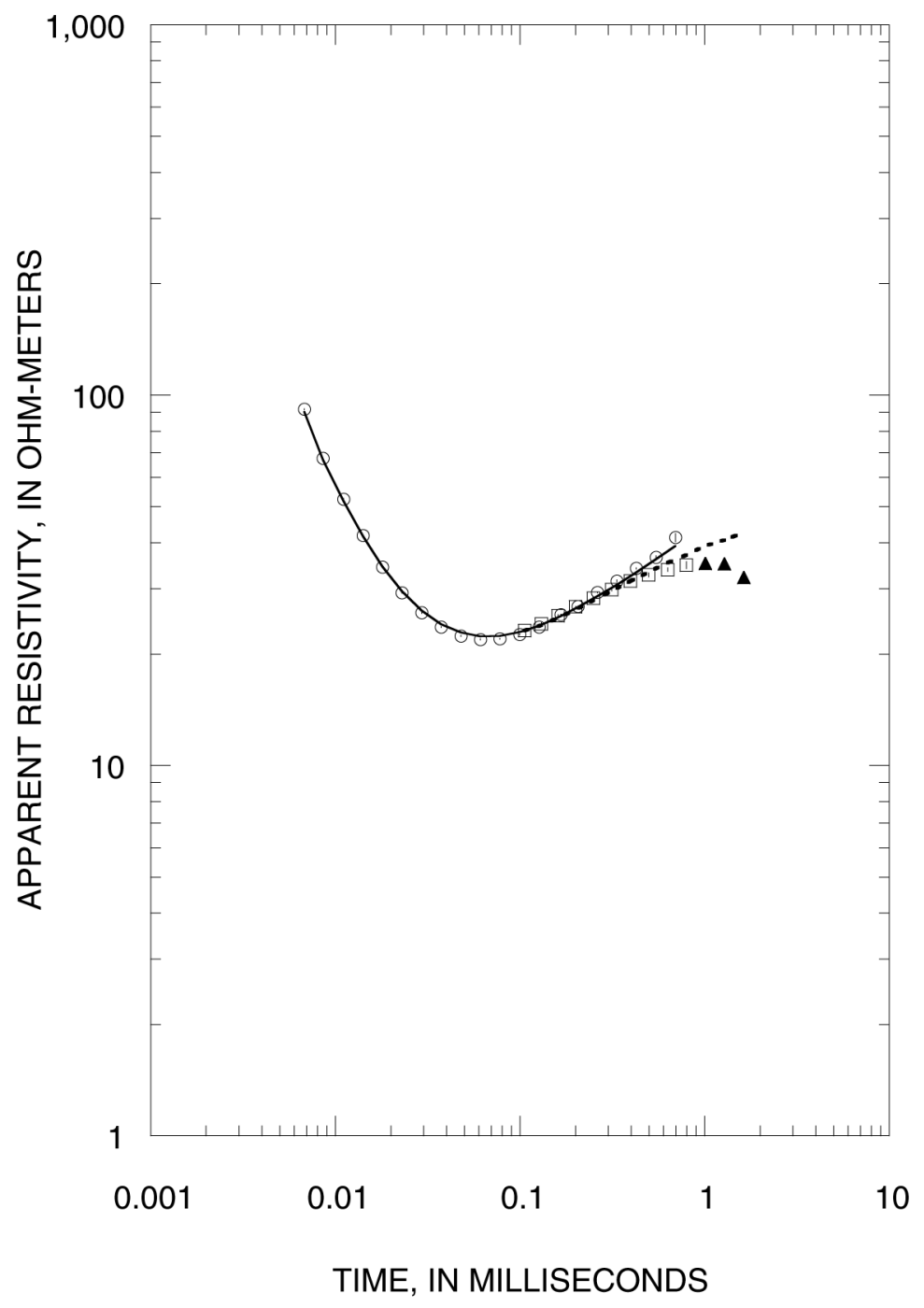

MIA233F3

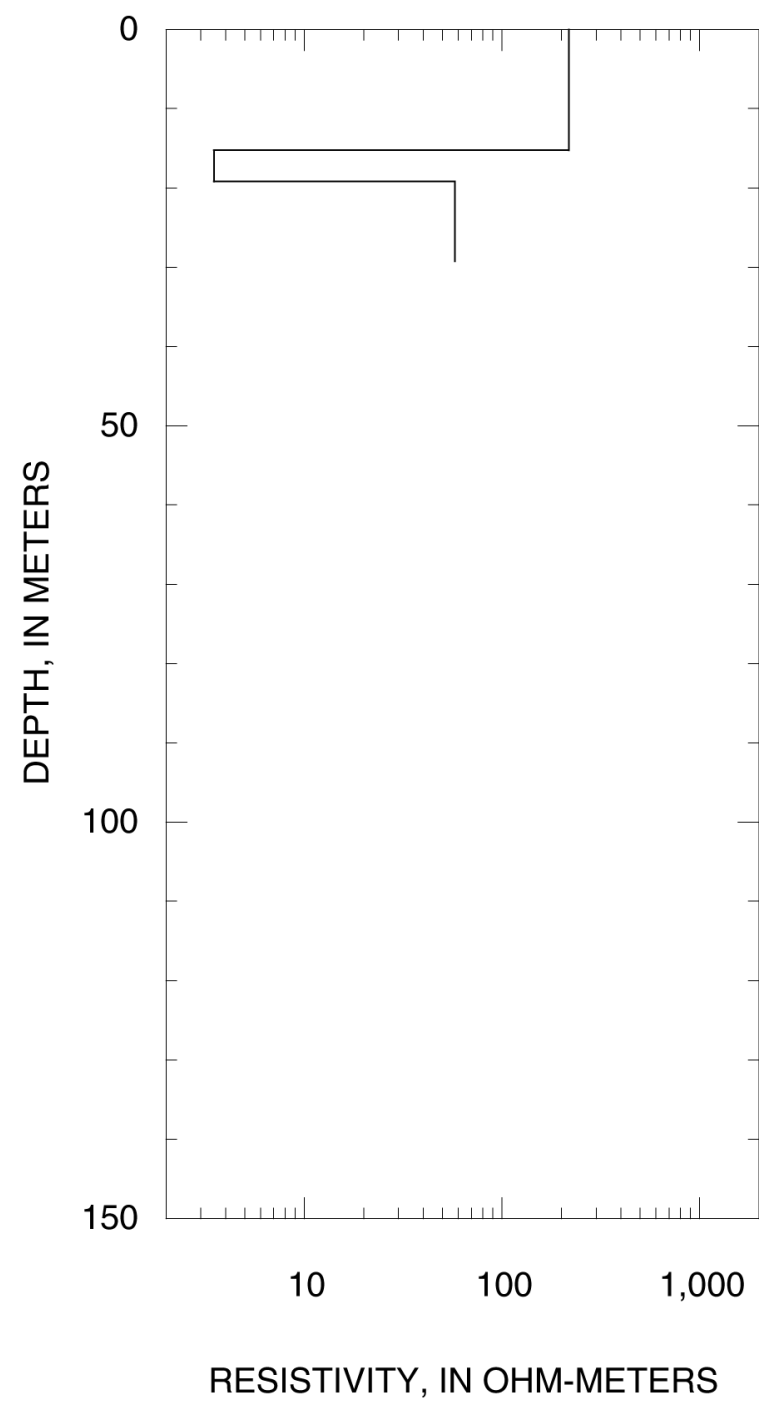

Figure 7-46. Sounding MIA233F3 
MIA234

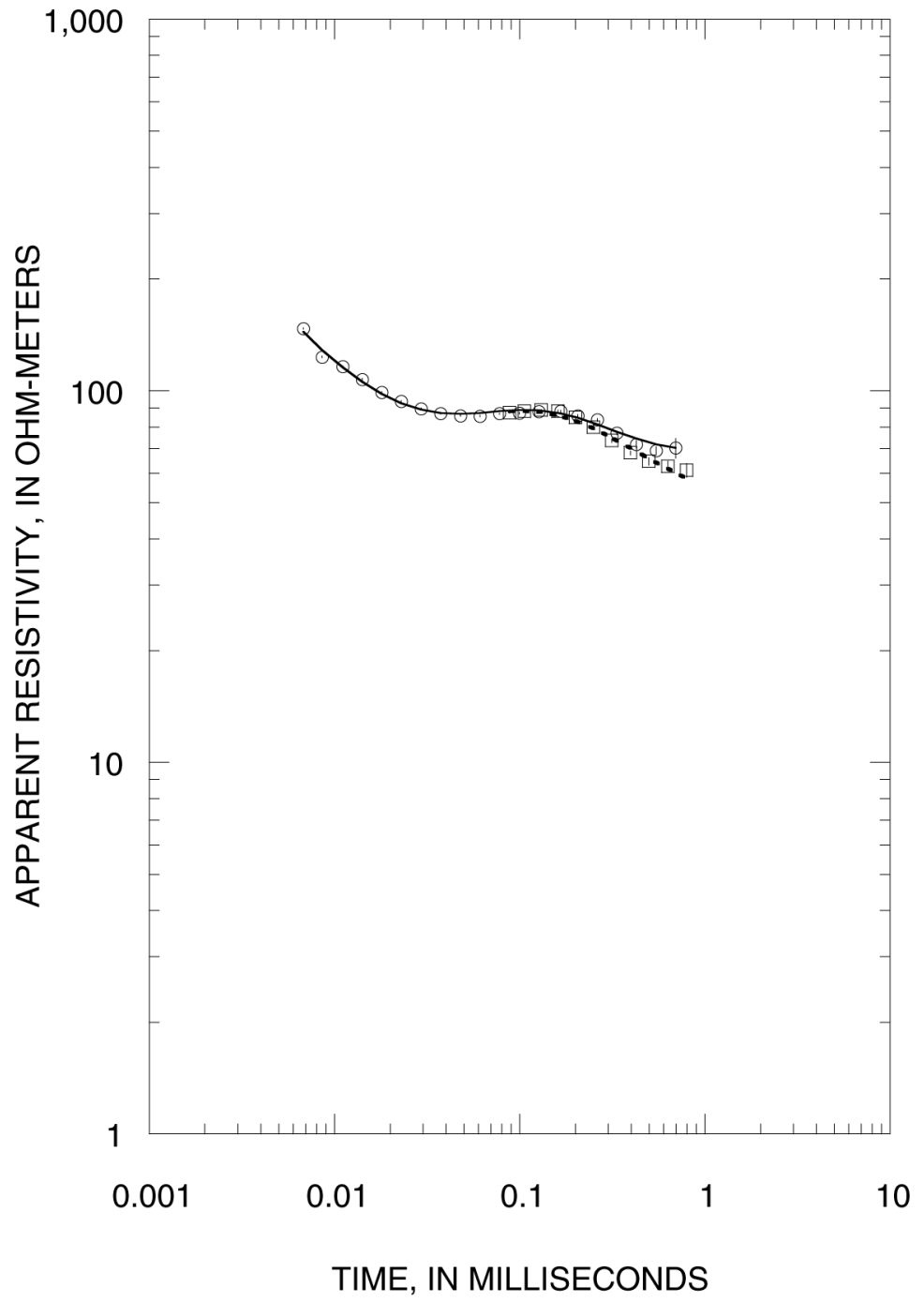

Figure 7-47. Sounding MIA234

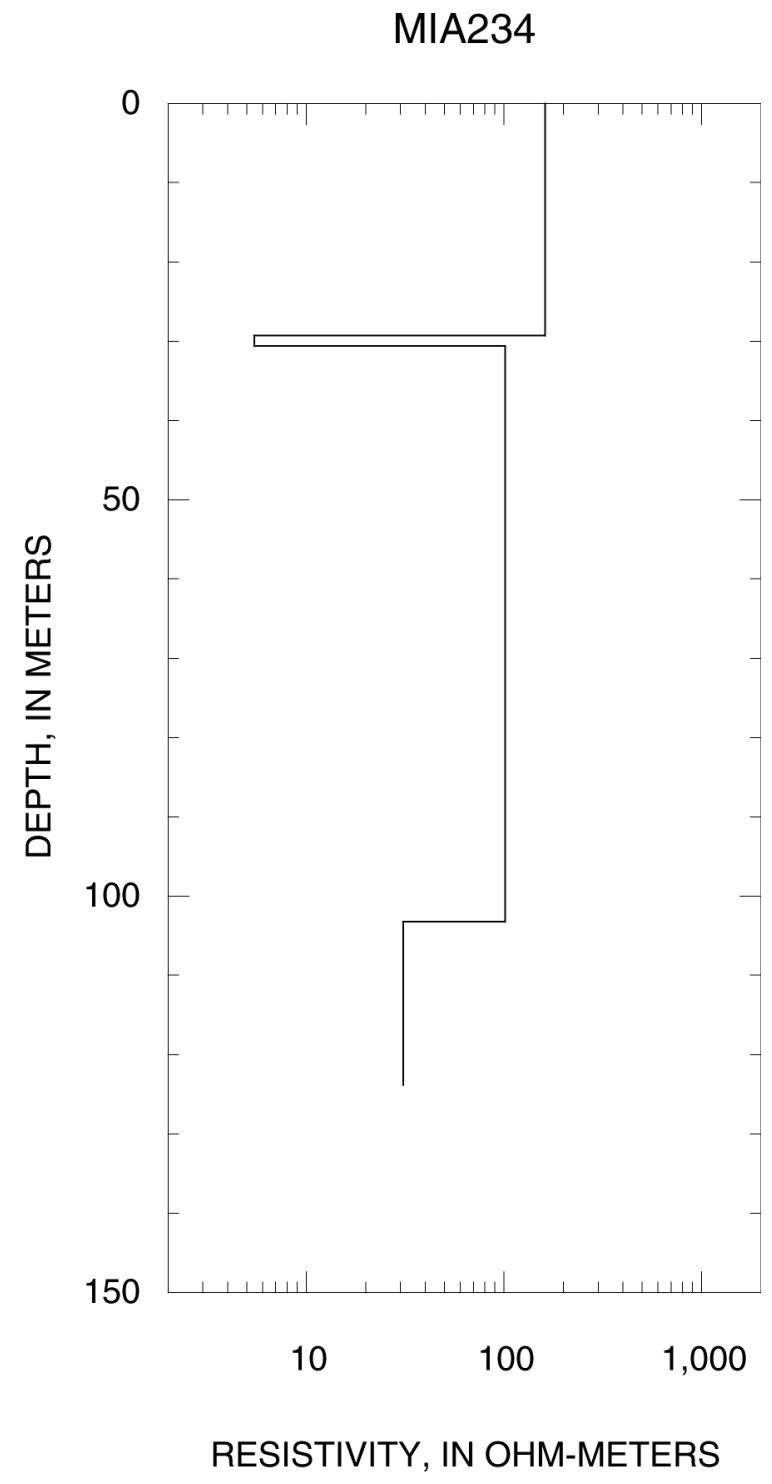



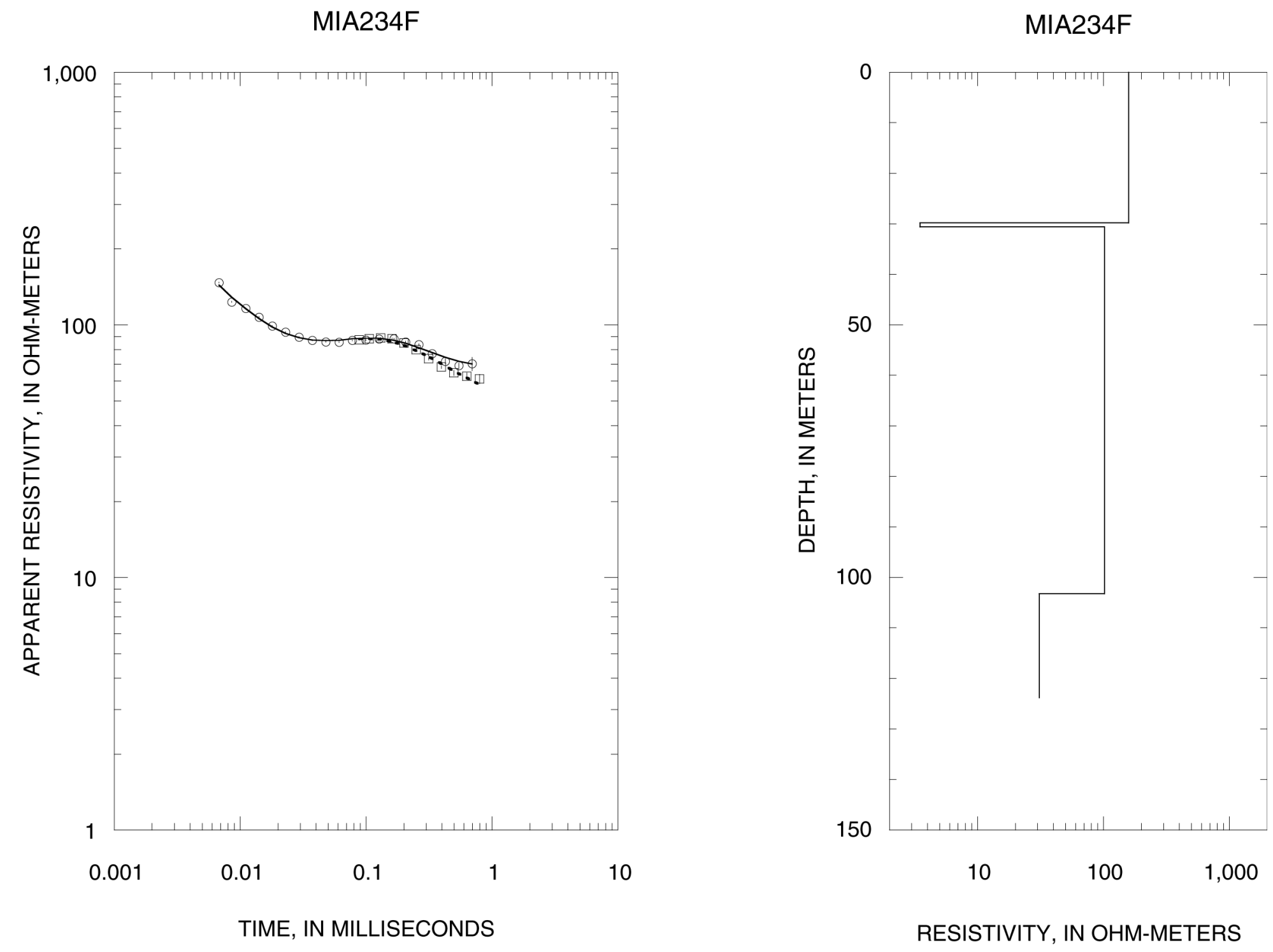

Figure 7-48. Sounding MIA234F 
MIA235

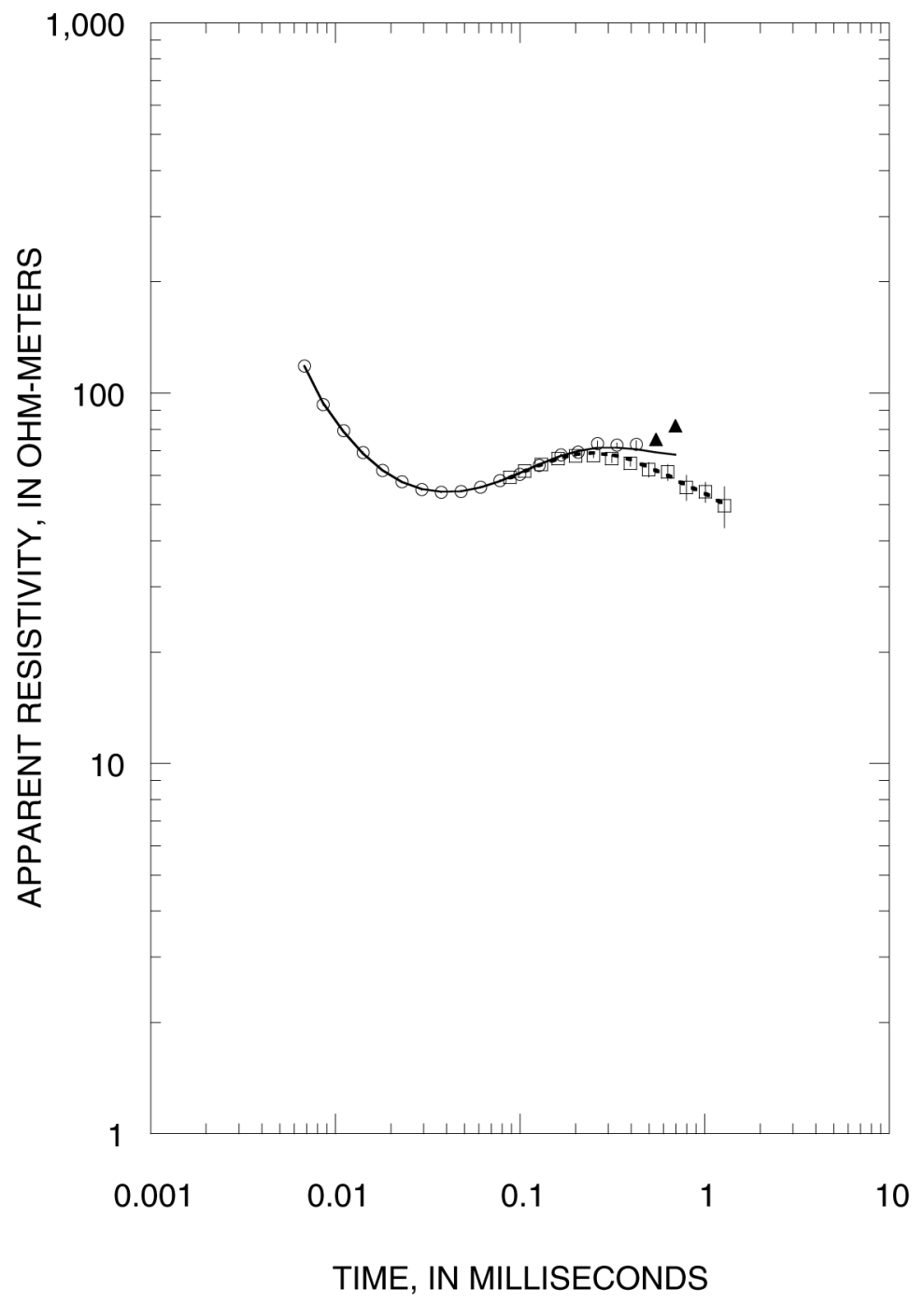

MIA235

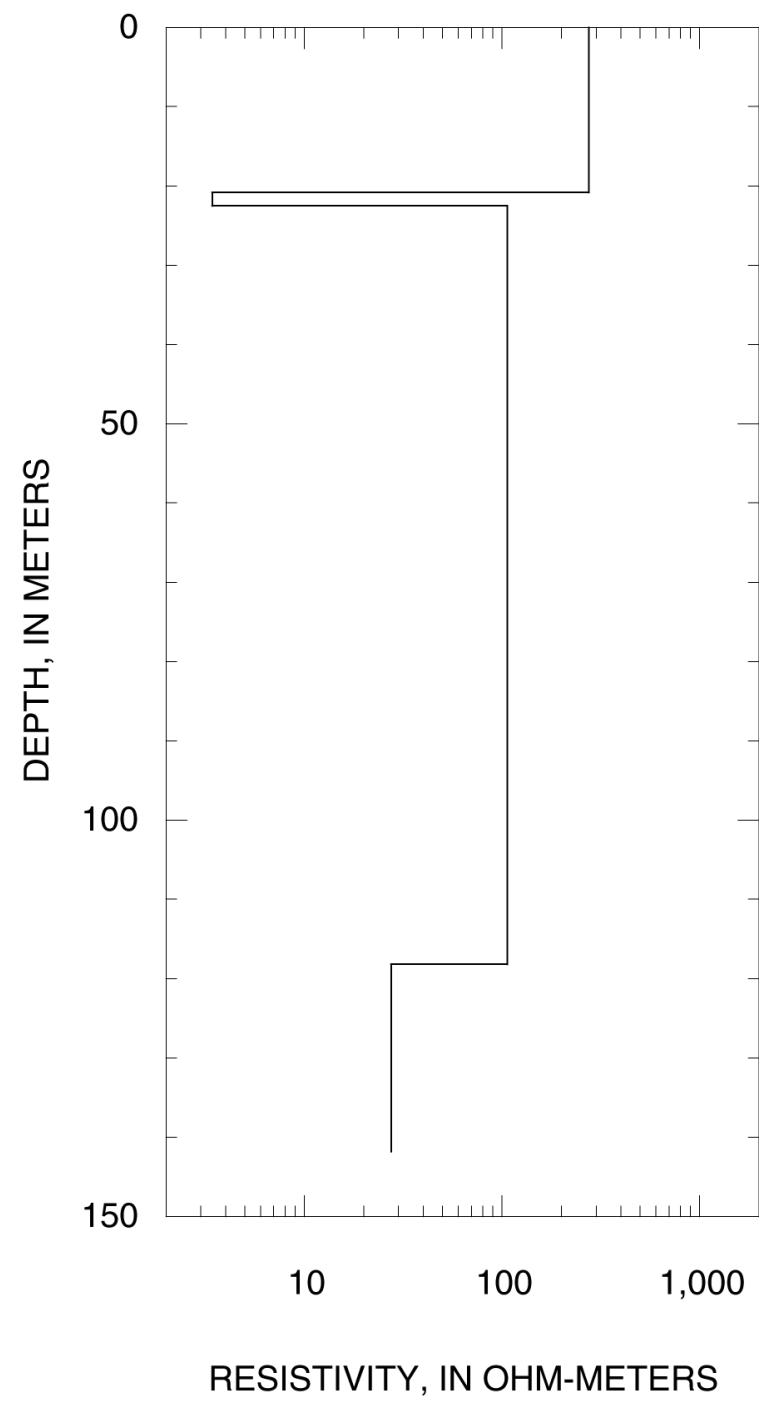

Figure 7-49. Sounding MIA235 

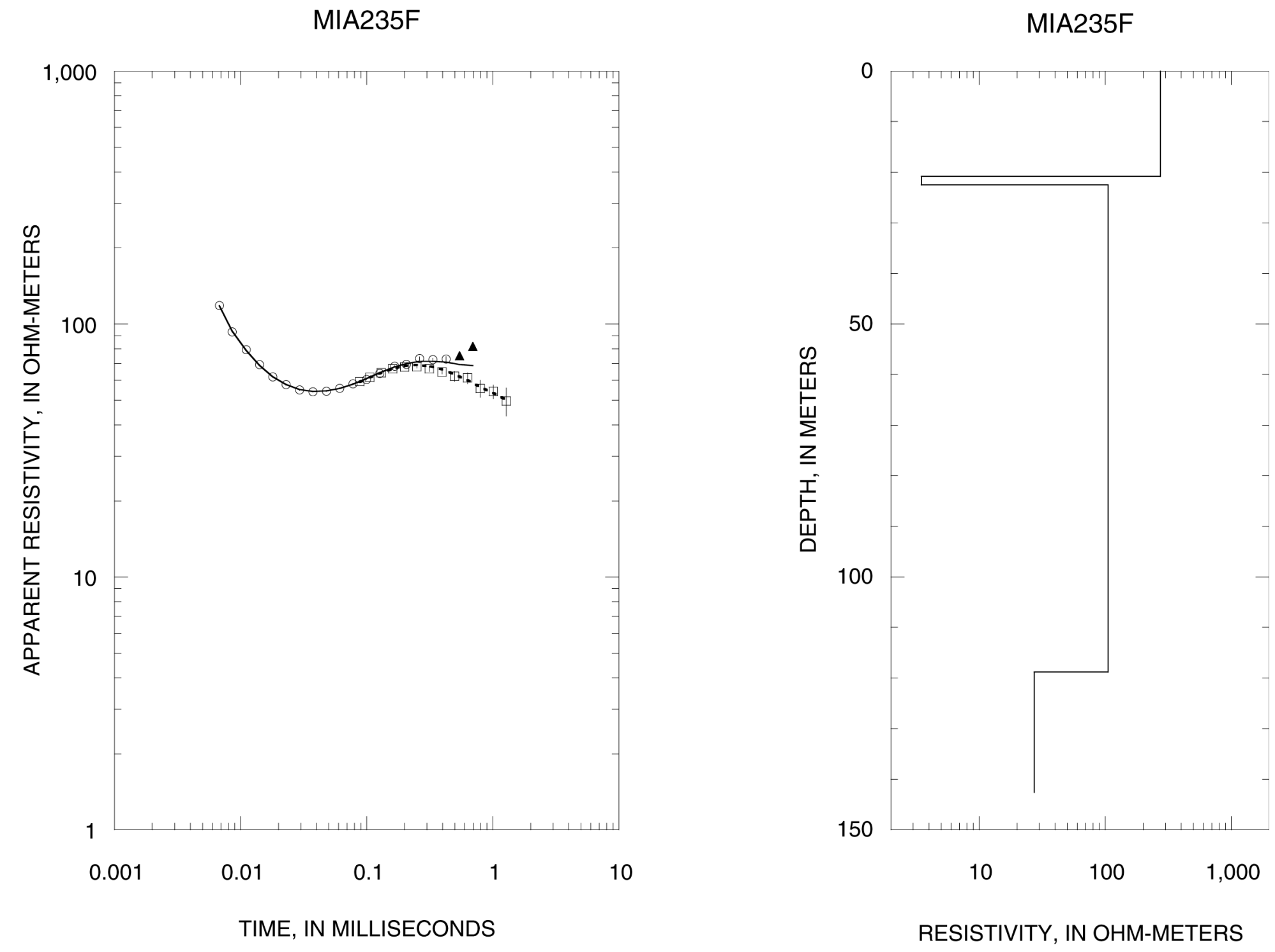

Figure 7-50. Sounding MIA235F 
MIA236

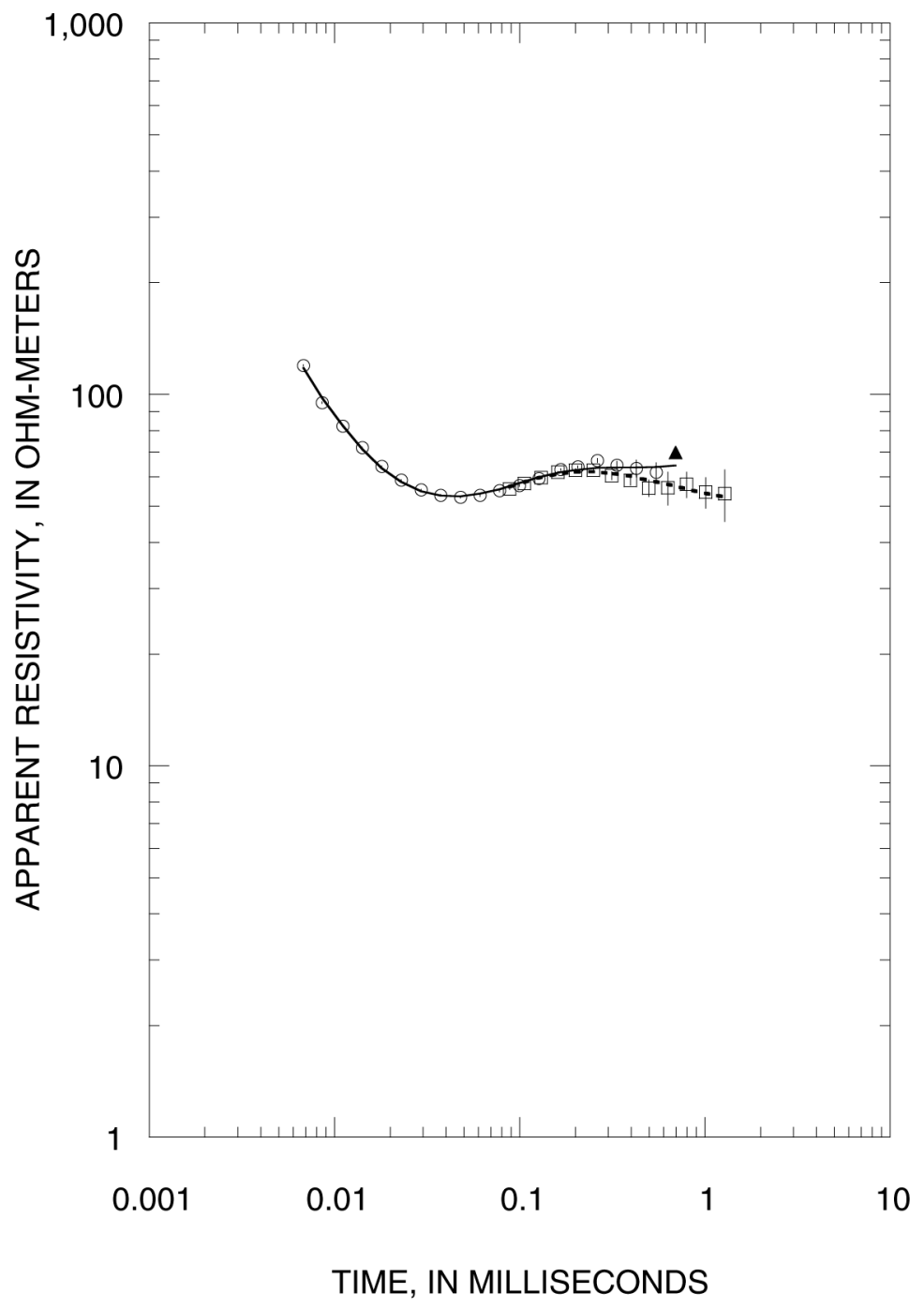

MIA236

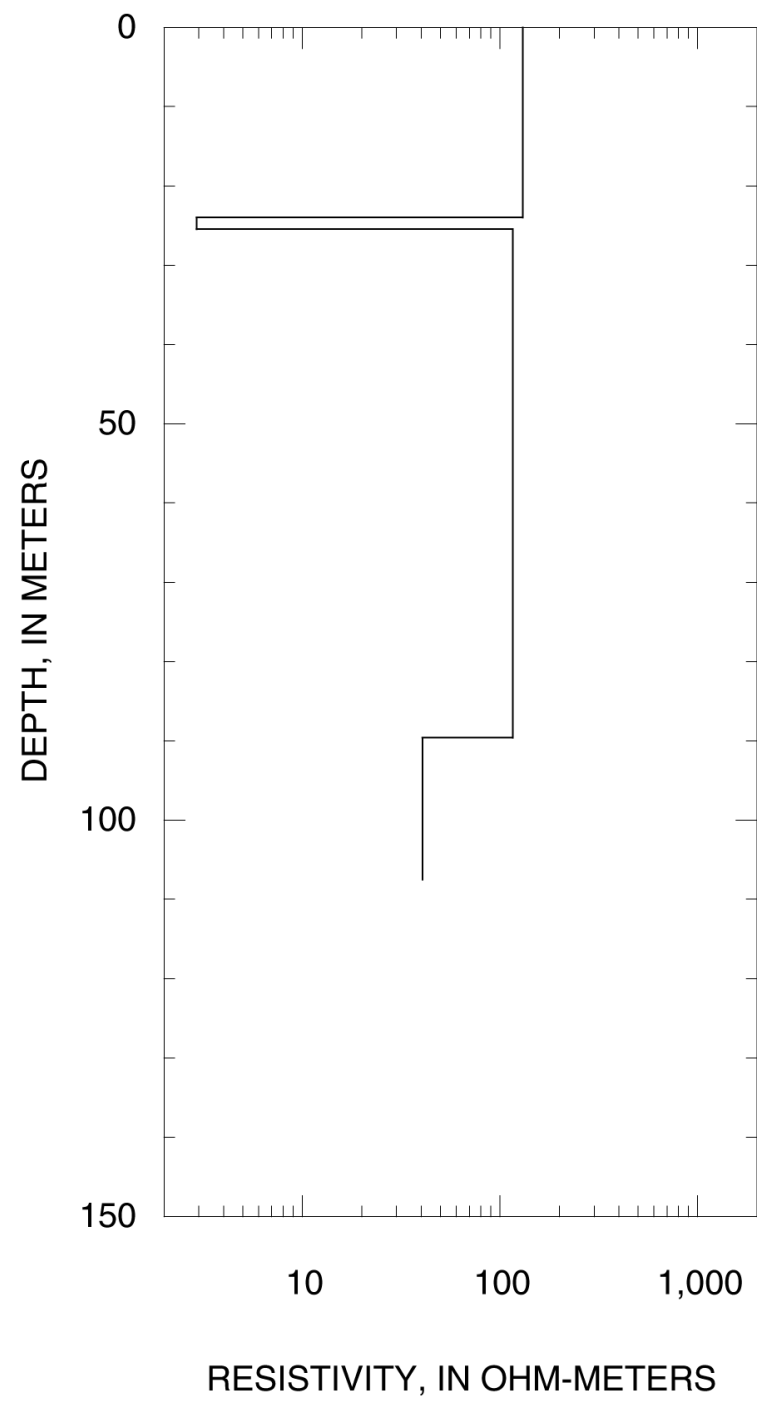

Figure 7-51. Sounding MIA236 

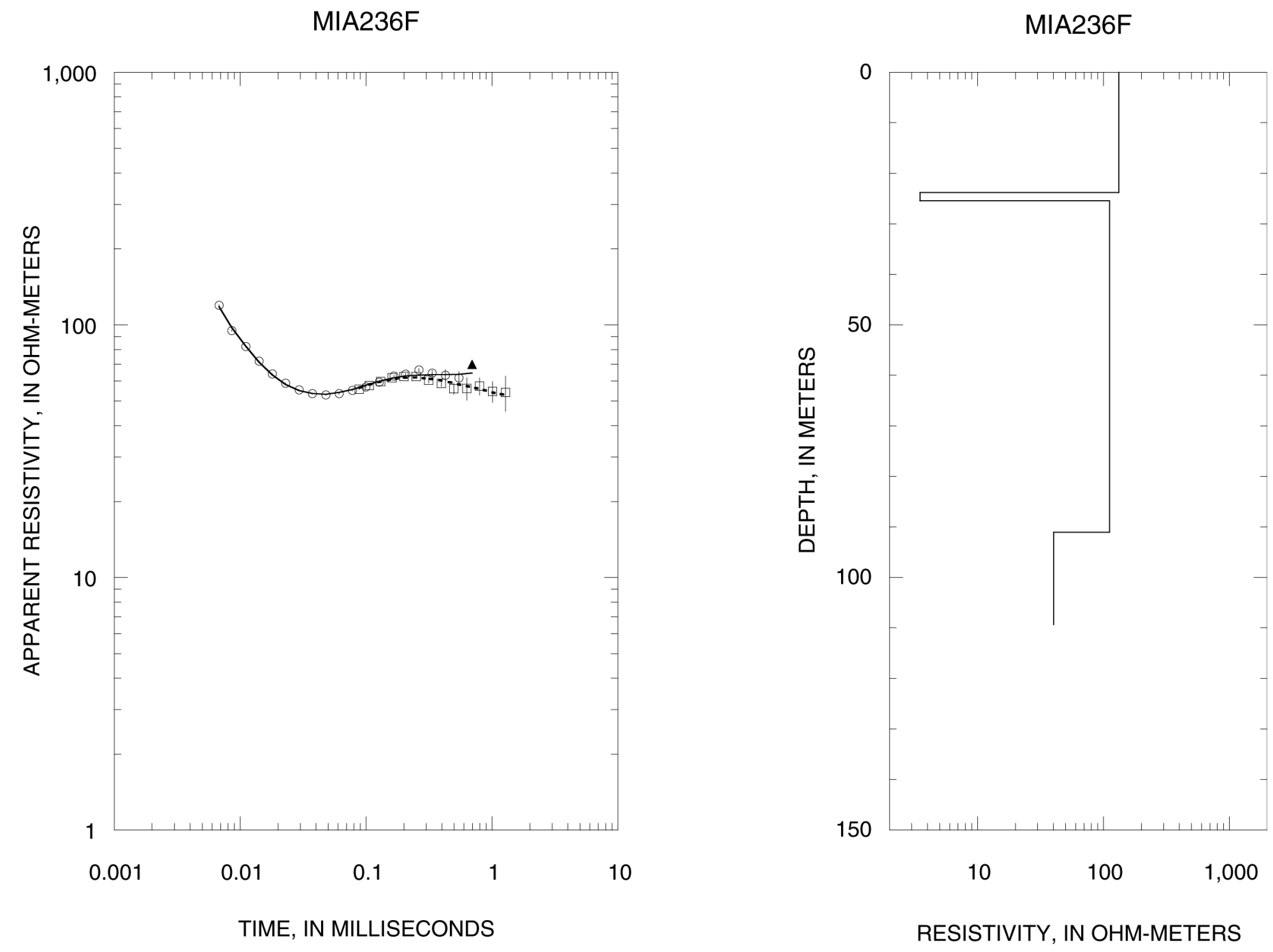

Figure 7-52. Sounding MIA236F 

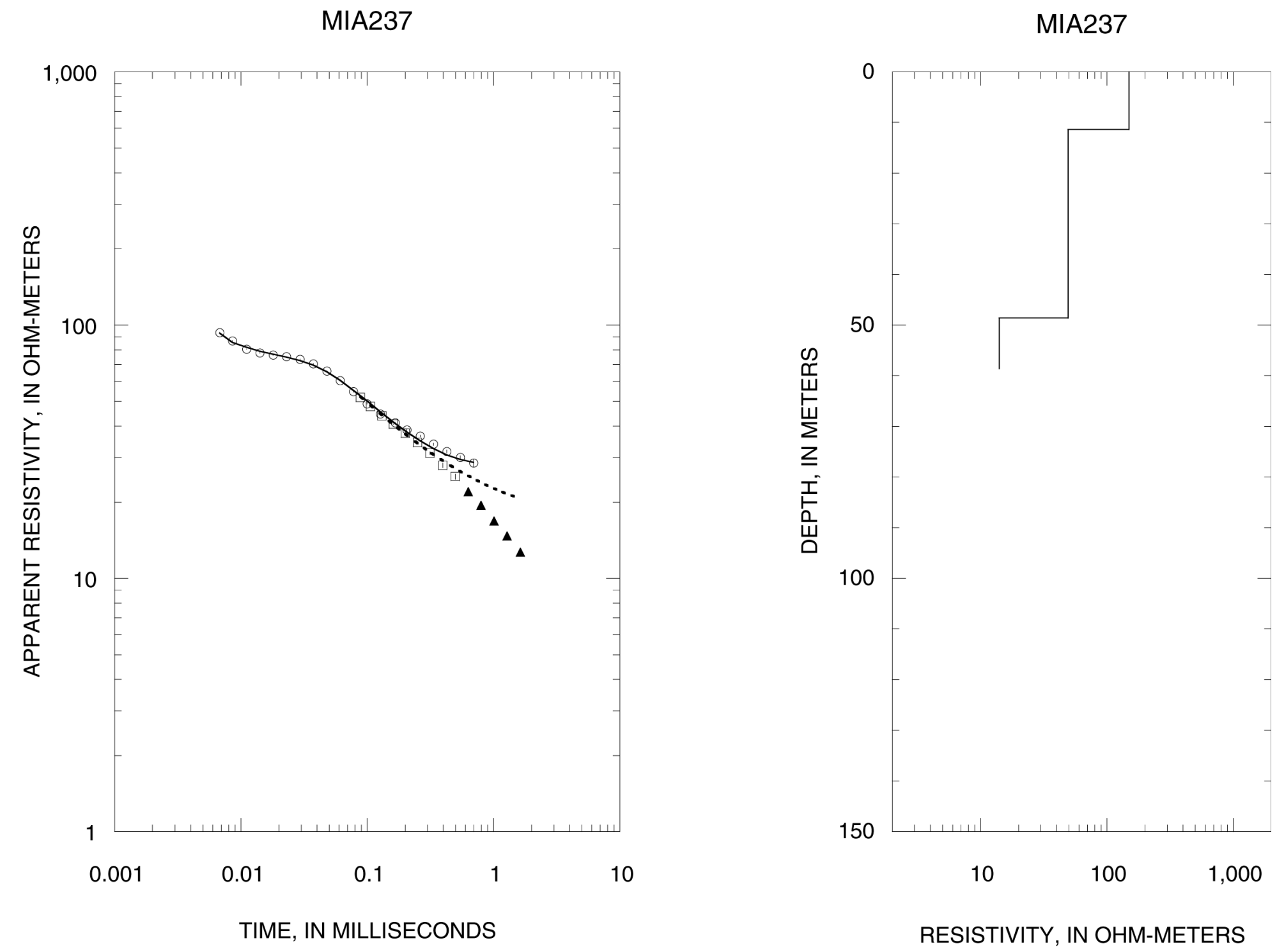

Figure 7-53. Sounding MIA237 
MIA238

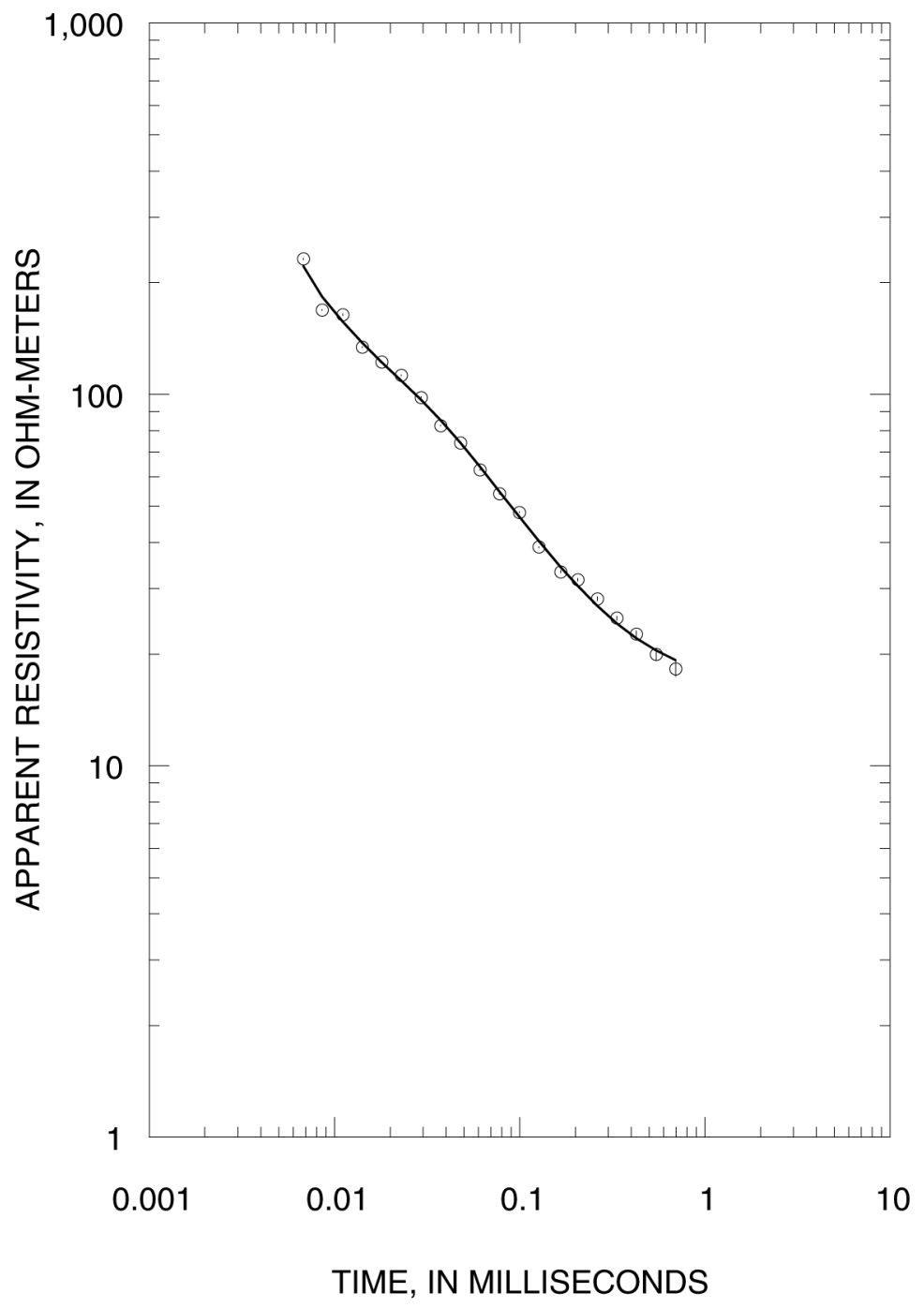

MIA238

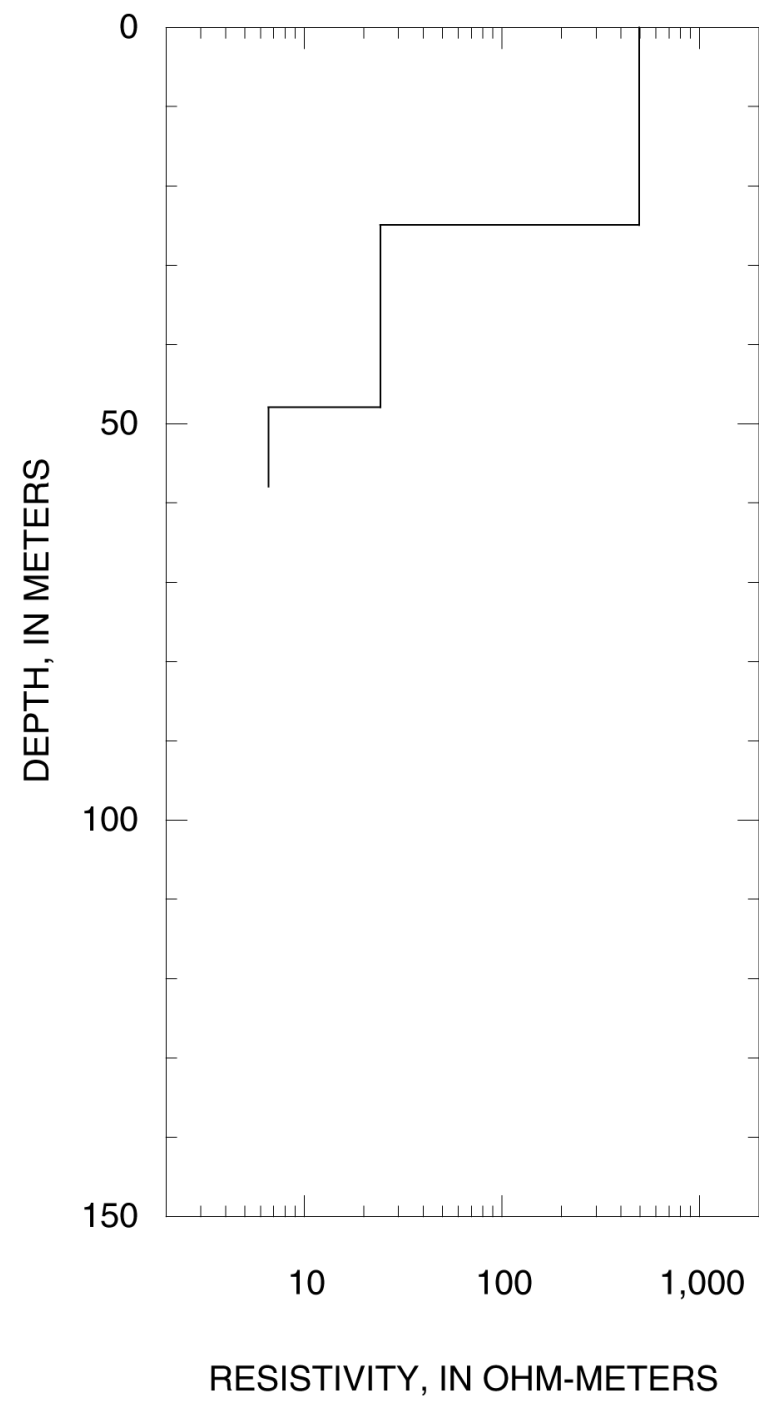

Figure 7-54. Sounding MIA238 
MIA239

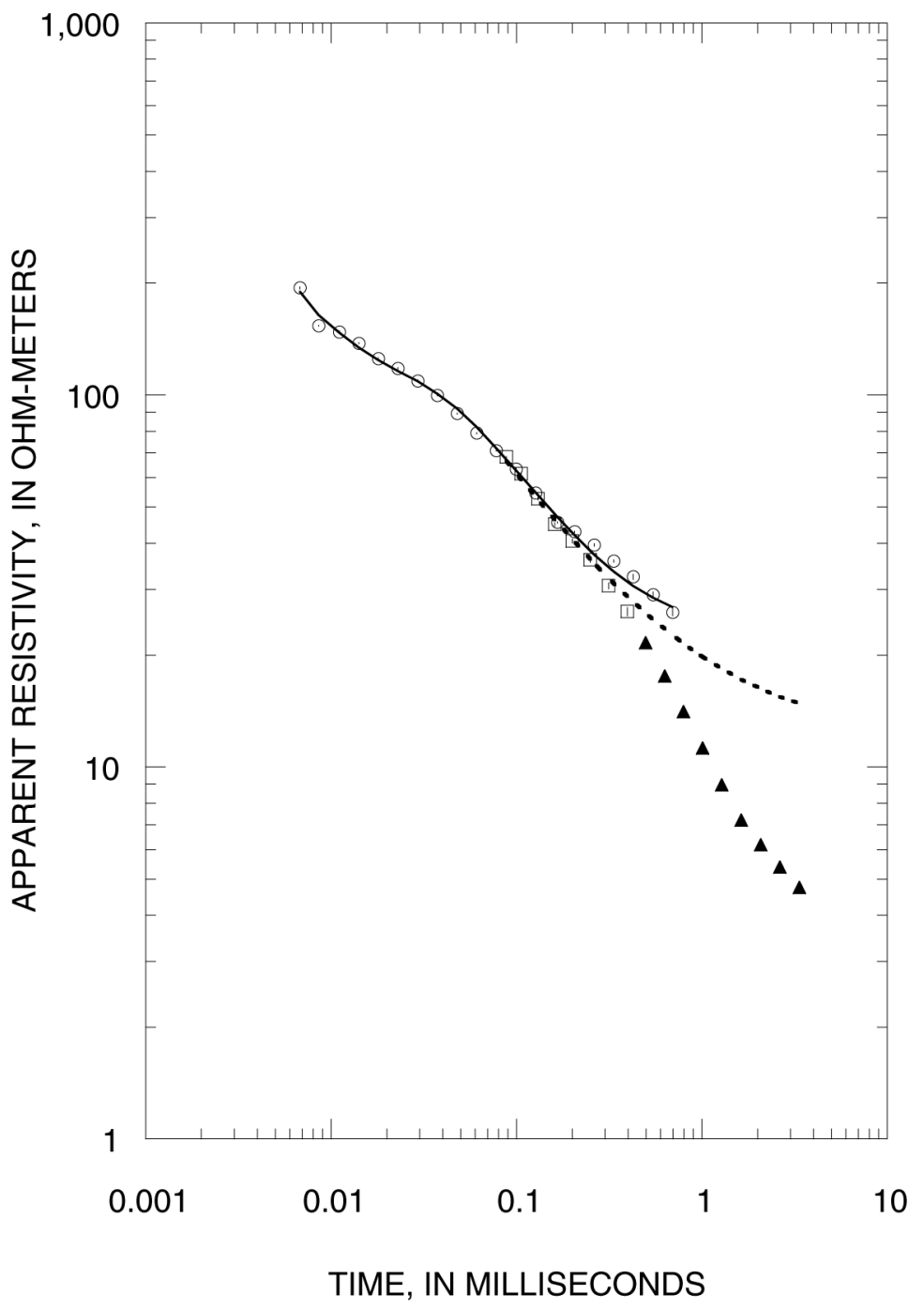

MIA239

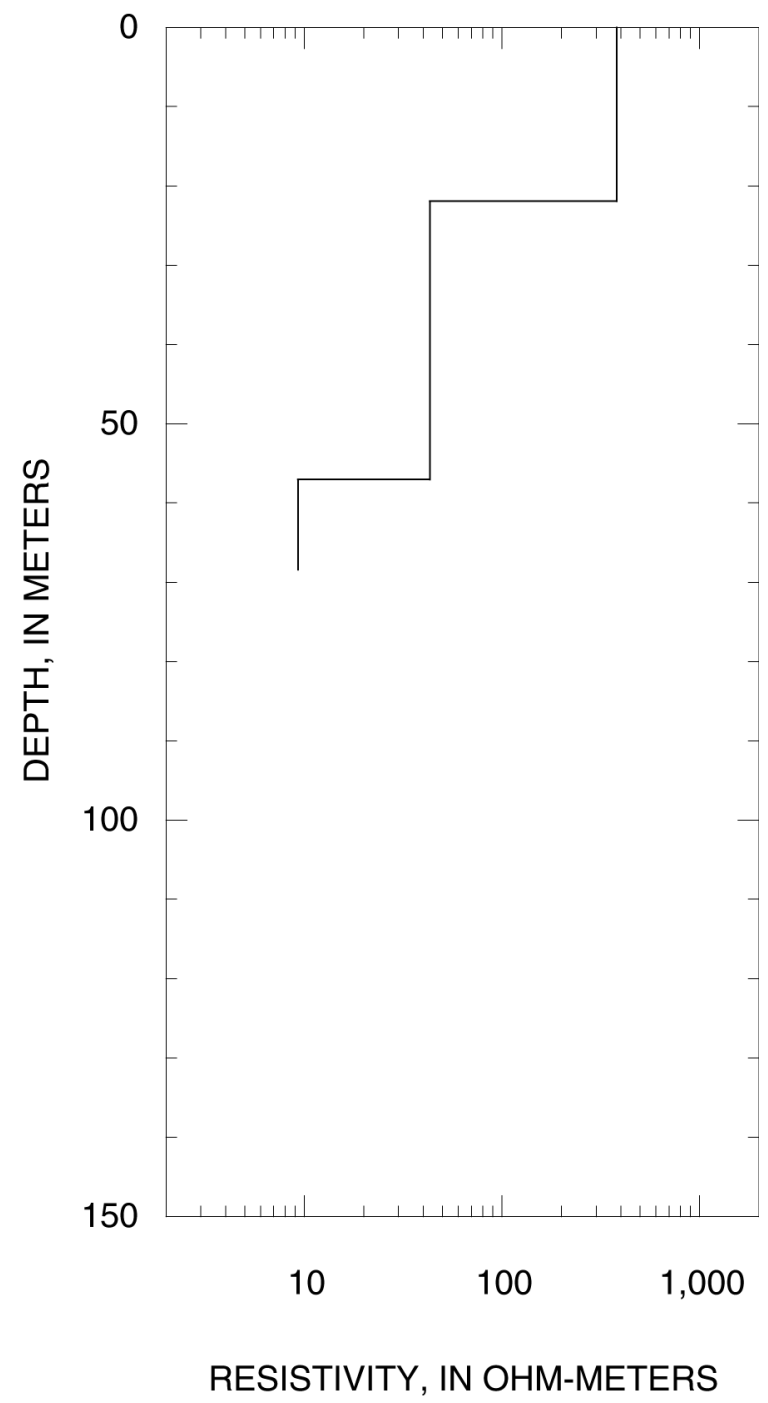

Figure 7-55. Sounding MIA239 

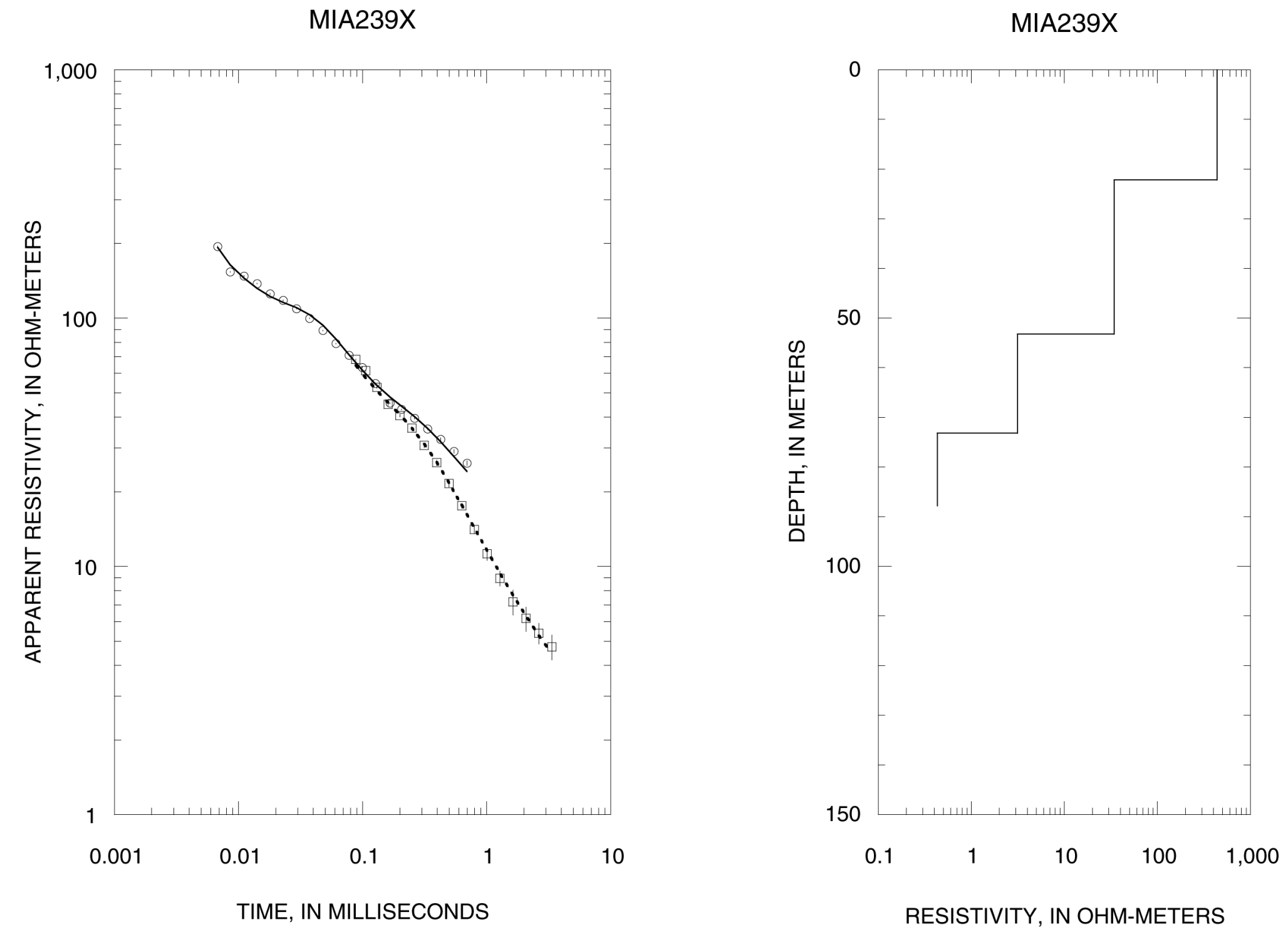

Figure 7-56. Sounding MIA239X 

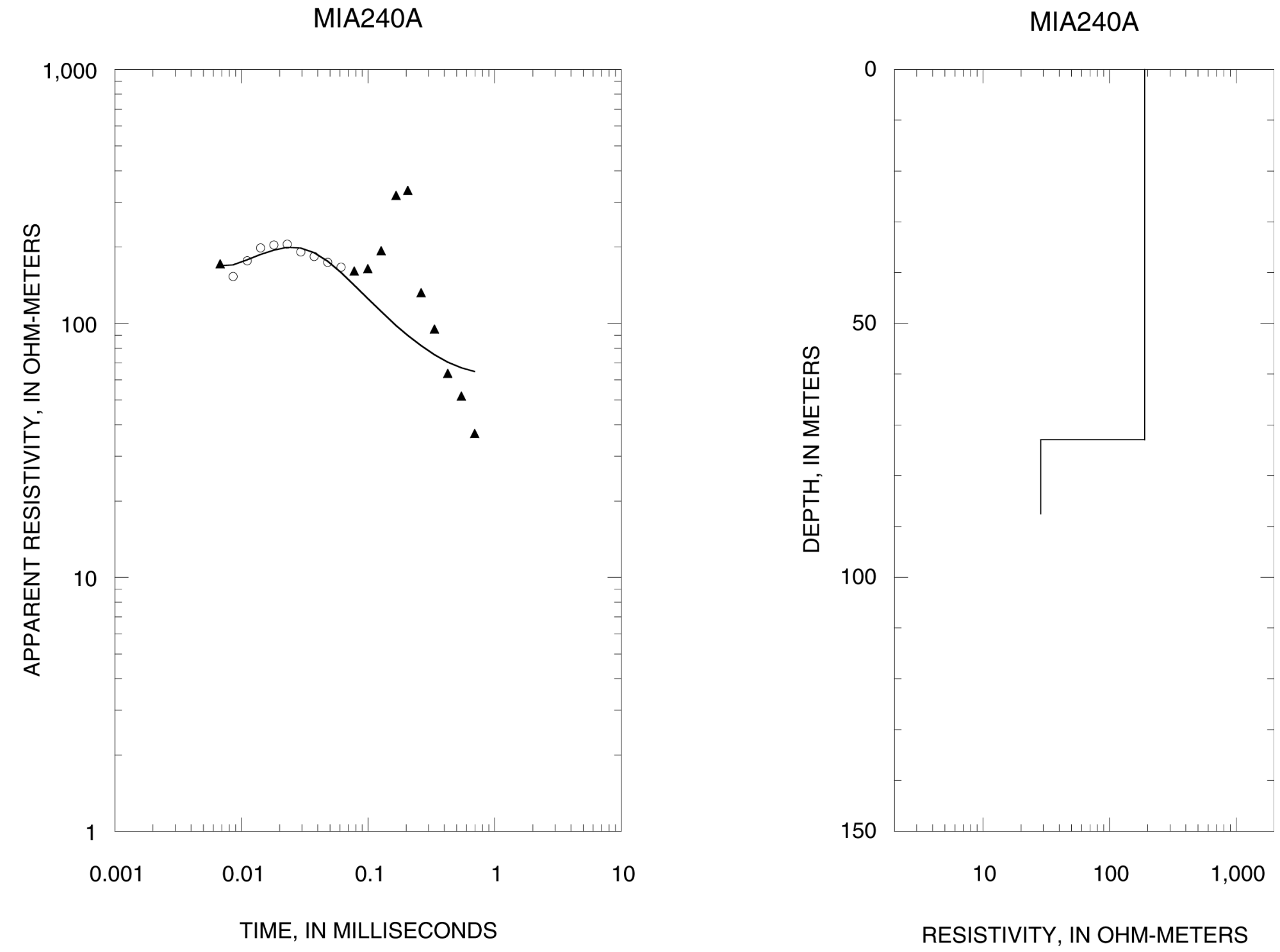

Figure 7-57. Sounding MIA240A 

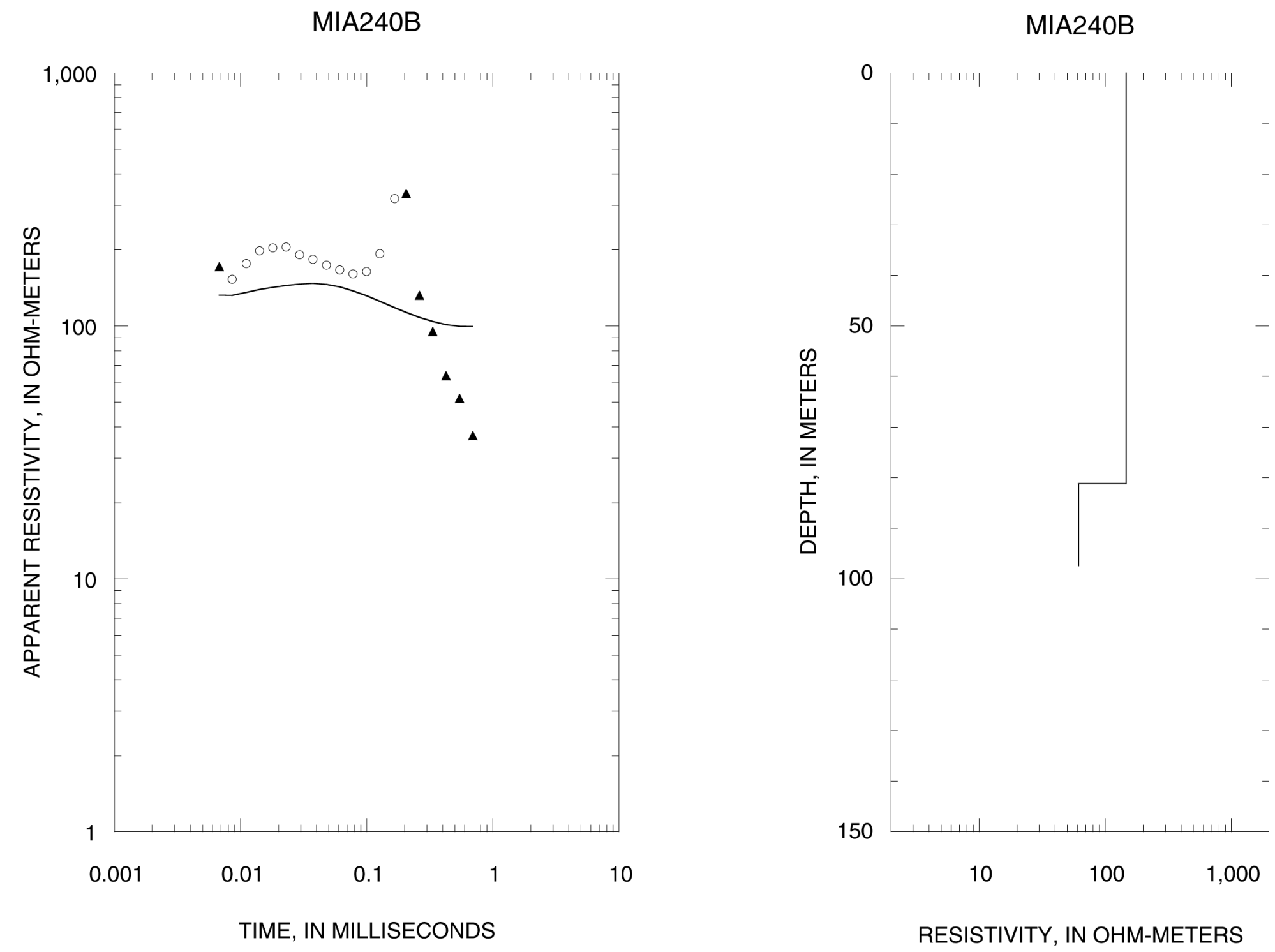

Figure 7-58. Sounding MIA240B 
MIA240C

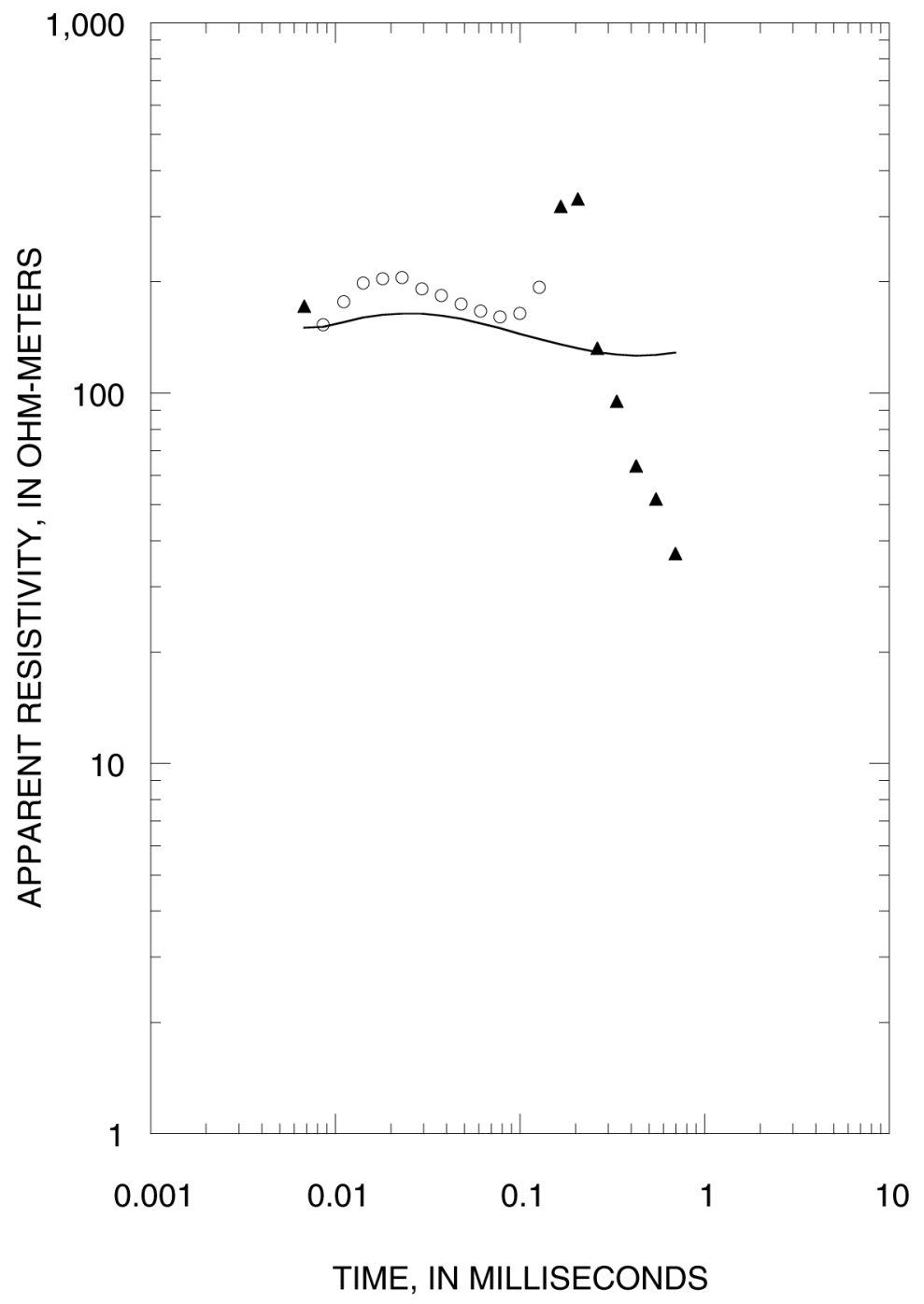

MIA240C

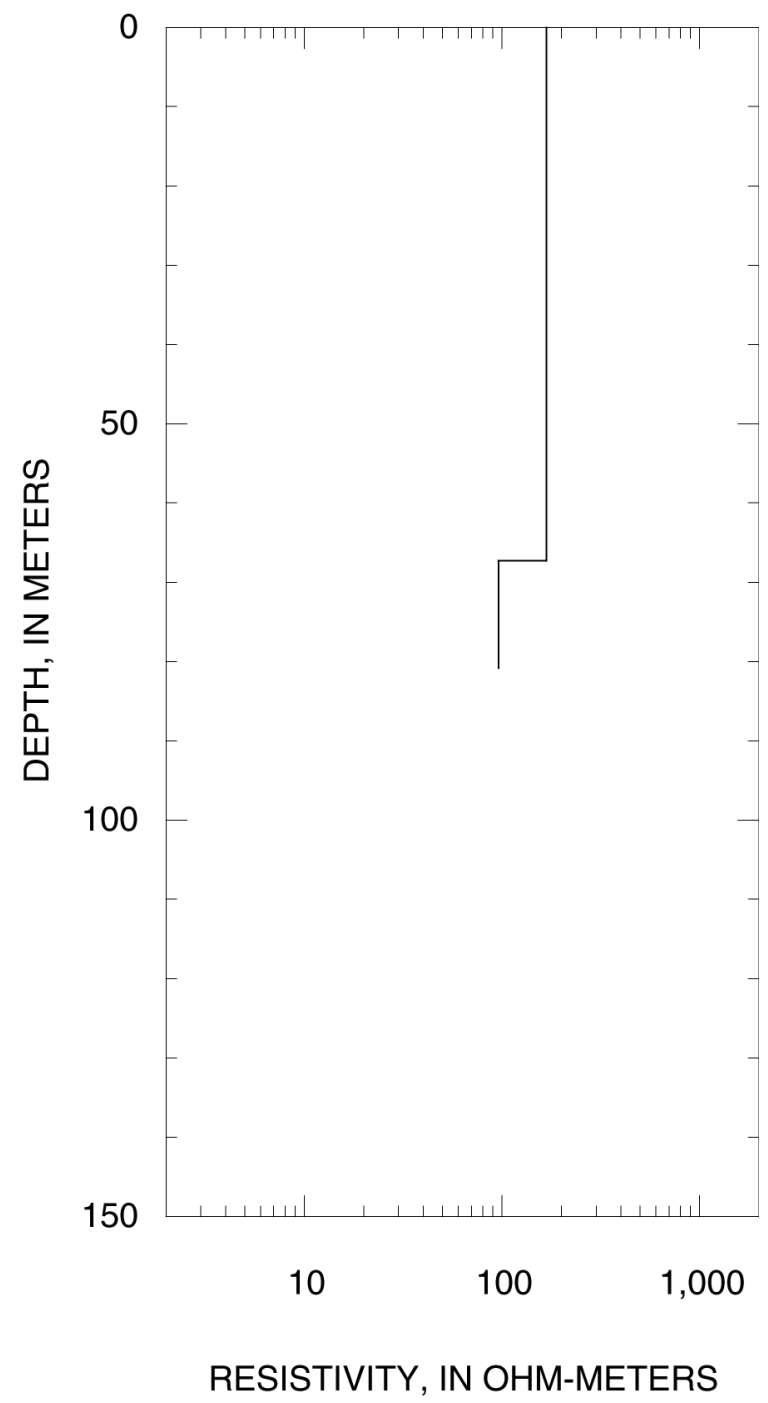

Figure 7-59. Sounding MIA240C 
MIA241

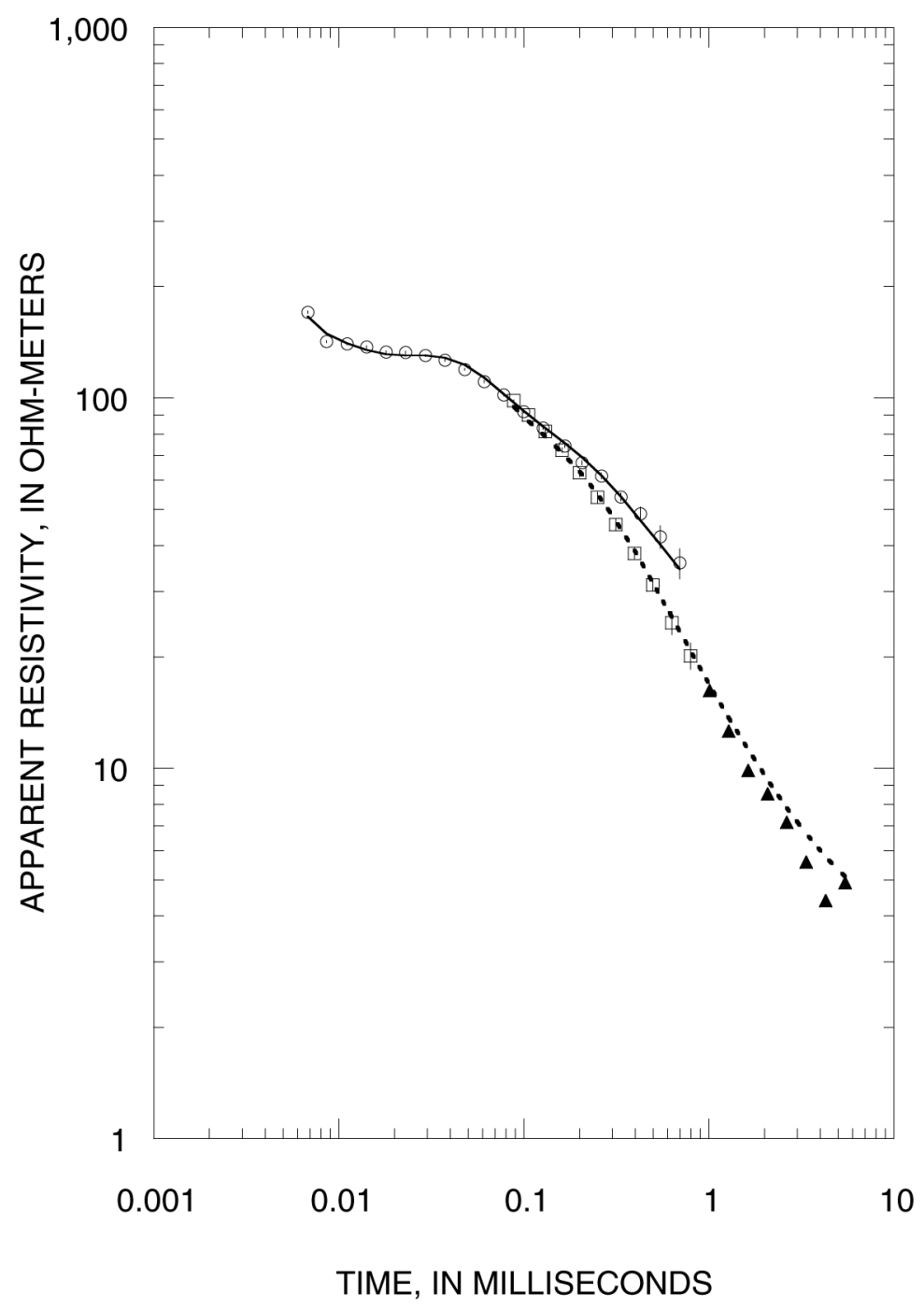

Figure 7-60. Sounding MIA241

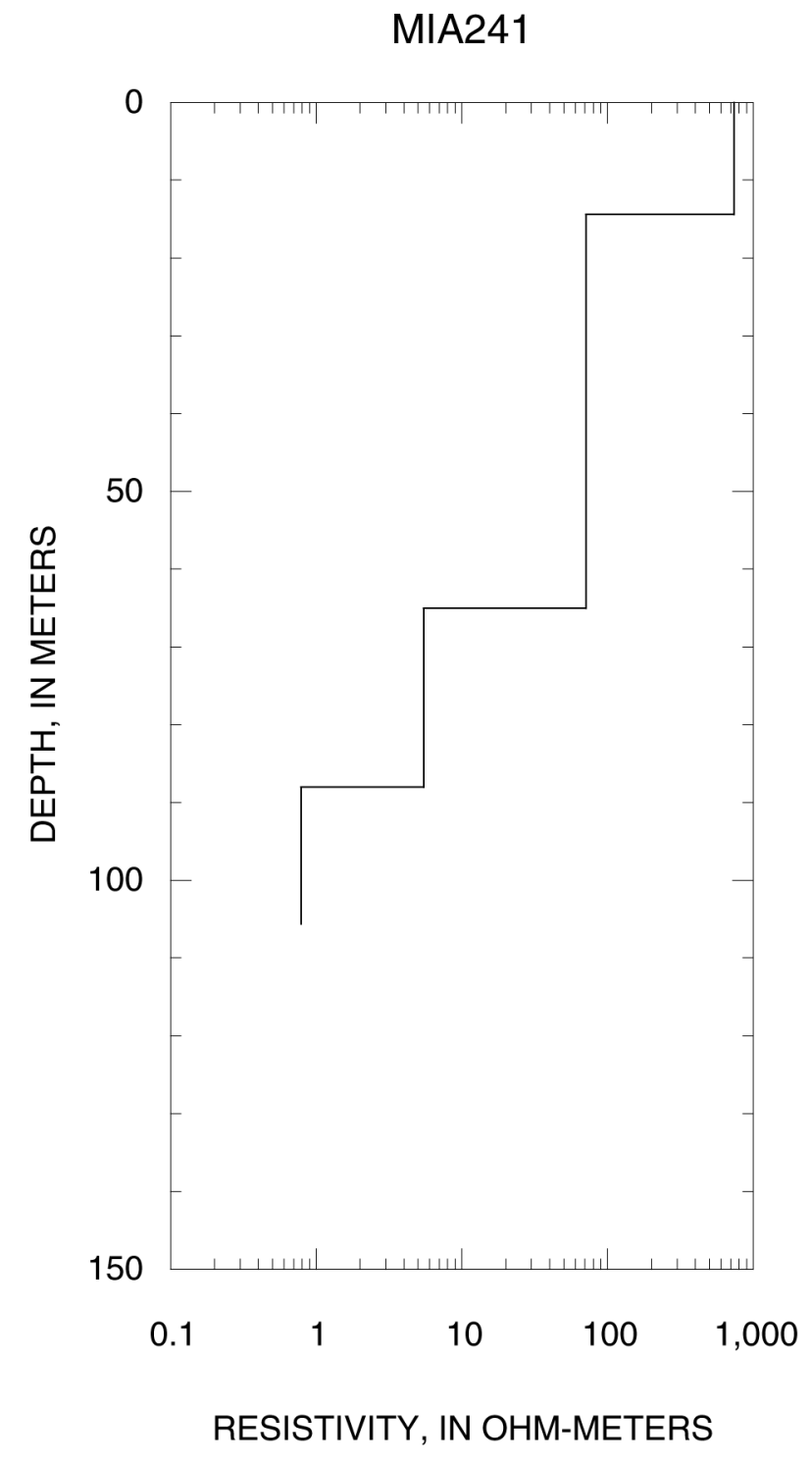



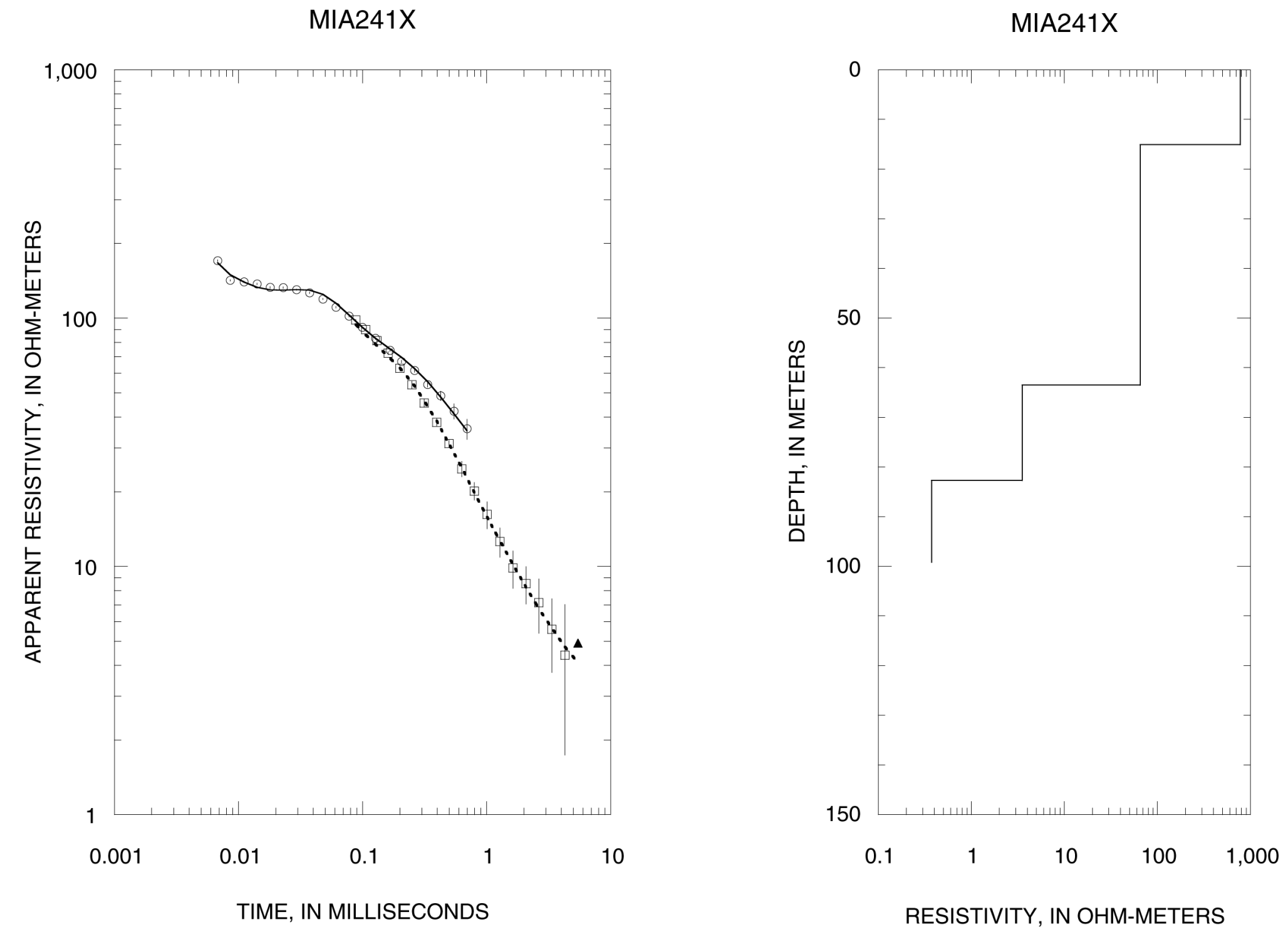

Figure 7-61. Sounding MIA241X 
MIA242

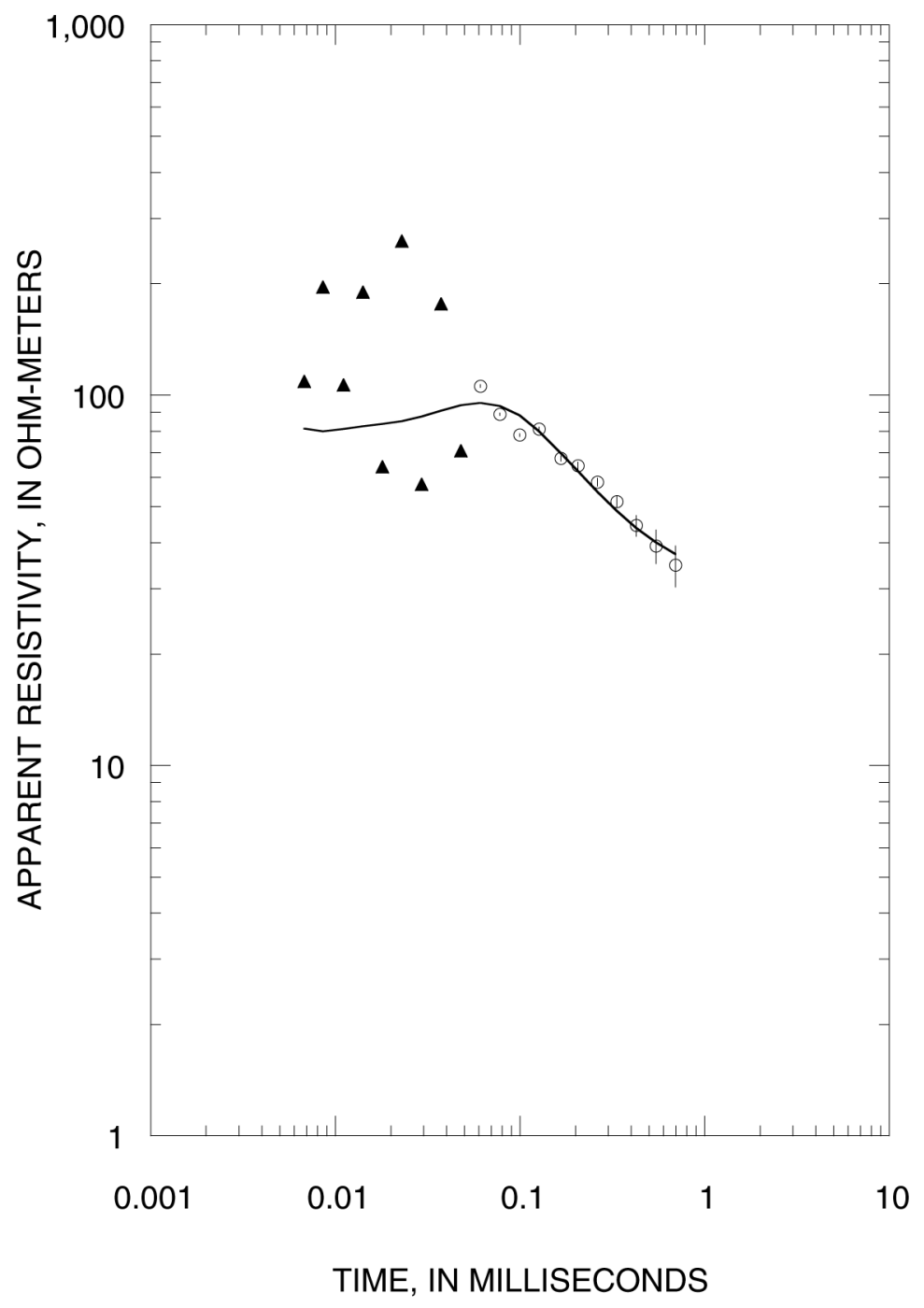

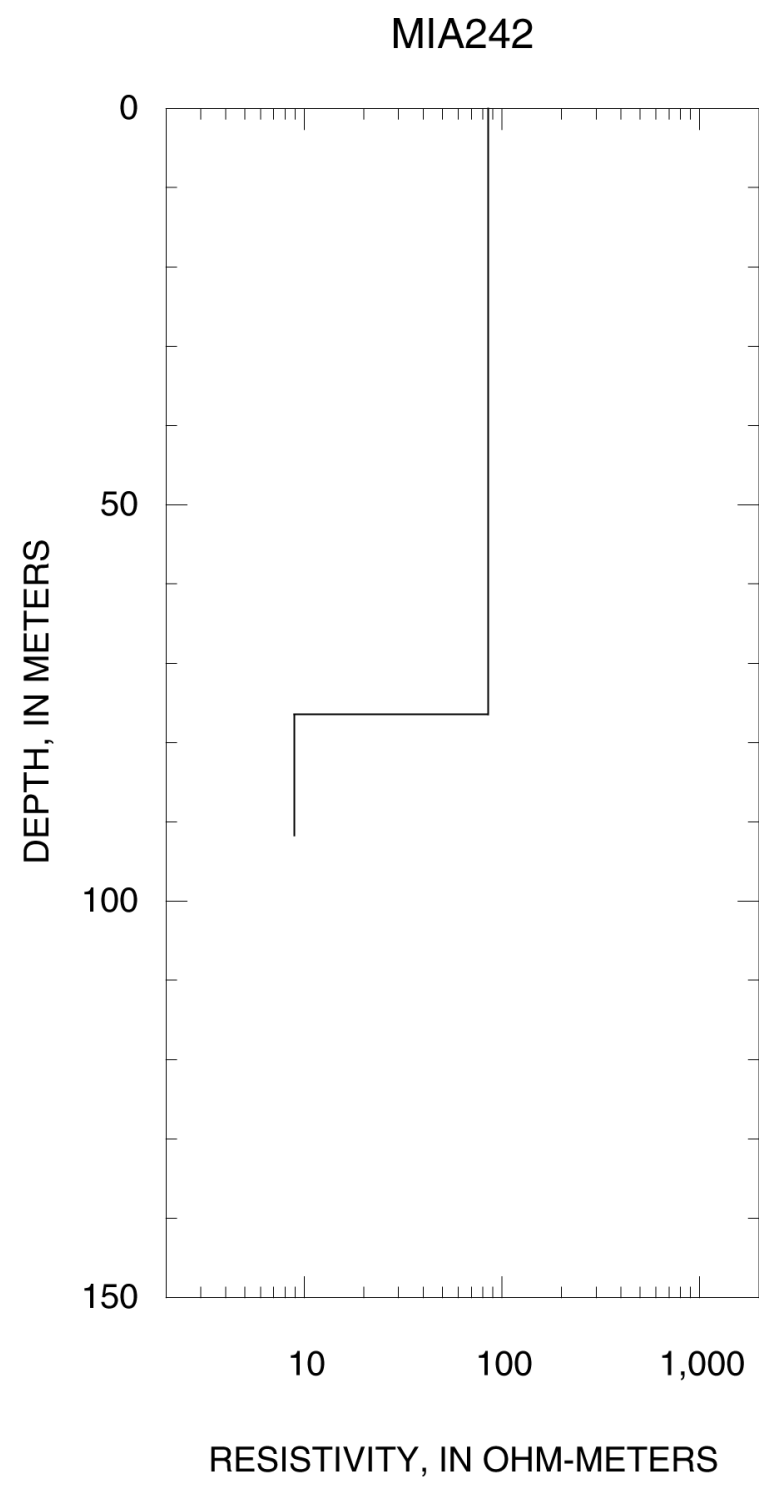

Figure 7-62. Sounding MIA242 

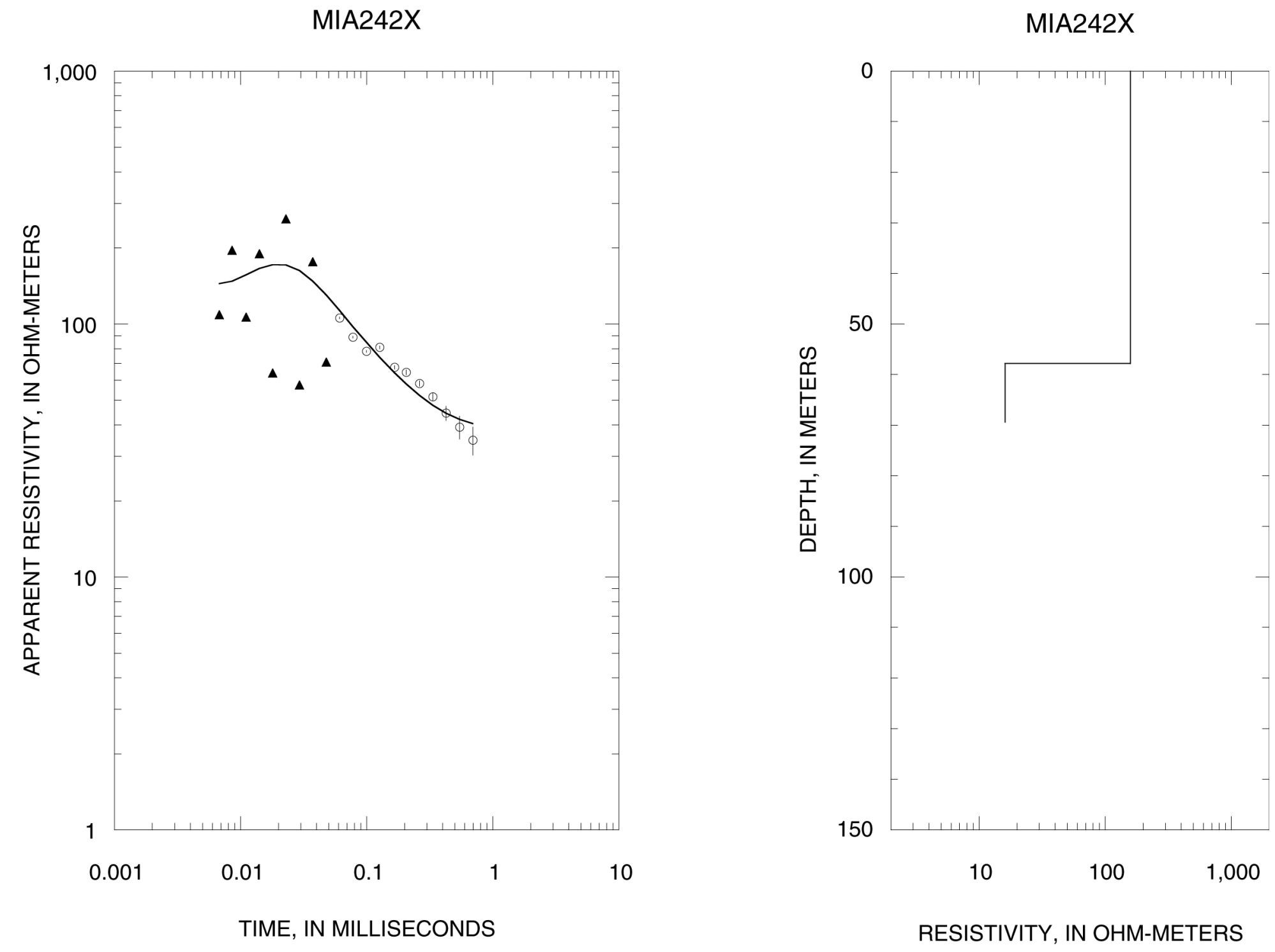

Figure 7-63. Sounding MIA242X 